\title{
Table of Nuclear Masses according to the 1994 Thomas-Fermi Model
}

\author{
W.D. Myers and W.J. Swiatecki \\ Nuclear Science Division \\ Lawrence Berkeley Laboratory \\ University of California \\ Berkeley, CA 94720
}

December 1994

This work was supported in part by the Director, Office of Energy Research, Office of High Energy and Nuclear Physics, Division of Nuclear Physics, and by the Office of Basic Energy Sciences, Division of Nuclear Sciences, of the U.S. Department of Energy under Contract No. DE-AC03-76SF00098. 


\section{DISCLAIMER}

This report was prepared as an account of work sponsored by an agency of the United States Government. Neither the United States Government nor any agency thereof, nor any of their employees, make any warranty, express or implied, or assumes any legal liability or responsibility for the accuracy, completeness, or usefulness of any information, apparatus, product, or process disclosed, or represents that its use would not infringe privately owned rights. Reference herein to any specific commercial product, process, or service by trade name, trademark, manufacturer, or otherwise does not necessarily constitute or imply its endorsement, recommendation, or favoring by the United States Government or any agency thereof. The views and opinions of authors expressed herein do not necessarily state or reflect those of the United States Government or any agency thereof. 


\section{DISCLAIMER}

Portions of this document may be illegible in electronic image products. Images are produced from the best available original document. 
This is a table of nuclear masses based on the Thomas-Fermi model developed in Ref. 1, but with parameters determined recently by fitting to nuclear masses and other nuclear properties as described in W.D. Myers \& W.J. Swiatecki, "Nuclear Properties According to the Thomas-Fermi Model", Lawrence Berkeley Laboratory preprint LBL-36557, December, 1994, submitted to Nuclear Physics A. This preprint is reproduced following the table.

The table is also available by "anonymous" ftp as pub/myers/ms-mass-table from nsdssd.lbl.gov.

The following quantities are listed in the table (all masses in $\mathrm{MeV}$ ):
Z Proton number.
$N \quad$ Neutron number.
A Mass number.
CHP Thomas-Fermi proton chemical potential.
CHN Thomas-Fermi neutron chemical potential.
TF Thomas-Fermi binding energy.
SHL Shell effect taken from Ref. 2, Table 1 , for $\mathrm{N}, \mathrm{Z} \leq 29$ and
from Ref.3, column $E_{\text {mic }}$, for the remaining nuclei.

EVOD Even-odd correction based on Ref. 3 viz.,
$4.8 / \mathrm{N}^{1 / \beta}+4.8 / \mathrm{Z}^{1 / 3}-6.6 / \mathrm{A}^{2 / 3}+30 / \mathrm{A}$
$\mathrm{N}=\mathrm{Z}$, odd
$4.8 / \mathrm{N}^{1 / 3}+4.8 / \mathrm{Z}^{1 / \beta}-6.6 / \mathrm{A}^{2 / 3}$
$\mathrm{N}$ and $\mathrm{Z}$ odd
$4.8 / \mathrm{Z}^{1 / 3}$
$N$ even, $Z$ odd
$4.8 / \mathrm{N}^{1 / 3}$
Nodd $Z$ even
0
$\mathrm{N}$ and $\mathrm{Z}$ even

CONG Congruence energy:

$\mathrm{CONG}=-10 \exp (-42 \mid \mathrm{I} / / 10)$, where $\mathrm{I}=(\mathrm{N}-\mathrm{Z}) / \mathrm{A}$

$\mathrm{BE}, \mathrm{TH}$ Theoretical binding energy:

$\mathrm{BE}, \mathrm{TH}=\mathrm{TF}+\mathrm{SHL}+\mathrm{EVOD}+\mathrm{CONG}$

M,TH Theoretical atomic mass (excess):

$$
\begin{aligned}
& M, T H=B E, T H+N \cdot M_{n}+Z \cdot M_{H}-0.00001433 \cdot Z^{2.39} \text {, } \\
& \text { where } M_{n}=8.071431 \text { and } M_{H}=7.289034
\end{aligned}
$$

M,EX Experimental atomic mass from Ref. 3 (and Ref. 4 for $N, Z<8$ ).

"SHL" "Experimental" shell effect i.e., what the shell effect would have to be to ensure exact agreement with experiment: "SHL"= SHL+(M,EX - M,TH)

EX-TH Difference between experimental and theoretical masses:

$\mathrm{EX}-\mathrm{TH}=\mathrm{M}, \mathrm{EX}-\mathrm{M}, \mathrm{TH}$.

\section{References}

1. W.D. Myers and W.J. Swiatecki, Ann. of Phys. 204 (1990) 401.

2. W.D. Myers and W.J. Swiatecki, Nucl. Phys. 81 (1966) 1.

3. P. Möller, J.R. Nix, W.D. Myers and W.J. Swiatecki, "Nuclear Ground State Masses and Deformations," Los Alamos preprint LA-UR-93-3083, Aug. 16,1993, to be published in the Atomic Data and Nuclear Data Tables.

4. G. Audi and A.H. Wapstra, Nucl. Phys. A565 (1993) 1. 
$$
1
$$

$$
\begin{array}{rrr}
2 & -4.8 & -5.5 \\
3 & -12.7 & -1.0 \\
4 & 0.0 & 0.0 \\
5 & 0.0 & 0.0 \\
6 & 0.0 & 0.0 \\
7 & 0.0 & 0.0 \\
8 & 0.0 & 0.0 \\
9 & 0.0 & 0.0 \\
3 & 0.1-13.1 \\
4 & -6.2 & -7.5
\end{array}
$$$$
5-11.8-3.7
$$$$
6-16.1-1.4
$$$$
7-19.1-0.2
$$$$
8 \quad 0.0 \quad 0.0
$$$$
\begin{array}{lll}
9 & 0.0 & 0.0
\end{array}
$$$$
\begin{array}{lll}
10 & 0.0 & 0.0
\end{array}
$$$$
\begin{array}{lll}
11 & 0.0 & 0.0
\end{array}
$$$$
\begin{array}{lll}
12 & 0.0 & 0.0
\end{array}
$$$$
\begin{array}{lll}
4 & 0.0 & 0.0
\end{array}
$$$$
5-1.9-13.1
$$$$
\begin{array}{llll}
6 & -6.7 & -8.6
\end{array}
$$$$
7-11.2-5.4
$$$$
8-14.8-3.2
$$$$
9-17.9-1.6
$$$$
10-20.2-0.5
$$$$
\begin{array}{lll}
11 & 0.0 & 0.0
\end{array}
$$$$
\begin{array}{lll}
12 & 0.0 & 0.0
\end{array}
$$$$
\begin{array}{lll}
13 & 0.0 & 0.0
\end{array}
$$$$
140.0 \quad 0.0
$$$$
\begin{array}{lll}
15 & 0.0 & 0.0
\end{array}
$$$$
5 \quad 0.0 \quad 0.0
$$$$
60.6-17.1
$$$$
7-3.1-13.0
$$$$
\text { 8. }-7.0-9.3
$$$$
9-10.6-6.6
$$$$
10-13.8-4.5
$$$$
11-16.6-2.9
$$$$
12-18.9-1.7
$$$$
13-20.9-0.8
$$$$
14-22.4-0.1
$$$$
\begin{array}{lll}
15 & 0.0 & 0.0
\end{array}
$$$$
\begin{array}{lll}
16 & 0.0 & 0.0
\end{array}
$$$$
\begin{array}{lll}
17 & 0.0 & 0.0
\end{array}
$$$$
\begin{array}{lll}
18 & 0.0 & 0.0
\end{array}
$$$$
\begin{array}{lll}
6 & 0.0 & 0.0
\end{array}
$$$$
\begin{array}{lll}
7 & 0.0 & 0.0
\end{array}
$$$$
8-0.6-16.5
$$$$
9-3.8-12.9
$$$$
\begin{array}{lll}
10 & -7.1 & -9.9
\end{array}
$$$$
11-10.2-7.4
$$$$
12-13.0-5.5
$$$$
13-15.5-4.0
$$$$
\text { 14-17.7 -2.7 }
$$$$
15-19.6-1.8
$$$$
16-21.3-1.0
$$$$
17-22.7-0.3
$$$$
\begin{array}{lll}
18 & 0.0 & 0.0
\end{array}
$$$$
\begin{array}{lll}
19 & 0.0 & 0.0
\end{array}
$$$$
20 \quad 0.0 \cdot 0.0
$$$$
210.0 \cdot 0.0
$$$$
\begin{array}{lll}
7 & 0.0 & 0.0
\end{array}
$$$$
80.0 \quad 0.0
$$$$
91.2-19.2
$$

$10-1.4-16.0$
$-6.10-1.7820 .4-10.00$

$\begin{array}{llllll}-9.00 & -1.59 & 4.80 & -2.47\end{array}$

$\begin{array}{lllll}0.00 & -0.40 & 5.51 & -1.22\end{array}$

$0.00 \quad 0.00 \quad 4.80-0.80$

$\begin{array}{lllll}0.00 & 1.23 & 5.61 & -0.61\end{array}$

$0.00-0.88 \quad 4.80-0.50$

$\begin{array}{lllll}0.00 & -1.32 & 5.66 & -0.43\end{array}$

$\begin{array}{lllll}0.00 & -0.66 & 4.80 & -0.38\end{array}$

$\begin{array}{llll}-8.01 & -1.59 \cdot 4.80 & -2.47\end{array}$

$-18.29-1.510 .00-10.00$

$\begin{array}{llll}-23.71 & -0.51 & 3.33 & -4.32\end{array}$

$\begin{array}{lllll}-26.18 & -0.14 & 0.00 & -2.47\end{array}$

$\begin{array}{lllll}-26.91 & 0.98 & 2.81 & -1.65\end{array}$

$\begin{array}{lllll}0.00 & -0.93 & 0.00 & -1.22\end{array}$

$\begin{array}{lllll}0.00 & -1.33 & 2.51 & -0.97\end{array}$

$0.00-0.72 \quad 0.00-0.80$

$\begin{array}{lllll}0.00 & 1.50 & 2.31 & -0.69\end{array}$

$\begin{array}{lllll}0.00 & 1.61 & 0.00 & -0.61\end{array}$

$\begin{array}{lllll}0.00 & -0.40 & 5.51 & -1.22\end{array}$

$\begin{array}{lllll}-22.14 & -0.51 & 3.33 & -4.32\end{array}$

$-32.88 \quad 0.249 .66-10.00$

$\begin{array}{lllll}-39.79 & 0.50 & 3.33 & -5.49\end{array}$

$\begin{array}{lllll}-44.00 & 1.47 & 4.49 & -3.50\end{array}$

$\begin{array}{lllll}-46.34 & -0.33 & 3.33 & -2.47\end{array}$

$\begin{array}{lllll}-47.39 & -0.75 & 4.42 & -1.86\end{array}$

$\begin{array}{lllll}0.00 & -0.21 & 3.33 & -1.48\end{array}$

$\begin{array}{lllll}0.00 & 1.85 & 4.38 & -1.22\end{array}$

$0.00 \quad 1.94 \quad 3.33-1.04$

$0.00 \quad 1.98 \quad 4.35-0.91$

$0.00 \quad 1.19 \quad 3.33-0.80$

$\begin{array}{lllll}0.00 & 0.00 & 4.80 & -0.80\end{array}$

$\begin{array}{lllll}-22.68 & -0.14 & 0.00 & -2.47\end{array}$

$\begin{array}{lllll}-37.70 & 0.50 & 3.33 & -5.49\end{array}$

$\begin{array}{lll}-48.78 & 0.71 & 0.00-10.00\end{array}$

$\begin{array}{lllll}-56.68 & 1.60 & 2.81 & -6.27\end{array}$

$\begin{array}{lllll}-62.16 & -0.09 & 0.00 & -4.32\end{array}$

$\begin{array}{lllll}-65.82 & -0.49 & 2.51 & -3.18\end{array}$

$\begin{array}{lllll}-68.10 & 0.00 & 0.00 & -2.47\end{array}$

$\begin{array}{lllll}-69.33 & 1.94 & 2.31 & -1.99\end{array}$

$\begin{array}{lllll}-69.77 & 2.02 & 0.00 & -1.65\end{array}$

$0.00 \quad 2.06 \quad 2.16-1.41$

$0.00 \quad 1.30 \quad 0.00-1.22$

$\begin{array}{lllll}0.00 & 1.47 & 2.04 & -1.08\end{array}$

$\begin{array}{lllll}0.00 & 0.03 & 0.00 & -0.97\end{array}$

$\begin{array}{lllll}0.00 & 1.23 & 5.61 & -0.61\end{array}$

$\begin{array}{lllll}0.00 & 0.98 & 2.81 & -1.65\end{array}$

$\begin{array}{lllll}-39.47 & 1.47 & 4.49 & -3.50\end{array}$

$\begin{array}{lllll}-54.14 & 1.60 & 2.81 & -6.27\end{array}$

$\begin{array}{lll}-65.49 & 2.367 .19-10.00\end{array}$

$\begin{array}{lllll}-74.11 & 0.74 & 2.81 & -6.83\end{array}$

$-80.55$

$0.314 .06-4.97$

$-85.26$

$\begin{array}{llll}0.73 & 2.81 & -3.79\end{array}$

$-88.60$

$2.55 \quad 3.98-3.01$

$\begin{array}{lllll}-90.83 & 2.60 & 2.81 & -2.47\end{array}$

$-92.18$

$\begin{array}{llll}2.62 & 3.93 & -2.07\end{array}$

$-92.82$

0.00

$\begin{array}{llll}1.86 & 2.81 & -1.77\end{array}$

$0.00 \quad 0.602 .81-1.37$

0.00

$0.59 \quad 3.86-1.22$

$0.00 \quad 1.15 \quad 2.81-1.11$

$0.00-0.88 \quad 4.80-0.50$

$0.00-0.93 \quad 0.00-1.22$

$\begin{array}{lllll}-39.11 & -0.33 & 3.33 & -2.47\end{array}$

$\begin{array}{llll}-56.72 & -0.09 & 0.00 & -4.32\end{array}$
$-8.25$

0.00

0.00

0.00

0.00

0.00

0.00

$-7.27$

$-29.80$

$-25.22$

$-28.79$

$-24.78$

0.00

0.00

0.00

0.00

0.00

0.00

$-23.64$

$-32.99$

$-41.45$

$-41.54$

$-45.81$

$-45.59$

0.00

0.00

0.00

0.00

0.00

0.00

$-25.29$

$-39.37$

$-58.08$

$-58.55$

$-66.56$

$-66.98$

$-70.56$

$-67.07$

$-69.40$

0.00

0.00

0.00

0.00

0.00

0.00

$-37.02$

$-56.01$

$-65.93$

$-77.38$

$-81.15$

$-85.52$

$-85.08$

$-87.89$

$-87.71$

$-89.93$

0.00

0.00

0.00

0.00

0.00

0.00

$-38.58$

$-61.13$

17. 92

15.18

0.00

0.00

0.00

0.00

0.00

0.00

15. 38

0.92

13.57

18.08

30.16

0.00

0.00

0.00

0.00

0.00

0.00

14.37

13.09

12.70 
$11-4.3-12.9$

$$
12-7.1-10.3
$$$$
\begin{array}{lll}
13 & -9.8-8.1
\end{array}
$$

$$
17-13.7-5.6-124.04
$$

$$
18-15.6-4.4-129.03
$$

$19-17.4-3.4-132.92$

$\begin{array}{llll}0.86 & 3.78 & -5.92 & -119.03\end{array}$ $\begin{array}{lllll}0.98 & 2.51 & -4.77 & -125.31\end{array}$ $\begin{array}{llll}1.06 & 3.71 & -3.93 & -128.19\end{array}$ $0.42 \quad 2.51-3.31-133.31$ $\begin{array}{lllllll}20-19.0 & -2.5 & -135.88 & 0.61 & 3.65 & -2.84 & -134.46\end{array}$ $\begin{array}{llllllll}21-20.4 & -1.8 & -138.04 & -0.67 & 2.51 & -2.47 & -138.66\end{array}$

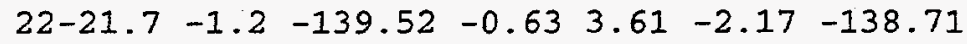
$\begin{array}{lllllll}23-22.9 & -0.6 & -140.43 & -0.07 & 2.51 & -1.93 & -139.92\end{array}$ $24-23.9-0.2-140.85$ $\begin{array}{llll}25 & 0.0 & 0.0 & 0.00\end{array}$ $\begin{array}{lll}26 & 0.0 & 0.0\end{array}$

$\begin{array}{lll}27 & 0.0 & 0.0\end{array}$

$\begin{array}{lll}11 & 0.0 & 0.0\end{array}$

$\begin{array}{lll}12 & 0.0 & 0.0\end{array}$

0.00

8.81

$13-0.2-18.0$

0.00

$\begin{array}{llll}0.77 & 3.56 & -1.44\end{array}$

0.00

0.00

$\begin{array}{llll}0.68 & 2.51 & -1.32\end{array}$

0.00

$-75.38$

$0.00 \quad 0.00-2.47$

0.00

0.00

$14-2.5-15.4$

$\begin{array}{llll}0.73 & 2.81 & -3.79\end{array}$

$-75.63$

$15-4.8-13.0-106.19$

$17-9.1-9.1-128.13$

$19-13.0-6.2-143.35$

$20-14.8-5.1-148.99$

$21-16.4-4.1-153.57$

$22-18.0-3.2-157.21$

$\begin{array}{lll}23-19.4 & -2.5 & -160.05 \\ 24-20.6 & -1.8 & -162.18\end{array}$

$25-21.8-1.2-163.70$

$26-22.9-0.8-164.70$

$27-23.8-0.3-165.24$

$\begin{array}{lll}28 & 0.0 & 0.0\end{array}$

0.00

$\begin{array}{lll}29 & 0.0 & 0.0 \\ 30 & 0.0 & 0.0\end{array}$

0.00

0.00

0.00

$\begin{array}{lll}31 & 0.0 & 0.0 \\ 32 & 0.0 & 0.0\end{array}$

0.00

$$
0.00
$$

$$
\begin{aligned}
& 0.00 \\
& 0.00 \\
& 0.00
\end{aligned}
$$

0.00

$0.0 \quad 0.0$

$-74.86$

$-74.86$

$\begin{array}{llll}-0.56 & 0.00 & -5.49\end{array}$

$-98.08$

$\begin{array}{llll}-0.87 & 2.51 & -7.56 & -112.11\end{array}$

$\begin{array}{lll}-0.45 & 0.00-10.00 & -128.57\end{array}$

$\begin{array}{lllll}1.19 & 2.31 & -7.81 & -132.44\end{array}$

$\begin{array}{lllll}1.30 & 0.00 & -6.27 & -141.43\end{array}$

$\begin{array}{lllll}1.36 & 2.16 & -5.15 & -144.98\end{array}$

$\begin{array}{lllll}0.73 & 0.00 & -4.32 & -152.58\end{array}$

$\begin{array}{lllll}0.90 & 2.04 & -3.68 & -154.30\end{array}$

$\begin{array}{lllll}-0.34 & 0.00 & -3.18 & -160.73\end{array}$

$\begin{array}{lllll}-0.31 & 1.95 & -2.79 & -161.20\end{array}$

$\begin{array}{lllll}0.23 & 0.00 & -2.47 & -164.42\end{array}$

$\begin{array}{lllll}0.48 & 1.87 & -2.20 & -163.56\end{array}$

$\begin{array}{lllll}0.81 & 0.00 & -1.99 & -165.88\end{array}$

$\begin{array}{lllll}1.02 & 1.80 & -1.81 & -164.23\end{array}$

$0.930 .00-1.65$

0.00

$1.92 \quad 1.74-1.52$

0.00

$1.94 \quad 0.00-1.41$

$1.54 \quad 1.69-1.31$

0.00

0.00

$\begin{array}{llll}0.97 & 0.00 & -1.22\end{array}$

0.00

$0.37 \quad 1.64-1.15$

0.00

0.00

$\begin{array}{lll}0.24 & 0.00 & -1.08 \\ 1.85 & 4.38 & -1.22\end{array}$

$1.942 .31-1.99$

$\begin{array}{llll}2.55 & 3.98 & -3.01\end{array}$

$\begin{array}{llll}1.23 & 2.31 & -4.32\end{array}$

0.00

0.00

$\begin{array}{lllll}0.86 & 3.78 & -5.92 & -111.21\end{array}$

$\begin{array}{lllll}1.19 & 2.31 & -7.81 & -128.33\end{array}$

$2.71 \quad 5.32-10.00-138.07$

$\begin{array}{lllll}2.76 & 2.31 & -8.02 & -149.37\end{array}$ $\begin{array}{lll}0.20 & 3.58 & -1.74 \\ 0.55 & 2.51 & -1.58\end{array}$

$-92.03$

$18-11.2-7.6-136.46$
9.67

$-1.25$

2.58

3.16

10.93

13.39

21.16

24.64

33.85

38.03

48.00

0.00

0.00

0.00

0.00

0.00

0.00

24.94

16.50

5.16

1.91

$-0.26$

4.64

6.42

11.61

14.57

21.50

25.36

33.38

40.24

49.43

0.00

0.00

0.00

0.00

0.00

23.03

8.66

2.70

$-5.69$

$-1.48$

$-2.40$

2.12

2. 59

8.94

10.59

18.18

23.03

31.96

37.72

47.43

0.00

0.00

0.00

0.00

0.00

0.00

0.00

0.00

0.00

34.61

19.65

10.89

1.84

0.17

$-3.06$

10.65

0.00

1.73

0.98

$\begin{array}{lll}3.13 & -0.48 & 0.55\end{array}$

$\begin{array}{llll}3.02 & -0.70 & -0.14\end{array}$

$\begin{array}{llll}9.87 & 0.18 & -1.06\end{array}$

$\begin{array}{lll}13.69 & 1.65 & 0.30\end{array}$

$\begin{array}{llll}21.04 & 1.29 & -0.12\end{array}$

$\begin{array}{lll}24.92 & 1.01 & 0.28\end{array}$

$32.83-0.10-1.02$

$\begin{array}{llll}37.56 & -0.88 & -0.47\end{array}$

$\begin{array}{llll}45.96 & -2.43 & -2.04\end{array}$

$\begin{array}{lll}52.58 & 0.00 & 0.00\end{array}$

$\begin{array}{llll}0.00 & 0.00 & 0.00\end{array}$

$\begin{array}{llll}0.00 & 0.00 & 0.00\end{array}$

$\begin{array}{llll}0.00 & 0.00 & 0.00\end{array}$

$\begin{array}{llll}0.00 & 0.00 & 0.00\end{array}$

$\begin{array}{llll}39.70 & 0.00 & 0.00\end{array}$

$\begin{array}{lll}24.96 & -0.48 & 0.02\end{array}$

$\begin{array}{lll}17.34 & 1.14 & 0.83\end{array}$

$\begin{array}{llll}5.34 & -0.84 & 0.19\end{array}$

$\begin{array}{llll}2.86 & -0.38 & 0.95\end{array}$

$\begin{array}{lll}0.10 & -0.51 & 0.36\end{array}$

$\begin{array}{lll}5.68 & 1.90 & 1.04\end{array}$

$\begin{array}{lll}7.87 & 2.43 & 1.45\end{array}$

$\begin{array}{lll}13.12 & 2.56 & 1.50\end{array}$

$\begin{array}{lll}15.86 & 1.70 & 1.29\end{array}$

$\begin{array}{lll}21.77 & 0.88 & 0.27\end{array}$

$\begin{array}{llll}25.23 & -0.79 & -0.13\end{array}$

$32.08-1.93-1.30$

$\begin{array}{llll}37.74 & -2.58 & -2.51\end{array}$

$\begin{array}{llll}47.04 & -2.19 & -2.39\end{array}$

$\begin{array}{lll}0.00 & 0.00 & 0.00\end{array}$

$\begin{array}{lll}0.00 & 0.00 & 0.00\end{array}$

$\begin{array}{llll}0.00 & 0.00 & 0.00\end{array}$

$\begin{array}{lll}0.00 & 0.00 & 0.00\end{array}$

$\begin{array}{llll}32.06 & 0.00 & 0.00\end{array}$

$\begin{array}{lll}23.11 & 0.81 & 0.08\end{array}$ 


\begin{tabular}{|c|c|c|c|c|c|c|c|c|c|c|c|c|c|}
\hline 9 & 11 & \multicolumn{2}{|c|}{$20-10.7$} & -8.1 & 19 & ( & 57 & -6.57 & -155.41 & -1.03 & -0.02 & 3.78 & 1.01 \\
\hline 9 & 12 & \multicolumn{2}{|c|}{$21-12.4$} & -6.8 & -162.59 & 2.12 & 2.31 & -5.49 & -163.65 & -1.20 & -0.05 & 3.27 & \\
\hline 9 & 13 & \multicolumn{2}{|c|}{$22-14.1$} & -5.7 & -168.81 & 25 & 51 & -4.66 & -167.72 & 2.81 & 79 & 2.22 & -0.02 \\
\hline 9 & 14 & \multicolumn{2}{|c|}{$23-15.6$} & -4.7 & -173.98 & 1.00 & 31 & -4.01 & -174.69 & 3.91 & 3.33 & 0.42 & \\
\hline 9 & 15 & \multicolumn{2}{|c|}{$24-17.1$} & -3.8 & -178.24 & 0.99 & 3.46 & -3.50 & -177.29 & 9.38 & 7.71 & 0.68 & \\
\hline 9 & 16 & \multicolumn{2}{|c|}{$25-18.4$} & -3.1 & -181.69 & 1.48 & 1 & -3.09 & -180.99 & 5 & 11.33 & 4 & 2 \\
\hline$y$ & 17 & \multicolumn{2}{|c|}{$26-19.6$} & -2.4 & -184.43 & 1.69 & 3.42 & -2.75 & -182.06 & .75 & 8.56 & .50 & -2.19 \\
\hline 9 & 18 & \multicolumn{2}{|c|}{$27-20.8$} & -1.8 & -186.54 & 1.99 & 2.31 & -2.47 & -184.71 & 26.17 & 25.62 & 1.43 & -0.55 \\
\hline 9 & 19 & \multicolumn{2}{|c|}{$28-21.9$} & -1.3 & -188.09 & 2.16 & 3.39 & -2.23 & -184.78 & 34.18 & & 0.00 & \\
\hline 9 & 20 & $29-$ & 22.8 & -0.8 & 9.16 & & & -2.03 & -186.84 & 40.18 & & & \\
\hline & 21 & & Zu & -0.4 & -189.81 & 2.99 & 3.36 & -1.86 & -185.31 & 49.79 & 0.00 & 0.00 & 0.00 \\
\hline & 22 & & 5 & -0.1 & .08 & 2.98 & 2.31 & -1.72 & .51 & 56.66 & 0.00 & 0.00 & 0.00 \\
\hline 9 & 23 & 32 & 0.0 & 0.0 & 0.00 & 2.57 & 3.34 & -1.59 & 0.00 & 0.00 & 0.00 & 0.00 & 0.00 \\
\hline 9 & 24 & 33 & 0.0 & 0.0 & 0.00 & 2.00 & 2.31 & -1.48 & 0.00 & 0.00 & 0.00 & 0.00 & 00 \\
\hline 9 & 25 & 34 & 0.0 & 0.0 & 0. & 1.39 & 3.32 & -1.39 & 0.00 & 0.00 & 0.00 & 0.00 & \\
\hline 9 & 26 & 35 & 0.0 & 0.0 & 0.00 & 0.77 & 2.31 & -1.30 & 0.00 & 0.00 & 0.00 & 0.00 & 0.00 \\
\hline$y$ & 27 & 36 & 0.0 & 0.0 & 0.00 & 0.00 & 3.30 & -1.22 & 0.00 & 0.00 & 0.00 & 0.00 & 0.00 \\
\hline$y$ & 28 & 37 & 0.0 & 0.0 & 0.00 & -1.04 & 2.31 & -1.16 & 0.00 & 0.00 & 0.00 & 0.00 & 0.00 \\
\hline & 29 & 38 & 0.0 & 0.0 & 0.00 & -0.64 & 3.29 & -1.10 & 0.00 & 0.00 & 0.00 & 0.00 & 0.00 \\
\hline & 4 & 14 & 0.0 & 0.0 & 0.00 & 2.02 & 0.00 & -1.65 & 0.00 & 0.00 & 0.00 & 0.00 & 0.00 \\
\hline & 5 & 15 & 0.0 & 0.0 & 0.00 & 2.60 & 2.81 & -2.47 & 0.00 & 0.00 & 41.39 & 0.00 & 0.00 \\
\hline & 6 & $\perp 0$ & $0.7-$ & 19.4 & -93.57 & 1.34 & 0.00 & -3.50 & -95.73 & 25.59 & 23.99 & -0.25 & -1.60 \\
\hline & 7 & 17 & -1.1 & -17.1 & -111.84 & 0.98 & 2.51 & -4.77 & -113.12 & 16.27 & 16.49 & 1.20 & 0.22 \\
\hline & 8 & 18 & $-3 . c$ & -15.0 & -127.91 & 1.30 & 0.00 & -6.27 & -132.89 & 4.57 & 5.32 & 2.05 & 0.75 \\
\hline & 9 & 19 & -4.9 & 13.1 & -141.96 & 2.76 & 2.31 & -8.02 & -144.91 & 0.62 & 1.75 & 3.89 & 1.13 \\
\hline & 10 & 20 & $-6.7-$ & -11.4 & -154.18 & 2.81 & $0 \cap 0$ & -10.00 & -161.37 & -7.77 & -7.04 & 3.54 & 0.73 \\
\hline & 11 & 21 & -8.5 & -9.8 & .77 & 2.82 & 2.16 & -8.19 & -167.98 & -6.31 & -5.73 & 3.40 & 0.58 \\
\hline & 12 & $22-$ & 10.2 & -8.5 & .91 & 2.19 & 0.00 & -6.83 & -178.55 & -8.81 & -8.03 & 2.96 & 0.78 \\
\hline & 13 & $23-$ & 11.9 & -7.3 & -181.77 & 2.31 & 2.04 & -5.78 & -183.20 & -5.39 & -5.16 & 2.53 & 0.23 \\
\hline & 14 & $24-$ & 13.4 & -6.2 & .49 & 1.10 & 0.00 & -4.97 & .35 & -6.47 & -5.95 &. .62 & 0.52 \\
\hline & 15 & $25-$ & 14.9 & -5.2 & 9 & 1.08 & 5 & -4.32 & .49 & -1.53 & -2.06 & 0.55 & -0.53 \\
\hline & 16 & $26-$ & 16.2 & -4.4 & .00 & 1.55 & 0.00 & -3.79 & -201.24 & 0.79 & 0.44 & 1.21 & -0.35 \\
\hline 0 & 17 & 27 & 17.5 & -3.6 & -203.01 & 1.76 & 1.87 & -3.37 & -202.75 & 7.3 & 6.96 & 1.37 & -0.40 \\
\hline & 18 & $28-$ & 18.7 & -3.0 & 30 & 2.05 & 0.00 & -3.01 & .27 & 10.90 & 11.12 & 2.26 & 0.22 \\
\hline & 19 & $29-$ & 9.9 & -2.4 & 6 & 2 . & 1.80 & -2 . & .66 & 18.58 & 0.00 & 0.00 & 0.00 \\
\hline & 20 & $30-$ & .9 & -1.8 & .05 & 2.11 & 0.00 & -2.47 & -211.41 & 22.90 & 0.00 & 0.00 & 0.00 \\
\hline & 21 & $31-$ & 21.9 & -1.3 & .63 & 3.03 & 1.74 & -2.25 & -210.11 & 32.2 & 0.00 & 00 & 0.00 \\
\hline & 22 & $32-$ & 22.8 & -0.9 & 6 & 3.02 & 0 & -2.07 & .81 & 37. & 0 & 10 & 0.00 \\
\hline & 23 & $33-$ & 23.6 & -0.5 & & 2 . & & -1 & -2 & 46.44 & 0.00 & 0.00 & 0.00 \\
\hline & 24 & $34-$ & 4.4 & -0.2 & .86 & 2.05 & 0.00 & -1.77 & -214.58 & 52.02 & 0.00 & 0.00 & 0.00 \\
\hline 0 & 25 & 35 & 0.0 & 0.0 & 0.00 & 1.46 & 1.64 & -1.65 & 0.00 & 0 . & 0.00 & 0.00 & 0.00 \\
\hline$C_{2}-1$ & 26 & 36 & 0.0 & 0.0 & 0.8 & 0. & 0 & -1 & 0 . & & & & 00 \\
\hline & 27 & 7 & 0 . & 0 & & & & & & 0 & 0 & 0.00 & 0.00 \\
\hline & 28 & 38 & 0.0 & 0.0 & 0.00 & -0.93 & 0.00 & -1.37 & 0.00 & 0. & 0.00 & 0.00 & 0.00 \\
\hline 0 & 29 & 39 & 0.0 & 0.0 & 0.00 & -0.54 & 1.56 & -1.29 & 0.00 & 0 . & 0.00 & 0.00 & 0.00 \\
\hline 0 & 30 & 40 & 0.0 & 0.0 & 0.00 & -0 . & 0 & -1 & 0.00 & & & & \\
\hline & 31 & 41 & 0. & 0.0 & & & & & & 0. & 0.00 & 0.00 & 0.00 \\
\hline & 4 & 15 & .4 & 0.0 & 0.00 & 6 & 2.16 & -1.41 & 0.00 & 0.00 & 0.00 & 0.00 & 0.00 \\
\hline 1 & 5 & 16 & 0.0 & 0.0 & 0.00 & 2.62 & 3.93 & -2.07 & 0.00 & 0 . & 0.00 & 0.00 & 0.00 \\
\hline 1 & 6 & 17 & $1.9-$ & -21.0 & 2.22 & 1.41 & 2.16 & -2.91 & 1.56 & 37. & 7 & & 1.88 \\
\hline & 7 & 18 & 0 . & 8.9 & & & & -3 & & & 2 & 7 & 0.01 \\
\hline & 8 & 19 & -1.4 & -16.9 & -130.07 & 1.36 & 2.16 & -5.15 & 1.71 & 13.04 & 12.93 & 1.25 & -0.11 \\
\hline & 9 & 20 & $-3.1-$ & -14.9 & 95 & 2.78 & 3.57 & -6.57 & -146.17 & 6.65 & 6.84 & 2.97 & 0.19 \\
\hline & 10 & 21 & $-4.9-$ & -13.2 & -1 & 2 . & 2 . & -8.19 & .18 & -2 & & & \\
\hline & 11 & 22 & $6.8>>$ & 6 & & & 4. & & -174.64 & -5.68 & -5 . & 3 & 0.50 \\
\hline & 12 & 23 & $-8.2-$ & -10.1 & .14 & 2.22 & 2.16 & -8.33 & -187.09 & -10.06 & -9.53 & 2.75 & 0.53 \\
\hline & 13 & 24 & -9.8 & -8.8 & 61 & 2.34 & 3.41 & -7.05 & 3.91 & -8.81 & -8.42 & .73 & 0.39 \\
\hline & 14 & & 11.4 & -7.7 & & 1. & 2 . & -6 & 8 & -10 & -9 & & 1.05 \\
\hline & 15 & & 2.8 & 6.7 & .02 & 1.1 & 3.35 & -5.24 & -208.77 & -7.52 & -6.90 & 1.77 & 0.62 \\
\hline & 16 & 27 & 14.2 & -5.7 & -214.21 & 1.60 & 2.16 & -4.59 & -215.04 & -5.72 & -5.60 & 1.73 & 0.12 \\
\hline & 17 & & 15.5 & -4.9 & $-219 \quad 51$ & 1.81 & 3.31 & -4.07 & -218.46 & -1.07 & -1.14 & 1.74 & -0.07 \\
\hline & 18 & & 6.7 & -4 . & 0 & 1 & 2.16 & 3. & & & 2.66 & 2.70 & 0.61 \\
\hline & 19 & & 7.9 & -3.5 & 7.84 & 2.25 & 3.27 & -3.26 & -225.58 & 7.95 & 8.29 & 2.58 & 0.34 \\
\hline & 20 & $31-$ & 9.0 & -2.9 & -231.00 & 2.14 & .16 & -2.95 & -229.66 & 11.94 & 12.52 & 2.72 & 0.58 \\
\hline & 21 & $32-$ & 0.0 & -2.3 & 233.59 & 3.05 & 3.24 & -2.69 & -230.00 & 19.68 & 18.37 & 1.74 & -1.31 \\
\hline & 22 & & $\cdots$ & .0 & & & & 2. & -232.93 & 24.81 & 25.50 & 3.72 & 0.69 \\
\hline
\end{tabular}




\begin{tabular}{|c|c|c|c|c|c|c|c|c|c|c|c|c|c|}
\hline & 3 & \multicolumn{2}{|c|}{$34-21.9$} & -1.4 & -237.26 & 2.64 & 22 & -2.27 & -233.68 & \pm & 0 & 10 & 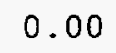 \\
\hline & 24 & \multicolumn{2}{|c|}{$35-22.8$} & -10 & $-238,44$ & & .16 & -2.10 & -236.30 & 59 & 0.00 & 0.00 & 0.00 \\
\hline & 25 & \multicolumn{2}{|c|}{$36-23.5$} & -0.6 & 39 & & & -1.95 & -236.50 & 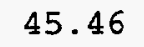 & 00 & 0.00 & .00 \\
\hline & 26 & \multicolumn{2}{|c|}{$37-24.3$} & -0.3 & 9.70 & 0.90 & 16 & -1.82 & -238.47 & 57 & 0.00 & 0.00 & 0.00 \\
\hline & 27 & \multicolumn{2}{|c|}{$38-24.9$} & 0.0 & 39.87 & 0.16 & 3.17 & -1.71 & -238.24 & 59.86 & 0.00 & .00 & .00 \\
\hline & 28 & 39 & 0.0 & 0.0 & 0.00 & -0.84 & 6 & -1.60 & 0.00 & 0 & & 00 & 0.00 \\
\hline & 29 & 40 & 0.0 & 0.0 & 0 & & & -1.51 & & 0.00 & 0.00 & 0.00 & 0.00 \\
\hline & 30 & 41 & 0.0 & 0.0 & 0.00 & 1.17 & 2.16 & -1.43 & 0.00 & 0.00 & 0.00 & 0.00 & 0.00 \\
\hline & 31 & 42 & 0.0 & 0.0 & 0.00 & 0.98 & 3.14 & -1.35 & 0.00 & 0.00 & & 0.00 & 0.00 \\
\hline & 32 & 43 & 0.0 & 0.0 & 0.00 & 0.39 & & -1.29 & 0.00 & 0.00 & & & \\
\hline & 33 & 44 & 0.0 & & .00 & .09 & & -1.22 & & 0.00 & 0.00 & .00 & 0.00 \\
\hline & 5 & 17 & 0.0 & 0.0 & 0.00 & 1.0 & 2.81 & -1.77 & 0.00 & 0.00 & 0.00 & 0.00 & 0.00 \\
\hline & 6 & 18 & 0.0 & 0.0 & 0.00 & 0.73 & 0.00 & -2.47 & 0.00 & 0.00 & 0.00 & 0.00 & .00 \\
\hline & 7 & 19 & $1.6-$ & -20.5 & -111.23 & 0.42 & & -3.31 & -111.61 & 32.35 & 31.95 & 0.01 & .40 \\
\hline & 8 & 20 & & -18.5 & -130.73 & 0.73 & 0 & -4.32 & -134.31 & 17.72 & 17.57 & 0.58 & 0.15 \\
\hline & 9 & 21 & $.6-$ & 16.0 & -148.28 & 2.12 & 2.31 & -5.49 & -149.34 & 10.76 & 10.91 & 2.27 & 0.15 \\
\hline & 10 & 22 & $-3.2-$ & -14.8 & -164.00 & 2.19 & 0.00 & -6.83 & -168.64 & -0.46 & -0.40 & 2.25 & .06 \\
\hline & 11 & 23 & $-4.8-$ & -13.2 & $-178: 01$ & 2.22 & & -8.33 & -181.96 & -5.71 & & .46 & .24 \\
\hline & 12 & 24 & & -11.7 & -190.46 & 1.64 & 0.00 & 10.00 & -198.82 & -14.50 & -13.93 & 2.21 & 0.57 \\
\hline & 13 & 25 & $-8.0-$ & -10.4 & -201.50 & 1.77 & 2.04 & -8.45 & -206.14 & $-13 \cdot 75$ & -13.19 & 2.33 & 0.56 \\
\hline & 14 & 26 & -9.5 & -9.2 & -211.26 & 0.64 & 0.00 & -7.24 & -217.86 & -17.40 & -16.21 & 1.83 & .19 \\
\hline & 15 & $27-$ & 10.9 & -8.1 & -219.87 & 0.63 & & -6.27 & -223.56 & -15.02 & & 07 & 0.43 \\
\hline & 16 & $28-$ & 12.3 & -7.1 & -227.42 & 1.10 & 0.00 & -5.49 & -231.81 & -15.21 & -15.02 & 1.29 & 0.19 \\
\hline & 17 & $29-$ & 13.6 & -6.2 & -234.04 & 1.31 & 1.87 & -4.85 & -235.71 & -11.03 & -10.66 & 1.68 & .37 \\
\hline & 18 & 30 & 14.8 & -5.4 & -239.79 & 1.59 & 0.00 & -4.32 & -242.52 & -9.77 & -9.07 & 29 & .70 \\
\hline & 19 & 31 & 16.0 & -4.6 & .78 & 1.76 & 1. & -3 & $-245: 09$ & -4.27 & -3.35 & 2.68 & 0.92 \\
\hline & 20 & $32-$ & 17.1 & -3.9 & .06 & 1.67 & 0.00 & -3.50 & -250.89 & -2.00 & -0.82 & 2.84 & 1.18 \\
\hline & 21 & $33-$ & 18.2 & -3.3 & -252.70 & 2.57 & 1.74 & -3.18 & -251.57 & 5.39 & 5.09 & 2.26 & 0.30 \\
\hline & 22 & 34 & 19.2 & -2.8 & .76 & 2.57 & 0.00 & -2.91 & -256.10 & 8.94 & 0.00 & .00 & 0.00 \\
\hline & 23 & 35 & 20.1 & -2.3 & & 9 & & & -257.09 & 16.02 & 0 & 0.00 & .00 \\
\hline & 24 & $36-$ & 21.0 & -1.8 & -2 & 1.65 & 0.00 & -2.47 & -261.15 & 20.02 & 0.00 & 0.00 & .00 \\
\hline & 25 & $37-$ & 21.9 & -1.4 & .96 & 1.09 & 1.64 & -2.29 & -261.52 & 27.73 & & 0 & 20 \\
\hline & 26 & $38-$ & 22.7 & -1.0 & -2 & 0.50 & 0.00 & -2.13 & 4.80 & 32.52 & & 0 & 00 \\
\hline & 27 & & 23.4 & -0 & 4 & & 1.60 & & & 75 & 0 & 0.00 & .00 \\
\hline & 28 & $40-$ & 24.1 & -0.4 & -2 & -1.20 & 0.00 & -1.86 & -267.64 & 45.82 & 0.00 & 0.00 & .00 \\
\hline$(2)$ & 29 & $41-$ & 24.7 & -0.1 & -264.83 & -0.82 & 1.56 & -1.75 & -265.84 & 55.69 & 0 & 0 & 0 \\
\hline 12 & 30 & 42 & 0.0 & 0.0 & 0.00 & 1.00 & 0 & 5 & & & & 0 & .00 \\
\hline & 31 & 43 & 0.0 & 0 & & & 1. & -1 & .00 & 0 & 0 & 0.00 & .00 \\
\hline & 32 & 44 & 0.0 & 0 . & 0 . & -4.93 & 0.00 & -1.48 & 0.00 & 0.00 & 0 . & 0.00 & .00 \\
\hline $12+$ & 33 & 45 & 0.0 & 0.0 & 0.00 & 1.21 & 1.50 & -1.4 & 0. & 0.00 & & 0 & 10 \\
\hline 12 & 34 & 46 & 0.0 & 0 . & 0 & 0 & & & & & & 0 & .00 \\
\hline & 35 & 47 & 0.0 & 0. & & 6 & 1. & -1.28 & 0 & 0 & 0 & 00 & 0.00 \\
\hline & 3 & 18 & 0.0 & 0.0 & 0.00 & 2.01 & 3.89 & -1.55 & 0.1 & 0.00 & 0 . & 0.00 & 0.00 \\
\hline 13 & 6 & 19 & 0.0 & 0.0 & 0 & 0.91 & 2.04 & -2.13 & 0. & 0 & & 0 & Do \\
\hline & 7 & 20 & 2.6 & -21.9 & -1 & 0.61 & 3 . & & -107 & & & 0.00 & 0.00 \\
\hline & 8 & 21 & 1.3 & 100 & & 0 & 4 & -3.68 & -13 & 28 & 26.12 & -1.49 & -2.39 \\
\hline 13 & 9 & 22 & $-0.2-$ & -18.2 & .17 & 2.25 & 3.51 & -4.66 & -148.08 & 19.32 & 18. & 0.97 & -1.28 \\
\hline 13 & 10 & 23 & $-1.7-$ & 6.4 & -1 & 2 & 2.04 & -5.78 & & & & & \\
\hline 25 & 11 & 24 & -3 & & & & & & -18 & & & 9 & -0.24 \\
\hline & 12 & 25 & $-4.8-$ & .3 & -1 & 1. & 2.04 & -8.45 & -200.69 & -9.08 & -8 . & 1.94 & 0.17 \\
\hline 13 & 13 & 26 & $-6.3-$ & 1.9 & .61 & 1.89 & 4.48 & 10.00 & -212.23 & -12.55 & -12.21 & 23 & 0.34 \\
\hline 13 & 14 & 27 & -7.7 & -10.6 & .84 & 0 . & 2 . & & & & & & 0.61 \\
\hline & + & 28 & 1 & & & 0. & & & -233.21 & -17 & -1 & 1.32 & 0.54 \\
\hline & 16 & 29 & 10.5 & -8.4 & -2 & 1. & 2.04 & -6.48 & -241.98 & -18.08 & -1 & 1.10 & -0.14 \\
\hline 13 & 17 & $30-$ & 11.8 & -7.4 & .69 & 1.43 & 3.22 & -5.71 & -247.74 & -15.78 & -1 & 1.34 & -0.09 \\
\hline 13 & 18 & 31 & 3.0 & $6 . \epsilon$ & -2 & 1. & 2.04 & -5 & .02 & -1 & & & \\
\hline & - & & & & & & & -4 & & -11 & -11 & 3 & 16 \\
\hline 13 & 20 & 33 & 5.3 & -5.1 & -2 & 1. & 2.04 & -4.10 & -265.55 & -9.37 & -8 & 56 & 0.78 \\
\hline 13 & 21 & 34 & 6.4 & -4.4 & -269.98 & 2 . & 3.15 & -3.72 & -267.89 & -3.64 & -3 & 3.09 & .43 \\
\hline 13 & 22 & & 7.4 & 3.18 & -2 & 2 . & & -3 & & & & 77 & 0.12 \\
\hline 13 & 23 & & & 3 & -2 & 2.2 & 3.12 & -3.11 & $-2^{\circ}$ & & & & 0.00 \\
\hline 13 & 24 & 37 & 9.3 & -2.7 & -28 & 1. & 2.04 & -2.87 & -279.60 & 8 & & 10 & 0.00 \\
\hline 13 & 25 & 38 & 0.2 & -2.3 & -283.02 & 1.20 & 3.10 & -2.65 & -281.37 & & & 0 & .00 \\
\hline 13 & 26 & & 1.1 & -18 & & & 4 & -2 & -284.85 & & & 0 & 0.00 \\
\hline 13 & 27 & & .9 & -1.4 & & -0.0 & 3.08 & -2.30 & .99 & & 0.00 & 0.00 & 0.00 \\
\hline & 28 & & 2.6 & . & 37.94 & & 04 & & -289.10 & 31.05 & 0.00 & 0.00 & 0 \\
\hline
\end{tabular}




\begin{tabular}{|c|c|c|c|c|c|c|c|c|c|c|c|c|}
\hline 3 & 29 & 3.3 & 0.8 & & -0.69 & 3.06 & -2.02 & -288.50 & 40.32 & 0.00 & 0.00 & 0.00 \\
\hline 3 & 30 & 4.0 & -0.5 & 289.46 & 1.66 & 2.04 & -1.90 & -287.66 & & 0.00 & 0.00 & 0.00 \\
\hline 2 & 31 & 4 & -0.2 & -28979 & 1.26 & 3.04 & -1.79 & -287.28 & 57.68 & .00 & .00 & 0.00 \\
\hline & 32 & 0.0 & 0.0 & 0 & -3 & & -1.70 & 0.00 & 0.00 & .00 & .00 & .00 \\
\hline 3 & 33 & 0.0 & 0.0 & 0.00 & 1.55 & 3.02 & -1.61 & 0.00 & 0.00 & 0.00 & 0.00 & 0.00 \\
\hline & 34 & 0.0 & 0.0 & 0.00 & 1.76 & 2.04 & -1.53 & 0.00 & & 00 & 00 & .00 \\
\hline & 35 & 0.0 & 0.0 & & & 3.01 & -1.46 & & & & & .00 \\
\hline & 36 & 49 & 0. & & 2.80 & & -1.39 & 0.00 & & & 00 & .00 \\
\hline & 7 & 0 & 0.0 & 0 & 1.93 & 3.00 & -1.33 & 0.00 & 0.00 & 0.00 & 0.00 & 0.00 \\
\hline & 38 & 0.0 & 0.0 & 0.00 & 1.35 & 2.04 & -1.28 & 0.00 & & & 00 & .00 \\
\hline & 6 & 20 & 0.0 & & & 0.00 & -1.86 & & & & & .00 \\
\hline & 7 & 21 & 0.0 & 0.5 & & 2.51 & -2.47 & 0.00 & 0.00 & 0.00 & 0.00 & 0.00 \\
\hline & 8 & 22 & 21.4 & -128.29 & -0.34 & 0.00 & -3.18 & -131.80 & 34.81 & 32.16 & -2.98 & -2.64 \\
\hline & 9 & $1.0-$ & 19.6 & -148.77 & 1.00 & 2.31 & -4.01 & -149.47 & & & & -1.44 \\
\hline & 10 & 24 & 17.9 & -167.51 & 1.10 & 0.00 & -4.97 & & 11.37 & 10.76 & 49 & -0.61 \\
\hline & 11 & $25-1$ & 6.3 & -184.57 & 1.16 & 2.16 & -6.04 & -187.30 & 3.53 & 3.83 & 1.46 & 0.30 \\
\hline & 12 & $26-3$ & -14.7 & -200.06 & 0.64 & 0.00 & -7.24 & -206.66 & -7.76 & -7.14 & 1.26 & 0.62 \\
\hline & 13 & $27-4.7-$ & -13.3 & -214.07 & 0.79 & 2.04 & -8.56 & -219.80 & -12.83 & -12.39 & 1.23 & 0.44 \\
\hline & 14 & $28-6$. & -12.0 & -226.72 & -0.26 & & & 6.98 & -21.94 & -21.49 & 0.19 & 0.45 \\
\hline & 15 & 29 & -10.8 & -2 & -0 & 1.95 & -8.65 & -245.07 & -21.96 & -21.90 & -0.18 & 0.06 \\
\hline & 16 & $30-8.8$ & -9.7 & -248.38 & 0.22 & 0.00 & -7.56 & -255.72 & -24.54 & -24.43 & 0.32 & 0.11 \\
\hline & 17 & $31-10.0$ & -8.7 & -257.58 & 0.44 & 1.87 & -6.66 & -261.93 & -22.68 & -22.95 & .17 & -0.27 \\
\hline & 18 & $32-11.3$ & -7.8 & -2 & 0.73 & 0.00 & -5.92 & -271.00 & -23.68 & & .33 & .40 \\
\hline & 19 & 33. & -6.9 & & & 1.80 & -5.29 & -275.75 & -2 & -2 & 0.78 & -0.13 \\
\hline & 20 & $34-13.6$ & -6.2 & -279.71 & 0. & 0.00 & -4.77 & -283.64 & -20.18 & -1 & 1.05 & 0.22 \\
\hline & 21 & $35-14.7$ & -5.5 & -285.52 & 1.72 & 1.74 & -4.32 & -286.38 & -14.84 & & 2.19 & .48 \\
\hline & 22 & $36-$ & -4.8 & & 4 & 0.00 & -3.93 & 2.83 & -13 & 48 & & .74 \\
\hline & 23 & 37 & -4.2 & & & 1.69 & -3.60 & 5.65 & -7 & -5 & 1 & 32 \\
\hline & 24 & $38-$ & -3.6 & -2 & 0 . & 0.00 & -3.31 & -301.46 & -5.71 & 0.00 & 0 & 0.00 \\
\hline & 25 & $39-18.6$ & -3.1 & -302.42 & 0.35 & 1.64 & -3.06 & -3 & 0.34 & & & 00 \\
\hline & 26 & 40 & -2.7 & & -0. & 0.00 & -2.84 & 4 & & & 10 & .00 \\
\hline & 27 & & -2 . & & & 1.60 & -2.64 & 57 & 10 & 0 & 00 & 00 \\
\hline & 28 & $42-21.1$ & -1.8 & .77 & -1.82 & 0.00 & -2.47 & -3 & 13 & 0. & 00 & 0.00 \\
\hline & 29 & $43-21.8$ & -1.5 & .41 & -1.45 & 1.56 & -2.31 & & & & & \\
\hline & 30 & .5 & -1.1 & & & 0.00 & -2.17 & & & & 00 & 00 \\
\hline & 31 & .2 & -0.8 & -3 & 0 . & 1.53 & -2.05 & -3 & 52 & 0. & 00 & 0.00 \\
\hline & 32 & $46-23.8$ & -0.5 & -314.33 & -1.97 & 0.00 & -1.93 & -3 & 42.09 & 0. & 10 & 0.00 \\
\hline & 33 & $47-24.4$ & -0.3 & -3 & 1. & 50 & -1.83 & & & & & \\
\hline & 34 & 9 & & 9 & & & -1 & & 7 & 10 & 00 & 00 \\
\hline & 35 & 0.0 & 0.0 & 0 & 1. & 1.47 & -1.65 & 0.00 & 0.00 & 0.00 & 0 & 0.00 \\
\hline & 36 & 0.0 & 0.0 & 0. & 2.13 & 0.00 & -1 & 0 . & 0. & 0 & 0 & 0.00 \\
\hline & 37 & 0.0 & 0. & 0. & 2 . & 4 & -1 & & & & & \\
\hline & 38 & 5 & & & & & & 00 & & 0 & 0 & 00 \\
\hline & 39 & 0.0 & 0.0 & 0 & 0 . & 1.42 & -1.38 & 0. & 0.00 & 0.00 & 0 & 0.00 \\
\hline & 40 & 0.0 & 0.0 & 0.00 & -0 . & 0.00 & -1 & 0 . & 0 & 0.00 & 0 & 00 \\
\hline & 6 & 0. & 0.1 & & & & & & & & & bo \\
\hline & 7 & 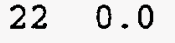 & & & & & -2 & & & & 0 & 00 \\
\hline & 8 & 0.0 & 0.0 & 0 & -0.31 & 1.95 & -2.79 & 0.00 & 10 & 0.00 & 00 & 0.00 \\
\hline & 9 & 2.1 & -20.9 & -147.24 & $0.99^{\circ}$ & 3.46 & -3.50 & 6.29 & 35 & 0.00 & 0 & 0.00 \\
\hline & 10 & 0.8 & .2 & & 1 & .95 & -5 & & & & & 00 \\
\hline & 11 & 26 & -1 & & 1. & 3.35 & -5.24 & & 1 & & 0 & 00 \\
\hline & 12 & $\begin{array}{ll}27 & -1.9\end{array}$ & -16.1 & -2 & 0 . & 1.95 & -6.27 & 32 & -0.14 & -0.75 & 0.02 & -0.61 \\
\hline & 13 & $28-3.3-$ & $-14 . .7$ & -2 & 0. & 3.27 & -7.41 & 38 & -7 & -7.16 & 75 & -0.03 \\
\hline & 14 & -4.6 & & & -0 . & 1.5 & -8.65 & 0 & & & & \\
\hline & 15 & ( $>2$ & & & -0 . & $4.21-$ & & 81 & & & -0 & 0.21 \\
\hline & 16 & $31-7.2-$ & -11.0 & -2 & 0.22 & 1.95 & -8.73 & -262.91 & -24.44 & -24.44 & 0.23 & 0.00 \\
\hline & 17 & $32-8.4-$ & -10.0 & -2 & 0.44 & 3.16 & -7.69 & 0.91 & -24.37 & -2 & 50 & 0.06 \\
\hline & 18 & -9.7 & & & & 1. & & & & & 20 & 52 \\
\hline & 19 & .0 .8 & -8 & -2 & 0. & 3.12 & -6 . & & -2 & -24 & & -0.35 \\
\hline & 20 & 2.0 & -7.3 & -2 & 0.83 & 1.95 & -5 & 5.20 & -24.44 & -24.86 & 0.41 & -0.42 \\
\hline & 21 & 13.0 & -6.5 & -2 & 1.6 & 3.08 & -4.97 & 9.57 & -2 & & & 0.49 \\
\hline & 22 & & & & & 95 & & 6.40 & -19.50 & -19.06 & 15 & 0.44 \\
\hline & 23 & 5.1 & -5.2 & -311.0 & 1.5 & 3.05 & -4.13 & .74 & & & 0.00 & 0.00 \\
\hline & 24 & 6.1 & -4.6 & -315.90 & 0.88 & 1.95 & -3.79 & -316.87 & -13 & 0 & 00 & 0.00 \\
\hline & 25 & 7.0 & -4.0 & -3 & 0.3 & 3.02 & -3 & & & & .00 & 0.0 \\
\hline & 26 & 1. & 2.2 & -323.97 & ... & & -3.24 & - & -6.27 & 0.00 & 0.00 & 0.00 \\
\hline
\end{tabular}




\begin{tabular}{|c|c|c|c|c|c|c|c|c|c|c|c|c|c|}
\hline 5 & 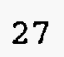 & \multicolumn{2}{|c|}{$42-18.7$} & 3.0 & -327.25 & 0 & 00 & -3.01 & -328 & -0 & 0 & 0 & 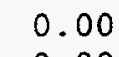 \\
\hline & 8 & \multicolumn{2}{|c|}{$43-19.5$} & 06 & 330.07 & -179 & 95 & -2.81 & -332 & & & & 00 \\
\hline & 9 & \multirow{2}{*}{\multicolumn{2}{|c|}{$44-20.3$}} & 2.2 & 332.47 & .42 & 8 & -2 & 23 & & & 0 & 0.0 \\
\hline & 30 & & & 1.8 & 334.48 & 64 & & -2 & & 18. & & .00 & .00 \\
\hline & 31 & \multicolumn{2}{|c|}{$46-21.8$} & 1.5 & -336.13 & 1.54 & 96 & -2.32 & -3 & & & & .00 \\
\hline & 32 & \multicolumn{2}{|c|}{$47-22.4$} & 1.2 & 15 & -0.26 & & -2.19 & & & bo & & \\
\hline & 33 & \multicolumn{2}{|c|}{$48-23.1$} & 0.9 & & & & -2 & & 39 & & & 0.00 \\
\hline & 34 & \multicolumn{2}{|c|}{$49-23.7$} & & & & & & & & & 0 & 0.00 \\
\hline & 35 & \multicolumn{2}{|c|}{$50-24.2$} & 0.3 & .64 & 2.71 & & -1.86 & .87 & & & & .00 \\
\hline & 36 & $51-$ & 24.7 & 0.1 & .86 & 3.07 & 5 & -1.77 & & & & 00 & 00 \\
\hline & 37 & 52 & 0.0 & & & 200 & & -1.69 & & & & & 0.00 \\
\hline & 38 & 53 & 0.0 & 0. & & & & -1 & & & & 00 & .00 \\
\hline & 39 & 54 & 0.0 & 0. & & & & -1.55 & 0.00 & 0.00 & & & .00 \\
\hline & 40 & 55 & 0.0 & 0. & 0.00 & 1.19 & 5 & -1.48 & 0 . & 0 . & & & .00 \\
\hline & 41 & 56 & 0.0 & 0. & .00 & 1.30 & 9 & -1 & & & & & 10 \\
\hline & 42 & 57 & 0.0 & & & 1.24 & 5 & -1 & & & & & .00 \\
\hline & 7 & 2 & 0.0 & 0.0 & & -0 . & & -1.93 & 0.00 & 0.00 & & 0.00 & .00 \\
\hline & 8 & 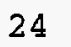 & 0.0 & 0.0 & 10 & 0.23 & & -2.47 & 0.00 & 0.00 & & & .00 \\
\hline & 9 & 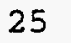 & 3.0 & -22.1 & 2 & & & -3.09 & & 5.24 & & & .00 \\
\hline & 10 & 26 & $1.8-$ & 20.5 & -16 & 1.55 & 0 & -3.79 & .25 & 29.08 & & 0.00 & 0.00 \\
\hline & 11 & 27 & 0.6 & .9 & -18 & 1.60 & & -4.59 & .55 & 18.84 & & 0.00 & 0.00 \\
\hline & 12 & 28 & $-0.7-$ & .4 & -20 & 1.10 & 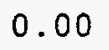 & -5.49 & 3.29 & & & & 0.98 \\
\hline & 13 & 29 & -1.9 & & & & & & & -2.27 & 6 & 0.35 & -0.89 \\
\hline & 14 & 30 & -3.2 & & -2 & 0.22 & 0 & -7.56 & .28 & -13.67 & -14.06 & -0.17 & -0.39 \\
\hline & 15 & 31 & -4.5 & .4 & -24 & 0.22 & 5 & -8.73 & -256.54 & -18.85 & & 0.03 & -0.20 \\
\hline & 16 & 32 & $-5.7-$ & .3 & & 0.66 & & -10.00 & 2.14 & -26.39 & & & 0.37 \\
\hline & $1 \%$ & 33 & $-6.9-$ & .2 & & & & - & & -26.75 & & & .16 \\
\hline & 18 & 34 & 8.1 & & & 1.13 & 0 & -7.81 & .84 & -29.94 & & 1.14 & 0.01 \\
\hline & 19 & 35 & -9.3 & -9.2 & -29 & 1.30 & .80 & -6.98 & .73 & -28.76 & & 1.21 & -0.09 \\
\hline & 20 & 3 & 10.4 & -8.4 & -3 & & & -6.27 & & & & & 01 \\
\hline & 21 & & & & & & & & & -27 & & & 49 \\
\hline & 22 & & .5 & -6.8 & -3 & 2 . & 0 & -5.15 & .92 & -27.73 & - & 2 . $>2>$ & 0.87 \\
\hline & 23 & 3 & 13.5 & -6.2 & -3 & 1.73 & .69 & -4.71 & .63 & -24.37 & & & 21 \\
\hline & 24 & & 4.5 & -5.5 & -3 & & & -4.32 & & & & & 42 \\
\hline & 25 & & & & & & & & & & & & 00 \\
\hline & 26 & & & -4.4 & & & 0 & -3.68 & -3 & $-1 \varepsilon$ & .00 & 0.00 & 0.00 \\
\hline & 27 & 43 & .2 & -3.9 & 21 & 0.49 & .60 & -3.41 & .51 & -12 & & & .00 \\
\hline & 28 & 4 & .0 & -3.4 & -3 & -1.41 & 0 & -3.18 & 14 & & & & 00 \\
\hline & 29 & & & & & 1 & & & & & & & 00 \\
\hline & 30 & & & -2 & & & 0 & -2 & & & & 0.00 & .00 \\
\hline & 31 & 47 & .3 & -2.2 & -3 & 1.70 & 3 & -2.62 & -3 & 10 & & & .00 \\
\hline & 32 & & .0 & -1.8 & & 1 & 0 & -2.47 & & & & & \\
\hline & 33 & & .7 & & & & & & & & & & 00 \\
\hline & 34 & & & -1 & & 4 & 0 & -2 & & & & 0 & .00 \\
\hline & 35 & 5 & .9 & -0.9 & -3 & 7 & 7 & -2.09 & 6 & & & & .00 \\
\hline & 36 & 5 & .5 & -0.6 & & & 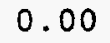 & - & & & & & \\
\hline & 37 & & 0 & & & & & & & & & & bo \\
\hline & 38 & 5 & .5 & -0.2 & & & 0 & -1.81 & & 5 & & & .00 \\
\hline & 35 & 5 & 0.0 & 0.1 & & & 2 & -1.73 & & & & & .00 \\
\hline & 40 & $-7+2>$ & 0.0 & 0. & & & & & & & & & \\
\hline & $x+2>$ & & & & & & & & & & & & \\
\hline & 42 & 5 & 0.0 & 0 & & & & -3 & & & & & 00 \\
\hline & 43 & 5 & 0.0 & 0 . & & & & -1 & 0 & & & & .00 \\
\hline & 44 & 6 & 0 & 0 & & & & & & & & & \\
\hline & 7 & 2 & 0. & 0. & & & & & & & & & .00 \\
\hline & 8 & 2 & 0.0 & 0. & & & & -2 & & & & & 00 \\
\hline & s & 2 & 0.0 & 0. & & & & -2 & & & & & .00 \\
\hline & 10 & 2 & . & . & & & & -3.37 & & & & & 0.00 \\
\hline & 11 & 28 & 1.7 & .1 & & & & -4.07 & & & & & 00 \\
\hline & 12 & 29 & 0.5 & & & & & & & 15 & & 00 & 0.00 \\
\hline & 13 & 30 & -0 . & & & & & & & & & 00 & .00 \\
\hline & 14 & 31 & - & 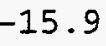 & & & & 56 & 2.87 & & & & -1.10 \\
\hline & 15 & 32 & & & & & & -7.69 & 7.88 & & & & -0.42 \\
\hline & 16 & 33 & & & & & & -8.80 & & & & 0.73 & -0.13 \\
\hline & 17 & 3 & & . & & 1.0 & & 10.00 & -285.70 & -24.58 & 44 & 1.19 & 0.14 \\
\hline & & & -6 & 1.3 & -292.57 & 1.32 & 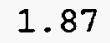 & -8.87 & -298.26 & -29.07 & -28.01 & 1.38 & 0.06 \\
\hline
\end{tabular}




\begin{tabular}{|c|c|c|c|c|c|c|c|c|c|c|c|c|}
\hline & 19 & -7.8 & 10.4 & -303.41 & 48 & 6 & -7 & -306.79 & -29.53 & -29 & 19 & 0.01 \\
\hline & 20 & 8.9 & .5 & -313.32 & .40 & 1.87 & -7.11 & -317.17 & -31.84 & -31.76 & 1.48 & 8 \\
\hline & 21 & 10.0 & -8.6 & -322.37 & 2.22 & 3.02 & -6.43 & -323.55 & -30.15 & -29.80 & .57 & \\
\hline & 22 & 10 & -7.9 & -330.62 & 2.23 & 1.87 & -5.84 & -332.36 & -30.88 & -29.80 & 3.32 & \\
\hline & 3 & 12.0 & -7.2 & -338.12 & 1.89 & 2.99 & -5.33 & -338.57 & -29.02 & & 3.35 & \\
\hline 7 & 24 & 13.0 & -6.5 & -344.93 & 1.41 & 1.87 & -4.88 & -346.54 & -28.92 & .40 & & \\
\hline 17 & 25 & -13.9 & -5.9 & -351.10 & 0.89 & 2.96 & -4.49 & -351.74 & -26.05 & -24.69 & 25 & \\
\hline 17 & 26 & -14.9 & -5.3 & -356.66 & 0.36 & 1.87 & -4.15 & -358.59 & -24.83 & 0.00 & Do & \\
\hline 17 & 27 & 5.7 & -4.7 & -361.66 & -0.31 & 2.94 & -3.85 & .88 & -21.05 & 0.00 & & \\
\hline 17 & 28 & 16.6 & -4.2 & -366.14 & -1.21 & 87 & -3.58 & .07 & -19.17 & & & \\
\hline 11 & 29 & $6-17.4$ & -3.8 & -370.13 & -0.86 & 2.92 & -3.34 & -371.42 & -13.45 & 0.00 & 0.00 & \\
\hline 17 & 30 & $9 ?$ & -3.3 & -373.67 & 1.53 & .87 & -3.13 & -373.40 & & & & \\
\hline 17 & 31 & 8.9 & -2.9 & -376.78 & 1.86 & 2.90 & -2.94 & -374.96 & -0.84 & 0.00 & & \\
\hline 7 & 32 & -19.6 & -2.5 & -379.49 & 1.95 & 37 & -2.76 & -378.43 & 3.75 & 0.00 & & \\
\hline r & 33 & $50-20.3$ & -2.2 & -381.82 & 2.19 & .88 & -2.61 & -379.37 & 10.89 & 0.00 & 0.00 & \\
\hline 17 & 34 & $51-21.0$ & -1.8 & -383.81 & 2.22 & 7 & -2.47 & -3 & & & & \\
\hline 17 & 35 & $52-21.6$ & -1.5 & -385.48 & 2.78 & 6 & -2.34 & -382.18 & 24.23 & 0.00 & 10 & \\
\hline & 36 & $53-22.2$ & -1.2 & -386.84 & 3.39 & .87 & -2.22 & -383.80 & 30.67 & & & \\
\hline & 37 & $54-22.8$ & -0.9 & -387.92 & 3.53 & .85 & -2.11 & -383.66 & 38.89 & 0.00 & 0.00 & \\
\hline 17 & 38 & $55-23.3$ & -0.7 & -388.74 & 401 & .87 & -2.01 & .87 & 45.74 & & 0 & \\
\hline 17 & 39 & $56-23.9$ & -0.5 & -389.31 & 3.8 & 3 & -1.92 & .56 & 54.13 & 10 & 10 & \\
\hline 17 & 40 & $57-24.4$ & -0.2 & -389.66 & 3. & .81 & -1.84 & -386.03 & 60.73 & 0.00 & 0.00 & \\
\hline & 41 & $58-24.8$ & 0.0 & 89.80 & 1.85 & .82 & -1.76 & 5.89 & 67.94 & 0.00 & & \\
\hline 7 & 42 & 59 & 0.0 & 0.00 & 0.95 & .87 & -1.69 & 0.00 & 0.00 & & & \\
\hline 17 & 43 & 0.0 & 0.0 & 0.00 & 0.2 & 1 & -1.62 & 0.00 & 0.00 & 0 & 10 & 0.0 \\
\hline 17 & 44 & 0.0 & 0.0 & 0.00 & -1.23 & .87 & -1.56 & 0.00 & 0.00 & 0.00 & 0.00 & 0. \\
\hline & 45 & 0.0 & 0. & 0.00 & -1.44 & 9 & -1.50 & 0.00 & 0.00 & 0.00 & & 0.00 \\
\hline 17 & 46 & 0.0 & 0. & 0.00 & -1.13 & 7 & -1.45 & 0.00 & 0.00 & & & \\
\hline 18 & 9 & 0.0 & 0.0 & 0.00 & 1.9 & 1 & -2.47 & 0.00 & 0.00 & 0 & 0 & 0. \\
\hline $1 c_{2}$ & 10 & 0.0 & 0.0 & 0.00 & 2.05 & .00 & -3.01 & 0.00 & 0.00 & 0 & 0.00 & 0.00 \\
\hline & 1. & 29 & 21. & -182.46 & & & -3 & -181.84 & 38.13 & & & bo \\
\hline 18 & 12 & 1.5 & 19.8 & -202.99 & 1.5 & 0 & -4.32 & .72 & 22.33 & 0 & 10 & 0.00 \\
\hline 18 & 13 & 0.4 & -18.4 & -222.11 & 1.7 & .04 & -5.08 & .44 & 12.68 & 0.00 & 0.00 & 0.00 \\
\hline 1 & 14 & 0.8 & 17.1 & -239.87 & 0.7 & 10 & -5.92 & .06 & -0.87 & -2.18 & -0.58 & -1.31 \\
\hline 18 & 15 & 33 & 5.8 & 5.32 & 0. & & -6.83 & 8 & -8.22 & & & \\
\hline 18 & 16 & $34-3.1$ & -14.6 & -271.55 & 1.1 & 0.00 & -7.81 & .23 & -17.90 & -1 & 0 . & -0.48 \\
\hline 18 & 17 & $35-4.2-$ & -13.5 & -285.62 & 1.32 & 1.87 & -8.87 & .30 & -22.90 & -2 & 1.17 & -0.15 \\
\hline $1 \varepsilon$ & 18 & 36 & 5 & .59 & 1.57 & & & 2 & -3 & & & 0.32 \\
\hline 18 & 19 & $37-6.5-$ & 5 & .55 & 1.72 & 1.80 & -8 & .95 & -31.41 & & 2 . & 0.46 \\
\hline $1 \varepsilon_{-}$ & 2 & $38-7.5-$ & -10.5 & -321.55 & 1.64 & 0.00 & -8.02 & -327.93 & -35.31 & -3 & 2.23 & 0.59 \\
\hline $1 \varepsilon$ & 21 & $39-8.6$ & -9.7 & -331.66 & 2.44 & 1.74 & -7.24 & -334.72 & -34.03 & .24 & 3.23 & 0.79 \\
\hline & 22 & 40 & -8.9 & & & & -6 & -3 & & & & 26 \\
\hline L $\varepsilon_{2}$ & 23 & $41-10.6$ & -8.1 & .44 & 2.7 & 1.69 & -5 & .63 & -34.80 & -3 & 5 & 1.73 \\
\hline $1 \xi$ & 2 & $42-11.6$ & -7.4 & -357.22 & 1.63 & 0.00 & -5.49 & -361.08 & -36.17 & .42 & 3.39 & 1.75 \\
\hline 18 & 2 & $43-12.5$ & -6.8 & 1.32 & 1.1 & 1.64 & -5.05 & .61 & -33.63 & -3 & 2.79 & 1.66 \\
\hline 18 & 2 & & & .79 & & & -4 & 6 & & & & 55 \\
\hline 18 & 2 & $45-14.3$ & -5.6 & -376.67 & -0 . & 1.60 & -4.32 & .45 & -30.33 & 72 & 5 & 0.61 \\
\hline 18 & 2 & $46-15.1$ & -5.1 & -381.99 & -0.9 & 0.00 & -4.01 & .96 & -29.77 & .72 & -0.90 & 0.05 \\
\hline 18 & 2 & -16.0 & -4.6 & .80 & -0.6 & 56 & -3 & -3 & -24 & 91 & -2.19 & -1.58 \\
\hline 18 & & & 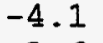 & & & & & & & & & \\
\hline 18 & 3. & $49-17.5$ & -3.6 & -3 & 1.7 & 53 & -3.28 & .00 & -1 & 0 & 0 & 00 \\
\hline 18 & 32 & -18.2 & -3.2 & -398.42 & 1.7 & 0.00 & -3.09 & .71 & -10 & 0.00 & 0.00 & 0.00 \\
\hline L & & $1-19.0$ & -2.8 & -4 & 2. & & 1 & & -3 & 0.0 & 0 & .8 \\
\hline 18 & 34 & $2-19.6$ & -2.5 & 1.12 & 2 . & 0.00 & -2.75 & .83 & 0. & & & \\
\hline 18 & 3 & $53-20.3$ & -2.1 & .43 & 2.3 & 1.47 & -2.60 & .17 & 8. & 0 & 0 & 00 \\
\hline 18 & - & -20.9 & -1.8 & 4.4 & 2.7 & 0 & -2.47 & -4 & 3 & 0 & 0 & 0.8 \\
\hline & & 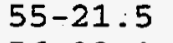 & & & & & & & & & & \\
\hline 18 & 38 & $6-22.1$ & -1.2 & .46 & 1.1 & 0 & -2.23 & .52 & 25 & & & 0.00 \\
\hline 18 & 35 & -22.7 & -1.0 & -412.57 & 1.5 & & -2.13 & .71 & 34 & n. & 0 & \\
\hline & 40 & $8-2$ & -0.7 & & & & & & & 0.0 & 0 & 0 \\
\hline 18 & 41 & -23.7 & -0.5 & -414.05 & 1.2 & & -1.95 & .33 & 48.78 & & & \\
\hline $18, \quad$ & 42 & -24.2 & -0.3 & -414.45 & 0.7 & & -1.86 & .58 & 5161 & & & \\
\hline 18 & 4 & -24.6 & -0.1 & 14.65 & 0.24 & & 179 & -415.31 & 62.95 & 0 & 0.00 & 0 \\
\hline & & 0.0 & & & & & & & & & & \\
\hline L) & 4 & 0.0 & 0. & 0. & -1 & & & 0 & & & & 0.00 \\
\hline & 46 & 0.0 & 0.0 & 0.00 & -1.59 & & -1.59 & 0.00 & 0.00 & 0.00 & 0.00 & 0.00 \\
\hline
\end{tabular}




\begin{tabular}{|c|c|c|c|c|c|c|c|c|c|c|c|c|c|}
\hline & 7 & 5 & 0.0 & 0.0 & 00 & -1.24 & 1.33 & -1.54 & .00 & 00 & 00 & 0.00 & 0.00 \\
\hline 18 & 48 & 6 & 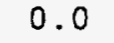 & 0.0 & & & 0.00 & & 00 & & & & \\
\hline & 49 & 67 & 0.0 & 0.0 & & - & 31 & - & 0 & & & & 0 \\
\hline & 10 & 29 & 0. & 0.0 & 0.00 & .22 & 1.80 & -2.72 & 0.00 & 0.00 & .00 & 00 & .00 \\
\hline & 1 & 0 & & 22.3 & -179.44 & 2.25 & 3.27 & -3.26 & -177.18 & 08 & .00 & 00 & .00 \\
\hline & 12 & 1 & & 9 & -201.02 & & & & & & & & \\
\hline & 3 & 2 & & & -221.23 & 1.88 & 3.19 & & -220.72 & & & 00 & 0 \\
\hline & 14 & 3 & 0.3 & 18.2 & -240.10 & 0.91 & 1.80 & -5.29 & -242.68 & 8.79 & 0.00 & .00 & 0.00 \\
\hline & 15 & 4 & $-0.8-$ & 17.0 & -257.69 & 0.90 & 3.12 & -6.10 & -259.77 & -0.22 & .00 & .00 & \\
\hline & 16 & 35 & & 58 & -274.04 & & .80 & -6.98 & -277.92 & $-10 \cdot 30$ & -11.17 & & \\
\hline & 7 & 6 & -3 & & -289.23 & 1.48 & 6 & -7.92 & -292.61 & -16.92 & 7.42 & & \\
\hline & 18 & 37 & -4 & .6 & 31 & 1.72 & 80 & -8.93 & -308.72 & -24 & -2 & 1.88 & 0.16 \\
\hline & 19 & 38 & $-5.2-$ & 12.5 & -316.36 & 1.87 & 38 & -10.00 & -320.68 & -28.85 & -28.80 & 1.92 & 0.05 \\
\hline & 20 & 39 & -6. & 11.6 & -328.43 & 1.79 & & & -333.82 & -33.92 & & & \\
\hline & 1 & 40 & -7.3 & 10.7 & -339.58 & 2.58 & 77 & -8.11 & -342.14 & -34 & -33.53 & 3.21 & 0.63 \\
\hline & 22 & 41 & 8.3 & .9 & -349.88 & 2.58 & & & 2.85 & -36.80 & -35.56 & & 1.24 \\
\hline & 3 & 2 & -9.3 & -9.1 & -359.37 & 2.25 & .94 & -6.70 & -360.89 & -36.77 & -3 & & 1.75 \\
\hline & 24 & & 0 & -8.4 & -368.11 & 1.78 & & & -370.67 & -38 & & & \\
\hline & 25 & 4 & 1.1 & -7.7 & -376.14 & 1.27 & 1 & -5.64 & -377.61 & -37.34 & -3 & 2.80 & 1.53 \\
\hline & 26 & & 2.0 & -7.1 & -383.52 & 0.75 & 0 & -5.20 & -386.17 & -37.84 & -3 & 1.98 & 1.23 \\
\hline & 27 & & 2.9 & -6.5 & .27 & 0.10 & .88 & -4.82 & -392.10 & -35.70 & & 0.38 & 0.28 \\
\hline & 28 & 4 & 3.8 & -5.9 & .44 & -0.79 & 0. & -4.47 & -399.90 & -35.43 & 59 & .05 & \\
\hline & 29 & 4 & .6 & -5.4 & -402.07 & -0.46 & 6 & -4.17 & -403.83 & -31.28 & -3 & -1.29 & -0.84 \\
\hline & 20 & & & -4.9 & 7.18 & 1.07 & 30 & -3.90 & -408.21 & -27.59 & .32 & .66 & .73 \\
\hline & 31 & & & -4.4 & -4 & 1.69 & & & .93 & -22.24 & & & \\
\hline & 32 & 5 & .9 & -4.0 & .99 & 2.33 & 0 & -3.43 & -415.29 & -18.53 & 0.00 & 10 & 0 . \\
\hline & 33 & & .6 & -3.5 & .14 & 2.78 & .82 & -3.23 & -417.37 & -12.53 & 0.00 & 0.00 & 0.00 \\
\hline & 34 & & & & & 3.09 & & -3.05 & 1.25 & -8 & & & \\
\hline & 35 & & & -2 & & 3 & & 38 & $-6<+3$ & & & & \\
\hline & 36 & 5 & .6 & -2.4 & .69 & 4.12 & 0 & -2.73 & 5.50 & 3 . & 0 . & 0 & 0. \\
\hline & 37 & & 0.3 & -2.1 & -4 & 3.49 & 9 & -2.59 & -427.29 & 9. & 0.00 & 0.00 & 0.00 \\
\hline & 38 & & & -1.8 & & & & 7 & 1 & & & & \\
\hline & 39 & & 21 & -1.5 & -4 & 5 & & & & & & & \\
\hline & 40 & & .0 & -1.3 & -436.02 & 2.51 & 0 & -2.24 & 3.96 & 27. & 0 & 10 & 10 \\
\hline & 41 & & 22.5 & -1.0 & .16 & 2.17 & 2.76 & -2.14 & -434.38 & 35 & 0 . & 0 & 0 \\
\hline & 4 & & 0 & -0 & & 1 & & & & & & & \\
\hline & 43 & & & -0 . & & 1 & & -1 & & & & & \\
\hline & 44 & - & 24.0 & -0.3 & -439.17 & 0.29 & 1.80 & -1.89 & 8.97 & 54 & 0.00 & 0 & Do \\
\hline & 4 & & 24.4 & -0.2 & .43 & 1.62 & $A$ & -1.82 & 6.89 & 6 & 0 & 0 & 0 \\
\hline & 4 & & & & & & & & & & & & \\
\hline & 47 & 6 & 0.0 & 0 . & 0. & 0 & 2 & -1 & 0. & 0. & & & \\
\hline & 4 & 6 & 0.0 & 0 . & 0.00 & -0.25 & 0 & -1 & 0. & 0. & 0 & & 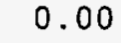 \\
\hline & 45 & & 0.0 & 0 . & 0.00 & & & -1 & 0 & & & & \\
\hline & & & & & & & & & & & & & \\
\hline & 5 & 7 & 0.0 & 0. & 0 . & -1.98 & & -1 & 0 & 0. & & & \\
\hline & 1 & & 0.0 & 0. & 0. & 2.11 & & -2 & 0. & 0. & & & \\
\hline & 1. & 31 & 0 & 0. & & & & & & & & & \\
\hline & 12 & 3 & $3.3-2$ & 21.9 & 3 . & 1.67 & 0 . & -3 & & & & & \\
\hline & 1 & 3 & 2.3 & 0.6 & -2 & 1. & 2.04 & -4 & -2 & 31.03 & 0 & & \\
\hline & 14 & 34 & 1.3 & 3 & -2 & 0 & & & - & 15 . & 0 & & \\
\hline & & & & & & & & & & & & & \\
\hline & 16 & 36 & -0.8 & & -2 & 2 & 0 & -6 & 0.44 & -5 & -6 & & 90 \\
\hline & 1 & 3 & -1.9 & .7 & -2 & 1 & 7 & -7 & 5.50 & -12.53 & 6 & 16 & -0 \\
\hline & 18 & 38 & $-2.9+5$ & & & 1.6 & & & 9 & -22 & & & \\
\hline & 19 & 39 & & .6 & & & & -8.98 & & & & & \\
\hline & $2 c$ & 40 & -5.0 & .6 & & 1 & 0. & & 2.32 & -35.13 & 35 & & \\
\hline & 2 & 41 & -6.0 & .7 & $-3+3+$ & $2.2+3$ & 1.7 & $-s$ & 1.00 & -35.74 & -4 & & \\
\hline & 2 & 42 & & & & & & & & & & & \\
\hline & 2 & 43 & $-8.0-$ & -10.1 & & 2. & & -7 & 1.58 & -4 & & & \\
\hline & 2 & 4 & -8.9 & & & & & & 2.78 & & & & \\
\hline & 25 & $4 !+2>$ & 9.8 & .6 & 5 & & 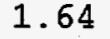 & & 0.05 & -42.50 & 1 & & \\
\hline & 2 & & & -7.9 & & t & & -5.78 & & & & & \\
\hline & 27 & & & 7.3 & & & & & 2 & -42. & & & \\
\hline & 28 & & & 67 & & -0.8 & & & 5.33 & -43.56 & & & \\
\hline & 29 & & & -6.2 & & -0.4 & & -4.62 & .54 & - & -41.29 & 2.07 & \\
\hline & 30 & & & & 1.5 & 36 & & $10^{\circ 2}$ & 25.36 & 37.45 & -39.57 & -1.26 & 2.12 \\
\hline
\end{tabular}




\begin{tabular}{|c|c|c|c|c|c|c|c|c|c|c|c|c|}
\hline 0 & 31 & 4.8 & -5.2 & -427.30 & 1.50 & 1.53 & -4.04 & -42 & -32.34 & 01 & -1.17 & 1 \\
\hline 20 & 2 & .6 & -4.7 & -432.23 & 2.13 & 0.00 & -3.79 & -433.89 & -29.84 & 0.00 & & \\
\hline & 33 & 16.3 & -4.3 & -436.71 & 2.48 & 50 & -3.57 & -436.30 & -24.18 & 0 & & \\
\hline & 34 & -17.0 & -3.8 & -440.76 & 2.68 & 0.00 & -3.37 & -441.44 & -21.25 & 00 & 0.00 & .00 \\
\hline & 35 & $5-17.7$ & -3.5 & -444.41 & 2.79 & 1.47 & -3.18 & -443.33 & -15.07 & 0.00 & 0.00 & 0.00 \\
\hline & 36 & 8.4 & -3.1 & -447.68 & 2.68 & 0.00 & -3.01 & .02 & -11.68 & 0.00 & & \\
\hline & 37 & 9.0 & -2.7 & -450.60 & 3.39 & 1.44 & -2 & .63 & -4.22 & & & \\
\hline & 38 & $58-19.6$ & -2.4 & -453.18 & 2.41 & 0.00 & -2.72 & -453.49 & -1.01 & 0.00 & 0.00 & 0 \\
\hline & 39 & $59-20.2$ & -2.1 & -455.45 & 2.52 & 1.42 & -2.59 & -454.10 & 6.45 & 0.00 & 0.00 & 0.00 \\
\hline & 40 & $60-20.8$ & -1.8 & -457.41 & 244 & 0.00 & -2.47 & .44 & 11.18 & 0.00 & 00 & \\
\hline & 41 & $61-21.3$ & -1.5 & .10 & 1.68 & 39 & & 38 & 18.31 & & & \\
\hline & 42 & $62-21.9$ & -1.3 & .51 & 1.42 & 0.00 & -2.25 & .35 & 23.42 & 0.00 & 0.00 & 00 \\
\hline & 43 & $63-$ & -1.0 & -461.68 & 1.64 & 1.37 & -2.16 & .83 & 32.01 & & & 0.00 \\
\hline & 44 & $64-22.9$ & -0.8 & -462.61 & 1.07 & 0.00 & -2.07 & 61 & 37.30 & 0 & 0 & 00 \\
\hline & 45 & 23.4 & -0.6 & .31 & 0.79 & .35 & -1.99 & -4 & 45.81 & & & \\
\hline & 46 & $66-23.8$ & -0.4 & -463.81 & 0.15 & 0.00 & -1.91 & .58 & 51.47 & 0.00 & & \\
\hline & 47 & $67-$ & -0.2 & .12 & -0.42 & .33 & -1.84 & .05 & 60.07 & & & 0.00 \\
\hline & 48 & $68-24.6$ & 0.0 & -464.25 & -1.17 & 0.00 & -1.77 & 7.19 & 66.00 & 10 & 0 & 0.00 \\
\hline & 49 & 0.0 & 0.0 & 0.00 & -1.85 & 31 & -1.71 & 0.00 & 0.00 & & 0.00 & \\
\hline & 50 & 0.0 & 0.0 & 0.00 & -3.12 & .00 & -1.65 & 0.00 & 0.00 & 0.00 & 0.00 & 0.00 \\
\hline & 51 & 0.0 & 0.0 & 0.00 & & .29 & -1.60 & 0.00 & 0.00 & & & 00 \\
\hline & 52 & 0.0 & 0.0 & 0.00 & -2.57 & 0.00 & -1 & 0.00 & 0.00 & 10 & 10 & 0 \\
\hline & 53 & 0.0 & 0.0 & 0.00 & -2.48 & 1.28 & -1.50 & 0.00 & 0.00 & 0.00 & 0.00 & 0.00 \\
\hline & 11 & 0.0 & 0.0 & 0.00 & 30 & 3.24 & -2.69 & 0.00 & 0.00 & 0.00 & & 0.00 \\
\hline & 12 & 0.0 & 0.0 & 0.00 & 7 & 1.74 & -3.18 & 0.00 & 0.00 & & & 0.00 \\
\hline & 13 & 3.2 & 21.5 & -216.64 & 2.66 & .15 & -3.72 & -214.55 & 43.43 & 0.00 & 0 & 0.00 \\
\hline & 14 & 2.2 & 20.3 & -237.55 & 1.72 & .74 & -4.32 & .41 & 27.64 & 0.00 & 0. & 0.00 \\
\hline & (כ) & 36 & 9.1 & -257.22 & 1.69 & 3.08 & -4.97 & .41 & 16.71 & 0 . & 0 & 0.00 \\
\hline & 16 & 37 & -17.9 & -275.68 & 2.06 & 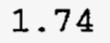 & -5.67 & .54 & 4.65 & & & \\
\hline & 17 & -0.8 & -16.7 & -292.97 & 2.22 & 3.02 & -6.43 & 1.16 & -3.89 & 0 & 0 & 0.00 \\
\hline & 18 & $39-1.8$ & -15.7 & -309.16 & 2.44 & 1.74 & -7.24 & .22 & -13.88 & -1 & 2.16 & -0.29 \\
\hline & 19 & $40-2$ & -14.6 & 4.29 & 2.5 & 7 & -8.11 & .84 & -20 & & & 9 \\
\hline & 20 & 41 & -13.6 & .42 & 2.4 & 4 & -9.03 & .22 & -28.74 & -2 & & 0 \\
\hline & 21 & $42-4.8$ & 12.7 & -351.59 & 3.24 & $.65-$ & -10.00 & -354.70 & -32.16 & .12 & 3. & 0.04 \\
\hline & 2 & $43-5.8-$ & -11.8 & -363.87 & 3.23 & 1.74 & -9.07 & .96 & -37.34 & -3 & 4.39 & 1.15 \\
\hline & 23 & 44 & -11.0 & & 2.90 & & & & -3 & & & \\
\hline 2 & 24 & $45-7$ & -10.2 & 5.93 & 2.4 & 1.74 & -7.56 & .31 & -42.55 & -8 & 3 . & 1.48 \\
\hline & 25 & $46-8.6$ & -9.5 & -395.80 & 1.93 & 2.87 & -6.94 & -397.95 & -43.11 & -41.76 & 3.28 & 1.35 \\
\hline & 26 & $47-9.5$ & -8.8 & -404.97 & 1.42 & 1.74 & -6.40 & .21 & -45.30 & -4 & 2 . & 0.97 \\
\hline & 27 & $48-10.3$ & -8.2 & .47 & & 4 & & .77 & -44 & & & \\
\hline & 28 & $49-11.2$ & -7.6 & .33 & -0. & 1.74 & -5.49 & .18 & -46.13 & 56 & -0.53 & -0.43 \\
\hline & 29 & -12.0 & -7.0 & -428.61 & 0.2 & 2.82 & -5.11 & .68 & -43.56 & 53 & -0.75 & -0.97 \\
\hline & 30 & 8 & -6.4 & & & & & & -42 & & & 3 \\
\hline 27. & 31 & -13.6 & -5.9 & .50 & 1.0 & 2.79 & -4.46 & .49 & -38.23 & 0 & 0. & 0. \\
\hline & 32 & -14.3 & -5.4 & .18 & 2.60 & 1.74 & -4.18 & .02 & -35.68 & 0.00 & 0. & 0.00 \\
\hline & 33 & .0 & -5.0 & -4 & 3.2 & 7 & -3 & 27 & -30 & & & 0. \\
\hline & 34 & .8 & -4 & -4 & & & & & & & & \\
\hline . & 35 & 6.4 & -4.1 & .48 & 4.5 & 2.76 & -3.50 & .22 & -22.67 & 0 & 0. & 0.00 \\
\hline & 36 & $57-17.1$ & -3.7 & .42 & 4.0 & 1.74 & -3.31 & . 91 & -19.28 & 0 & 0.00 & 0.00 \\
\hline & 3 & -17.8 & -3.4 & -4 & 4 . & 2.74 & -3.14 & .16 & -13 . & 0.00 & 0 & 0.1 \\
\hline & 3 & .4 & -3 & I & 5 & & & -4 & & & & 0.0 \\
\hline & 39 & 9.0 & -2.7 & .06 & 52 & 2.72 & -2.84 &. .65 & -3 & 0 & 0.00 & \\
\hline ?. & 40 & $61-19.6$ & -2.4 & -477.61 & 2.56 & 1.74 & -2.70 & -4 & -0.10 & 0.00 & 0.00 & 0 \\
\hline & 41 & -20.2 & -2.1 & 8 & 2 & 1 & -2.58 & 0 & 7 & 0.00 & 0 & 0. \\
\hline & 42 & & & & & & & & & & & \\
\hline - & 43 & .2 & -1.6 & -4 & 2.4 & 2.70 & -2 & .67 & 5 & & 10 & 0 \\
\hline $21+1$ & 44 & -21.8 & -1.3 & -484.9 & 19 & 74 & -2.26 & .55 & 24.64 & 0 & 0.00 & 0 \\
\hline & 45 & .3 & 1.1 & & 5 & & 7 & & & & & 0.00 \\
\hline & 46 & 2.7 & -0.8 & -487.08 & 0.79 & 1.74 & 99 & .63 & 37.70 & & 0 . & \\
\hline 8. & 47 & -23.2 & -0.6 & -487.82 & 0.25 & 2.67 & -2 & .91 & 45.50 & 0 & 0. & \\
\hline & 48 & $69-23.6$ & 0.4 & -488.37 & 0.55 & .74 & 1.93 & .11 & 51.37 & 0.00 & 0.00 & 0.00 \\
\hline & 4 & & -0.3 & & & & & & & & & \\
\hline & 50 & $71-24.4$ & -0.1 & -488.9 & -1.9 & 74 & 0 & -490.89 & 65. & & & \\
\hline 3. & 51 & 0.0 & 0.0 & 0.00 & 208 & & & 0.00 & 0.00 & & & \\
\hline & 5 & 0.0 & 0.0 & .00 & & & & & & & & \\
\hline & 53 & 0.0 & 0.0 & 0.00 & -2.17 & & -1.63 & 0.00 & 0.00 & 0.00 & 0.00 & 0.00 \\
\hline
\end{tabular}




\begin{tabular}{|c|c|c|c|c|c|c|c|c|c|c|c|c|c|}
\hline & & 75 & 0.0 & 0.0 & & -1.72 & 1.74 & 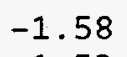 & 30 & 0 & 0 & 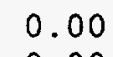 & \\
\hline & 5 & 6 & 10 & 0 & & & 2.63 & -153 & & 0.00 & 0.00 & Do & 0 \\
\hline & 2 & 4 & 0. & 0. & 0 & & 00 & & & & 0 & po & \\
\hline & 13 & 35 & 3 & & 213.09 & & & & .78 & - . & .00 & 00 & 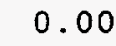 \\
\hline & & 6 & 3. & -21.2 & 234 & & 00 & 3.93 & .237 .11 & & & & \\
\hline & 15 & 7 & & & & & & & & & & & \\
\hline & 6 & 8 & 1 & & -21 & & & & 2 & & & .00 & 0.00 \\
\hline & 7 & y & 0.2 & .7 & -293. & & 1.87 & & -295.00 & & & .00 & .00 \\
\hline & 18 & 0 & 0.8 & -16.6 & -310.44 & 2 . & 0.00 & 57 & -314.56 & -8 & & & -0.12 \\
\hline & & 41 & 1.7 & & -326.56 & & & & & -15.84 & & & \\
\hline & 20 & 42 &. & 6 & -32 & & 0.00 & & -347.36 & 50 & & & \\
\hline & 21 & 3 & 3.1 & .7 & -355.82 & & & & 9.91 & -30.07 & -29 & .99 & 75 \\
\hline & & 4 & 4.6 & .8 & & 3. & & & -375.82 & -37.91 & -3 & & 0.36 \\
\hline & & 45 & $-5.5-$ & -12.0 & -381.42 & 2.90 & 9 & & & & & & \\
\hline & $z$ & 46 & & -11.2 & 0 & & & & & 32 & & & \\
\hline & 25 & 47 & -7 & -10.4 & -76 & & 4 & & -4 & & & & \\
\hline & & 48 & -8.2 & -9.7 & -41 & 1 . & 0 & -7 & .41 & -4 & & 2.17 & 0.73 \\
\hline & & 9 & -9.1 & -9.0 & -423.17 & 0.81 & 1.60 & & & -4 & & & 45 \\
\hline & 8 & & 9.9 & -8.4 & -43 & & & & & & & & \\
\hline & 29 & & 10.7 & -7.8 & -439.97 & 0. & 6 & -5 & .76 & -4 & -4 & -0.12 & \\
\hline & 30 & & 1.5 & -7.2 & . & 1. & 0 & -5 & -4 & -4 & & & -0.26 \\
\hline & & & 2.3 & -6.7 & & 1 & & & & & & & 30 \\
\hline & 32 & & & -6.2 & & & & & & & & & 00 \\
\hline & 33 & 55 & 3.8 & -5.7 & -4 & 2 . & 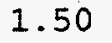 & -4 & & -4 & & & 10 \\
\hline & 34 & & 4.5 & -5.3 & .28 & 3. & & -4 & & & & & 0 \\
\hline & & & 5.2 & -4.8 & & & & & & & & & 00 \\
\hline & 36 & & & -4. & - & & & & & & & & \\
\hline & $3^{\prime}$ & & .6 & -4.0 & -4 & 3. & & -3 & & -2 & & & Uu \\
\hline & 38 & & 7.2 & -3.7 & 8 & 3. & & 6 & & & & & 00 \\
\hline & 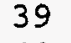 & & & -3.3 & & & & & & & & & 00 \\
\hline & 40 & & & -3.0 & & 2 . & & & & -1 & & & 00 \\
\hline & 41 & 63 & .0 & -2.7 & -4 & 3. & & -2 & -4 & -6 & & & 00 \\
\hline & 42 & & 9.6 & -2.4 & & 2 . & & & & & & & \\
\hline & 43 & & & -2.1 & & & & & & & & & 0 \\
\hline & 44 & & & -1.8 & & 1 . & 0 & -2 & & & & & bo \\
\hline & 45 & & 21.1 & -1.6 & & 1. & 5 & -2 & & 15 & & & 00 \\
\hline & 4 & & 21.6 & -1.3 & & 0. & & & & & & & \\
\hline & 4 & & & -1.1 & & & & & & & & & \\
\hline & 4 & & 2.6 & -0.9 & & -0 & & -2 & & & & & 0 \\
\hline & 4 & & 23.0 & -0.7 & & -1 & 1 & -2 & & & & & \\
\hline & 5 & & .4 & -0.5 & & -2 & & & & & & & \\
\hline & 5 & & & -0.3 & & & & & & & & & \\
\hline & 5 & 7 & -24.2 & -0.1 & 5 & -2 & 0 & -1 & & 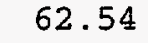 & & & 00 \\
\hline & 5 & 75 & 0.0 & 0.0 & 0 & -1 & 8 & -1 & & & & & 00 \\
\hline & 5 & & 0.0 & 0.0 & 0 & & & & & & & & \\
\hline & & & & & & & & & & & & & \\
\hline & & & & 0.0 & & & & & & & & & 00 \\
\hline & 5 & 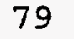 & 0 . & 0.0 & 0 & -1 & & & & & & & 00 \\
\hline & 5 & & 0. & 0 & & & & & & & & & \\
\hline & 1 & 3 & & & & & & & & & & & \\
\hline & 1 & 3 & 3.8 & -22.1 & -2 & & & -3 & & & & & 00 \\
\hline & 1 & & 30 & -20.9 & & & & & & & & & \\
\hline & 1 & 3 & & & & & & & & & & & \\
\hline & 1 & 4 & 1. & 7 & & & & & & & & & \\
\hline & 1 & 4 & & 6 & 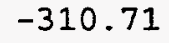 & & & & & & & & 00 \\
\hline & 1 & 42 & -0.7 & 6 & & & & & & & & & \\
\hline & 2 & & & & & & & & & & & & \\
\hline & & & -2 & & & & & & & & & & \\
\hline & 2 & & -3 & & & & & & & & & & \\
\hline & 2 & -5 & -4 & & 6 & & & & & 4 & & & \\
\hline & 2 & 4 & & & & & & & & & & & \\
\hline & 2 & 4 & -6 & & & & & & & & & & \\
\hline & 2 & 4 & $-7.1-$ & & & & & & & & & & \\
\hline & 2 & 50 & & & & & & & & 2 & & & \\
\hline & 2 & 5 & & & & & & & 45 & 33 & & & \\
\hline & 2 & 2 & & 8.6 & 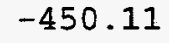 & & . & & 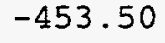 & 1.80 & -1 & 0 & 0.3 \\
\hline
\end{tabular}




\begin{tabular}{|c|c|c|c|c|c|c|c|c|c|c|c|c|c|}
\hline & 30 & \multicolumn{2}{|c|}{$53-10.4$} & 8.0 & 2 & 36 & & 4 & 1 & $-5]$ & 85 & 86 & 0 \\
\hline & 31 & \multicolumn{2}{|c|}{$54-11.1$} & -7.5 & -466.15 & 2.12 & 2.75 & -5.37 & -466.64 & -48.81 & & .04 & -1.08 \\
\hline & 32 & \multirow{2}{*}{\multicolumn{2}{|c|}{$\begin{array}{l}55-11.9 \\
56-12.6\end{array}$}} & -6.9 & -473.34 & 253 & 1.69 & -5.03 & -474.15 & -48.24 & -49.15 & 1.62 & -0.91 \\
\hline & 33 & & & & -480.02 & & & & & .77 & .00 & .00 & 0.00 \\
\hline & 34 & \multicolumn{2}{|c|}{$57-13.3$} & -6.0 & -486.21 & 3.49 & .69 & -4.45 & -485.48 & -43.43 & 00 & 00 & 0.00 \\
\hline & 35 & \multicolumn{2}{|c|}{$58-14.0$} & -5.5 & -491.94 & 4.03 & .71 & -4.19 & -489.39 & -39.27 & & 00 & .00 \\
\hline & 36 & \multicolumn{2}{|c|}{$59-14.7$} & -5.1 & & & .69 & -3.96 & & -37.43 & & & .00 \\
\hline & 37 & \multicolumn{2}{|c|}{$60-15.4$} & & & & & -3.75 & 98.75 & -32.49 & & 00 & .00 \\
\hline & 38 & \multicolumn{2}{|c|}{$61-16.0$} & 4.3 & & & 69 & -3.56 & 43 & $-3 c$ & 0.00 & .00 & 0.00 \\
\hline & 39 & \multicolumn{2}{|c|}{$62-16.6$} & -3.9 & & 3.89 & .68 & -3.38 & & -25.09 & 0.00 & .00 & .00 \\
\hline & 40 & $63-$ & 17.3 & 3.6 & 44 & 3.26 & & -3.22 & & & & & 00 \\
\hline & 41 & & & & & & & 7 & & 86 & & & .00 \\
\hline & 42 & & & & & & 69 & -2.93 & & 51 & 0.00 & .00 & .00 \\
\hline & 43 & & .0 & 2.6 & & 2.25 & 65 & -2.80 & & -6.91 & 0.00 & .00 & .00 \\
\hline & 44 & $6^{\circ}$ & 19.5 & -2.3 & -5 & 2.15 & .69 & -2.68 & & & & & .00 \\
\hline & 45 & & & 1 & & 9 & 64 & & & & & & 00 \\
\hline & 46 & 69 & .5 & 1.8 & & 0.85 & 9 & -2.47 & & 8.62 & 0.00 & Do & .00 \\
\hline & 47 & & .0 & 1.6 & -5 & 0.33 & 63 & -2.37 & & 15.52 & 0.00 & .00 & 0.00 \\
\hline & 48 & $71-$ & 21.5 & 1.3 & & -0.33 & .69 & -2.28 & & & & & .00 \\
\hline$z$ & 49 & 72 & 0 & & & & 62 & -2.19 & & & & Do & 0.00 \\
\hline & 50 & 73 & .4 & -0.9 & -5 & -2 . & 9 & -2.12 & & 32.97 & 10 & .00 & 0.00 \\
\hline & 51 & 74 & .8 & -0.7 & 58 & -1.85 & .61 & -2.04 & & 41.41 & & & .00 \\
\hline & $2 \Delta$ & 75 & .3 & -0.5 & & -1. & .69 & -1.97 & & & 0 & & .00 \\
\hline & 53 & $76-$ & .7 & 0.4 & & & 0 & -1.91 & & & & 0 & 0.00 \\
\hline 25 & 54 & 77 & 24.0 & -0.2 & 92 & -0. & 9 & -1.84 & 0 & 78 & 10 & 0.00 & 0.00 \\
\hline & 55 & $78-$ & 24.4 & 0.0 & -538.03 & -0.4 & .59 & -1.79 & 3 & 73.92 & 0.00 & 20 & 0.00 \\
\hline & 30 & 79 & 0.0 & 0.0 & & & 9 & -1.73 & & & & 0 & .00 \\
\hline & 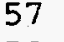 & 80 & 0.0 & 0 & & & & -1 & & & & & 00 \\
\hline 33 & 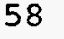 & 81 & 0.0 & 0. & 0.00 & - & 9 & -1 & 0. & 0.00 & 0 & 0 & 0.00 \\
\hline$=$ & - & 82 & 0.0 & 0.0 & 0.00 & 2 & 7 & -1.58 & 0. & 0.00 & 0 & 0 & 0.00 \\
\hline & 60 & 83 & 0.0 & 0.0 & & & & -1 & & & & & 00 \\
\hline & 1 & 38 & 5 & 2.9 & -22 & & & & & & & & 00 \\
\hline & 1 & 39 & .7 & .8 & -2 & 0. & 5 & -3.79 & -2 & 7 & 0 & 0 & 0.00 \\
\hline & s & 40 & 2.9 & 0.7 & -27 & 1.2 & .00 & -4.32 & -2 & 3 & & & 0.00 \\
\hline & 17 & 41 & 2.0 & .6 & & & & & & & & & \\
\hline & 1 & 42 & & & & & & & & & & & \\
\hline 14 & 19 & 43 & $0.2-$ & .5 & -3 & 1 & 0 & -6 & -3 & -2 & 0 & 0 & 0.00 \\
\hline & 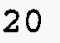 & 44 & $-0.7-$ & 5.5 & -3 & .70 & .00 & -6.83 & -3 & -13 & -1 & 4 & 24 \\
\hline & 21 & 45 & -1.6 & & & & & 6 & & & & & \\
\hline & 22 & 46 & & & & & & & & & & & 13 \\
\hline 4 & 23 & 47 & -3.4 & 8 & -3 & 2 & .69 & -9.15 & -3 & 25 & 55 & 4 & 0.50 \\
\hline & 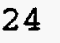 & 48 & 4.2 & .9 & -4 & 1. & & -10 & -4 & -4 & & & 19 \\
\hline & 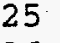 & 9 & & & & & & & & & & & \\
\hline \pm & 26 & 50 & & + & & & & -8 & 7 & 0 & & & 54 \\
\hline & 27 & 51 & $6.8-$ & 7 & -4 & 0.1 & 0 & -7.81 & -4 & -5 & -5 & 4 & 80 \\
\hline & 2 & 52 & $7.6-$ & 0 & -4 & .6 & .00 & -7 & 1 & & & & 9 \\
\hline & 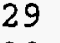 & 53 & 4 & & & & & & & & & & \\
\hline & 30 & 54 & & & & & & -6 & 2 & -56 & & 8 & 6 \\
\hline & 31 & & 10.0 & -8.2 & -4 & 1. & .53 & -5.86 & 5 & -54. & & 0 & 77 \\
\hline 28 & 32 & & .7 & -7.7 & -4 & 1. & 0.00 & -5 & 4 & 5 & & 4 & 4 \\
\hline 4 & 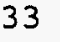 & & & 2 & & & & & & & & & \\
\hline & 4 & & & 7 & & 2 . & & -4 & & 7 & & & \\
\hline 24 & 35 & & & -6.2 & & 3 & .47 & -4.57 & & -4 & & & 0 \\
\hline 24 & 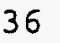 & & .6 & 5.8 & -5 & 3 & 0.00 & -4 & 2 & -4 & & & \\
\hline 24 & ( & & .2 & .3 & & & & & & & & & \\
\hline 4 & 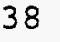 & & & & & & & -3 & & & & & \\
\hline & 39 & & & .5 & & & & -3.68 & & & & & \\
\hline 2 & 40 & & .1 & 4.2 & -5 & 2 & 0.00 & -3.50 & 8 & -3 & & & \\
\hline 24 & 41 & & & .8 & & & & & & & & & \\
\hline & -7 & & & & & & & -3.18 & & & & & \\
\hline & 43 & & & 2 & & & & & & & & & \\
\hline$?$ & 44 & 68 & 4 & & 1 & & & 2 & 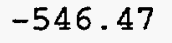 & -1 & & & \\
\hline 24 & 45 & & & & & & & & & & & & \\
\hline & 46 & & & .3 & & & & & & -6 & & & \\
\hline & 47 & & & & & 1 & & & & & & & 0.00 \\
\hline & & & & & & & & & & & & & 0.0 \\
\hline
\end{tabular}




\begin{tabular}{|c|c|c|c|c|c|c|c|c|c|c|c|c|}
\hline & 9 & $73-20.9$ & 1.6 & 20 & -1.62 & & -2.37 & -558. & .53 & 0 & 0 . & 0 . \\
\hline & 0 & $74-21.4$ & 1.4 & 67 & -2.41 & 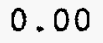 & -2.29 & -562.36 & 16.12 & 0.00 & 0.00 & 0.00 \\
\hline & 1 & \multirow{2}{*}{$75-21.8$} & -1.1 & 58.92 & -2.13 & 1.29 & -2.20 & -561.96 & 24.59 & 0.00 & 0.00 & 0.00 \\
\hline & 2 & & -0.9 & 559.96 & -1.74 & 0.00 & -2.13 & -563.83 & & .00 & .00 & .00 \\
\hline & 3 & $\begin{array}{l}10-22.3 \\
77-22.7\end{array}$ & & 81 & -1.39 & 28 & & 98 & & & & 00 \\
\hline & & $78-23.1$ & -0.6 & 61.47 & -1.09 & 0.00 & -1.99 & 4.55 & .22 & 0.00 & 0.00 & 0.00 \\
\hline & 5 & $79-23.5$ & -0.4 & 561.95 & -0.85 & 1.26 & -1.92 & -563.47 & 55.37 & 00 & 0.00 & 0.00 \\
\hline 4 & 6 & $80-23.8$ & -0.2 & -562.27 & & 0.00 & -1.86 & 3.69 & 21 & & & \\
\hline & 5 & $81-24.2$ & 0.1 & .43 & 0.41 & & -1.81 & .58 & 72.40 & 0.00 & & \\
\hline & 5 & 0.0 & 0.0 & 0.00 & 0.76 & 0.00 & -1.75 & 0.00 & 0.00 & 0.00 & 0.00 & 0.00 \\
\hline & 59 & 0.0 & 0.0 & 0.00 & 0.46 & 1.23 & -1.70 & 0.00 & 0.00 & 0.00 & 0.00 & 0.00 \\
\hline & 60 & 84 & 0.0 & 0.00 & 0 & & -1.65 & 0 & .00 & & & \\
\hline 4 & 61 & 85 & 0.0 & 0.00 & 0.83 & 2 & -1.61 & 0.00 & 0.00 & & & \\
\hline & 62 & 0.0 & 0.0 & 0.00 & 1.15 & .00 & -1.56 & 0.00 & 0.00 & 0.00 & 0.00 & .00 \\
\hline & & 4.4 & 22.6 & -245.54 & 0.35 & 3.02 & -3.50 & -245.67 & 57.60 & 0.00 & 0.00 & 0.00 \\
\hline & 16 & 41 & 21.5 & -267.59 & 0.72 & 1.64 & -3.98 & -269.21 & & & & \\
\hline 5 & 17 & 42 & 20.4 & -288.56 & 0.89 & 2.96 & -4.49 & -289.20 & 30.20 & 0 & 0 & 00 \\
\hline 5 & 18 & 43 & 19.4 & -308.47 & 1.12 & 1.64 & -5.05 & -310.75 & 16.73 & 0.00 & 0.00 & \\
\hline & 19 & 44 & 18.4 & 327.34 & 1.27 & 2.91 & -5.64 & -328.80 & 6.75 & & 0.00 & 0.00 \\
\hline 5 & 20 & 45 & 17.4 & -345.20 & 1.20 & 1.64 & -6.27 & & & & & \\
\hline 5 & 21 & $46-0.6$ & 6.4 & -362.09 & 1.93 & .87 & -6.94 & -364.23 & -12.54 & 0 & 0 & 0.00 \\
\hline & 22 & $\begin{array}{ll}47 & -1.5\end{array}$ & .5 & -378.05 & 1.95 & 1.64 & -7.65 & -382.11 & -22.35 & 0.00 & 00 & 0.00 \\
\hline & 23 & $48-2.3-$ & 14.6 & -393.12 & 1.65 & 2.83 & -8.39 & .04 & -29 & 20 & & \\
\hline & 24 & 49 & 13.8 & .34 & 1.23 & L. 64 & -9.18 & -41 & -37.74 & -3 & 36 & 13 \\
\hline & 25 & $50-4.0$ & 13.0 & -420.74 & 0.77 & 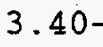 & 10.00 & -426.57 & -42.59 & -42 & 0.74 & -0.03 \\
\hline & 26 & $51-4.9-$ & 12.3 & -433.37 & 0.30 & .64 & -9.21 & -440.64 & -48.58 & & 0.64 & 0.34 \\
\hline & 27 & 52 & 11.5 & -44 & -0.29 & & -8.51 & $-4 !$ & -51.17 & & & \\
\hline & 28 & $53-6.5-$ & 10.8 & -456.44 & -1.11 & 1.64 & -7.88 & -463.78 & -55.59 & -5 & -0 & 0.90 \\
\hline & 2 & $54-7.3-$ & 10.2 & -466.95 & -0.80 & 2.74 & -7.33 & -472.33 & -56.06 & 55 & -0.28 & 0.51 \\
\hline & 30 & -8.1 & -9.6 & & 0.68 & 54 & -6.83 & .33 & & & & \\
\hline & 31 & 56 & -9.0 & & 0 & 2.72 & 38 & 3.25 & 84 & & 3 & 7 \\
\hline & 32 & $57-9.6$ & -8.4 & -494.79 & 1.96 & 1.64 & -5.97 & -497.16 & -56.68 & -5 & 1.15 & -0.81 \\
\hline & 33 & $58-10.3$ & -7.9 & .93 & 2.56 & 2.70 & -5.60 & -503.28 & -54.73 & 3 & 1.46 & -1.10 \\
\hline & 3 & & -7.4 & & 2.80 & .64 & -5.27 & .39 & & & & \\
\hline & 35 & 60 & -6.9 & .69 & 3.16 & 2.68 & -4.97 & -516.81 & -52.12 & & 1 & 05 \\
\hline & 36 & $61-12.4$ & -6.4 & -524.34 & 3.34 & 1.64 & -4.69 & -524.05 & -51.28 & 10 & 0.00 & 0.00 \\
\hline & 37 & $62-13.1$ & -6.0 & -5 & 3.96 & 2.66 & -4.44 & .36 & 52 & & & 0 \\
\hline & 38 & .7 & -5.6 & & & & & -5 & & & & \\
\hline & 35 & & -5.2 & -5 & 4.02 & 2 . & -3.99 & .01 & 13 & & & \\
\hline & 40 & $65-15.0$ & -4.8 & .66 & 3.79 & 1.64 & -3.79 & -545.02 & -39.97 & 0 & 10 & 00 \\
\hline & 41 & 66 & -4.4 & -5 & 3 . & 2.63 & -3 & 39 & & & & 10 \\
\hline & 42 & & -4 & & & & & & & & & \\
\hline & 43 & $68-$ & -3.7 & -5 & 2.52 & 2.62 & -3.29 & .58 & -2 & & & \\
\hline & 44 & $69-17.3$ & -3.4 & .02 & 1.75 & 1.64 & -3.15 & .77 & -2 & 0 & 0 & 00 \\
\hline & 45 & 70 & -3.1 & & 1. & 2 . & -3 & 14 & & & & \\
\hline & 4 & & & & & & & & & & & \\
\hline & 47 & $72-$ & -2.6 & .99 & -0.12 & 2.59 & -2.77 & .29 & -1 & & & 0 \\
\hline & 48 & $73-19.4$ & -2.3 & .43 & -0. & 1.64 & -2.66 & .32 & -6 & & 0 & 00 \\
\hline & 49 & & -2.1 & & & & & & & & & \\
\hline & 50 & $75-$ & -1.8 & -5 & -2 & 1. & -2.47 & -5 & & & & \\
\hline & 51 & $76-$ & -1.6 & .25 & -2.16 & 2.57 & -2.38 & 22 & 11 & & & 00 \\
\hline & 5 & 3 & 1.4 & & & 1. & -2 & & & & & \\
\hline & 5 & & & & & & & & & & & \\
\hline & 54 & 79 & -1.0 & -5 & -0 & 1 & -2 & -5 & & & & \\
\hline & $5:$ & $80-$ & -0.8 & -5 & -0 & 2 . & -2.07 & 7 & & & & 0 \\
\hline & 56 & & -0 & & & & & & & & & \\
\hline & 57 & $82-$ & -0.4 & & 0 . & 2 . & -1 & & & & & \\
\hline & 58 & $83-$ & -0.3 & .52 & 1.47 & 1. & -1.88 & 29 & 65 & & & \\
\hline & 59 & $84-24.0$ & -0.1 & -586.73 & 1.37 & 3 & -1.83 & 84.65 & 73 & & 10 & 0 \\
\hline & 6 & 85 & 0 . & & & & & & & & & \\
\hline 25 & 61 & 0.0 & 0.0 & & 1. & & -1 & & & & & \\
\hline & 62 & 0.0 & 0.0 & 0 & 1. & 1.64 & -1 & & & & & \\
\hline 25 & 63 & 0.0 & 0. & & 1 & & -1.63 & & & & & \\
\hline 25 & 64 & 89 & 0.0 & 0.00 & 1.1 & 1.64 & -1.59 & & & & & \\
\hline 26 & 16 & 4.4 & 22.3 & 57 & 0. & & -3.68 & 7 & & & & \\
\hline & 17 & 43 & 1.2 & 5.34 & 0.3 & .87 & & 7.27 & 39.43 & 0.00 & 0.00 & 0.00 \\
\hline
\end{tabular}




\begin{tabular}{|c|c|c|c|c|c|c|c|c|c|c|c|c|}
\hline 6 & 8 & 4 & -20.2 & -306.07 & 50 & 10 & .66 & -310.14 & 24.63 & 0.00 & 0.00 & 0.00 \\
\hline 26 & 19 & 5 & -19.2 & -325.78 & 0.75 & 1.80 & -5.20 & -328.44 & 14.40 & 0.00 & 0.00 & 0.00 \\
\hline 26 & 0 & 6 & 18.2 & -344.49 & 69 & 0.00 & & -349.58 & & .00 & & .00 \\
\hline & 1 & 7 & -17.3 & -362.24 & 42 & 74 & 40 & -365.48 & 0 & 0.00 & oo & 0 \\
\hline & 22 & 48 & $-0.5-16.4$ & -379.06 & .44 & 0.00 & .05 & 66 & -17.61 & -18.12 & 0.93 & -0. \\
\hline & 23 & 49 & 15.5 & -394.98 & 1.15 & 1.69 & -7.73 & -399.87 & -24.74 & -24.58 & 32 & 0 . \\
\hline 26 & 24 & 50 & -2 . & -410.04 & & 0.00 & & -417.75 & & & & \\
\hline 26 & 5 & 1 & $-3.0-13.8$ & -424.28 & 0.30 & 1.64 & & -431.55 & & -40.22 & & \\
\hline & 26 & 52 & $-3.9-13.1$ & -437.73 & -0.16 & 00 & -10.00 & -447.89 & -48.56 & -48.33 & 0.07 & \\
\hline & 27 & 3 & $-4.7-12.3$ & -450.43 & -0.74 & 1.60 & -9.24 & -458.81 & -51.40 & -50.94 & -0.28 & 0.46 \\
\hline 26 & 28 & 54 & -5 & -462.42 & 4 & 0.00 & 56 & -472.52 & -57 & -5 & & 0 . \\
\hline 26 & 29 & 55 & $-6.2-11.0$ & -473.72 & -1.22 & 1.56 & -7.95 & -481.34 & -57.79 & -57.47 & -0.91 & \\
\hline & 30 & 56 & $-7.0-10.3$ & -484.37 & 0.05 & 0.00 & -7.41 & -49 & -60.11 & -60.60 & & -0 \\
\hline & 31 & 7 & $\begin{array}{ll}-7.8 & -9.7\end{array}$ & -494.40 & 0.72 & 1.53 & -6.92 & -499.07 & -59.38 & -60.18 & -0.08 & -0.80 \\
\hline 26 & 32 & 58 & -8.5 & -50 & 1.22 & 0.00 & -6 & 9.10 & -61.33 & -62 & 40 & $-c$ \\
\hline 5 & 33 & 59 & $-9.2-8.6$ & -512.72 & 1.94 & 1.50 & -6.08 & -515.35 & -59.52 & -60.66 & 0.80 & \\
\hline & 34 & & $-10.0-8.1$ & -521.05 & 2.07 & 0.00 & -5.71 & -524.69 & -60.79 & -61.41 & 1.45 & -0.62 \\
\hline & 35 & & $0.6-7.6$ & -528.88 & 2.38 & 1.47 & -5.38 & -530.41 & -58.43 & -58.92 & 1.89 & -0.49 \\
\hline 6 & 36 & 62 & 7.1 & -536.21 & 2.59 & 0.00 & -5 & - & -58 & 9 & & \\
\hline 6 & 37 & 63 & -6.6 & -543.08 & 3.10 & 1.44 & -4.80 & -543.35 & -55.22 & -55.98 & 34 & -0.76 \\
\hline & 38 & & $2.6-6.2$ & -549.51 & 2.70 & 0.00 & -4.55 & -551.36 & -55.16 & 0.00 & 0.00 & 0 \\
\hline & 39 & & $3-58$ & .51 & 2.60 & 1.42 & $-4,32$ & .81 & -51.55 & & & \\
\hline 26 & 40 & 66 & -5.4 & 1 & 2.24 & 0.00 & - & - & 53 & 0 & & 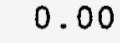 \\
\hline 26 & 41 & & $4.5-5.0$ & -566.32 & 2.81 & 1.39 & -3.91 & -566.02 & -45.61 & 0.00 & 10 & 00 \\
\hline & 42 & & $\begin{array}{lll}5.1 & -4.7\end{array}$ & .16 & 1.97 & 0.00 & -3.72 & -572.91 & -44.43 & 0.00 & 0.00 & 10 \\
\hline & 43 & & -4.3 & .64 & 1.99 & 1.37 & -3.55 & .84 & -39.28 & 0.00 & & 0 \\
\hline & 44 & 7 & 4.6 & 9 & 1.26 & .00 & 0 & - & 31 & 0 & & \\
\hline 26 & 45 & 71 & $\begin{array}{lll}6.8 & -3.7\end{array}$ & -583.62 & 0.67 & 1.35 & -3.25 & -584.85 & -32.16 & 0.00 & 0.00 & 0 \\
\hline 26 & 46 & 12 & $-17.3-3.4$ & -587.14 & -0.17 & 0.00 & -3.11 & -590.42 & -29.66 & 0.00 & 0.00 & 0.00 \\
\hline & 47 & & 3.1 & .36 & -0.69 & 1.33 & -2 . & .71 & & & & \\
\hline & 48 & & 2.8 & 31 & & 0.00 & -2 . & & -2 & & & \\
\hline 26 & 49 & 1 & $-18.9-2.5$ & -595.98 & -2.42 & 1.31 & -2.76 & -599.85 & -14.87 & .00 & 0.00 & 0.00 \\
\hline 26 & 50 & 1 & $9.3-2.3$ & 0 & -3.36 & 0.00 & -2.65 & .41 & -11.36 & 0.00 & 0.00 & 0.00 \\
\hline & 51 & & 2.0 & & & 1.29 & & & -3.47 & & & \\
\hline & 52 & 78 & 1.8 & & & 0.00 & -2 . & & 3 & & & \\
\hline 26 & 53 & 7 & $-20.7-1.6$ & .21 & -1.71 & 1.28 & -2.38 & -607.02 & 10.25 & .00 & 0 & 0.00 \\
\hline 26 & 54 & $80-$ & $-21.1-1.4$ & .70 & -0.82 & 0.00 & -2.30 & .82 & 16.52 & 0.00 & 0 & 0.00 \\
\hline & 55 & 81 & 1.2 & & & 1.26 & -2 & & & & & 0.00 \\
\hline 26 & 56 & $2-$ & -1.0 & -6 & 0.18 & 0.0 & -2.15 & 5 & 31.43 & & 0 & 0. \\
\hline 2 & 57 & - & $-22.4-0.8$ & .99 & 0.58 & 1.25 & -2. & 9.24 & 40.31 & 0.00 & 0.00 & 0.00 \\
\hline & 58 & 84 & -22 & 1 & 9 & 0.00 & -2 & -6 & 47 & 0 & 0 & 0.00 \\
\hline & 50 & & 0.5 & & & & & & & & & \\
\hline 2 & 60 & $6-$ & $-23.5-0.3$ & .67 & 1.26 & 0.00 & -1.90 & .31 & 62.46 & 0.00 & 0.00 & 0.00 \\
\hline 26 & 6 & - & -0.2 & 91 & 1.28 & 1.22 & -1.85 & .26 & 71.58 & 0.00 & 0.00 & 0.00 \\
\hline & & & 0.0 & & & 0 . & & 6 & 5 & & & \\
\hline 26 & 63 & 89 & 0.0 & .00 & 1.21 & 1.21 & -1.74 & 0. & 0. & 0 & 0 & 0.00 \\
\hline 2 & 6 & 90 & 0.0 & 0.00 & 0.98 & 0.00 & -1.70 & 0.00 & 0.00 & 0.00 & 0.00 & 0.00 \\
\hline $2 t$ & 6 & -1 & 0.0 & b & 47 & .1 & -1 & 0 . & 0 & 0 & 0 & 0.00 \\
\hline & & & 0.0 & & & 0 & & & & & & \\
\hline 2 & 1 & 44 & $4.3-22.0$ & -281.38 & -0.31 & 2.94 & -3.85 & -282.60 & 51.38 & 0 & 0.00 & 0.00 \\
\hline 2 & 18 & 45 & $3.6-21.0$ & 302.89 & -0.06 & 1.60 & -4.32 & -305.67 & 36.38 & 0.00 & 0.00 & 0.00 \\
\hline & 19 & 46 & .0 & .41 & 0 & 2.8 & -4 & 5.24 & 8 & 0 & 0 & .00 \\
\hline 2 & & 47 & 19.0 & & & 1.60 & & & 11 . & & & \\
\hline 2 & 2 & 48 & $1.2-18.1$ & .50 & 0.78 & 2.84 & -5 . & 3.80 & 6 & 0 & 0.00 & 0.00 \\
\hline 2 & 22 & 49 & 7.2 & 79.14 & 0.8 & 1.60 & -6.51 & -383.25 & -8.91 & 0.00 & 0.00 & 0.00 \\
\hline & & 0 & .3 & 9 & 0.54 & 2.8 & -7 & 0 & -17. & 0. & .00 & .00 \\
\hline 27 & 2 & & 5.5 & & & & & & & & & \\
\hline 2 & 25 & 52 & 4.7 & 83 & -0 . & 2.77 & -8 & -4 & -34 & -34 & -0.33 & -0.03 \\
\hline 2 & 26 & 53 & $-2.9-13.9$ & 441.10 & -0.74 & 1.60 & 4 & -449.47 & -42.85 & -42.64 & -0.53 & 0.21 \\
\hline & & & & & & & & & & & & \\
\hline 2 & 28 & 55 & $-4.5-12.4$ & .38 & -2.09 & 1.60 & -9 & -477.13 & -54.36 & 02 & -1.75 & 0.34 \\
\hline 2 & 29 & 56 & $-5.2-11.7$ & 179.46 & -1.77 & 2.71 & -8.61 & -487.12 & -56.28 & 04 & -1.53 & 0.24 \\
\hline & & 57 & .1 & & -0.89 & & & 98.18 & .27 & & -0 & \\
\hline 27 & 3 & 58 & $-6.7-10.5$ &. .65 & 0.1 & 2.69 & -7.49 & 6.34 & -59.36 & 84 & -0.37 & -0 \\
\hline 27 & 3 & 5 & -7.5 & 511.83 & 0.77 & 1.60 & -7.01 & -516.46 & -61.41 & -62.22 & -0.04 & -0.81 \\
\hline 20 & 33 & 60 & - & 1.43 & ...39 & 1 & & 523.94 & 32 & 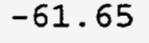 & 0.30 & \\
\hline
\end{tabular}




\begin{tabular}{|c|c|c|c|c|c|c|c|c|c|c|c|c|c|}
\hline & 4 & 51 & 3.9 & 8.8 & 530.47 & & 60 & -6.1 & -53 & -6 & & & \\
\hline & 35 & $6^{\circ}+2+$ & & & & & & & & 4 & & & \\
\hline & 6 & & .3 & 7.8 & $\$ 47.01$ & & .60 & & 48 & -61.15 & -61.84 & & -069 \\
\hline & 37 & & 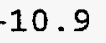 & 7.3 & 554.54 & 2.89 & .63 & -5.19 & -554.21 & -58.80 & -59.79 & 90 & 0.99 \\
\hline & 38 & & 1.6 & -0. & .62 & 56 & 60 & & 52.37 & 3.89 & & & \\
\hline & 39 & & .2 & $c$ & & & 61 & -4.66 & .71 & .16 & & .99 & .60 \\
\hline & 40 & & -12.8 & 6.0 & .47 & 2 & .60 & -4.43 & -575.07 & -55.45 & & & 0.00 \\
\hline & 41 & & $73 \quad 1$ & -56 & & & & & -579.46 & & & & 00 \\
\hline 27 & 42 & & .0 & *. & & & 50 & & -586.36 & 59 & & .00 & .00 \\
\hline & 43 & & & 1 & & 1 & & -3.83 & .34 & & 0.00 & 0.00 & .00 \\
\hline & 44 & & 5.2 & 4.5 & .51 & 1.0 & 60 & -3.66 & -596.52 & -44 & 0.00 & 0.00 & .00 \\
\hline & 45 & $72-$ & -15.7 & 4.2 & .89 & 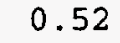 & .57 & -3.50 & & & & & \\
\hline 27 & 46 & & -163 & -3.9 & .94 & .2 & 0 & & 99 & -37.93 & & & \\
\hline 27 & 47 & & .8 & -3.6 & .69 & & & -3.21 & & -33.14 & Do & 0.00 & .00 \\
\hline & 48 & 75 & -17.3 & -3.3 & .15 & 1.8 & 50 & -3.09 & .52 & 32 & & 0.00 & .00 \\
\hline & 4 & $76-$ & -17.8 & -3.0 & .32 & 2.7 & .54 & -2.96 & 53 & & & & \\
\hline & 50 & & -18.3 & 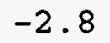 & & 3.60 & & -2.85 & -6 & -21.74 & & & \\
\hline 27 & 51 & $78-$ & -18.8 & -2 & & -3 & 3 & & 11 & -14.70 & & & \\
\hline & 52 & & .3 & -2 & .27 & -2 . & 60 & -2.65 & & -9.13 & & 0.00 & .00 \\
\hline & 5 & $80-$ & -19.7 & -2 & .42 & -1.7 & .52 & -2.55 & .17 & -1.62 & & & \\
\hline & 5 & $81-$ & -20.2 & & & & & & 7 & & & 0 & .00 \\
\hline 27 & 55 & $82-$ & -20.6 & -1.6 & .07 & -0.3 & .51 & -2.38 & 26 & 12.44 & 0 & 0.00 & .00 \\
\hline & 5 & $83-$ & -21.0 & -1.4 & .57 & 0.1 & 60 & -2.31 & -6 & 18.61 & & 0.00 & .00 \\
\hline & 3 & & -21.4 & 1. & 87 & & 50 & -2.23 & & & & 0.00 & .00 \\
\hline & 58 & & -21.8 & -1 & & 1 & & -2.16 & & & & & .00 \\
\hline 27 & 59 & $86-$ & -22.2 & -0.8 & -6 & 1.7 & 9 & -2.10 & -6 & 42.19 & 0. & 0 . & 00 \\
\hline 2 & 60 & 8 & -22.6 & -0.7 & -6 & 1.7 & 0 & -2.03 & -6 & & & & 00 \\
\hline & 6 & & .9 & 0 & & & & -1.97 & & & & & 00 \\
\hline & 62 & & -23.3 & -0 & & $\perp$. & & -1.92 & -6 & & 0 & 0 & 00 \\
\hline 27 & 63 & 9 & -23.6 & -0.2 & -6 & $1.5^{\circ}$ & 48 & -1.86 & .80 & 72 & 0. & 0 . & .00 \\
\hline $27-3$ & 6 & $91-$ & -23.9 & -0.1 & -63 & 1.1 & 0 & -1.81 & & & & & Do \\
\hline 2 & 6 & 92 & 0 & & & & & 76 & & & & & 0 \\
\hline 27 & 6 & 93 & 0.0 & 0. & & 1. & & -1 & & 0 & & 0 & 0 \\
\hline 27 & 6 & 94 & 0.0 & 0. & 0.0 & 1.1 & .46 & -1.67 & 0.00 & 0 . & & 0. & 00 \\
\hline 27 & 6 & 95 & 0.0 & 0. & 0.0 & 1. & 0 & - & & & & & bo \\
\hline 2 & 6 & 9 & 0.0 & 0. & & & & -1 & & & & & 00 \\
\hline 28 & 18 & 46 &. & 21. & -2 & -0.5 & .00 & -4.01 & -303.95 & 45 & 0 & 0.00 & .00 \\
\hline 28 & 19 & 47 & 3.5 & 0.8 & .26 & 0.7 & .80 & -4.47 & .72 & 59 & 0 & 0 . & 0.00 \\
\hline $2 \varepsilon+\gamma$ & 2 & 48 & . & & & & & & & & & & \\
\hline & 2 & 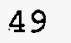 & & & & & & -5 & 77 & & & & 00 \\
\hline 28 & 22 & 50 & .2 & 8.0 & -3 & -0 . & 0 & -6.04 & 4.46 & -2.83 & 0 . & 0. & 0.00 \\
\hline $28-2=$ & 23 & 51 & 0.4 & 7.1 & .91 & 0. & .69 & -6.62 & -401.16 & -11.46 & 0 & 10 & 0 \\
\hline $2 \varepsilon-\gamma$ & 2 & 52 & $-0.4+3$ & 6.3 & & 0 & & & & & & & \\
\hline & 25 & 5 & 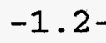 & & & & & -7 & 30 & 6 & -29.38 & & \\
\hline & 2 & 54 & -1.9 & & -4 & -1 & .00 & -8.56 & .60 & -39.69 & -39.21 & -1.06 & .48 \\
\hline & 2 & 55 & -2.7 & $\begin{array}{lll}3 & 9\end{array}$ & .79 & -2 . & 0 & -9.26 & 7.54 & -45 & -4 & 35 & 23 \\
\hline 2 & 2 & 56 & -3 & & & & & & & & & & \\
\hline & 29 & 5 & -4.2 & & & & & & 14 & & & & \\
\hline & 30 & 58 & -5.0 & 8 & -4 & -1 & .00 & -8.65 & 5.59 & -60.39 & -60 & -1.41 & 17 \\
\hline & 31 & 59 & -5.7 & 12 & -5 & -0 & .53 & -8 & 11 & 34 & -6 & & 31 \\
\hline & 32 & 60 & -6 . & & & & & & & & & & \\
\hline & 33 & 61 & -7 . & & & & & - & .0 & 9 & & 6 & 53 \\
\hline & 3 & 62 & -7.9 & - & & & .00 & -6 & -544.41 & 3 & & 0. & -0.81 \\
\hline & 3 & 63 & -8.6 & 0 & & & & & & & & & \\
\hline & & 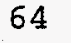 & & & & & & & & & & & \\
\hline & 3 & 6 & -9.9 & -8 & & & & & 74 & & & 128 & -0.48 \\
\hline & 38 & 6 & -10.5 & -7.5 & & & .00 & -5 & 6.42 & & & 1.17 & 38 \\
\hline 2 & 39 & & 12 & & & & & & & & & & \\
\hline & 40 & & & & & & 0. & & 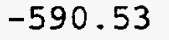 & & & & \\
\hline 28 & 41 & 6 & 4 & -6 & & 1 . & & & & & -6 & & \\
\hline 28 & 42 & & 30 & & & 0. & 0.00 & & -6 & & & 00 & .00 \\
\hline 28 & 43 & & 26 & & & & & -4.12 & -6 & & & & .00 \\
\hline 2 & 44 & 72 & & & & & & & & & & & \\
\hline 28 & 45 & 73 & & & & & & & & & & & 00 \\
\hline 2 & 46 & & & -4 & & 0. & 0.00 & -3.60 & -624.01 & -48.67 & 0.00 & 0.00 & 0.00 \\
\hline & & & & & 00 & 1.57 & 33 & -3.45 & -627.69 & 4.28 & 0.00 & 0.00 & 0.00 \\
\hline
\end{tabular}




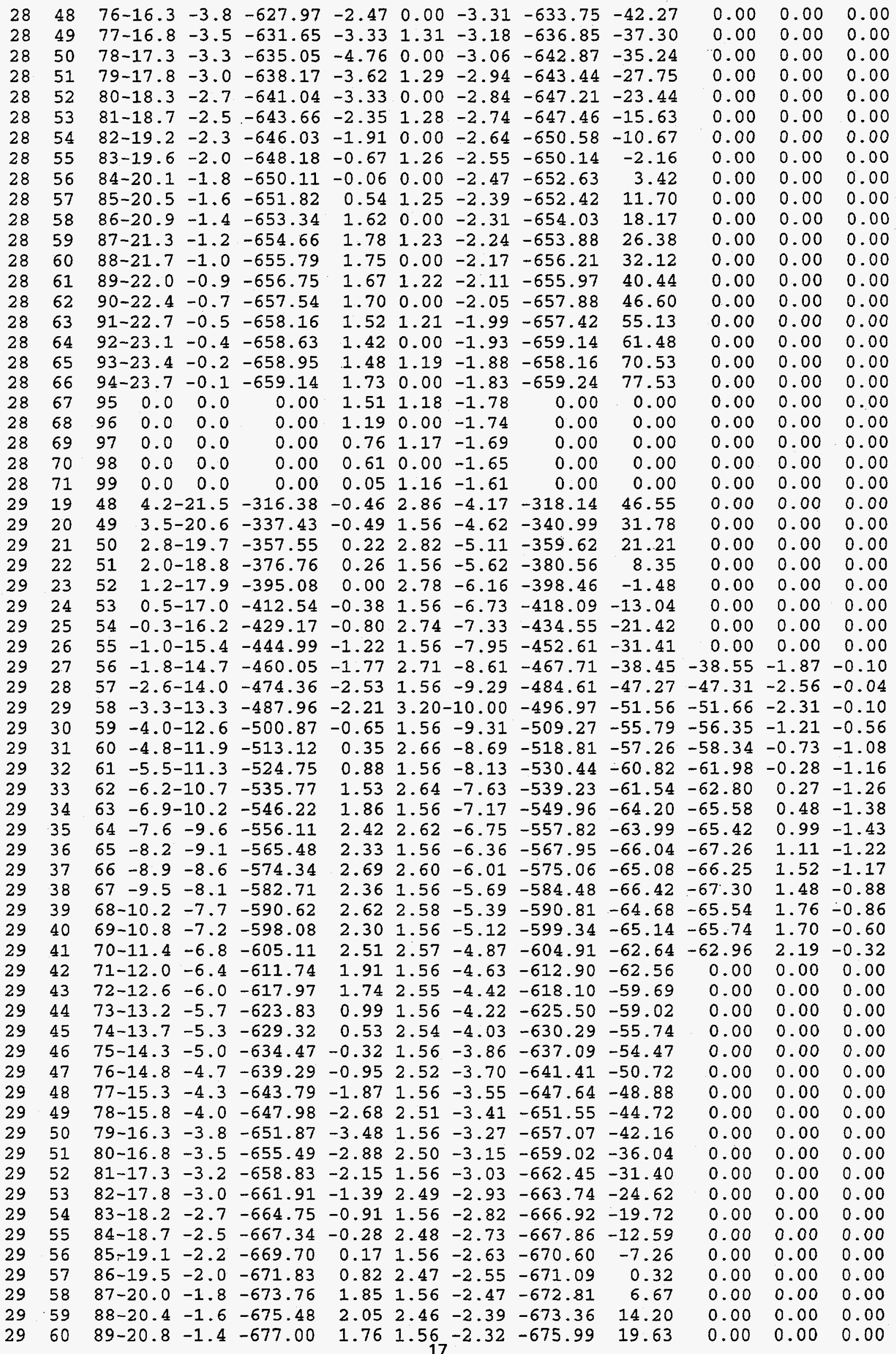




\begin{tabular}{|c|c|c|c|c|c|c|c|c|c|c|c|c|c|}
\hline & & \multirow{2}{*}{\multicolumn{2}{|c|}{-21.1}} & 2 & & & 5 & & & & & & \\
\hline & 2 & & & L. 1 & -679. & 97 & 56 & -2.18 & -67 & & .00 & .00 & \\
\hline & 3 & \multicolumn{2}{|c|}{$\begin{array}{l}91-21.5 \\
92-21.9\end{array}$} & 0.9 & 47 & & & -2.12 & -678.41 & & .00 & .00 & .00 \\
\hline & 4 & \multicolumn{2}{|c|}{$93-22.2$} & 0.7 & 31.29 & & & & -680.12 & & & & \\
\hline & 5 & \multicolumn{2}{|c|}{$94-22.6$} & 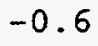 & 601 & 7 & & & 4 & & & & \\
\hline & 66 & \multicolumn{2}{|c|}{$95-22.9$} & & & & & -1.95 & & & & & 00 \\
\hline & 67 & \multicolumn{2}{|c|}{$96-23.2$} & 0.3 & 2.81 & 2 . & & -1.90 & 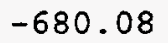 & & 00 & .00 & .00 \\
\hline & 68 & \multicolumn{2}{|c|}{$97-23.5$} & 0.2 & 3.03 & .92 & & -1.85 & & & & & .00 \\
\hline & 69 & \multicolumn{2}{|c|}{$98-23.8$} & & & 58 & & 30 & 2 & & & & \\
\hline & 70 & 99 & & 0 & 0.00 & & & -1.76 & 0.00 & 0 & 0.00 & & 0.00 \\
\hline & 71 & 100 & 0.0 & 0. & 0.00 & 9. & & -1.71 & 0.0 & & 0.00 & .00 & 0.00 \\
\hline & 72 & 01 & 0.0 & 0. & 0.00 & 1.5 & 6 & -1.6 & 0. & & & 10 & 00 \\
\hline & 73 & 102 & 0.0 & & & & & & & & & & \\
\hline & 21 & 51 & 3 & 20 & -354 & * & & -4.81 & -320.40 & & & & \\
\hline & 2 & 52 & 2. & & -3 & 1 & & -5.24 & -3 & 17.77 & & & .00 \\
\hline & 23 & 53 & 2. & & .44 & 1.8 & 59 & -5.74 & -395.67 & & & & 00 \\
\hline & 24 & 54 & 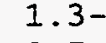 & & & 1 & & & & & & & 00 \\
\hline & 25 & 55 & 0.5 & & .03 & 1.12 & 4 & -6.83 & .10 & 9 & & & 0.00 \\
\hline & 6 & 56 & 0.2 & & -445.61 & 2 & & & .80 & 32 & 5 & 1 & -1.63 \\
\hline & 27 & 57 & 0.9 & & -461.41 & 0.30 & 0 & -8.02 & .13 & -31.58 & 70 & -1.42 & -1.12 \\
\hline & 28 & 58 & -1.7 & & & .01 & & & .13 & -41.50 & 29 & & -0.79 \\
\hline & 29 & 59 & -2 . & & & 32 & & & 8.87 & -46.18 & 6 & 10 & -1.08 \\
\hline & 30 & 60 & -3.1 & & -5 & 0.35 & - & 10.00 & -514.09 & -53.33 & -54.18 & -0.50 & -0.85 \\
\hline & 31 & 61 & -3.8 & .7 & -5 & $1 .$. & & -9.33 & -5 & 23 & 34 & & -1.11 \\
\hline & 32 & 62 & -4.5 & & & 5 & & & 22 & 01 & & & \\
\hline & כנ & 63 & 5.2 & & & & & & & -6 & - & & 28 \\
\hline & 34 & 64 & 5.9 & & .62 & 2.53 & 00 & -7.69 & -5 & -64.73 & -6 & 1.26 & -1.27 \\
\hline & 35 & 65 & -6.6 & & .19 & 2.88 & 47 & -7.24 & -5 & -6 & 91 & & \\
\hline & & 66 & 7.3 & & & & & & 6 & & & & \\
\hline & & 67 & 7.9 & & & & & & 8 & -6 & & & \\
\hline & 8 & 68 & 8.6 & -8.8 & -5 & .99 & .00 & -6.10 & .86 & -6 & - & 2.52 & -0.47 \\
\hline & & 69 & 9.2 & -8.3 & -6 & 3. & 2 & -5.78 & .33 & & & & \\
\hline & & 70 & 9.8 & & & & & & & & & & \\
\hline & $\perp$ & & & & & & & & & & & & \\
\hline & 42 & 72 & .0 & -7.0 & -6 & 59 & 0 & -4 & 1 & -6 & & & 14 \\
\hline & 43 & 73 & 1.6 & -6.6 & & 3 & 7 & -4 & & & & & \\
\hline & & & & & & & & & & & & & 23 \\
\hline & 5 & & .7 & & & 0 & & -4.32 & 1 & & & & 30 \\
\hline & 6 & 76 & 3.3 & -5.5 & .24 & 0. & 0 & -4.13 & -6 & -6 & & & 16 \\
\hline & & & 3.8 & -5.2 & -6 & .48 & 3 & - & & & & & \\
\hline & & & & & & & & & & & & & \\
\hline & & 7 & & & & & & -3 & -6 & -5 & & -2 & -0 \\
\hline & 0 & $8(5>-2$ & .4 & -4.3 & -6 & 2.83 & 10 & -3.50 & .05 & -5 & & & 30 \\
\hline & & & 5.9 & -4.0 & -6 & 2.30 & 9 & & & & & & \\
\hline & & & & & & & & & & & & & \\
\hline & 3 & & & & & & & & & & & & \\
\hline & 4 & 84 & .3 & -3.2 & -6 & 47 & 0 & 1 & -6 & & & & 0 \\
\hline & $-7+2$ & 85 & 7.7 & -2.9 & & & 6 & & & & & & \\
\hline & & & 2 & & & & & & & & & & \\
\hline & & & & & & & & & & & & & \\
\hline & & & & -2 & & & .00 & 3 & & & & & 00 \\
\hline & & 8 & 9.4 & -2.0 & & & & & & & & & \\
\hline & & & & -1 & & & & & & & & & \\
\hline & & & & & & & & & & & & & \\
\hline & 62 & & .6 & -1.4 & & & 0 & -2 . & & & & & \\
\hline & & & 0 & $-1,3$ & & & 1 & & & & & & 00 \\
\hline & & & & & & & & & & & & & \\
\hline & & & & & & & & & & & & & \\
\hline & 66 & 96. & .1 & -0.8 & & & & & & & & & 00 \\
\hline$(-2+3$ & & & .4 & 0.6 & & & & & & & & & \\
\hline & & & & & & & & & & & & & \\
\hline & 69 & & - & & & & & & & & & & \\
\hline & 70 & (2) & & & & & & & & & & & \\
\hline t & 71 & . & 23.6 & 0. & 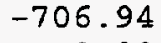 & & & & 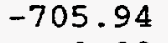 & & & & \\
\hline 30 & 72 & 02 & 0. & . & & & & & 0.00 & & & & .0 \\
\hline & 73 & & 0. & $a_{0}+2$ & & & & & 0.00 & 0 & 00 & 00 & 0.0 \\
\hline
\end{tabular}




\begin{tabular}{|c|c|c|c|c|c|c|c|c|c|c|c|c|c|}
\hline 0 & 4 & 04 & .0 & 0.0 & 0 & 8 & .00 & -1.69 & 0.00 & 00 & 0 & 0 & 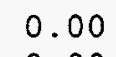 \\
\hline U & 75 & 05 & 0.0 & 0 & & & 1.14 & -1.65 & 0.00 & 00 & 00 & & \\
\hline 1 & 22 & 53 & & 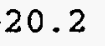 & -371.25 & 68 & 53 & 90 & -372.94 & 30.54 & 00 & Do & 0.00 \\
\hline 1 & 3 & 4 & & & -391.03 & 2.27 & 75 & -5 & .38 & 20.17 & 0 & & 0.00 \\
\hline 1 & 4 & 55 & 2 . & 8.5 & -409.97 & 1.72 & .53 & -5.86 & -412.58 & 7.04 & 0 & & 00 \\
\hline & 25 & 56 & 1.3 & 17.7 & -428.08 & 1.77 & 2.72 & -6.38 & -429.97 & -2.27 & & & \\
\hline & 6 & 57 & & 60 & -445.39 & & 3 & & -449 . & -13 & & & \\
\hline & 27 & 58 & -0 & & -46 & & 2.69 & 19 & -466.10 & -22. & & & \\
\hline & 8 & 9 & & & -477.70 & -0.20 & .53 & -8.08 & $-4 \varepsilon$ & -32.54 & 0.00 & 0.00 & 0.00 \\
\hline & 9 & 60 & $-1.5-$ & 14.7 & -492.75 & 0.69 & .66 & -8.69 & -498.10 & -38.12 & 39.82 & 1.01 & \\
\hline & 0 & 61 & $-2.2-$ & 14.0 & -507.11 & 1.21 & 1.53 & -9.33 & -513.71 & -45.66 & & & \\
\hline & 1 & 62 & -2 & .4 & -520 & 1.94 & & & -5 & & 52.00 & 5 & 2 \\
\hline & 2 & 63 & -3.6 & 12.7 & -533.83 & 2.3 & & & -53 & 7 & & & • \\
\hline & 3 & 64 & $-4 \cdot 3-$ & 12.1 & -546.25 & 2.76 & 1 & -8.77 & -549.65 & -57.38 & & & -1.46 \\
\hline & 4 & 65 & $-5.0-$ & 11.5 & -558.07 & 2.98 & 1.53 & -8.24 & -561.80 & -61.46 & -6 & & 19 \\
\hline & 5 & 66 & $5.7 \quad-2$ & 11.0 & -569.31 & & 2.59 & & & -62.67 & -6 & & ? \\
\hline & 36 & 67 & $-6.3-$ & -10.4 & -580.01 & 3.69 & 1.53 & -7.31 & -582.10 & -65.62 & 38 & 2.43 & 1.200 \\
\hline & 7 & 68 & -7.0 & -9.9 & -590.17 & & 7 & -6 & -590.61 & -66.06 & -67 & & -1.02 \\
\hline & 8 & 69 & -7.6 & -9.4 & -599.83 & 379 & 53 & -6 & -601.04 & -68.42 & -6 & & \\
\hline & 9 & 70 & 8.2 & -8.9 & -6 & & 2. & & 9 & & -6 & & 92 \\
\hline & 40 & 71 & -8.8 & -8.5 & -617.69 & 3.7 & 1.53 & -5.87 & -618.33 & -69.56 & 4 & 3 & -0.58 \\
\hline & 41 & 72 & -9.5 & -8.0 & -625.94 & 3.92 & 2.54 & -5.58 & -625.06 & -68.23 & & & 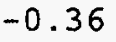 \\
\hline & 42 & 73 & 0.0 & -7.6 & -633.76 & 3.3 & 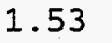 & -5 & & -69 & & & \\
\hline & 3 & & & -7.2 & -6 & & 2 & & & 36 & 5 & & -0.19 \\
\hline & 44 & & 1.2 & -6.8 & -648.1 & 2.09 & 1.53 & -4.83 & -649 . & -68.32 & -6 & 5 & -0.14 \\
\hline & 45 & 76 & 11.8 & -6.4 & -654.78 & 1.71 & 1 & -4.61 & -6 & -6 & & & 37 \\
\hline & 6 & & 2.3 & -6.1 & -6 & $0 . \varepsilon$ & 1.53 & & & & & & 0.02 \\
\hline & 7 & & & -5.7 & & 0 & 2. & & & & & & 0.00 \\
\hline & 48 & $79-$ & 3.4 & -5.4 & -672.47 & -0 . & 1. & -4.05 & 5.64 & -62.30 & -62.72 & -1 & -0.42 \\
\hline & 49 & $80-$ & 13.9 & -5.1 & -677.70 & -1 & 2.48 & -3.89 & 0.46 & -59.05 & 5 & 6 & -0.40 \\
\hline & 50 & 81. & 4.4 & -4.8 & .61 & -1 & 1. & 3 & & & & & \\
\hline & 1 & & & -4.5 & & & 2. & & & 6 & & & 0 \\
\hline & 2 & & & -4.2 & 52 & -0 & 1.53 & -3 & -6 & -48.44 & 0 & 0 & 0.00 \\
\hline & 53 & $4-$ & 15.9 & -3.9 & -695.55 & -0.3 & 2.46 & -3.33 & -6 & -42 . & 0 & 0 & 0. \\
\hline & 54 & $85-$ & 16.3 & -3.6 & -6 & & 1. & 1 & & & & & \\
\hline & 5 & & & -3 & & & & & & & & & 00 \\
\hline & & & & -3 & -7 & 1. & 1 . & -2 & -7 & -2 & 0 & 0 & 0.00 \\
\hline & 57 & $88-$ & 17.7 & -2.9 & -709.06 & 1. & 2.44 & -2.89 & .72 & -21 & 0 & 0 & 0.00 \\
\hline & 58 & $89-$ & 18.1 & -2.7 & -7 & 2 . & 1. & 0 & - & & & & \\
\hline & 59 & & & -2 & & & & & & & & & \\
\hline & & & & -2.2 & -7 & 2.5 & 1.53 & -2.62 & -7 & -5.08 & 0 & 0 & 0.00 \\
\hline & 61 & $92-$ & 19.3 & -2.0 & .82 & 2 . & 2.42 & -2.54 & -7 & 1. & & & 0.00 \\
\hline & 62 & $93-$ & .7 & -1.8 & -7 & 2 . & 1.5 & 7 & & & & & \\
\hline & 63 & & .1 & -1 & & & & & & & & & - \\
\hline & & 95 & & -1.4 & -7 & & 1 . & -2 . & -7 & 20 & & & 0 \\
\hline & 65 & $96-$ & 20.9 & -1.3 & -7 & 3. & 2.41 & -2.26 & -7 & 28 & 0 & & 0 \\
\hline & 66 & $97-$ & .2 & -1.1 & -7 & & 1 & 0 & & & & & \\
\hline & & & & -0 & & & & & -7 & 42 & & & 0.00 \\
\hline & 68 & $99-$ & 9 & -0.8 & -7 & & 1.53 & -2 & .27 & 48. & 0 & 0 & 0. \\
\hline & 69 & 00 & 22.2 & -0.6 & -7 & 2 . & 2. & -2 & -7 & 56 & & & \\
\hline & 70 & & .5 & -0 . & & & 1.5 & & & & & & \\
\hline & 71 & & 2.8 & -0 & & & 3 & & -7 & & & & 0.00 \\
\hline & & & 2 & -0.2 & 7 & 2 . & 1.53 & -1.88 & 8.74 & 78.31 & 0 & 0 & 0.00 \\
\hline & 73 & & 23.4 & -0.1 & 30.6 & 1 . & 2.38 & -1.83 & -728.26 & 86. & 0 & 0 & 0. \\
\hline & 74 & 105 & 0.0 & 0.0 & & & 1. & 9 & & & & & \\
\hline & 75 & & 0. & • & & & & & & & & & \\
\hline & 76 & & 0. & 0. & 0 . & & 1.53 & & 0. & 0. & & 0 & 0 \\
\hline & 77 & 10 & 0.0 & 0.0 & 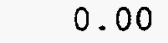 & -0 . & 2.37 & -167 & 0 & 0. & 0 . & 0 & 0.8 \\
\hline & 23 & 55 & 3.5. & 01 & 8789 & & 1.69 & 3 & 6 & 7 & & & \\
\hline & 24 & 6 & 2. & & & & 0 & & & & & & \\
\hline & 25 & 5. $>3>-$ & 2.1 & $8.4 .25 y$ & .3 & & 1.64 & & -4 & 6 & & & \\
\hline & 26 & 58 & 1.4 & 7 . & -444.3 & & 0.00 & -6.48 & -449.37 & -6.32 & 0 & 0 & \\
\hline & 27 & 59 & & 0 & 1.0 & 1. & 1.60 & & & & & & \\
\hline & 28 & 60 & & $\ldots$ & 8.0 & & 0.00 & -7.56 & 5.08 & 88 & 7 & 2 & -1 \\
\hline & 2. & 61 & -0.7 & & & & & & & & & & \\
\hline & & 62 & & & & & & & & & & & \\
\hline
\end{tabular}


$\begin{array}{llllll}32 & 31 & 63 & -2.1-14.1 & -523.29\end{array}$

$\begin{array}{llllll}32 & 32 & 64 & -2.8-13.4 & -537.02\end{array}$

$\begin{array}{llllll}32 & 33 & 65 & -3.4-12.8 & -550.12\end{array}$

$\begin{array}{llllll}32 & 34 & 66 & -4.1-12.2 & -562.61\end{array}$

$\begin{array}{llllll}32 & 35 & 67 & -4.8-11.6 & -574.52\end{array}$

$\begin{array}{llllll}32 & 36 & 68 & -5.4-11.1 & -585.86\end{array}$

$\begin{array}{llllll}32 & 37 & 69 & -6.0-10.5 & -596.67\end{array}$

$\begin{array}{llllll}32 & 38 & 70 & -6.7-10.0 & -606.96\end{array}$

$\begin{array}{lllllll}32 & 39 & 71 & -7.3 & -9.6 & -616.76\end{array}$

$\begin{array}{lllllll}32 & 40 & 72 & -7.9 & -9.1 & -626.07\end{array}$

$\begin{array}{lllllll}32 & 41 & 73 & -8.5 & -8.6 & -634.92\end{array}$

$\begin{array}{lllllll}32 & 42 & 74 & -9.1 & -8.2 & -643.33\end{array}$

$\begin{array}{lllllll}32 & 43 & 75 & -9.7 & -7.8 & -651.31\end{array}$

$\begin{array}{llllll}32 & 44 & 76-10.3 & -7.4 & -658.89\end{array}$

$\begin{array}{llllll}32 & 45 & 77-10.8 & -7.0 & -666.07\end{array}$

$32 \quad 46 \quad 78-11.4 \quad-6.6 \quad-672.86$

$\begin{array}{llllll}32 & 47 & 79-11.9 & -6.3 & -679.30\end{array}$

$\begin{array}{llllll}32 & 48 & 80-12.4 & -5.9 & -685.38\end{array}$
$2.37 \quad 1.53-9.36-528.75-45.34$ 0.00 $2.68 \quad 0.00-10.00 \quad-544.34-52.86-54.43$ $\begin{array}{lllllll}3.07 & 1.50 & -9.37 & -554.92 & -55.37 & -56.41\end{array}$ $\begin{array}{lllllll}3.23 & 0.00 & -8.80 & -568.18 & -60.56 & -61.62\end{array}$ $\begin{array}{lllllll}3.74 & 1.47 & -8.29 & -577.59 & -61.90 & -62.65\end{array}$ $\begin{array}{lllllll}3.67 & 0.00 & -7.81 & -590.01 & -66.24 & -66.98\end{array}$ $\begin{array}{lllllll}4.19 & 1.44 & -7.38 & -598.42 & -66.58 & -67.10\end{array}$ $\begin{array}{lllllll}4.13 & 0.00 & -6.98 & -609.81 & -69.90 & -70.56\end{array}$ $\begin{array}{lllllll}4.30 & 1.42 & -6.61 & -617.65 & -69.67 & -69.91\end{array}$ $\begin{array}{lllllll}4.08 & 0.00 & -6.27 & -628.26 & -72.21 & -72.58\end{array}$ $\begin{array}{lllllll}4.19 & 1.39 & -5.96 & -635.30 & -71.18 & -71.30\end{array}$ $\begin{array}{lllllll}3.82 & 0.00 & -5.67 & -645.18 & -72.99 & -73.42\end{array}$ $\begin{array}{llllllll}3.75 & 1.37 & -5.40 & -651.59 & -71.33 & -71.86\end{array}$

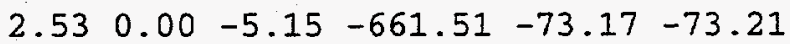
$\begin{array}{lllllll}2.15 & 1.35 & -4.92 & -667.49 & -71.08 & -71.21\end{array}$ $\begin{array}{lllllll}1.23 & 0.00 & -4.71 & -676.34 & -71.86 & -71.86\end{array}$ $0.671 .33-4.50-681.80-69.25-69.49$

$\begin{array}{lllllll}-0.18 & 0.00 & -4.32 & -689.88 & -69.26 & -69.45\end{array}$ $-66.30$

$0.00 \quad 0.00$ $1.11-1.57$

$2.03-1.04$

$2.17-1.06$

$2.99-0.75$

$2.93-0.74$

$3.67-0.52$

$3.47-0.66$

$4.06-0.24$

$3.71-0.37$

$4.07-0.12$

$3.39-0.43$

$3.22-0.53$

$2.49-0.04$

$2.02-0.13$

1.230 .00

$0.43-0.24$

$\begin{array}{lllllllll}-1.11 & 0.00 & -3.98 & -701.63 & -64.87 & -65.54 & -1.78 & -0.67\end{array}$

$\begin{array}{llllllll}-0.78 & 1.29 & -3.82 & -704.96 & -60.12 & 0.00 & 0.00 & 0.00\end{array}$

$\begin{array}{lllll}-0.17 & 0.00 & -3.68 & -710.30 & -57.39\end{array}$

0.00

0.00

$0.451 .28-3.54-712.77-51.79$

$1.401 .26-3.29-719.78-42.66$

$\begin{array}{llllll}1.83 & 0.00 & -3.18 & -724.19 & -39.00\end{array}$

$\begin{array}{llllll}2.25 & 1.25 & -3.07 & -725.86 & -32.60\end{array}$

$\begin{array}{llllll}2.61 & 0.00 & -2.97 & -729.85 & -28.52\end{array}$

$\begin{array}{llllll}3.00 & 1.23 & -2.88 & -731.10 & -21.69\end{array}$

$\begin{array}{llllll}3.09 & 0.00 & -2.79 & -734.90 & -17.42\end{array}$

$\begin{array}{llllll}2.93 & 1.22 & -2.70 & -736.28 & -10.73\end{array}$

$\begin{array}{llllll}3.00 & 0.00 & -2.62 & -739.66 & -6.04\end{array}$

$\begin{array}{llllll}3.12 & 1.21 & -2.54 & -740.37 & 1.32\end{array}$

$\begin{array}{lllll}3.26 & 0.00 & -2.47 & -743.28\end{array}$

6.48

$3.37 \quad 1.19-2.40-743.64$

14.20

$\begin{array}{lllll}3.26 & 0.00 & -2.33 & -746.43\end{array}$

$2.93 \quad 1.18-2.27 \quad-746.89$

$2.58 \quad 0.00-2.20-749.56$

$\begin{array}{lllll}2.04 & 1.17 & -2.15 & -749.92\end{array}$

$\begin{array}{lllll}1.85 & 0.00 & -2.09 & -752.12\end{array}$

$\begin{array}{lllll}1.84 & 1.16 & -2.04 & -751.66\end{array}$

$\begin{array}{lllll}2.02 & 0.00 & -1.99 & -753.18\end{array}$

$\begin{array}{lllll}1.66 & 1.15 & -1.94 & -752.81\end{array}$

$1.54 \quad 0.00-1.89-754.36$

$\begin{array}{lllll}1.13 & 1.14 & -1.85 & -753.79\end{array}$

$\begin{array}{lllll}0.95 & 0.00 & -1.81 & -755.15\end{array}$

19.48

27.09

32.48

40.20

46.07

54.61

61.15

69.60

76.12

84.76

91.47

$\begin{array}{llll}-0.37 & 1.13 & -1.77\end{array}$

0.00

0.00

$\begin{array}{llll}-0.59 & 0.00 & -1.73\end{array}$

0.00

0.00

$\begin{array}{rrr}-1.43 & 1.12 & -1.69 \\ -1.67 & 0.00 & -1.65\end{array}$

0.00

0.00

0.00

0.00

0.00

0.00

0.00

0.00

0.00

0.00

0.00

0.00

0.00

0.00

0.00

0.00

0.00

0.00

0.00

0.00

0.00

0.00

0.00

0.00

0.00

$2.37 \quad 1.50-5.15-405.65$

$2.40 \quad 2.70-5.60-424.37$

0.00

$1.90 \quad 1.50-6.08-445.25$

17.89

$1.742 .67-6.57-462.66$

5.09

$\begin{array}{llllll}1.24 & 1.50 & -7.09 & -482.03 & -15.55\end{array}$

$\begin{array}{llllll}1.95 & 2.64 & -7.63 & -497.17 & -22.62\end{array}$

$2.341 .50-8.19-514.22-31.60$

$2.85 \quad 2.61-8.77-528.24 \quad-37.55$

0.00

0.00

0.00

0.00

0.00

0.00

0.00

0.00

0.00

0.00

0.00

0.00

0.00

0.00

0.00

0.00

0.00

0.00

0.00

0.00

0.00

0.00

0.00

0.00

0.00

0.00

0.00

0.00

0.00

0.00

0.00

0.00

0.00

0.00

$\begin{array}{llllll}3.05 & 1.50 & -9.37 & -544.17 & -45.41\end{array}$

0.00

$3.393 .04-10.00 \quad-556.67-49.84-52.07$

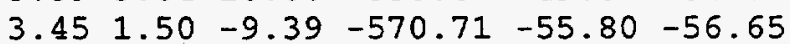

$\begin{array}{lllllll}3.83 & 2.57 & -8.84 & -581.27 & -58.29 & -58.88\end{array}$

$\begin{array}{lllllll}3.76 & 1.50 & -8.33 & -593.90 & -62.85 & -63.08\end{array}$

$\begin{array}{lllllll}4.32 & 2.55 & -7.87 & -603.27 & -64.15 & -64.34\end{array}$

$\begin{array}{lllllll}4.35 & 1.50 & -7.44 & -614.78 & -67.59 & -67.89\end{array}$

$\begin{array}{lllllll}4.48 & 2.53 & -7.05 & -623.64 & -68.37 & -68.23\end{array}$ 20 


\begin{tabular}{|c|c|c|c|c|c|c|c|c|c|c|c|c|c|}
\hline 33 & 40 & 73 & -7.0 & -9.7 & -633.52 & 37 & 1.50 & -6 & -634.34 & -71.01 & -70.96 & 42 & 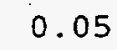 \\
\hline 32 & 11 & 79 & -7.6 & & -642.98 & 4.41 & 2.51 & -6.35 & -642.40 & -71.00 & -70.86 & 4.55 & .14 \\
\hline & 42 & 75 & -8.2 & .8 & -651.98 & 4.08 & & & .44 & & & & \\
\hline & 43 & 76 & -8.8 & -8.4 & -660.54 & 4.03 & & & 76 & -72.22 & -72.29 & 96 & -0.07 \\
\hline & 44 & 77 & -9.3 & -7.9 & -668.68 & 3.02 & 1.50 & -5.49 & -669.65 & -74.03 & -73.92 & & \\
\hline & 45 & 78 & & -7.5 & -676.42 & 2 & & & 6 & -72.87 & -72.82 & & \\
\hline & 46 & & . & -7.2 & -683.77 & 1.65 & & & & -73.87 & -73.64 & 1.88 . & \\
\hline & 47 & 8 & 1.0 & -6.8 & -690.75 & 1.04 & 47 & -4.80 & -692.03 & -72.19 & -72.12 & 1.11 & 0.07 \\
\hline & 48 & & 11.5 & -6.4 & -697.36 & 0.32 & 1.50 & -4.59 & -700.14 & -72.23 & -72.53 & 0.02 & -0.30 \\
\hline & 49 & & & 61 & -703.62 & -0.42 & & & -705.99 & & & & \\
\hline & 50 & & . & -5.8 & .55 & & .50 & -4.23 & -713.09 & -69.04 & .88 & -1.64 & \\
\hline & 51 & & 3.1 & -5.4 & -715.16 & -0.44 & .45 & -4.07 & -717.22 & -65.10 & 0.00 & 0.00 & \\
\hline & 52 & & 3.5 & -5.1 & -720.45 & 0.22 & .50 & -3.91 & -722.65 & -62.45 & 00 & 00 & ov \\
\hline & 53 & & 14.0 & -4.8 & -7 & 93 & 4 & & 5.84 & -5 & .00 & & \\
\hline & 54 & & . & -4.6 & -7 & 1.45 & .50 & -3.63 & .83 & 9 & .00 & & \\
\hline & 55 & & 5.0 & -4.3 & .57 & 1.98 & 2.43 & -3.50 & .66 & -4 & 0.00 & & \\
\hline & 56 & 89 & & -4.0 & -7 & 2.38 & .50 & -3.38 & 3.22 & & .00 & 10 & 00 \\
\hline & 57 & & -15.9 & -3.8 & & 6 & 2 & -3 & .70 & -40 & 00 & 0 & \\
\hline & 58 & & -16.3 & -3.5 & -7 & 3.05 & .50 & -3.15 & -744.86 & -36 & 0.00 & 0.00 & \\
\hline & 59 & & -16.7 & -3.3 & -7 & 3.38 & .41 & -3.05 & .91 & -30 & 0.00 & 00 & \\
\hline & 60 & $93-$ & -1 & 30 & & 353 & 0 & -2 . & & & & & 10 \\
\hline & 61 & 9 & -17.6 & -2.8 & -7 & 3.4 & .40 & -2.86 & 2.75 & -1 & .00 & 10 & 00 \\
\hline & 62 & & -18.0 & -2.6 & -7 & 3.47 & .50 & -2.77 & 5.27 & -15 & 0.00 & 0.00 & 00 \\
\hline & 63 & $96-$ & -18. & -2.4 & & 3.57 & .39 & -2.69 & .70 & & 0 & & \\
\hline & 64 & $97-$ & -1 & -2.2 & & 3.72 & & & & -3 & 00 & 10 & \\
\hline 33 & 65 & $98-$ & -19.1 & -2.0 & .38 & 3.59 & .38 & -2.54 & .95 & 3. & .00 & 10 & 00 \\
\hline & 66 & 9y- & -19.5 & -1.8 & -7 & 3.38 & .50 & -2.47 & -7 & & & 0 & 0 \\
\hline & 67 & 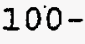 & -1 & -1.6 & & & 7 & -2.40 & 2 & & & & \\
\hline 33 & 68 & - & -20.2 & -1.5 & -7 & 2.83 & 0 & -2.33 & .60 & 20 & 00 & 10 & 00 \\
\hline 3. & 69 & $102-$ & -20.6 & -1.3 & -77 & 2.32 & .36 & -2.27 & .57 & 27 & 0 & 1.00 & 0 \\
\hline & 70 & נ5 & -20.9 & -1.1 & -7 & 1.5 & 0 & -2.21 & & & & & \\
\hline & 71 & - & 2 & -1.0 & & 1.92 & 36 & -2.16 & & & & & \\
\hline 33 & 72 & $05-$ & 21.6 & -0.8 & -7 & 1.99 & 0 & -2.10 & .80 & 47 & 00 & 10 & 00 \\
\hline 3. & 73 & $6-$ & -21.9 & -0.7 & -7 & 1.7 & 2.35 & -2.05 & .92 & b5 & 10 & 0 & 0 \\
\hline & 7 & - & .2 & -0.6 & 8 & 1 . & 50 & 0 & 0 & & & & \\
\hline & 75 & $08-$ & -22 & -0.4 & & & & & 4 & & 10 & 0 & U \\
\hline 33 & 76 & $09-$ & -22.8 & -0.3 & .43 & 0.94 & 1.50 & -1.91 & .90 & 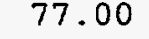 & .00 & .00 & 0.00 \\
\hline $3=$ & 77 & $110-$ & -23.0 & -0.2 & .67 & -0.01 & 2.34 & -1.86 & .21 & 84.77 & 0 & 0 & 1.00 \\
\hline & 7 & $1-$ & -23.3 & 0.1 & 9 & 5 & 0 & 2 & 7 & & & & \\
\hline & 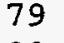 & 12 & & 0.0 & & & & 8 & 0.00 & & 0 & 0 & 0.0 \\
\hline 33 & 8 & 113 & 0.0 & 0.0 & .00 & -1.44 & 1.50 & -1.74 & 0.00 & 0. & 0 & 0 & .00 \\
\hline 33 & 8 & 114 & 0.0 & 0.0 &. & -4.51 & 2.33 & -1.7 & 0.00 & 0. & & & 0 \\
\hline 3. & 8 & 115 & 0.0 & 0.0 & & & & & & & & & \\
\hline 34 & 25 & 59 & 3.5 & 19.8 & $-42 C$ & 2 . & 1.64 & -5.27 & -421.96 & 27 & 0 & 0.00 & 0.00 \\
\hline 34 & 2 & 60 & 2.9 & 9.0 & -44 & 1.8 & 0.00 & -5.71 & .87 & 13 & 0 & 0 & 0.00 \\
\hline & 2 & 6 & .2 & 2 & & & 1 & 8 & & & & & \\
\hline & & 62 & & & & & & & 1.60 & -7 & 0 & 0 & 0.00 \\
\hline 34 & 29 & 63 & 0.9 & .8 & -4 & 2.10 & 1. & -7.17 & -497.12 & -15 & 0 & 0 & 0.00 \\
\hline & -1 & 64 & 0.2 & .1 & -5 & 2.5 & 0. & -7 . & 9 & & .00 & & 0.00 \\
\hline & & 65 & -0.4 & & & & & & & & & & \\
\hline 36 & 32 & 66. & -1.1 & 7 & -5 & 3 & 0 & -8.80 & -5 & -4 & 10 & 0 & 0.00 \\
\hline $3<$ & 33 & 67 & $-1.7-$ & .1 & -5 & 3 . & 1 . & -9.39 & 74 & -4 & 0 & 0 & 0.00 \\
\hline 34 & 34 & 68 & -2. & .5 & & 3. & & -10 & & & & & \\
\hline & & 69 & & & & & & & -5 & -5 & -56 & 3 . & -0.08 \\
\hline 34 & 36 & 70 & -3 & 4 & -5 & 3 & 0 & -8 & 0.08 & -6 & 0. & 0. & 0.00 \\
\hline 34 & 3 & 71 & 1.3 & .8 & -6 & 4 & 1.44 & -8.37 & 57 & -6, & 0.00 & 0.0 & .00 \\
\hline & 38 & 72 & & & & & & & .12 & & & & \\
\hline 34 & 39 & 73 & $-5.5-$ & .8 & & 4. & 1.42 & -.7 .50 & 0.97 & -68 & .22 & 4. & 0.20 \\
\hline 34 & 40 & 74 & $-6.1-$ & -10.3 & -6 & 4. & 00 & -7.11 & -642.78 & -72.16 & -72.21 & 4 . & -0.05 \\
\hline 3 & 41 & 75 & 6.7 & .8 & $-c+c$ & 4.4 & $=$ & 6.76 & & 3 & & & \\
\hline & & 76 & & & & & & & -662.07 & -75.30 & -75.25 & & 0.05 \\
\hline 34 & 43 & 77 & -7.9 & -8.9 & -6 & 4. & 1.37 & -6 & .55 & -74.72 & -74.60 & & 0.12 \\
\hline 3620 & 44 & 78 & -8.4 & 8.5 & -677.57 & 3.27 & 0.00 & .5 .84 & -680.14 & -77.23 & -77.03 & 7 & 0.2 \\
\hline & & 79 & & & & & & & 7.22 & -76.24 & 92 & & 0.32 \\
\hline $3 \leq$ & 46 & $80^{\circ}$ & -9.6 & .7 & -6 & 1.89 & 0.00 & -5.33 & .20 & -78 & & 2.29 & 0.40 \\
\hline 34 & 47 & $81-$ & -10.1 & 7.3 & -701.28 & 1.25 & & & -703.80 & -76.68 & -76.39 & 1.54 & 0.29 \\
\hline
\end{tabular}




\begin{tabular}{|c|c|c|c|c|c|c|c|c|c|c|c|c|c|}
\hline & & \multirow{2}{*}{\multicolumn{2}{|c|}{$\begin{array}{l}82-10 \cdot 6 \\
83-11.1\end{array}$}} & .0 & & & & & & -77.74 & 0 & & \\
\hline & & & & .6 & & & & & & & & & \\
\hline & 50 & \multicolumn{2}{|c|}{$84-11.7$} & • & & & & & & & & & \\
\hline & & \multicolumn{2}{|c|}{$85-12.2$} & & & & & & & & & & \\
\hline & & \multicolumn{2}{|c|}{$86-12.6$} & 56 & & & & & & -69.67 & & & \\
\hline & & \multirow{2}{*}{\multicolumn{2}{|c|}{$\begin{array}{l}87-13 \cdot 1 \\
88-13 \cdot 6\end{array}$}} & & & & & & & & & & \\
\hline & 54 & & & .0 & & & & & & & & & \\
\hline & 55 & \multicolumn{2}{|c|}{$89-14.1$} & 4.7 & .09 & 2.29 & & -3.71 & & -57.56 & & & \\
\hline & & \multirow{2}{*}{\multicolumn{2}{|c|}{$\begin{array}{l}90-14.5 \\
91-15.0\end{array}$}} & 4.5 & & $? 67$ & bo & -3.58 & -754.61 & -54 & & & \\
\hline & 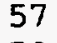 & & & 42 & & & & & & & & & \\
\hline & 58 & \multicolumn{2}{|c|}{$92-15.4$} & \pm .0 & & & & & & & & & \\
\hline & קנת & \multicolumn{2}{|c|}{$93-15.9$} & 3.7 & & & & -3.23 & & & & & \\
\hline & 60 & 94 & -16.3 & 5 & & & & & & & & & \\
\hline & 61 & & & 2 & & & & & & & & & \\
\hline & 62 & & & 3.0 & & 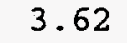 & 0 & & & $-2-2$ & & & \\
\hline & 6 & & & .8 & & & & 5 & & & & & \\
\hline & 64 & & & 2.6 & & & 0 & -2.76 & & 8 & & & \\
\hline & 65 & & & 2.4 & & & & & & & & & \\
\hline & 66 & & & -2.2 & & 3. & 0 & 1 & & -3. & & & \\
\hline & 67 & & & -2.0 & & 2 . & .18 & -2.34 & & & & & \\
\hline & 68 & & & -1.8 & & & 0 & 7 & & & & & \\
\hline & 69 & & & -1.6 & & & & & & & & & \\
\hline & 70 & & & -1.5 & -7 & 1 . & 0 & 4 & & & & & \\
\hline & 1 & 1 & & -1.3 & & 2. & 6 & -2.28 & & & & & \\
\hline & 72 & & & -1.2 & & 1 & & & & & & & \\
\hline & 73 & & & -1 & & & & & & & & & \\
\hline & 4 & 1 &. & -0.9 & & 1. & 0 & 1 & & & & & \\
\hline & 5 & & & -0.7 & & 1.0 & .14 & -2.06 & & & & & \\
\hline & 6 & & & 0 & & & & & & & & & \\
\hline & 77 & & & & & & & & & & & & \\
\hline & 78 & 1 & & -0.3 & & s & 0 & -1 & & & & & \\
\hline & 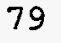 & 3 & & -0.2 & & & 2 & -1.88 & & & & & \\
\hline & & & & & & & & & & & & & \\
\hline & & & 0 & & & & & & & & & & \\
\hline & & 6 & 0.0 & 0 . & 0 & & & -1 & 0 & & & & \\
\hline & & &. & 0. & & & & -1 & & & & & \\
\hline & & & & & & & & & & & & & \\
\hline & & 6 & & 9.6 & $=$ & & & & & & & & \\
\hline & & 6 & 2. & .9 & & & & -5 & & & & & \\
\hline & & & & & & & & -6 & & & & & \\
\hline & & & & & & & & & & & & & \\
\hline & & 6 & 1. & & & & & & & & & & \\
\hline & & 6 & 0.1 & & & & 9 & -7 & & & & & \\
\hline & & 6 & . & & & & & & & & & & \\
\hline & & & & & & & & & & & & & \\
\hline & & 69 & -1 & & & & & & & & & & \\
\hline & & 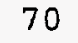 & -2 & & & & & & & & & & \\
\hline & & 7 & -2 & & & & & & & & & & \\
\hline & & 7 & & & & & & & & & & & \\
\hline & 3 & 7 & -4 & & & & & & & & & & \\
\hline & & & 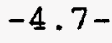 & & & & & -7 & & & & & \\
\hline & & & & & & & & & & & & & \\
\hline & & & & & & & & & & & & & \\
\hline & & & & & & & & & & & & & \\
\hline & & & -7.0 & & & & & & & & & & \\
\hline & & & -7.6 & & & & & & & & & & \\
\hline & & & & & & & & & & & & & \\
\hline & 4 & 8 & -8.7 & & & & & & & & & & \\
\hline & $=$ & 8 & 92 & & & & & & & & & & \\
\hline & & & & & & & & & & & & & \\
\hline & 4 & & & 7 & & & & & & & & & \\
\hline & & & & & & & & & & & & & \\
\hline & 51 & & & & & & & & & & & & \\
\hline & - & &. .8 & -6.1 & 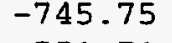 & & & & .85 & -73.09 & 36 & & - \\
\hline & 53 & & & - & & & & & & & & & \\
\hline & & & & & -757.37 & 35 & & & & & & 0 & 2.0 \\
\hline
\end{tabular}




\begin{tabular}{|c|c|c|c|c|c|c|c|c|c|c|c|c|c|}
\hline & 55 & \multirow{2}{*}{\multicolumn{2}{|c|}{$\begin{array}{l}90-13.2 \\
91-13.7\end{array}$}} & -5.2 & -762.73 & 2.79 & 2.40 & -3.93 & -761.47 & -62.49 & -64.68 & 0.60 & -2.19 \\
\hline 35 & 56 & & & -4.9 & -767.80 & 3.09 & 47 & -3.79 & -767.03 & -59.99 & -61.56 & 1.52 & -1 \\
\hline & 57 & & & -4.7 & -772.59 & & 39 & -3.66 & -770.40 & -55.28 & & 19 & -1. \\
\hline & 58 & & 1.6 & -4.4 & -777.11 & 3.73 & .47 & -3.54 & -775.46 & -52.27 & .00 & 00 & .00 \\
\hline & 59 & & & -4.1 & -781.38 & 3.67 & 2.38 & & -778.75 & -47.49 & 0.00 & 00 & \\
\hline & 60 & & & -3.9 & -785.39 & 3.73 & & & -783.50 & -44.17 & & & \\
\hline & 61 & & -15.8 & -3.6 & -789.16 & 3.60 & 37 & -3.21 & -786.40 & -38.99 & & & \\
\hline & 62 & $97-$ & -16.3 & -3.4 & -792.70 & 3.62 & .47 & -3.11 & -790.72 & -35.24 & 0.00 & 00 & 0.00 \\
\hline 35 & 63 & & 6.7 & -3.2 & -796.01 & 3.70 & 36 & -3.01 & -792.96 & & 0.00 & 0.00 & \\
\hline & 64 & & & -3.0 & -799.10 & 3.83 & & & -7 & & & & \\
\hline & 65 & 100 & -17.5 & -2.8 & -801.97 & 3.66 & 35 & -2 & -798.79 & -19.10 & & & \\
\hline & 66 & 101 & -17.8 & -2.6 & -804.64 & 3.37 & .47 & -2.76 & -802.56 & -14.80 & 0.00 & 0.00 & 0 \\
\hline & 67 & 102 & 0 & -2.4 & -807.12 & 2.97 & 2.35 & -2.68 & -804.48 & -8.65 & 0.00 & 0.00 & 0.00 \\
\hline & 68 & 103 & .6 & -2.2 & -809.40 & 2.69 & 47 & -2.60 & -807.85 & -3.94 & 00 & & \\
\hline & 69 & 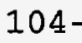 & 3.9 & -2.0 & -81 & 2.31 & 34 & -2.53 & & 2.60 & & & \\
\hline & 70 & 105 & 19.3 & -1.8 & -813.41 & 2.12 & .47 & -2.47 & -812.29 & 7.76 & 0.00 & & \\
\hline 3 & 71 & 1 & 19.6 & -1.7 & -815.15 & 2.00 & .33 & -2.40 & -813.22 & 39 & 0 & 0 & 0.00 \\
\hline & 72 & 107 & .0 & -1.5 & -816.73 & 2.11 & .47 & -2.34 & .49 & 20.70 & 10 & & 0.00 \\
\hline & 73 & $108-$ & .3 & -1.3 & -818.14 & 1.90 & 32 & -2.28 & -816.20 & 28 & & & \\
\hline & 74 & 109 & .6 & -1.2 & -819.39 & 1.92 & .47 & -2.23 & -818.23 & 34.10 & 0.00 & 0.00 & 0.00 \\
\hline & 75 & $110-$ & 20.9 & -1.0 & -820.50 & 8 & .32 & -2.17 & -817.67 & & & & 0.00 \\
\hline & 76 & 111 & .2 & -0.9 & -821.46 & 2.66 & .47 & -2.12 & -819.45 & 49 . & 0 & 0 & 0.00 \\
\hline & 77 & 112 &. .5 & -0.7 & -822.27 & 0.38 & 2.31 & -2.07 & -821.65 & 54.89 & 0.00 & 0 . & 0.00 \\
\hline & 78 & 113 & -21.8 & -0.6 & -822.95 & 0.01 & 47 & -2.02 & -823.52 & 61.10 & & & 0.00 \\
\hline & 79 & 114 & .1 & -0.5 & -823.50 & -0.92 & 1 & -1.98 & 4.10 & 68 & & & .00 \\
\hline & 80 & $115-$ & .4 & -0.4 & -823.93 & -1.3 & .47 & -1.93 & 5.72 & 75 . & 0.00 & 0 & 0.00 \\
\hline & 81 & $116-$ & .7 & -0.2 & 1.23 & -4.44 & .30 & -1.89 & -828.26 & 80.57 & 0.00 & 0.00 & 0.00 \\
\hline & 82 & 11 & 9 & -0.1 & -824.42 & & & -1.85 & 9.87 & 3 & 0 & & 0 \\
\hline 35 & 83 & $118-$ & 23.2 & 0.0 & -824.50 & -4.83 & .29 & -1.81 & -828.85 & 96. & 0. & 0. & 0.00 \\
\hline & 84 & 119 & 0.0 & 0.0 & 0.00 & -3.93 & .47 & -1.77 & 0.00 & 0 . & 0.00 & 0.00 & 0.00 \\
\hline & 85 & 120 & 0.0 & 0.0 & 0.00 & & 9 & -1.74 & 0.00 & & & & 0.00 \\
\hline 5 & 86 & 121 & 0.0 & 0.0 & 0.00 & -1.91 & 7 & -1.70 & 0.00 & 0 & & & .00 \\
\hline 3 & 27 & 63 & 3.6 & 19.5 & -452.79 & 2.6 & 1.60 & -5.49 & -454.03 & 26 . & 0 & 0 & 0.00 \\
\hline & 28 & 64 & 3. & 8.8 & -471.93 & 1.9 & 0.00 & -5.92 & -475.90 & 12.43 & 0.00 & 0.00 & 0.00 \\
\hline & 29 & 65 & & & 0.35 & & & & .66 & & & & 0 . \\
\hline 36 & 30 & 66 & 1. & 7.4 & -508.05 & 2 & .00 & -6.83 & 2.10 & -7 & & & \\
\hline & 31 & 67 & 1.1 & 6.7 & -525.08 & 3.4 & 53 & -7.31 & -527.46 & -14 & 0 & 0 . & 0.00 \\
\hline 3 & 32 & 68 & 0.5 & 6.0 & -541.43 & 3.4 & 00 & -7.81 & -545.76 & -25 & 0.00 & 0.00 & 0.00 \\
\hline 3 & 33 & 69 & -0. & 5.4 & .1 & & & & .29 & & & & 0 \\
\hline $3 t$ & 34 & 70 & -0.8 & 4.8 & -572.22 & . & & -8 & 7.62 & & 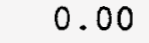 & & 0 . \\
\hline 3 & 35 & 71 & -1.4 & 4.2 & -586.69 & 3.7 & 77 & -9.43 & -590.89 & -46 & 0 & 0.00 & 0.00 \\
\hline 3 & 3 & 72 & -2.0 & .6 & -600.58 & 3.6 & & -10.00 & 6.92 & -54 & 0.00 & 0.00 & 0.00 \\
\hline & 37 & 73 & -2.6 & .0 & .8 & 4 & & & & & & & 0.06 \\
\hline & 38 & 74 & -3.2 & .5 & 5.66 & 2. & 0 & -8.93 & 1.88 & 3 & & 4 & 0 . \\
\hline & 39 & 75 & -3.8 & .0 & -638.89 & 4.0 & 2 & -8.45 & -641.90 & -64.79 & - & 4. & 0.5 \\
\hline & 40 & 76 & & 5 & & & & -8 & & & & 4 & 0 . \\
\hline 36 & 41 & 77 & $-5.0-$ & .0 & -661.84 & 4.8 & & -7.61 & .17 & -6 & & & \\
\hline & 4 & 78 & $-5.6-$ & -10.5 & 2.59 & 4.4 & 0 & -7.24 & .43 & -74.10 & & 4 & -0.06 \\
\hline & 4 & 79 & & 1 & .8 & 4.2 & & -6.89 & .11 & 4.70 & & & 0.2 \\
\hline & 44 & 80 & -6.7 & & .7 & 4.3 & & -6 & 9 & & & & \\
\hline & 4 & 81 & -7.3 & -9.2 & 2.1 & 3.7 & & -6.27 & .27 & -77.73 & -7 & 3 & 0.04 \\
\hline 3 & - & 82 & -7.8 & -8.8 &. .1 & 2.7 & & -5.99 & 4.36 & -80.75 & $-\varepsilon$ & & 0.16 \\
\hline 36 & 47 & 83 & & & .7 & & & & & & & & 0. \\
\hline 36 & 4 & 84 & -8.9 & -8.0 & 27.90 & 0.9 & 10 & -5 & 2.43 & -82.67 & & & \\
\hline 36 & 2 & 85 & 9.4 & 7.6 & 5.7 & 02 & 1 & -5.26 & 9.30 & -81.47 & -8 & & 01 \\
\hline & 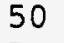 & 86 & -9.9 & 3 & 3.19 & 0.40 & 0 & 5.05 & 4 & -82.74 & -8 & 92 & -0.52 \\
\hline 36 & 51 & $87-$ & -10.4 & -6.9 & 0.31 & 25 & & & & & & & \\
\hline 3 & 5 & $88-$ & 10.9 & -6.6 & 7.08 & 0.93 & & -4.66 & .81 & -78.77 & & & -0.92 \\
\hline 36 & 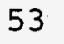 & $89-$ & .4 & 5.3 & 3.53 & 7 & 28 & -4.48 &. .99 & -74.87 & -7 & -0.10 & -1.85 \\
\hline & 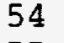 & & -11.9 & & 6 & 5 & 0 & 432 & 1.48 & 3.30 & -7 & & .66 \\
\hline & 55 & $91-$ & -12.3 & -5.7 & 75.49 & 96 & & & & & & & \\
\hline$(6$ & 5 & $92-$ & .8 & .4 & 1.03 & 3.2 & & -4.01 & 1.83 & -67.50 & & & -1.21 \\
\hline 36 & 57 & & -13.3 & & 86.2 & & & 87 & 5.26 & -62.86 & -6 & & -1.23 \\
\hline & 58 & & & & & & & & & & .00 & 0,00 & 0.00 \\
\hline & 5 & & & 4.6 & 5.94 & 3.59 & & -3.62 & 4.74 & -56.19 & & & \\
\hline & 60 & 96 & -14.6 & .3 & 00.39 & 3.62 & & 3.50 & 00.27 & -53.65 & 0.00 & 0.00 & 0. \\
\hline
\end{tabular}




\begin{tabular}{|c|c|c|c|c|c|c|c|c|c|c|c|c|c|}
\hline & 1 & \multirow{2}{*}{\multicolumn{2}{|c|}{$\begin{array}{l}97-15.0 \\
98-15.4\end{array}$}} & 4.1 & 8 & .46 & 2 & -3 & 29 & - & & 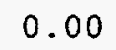 & \\
\hline & 62 & & & 38 & -808 & & 0.00 & 28 & -808.32 & $-4 !$ & 00 & & \\
\hline & 63 & \multicolumn{2}{|c|}{$99-15.8$} & & 12.25 & & & & & & & & \\
\hline & 64 & \multicolumn{2}{|c|}{$100-16.2$} & 3.4 & -815.74 & & & & -815.20 & -36.30 & 00 & 00 & 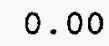 \\
\hline & 65 & \multicolumn{2}{|c|}{$101-16.6$} & 3.2 & -819.01 & 7 & 9 & -2.99 & -8 & & & & \\
\hline & 66 & \multicolumn{2}{|c|}{$102-17.0$} & 2 & & & & & & & & & \\
\hline & 67 & \multirow{2}{*}{\multicolumn{2}{|c|}{$\begin{array}{l}103-17.4 \\
104-17.8\end{array}$}} & & 1 & & & & & & & & \\
\hline & 68 & & & -2.5 & -82 & & & -2.75 & .85 & & & & 0 \\
\hline & 69 & \multicolumn{2}{|c|}{$105-18.1$} & 2.4 & 02 & 2.21 & 17 & -2.67 & -829.31 & -1 & & & .00 \\
\hline & 70 & \multicolumn{2}{|c|}{$106-18.5$} & 2 & & & & & & & & & \\
\hline & 71 & \multicolumn{2}{|c|}{$107-18.8$} & 0 & & & & 3 & 0 & & & & \\
\hline & 72 & $108-$ & 19.2 & -1.8 & & & & -2.47 & & 2 & & & 00 \\
\hline & 73 & 109 & & 1.7 & & 1.98 & 5 & -2.40 & .32 & & & & .00 \\
\hline & 74 & 11 & & 1.5 & & $=90$ & & & & & & & 00 \\
\hline & 75 & 11. & & 3 & & & & 9 & & & & & \\
\hline & 76 & 112 & & & & & & -2.23 & & & & & \\
\hline & 77 & 113 & & 1.0 & & 0.45 & 3 & -2.18 & & 79 & & & .00 \\
\hline & 78 & 1 & & 0 & & & & & & & & & 00 \\
\hline & 79 & 11 & & & & & & & & & & & \\
\hline & 80 & 11 & & -0. & & 9 & 0 & -2.03 & .69 & 35 & & 10 & 00 \\
\hline & 81 & 11 & & 0.130 & & 4.20 & & -1.99 & 1.02 & & & & 00 \\
\hline & 82 & 118 & & 0 & & & & -1.95 & & & & & oo \\
\hline & 83 & 11 & & & & & & & & & & & \\
\hline & 84 & 120 & & -0.2 & & 3 . & 0 & -1.86 & - & 8 & & 0 & 0.00 \\
\hline & 85 & 121 & .0 & 0.1 & .67 & 2.97 & .09 & -1.83 & $r$ & 03 & & 00 & 0.00 \\
\hline & 86 & 122 & 0 & & & -1.88 & & -1.79 & & & & & \\
\hline & 7 & 123 & .0 & & & & & & & & & & \\
\hline & 88 & 124 & 0.0 & 0.0 & 0 & -0.07 & & -1.72 & 0. & & & 0 & 00 \\
\hline & & 66 & $3.0-$ & 18.7 & -48 & 3.25 & 0 & -6.01 & -487.81 & 15.88 & & 0 & 00 \\
\hline & & 67 & & & & & & & & & & & \\
\hline & 1 & 68 & & & & & & & & & & & \\
\hline & 32 & 69 & 1.20 & 5.7 & & 4 & 4 & -7 & - & -1 & 0. & 0 & 1.00 \\
\hline & 2 & 70 & 0.6 & - & & 4.08 & 5 & -7.87 & 7 & -2 & 0 . & 0 & 10 \\
\hline & & 71 & U. & & & & & & & & & & \\
\hline & 5 & 72 & & & & & & & & & & & \\
\hline & 36 & 73 & 1 & & & & & -5 & & -4 & 0 . & 0 & 00 \\
\hline & 2 & 74 & $1 . \varepsilon$ & .6 & & 3 & & & 3 & -5 & 51. & 3.20 & 59 \\
\hline & & 75 & 2. & & & & & & & & & & \\
\hline & 39 & 76 & & & & & & & & & & & \\
\hline & 40 & 77 & 3. & -12.1 & & 4 & & $-\varepsilon$ & 15 & -65.67 & & 9 & 0.75 \\
\hline & 41 & 78 & 4.2 & 1.6 & & & & $-\varepsilon$ & & -67.65 & & 5 & 0. \\
\hline & 42 & 79 & \pm & & & & & & & & & & \\
\hline & 43 & 80 & & & & & & & & & & 4.61 & \\
\hline & 44 & 81 & -7 & 2 & & &. .44 & -6 & 97 & -7 & & 4.31 & -0.24 \\
\hline & 45 & 82 & 625 & .7 & & & & & & -7 & & & 0.11 \\
\hline & & 83 & & & & & & & & & & & \\
\hline & & 84 & & & & & & & & & & & \\
\hline & 48 & 85 & 3.0 & & & & & -5 & & -82.55 & & 51 & 38 \\
\hline & 4 & 86 & 8.5 & 8.2 & & & & -5 & & -82.30 & & 0.01 & \\
\hline & & 87 & 9.1 & 7.8 & & & & & & & & & \\
\hline & & & & & & & & & & & & & \\
\hline & 5 & 8 & & 1 & & & 4 & -4 & & $-\varepsilon$ & & & \\
\hline & 53 & 90 & & .8 & & & & -4 & & -7 & & & \\
\hline & $=$ & & & 55 & & & & & & & & & \\
\hline & & & & & & & & & & -7 & & & \\
\hline & 56 & 9 & & -5.8 & & & & -4 & & -7 & & & \\
\hline & 57 & 94 & & -5.6 & & & & & - & 8 & & 2 & \\
\hline & & & & & & & & & & & & & \\
\hline & & & & & & & & & & -61.81 & & & \\
\hline & 6 & & & -4.7 & & & & & & & & & \\
\hline & 61 & 98 & & 5 & & & & & 8 & -55.11 & & & 94 \\
\hline & ( & & & & & & & & & & & & \\
\hline & 63 & & & -4 & & & & & & & & & $0 C$ \\
\hline & 6 & & & & & & & & & & & & \\
\hline $3^{\circ}$ & 65 & 10 & & & & & & & & & & & 00 \\
\hline & $\epsilon$ & & & & & & & & & & & & \\
\hline
\end{tabular}




\begin{tabular}{|c|c|c|c|c|c|c|c|c|c|c|c|c|c|}
\hline & 67 & \multirow{2}{*}{\multicolumn{2}{|c|}{$\begin{array}{l}104-16.6 \\
105-16.9\end{array}$}} & 3.1 & -841 & 87 & 2 & -2.98 & -83 & -29 & 10 & 0 & 0 \\
\hline & 68 & & & -2.9 & -844.91 & 2.46 & 44 & -2.89 & 843.90 & -25.43 & 0.00 & 0.00 & 0.00 \\
\hline & 6 & $106-$ & 17.3 & -2.7 & -847.74 & 2.08 & .32 & -2.81 & -846.15 & -19.61 & 00 & 00 & .00 \\
\hline & 70 & $10 \%$ & 17.7 & -2.5 & -850.37 & 2.08 & 1.44 & -2.74 & -849.59 & -14.97 & .00 & 0.00 & 0.00 \\
\hline 37 & 71 & & 20 & -23 & -852.81 & 73 & & -2.67 & & -8.75 & .00 & Do & bo \\
\hline 7 & 72 & & & -2.2 & -855.07 & 2.04 & & -2.60 & .19 & -3.43 & 0.00 & 00 & 0.00 \\
\hline 3 & 73 & 1 & 18.7 & -2.0 & -857.16 & 2.18 & .30 & -2.53 & -855.21 & 3.62 & 0.00 & .00 & 0.00 \\
\hline 37 & 74 & $111-$ & 19.1 & -1.8 & -859.08 & 3.19 & 1.44 & -2.47 & -856.91 & 9.99 & 0.00 & 0.00 & 0.00 \\
\hline 37 & 75 & 112 & 9.4 & -1.7 & -860.83 & 9 & 2.29 & -2.41 & -857.75 & .23 & 00 & 00 & 00 \\
\hline 37 & 76 & $\perp$ & 19.7 & -1.3 & -862.41 & - & & -2.35 & 1.78 & 21.26 & 0 & & 00 \\
\hline 37 & 77 & .14 & 20.0 & -1.4 & -863.85 & 0.60 & 2.29 & -2.29 & -863.25 & 27.86 & 0.00 & 0.00 & 0.00 \\
\hline 37 & 78 & 15 & 20.3 & -1.2 & -865.13 & 0.0 & 1.44 & -2.24 & -865.90 & 33.29 & 0.00 & .00 & .00 \\
\hline 37 & 79 & 116 & .6 & -1.1 & 27 & -0.88 & 2.28 & -2.19 & & 40.20 & 00 & 00 & .00 \\
\hline 31 & 80 & 1 & 20.9 & -0.9 & 27 & & .44 & -2.14 & 68 & 45.65 & .00 & 0.00 & 0.00 \\
\hline 37 & 81 & 110 & 21.2 & -0.8 & -868.13 & -4.19 & .28 & -2.09 & -872.14 & 51.26 & 0.00 & 0.00 & 0.00 \\
\hline 37 & 82 & $19-$ & 21.5 & -0.7 & .87 & -5.13 & 1.44 & -2.04 & -874.60 & 56.87 & 0.00 & 0.00 & 0.00 \\
\hline 37 & 83 & 2 & 21.8 & -0.5 & 77 & & 2.27 & -2.00 & -873.89 & & 0.00 & 00 & 10 \\
\hline 37 & 84 & 21 & 22.0 & -0.4 & .95 & -3.8 & 1.44 & -1.96 & -874.33 & 73.29 & 0.00 & 0.00 & 0.00 \\
\hline 37 & 85 & 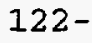 & .3 & -0.3 & -870.32 & -2.92 & 2.26 & -1.92 & -872.89 & 82.80 & 0.00 & 0.00 & 0.00 \\
\hline 37 & 86 & 23 & 22.6 & -0.2 & .57 & -2.00 & 1.44 & -1.88 & -873.01 & 90.75 & 0.00 & 1.00 & 0.00 \\
\hline 37 & 87 & 24 & 22.8 & -0.1 & 72 & -1 & 26 & -1.84 & -871.67 & 100.16 & 0.00 & 10 & 0. \\
\hline 37 & 88 & 125 & 0.0 & 0.0 & 0.00 & -0.05 & 1.44 & -1.80 & 0.00 & 0.00 & 0.00 & 0.00 & 0.00 \\
\hline 37 & 89 & 126 & 0.0 & 0.0 & 0.00 & 1.30 & 2.25 & -1.77 & 0.00 & 0.00 & 0.00 & 0.00 & 0.00 \\
\hline 37 & 90 & 127 & 0.0 & 0.8 & 0.00 & .22 & .44 & -1.73 & 0 & 0 & 0.00 & 0.00 & 00 \\
\hline 37 & 91 & 128 & 0.0 & 0.0 & 0.00 & 1.09 & 2.25 & -1.70 & 0.00 & 0.00 & 0.00 & 0.00 & 0.00 \\
\hline 38 & 30 & 68 & 3.1 & 18.6 & -503.21 & 2.95 & 0.00 & -6.10 & -506.36 & 12.68 & 0.00 & 0.00 & 0.00 \\
\hline 38 & 31 & 69 & 2.5 & 7.3 & 46 & 3.68 & 1.53 & -6.53 & 78 & & & & .00 \\
\hline 38 & 32 & 70 & 5 & 77 & 4 & 9 & 0 & -6.98 & .23 & -7 & 00 & 0.00 & 0.00 \\
\hline 38 & 33 & 71 & 1.3 & 16.6 & -555.98 & 4.23 & 1.50 & -7.44 & -557.70 & -14.44 & 0.00 & 0.00 & 0.00 \\
\hline 38 & 34 & 72 & 0.7 & 6.0 & -572.29 & 3.9 & 0.00 & -7.92 & .27 & -24 & 0.00 & 00 & 0.00 \\
\hline 8 & 35 & 73 & 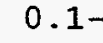 & & & & 1.47 & -8.41 & 3 & -3 & & & Do \\
\hline 38 & 36 & 74 & -0.5 & .8 & -6 & 3. & 0.00 & -8.93 & .54 & -41 & 0.00 & 0.00 & 0.00 \\
\hline 38 & 37 & 75 & -1.1 & -14.2 & -617.59 & 3.28 & 1.44 & -9.46 & -622.33 & -46.79 & 0.00 & 0.00 & 0.00 \\
\hline 38 & 38 & 76 & -1.7 & 3.7 & .55 & 2.92 & $.00-$ & -10.00 & .63 & -5 & 0 & 0 & 0.00 \\
\hline 38 & 39 & 77 & 2 . & .2 & 37 & & 1.42 & -9 . & & & & & 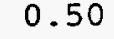 \\
\hline 8 & 40 & 78 & . & 6 & -6 & 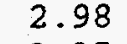 & .00 & -8.98 & .85 & -6 & 0.00 & 0.00 & 0.00 \\
\hline 38 & 41 & 79 & $-3.4-$ & -12.1 & -670.24 & 3.3 & 1.39 & -8.53 & -674.02 & -66.19 & 0.00 & 0.00 & 0.00 \\
\hline 38 & 4 & 80 & -4.0 & 1.6 & 12 & 5 & 0.00 & -8.11 & 8 & -6 & 0 & 3 & 32 \\
\hline 38 & 43 & 81 & -4. & 11.2 & & & 7 & -7 & & & & & \\
\hline 38 & 44 & 82 & $5.1-$ & -10.7 & 48 & 4.2 & 0.00 & -7.35 & .58 & -75.54 & .00 & 3.80 & -0.46 \\
\hline 38 & 4 & 83 & $-5.6-$ & -10.3 & .99 & 3.7 & 1.35 & -7.02 & 5.90 & -76.78 & -76.80 & 3.74 & -0.02 \\
\hline 38 & 46 & 84 & -6.2 & -9 & & 2.7 & 00 & -6 & 6 & 8 & 4 & & 4 \\
\hline 8 & & 5 & & & & & & & & -8 & & 2.44 & 0.49 \\
\hline 38 & 4 & 86 & -7.2 & -9.1 & -7 & 0 . & 0.00 & -6.14 & .32 & -84.99 & .52 & 1.26 & 0.47 \\
\hline 38 & 49 & 87 & -7.7 & -8.7 & .83 & 0.0 & .31 & -5.88 & 5 & 5 & 8 & 2 & 07 \\
\hline 8 & ( & 88 & 2 & & & & & & .92 & 5 & 2 & & \\
\hline 38 & 51 & 89 & -8.7 & -7.9 & .43 & -0.08 & 1.29 & -5.41 & -773.63 & -85.09 & .21 & -1.20 & -1.12 \\
\hline 38 & 5 & 90 & -9.2 & -7.6 & .20 & 0. & 0.00 & -5.20 & L. 58 & -84 & .94 & -0.15 & .97 \\
\hline 38 & - & 91 & -9.7 & -7 & & & 28 & & & & & & \\
\hline 38 & 54 & 92 & 0.2 & -6.9 & -7 & 2.76 & 0.00 & -4.82 & 3.77 & -81.01 & 2 & 0.85 & -1.91 \\
\hline 38 & 5 & 93 & 10.7 & -6.6 & .48 & 3.3 & 1.26 & -4.64 & 8.56 & -77.73 & $-\varepsilon$ & 0.87 & -2.43 \\
\hline 38 & 5 & & .1 & -6.3 & & & 00 & -4. & -8 & 5 & & & 0 \\
\hline & & & & & & & & & & & & & \\
\hline 38 & 5 & 96 & 0 & -5.7 & -8 & 3. & 0.00 & -4.17 & -8 & -7 & 3 & 3.16 & -0.28 \\
\hline 38 & 59 & 97 & 2.5 & -5.4 & -8 & 3 . & 23 & -4.03 & -8 & 3 & 0 & 3 & 0.13 \\
\hline 38 & 6 & & & -5 . & & & .00 & -3. & & & & & \\
\hline & & & .3 & -4.9 & & 3 . & .22 & -3.77 & -8 & & & 4. & 0.88 \\
\hline 38 & 62 & 100 & 3.8 & -4.7 & -8 & 3.1 & 0.00 & -3.65 & $-\varepsilon$ & -6 & 2 & 9 & 0.64 \\
\hline 38 & 63 & 101 & .2 & -4.4 & -8 & 3. & 21 & -3.54 & .44 & -56. & 0 & 0.00 & 0.00 \\
\hline 38 & 64 & & & & & & 00 & & & & & & \\
\hline 38 & 65 & 103 & 5.0 & -3.9 & & 3.07 & .19 & -3.33 & 5 & -4 & 0. & 0. & 0.00 \\
\hline 38 & 66 & 104 & 5.4 & -3.7 & -8 & 2.9 & 0.00 & -3.23 & 1.68 & -4 & 0.00 & 0.00 & 0.00 \\
\hline 38 & 67 & 105 & 5.8 & -3 & -8 & & 18 & -3 & & & .00 & 0.00 & 00 \\
\hline 8 & & & 6.1 & -3.3 & .45 & 2.29 & .00 & -3.05 & .20 & -36 & 0.00 & 0.00 & 0.00 \\
\hline 38 & 69 & 107 & & -3.1 & & 1. & 17 & & 17 & -30 & 0.00 & 0 & 0.00 \\
\hline 8 & 70 & - & .9 & .9 & 4 & .97 & 00 & 38 & & & 00 & 00 & \\
\hline
\end{tabular}




\begin{tabular}{|c|c|c|c|c|c|c|c|c|c|c|c|c|}
\hline & 1 & & 2.7 & -870.44 & 1.85 & & -2.80 & 70.24 & -20.27 & 0.00 & 0.00 & 0.0 \\
\hline & 2 & 7 & .5 & 73 & 35 & 00 & -2.73 & -873.44 & -15.40 & .00 & .00 & 0.00 \\
\hline & 3 & $1-17.9$ & -2.3 & -875.49 & 32 & 15 & -2.66 & 74.68 & 57 & .00 & .00 & .00 \\
\hline & 4 & $12-18.3$ & -2.2 & -877.74 & 3.05 & Do & -2.59 & -877.24 & -3.06 & 00 & .00 & .00 \\
\hline & 5 & 8.6 & -2.0 & -879.82 & 10 & & -2.53 & -879.28 & & & & \\
\hline & 76 & .9 & 1 & & & & & & 7.39 & & & 00 \\
\hline & 77 & $.5-19.3$ & -1.7 & -883.49 & & 1.13 & -2.41 & .43 & 13.97 & .00 & .00 & .00 \\
\hline & 78 & $116-19.6$ & -1.5 & -885.09 & -0.09 & 0.00 & -2.35 & -88 & 18.94 & 0.00 & .00 & 0.00 \\
\hline & 79 & 19.9 & -1.4 & -886.53 & 1.00 & 1.12 & -2.30 & & & & & 00 \\
\hline & 80 & 118 & -1 & & 6 & & -2 & & & & & \\
\hline & 81 & $119-20.5$ & -1.1 & -8 & -4.34 & & -2.19 & 1 & 36.27 & & .00 & 0.00 \\
\hline & 82 & $120-20.8$ & -0.9 & .01 & -5.18 & 0.00 & -2.14 & -89 & 41.43 & 0.00 & 0.00 & 0.00 \\
\hline & 8 & $121-21.1$ & -0.8 & -890.89 & -4.67 & 1.10 & -2.10 & & 50 & 0 & 0 & .00 \\
\hline & 84 & $122-$ & -0.7 & & -3.74 & & & & & & & \\
\hline & 85 & $123-21.6$ & -0.6 & 2.28 & -3.14 & 9 & -2.01 & -896.33 & 66.64 & & & \\
\hline & 8 & $124-21.9$ & -0.4 & .79 & -1.97 & 0.00 & -1.97 & -896.72 & 74.32 & 0.00 & 0.00 & 0.00 \\
\hline & 0 & $225-22.1$ & -0.3 & 3.18 & -1.45 & 1.08 & -1.93 & .47 & 83.64 & 0.00 & 0.00 & 0.00 \\
\hline & 88 & 2.4 & -0.2 & .46 & 0.48 & 0. & - & & & & & 10 \\
\hline & 89 & $127-22.6$ & -0.1 & -893.64 & 1.45 & 1.08 & -1.85 & -892.97 & 102.29 & 0.00 & 0.00 & bo \\
\hline & 9 & 0.0 & 0.0 & 0.00 & 1.37 & 0.00 & -1.82 & 0.00 & 0.00 & 0.00 & 0.00 & 0.00 \\
\hline & 9 & 0.0 & 0. & 0.00 & 1.60 & 1.07 & -1.78 & 0.00 & 0.00 & 0.00 & 0.00 & .00 \\
\hline & 3 & 130 & 0. & 0.8 & 1.58 & 0 & s & & & & & \\
\hline & 93 & 131 & 0.0 & 0.00 & 1.3 & 1.06 & -1.71 & 0. & 0.00 & 0 . & 0.00 & 0 \\
\hline & 31 & 70 & 18.5 & -518.62 & 3. & 2.55 & -6.19 & -518.58 & 15.81 & 0.00 & 0.00 & 0.00 \\
\hline & 32 & 71 & 17.9 & -536.80 & 3.88 & 1.42 & -6.61 & -538.11 & 4.36 & 0.00 & .00 & 0.00 \\
\hline & 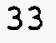 & 72 & $7 ?$ & -5 & 414 & 2.53 & 05 & -554.70 & -4.16 & & & .00 \\
\hline & 34 & 73 & & & 05 & & & -573.26 & -14.65 & 0 . & 0.00 & 0.00 \\
\hline & 3 & 74 & 16.0 & -587.51 & 4 & 2.51 & -7.97 & -588.95 & -22.27 & 0 . & 0.00 & 0.00 \\
\hline & 3 & 75 & 15.4 & -603.20 & 3.5 & 1.42 & -8.45 & -606 & -31.96 & 0. & 0.00 & 0.00 \\
\hline & & 76 & 4.8 & -6 & & & & & -38 & & & \\
\hline & 38 & 77 & .3 & -6 & 2 & 1. & -5 & 9 & -47.20 & 0 . & 0.00 & 0 \\
\hline & 39 & $\begin{array}{ll}78 & -1.5\end{array}$ & 13.7 & -646.83 & 2.8 & 2. & 10.00 & -651.16 & -52.19 & 0. & 0.00 & 0.00 \\
\hline & 45 & 79 & 13.2 & -6 & 2.7 & 1.42 & -9.48 & - & -58.57 & 0. & 0.00 & 0.00 \\
\hline & & 80 & .7 & & & & & & 1 & & & \\
\hline & 42 & 81 & .2 & & 3 & & -8 & 9 & -66.21 & 5.95 & 3.71 & 0. \\
\hline & 43 & $\begin{array}{lll}82 & -3.7\end{array}$ & 11.7 & .66 & 5.2 & 2. & -8.15 & 3.11 & -66.86 & -68. & 3.94 & -1.32 \\
\hline & 44 & 4.3 & -11.3 & -7 & 4.3 & 1.42 & -7.76 & - & -71.88 & - & 3.84 & 0.46 \\
\hline & & 84 & 0.8 & -1 & & 2 & & & -74.00 & & & \\
\hline & & 5 & .4 & & 2.73 & & 8 & 4 & -78.28 & -77.85 & 3.16 & 0 \\
\hline & 47 & $5.9-$ & 10.0 & .00 & 1.5 & 2.41 & -6.77 & 3.41 & -79.87 & -79. & 2.54 & 0.59 \\
\hline & & 6.4 & -9.6 & .77 & 0. & 1.42 & -6 . & & -83.44 & 1 & 1.21 & 43 \\
\hline & & 88 & -9.2 & & & & & & & & & \\
\hline & & 7.4 & -8.8 & .14 & -1.19 & 1.42 & -5.95 & 4.86 & -87.11 & -87.70 & -1.78 & -0.59 \\
\hline & & 7.9 & -8.4 & -777.75 & -0.11 & 2.38 & -5.71 & .20 & -85.37 & -86.49 & -1.23 & -1.12 \\
\hline & & 8.4 & -8.1 & .01 & & 1.42 & -5 & & -8 & 5 & 6 & -1.01 \\
\hline & & 92 & -7 & & & & & & -82.97 & 33 & 0.03 & \\
\hline & 54 & -9.4 & -7.4 &. .49 & 2.68 & 1.42 & -5.08 & -8 & -82.43 & .25 & 0.86 & -1.82 \\
\hline & & -9.8 & -7.1 & -808.73 & 3.4 & 2.36 & -4.89 & $-\varepsilon$ & -79.73 & 5 & 30 & -2.62 \\
\hline & & 10.3 & -6.8 & .65 & 3 & 1.42 & -4 & & -79 & - & 36 & -1.98 \\
\hline & 5 & 96 & -6.5 & -8 & 3 & 2 . & & & & & 39 & \\
\hline & 58 & & 5.2 & & & & -4 & & -75.79 & 7 & & -0 \\
\hline & 59 & .7 & -5.9 & .61 & 3 & 2 . & -4.24 & $-\varepsilon$ & -72.79 & -7 & 9 & 46 \\
\hline & 6 & 2.1 & -5.6 & -8 & 20 & 1.42 & -4 & 4 & -71.37 & - & 7 & 17 \\
\hline & $\epsilon$ & .5 & -5.3 & -8 & & 2 & & & & & & \\
\hline & & & & & & & -3 & & -65. & 0 & & 50 \\
\hline & 6 & 4 & -4.8 & -8 & 3 . & 2 & -3.72 & -8 & -61.62 & 0 & 0 & 0.00 \\
\hline & 6 & 103 & -4.6 & -8 & 3.12 & 1. & -3.61 & -8 & -58 & 0 & 0 & 00 \\
\hline & 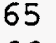 & 10 & & & & & & & & & & \\
\hline & 66 & 105 & -4.1 & -8 & 2 . & 1. & -3 & & & 0 & & 0 \\
\hline & 6 & 106 & -3.9 & & & & & & & .00 & 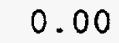 & \\
\hline & 6 & 107 & & -8 & & 1.4 & & & & .00 & 0 & 0 \\
\hline & 6 & & -3.5 & & & & & & & & & \\
\hline & 7 & 109 & -3.3 & 4.12 & 1. & 1. & -3 & & -3 & & & 0.00 \\
\hline & 71 & $110-16.4$ & -3.1 & -887.28 & 1. & & & -8 & -2 & 0 & 10 & 0.00 \\
\hline & 72 & 111. & -2.9 & -890.25 & 2.24 & 1.42 & -2.87 & -889. & -24.14 & .00 & .00 & 0.00 \\
\hline & 7 & $12-17.2$ & 2.7 & 3 & & 2.28 & $\overline{2}$ & & & 00 & 00 & 00 \\
\hline
\end{tabular}




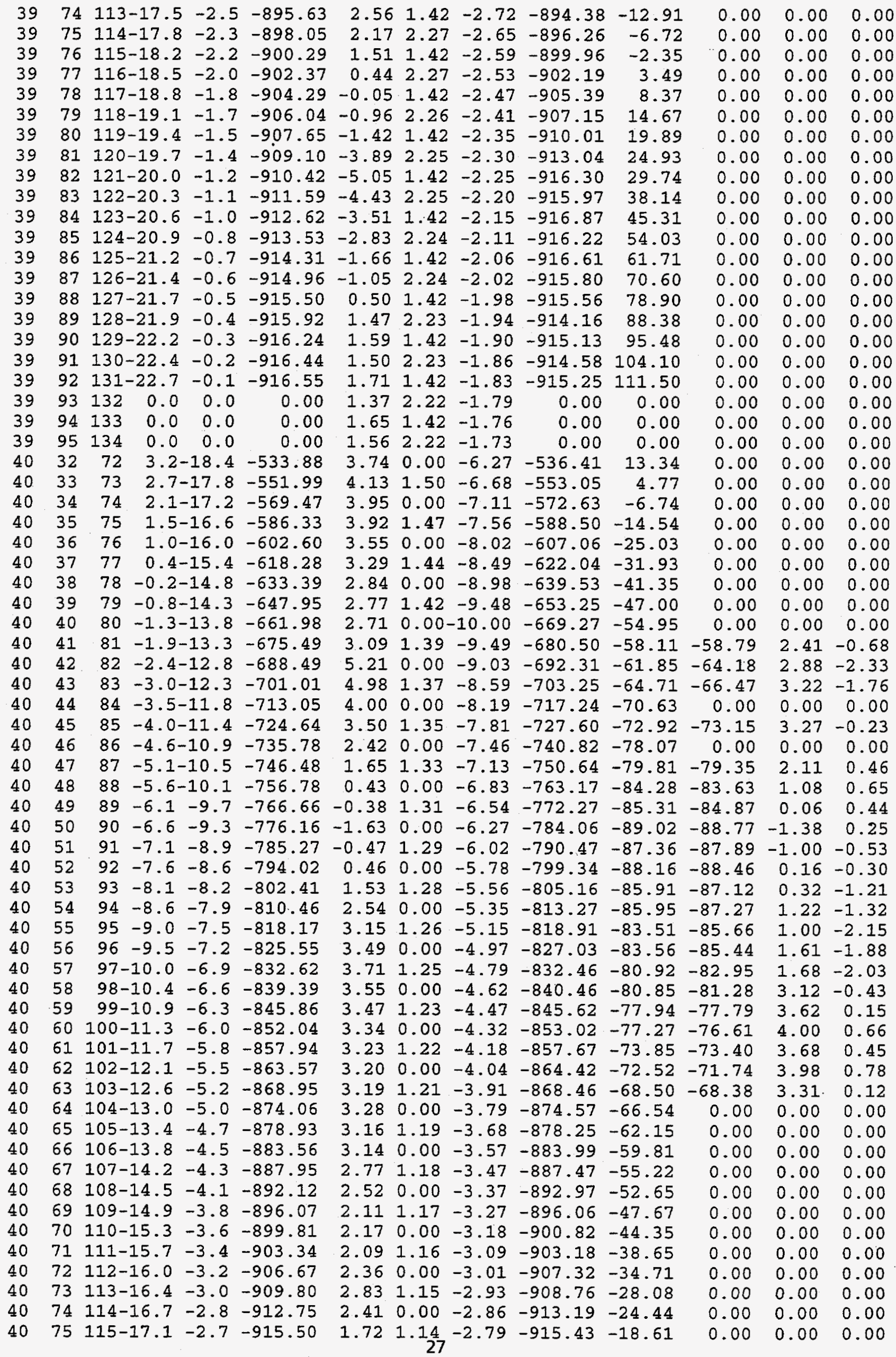




\begin{tabular}{|c|c|c|c|c|c|c|c|c|c|c|c|c|c|}
\hline & 6 & \multicolumn{2}{|c|}{$116-17.4$} & 2.5 & 19 & 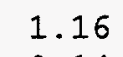 & 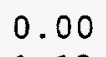 & -2 & $z$ & -14 & 0 & & \\
\hline & 7 & \multicolumn{2}{|c|}{$117-17.7$} & 2.3 & 320.49 & 14 & 13 & -2 & -921.87 & 1 & & & 0 \\
\hline & 8 & \multicolumn{2}{|c|}{$118-18.1$} & 2 & 3 & & 0 & & & & 00 & 00 & 8 \\
\hline & 79 & \multicolumn{2}{|c|}{$119-18.4$} & 2.0 & 4.81 & .34 & 2 & -2.52 & -927.55 & .56 & 00 & 00 & .00 \\
\hline & 80 & \multicolumn{2}{|c|}{$120-18.7$} & 1.8 & 6.72 & 1.77 & 00 & -2.47 & -930.96 & & & & 00 \\
\hline & 81 & \multicolumn{2}{|c|}{$121-19.0$} & 17 & & -4.20 & & -2 & -933.98 & & & & 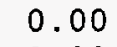 \\
\hline & 82 & \multicolumn{2}{|c|}{$122-19.3$} & .5 & & & .00 & 6 & & & 0 & & .00 \\
\hline & 83 & \multicolumn{2}{|c|}{$123-19.6$} & -1.4 & 56 & & 10 & -2.30 & -937.47 & 92 & & 00 & 0.00 \\
\hline & 84 & \multicolumn{2}{|c|}{$124-19.9$} & 1.3 & .88 & -3.78 & .00 & -2.25 & 8.92 & & 0 & 00 & \\
\hline & 85 & $125-$ & 20.2 & 1.1 & .07 & -3.05 & & -2.20 & & & & & 0.00 \\
\hline & 86 & & & -1.0 & 3 & 1 & & 6 & 9 & & & 00 & 0.00 \\
\hline & & 127 & .7 & -0 & & -0 & & -2.11 & -9 & & & & .00 \\
\hline & 88 & - & 21.0 & -0.7 & 85 & 0.78 & .00 & -2.07 & 8.14 & & & & \\
\hline 4 & 89 & - & 21.3 & -0.6 & & 1.48 & & & & 72 & & & \\
\hline 40 & 90 & 30 & 21.5 & -0 & & 1.82 & 0 & -1.99 & - & & 0 & 0 & .00 \\
\hline \pm & & - & 21.8 & -04 & & 1. & & -1.95 & 94 & & & & .00 \\
\hline & 9 & - & .0 & -0.3 & 9 & 1.8 & .00 & -1.91 & 8.96 & & & & \\
\hline & - & 33 & .2 & -0.2 & & & 6 & & 31 & 103 & & & 0 \\
\hline 40 & 9 & 34 & .5 & -0.1 & $\cdots$ & 1.8 & 10 & -1.84 & 26 & 10.92 & 0.00 & 0.00 & .00 \\
\hline & 95 & 135 & 0.0 & 0.0 & 0 & 1.80 & .05 & -1.81 & 0.00 & 0.00 & .00 & 00 & .00 \\
\hline & & 36 & 0.0 & 0. & 0 & 2.00 & 0 & -1.77 & 0.00 & & & & 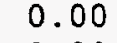 \\
\hline & 9 & 137 & 0.0 & 0. & & & & & 0.00 & 0 & 10 & & 0.00 \\
\hline 1 & 3 & 74 & .3 & 8.4 & -548 & 4. & 1 & -6.35 & -548.76 & 16.35 & 0 & 0 & 0.00 \\
\hline \pm 1 & 34 & 75 & 2.8 & 1.7 & 4 & 4. & .39 & -6.76 & .96 & & & & .00 \\
\hline & 3 & 76 & 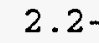 & & & & & -7.18 & 71 & & & & .00 \\
\hline & 3 & 77 & .6 & & & & & & 3.53 & -14 & & 10 & 0.00 \\
\hline 11 & 37 & 78 & L. & .0 & -6 & 3 & 7 & -8.06 & 9.35 & -21.96 & 10 & 10 & 0.00 \\
\hline 41 & 3 & 79 & 0.5 & . & -6 & 3. & 9 & -8.53 & 16 & & & & .00 \\
\hline. \pm & 35 & 80 & 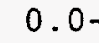 & & & & & -9 & 31 & & & & .00 \\
\hline & 40 & 81 & -0 . & & & 3 & & & 3.00 & 40 & 0 & 10 & 0.00 \\
\hline 1 & 41 & 82 & -1 & .8 & -6 & 3. & .8 & -10.00 & -680.83 & -51.15 & 0. & 0 & 0.00 \\
\hline 41 & 42 & 83 & -1.7 & .3 & -6 & 3.8 & 9 & -9.51 & .77 & -5 & 7 & & -1.95 \\
\hline & 4 & 84 & -2. & & & & & & 20 & & & & .00 \\
\hline & & 85 & -2.8 & & & 4. & & $-\varepsilon$ & .38 & 18 & -67 & 37 & -1.67 \\
\hline & 45 & 86 & $-3.3-$ & .9 & -7 & 3.5 & .40 & -8.23 & .60 & -68.64 & 0 . & 10 & 0.00 \\
\hline 41 & 46 & 87 & -3.8 &. .4 & -7 & 2. & 9 & -7.86 & .97 & & -74 & & \\
\hline \pm 1 & $4^{\circ}$ & 88 & -4 & & & & & & & & 0.00 & 0 & 00 \\
\hline & 48 & 89 & $-4.8-$ & & & 0 & .39 & -7 & 7.34 & -81.16 & -80.58 & 1.04 & 0.58 \\
\hline 1 & 45 & 90 & -5.4 & .2 & .40 & 0.3 & 2.38 & -6.88 & 7.25 & -83 & -82 & 0 & 0 . \\
\hline 11 & 5 & 91 & -5.9 & .8 & & 1.44 & 9 & -6 & 4 & & & & \\
\hline & 51 & 9 & -6.3 & & & & & & & 6 & 15 & 6 & -0.29 \\
\hline & 52 & 93 & 6.8 & & & 0 & 9 & -6 & .49 & -87.03 & -87.21 & 26 & -0.18 \\
\hline & 5 & 94 & -7.3 & -8.7 & & 1.5 & 2.35 & -5.85 & 2.10 & 57 & -8 & 71 & -0.80 \\
\hline 4$]$ & 5 & 95 & -7.8 & . & & 2 & 39 & -5 & & & & & \\
\hline & 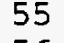 & 96 & 8.3 & & & & & & & & & 51 & 36 \\
\hline & 5 & 97 & -8.7 & -7 & & 3 & 9 & -5 & .14 & -8 & -8 & 14 & -1.21 \\
\hline 7. & 5 & 98 & -9.2 & -7 & & 3 . & 2.33 & -5 . & 6 & & & & 19 \\
\hline 43 & 5 & 9 & -9.6 & & & & & & & & & & \\
\hline & 59 & 10 & 0.1 & -6. & & 3 . & 2 . & -4 & 18 & -8 & -7 & & 0.08 \\
\hline & 60 & 01 & .5 & -6 . & & 3. & 1.39 & -4 & .58 & -79.55 & -7 & & 63 \\
\hline 4$]$ & 61 & 102 & 0.9 & 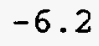 & & 3. & 2 . & -4 & - & & - & & 52 \\
\hline 41 & 62 & & & & & & & & & & & & \\
\hline & 63 & & . & -5 & & 3 . & 2 . & -4 & 1 & 37 & 3 & 15 & 0.14 \\
\hline & 64 & 105 & 2.2 & -5.4 & & 3. & 1. & -3 & 5.79 & -7 & -70 & 5 & -0.39 \\
\hline 41 & 65 & 106 & 6 & -51 & & & 2 . & & -8 & & & & 00 \\
\hline 41 & 66 & & & & & & & & & & & & \\
\hline & 67 & & 13.4 & -4.7 & & & & & & & & & 00 \\
\hline & 68 & 10 & .8 & $-z$ & & 2 & 1 & -3 & 53 & -5 & 0.00 & .00 & .00 \\
\hline 41 & 69 & & & & & & & & & & & & .00 \\
\hline & 70 & & & -10 & & & & & 8 & & & & \\
\hline 4 & 71 & & 4.9 & -3.8 & & 2. & 2 & -3 & & & & & 00 \\
\hline 47 & 72 & 13 & 15.3 & 6 & -9 & 3. & 1. & -3.16 & 0.89 & & & & 08 \\
\hline 4 & 73 & & & & & & 2 . & & 3.68 & & & & .0 \\
\hline 4. & 74 & 11 & 5.0 & -3.2 & & 2 . & 1. & -3 & 3.18 & & & & 00 \\
\hline 41 & 75 & 11 & 6.3 & 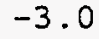 & & 1. & & -2.92 & 1.15 & 04 & 0 . & 0 & .00 \\
\hline 41 & 76 & 17 & 6.7 & r & -935.12 & 1.10 & & & 25 & & 0.00 & 0.00 & \\
\hline
\end{tabular}




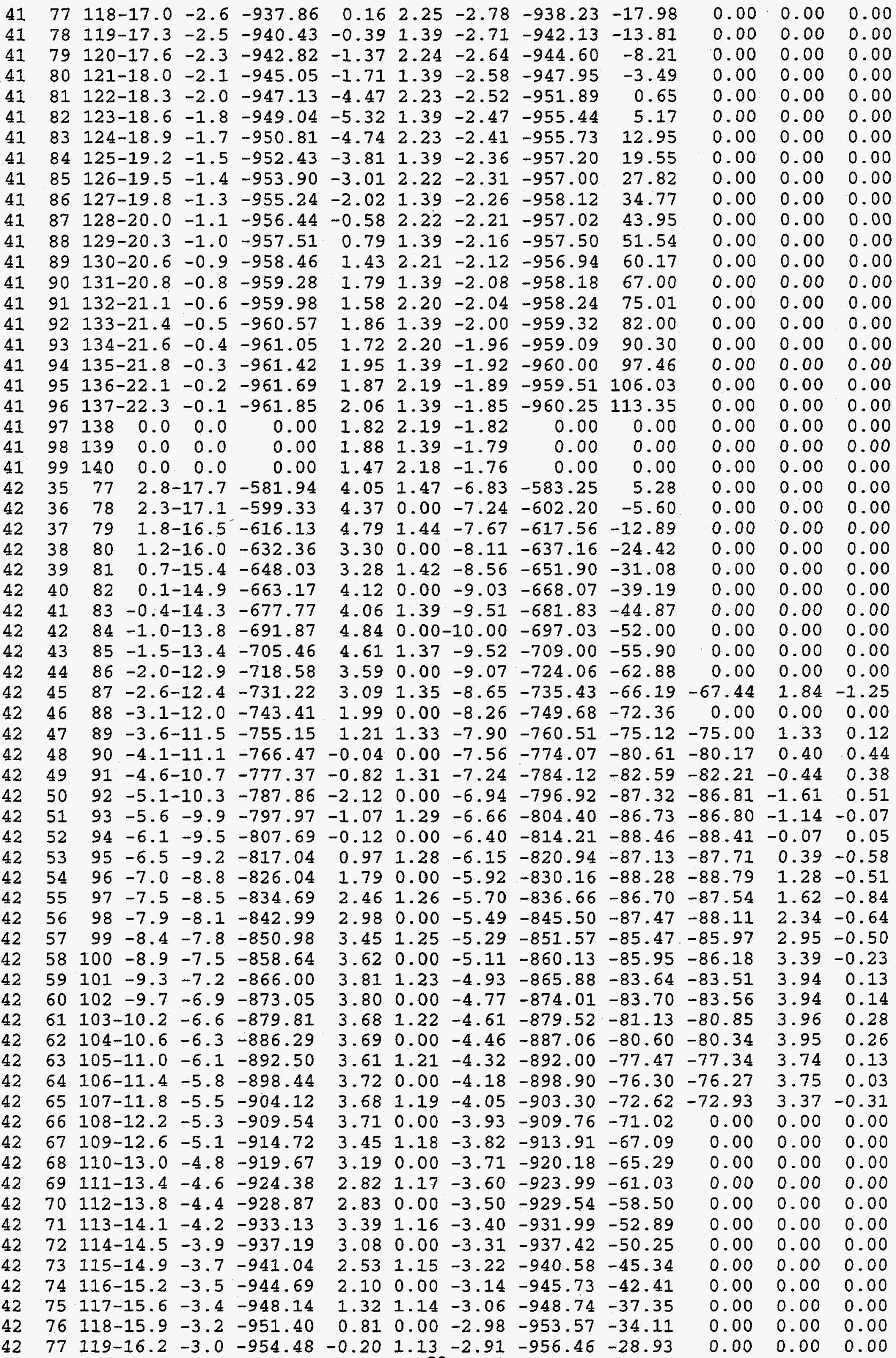




\begin{tabular}{|c|c|c|c|c|c|c|c|c|c|c|c|c|c|}
\hline & 78 & \multicolumn{2}{|c|}{$120-16.6$} & -2.8 & 37 & -0 & 00 & -2.84 & -960 & -25 & 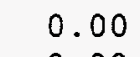 & 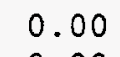 & \\
\hline & 79 & & & -2.6 & -960.10 & -1.78 & 12 & -2.77 & -963.53 & -19 & 10 & 10 & 0 \\
\hline & 80 & & & -2.5 & -962.65 & -2.14 & 00 & -2.70 & & & 0 & & \\
\hline & 81 & 123 & 7.5 & -2.3 & -965.03 & -4.86 & 11 & -2.64 & -971.43 & -11.61 & Do & 00 & \\
\hline & 82 & 25 & -17.9 & -2.1 & 7.26 & -5.93 & 0 & -2.58 & -975.77 & -7.88 & 0 & & \\
\hline & 83 & 125 & 2 & -2 & -5 & -5.33 & 0 & -2.52 & .08 & -0.12 & 0 & 10 & \\
\hline & 84 & & 3.5 & -1.8 & 25 & 25 & & -2.47 & & & & & \\
\hline & 85 & 127 & 3.8 & -1.7 & -973.02 & -3.50 & 09 & -2.41 & -977.84 & 14.27 & .00 & 10 & 0 \\
\hline & 86 & $128-$ & -19.0 & -1.6 & -974.64 & -2.32 & 10 & -2.36 & -97 & 20.85 & 00 & 00 & 0 \\
\hline & 87 & $129-$ & .3 & -1 & -9 & & 8 & -2.31 & & & 10 & & \\
\hline & 88 & 136 & 6 & -1.3 & -9 & 0 & 0 & .26 & 24 & 37.08 & 0 & & \\
\hline & 89 & & .9 & -1.1 & -9 & 1.15 & & -2.22 & .68 & 45.70 & 0 & 0 & \\
\hline & 90 & $132-$ & .2 & -1.0 & -979.78 & 1.63 & Do & -2.17 & .32 & 52.14 & 10 & 10 & Do \\
\hline & 91 & 133 & .4 & -0. & -9 & 1.59 & 7 & -2.13 & & & & & 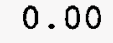 \\
\hline & 92 & 134 & & -0.8 & -9 & 1 & & 9 & -5 & & & & \\
\hline & 93 & & 1.9 & -0.7 & -982.32 & 1.81 & & -2.05 & & .18 & .00 & & \\
\hline & 94 & $136-$ &. .2 & -0.6 & -982.93 & 2.10 & .00 & -2.01 & -9 & 81.91 & 0.00 & & 00 \\
\hline & 9 & $137-$ &. .4 & -0.4 & -9 & 2.05 & & -1.97 & & 90.52 & & & 0 \\
\hline & 96 & 1. & & & & & & 3 & & & & & \\
\hline 42 & 97 & 13 & .9 & -0.2 & 1.12 & 2.06 & 4 & -1.90 & .92 & 106.04 & 0.00 & 0 & 0 \\
\hline & 98 & 140 & .1 & -0.1 & -9 & 2.18 & 10 & -1.86 & 0 & .03 & 10 & 10 & .00 \\
\hline & 9 & 1 & .3 & -0.1 & .42 & 1.80 & & -1.83 & 11 & 1.69 & & & 10 \\
\hline & 10 & 142 & 0 & 0.0 & & 4 & & .80 & 0 & & & & \\
\hline 4 & .01 & 143 & 0.0 & 0.0 & 0. & 1.06 & 3 & -1.77 & 0.00 & 0. & 0 & 10 & .00 \\
\hline 42 & 106 & 144 & 0.0 & 0.0 & 0 & 0.80 & 0 & -1.74 & 0. & 0.00 & 0 & 10 & 10 \\
\hline & 36 & 79 & 28 & 7. & -5 & & & -6.89 & -5 & & & & \\
\hline & 37 & 80 & 1. & & & & & & & & & & \\
\hline 4. & 38 & 81 & 1.9 & -16.5 & -63 & 4.18 & 7 & -7.72 & .99 & -12.96 & 0 & 0 & 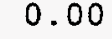 \\
\hline & 39 & 82 & 1.3 & 5.9 & -64 & 4.31 & 4 & -8.15 & .44 & -20.34 & 0.00 & 1.00 & .00 \\
\hline & 40 & 83 & 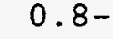 & & & & & & & & & & \\
\hline & 41 & 84 & & & & & & & & & 0 & 0 & \\
\hline 43 & 42 & 85 & -0.3 & -14.4 & -6 & 4.52 & 7 & -9.52 & .11 & -43.79 & 0.00 & .00 & 0 \\
\hline 4. & 43 & 86 & .0 .8 & 3.9 & -7 & 4.27 & 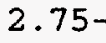 & -10.00 & .58 & -49.20 & 0. & 0 & .00 \\
\hline & 44 & 87 & 1. & & & & & & & & & & \\
\hline & 45 & 88 & 1. & -12.9 & & & & & & & & & \\
\hline & 46 & 89 & $2.3-$ & $-12 \cdot 5$ & -7 & 1 . & 7 & -8 . & -7 & -67.20 & 0 . & 0 & 0 \\
\hline & 47 & 90 & $-2.9-$ & -12.0 & -7 & 4 & 7 & -8.30 & -7 & -70.78 & 0. & 0.00 & .00 \\
\hline & 48 & 91 & 3.4 & .6 & & & & & & 1 & 9 & & .42 \\
\hline & 49 & 2 & & 2 & & & 6 & -7.60 & & -79.19 & 4 & 2 & \\
\hline 2. & 50 & 93 & $-4.4-$ & -10.8 & .58 & -2.45 & .37 & -7.29 & $-\varepsilon$ & -84.07 & 61 & -1.99 & 0.46 \\
\hline & 51 & 94 & $-4.8-$ & -10.4 & -8 & -1.37 & .35 & -6.99 & $-\varepsilon$ & -84.24 & 6 & -1.29 & 0.08 \\
\hline & 5 & 95 & 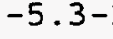 & 0 & & & & & & 0 & & & \\
\hline & 53 & 96 & & & $-\varepsilon$ & & 2 & -6 & 4 & 54 & & 42 & \\
\hline 18 & 54 & 97 & 6.3 & -9.3 & -8 & 1.26 & .37 & -6.21 & $-\varepsilon$ & -87.09 & 22 & 1.13 & -0 \\
\hline & 55 & 98 & 6.7 & -8.9 & -8 & 2. & .32 & -5.98 & $-\varepsilon$ & -86.16 & 3 & .78 & 7 \\
\hline & 56 & 99 & .2 & -8.6 & & & & & & 0 & & 2 & \\
\hline & & & & & & & & -5.55 & & & & & \\
\hline 3 & 58 & 101 & -8.1 & -8.0 & -8 & 3. & .37 & -5.36 & -8 & -86.12 & 4 & 3.30 & -0.22 \\
\hline & 59 & 02 & 8.5 & -7.6 & 9.5 & 3 . & 0 & -5 & $-\varepsilon$ & -84.53 & & .68 & -0.04 \\
\hline & 60 & 3 & .0 & -7.3 & & & 1.37 & -5 & & 3 & & 83 & 3 \\
\hline & & & & & & & & & & & & & \\
\hline & 62 & 05 & -9.8 & -6.8 & -8 & & 7 & -4.68 & $-\varepsilon$ & -82.23 & 9 & 3.77 & \\
\hline & 63 & $6-$ & 10.2 & -6.5 & -9 & 7 & 2.28 & -4 & -9 & -79.68 & 8 & 3.77 & -0.10 \\
\hline & 64 & 107 & .7 & -6.2 & -5 & & 1.3 & -4 & 9 & 71 & & 51 & \\
\hline & & & & & & & & & & & & & \\
\hline & 66 & 109 & & -5.7 & -9 & 4. & 1.37 & -4.12 & -9 & -73.50 & 1 & 3.50 & -1.11 \\
\hline 17. & 67 & $10-$ & .9 & -5.4 & -9 & & 2 & -4.00 & -9 & -70.24 & 0 & 0 & 10 \\
\hline & 68 & 1 & .2 & -5.2 & & & & & & & & & \\
\hline 13 & 69 & 112 & 6 & -5.0 & -9 & 3. & 2.26 & -3.77 & & -6 & & & \\
\hline 43 & 70 & 13 & & -4.7 & & & & & & & & & \\
\hline 3 & 71 & $114-$ & 4 & 4.5 & 9 & & & & & 5 & & & .00 \\
\hline & 72 & & & & & & & & & & & & \\
\hline 43 & 73 & 11 & .1 & -4.1 & -9 & 6 & 2.24 & -3.37 & & -51 & & & 10 \\
\hline 43 & 74 & 117 & & -3.9 & -959.5 & 1. & & -3.29 & & $-4 \varepsilon$ & & 10 & 0.00 \\
\hline 43 & 75 & 118 & & -3.7 & -96 & & 2.2 & -3.20 & & -44.48 & .00 & 0.00 & 0.00 \\
\hline$x-2$ & 76 & & 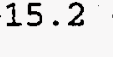 & -3.5 & & & 1.37 & 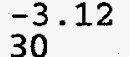 & 0 & & & & \\
\hline
\end{tabular}



$89132-19.2-1.4 \quad-998.24$ $92135-20.0-1.0-1001.94$ $93136-20.3-0.9-1002.92$ $\begin{array}{lll}94 & 137-20.5 & -0.8-1003.78 \\ 95 & 138-20.8 & -0.7-1004.53\end{array}$ $96139-21.0-0.6-1005.17$ $\begin{array}{lll}97 & 140-21.3 & -0.5-1005.70 \\ 98 & 141-21.5 & -0.4-1006.12\end{array}$ $99142-21.7-0.3-1006.44$ $\begin{array}{lll}100 & 143-21.9 & -0.2-1006.67 \\ 101 & 144-22.1 & -0.1-1006.79\end{array}$ $\begin{array}{llll}37 & 81 & 3.0-17.6 & -611.32\end{array}$ $4993-3.1-11.7-785.09$ $5094-3.6-11.3-796.57$ $\begin{array}{lllll}51 & 95 & -4.1-10.9 & -807.65\end{array}$ $5296-4.6-10.5-818.34$

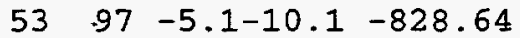
$\begin{array}{llllll}54 & 98 & -5.5 & -9.8 & -838.58\end{array}$ $\begin{array}{llllll}55 & 99 & -6.0 & -9.4 & -848.15\end{array}$ $\begin{array}{llllll}56 & 100 & -6.5 & -9.1 & -857.38\end{array}$ $\begin{array}{llllll}57 & 101 & -6.9 & -8.7 & -866.27\end{array}$ $\begin{array}{llllll}58 & 102 & -7.3 & -8.4 & -874.83\end{array}$ $\begin{array}{llllll}59 & 103 & -7.8 & -8.1 & -883.07\end{array}$ $\begin{array}{lllll}60 & 104 & -8.2 & -7.8 & -890.99\end{array}$ $\begin{array}{llllll}61 & 105 & -8.7 & -7.5 & -898.61\end{array}$ $\begin{array}{lllllll}62 & 106 & -9.1 & -7.2 & -905.94\end{array}$ $\begin{array}{lllll}63 & 107 & -9.5 & -6.9 & -912.98\end{array}$ $\begin{array}{llllll}64 & 108 & -9.9 & -6.6 & -919.75\end{array}$ $65109-10.3-6.4-926.24$ $\begin{array}{lllll}66 & 110-10.7 & -6.1 & -932.47\end{array}$ $67 \quad 111-11.1-5.8-938.44$ $68 \quad 112-11.5 \quad-5.6 \quad-944.16$ $69113-11.9-5.4 \quad-949.64$ $70 \quad 114-12.3 \quad-5.1 \quad-954.89$ $71 \quad 115-12.6 \quad-4.9 \quad-959.90$ $72116-13.0 \quad-4.7-964.69$ $73 \quad 117-13.4 \quad-4.5 \quad-969.27$ $74118-13.7-4.3-973.63$ $\begin{array}{lllllllllll}77 & 120 & -15.5 & -3.3 & -970.35 & -0.32 & 2.23 & -3.04 & -971.49 & -36.67\end{array}$ $\begin{array}{llllllllll}78 & 121-15.8 & -3.1 & -973.59 & -0.91 & 1.37 & -2.97 & -976.09 & -33.21\end{array}$ $\begin{array}{lllllllll}122-16.2 & -3.0 & -976.64 & -1.89 & 2.22 & -2.90 & -979.20 & -28.24\end{array}$ $\begin{array}{lllllll}17.7 & -2.1 & -989.35 & -4.38 & 1.37 & -2.58 & -994.94\end{array}$

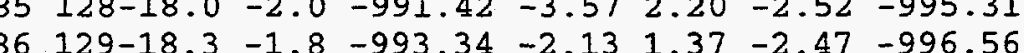
$87130-18.6-1.7-995.11-0.592 .20-2.41-995.92$ $90 \quad 133-19.5-1.3-999.60$
$0.311 .37-2.36-997.42$

$0.962 .19-2.31 \quad-997.40$

$\begin{array}{llll}1.56 & 1.37 & -2.27 & -998.94\end{array}$

$\begin{array}{llll}1.55 & 2.19 & -2.22 & -999.32\end{array}$

$1.74 \quad 1.37-2.18-1001.00$

$1.812 .18-2.14-1001.06$

$2.08 \quad 1.37-2.09-1002.43$

$2.042 .17-2.05-1002.37$

$2.141 .37-2.02-1003.67$

$2.03 \quad 2.17-1.98-1003.48$

$2.22 \quad 1.37-1.94-1004.47$

$1.842 .17-1.91-1004.35$

$1.761 .37-1.87-1005.41 \quad 115.05$

$\begin{array}{lllll}1.17 & 2.16 & -1.84-1005.31 & 123.22\end{array}$

$\begin{array}{lllll}1.01 & 1.37 & -1.81 & 0.00 & 0.00\end{array}$

$0.832 .16-1.78 \quad 0.00 \quad 0.00$

$\begin{array}{lllll}1.44 & 1.37 & -1.75 & 0.00 & 0.00\end{array}$

$\begin{array}{lllll}4.02 & 1.44 & -6.96 & -612.82 & 6.42\end{array}$

$\begin{array}{llllll}3.80 & 0.00 & -7.35 & -632.18 & -4.87\end{array}$

$\begin{array}{llllll}5.23 & 1.42 & -7.76 & -646.49 & -11.11\end{array}$

$\begin{array}{llllll}4.74 & 0.00 & -8.19 & -665.02 & -21.57\end{array}$

$\begin{array}{lllllll}4.64 & 1.39 & -8.62 & -679.84 & -28.31\end{array}$

$\begin{array}{llllll}3.77 & 0.00 & -9.07 & -697.70 & -38.10\end{array}$

$\begin{array}{llllll}3.52 & 1.37 & -9.53 & -711.69 & -44.02\end{array}$

$\begin{array}{llllll}2.49 & 0.00-10.00 & -728.72 & -52.98\end{array}$

$\begin{array}{lllllll}1.98 & 1.35 & -9.54 & -741.10 & -57.29\end{array}$

$\begin{array}{lllllll}0.85 & 0.00 & -9.11 & -756.36 & -64.48\end{array}$

$\begin{array}{llllll}0.08 & 1.33 & -8.71 & -768.16 & -68.21\end{array}$

$\begin{array}{llllll}-1.20 & 0.00 & -8.33 & -782.72 & -74.70\end{array}$

$\begin{array}{llllll}-1.95 & 1.31 & -7.98 & -793.70 & -77.61\end{array}$

$\begin{array}{llllll}-3.24 & 0.00 & -7.65 & -807.46 & -83.29\end{array}$

$\begin{array}{lllllll}-2.08 & 1.29 & -7.34 & -815.77 & -83.53\end{array}$

$\begin{array}{llllll}-1.11 & 0.00 & -7.05 & -826.49 & -86.18 \\ 0.01 & 1.28 & -6.77 & -834.13 & -85.74\end{array}$

$\begin{array}{llllll}0.01 & 1.28 & -6.77 & -834.13 & -85.74 \\ 0.57 & 0.00 & -6.51 & -844.52 & -88.07\end{array}$

$1.36 \quad 1.26-6.27 \quad-851.80-87.28$

$2.00 \quad 0.00-6.04-861.42-88.83$

$2.54 \quad 1.25 \quad-5.82 \quad-868.31 \quad-87.64$

$\begin{array}{llllll}2.98 & 0.00 & -5.62 & -877.47 & -88.73\end{array}$

$\begin{array}{llllll}3.31 & 1.23 & -5.42 & -883.95 & -87.14\end{array}$

$\begin{array}{lllllll}3.49 & 0.00 & -5.24 & -892.74 & -87.86\end{array}$

$\begin{array}{lllllll}3.70 & 1.22 & -5.07 & -898.76 & -85.81 & -85\end{array}$

$\begin{array}{lllllll}3.83 & 0.00 & -4.90 & -907.01 & -85.99\end{array}$

$3.90 \quad 1.21-4.74-912.62-83.53$

$\begin{array}{llllll}3.99 & 0.00 & -4.59 & -920.35 & -83.18\end{array}$

$3.94 \quad 1.19-4.45-925.56-80.32$

$\begin{array}{lllllll}4.20 & 0.00 & -4.32 & -932.58 & -79.27\end{array}$

$3.93 \quad 1.18-4.19-937.52 \quad-76.13$

$\begin{array}{lllllll}3.60 & 0.00 & -4.07 & -944.63 & -75.18\end{array}$

$\begin{array}{llllll}3.28 & 1.17 & -3.95 & -949.14 & -71.62\end{array}$

$\begin{array}{llllll}3.01 & 0.00 & -3.84 & -955.71 & -70.12\end{array}$

$2.771 .16-3.73-959.70-66.03$

$2.49 \quad 0.00-3.63 \quad-965.83 \quad-64.09$

$\begin{array}{llllll}1.99 & 1.15 & -3.53 & -969.66 & -59.85\end{array}$

$\begin{array}{llllll}1.55 & 0.00 & -3.44 & -975.51 & -57.63\end{array}$

0.00

0.00

0.00

0.00

0.00

0.00

0.00

0.00

0.00

0.00

0.00

0.00

0.00

0.00

0.00

0.00

0.00

0.00

0.00

0.00

0.00

0.00

0.00

0.00

0.00

0.00

0.00

0.00

0.00

0.00

0.00

0.00

0.00

0.00

0.00

0.00

0.00

0.00

0.00

0.00

0.00

0.00

0.00

0.00

0.00

0.00

0.00

0.00

0.00
0.00

0.00

0.00

0.00

0.00

0.00

0.00

0.00

0.00

0.00

0.00

0.00

0.00

0.00

0.00

0.00

0.00

0.00

0.00

0.00

0.00

0.00

0.00

0.00

0.00

0.00
0.00

0.00

0.00

0.00

0.00

0.00

0.00

0.00

0.00

0.00

0.00

0.00

0.00

0.00

0.00

0.00

0.00

0.00

0.00

0.00

0.00

0.00

0.00

0.00

0.00

0.00

0.00

0.00

0.00

0.00

0.00

$-77.27-1.61$

$-82.57-2.52$

0.00

0.34

0.72

$\begin{array}{llll}-83.45 & -2.00 & 0.08\end{array}$

$\begin{array}{llll}-86.07 & -1.00 & 0.11\end{array}$

$-86.11-0.36-0.37$

$-88.22$

$0.42-0.15$

$1.02-0.34$

$\begin{array}{lll}1.61 & -0.39\end{array}$

$2.23-0.31$

$2.61-0.37$

$3.19-0.12$

$3.26-0.23$

$3.58-0.12$

$3.49-0.34$

$3.52-0.38$

$3.52-0.47$

$3.41-0.53$

$\begin{array}{llll}0.00 & 0.00 & 0.00\end{array}$

0.00

0.00 


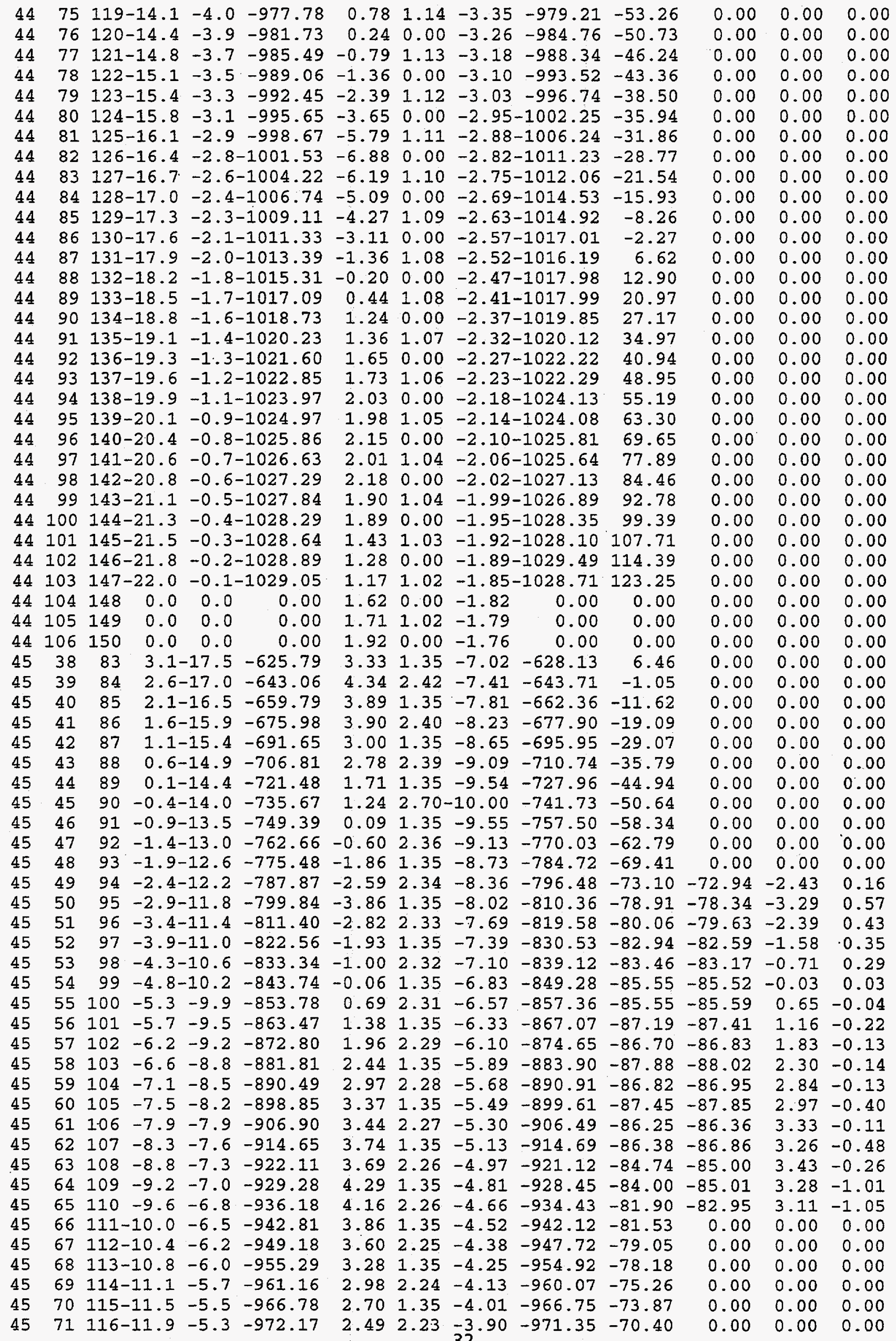




\begin{tabular}{|c|c|c|c|c|c|c|c|c|c|c|c|c|c|}
\hline 45 & 72 & \multicolumn{2}{|c|}{$117-12.3$} & 5.0 & -977.33 & \pm 1 & 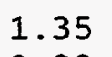 & -3 & $-97^{\circ}$ & -6 & 0 & 00 & 0 \\
\hline 45 & 73 & & & 8 & -982.27 & 1.72 & 2.22 & -3.69 & -982.02 & -64.93 & 0.00 & 0.00 & 0.00 \\
\hline 5 & 74 & & & 4.6 & 36.99 & .29 & 35 & -3.59 & -98 & -62.78 & 00 & 00 & 0.00 \\
\hline & 75 & 126 & 13.4 & -4.4 & -991.50 & 0.51 & 2.22 & -3.50 & -992.27 & -59.04 & 00 & 0.00 & 0.00 \\
\hline & 76 & 121 & -13.7 & -4.2 & .80 & -0.10 & 1.35 & -3.41 & -997.96 & -56.66 & & 00 & .00 \\
\hline 15 & 77 & 12 & 4.0 & 1 & 91 & 19 & 21 & -3 & 02.11 & -52 & & & Do \\
\hline 5 & 78 & & 4.4 & -3 & .003 .81 & -1.74 & .35 & -3.2 & 45 & -50.00 & .00 & 0.00 & 0.00 \\
\hline 45 & 79 & 124 & 4.7 & -3 & 007.53 & -2.74 & 20 & -3.1 & 23 & -45.71 & 0.00 & 0.00 & 0.00 \\
\hline 45 & 80 & $125-$ & .1 & -3 & .06 & -4.01 & .35 & -3 & .81 & -43.21 & & & .00 \\
\hline 45 & 81 & & & & & -6 & .20 & -3 & & & & & \\
\hline 5 & 82 & 127 & & -3. & 7.59 & -1.28 & 35 & & 026.45 & -36.71 & 0 & & 0.00 \\
\hline 15 & 83 & 128 & 5.0 & -2.5 & 0.59 & -6.47 & 19 & -2 & .75 & -29.94 & 00 & 0.00 & 0.00 \\
\hline 15 & 84 & 129 & .3 & $-2 . \varepsilon$ & .43 & -5.44 & .35 & -2. & 0.33 & 45 & 00 & 0 & 00 \\
\hline$=5$ & 85 & 30 & .6 & -2.6 & .10 & -4.6 & .18 & -2 & & & & & 0 \\
\hline 15 & 86 & 131 & 9 & -2 & 62 & -2.93 & .35 & -2 & .88 & -10.86 & & & .00 \\
\hline 5 & 87 & 134 & .2 & -2.3 & -1030.98 & -1.62 & .18 & -2.6 & 3.05 & -2.95 & 00 & 0.00 & 0.00 \\
\hline 15 & 88 & $133-$ & -17.5 & $-2.1-$ & -1033.19 & -0.52 & .35 & -2.5 & .93 & 24 & 0.00 & 00 & 0.00 \\
\hline \pm- & 89 & 34 & .8 & $-2.0-$ & .25 & 0.18 & .17 & -2.5 & .42 & & & & 00 \\
\hline 45 & 90 & 135 & & $-1.8-$ & 17 & .93 & .35 & -2 & & & 10 & 0 & 4 \\
\hline 45 & 91 & 136 & .4 & $-1.7-$ & .95 & 1.2 & 2.17 & -2.4 & .95 & 43 & 0.00 & 0.00 & 0.00 \\
\hline 45 & 92 & $137-$ & -18.7 & $-1.6-$ & -1040.60 & 1.48 & .35 & -2.3 & .13 & 30.32 & 0.00 & 0.00 & 0.00 \\
\hline 15 & 93 & 38 & .9 & $-1.4-$ & .11 & 1.48 & 2.16 & -2.3 & .79 & & & & bo \\
\hline 15 & 94 & 139 & .2 & $-1.3-$ & 9 & 1.86 & .35 & -2.2 & & & & 0 & 0 \\
\hline 45 & 95 & 140 & .4 & $-1.2-$ & .75 & 1.8 & .16 & -2.2 & .99 & 58 & 10 & 0 & 0.00 \\
\hline 45 & 96 & $\perp 4 \perp$ & .7 & $-1.1-$ & .89 & 1.9 & .35 & -2.1 &. .78 & 95 & 10 & 10 & .00 \\
\hline & 97 & $\$ 2$ & .0 & $-1.0-$ & 91 & 18 & 2.15 & -2.1 & 7 & & & & 00 \\
\hline 5 & 98 & 33 & .2 & $-0.8-$ & -1 & 2. & 1.35 & -2 . & 6 & 2 & 0 & 0 & 00 \\
\hline 5 & 99 & 44 & -20.4 & $-0.7-$ & .61 & 1.6 & 2.15 & -2.0 & 5.87 & 8 & 0 & 0 & 0.00 \\
\hline 5 & 00 & $45-$ & -20.7 & $-0.6-$ & 9.29 & 1.70 & .35 & -2.0 & 3.27 & & & & 0.00 \\
\hline & 1 & 46 & & $-0.5-$ & -1 & $1=$ & 2.14 & -2 & 40 & & & & \\
\hline 5 & 102 & $7-$ & .1 & -0 . & -1 & 1.2 & 5 & -1.5 & .69 & 7 & 0 & 10 & 0.00 \\
\hline 5 & 103 & 148 & -21.4 & $-0.3-$ & 0.71 & 1.16 & 2.14 & -1.9 & 9.34 & 39 & 0 & 10 & 0.00 \\
\hline 15 & 104 & $149-$ & -21.6 & $-0.2-$ & .99 & 1.67 & .35 & -1. & .87 & & & & 0 \\
\hline 5 & 5 & 50 & .8 & $-0.1-$ & 8 & 16 & 2.13 & -1 & .29 & & & & \\
\hline 5 & 106 & 151 & .0 & & 27 & & 5 & -1 & .92 & 3 & 0 & 0.00 & 0.00 \\
\hline 5 & 107 & 152 & 0.0 & 0.0 & 0.00 & 1.55 & 2.13 & -1.80 & 0.00 & 0.00 & 0.00 & 10 & 0.00 \\
\hline 5 & 108 & 153 & 0.0 & 0.0 & 0.00 & 1.51 & 1.35 & -1.77 & 0.00 & 0 & & & 0 \\
\hline 0 & 40 & 86 & & 7. & -6 & 2 & 0 & -7.46 & 9 & & & & \\
\hline 6 & 41 & 87 & & & & & 39 & -7 & .40 & 1 & 0 & 10 & 0.00 \\
\hline 6 & 42 & 88 & 1.7 & 15.9 & .25 & 2.2 & 0.00 & -8.26 & -696.25 & -22.09 & 0.00 & 0.00 & 0.00 \\
\hline 6 & 43 & 89 & 1.2 & 5.4 & -705.92 & 2.04 & 1.37 & -8.68 & .19 & -2 & 0 & 0 & 0. \\
\hline 6 & 44 & 90 & - & 4.9 & -7 & 0 . & 0 & 1 & 4 & & & & \\
\hline$=0$ & 45 & 91 & & & & & & & & & 0 & 0 & 0 \\
\hline 6 & 46 & 92 & $-0.3-$ & -14.0 & .00 & -0.70 & $0-$ & -10.00 & .70 & -5 & 0.00 & 0.00 & 0.00 \\
\hline 6 & 47 & 93 & -0.8 & 3.5 & .76 & -1.43 & 1.33 & -9.56 & .42 & -5 & 0 . & 0.00 & 0.00 \\
\hline$=6$ & 48 & 94 & -1 & 3.1 & 7 & -2 & 0 & -9 & 9 & & & & 0 \\
\hline 6 & 49 & 95 & -1 & & & & & & & & & & \\
\hline 6 & 50 & 96 & .2 & -12.2 & 10 & -4 & 0 & -8.39 & .67 & 4 & 8 & -4 & 0.76 \\
\hline 6 & 51 & 97 & -2.7 & 8 & 44 & -3.6 & 1.29 & -8.05 & .89 & -7 & 30 & -3.40 & 0.29 \\
\hline 6 & 52 & 98 & -3 & 1.4 & 0 & -2.8 & .00 & -7 & .67 & & & & \\
\hline & 53 & 99 & & & & & & & & & & & \\
\hline 6 & 54 & 100 & -4.1 & .7 & .20 & -0.81 & 0.00 & -7.15 & 15 & -8 & 3 & -0 & -0 \\
\hline 6 & 55 & 101 & -4.6 & .3 & .69 & -0.0 & 26 & -6.88 & 37 & 8 & & 1 & -0 \\
\hline & 56 & 102 & & & & 0 . & 0 & & & & & & \\
\hline & 57 & 103 & -5.5 & 9. & 62 & 1.2 & 25 & -6.39 & 2.47 & 3 & & 1.04 & -0 \\
\hline 6 & 58 & 104 & -5.9 & -9.3 & .06 & 1.83 & 0.00 & -6.16 & 2.39 & 09 & 9 & 1.53 & -0.30 \\
\hline 6 & 59 & 105 & -6.3 & 9.0 & 1 & 2.3 & 23 & -5.95 & 0 & 3 & & 2.10 & -0.29 \\
\hline$E$ & 60 & 106 & & & & & & & & & & & \\
\hline$E$ & 61 & 107 & -7.2 & -8.3 & .45 & 3. & .22 & -5.55 & -915.70 & & & 2.89 & -0.19 \\
\hline 6 & 62 & 108 & 7.6 & -8.0 & 63 & 3.1 & .00 & -5.37 & -924.65 & 6 & 2 & 2.88 & -0.46 \\
\hline 46 & 63 & 109 & 8.0 & 7.7 & 50 & 336 & 21 & -5.19 & 1.13 & 47 & & 2 & 0.14 \\
\hline & 64 & $1: 10$ & 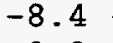 & 4 & & & 00 & & & & & & \\
\hline & 65 & 111 & 8.9 & 7.2 & -9 & 3.7 & 19 & -4.87 & 29 & & & 3.24 & \\
\hline 46 & 66 & 112 & 9.3 & -6.9 & -952.4 & 3.48 & 0.00 & -4.72 & 3.67 & -85.80 & & 2.95 & -0.53 \\
\hline 16 & 67 & 113 & 9.6 & -6.6 & -959.19 & 3.23 & 1.18 & -4.58 & -959.36 & -83.41 & 8 & 2.96 & -0.27 \\
\hline & 68 & & $\theta_{4}$ & 0 & . & & 3 & & 2 & 21 & 6 & 6 & 8.20 \\
\hline
\end{tabular}




\begin{tabular}{|c|c|c|c|c|c|c|c|c|c|c|c|c|c|}
\hline & 69 & \multirow{2}{*}{\multicolumn{2}{|c|}{$\begin{array}{l}115-10.4 \\
116-10.8\end{array}$}} & -6.1 & -971.95 & 2.68 & .17 & -4.32 & -972.41 & -80.32 & 00 & 0.00 & 00 \\
\hline 46 & 70 & & & -5.9 & 977.95 & 2.34 & 0.00 & -4.19 & -979.81 & -79.65 & -80.14 & 1.85 & -0.49 \\
\hline 46 & 71 & & 11.2 & -5.6 & 83.72 & 17 & .16 & & -984.46 & -76.23 & 0.00 & .00 & 0.00 \\
\hline 40 & 72 & - & -11.6 & -5.4 & -989.25 & 1.85 & 0.00 & -3.96 & -991.36 & -75.06 & -75.64 & .27 & -0.58 \\
\hline 46 & 73 & & & -5.2 & & & .15 & & -995.90 & -71.52 & 0.00 & .00 & 0.00 \\
\hline & 74 & 120 & -12.3 & -1 & 53 & 0.78 & 00 & & & & & & \\
\hline 0 & 75 & & -12.6 & -4.8 & .50 & 0.04 & 1.14 & -3 & & & .00 & & 0.00 \\
\hline & 76 & $122-$ & -13.0 & -4 & 009.15 & -0.58 & 0.00 & -3 & & -64.70 & 0.00 & 0.00 & 0.00 \\
\hline 40 & 77 & & & & & & 1.13 & & & -60.74 & 0.00 & 0.00 & 0.00 \\
\hline & 78 & & & -4 & & -2.46 & 0.00 & & & $-5 \varepsilon$ & & 00 & .00 \\
\hline & 79 & 125 & .0 & -4 & .90 & -3.84 & 1.12 & & & .12 & .00 & .00 & .00 \\
\hline 40 & 80 & $126-$ & .3 & -3 & 025.76 & -5.26 & 0.00 & -3.2 & 4.24 & -53.37 & 0.00 & 0.00 & 0.00 \\
\hline 40 & 81 & & .7 & & & -6.98 & .11 & & & -49 & 0.00 & 00 & .00 \\
\hline 6 & 82 & 128 & .0 & -3 & 032.94 & -7.97 & 0.00 & -3 & 8 & -46.96 & 0.00 & .00 & 0.00 \\
\hline & 83 & 129 & .3 & -3.2 & -1036.26 & -7.36 & 1.10 & -3 & 5.52 & -40.43 & 0.00 & .00 & 0.00 \\
\hline & 84 & $130-$ & .6 & -3.1 & 39.41 & -6.32 & 0.00 & -2. & 0.56 & -35.50 & 0.00 & .00 & 0.00 \\
\hline 16 & 85 & $131-$ & .9 & -2.5 & .042 .39 & -5.39 & 1.09 & -2 . & & -28 & 0 & 00 & 0.00 \\
\hline & 6 & 125 & .2 & -2 & 45.21 & -4.21 & 0.00 & -2 & 2 & -22.92 & 0.00 & 0.00 & 0.00 \\
\hline & 87 & - & .5 & -2 & 7.87 & -2.42 & 1.08 & -2.7 & 51.94 & -14.57 & 0.00 & 0.00 & 0.00 \\
\hline 16 & 88 & $4-$ & .8 & -2 & 50.37 & -1.18 & 0.00 & -2.68 & .23 & -8.79 & 0.00 & 0.00 & 0.00 \\
\hline 46 & 89 & $135-$ & -17.1 & -2.3 & 1052.72 & -0.31 & .08 & -2 & & -1 & 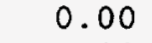 & 10 & 0.00 \\
\hline 46 & 90 & $130-$ & .4 & -2.1 & -1054.93 & 0.38 & 0.00 & $-2.57-$ & .12 & 4.47 & 0.00 & 0.00 & 0.00 \\
\hline & 91 & $37-$ & .7 & $-2.0-$ & 056.99 & 0.89 & 1.07 & $-2.52-$ & 7.55 & 12.11 & 0.00 & 0.00 & 0.00 \\
\hline & 92 & - & .0 & $-1.8-$ & .91 & 1 & 0.00 & -2 & -1 & 17. & & & 00 \\
\hline 16 & 93 & 135 & -18.3 & -1.7 & -1060.70 & 1.25 & 1.06 & $-2.42-$ & 1 & 25 . & 10 & 0 & 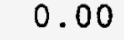 \\
\hline \pm 0 & 94 & $140-$ & -18.5 & -1.6 & -1062.35 & 1.53 & 0.00 & $-2.37-$ & .19 & 30.69 & 0.00 & 0.00 & 0.00 \\
\hline 6 & 95 & $11-$ & .8 & $-1.5-$ & .87 & 1.60 & 1.05 & -2 & 4 & 38.40 & 0.00 & 0.00 & 0.00 \\
\hline 46 & 96 & $142-$ & .0 & -1.3 & 5.27 & 1.81 & 0.00 & 2. & -1 & & 0.00 & 0 & .00 \\
\hline 6 & 97 & $143-$ & -19.3 & $-1.2-$ & -1066.54 & 1.74 & 1.04 & $-2.24-$ & .99 & 52. & 0 & 0 & 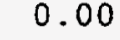 \\
\hline 16 & 98 & $\pm 4-$ & -19.6 & -1.1 & -1067.69 & 1.93 & 0.00 & -2.19 & 7.96 & 58.20 & 0.00 & 0.00 & 0.00 \\
\hline 6 & 99 & $5-$ & .8 & $-1.0-$ & 3.73 & 1.66 & 4 & -2 & 9 & 66. & 0 & 0 & 0.00 \\
\hline 6 & .00 & $146-$ & -20.0 & -0 & 9.65 & 1.74 & 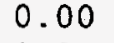 & -2 & & & & & 0 \\
\hline 6 & 1 & $147-$ & -20.3 & $-0.8-$ & -1070.47 & 1.47 & 1.03 & $-2.08-$ & .04 & 8 & 0 & 10 & 0 \\
\hline 6 & 02 & $3-$ & -20.5 & -0.6 & 1.17 & 1.47 & .00 & $-2.04-$ & .7 & 86.71 & 0 & 0 & 0.00 \\
\hline 6 & 3 & - & .7 & -0 . &. .77 & 1.35 & 2 & -2 & 0 & 95.12 & 0 & 0 & 0.00 \\
\hline 6 & 104 & 15 & -21.0 & -0. & 2.27 & 1.74 & 0.00 & -1. & - & & & & \\
\hline 6 & 05 & $51-$ & -21.2 & $-0.3-$ & -1072.67 & 1.70 & 1.02 & -1.5 &.$\varepsilon$ & 110.77 & 0 & 10 & Do \\
\hline 6 & 06 & $2-$ & 4 & -0.3 & .97 & 1.94 & 0.00 & -1.5 & .94 & 117.79 & 0 & bo & 0.00 \\
\hline 6 & 7 & t & 6 & -0. & & 1.66 & 1 & -1 & -1 & $\perp$ & & & 0 \\
\hline 6 & 108 & - & -21.8 & $-0.1-$ & 73.31 & 1.59 & 0.00 & -1 & 3.56 & & & & \\
\hline 6 & 109 & 155 & 0.0 & 0.0 & 0.00 & 1.12 & .00 & -1.81 & 0.00 & 0.00 & 0 & 00 & 0.00 \\
\hline 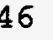 & 110 & 156 & & 0.0 & 0.00 & 0.90 & 0 & -1.7 & 0 . & 0 . & 0.00 & 0 & 0.00 \\
\hline 47 & 41 & 88 & $2.8-$ & 16.9 & -671.52 & 2.03 & & - & - & & & & \\
\hline 47 & 42 & 89 & 2.4 & 16.4 & -688.20 & 1.12 & 1.33 & -7.90 & .65 & -12.21 & 0 & 10 & 0 \\
\hline 7 & 43 & 90 & & 0 & -704.38 & 0.91 & .3 & -8.30 & 9.39 & -19 & 0 & .00 & 0.00 \\
\hline & 4 & 91 & & & & & & & & - & 0 & 0 & Do \\
\hline 47 & 45 & 92 & 0.9 & .4 .9 & -735.23 & -0.57 & 2.36 & -9.13 & -7 & & & & \\
\hline 47 & 46 & 93 & $0.4 x-2$ & 4.5 & -749.95 & -1.78 & .33 & -9.56 & 9.95 & -46.23 & 10 & 00 & 0.00 \\
\hline 17 & 47 & 94 & -0. & .0 & .20 & .52 & 2.6 & 10. & - & -52.26 & 0 . & 00 & 0.00 \\
\hline 7 & 48 & 95 & & & & & & & & - & 0 & 0 & 0.00 \\
\hline 47 & 49 & 96 & -1. & .1 & .35 & -4.55 & 2.33 & -9.16 & & & & & \\
\hline 47 & 50 & 97 & -1.5 & 2.7 & -804.28 & -5.67 & .33 & -8.78 & .40 & -7 & 0 & 0 & 0.00 \\
\hline 47 & 5 & 98 & 2.0 & .3 & .80 & -4.81 & 2.31 & -8 & $-\varepsilon$ & -73.63 & 0.00 & .00 & 0.00 \\
\hline 47 & 52 & 99 & & 1.9 & & & & & & .35 & 6 & 5 & 9 \\
\hline 7 & 53 & 100 & -2 & 1.5 & 62 & -2.72 & 30 & -7 & -8 & 8 & & & \\
\hline 17 & 5 & 101 & 4 & 1.1 & -851.95 & -1.82 & 3 & -7.47 & -8 & -81 & 9 & 10 & 42 \\
\hline 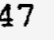 & 55 & 2 & & & 0 & 1.12 & .2 & -7 & $-\varepsilon$ & -82.55 & -82.09 & 0.66 & 0.46 \\
\hline 7 & 56 & 103 & $-4.3-$ & -10.4 & -873.49 & 28 & & & & & & & \\
\hline 7 & 57 & 104 & & -10.1 & -883.72 & 0.50 & 2.28 & -6 . & .62 & -85 & 1 & 0 & \\
\hline$y$ & 58 & 105 & -5.2 & 9.7 & -8 & 1.07 & 3 & -6.44 & -897.65 & -87.06 & -87.08 & .05 & -0.02 \\
\hline 8 & 5 & & & & -903. & 1.68 & & & & & 4 & .51 & \\
\hline 7 & 60 & 107 & -6.2. & 9.1 & -912.38 & 2.20 & 1.33 & -6.00 & 4.86 & -88.13 & & .92 & -0.28 \\
\hline 7 & 61 & 108 & 6.5 & 8.8 & -921.2 & 2.63 & 2 & 90 & & -87.40 & $-\varepsilon$ & 2.42 & \\
\hline & ( & & & & & & & & .26 & -88.38 & -88.72 & 2.57 & \\
\hline 7 & 63 & 110 & -7.3 & -8.1 & -938.18 & 3.12 & 2.25 & & -938.24 & -87.30 & & 2. & \\
\hline 1 & 64 & 111 & -7.7 & .9 & -946.18 & 3.18 & 3 & $\begin{array}{l}-5.26 \\
34\end{array}$ & -946.92 & 37.91 & -88.22 & 2.87 & 1 \\
\hline
\end{tabular}


$\begin{array}{lllllll}47 & 65 & 112 & -8.1 & -7.6 & -953.89\end{array}$

$\begin{array}{llllll}47 & 66 & 113 & -8.5 & -7.3 & -961.32\end{array}$

$\begin{array}{lllllll}47 & 67 & 114 & -8.9 & -7.0 & -968.48\end{array}$

$\begin{array}{llllll}47 & 68 & 115 & -9.3 & -6.8 & -975.38\end{array}$

$\begin{array}{llllll}47 & 69 & 116 & -9.7 & -6.5 & -982.02\end{array}$

$\begin{array}{llllll}47 & 70 & 117 & -10.1 & -6.3 & -988.40\end{array}$

$\begin{array}{llllll}47 & 71 & 118 & -10.5 & -6.0 & -994.54\end{array}$

$47 \quad 72 \quad 119-10.8 \quad-5.8-1000.44$

$47 \quad 73 \quad 120-11.2 \quad-5.6-1006.11$

$47 \quad 74 \quad 121-11.6 \quad-5.3-1011.56$

$\begin{array}{lllll}47 & 75 & 122 & -11.9 & -5.1-1016.78\end{array}$

$47 \quad 76 \quad 123-12.3 \quad-4.9-1021.78$

$\begin{array}{lllll}47 & 77 & 124-12.6 & -4.7-1026.58\end{array}$

$47 \quad 78 \quad 125-13.0 \quad-4.5-1031.17$

$47 \quad 79 \quad 126-13.3-4.3-1035.56$

$47 \quad 80 \quad 127-13.6-4.1-1039.76$

$47 \quad 81 \quad 128-14.0 \quad-3.9-1043.77$

$47 \quad 82 \quad 129-14.3 \quad-3.7-1047.59$

$4783 \quad 130-14.6-3.5-1051.23$

$47 \quad 84$ 131-14.9 $-3.4-1054.69$

$47 \quad 85 \quad 132-15.2-3.2-1057.98$

$47 \quad 86 \quad 133-15.6 \quad-3.0-1061.11$

$47 \quad 87 \quad 134-15.9-2.9-1064.07$

$47 \quad 88 \quad 135-16.2 \quad-2.7-1066.87$

$47 \quad 89 \quad 136-16.4 \quad-2.6-1069.52$

$47 \quad 90 \quad 137-16.7 \quad-2.4-1072.01$

$47 \quad 91 \quad 138-17.0-2.3-1074.36$

$47 \quad 92 \quad 139-17.3 \quad-2.1-1076.56$

$47 \quad 93 \quad 140-17.6 \quad-2.0-1078.62$

$47 \quad 94 \quad 141-17.9 \quad-1.9-1080.54$

$4795 \quad 142-18.1-1.7-1082.33$

$47 \quad 96 \quad 143-18.4-1.6-1083.99$

$\begin{array}{lllll}47 & 97 & 144-18.7 & -1.5-1085.52\end{array}$

$47 \quad 98 \quad 145-18.9-1.3-1086.93$

$47 \quad 99 \quad 146-19.2 \quad-1.2-1088.21$

$47 \quad 100 \quad 147-19.4 \quad-1.1-1089.38$

$47 \quad 101 \quad 148-19.6-1.0-1090.43$

$47 \quad 102 \quad 149-19.9-0.9-1091.37$

$47 \quad 103 \quad 150-20.1-0.8-1092.21$

$47 \quad 104 \quad 151-20.4 \quad-0.7-1092.93$

$47 \quad 105 \quad 152-20.6 \quad-0.6-1093.55$

$47 \quad 106 \quad 153-20.8 \quad-0.5-1094.08$

$47107154-21.0-0.4-1094.50$

$47108 \quad 155-21.2-0.3-1094.83$

$47 \quad 109 \quad 156-21.4 \quad-0.2-1095.07$

$47 \quad 110157-21.6 \quad-0.1-1095.22$

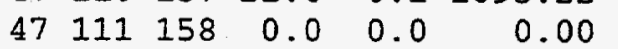

$\begin{array}{llllll}47 & 112 & 159 & 0.0 & 0.0 & 0.00\end{array}$

$\begin{array}{llllll}47 & 113 & 160 & 0.0 & 0.0 & 0.00\end{array}$

$\begin{array}{llllll}48 & 42 & 90 & 3.0-16.9 & -685.54\end{array}$

$\begin{array}{lllll}48 & 43 & 91 & 2.5-16.4 & -702.21\end{array}$

$\begin{array}{lllll}0.19 & 0.00 & -7.56 & -692.91 & -4.19\end{array}$

$\begin{array}{lllllllllll}48 & 45 & 93 & 1.5 & -15.4 & -734.04 & -1.61 & 1.35 & -8.73 & -743.04 & -30.10\end{array}$

$\begin{array}{lllllllllll}48 & 46 & 94 & 1.0 & -15.0 & -749.24 & -2.81 & 0.00 & -9.15 & -761.20 & -40.19\end{array}$

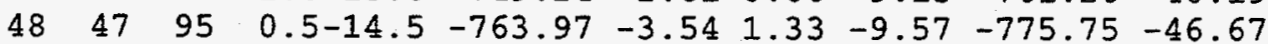

$\begin{array}{llllllllll}48 & 48 & 96 & 0.1-14.1 & -778.25 & -5.00 & 0.00-10.00 & -793.25 & -56.10\end{array}$

$\begin{array}{llllllllll}48 & 49 & 97 & -0.4-13.6 & -792.09 & -5.64 & 1.31 & -9.58 & -805.99 & -60.77\end{array}$

$\begin{array}{lllllllllll}48 & 50 & 98 & -0.9-13.2 & -805.49 & -7.05 & 0.00 & -9.18 & -821.72 & -68.43\end{array}$

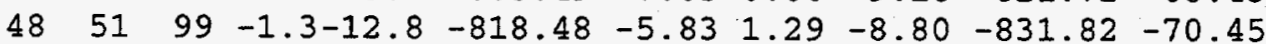

$\begin{array}{lllllllllll}48 & 52 & 100 & -1.8 & -12.4 & -831.05 & -5.10 & 0.00 & -8.45 & -844.60 & -75.16\end{array}$

48

48

48

48

48
48

$\begin{array}{lllllll}3.40 & 2.24 & -5.09 & -953.34 & -86.25 & -86.62\end{array}$ $\begin{array}{lll}-86.62 & -87.04\end{array}$ $.92-84.96$ $-75.77$ $-74.55$ 0.00 0.00 0.00 0.00 0.00 0.00

0.00

0.00

0.00

0.00

0.00

0.00

0.00

0.00

0.00

0.00

0.00

0.00

0.00

0.00

0.00

0.00

0.00

0.00

0.00

0.00

0.00

0.00

0.00

0.00

0.00

0.00

0.00

0.00

0.00

0.00

0.00

0.00

0.00

0.00

0.00

0.00

0.00

0.00

0.00

0.00

0.00

0.00

0.00

$\begin{array}{llll}53 & 101 & -2.3-12.0 & -843.22\end{array}$

$\begin{array}{llllll}-3.67 & 1.28 & -8.12 & -853.74 & -76.23\end{array}$

54102

55103

$-843.22$

$-853.74-76.23$
$-865.38-79.80$

$-74.29$

$-75.66$

0.00

$\begin{array}{llll}56 & 104 & -3.6-10.9 & -877.45\end{array}$

$57 \quad 105-4.1-10.5-888.13$

$58 \quad 106-4.5-10.2-898.46$ $\begin{array}{lllll}-1.86 & 1.26 & -7.52 & -874.53 & -80.88\end{array}$

$-80.65-1.63$

$\begin{array}{llll}-885.64 & -83.92 & -83.98 & -1.01\end{array}$

$\begin{array}{llllllll}-0.35 & 1.25 & -6.98 & -894.21 & -84.41 & -84.34 & -0.28\end{array}$

$\begin{array}{lllllll}0.31 & 0.00 & -6.73 & -904.87 & -87.01 & -87.14\end{array}$ 35
$3.03-0.37$

$2.73-0.42$

$2.85-0.04$

$2.44-0.13$

$2.17-0.21$

$1.76-0.27$

$1.43-0.49$

$1.08-0.36$

$0.51-0.67$

$-0.13-0.72$

$0.00 \quad 0.00$

$0.00 \quad 0.00$

$0.00 \quad 0.00$

$0.00 \quad 0.00$

$0.00 \quad 0.00$

$0.00 \quad 0.00$

$0.00 \quad 0.00$

$0.00 \quad 0.00$

$0.00 \quad 0.00$

$0.00 \quad 0.00$

$0.00 \quad 0.00$

$0.00 \quad 0.00$

$0.00 \quad 0.00$

$0.00 \quad 0.00$

$0.00 \quad 0.00$

$0.00 \quad 0.00$

$0.00 \quad 0.00$

$0.00 \quad 0.00$

$0.00 \quad 0.00$

$0.00 \quad 0.00$

$0.00 \quad 0.00$

$0.00 \quad 0.00$

$0.00 \quad 0.00$

$0.00 \quad 0.00$

$0.00 \quad 0.00$

$0.00 \quad 0.00$

$0.00 \quad 0.00$

$0.00 \quad 0.00$

$0.00 \quad 0.00$

$0.00 \quad 0.00$

$0.00 \quad 0.00$

$0.00 \quad 0.00$

$0.00 \quad 0.00$

$0.00 \quad 0.00$

$0.00 \quad 0.00$

$0.00 \quad 0.00$

$0.00 \quad 0.00$

$0.00 \quad 0.00$

$0.00 \quad 0.00$

$\begin{array}{lll}0.00 & 0.00\end{array}$

$0.00 \quad 0.00$

$0.00 \quad 0.00$

$0.00 \quad 0.00$

$0.00 \quad 0.00$

$0.00 \quad 0.00$

0.00

$0.00 \quad 0.00$

$0.00 \quad 0.00$

0.87

0.57

0.00

0.23

$-0.06$

0.07

$0.18-0.13$ 
$\begin{array}{lllllll}48 & 59 & 107 & -4.9 & -9.8 & -908 & 44\end{array}$

$\begin{array}{lllllll}48 & 60 & 108 & -5.4 & -9.5 & -918 & 10\end{array}$

$\begin{array}{lllllll}48 & 61 & 109 & -5.8 & -9.2 & -927.43\end{array}$

$\begin{array}{llllll}48 & 62 & 110 & -6.2 & -8.9 & -936.44\end{array}$

$\begin{array}{lllllll}48 & 63 & 111 & -6.6 & -8.6 & -945.15\end{array}$

$\begin{array}{llllll}48 & 64 & 112 & -7.0 & -8.3 & -953.56\end{array}$

$\begin{array}{lllllll}48 & 65 & 113 & -7.4 & -8.0 & -961.68\end{array}$

$\begin{array}{lllllll}48 & 66 & 114 & -7.8 & -7.7 & -969.51\end{array}$

$\begin{array}{lllllll}48 & 67 & 115 & -8.2 & -7.4 & -977.06\end{array}$

$\begin{array}{lllllll}48 & 68 & 116 & -8.6 & -7.1 & -984.35\end{array}$

$\begin{array}{lllllll}48 & 69 & 117 & -9.0 & -6.9 & -991.38\end{array}$

$\begin{array}{lllllll}48 & 70 & 118 & -9.4 & -6.6 & -998.14\end{array}$

$\begin{array}{lllll}48 & 71 & 119 & -9.8 & -6.4-1004.66\end{array}$

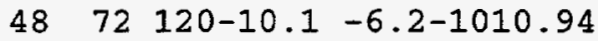

$\begin{array}{llll}48 & 73 & 121-10.5 & -5.9-1016.97\end{array}$

$48 \quad 74 \quad 122-10.9 \quad-5.7-1022.78$

$48 \quad 75 \quad 123-11.2-5.5-1028.36$

$48 \quad 76 \quad 124-11.6 \quad-5.2-1033.72$

48

48

48

48

48

48

48

48

48
48

48

.48

48

48

48

48

48

48

48

48

48

48

48 148-18.8-1.4-1108.47

$48102150-19.3-1.1-1110.95$

$48 \quad 103 \quad 151-19.5-1.0-1112.02$

$48 \quad 104 \quad 152-19.7-0.9-1112.98$

$48105 \quad 153-20.0-0.8-1113.83$

$48706 \quad 154-20.2-0.7-1114.57$

$48107155-20.4-0.6-1115.22$

$48108 \quad 156-20.6-0.5-1115.77$

$48109 \quad 157-20.8-0.4-1116.21$

$48110158-21.1-0.3-1116.57$

$48 \quad 111159-21.3-0.2-1116.84$

$\begin{array}{llll}48 & 112 & 160-21.5 & -0.1-1117.02\end{array}$

$48 \quad 113 \quad 161-21.6 \quad 0.0-1117.11$

$\begin{array}{llllllll}0.86 & 1.23 & -6.49 & -912.84 & -86.90 & -86.99\end{array}$

$\begin{array}{lllllll}1.35 & 0.00 & -6.27 & -923.02 & -89.01 & -89.25\end{array}$

$\begin{array}{lllllll}1.76 & 1.22 & -6.06 & -930.51 & -88.43 & -88.51\end{array}$

$\begin{array}{llllllll}2.11 & 0.00 & -5.86 & -940.19 & -90.04 & -90.35\end{array}$

$\begin{array}{llllllll}2.42 & 1.21 & -5.67 & -947.19 & -88.97 & -89.25\end{array}$

$\begin{array}{llllllll}2.52 & 0.00 & -5.49 & -956.53 & -90.23 & -90.58\end{array}$

$\begin{array}{lllllll}2.61 & 1.19 & -5.32 & -963.19 & -88.82 & -89.05\end{array}$

$2.50 \quad 0.00 \quad-5.15 \quad-972.16 \quad-89.72 \quad-90.02$

$\begin{array}{llllllll}2.48 & 1.18 & -5.00 & -978.40 & -87.89 & -88.09\end{array}$

$\begin{array}{llllllll}2.26 & 0.00 & -4.85 & -986.94 & -88.36 & -88.72\end{array}$

$\begin{array}{llllllll}2.12 & 1.17 & -4.71 & -992.79 & -86.14 & -86.42\end{array}$

$1.740 .00-4.57-1000.97-86.25-86.71$

$\begin{array}{llllll}1.65 & 1.16 & -4.44-1006.29 & -83.50 & -83.94\end{array}$

$\begin{array}{lllllll}0.78 & 0.00 & -4.32-1014.47 & -83.60 & -83.97\end{array}$

$\begin{array}{llllll}0.29 & 1.15 & -4.20-1019.73 & -80.79 & -80.95\end{array}$

$\begin{array}{llllll}-0.49 & 0.00 & -4.09-1027.35 & -80.34 & 0.00\end{array}$

$\begin{array}{llllll}-1.07 & 1.14 & -3.98-1032.27 & -77.18 & -77.31\end{array}$

$\begin{array}{llllll}-2.42 & 0.00 & -3.87-1040.01 & -76.86 & -76.71\end{array}$

$\begin{array}{lllllll}-3.48 & 1.13 & -3.77-1044.99 & -73.76 & -73.32\end{array}$

$\begin{array}{lllllll}-4.52 & 0.00 & -3.68-1051.99 & -72.70 & -72.33 \\ -5.74 & 1.12 & -3.59-1056.73 & -69.37 & -68.52\end{array}$

$-7.130 .00-3.50-1063.69$

$-68.25-6$

$\begin{array}{llll}-8.83 & 1.11 & -3.41-1068.53 & -65.02\end{array}$

$\begin{array}{lllll}-9.85 & 0.00 & -3.33-1074.72 & -63.14\end{array}$

$\begin{array}{lllll}-9.15 & 1.10 & -3.26-1076.81 & -57.15\end{array}$

$\begin{array}{lllll}-8.06 & 0.00 & -3.18-1080.52 & -52.80\end{array}$

$\begin{array}{lllll}-7.02 & 1.09 & -3.11-1081.93 & -46.13\end{array}$

$\begin{array}{llllll}-5.78 & 0.00 & -3.04-1085.14 & -41.27\end{array}$

$\begin{array}{lllll}-4.78 & 1.08 & -2.97-1086.25 & -34.32\end{array}$

$\begin{array}{lllll}-3.72 & 0.00 & -2.91-1089.32 & -29.31\end{array}$

$-2.011 .08-2.85-1089.41-21.33$

$\begin{array}{lllll}-1.08 & 0.00 & -2.79-1092.28 & -16.13\end{array}$

$0.151 .07-2.73-1092.56$

$0.74 \quad 0.00-2.67-1095.46$

$0.801 .06-2.62-1096.63$

$-8.33$

$-3.17$

3.74

$1.05 \quad 0.00-2.57-1099.58$

$1.231 .05-2.51-1100.36$

$1.50 \quad 0.00-2.47-1103.02$

$1.48 \quad 1.04-2.42-1103.74$

$1.68 \quad 0.00-2.37-1106.20$

$1.471 .04-2.33-1106.87$

$1.550 .00-2.29-1109.20$

$1.311 .03-2.24-1109.67$

$1.430 .00-2.20-1111.72$

$1.211 .02-2.17-1111.95$

$1.520 .00-2.13-1113.58$

$1.491 .02-2.09-1113.41$

$1.730 .00-2.06-1114.90$

$1.48 \quad 1.01-2.02-1114.75$

8.86

16.15

21.56

28.91

34.52

41.92

47.66

55.27

61.29

69.13

75.57

83.81

90.40

$\begin{array}{lllll}1.36 & 0.00 & -1.99-1116.39 & 105.05\end{array}$

$0.851 .00-1.96-1116.32113 .20$

$0.650 .00-1.92-1117.85119 .74$

$\begin{array}{lllll}-0.02 & 1.00 & -1.89-1117.75 & 127.90\end{array}$

$-0.290 .00-1.86-1119.17134 .56$

48

\section{8}

49

49

49

49

49

49

49

49
0.0

0.00

0.00

$-0.77$

$70.99-1.83-1118.72143 .07$

115163

$$
0.0 \quad 0.0
$$

$3.1-16.9-699.43$
$2.6-16.4-716.07$

$-0.68 \quad 0.00-1.81$

0.00

0.00

0.00

$43 \quad 92$

$\begin{array}{llll}.54 & 0.99 & -1.78\end{array}$

$60-706.27$

0.00

$-2.20$

$45 \quad 94$

$2.6-16.4-716.07$
$2.1-15.9-732.23$

$\begin{array}{llllll}2.75 & 1.31 & -7.98 & -725.49 & -13.34 \\ 3.23 & 2.34 & -8.36 & -741.48 & -21.26\end{array}$

$46 \quad 95$

$47 \quad 96$

$1.7-15.4-747.90$

$1.2-15.0-763.11$

$48 \quad 97$

$49 \quad 98$

$0.2-14.1-792.17$

$-5.182 .31$

$-8.76-759.79-31.50$

$50 \quad 99-0.2-13$.

$7-806.05$

$\begin{array}{lllll}-6.53 & 1.31 & -9.58 & -792.66 & -48.23 \\ -7.33 & 2.62 & -10.00 & -806.88 & -54.38\end{array}$

$\begin{array}{llllllll}5 & -8.69 & 1.31 & -9.58 & -823.01 & -62.43\end{array}$

$\begin{array}{lllllll}51 & 100 & -0.7-13.2 & -819.49 & -7.48 & 2.30 & -9\end{array}$

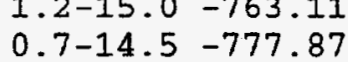

$-67.27$

0.00

0.00

0.00

0.00

0.00

0.00

0.00

0.00

0.00

0.00

0.00

0.00

0.00

0.00

0.00

0.00

0.00

0.00

0.00

0.00

0.00

0.00

0.00

0.00

0.00

0.00

0.00

0.00

0.00

0.00

0.00

0.00

0.00

0.00

0.00

0.00

0.00

0.00

0.00

0.00

0.00

0.00

0.00

0.00
$0.77-0.09$

$\begin{array}{lll}1.11 & -0.24\end{array}$

$1.68-0.08$

$1.80-0.31$

$2.14-0.28$

$2.17-0.35$

$2.38-0.23$

$2.20-0.30$

$2.28-0.20$

$1.90-0.36$

$1.84-0.28$

$1.28-0.46$

$1.21-0.44$

$0.41-0.37$

$0.13-0.16$

$0.00 \quad 0.00$

$-1.20-0.13$

$\begin{array}{ll}-2.27 & 0.15\end{array}$

$\begin{array}{ll}-3.04 & 0.44\end{array}$

$\begin{array}{ll}-4.15 & 0.37\end{array}$

$\begin{array}{ll}-4.89 & 0.85\end{array}$

$-6.15$

0.98

0.00

$0.00 \quad 0.00$

$0.00 \quad 0.00$

$0.00 \quad 0.00$

$0.00 \quad 0.00$

$0.00 \quad 0.00$

$0.00 \quad 0.00$

$0.00 \quad 0.00$

$0.00 \quad 0.00$

$0.00 \quad 0.00$

$0.00 \quad 0.00$

$0.00 \quad 0.00$

$0.00 \quad 0.00$

$0.00 \quad 0.00$

$0.00 \quad 0.00$

$0.00 \quad 0.00$

$0.00 \quad 0.00$

$0.00 \quad 0.00$

$0.00 \quad 0.00$

$0.00 \quad 0.00$

$0.00 \quad 0.00$

$0.00 \quad 0.00$

$0.00 \quad 0.00$

$0.00 \quad 0.00$

$0.00 \quad 0.00$

$0.00 \quad 0.00$

$0.00 \quad 0.00$

$\begin{array}{lll}0.00 & 0.00\end{array}$

$0.00 \quad 0.00$

$0.00 \quad 0.00$

$0.00 \quad 0.00$

$0.00 \quad 0.00$

$0.00 \quad 0.00$

$0.00 \quad 0.00$

$0.00 \quad 0.00$

$0.00 \quad 0.00$

$0.00 \quad 0.00$

$0.00 \quad 0.00$

$0.00 \quad 0.00$

$0.00 \quad 0.00$

$0.00 \quad 0.00$

$0.00 \quad 0.00$

$0.00 \quad 0.00$

$0.00 \quad 0.00$ 
49

$49100149-18.1-1.6-1126.92$

$49101 \quad 150-18.4-1.5-1128.46$ $49102 \quad 151-18.6-1.4-1129.89$ $49103 \quad 152-18.9-1.3-1131.20$ $49 \quad 104 \quad 153-19.1-1.1-1132.40$ $49105 \quad 154-19.3-1.0-1133.49$ $49106155-19.6-0.9-1134.46$ $49107 \quad 156-19.8-0.8-1135.33$ $49108 \quad 157-20.0-0.7-1136.10$ $49109158-20.3-0.6-1136.77$ $49110 \quad 159-20.5 \quad-0.5-1137.33$ $\begin{array}{lllll}49 & 111 & 160-20.7 & -0.4-1137.81\end{array}$ $49 \quad 112 \quad 161-20.9-0.3-1138.19$ $49113 \quad 162-21.1-0.2-1138.48$ $49114 \quad 163-21.3-0.2-1138.68$ $49115164-21.5 \quad-0.1-1138.81-1.672 .08-1.84-1140.24144 .98$

$-6.491 .31-8.83-846.53-69.81$ $\begin{array}{lllllll}6 & -5.25 & 2.29 & -8.48 & -856.61 & -71.81 \\ 0 & -4.12 & 1.31 & -8.16 & -868.36 & -75.50\end{array}$ $\begin{array}{lllll}-3.15 & 2.28 & -7.85 & -877.98 & -77.04\end{array}$ $4-2.111 .31-7.56-88$ $-889.09-80.09$ $\begin{array}{lllll}-1.42 & 2.26 & -7.28 & -89 \\ -0.72 & 1.31 & -7.02 & -90\end{array}$

$-0$
$1.012 .24-6.32$

$\begin{array}{lllllll}1.73 & 2.23 & -5.92 & -953.38 & -87.87 & -88.00\end{array}$ $\begin{array}{lllllll}1.83 & 1.31 & -5.73 & -962.83 & -89.25 & -89.37\end{array}$ $\begin{array}{lllllll}2.04 & 2.22 & -5.55 & -970.05 & -88.40 & -88.57\end{array}$ $\begin{array}{lllllll}1.97 & 1.31 & -5.37 & -979.09 & -89.37 & -89.54\end{array}$ $\begin{array}{lllllll}2.07 & 2.22 & -5.21 & -985.88 & -88.09 & -88.25\end{array}$ $\begin{array}{llllllll}1.73 & 1.31 & -5.06 & -994.64 & -88.78 & -88.94\end{array}$ $\begin{array}{lllllll}1.59 & 2.21 & -4.91-1001.15 & -87.21 & -87.23\end{array}$ $\begin{array}{lllllll}1.05 & 1.31 & -4.77-1009.59 & -87.58 & -87.73\end{array}$ $\begin{array}{lllllll}0.71 & 2.20 & -4.63-1015.80 & -85.72 & -85.73\end{array}$ $\begin{array}{lllllll}-0.02 & 1.31 & -4.50-1023.94 & -85.79 & -85.84\end{array}$ $\begin{array}{lllllll}1.34 & 1.31 & -6.11 & -945.77 & -88.33 & -88.39\end{array}$

$\begin{array}{ll}0.00 & 0.00 \\ 0.00 & 0.00\end{array}$ $-74.60-3.22$ $-76.08-2.19$ $-79.49-1.51$ $-80.61-0.81$ $-0.38$

0.23

0.57

1.12

1. -0.06

$1.60-0.13$

$1.71-0.12$

$1.87-0.17$

$1.80-0.17$

$1.91-0.16$

$1.57-0.16$

$1.57-0.02$

$0.90-0.15$

$0.70-0.01$

$-0.07-0.05$

$\begin{array}{rr}-0.48 & 0.00\end{array}$

$-83.43-1.48-0.03$

$-2.102 .18-4.15-1043.30-80.93-80.88 .-2.05 .0 .05$

$\begin{array}{lllllll}-3.34 & 1.31 & -4.04-1051.01 & -80.58 & -80.48 & -3.24\end{array}$ $\begin{array}{llllll}-5.68 & 1.31 & -3.83-1063.92 & -77.35 & -76.99 & -5.32\end{array}$

$\begin{array}{lllllll}-6.83 & 2.17 & -3.74-1069.19 & -74.54 & -74.34 & -6.63\end{array}$

$\begin{array}{lllllll}-8.31 & 1.31 & -3.64-1076.31 & -73.59 & -72.97 & -7.69\end{array}$

$\begin{array}{lllllll}-9.60 & 2.16 & -3.56-1081.32 & -70.53 & -69.88 & -8.95\end{array}$

$-68.19-10.09$

$-63.01-9.62$

$-9.972 .16-3.39-1090.29-63.36$

$-8.921 .31-3.31-1094.11-59.10$

0.00

0.00

0.00

$-6.551 .31-3.16-1099.25-48.11$

0.00

0.00

0.00

$\begin{array}{llll}-5.45 & 2.15 & -3.09-1100.82 & -41.60\end{array}$

$\begin{array}{lllll}-4.32 & 1.31 & -3.03-1103.86 & -36.57\end{array}$

$\begin{array}{llllll}-2.73 & 2.14 & -2.96-1104.62 & -29.25\end{array}$

0.00

0.00

0.00

0.00

0.00

0.00

$-2.001 .31-2.90-1107.73-24.30$

$\begin{array}{lllll}-1.03 & 2.13 & -2.84-1108.80 & -17.30\end{array}$

$0.351 .31-2.78-1110.96-11.38$

$0.492 .13-2.72-1112.56$

$0.861 .31-2.67-1115.43$

$-4.91$

$1.112 .12-2.61-1116.65$

$1.331 .31-2.56-1119.38$

$1.35 \quad 2.12-2.51-1120.57$

$1.591 .31-2.47-1123.01$

$1.392 .11-2.42-1124.16$

$1.501 .31-2.38-1126.48$

$1.262 .11-2.33-1127.43$

$1.391 .31-2.29-1129.48$

$1.262 .10-2.25-1130.09$

$1.531 .31-2.21-1131.77$

$1.382 .10-2.17-1132.18$

$1.461 .31-2.13-1133.82$

$1.242 .10-2.10-1134.09$

0.29

7.14

12.48

19.37

24.99

31.92

37.67

44.79

50.81

58.27

64.67

72.33

78.75

$1.201 .31-2.06-1135.65 \quad 93.07$

$0.772 .09-2.03-1135.93 \quad 100.86$

$\begin{array}{lllll}0.53 & 1.31 & -2.00-1137.49 & 107.37\end{array}$

$0.182 .09-1.96-1137.51115 .43$

$0.091 .31-1.93-1138.72 \quad 122.28$

$-0.392 .08-1.90-1138.69130 .39$

$-0.861 .31-1.87-1140.11 \quad 137.04$

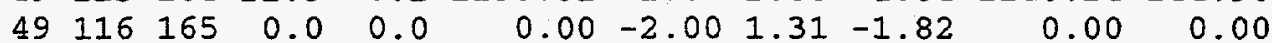

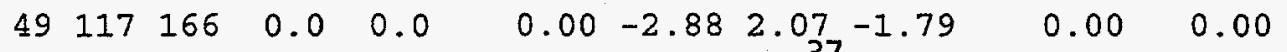

0.00

0.00

0.00

0.00

0.00

0.00

0.00

0.00

0.00

0.00

0.00

0.00

0.00

0.00

0.00

0.00

0.00

0.00

0.00

0.00

0.00

0.00

0.00

0.00

0.00

0.00

0.00
0.10

0.12

0.36

0.20

0.62

0.65

0.50

0.35

0.00

$0.00 \quad 0.00$

$0.00 \quad 0.00$

$0.00 \quad 0.00$

$0.00 \quad 0.00$

$0.00 \quad 0.00$

$0.00 \quad 0.00$

$0.00 \quad 0.00$

$0.00 \quad 0.00$

$0.00 \quad 0.00$

$0.00 \quad 0.00$

$0.00 \quad 0.00$

$0.00 \quad 0.00$

$0.00 \quad 0.00$

$0.00 \quad 0.00$

$0.00 \quad 0.00$

$0.00 \quad 0.00$

$0.00 \quad 0.00$

$0.00 \quad 0.00$

$0.00 \quad 0.00$

0.00 .0 .00

$0.00 \quad 0.00$

$0.00 \quad 0.00$

$0.00 \quad 0.00$

$0.00 \quad 0.00$

$0.00 \quad 0.00$

$0.00 \quad 0.00$

$0.00 \quad 0.00$ $0.00 \quad 0.00$ 


\begin{tabular}{|c|c|c|c|c|c|c|c|c|c|c|c|c|c|}
\hline & 44 & 94 & 3.2 & 9 & 17 & 15 & & & 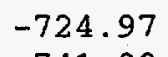 & 4 & 0.00 & 0.00 & \\
\hline & 45 & 95 & & & -729.80 & -4.62 & 35 & -8.02 & -741.09 & 9 & 0.00 & 10 & .00 \\
\hline & 46 & & & & -745.95 & & 00 & & -760.20 & & .00 & .00 & .00 \\
\hline & 47 & 97 & & .4 & -761.63 & 6.61 & & -8.78 & -775.69 & -32 & 00 & 00 & 0 \\
\hline & 48 & 98 & & & -776.85 & -8 & 10 & -9.18 & -794 & & & & \\
\hline & 49 & 99 & & & -791.62 & & 1 & 8 & $-80 \varepsilon$ & & & & 00 \\
\hline & 50 & 100 & & & & & & & & & & & 00 \\
\hline & 51 & 101 & -0.1 & & & & 29 & -9.59 & -8 & & & 00 & 0.00 \\
\hline & 52 & 102 & -0.5 & & -83 & -8.09 & 00 & -9.21 & & -6 & 0.00 & 00 & .00 \\
\hline & 53 & 103 & & & & & & & & & & & \\
\hline & 54 & 104 & - & & -8 & & & 51 & -8 & 99 & 1.53 & & \\
\hline & 55 & 105 & -1 & & & & & & & & & & .32 \\
\hline & 56 & 106 & -2.3 & & 36 & -3.34 & 0 & -7.88 & 58 & -78.30 & 77.42 & & .88 \\
\hline & 57 & 107 & $-2.7-$ & .4 & -894.91 & -2.25 & & -7.60 & 51 & -79.16 & 0.00 & 10 & .00 \\
\hline & 58 & 108 & -3.2 & 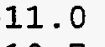 & -9 & -1.50 & & & -91 & -82 & 1.99 & & 52 \\
\hline & 59 & 109 & -3.6 & & & & & & & -83.02 & 3 & & 39 \\
\hline & 60 & 110 & $-4.0-$ & 10.3 & -927 & -0.41 & & -6.83 & & -86.13 & 33 & & .30 \\
\hline & 61 & 111 & -4. & & -937.64 & 0.13 & & -6.60 & & -86.24 & & & .30 \\
\hline & 62 & 112 & -4 & & & 0. & & & & & & & 12 \\
\hline & 63 & 113 & -5.3 & -9 & $-9 !$ & 0.89 & & -6.17 & -961.10 & -88.31 & -88.33 & 0.87 & -0.02 \\
\hline & 64 & 114 & -5.7 & -9. & -9 & 0.81 & & -5.97 & & -90.55 & -90.56 & & 0.01 \\
\hline & 65 & 115 & -6.1 & & & 1.03 & & & & -89.80 & & & \\
\hline & 66 & 116 & -6 & & & 4 & & & & -91.47 & 3 & 0.88 & -0 \\
\hline & 67 & 117 & -6.9 & -8 & -9 & 0.96 & 8 & -5.43 & -9 & -90.37 & 10 & 0.93 & -0.03 \\
\hline & 68 & 118 & -7.2 & -7 & .22 & 0.75 & & -5 & & -91.59 & 5 & & \\
\hline & 69 & 119 & & & & 070 & & & & -9 & & & \\
\hline & 70 & 120 & & & & 19 & & & 2 & 3 & & 12 & -0.07 \\
\hline & 71 & 121 & -8.4 & -7 . & 1 & -0.28 & 6 & -4 & 6 & -89.40 & -89.20 & -0.08 & 0.20 \\
\hline & 72 & 122 & 8.8 & -6. & & -0.99 & & -4 & & 18 & 94 & 35 & .14 \\
\hline & 73 & 123 & & & & & & & & & & & \\
\hline & 74 & 124 & & & & -2.51 & 0 & -4 & 8 & -88.51 & 24 & -2 & 27 \\
\hline & 75 & 125 & -9.9 & -6 . & 3 & -3.23 & 4 & -4 & 34 & -86.20 & .90 & -2 & 30 \\
\hline & 76 & 12 & -10.2 & -6 . & 0 & -4.36 & 0 & - & & 5 & & & 33 \\
\hline & 77 & 12 & 10. & & & & & & & & & & \\
\hline & 78 & 12 & & & & & & & & & & & 35 \\
\hline & 79 & 12 & -11.2 & -5 . & & -7.67 & 2 & -3 & & -8 & 62 & -7 & 27 \\
\hline & 80 & 13 & 11.6 & 5 . & & -9.14 & 0 & -3 & & -8 & & $-\varepsilon$ & 39 \\
\hline & 81 & 13 & 11.9 & 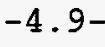 & & & & & & & & & \\
\hline & 82 & & .2 & & & & & & & & & & \\
\hline & 83 & & & & & & 0 & -3 & & -7 & -71 & 98 & -0 \\
\hline & 84 & 13 & 12.9 & -4 & & -9.67 & 0 & -3 & & -6 & 00 & .00 & 00 \\
\hline & 85 & 3 & .2 & 4 & & & & & & & & & \\
\hline & 86 & 13 & & & & & & & & & & & 00 \\
\hline & 87 & $13^{\circ}$ & -13.8 & -3 & 9 & -6.18 & 1.08 & -3 & & -5 & & & 00 \\
\hline & 88 & 38 & 14.1 & 3. & & -5.08 & 0 & - & & -4 & & & \\
\hline & 8 & 3 & & & & & & & & & & & \\
\hline & & 4 & 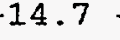 & & & & & & & & & & \\
\hline & 9 & 141 & .0 & -3 & & -1.76 & 1.07 & & & -2 & & & 00 \\
\hline & 9 & & .3 & 3 . & & -1 & & & & & & & \\
\hline & 9 & & . & & & & & & & & & & \\
\hline & & & & & & & & & & & & & \\
\hline & 9 & 145 & 16.2 & -2 . & & & 5 & & & & & & \\
\hline & 9 & & 5.4 & 2. & & & & & & & & & \\
\hline & 97 & & & -2 & & & & & & & & & \\
\hline & & & & & & & & & & & & & \\
\hline & 99 & 149 & 17.2 & -2 . & & 1 & 1. & & & & & & \\
\hline & 00 & & 5 & 1 & & 1 & & & & & & & .00 \\
\hline & 01 & & 7 & -1 & & & & & & & & & \\
\hline & & & & & & & & & & & & & \\
\hline & & 5 & & & & & & & & & & & \\
\hline & 04 & & & -1 & & & & & & & & & 00 \\
\hline & & & 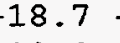 & & & 1.50 & & & & & & & \\
\hline & 06 & 15 & 19. & -1.2 & & & & & & & & & 00 \\
\hline & & & & & & & & & & & & & \\
\hline & & & & & & & & & & & & & \\
\hline & & & 107 & 00 & & & 0 & & & 7.37 & 0.00 & 0.00 & 0 \\
\hline
\end{tabular}


$50110 \quad 160-19.9-0.7-1157.51$ $50111 \quad 161-20.1-0.6-1158.19$

$50 \quad 112 \quad 162-20.3-0.5-1158.78$

$50 \quad 113 \quad 163-20.5-0.4-1159.28$

$50114 \quad 164-20.7 \quad-0.4-1159.69$

$50115 \quad 165-20.9-0.3-1160.00$

$50116 \quad 166-21.1-0.2-1160.23$

$50117 \quad 167-21.3-0.1-1160.38$

50118168

$0.0 \quad 0.0$

0.00

$0.00-4$.

0.91

$\begin{array}{llll}0.91 & 0.00 & -2.07-1158.67 & 93.48\end{array}$

$\begin{array}{llll}1.00 & -2.04-1158.60 & 101.61\end{array}$

$\begin{array}{lllll}0.32 & 0.00 & -2.00-1160.47 & 107.82\end{array}$

$\begin{array}{lll}50 & 119 & 169\end{array}$

$\begin{array}{lll}51 & 46 & 97\end{array}$

$0.0 \quad 0.0$

$-743.39$

$\begin{array}{ll}2.4-15.9 & -759.54 \\ 1.9-15.5 & -775.22\end{array}$

$\begin{array}{lll}51 & 47 & 98\end{array}$

$\begin{array}{lllll}0.24 & 0.99 & -1.97-1160.50 & 115.86\end{array}$

$\begin{array}{lll}51 & 48 & 99\end{array}$

$1.5-15.0-790.45$

$1.0-14.6-805.24$

50101

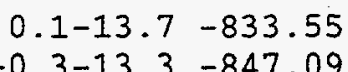

$-1.30$

$0.00-1.94-1162.20 \quad 122.23$

$\begin{array}{lllll}-1.87 & 0.00 & -1.88-1163.99 & 136.59\end{array}$

$\begin{array}{lll}51 & 51 & 102\end{array}$

$51 \quad 52103$

$-3.27$

$\begin{array}{lll}6 & 0.98\end{array}$

1.85

$\begin{array}{llll}-4.25 & 0.98 & -1.80\end{array}$

0.00

0.00

51

51

51

51

51

51

51

51

51

51

51

51

51

51

51

51

51

51

51

51
51

51

51

51

51

51

51

51

51

51

51

51

51

51

51

51

51

51

51

51

51

51

51

$\begin{array}{llll}51 & 98 & 149-16.3 & -2.4-1157.39\end{array}$

$\begin{array}{lllll}51 & 99 & 150-16.6 & -2.3-1159.72\end{array}$

$\begin{array}{llll}51 & 100 & 151-16.9 & -2.1-1161.90\end{array}$

$51 \quad 101 \quad 152-17.1-2.0-1163.96$

$\begin{array}{lllll}-4.82 & 2.31 & -8.42 & -770.47 & -19.54\end{array}$

$\begin{array}{lllll}-6.10 & 1.29 & -8.80 & -788.83 & -29.83\end{array}$

$\begin{array}{lllll}-6.83 & 2.30 & -9.19 & -804.18 & -37.11\end{array}$

$\begin{array}{lllll}-8.22 & 1.29 & -9.59 & -821.76 & -46.62\end{array}$

$2.58-10.00-834.13-50.92$

$\begin{array}{llllll}-6.18 & 1.29 & -9.60 & -848.04 & -56.76\end{array}$

$\begin{array}{llllll}-4.94 & 2.27 & -9.22 & -858.98 & -59.62\end{array}$

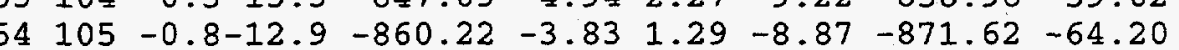

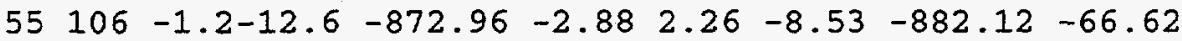

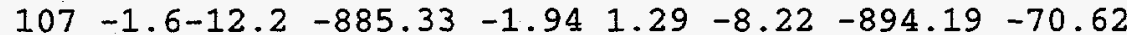

$\begin{array}{llllllll}108 & -2.1-11.8 & -897.32 & -1.11 & 2.25 & -7.92 & -904.10 & -72.46\end{array}$

$59110-2.9-11.1-920.22$

$60111-3.4-10.8-931.15$

$61 \quad 112-3.8-10.4-941.74$

$\begin{array}{lllll}63 & 114 & -4.6 & -9.8 & -961.95\end{array}$

$64.115-5.0-9.5-971.58$

$\begin{array}{lllll}65 & 116 & -5.4 & -9.2 & -980.91 \\ 66 & 117 & -5.8 & -8.9 & -989.94\end{array}$

$\begin{array}{lllll}67 & 118 & -6.2 & -8.6 & -998.68\end{array}$

$68 \quad 119-6.6-8.3-1007.13$

$70 \quad 121-7.3-7.8-1023.22$

$\begin{array}{lllll}71 & 122 & -7.7 & -7.5-1030.87\end{array}$

$\begin{array}{llll}72 & 123 & -8.1 & -7.3-1038.26 \\ 73 & 124 & -8.5 & -7.0-1045.40\end{array}$

$\begin{array}{lllll}-1.11 & 2.25 & -7.92 & -904.10 & -72.46 \\ -0.42 & 1.29 & -7.64 & -915.71 & -76.00\end{array}$

$0.17 \quad 2.24-7.37-925.18-77.40$

$\begin{array}{llllll}0.73 & 1.29 & -7.11 & -936.24 & -80.39\end{array}$

$\begin{array}{llllll}1.23 & 2.23 & -6.87 & -945.16 & -81.23\end{array}$

$\begin{array}{lllll}1.54 & 1.29 & -6.64 & -955.82 & -83.82\end{array}$

$\begin{array}{llllll}1.85 & 2.22 & -6.43 & -964.31 & -84.24\end{array}$

$1.831 .29-6.22-974.68-86.54-87.00$

$\begin{array}{lllllll}1.92 & 2.21 & -6.02 & -982.80 & -86.59 & -86.82\end{array}$

$\begin{array}{lllllll}1.70 & 1.29 & -5.84 & -992.78 & -88.50 & -88.64\end{array}$

$\begin{array}{llllll}1.65 & 2.20 & -5.66-1000.48 & -88.13 & -88.00\end{array}$

$\begin{array}{llllll}1.36 & 1.29 & -5.49-1009.97 & -89.54 & -89.47\end{array}$

$\begin{array}{llllll}1.22 & 2.19 & -5.33-1017.23 & -88.73 & -88.42\end{array}$

$\begin{array}{llllll}0.76 & 1.29 & -5.17-1026.34 & -89.77 & -89.59\end{array}$

$\begin{array}{llllll}0.52 & 2.19 & -5.02-1033.19 & -88.55 & -88.32\end{array}$

$\begin{array}{llllll}-0.16 & 1.29 & -4.88-1042.01 & -89.30 & -89.22 \\ -0.61 & 2.18 & -4.75-1048.58 & -87.80 & -87.62 \\ -1.57 & 1.29 & -4.62-1057.19 & -88.33 & -88.26\end{array}$

$-88.26$

$\begin{array}{ll}-86.50 & -86.40 \\ -86.88 & -86.71\end{array}$

$-84.61-4$.

$\begin{array}{lll}-84.62 & -5.37\end{array}$

$-82.33-6.36$

$-82.02-7.82$

$-79.81-9.29$

$-79.02-10.68$

$-74.03-9.76$

$-69.74-8.04$

$0.00 \quad 0.00$

$0.00 \quad 0.00$

$0.00 \quad 0.00$

0.00

0.00

$-3.841 .29-3.27-1131.92-50.06$
0

$\begin{array}{lllll}-2.89 & 2.12 & -3.20-1133.91 & -43.98\end{array}$

$\begin{array}{llll}-2.12 & 1.29 & -3.13-1137.57 & -39.58\end{array}$

$-0.772 .12-3.06-1138.84-32.77$

$\begin{array}{llll}-0.32 & 1.29 & -3.00-1142.50 & -28.36\end{array}$

$\begin{array}{llll}0.04 & 2.11 & -2.94-1144.45 & -22.24\end{array}$

$0.38 \quad 1.29-2.88-1147.91-17.63$

$0.502 .11-2.82-1149.81-11.45$

$0.78 \quad 1.29-2.76-1153.03$

$0.912 .10-2.71-1154.63$

$1.161 .29-2.66-1157.60$

$1.092 .10-2.61-1159.14$

$1.28 \quad 1.29-2.56-1161.89$

$1.112 .09-2.51-1163.27$
$-6.60$

$-0.13$

4.97

11.50

16.82

23.51
0.00

0.00

0.00

0.00

0.00

0.00

0.00

0.00

0.00

0.00

0.00

0.00
0.00

0.00

0.00

0.00

0.00

0.00

0.00

0.00

0.00

0.00

0.00

0.00

0.00

0.00

0.00

0.00

0.00

0.00

0.00

0.00

0.00

0.00

0.00

0.25

0.00

0.00

$0.86-0.37$

$94-0.60$

$1.37-0.46$

$1.69-0.23$

$1.56-0.14$

$\begin{array}{ll}1.78 & 0.13\end{array}$

$\begin{array}{ll}1.43 & 0.07\end{array}$

$\begin{array}{ll}53 & 0.31\end{array}$

0.18

0.23

0.08

0.18

0.07

0.10

0.17

0.25

0.50

0.50

0.61

0.31

$-0.04$

0.28

0.74

0.00

0.00

0.00

$0.00 \quad 0.00$

$0.00 \quad 0.00$

$0.00 \quad 0.00$

$0.00 \quad 0.00$

$0.00 \quad 0.00$

$0.00 \quad 0.00$

$0.00 \quad 0.00$

$0.00 \quad 0.00$

$0.00 \quad 0.00$

$0.00 \quad 0.00$

$0.00 \quad 0.00$

$0.00 \quad 0.00$

$0.00 \quad 0.00$ 
$51 \quad 102 \quad 153-17.4-1.9-1165.89$ $\begin{array}{lllll}51 & 103 & 154-17.6 & -1.7-1167.70\end{array}$

$51 \quad 104 \quad 155-17.9-1.6-1169.38$

51 105 156-18.1 -1.5-1170.95

$51106 \quad 157-18.4-1.4-1172.40$

$51107158-18.6-1.3-1173.73$

$51108159-18.8-1.2-1174.95$

$51109 \quad 160-19.0-1.1-1176.07$

$51110161-19.3-1.0-1177.08$

$51111162-19.5-0.9-1177.99$

$\begin{array}{lllll}51 & 112 & 163-19.7 & -0.8-1178.79\end{array}$

$51 \quad 113 \quad 164-19.9-0.7-1179.50$

$51114165-20.1-0.6-1180.11$

$51115166-20.3-0.5-1180.63$

$51116 \quad 167-20.5-0.4-1181.06$

$51117168-20.7-0.3-1181.40$

$51 \quad 118 \quad 169-20.9-0.2-1181.66$

$51119170-21.1-0.1-1181.83$

$\begin{array}{rrrrrr}51 & 120 & 171 & 0.0 & 0.0 & 0.00\end{array}$

$\begin{array}{rrrlrr}51 & 121 & 172 & 0.0 & 0.0 & 0.00 \\ 52 & 47 & 99 & 3.0-16.4 & -756.85\end{array}$

$\begin{array}{rrrrrr}52 & 47 & 99 & 3.0-16.4 & -756.85 \\ 52 & 48 & 100 & 2.5-15.9 & -772.99\end{array}$

$1.281 .29-2.47-1165.78$

$1.122 .09-2.42-1166.91$

$1.421 .29-2.38-1169.05$

$1.32 \quad 2.08-2.34-1169.88$

$1.501 .29-2.30-1171.90$

$1.372 .08-2.26-1172.54$

$1.521 .29-2.22-1174.36$

$1.112 .08-2.18-1175.07$

$1.091 .29-2.15-1176.84$

$0.742 .07-2.11-1177.29$

$0.521 .29-2.08-1179.06$

$-0.042 .07-2.04-1179.52$

$-0.461 .29-2.01-1181.29$

$-1.112 .06$

$-1.98-1181.65118 .12$

$\begin{array}{lllll}-1.76 & 1.29 & -1.95-1183.48 & 124.37\end{array}$

$-2.532 .06-1.92-1183.80132 .13$

$\begin{array}{lllll}-3.03 & 1.29 & -1.89-1185.29 & 138.71\end{array}$

52

49101

$2.1-15.5-788.67$

1. $6-15.0-803.92$

$-3.72$

2.06

$-1.86-1185.36146 .71$

50102

51103

$1.2-14.6-818.73$

$0.7-14.2-833.12$

$0.3-13.8-847.10$

3.97

$1.29-1.84$

0.00

0.00

52104

53105

54106

$-0.1-13.4-860.68$

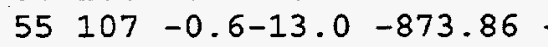

$56108-1.0-12.6-886.66$

$\begin{array}{llll}57 & 109 & -1.4-12.2 & -899.08\end{array}$

$\begin{array}{llll}58 & 110 & -1.9-11.9 & -911.13\end{array}$

$\begin{array}{lllll}59 & 111 & -2.3-11.5 & -922.83\end{array}$

$\begin{array}{lllll}60 & 112 & -2.7-11.2 & -934.18\end{array}$

$61 \quad 113-3.1-10.8-945.19$

$\begin{array}{llll}62 & 114 & -3.5-10.5 & -955.87\end{array}$

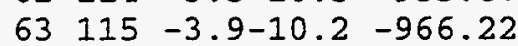

$\begin{array}{lllll}64 & 116 & -4.3 & -9.9 & -976.26\end{array}$

$\begin{array}{llllll}65 & 117 & -4.7 & -9.6 & -985.98\end{array}$

$\begin{array}{lllll}66 & 118 & -5.1 & -9.3 & -995.41\end{array}$

$\begin{array}{lllll}67 & 119 & -5.5 & -9.0-1004.54\end{array}$

$\begin{array}{lllll}68 & 120 & -5.9 & -8.7-1013.38\end{array}$

$\begin{array}{lllll}69 & 121 & -6.3 & -8.4-1021.95\end{array}$

$\begin{array}{lllll}70 & 122 & -6.7 & -8.2-1030.24\end{array}$

$\begin{array}{lllll}71 & 123 & -7.1 & -7.9-1038.26\end{array}$

$\begin{array}{lllll}72 & 124 & -7.4 & -7.6-1046.02\end{array}$

$\begin{array}{lllll}73 & 125 & -7.8 & -7.4-1053.52\end{array}$

3.731 .33

$-1.81$

0.00

0.00

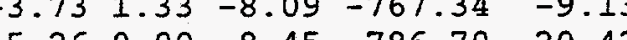

$\begin{array}{llllll}-5.64 & 1.31 & -8.83 & -801.83 & -27.48\end{array}$

$\begin{array}{llllll}-7.11 & 0.00 & -9.21 & -820.24 & -37.82\end{array}$

$\begin{array}{llllll}-5.89 & 1.29 & -9.60 & -832.93 & -42.44\end{array}$

$\begin{array}{llllll}-5.03 & 0.00-10.00 & -848.15 & -49.59\end{array}$

$\begin{array}{llllll}-3.72 & 1.28 & -9.61 & -859.15 & -52.52\end{array}$

$\begin{array}{llllll}-3.01 & 0.00 & -9.24 & -872.92 & -58.22\end{array}$

$\begin{array}{llllll}-1.94 & 1.26 & -8.89 & -883.42 & -60.65\end{array}$

$\begin{array}{llllll}-1.18 & 0.00 & -8.56 & -896.40 & -65.55\end{array}$

$\begin{array}{llllll}-0.41 & 1.25 & -8.25 & -906.49 & -67.57\end{array}$

$\begin{array}{lllllll}0.25 & 0.00 & -7.95 & -918.84 & -71.84 & -7\end{array}$

$\begin{array}{lllllll}0.85 & 1.23 & -7.67 & -928.42 & -73.36 & -7\end{array}$

$\begin{array}{llllll}1.33 & 0.00 & -7.41 & -940.26 & -77.13 & -7\end{array}$

$\begin{array}{llllll}1.67 & 1.22 & -7.16 & -949.46 & -78.25\end{array}$

$2.04 \quad 0.00-6.92 \quad-960.75 \quad-81.47$

$\begin{array}{llllll}2.09 & 1.21 & -6.69 & -969.62 & -82.27 & -8\end{array}$

$2.28 \quad 0.00-6.48-980.45-85.03$

$\begin{array}{lllllll}2.66 & 1.19 & -6.27 & -988.40 & -84.91 & -8\end{array}$

$2.46 \quad 0.00-6.08-999.02-87.46-8$

$2.381 .18-5.89-1006.87-87.23$

$2.08 \quad 0.00-5.71-1017.01-89.31$

$1.891 .17-5.54-1024.43$.

$1.550 .00-5.38-1034.07$

$-89.39$

$1.301 .16-5.23-1041.03$

$0.660 .00-5.08-1050.44$

$0.251 .15-4.94-1057.06-8$

$90.22-9$.

$-89.10-89.17$

$-90.45-90.52$

$74126-8.2-7.1-1060.78$

$-0.62$

0.00

$-4.80-1066.20$

$-89.00-8$

$-89.03$

$-90.07-90.07$

$75 \quad 127-8.5-6.9-1067.80$

$\begin{array}{lllll}76 & 128 & -8.9 & -6.7-1074.57\end{array}$

$\begin{array}{llll}77 & 129 & -9.2 & -6.4-1081.12\end{array}$

$-1.22$

1.14

$-4.67-1072.55$

$\begin{array}{llll}78 & 130 & -9.6 & -6.2-1087.44\end{array}$

3.521 .00

$-$

$\begin{array}{llll}79 & 131 & -9.9 & -6.0-1093.53\end{array}$

4.74

$-5.791 .12$

$2-4$

$\begin{array}{llll}80 & 132-10.2 & -5.8-1099.41 \\ 81 & 133-10.6 & -5.6-1105.08\end{array}$

7.33

.00

$-4.21-1102.41$

$82134-10.9-5.4-1110.55$

$-9.57$

1.11

$-4.00-1116.51$

$-88.34-8$

$-88.29$

$-88.99$

$-87.59-87.00$

$-88.07-8$

$-87.35$

$-85.92-85.21-5.08$

$-86.28-85.22-6.27$

$-83.87-82.97-7.63$

$83135-11.2-5.2-1115.81$

$-8.91$

1.10

$-3.91-1124.02$

$-83.32-8$

-7.63
-8.69

$-77.86-8.12$

$84136-11.6-5.0-1120.87-7.790 .00-3.72-1132.39$

$85 \quad 137-11.9-4.8-1125.75-6.641 .09-3.64-1134.93$

78.65

$5.54-74.46$

$-69.56$

0.00

$-6.71$

$86138-12.2-4.6-1130.43-5.390 .00-3.55-1139.37-66.38$

$87 \quad 139-12.5-4.4-1134.93-3.82 \quad 1.08-3.47-1141.14-60.08$

$\begin{array}{lllllll}88 & 140-12.8 & -4.2-1139.25 & -2.87 & 0.00 & -3.40-1145.52 & -56.38\end{array}$

$89 \quad 141-13.1-4.1-1143.40-1.87 \quad 1.08-3.32-1147.51-50.31$

$90-142-13.4-3.9-1147.37-1.270 .00-3.25-1151.89-46.61$

$91143-13.7-3.7-1151.17-0.77 \quad 1.07-3.18-1154.06-40.71$

$-3$

40
0.00

$0.00 \quad 0.00 \quad 0.00$

$\begin{array}{llll}0.00 & 0.00 & 0.00\end{array}$

$0.00 \quad 0.00 \quad 0.00$

$0.00 \quad 0.00 \quad 0.00$ 


\section{2}

\section{2}

53

53

53

53
53

53

53

53

53
53

53
53

53
53

53
53

53

53

53

53

53

53

53

53

53

53

53

53

53

53

53

53

53

53

53

53

53
$93145-14.3-3.4-1158.29$ $94146-14.6-3.2-1161.62$

$\begin{array}{llll}52 & 95 & 147-14.9 & -3.1-1164.79 \\ 52 & 96 & 148-15.1 & -2.9-1167.80\end{array}$

$5297 \quad 149-15.4 \quad-2.8-1170.68$

$5298 \quad 150-15.7-2.7-1173.41$

5299 151-16.0 -2.5-1175.99

$52100 \quad 152-16.2-2.4-1178.45$

$\begin{array}{llll}52 & 101 & 153-16.5 & -2.2-1180.76\end{array}$

$52102154-16.7-2.1-1182.95$

$52103 \quad 155-17.0-2.0-1185.01$

$52104156-17.2-1.9-1186.94$

$52105 \quad 157-17.5-1.7-1188.75$

$52106 \quad 158-17.7-1.6-1190.44$

$52107159-18.0-1.5-1192.01$

$52108 \quad 160-18.2-1.4-1193.47$

$52109161-18.4-1.3-1194.82$

$52110 \quad 162-18.7-1.2-1196.06$

52111 163-18.9 $-1.1-1197.19$

$52112164-19.1-1.0-1198.21$

$52113165-19.3-0.9-1199.14$

$52 \quad 114 \quad 166-19.6-0.8-1199.96$

$52115 \quad 167-19.8-0.7-1200.69$

$-0.121 .06-3.05-1160.40-30.91$

$0.250 .00-2.99-1164.35-26.79$

$0.371 .05-2.93-1166.29-20.66$

$0.66 \quad 0.00-2.87-1170.01-16.31$

$0.71 \quad 1.04-2.81-1171.74-9.96$

$0.990 .00-2.76-1175.17 \quad-5.33$

$0.991 .04-2.71-1176.67$

1. 25

$1.220 .00-2.65-1179.88$

6.11

$1.04 \quad 1.03-2.61-1181.30$

12.76

$1.200 .00-2.56-1184.31$

17.83

$0.931 .02-2.51-1185.56$

24.64

$1.290 .00-2.47-1188.12$

30.16

$1.241 .02-2.42-1188.92$

37.43

$1.50 \quad 0.00-2.38-1191.32$

$1.381 .01-2.34-1191.96$

43.10

$1.580 .00-2.30-1194.19$

50.53

$1.241 .00-2.26-1194.83$

56.37

$\begin{array}{llll}1.28 & 0.00 & -2.22-1197.00\end{array}$

63.80

$0.821 .00-2.19-1197.56$

69.71

$0.720 .00-2.15-1199.64$

77.22

$0.310 .99-2.12-1199.95$

83.20

90.97

$\begin{array}{lllll}-0.05 & 0.00 & -2.08-1202.10 & 96.89\end{array}$

$52116 \quad 168-20.0-0.6-1201.33$

$-0.740 .99-2.05-1202.50$

104.57

$52117 \quad 169-20.2-0.5-1201.87$

$\begin{array}{lllll}-1.03 & 0.00 & -2.02-1204.38 & 110.76\end{array}$

$52 \quad 118 \quad 170-20.4-0.4-1202.32$

$52 \quad 119 \quad 171-20.6-0.3-1202.68$

$\begin{array}{lllll}-2.12 & 0.98 & -1.99-1204.99 & 118.21\end{array}$

$\begin{array}{lllll}-2.52 & 0.00 & -1.96-1206.80 & 124.48\end{array}$

$52 \quad 120172-20.8-0.2-1202.97$

$52121 \quad 173-21.0-0.2-1203.16$

$\begin{array}{lllll}-3.05 & 0.98 & -1.93-1206.69 & 132.66\end{array}$

$52 \quad 122 \quad 174-21.1-0.1-1203.28$

$\begin{array}{lllll}-3.76 & 0.00 & -1.90-1208.63 & 138.79\end{array}$

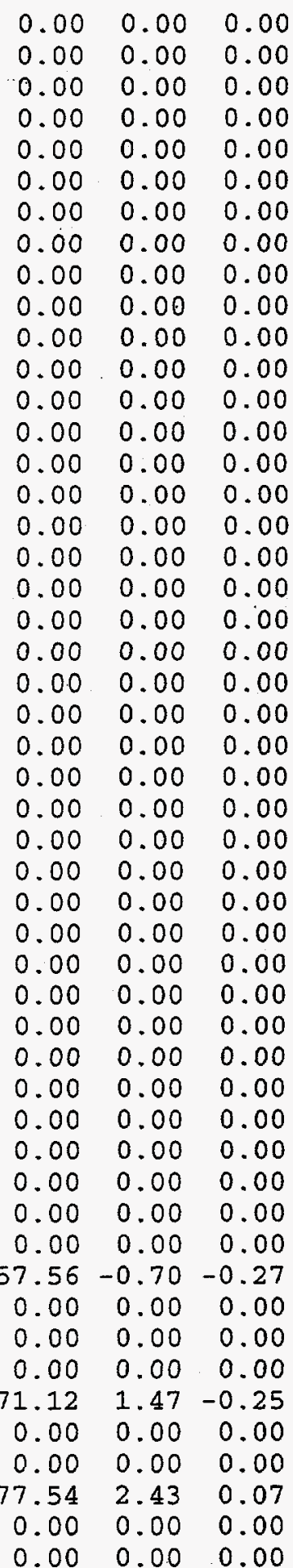

$-5.540 .00-1.85-1210.67152 .90$

$\begin{array}{lllll}-7.05 & 0.97 & -1.82 & 0.00 & 0.00\end{array}$

$\begin{array}{llllll}0.00 & -8.02 & 0.00 & -1.79 & 0.00 & 0.00\end{array}$

$\begin{array}{llll}48 & 101 & 3.1-16.4 & -770.17\end{array}$

$\begin{array}{llllll}-3.74 & 1.28 & -8.12 & -780.75 & -7.20\end{array}$

49102

$2.7-15.9-786.30$

$\begin{array}{llllll}-4.31 & 2.29 & -8.48 & -796.81 & -15.18\end{array}$

50103

$2.2-15.5-801.99$

51104

$1.8-15.0-817.25$

52105

$1.3-14.6-832.08$

$\begin{array}{llllll}30 & 1.28 & -8.85 & -814.86 & -25.16\end{array}$

$\begin{array}{llllll}-4.60 & 2.27 & -9.22 & -828.80 & -31.03\end{array}$

53106

$0.9-14.2-846.50$

$\begin{array}{lll}-3.89 & 1.28 & -9.61\end{array}$

$-844.30-38.46$

54107

$0.5-13.8-860.51$

$\begin{array}{lllll}2.67 & 2.54-10.00 & -856.63 & -42.71\end{array}$

$\begin{array}{lllll}55 & 108 & 0.0-13.4 & -874.13\end{array}$

$\begin{array}{lllll}56 & 109 & -0.4-13.0 & -887.36\end{array}$

$57110-0.8-12.7-900.21$

$\begin{array}{llll}58 & 111 & -1.2-12.3 & -912.69\end{array}$

$\begin{array}{lllll}59 & 112 & -1.7-11.9 & -924.81\end{array}$

$\begin{array}{lllll}60 & 113 & -2.1-11.6 & -936.57\end{array}$

$61114-2.5-11.3-948.00$

$\begin{array}{lllll}62 & 115 & -2.9-10.9 & -959.08\end{array}$

$\begin{array}{lllll}63 & 116 & -3.3-10.6 & -969.84\end{array}$

$\begin{array}{llll}64 & 117 & -3.7-10.3 & -980.28\end{array}$

$65118-4.1-10.0-990.41$

$\begin{array}{llll}66 & 119 & -4.5 & -9.7-1000.23\end{array}$

$\begin{array}{lllll}67 & 120 & -4.9 & -9.4-1009.75\end{array}$

$\begin{array}{llll}68 & 121 & -5.3 & -9.1-1018.98\end{array}$

$\begin{array}{llll}69 & 122 & -5.7 & -8.8-1027.92\end{array}$

$70123-6.0-8.5-1036.59$

$\begin{array}{lllll}71 & 124 & -6.4 & -8.3-1044.99\end{array}$

$72 \quad 125-6.8-8.0-1053.12$

$\begin{array}{lllll}73 & 126 & -7.1 & -7.7-1060.99\end{array}$

$\begin{array}{lllll}74 & 127 & -7.5 & -7.5-1068.61\end{array}$

$\begin{array}{lllll}75 & 128 & -7.9 & -7.2-1075.98\end{array}$

$\begin{array}{llllll}-1.15 & 2.25 & -9.25 & -882.28 & -52.22\end{array}$

$\begin{array}{lllll}-0.43 & 1.28 & -8.91 & -895.42 & -57.29\end{array}$

$\begin{array}{llllll}0.19 & 2.24 & -8.58 & -906.36 & -50.16\end{array}$

$\begin{array}{llllll}0.80 & 1.28 & -8.28 & -918.89 & -64.61\end{array}$

$\begin{array}{llllll}1.22 & 2.23 & -7.99 & -929.35 & -67.00\end{array}$

$\begin{array}{llllll}1.72 & 1.28 & -7.71 & -941.29 & -70.87\end{array}$

$\begin{array}{llllll}1.88 & 2.22 & -7.45 & -951.35 & -72.86\end{array}$

$2.28 \quad 1.28 \quad-7.20 \quad-962.73 \quad-75.17$

$2.36 \quad 2.21-6.96-972.24-77.61$

$2.56 \quad 1.28-6.74-983.18-80.48$

$2.58 \quad 2.20-6.52-992.15-81.38$

$2.611 .28-6.32-1002.66-83.81-83.78$

$2.512 .19-6.13-1011.18-84.26-83.77$

$2.601 .28-5.94-1021.04-86.05-86.27$

$2.43 \quad 2.18-5.76-1029.08-86.02-86.07$

$2.141 .28-5.60-1038.77-87.64-87.93$

$\begin{array}{lllllll}1.93 & 2.17 & -5.44-1046.32 & -87.12 & -87.37\end{array}$

$1.461 .28-5.28-1055.66-88.39-88.84$

$\begin{array}{llllll}1.04 & 2.16 & -5.13-1062.92 & -87.58 & -87.91\end{array}$

$\begin{array}{lllllll}0.35 & 1.28 & -4.99-1071.98 & -88.56 & -88.99\end{array}$

$2.64 \quad 0.03$

$3.00 \quad 0.49$

$2.38-0.22$

$2.38-0.05$

$1.85-0.29$

$1.68-0.25$

$1.01-0.45$

$0.71-0.33$

$\begin{array}{llll}76 & 129 & -8.2 & -7.0-1083.12\end{array}$

$77130-8.6-6.8-1090.01$

$-0.312 .16-4.86-1079.00$

$-87.51$

$-87.74$

$-0.54$

$-0.23$

$\begin{array}{llllll}-1.29 & 1.28 & -4.73-1087.86 & -88.30 & -88\end{array}$

$-88.50-1.49$

$-0.20$

$\begin{array}{lllll}79 & 132 & -9.3 & -6.3-1103.12\end{array}$

$-3.441 .28-4.49-1103.33$

$-87.62$

$-87.46-3.28$

0.04

0.16

0.43

$80 \quad 133-9.6-6.1-1109.34$

$4.562 .14-4.37-1109.91$

$-86.14-85.71-4.13$

0.73

0.72 
$\begin{array}{llllllllll}82 & 135-10.3 & -5.7-1121.14 & -8.39 & 1.28 & -4.06-1132.31 & -84.32 & -83.82 & -7.89\end{array}$ $\begin{array}{lllllllll}83 & 136-10.6 & -5.5-1126.73 & -7.74 & 2.13 & -3.96-1136.30 & -80.24 & -79.57 & -7.07\end{array}$

$\begin{array}{lllllll}88 & 141-12.2 & -4.5-1151.75 & -2.22 & 1.28 & -3.53-1156.21 & -59.80\end{array}$

$89142-12.5-4.4-1156.20-1.462 .11-3.45-1158.99-54.51$

\section{3}

$91144-13.1-4.0-1164.57$

\section{3}

\section{3}

$53101154-15.9-2.5-1196.94$

$53 \quad 102 \quad 155-16.1-2.4-1199.38$

$53103156-16.4-2.2-1201.70$

$53 \quad 104 \quad 157-16.6 \quad-2.1-1203.88$

$53 \quad 105 \quad 158-16.9-2.0-1205.94$

$53 \quad 106 \quad 159-17.1-1.9-1207.87$

$\begin{array}{lllll}53 & 107 & 160-17.4 & -1.8-1209.69\end{array}$

$53108 \quad 161-17.6-1.6-1211.38$

$53 \quad 109 \quad 162-17.8-1.5-1212.97$

$53 \quad 110 \quad 163-18.1-1.4-1214.43$

$53 \quad 111 \quad 164-18.3-1.3-1215.79$

$53 \quad 112165-18.5-1.2-1217.04$

$53 \quad 113166-18.8-1.1-1218.19$

$53 \quad 114 \quad 167-19.0-1.0-1219.23$

$53 \quad 115 \quad 168-19.2 \quad-0.9-1220.17$

$53116 \quad 169-19.4-0.8-1221.02$

$53 \quad 117 \quad 170-19.6-0.7-1221.76$

$53 \quad 118 \quad 171-19.8-0.6-1222.42$

$\begin{array}{llll}53 & 119 & 172-20.0 & -0.5-1222.98\end{array}$

$\begin{array}{llll}53 & 120 & 173-20.2 & -0.4-1223.46\end{array}$

$\begin{array}{llll}53 & 121 & 174-20.4 & -0.3-1223.85\end{array}$

$\begin{array}{lllll}53 & 122 & 175-20.6 & -0.3-1224.15 & -4\end{array}$

$53123176-20.8-0.2-1224.37$

$\begin{array}{llll}53 & 124 & 177-21.0 & -0.1-1224.52\end{array}$

$\begin{array}{llllll}53 & 125 & 178 & 0.0 & 0.0 & 0.00\end{array}$

$\begin{array}{lll}53 & 126 & 179\end{array}$

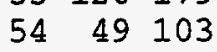

$54 \quad 50104$

$54 \quad 51105$

$54 \quad 5.2106$

$\begin{array}{lll}54 & 53 & 107\end{array}$

$\begin{array}{lll}54 & 54 & 108\end{array}$

$\begin{array}{lll}54 & 55 & 109\end{array}$

$\begin{array}{lll}0.0 & 0.0\end{array}$

0.00

$3.2-16.3-783.35$

$2.8-15.9-799.48$

$2.4-15.5-815.18$

$\begin{array}{ll}1.9-15.1 & -830.45 \\ 1.5-14.6 & -845.30\end{array}$

$\begin{array}{ll}1.5-14.6 & -845.30 \\ 1.1-14.2 & -859.74\end{array}$

$0.6-13.8-873.79$

$\begin{array}{llllll}54 & 56 & 110 & 0.2-13.5 & -887.44\end{array}$

$\begin{array}{lllll}54 & 57 & 111 & -0.2-13.1 & -900.72\end{array}$

$\begin{array}{llllll}54 & 58 & 112 & -0.6-12.7 & -913.62\end{array}$

$\begin{array}{llllll}54 & 59 & 113 & -1.0 & -12.4 & -926.16\end{array}$

$\begin{array}{llllll}54 & 60 & 114 & -1.5-12.0 & -938.34\end{array}$

$\begin{array}{lllll}54 & 61 & 115 & -1.9-11.7 & -950.17\end{array}$

$\begin{array}{lllll}54 & 62 & 116 & -2.3-11.3 & -961.67\end{array}$

$\begin{array}{llllll}54 & 63 & 117 & -2.7 & -11.0 & -972.83\end{array}$

$\begin{array}{lllll}54 & 64 & 118 & -3.1-10.7 & -983.67\end{array}$

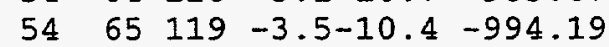

$54 \quad 66 \quad 120-3.9-10.1-1004.40$

$\begin{array}{llllll}54 & 67 & 121 & -4.2 & -9.8 & -1014.31\end{array}$

$\begin{array}{llllll}54 & 68 & 122 & -4.6 & -9.5 & -1023.93\end{array}$

$\begin{array}{lllll}54 & 69 & 123 & -5.0 & -9.2-1033.26\end{array}$ $\begin{array}{llll}-0.96 & 1.28 & -3.37-1163.53 & -50.97\end{array}$

$\begin{array}{llll}-0.48 & 2.10 & -3.30-1166.25 & -45.62\end{array}$

$-0.111 .28-3.23-1170.57-41.87$

$0.052 .10-3.16-1173.29-36.52$

$0.431 .28-3.10-1177.28-32.43$

$\begin{array}{lllll}0.51 & 2.09 & -3.04-1179.77 & -26.85\end{array}$

$0.78 \quad 1.28-2.98-1183.56-22.57$

$\begin{array}{llll}0.88 & 2.09 & -2.92-1185.73 & -16.68\end{array}$

$1.10 \quad 1.28-2.86-1189.27-12.14$

$0.972 .08-2.81-1191.40 \quad-6.19$

$1.201 .28-2.75-1194.64 \quad-1.36$

$\begin{array}{llll}0.99 & 2.08 & -2.70-1196.57 & 4.77\end{array}$

$\begin{array}{llll}1.16 & 1.28 & -2.65-1199.60 & 9.82\end{array}$

$0.812 .07-2.60-1201.42$

$1.151 .28-2.56-1204.01$

$1.152 .07-2.51-1205.23$

$1.391 .28-2.47-1207.67$

$1.28 \quad 2.06-2.42-1208.77$

$1.431 .28-2.38-1211.06$

$1.172 .06-2.34-1212.08$

$1.161 .28-2.30-1214.30$

$0.832 .06-2.26-1215.17$

$0.901 .28-2.23-1217.09$

$0.46 \quad 2.05-2.19-1217.87$

$0.24 \quad 1.28-2.16-1219.87$

$-0.512 .05-2.12-1220.76$

$-0.731 .28-2.09-1222.56$

$-1.442 .04-2.06-1223.22$

$-2.171 .28-2.03-1225.34113 .22$

$\begin{array}{lllll}-2.70 & 2.04 & -2.00-1225.64 & 120.99\end{array}$

$\begin{array}{lllll}-3.21 & 1.28 & -1.97-1227.36 & 127.34\end{array}$

$\begin{array}{lllll}-4.09 & 2.04 & -1.94-1227.84 & 134.94\end{array}$

$\begin{array}{llll}-4.84 & 1.28 & -1.91-1229.62 & 141.22\end{array}$

$\begin{array}{lllll}-6.42 & 2.03 & -1.88-1230.64 & 148.27\end{array}$

$\begin{array}{llll}-7.36 & 1.28 & -1.85-1232.45 & 154.53\end{array}$

$\begin{array}{lllll}-8.95 & 2.03 & -1.83 & 0.00 & 0.00\end{array}$

$\begin{array}{lllll}-9.69 & 1.28 & -1.80 & 0.00 & 0.00\end{array}$

$\begin{array}{llllll}-3.14 & 1.31 & -8.16 & -793.34 & -4.43\end{array}$

$\begin{array}{llllll}-4.22 & 0.00 & -8.51 & -812.21 & -15.23\end{array}$

$\begin{array}{lllll}-3.40 & 1.29 & -8.87 & -826.15 & -21.10\end{array}$

$\begin{array}{llllll}-2.73 & 0.00 & -9.24 & -842.42 & -29.29\end{array}$

$\begin{array}{lllll}-1.83 & 1.28 & -9.62 & -855.47 & -34.27\end{array}$

$\begin{array}{lllll}-1.15 & 0.00-10.00 & -870.89 & -41.63\end{array}$

$\begin{array}{lllll}-0.47 & 1.26 & -9.62 & -882.62 & -45.28\end{array}$

$\begin{array}{llllll}0.12 & 0.00 & -9.26 & -896.59 & -51.18\end{array}$

$\begin{array}{llllll}0.78 & 1.25 & -8.93 & -907.62 & -54.14\end{array}$

$\begin{array}{llllll}1.18 & 0.00 & -8.61 & -921.05 & -59.50\end{array}$

$\begin{array}{lllll}1.55 & 1.23 & -8.30 & -931.68 & -62.05\end{array}$

$\begin{array}{llllll}1.89 & 0.00 & -8.02 & -944.47 & -66.77\end{array}$

$\begin{array}{llllll}2.07 & 1.22 & -7.74 & -954.63 & -68.86\end{array}$

$\begin{array}{llllll}2.38 & 0.00 & -7.49 & -966.77 & -72.93\end{array}$

$\begin{array}{llllll}2.56 & 1.21 & -7.24 & -976.30 & -74.39\end{array}$

$\begin{array}{llllll}2.69 & 0.00 & -7.01 & -987.98 & -78.00\end{array}$

$\begin{array}{lllllll}2.75 & 1.19 & -6.78 & -997.03 & -78.98 & -78.74\end{array}$

$2.750 .00-6.57-1008.22 \quad-82.10-81.81$

$\begin{array}{llllll}2.70 & 1.18 & -6.37-1016.80 & -82.60 & -82.49\end{array}$

$\begin{array}{llllll}2.59 & 0.00 & -6.18-1027.51 & -85.25 & -84.99\end{array}$

$\begin{array}{llllll}2.42 & 1.17 & -5.99-1035.66 & -85.32 & -85.25\end{array}$
0.50

0.67

0.68

$-4.42-0.55$

$0.00 \quad 0.00$

0.00

$0.00 \quad 0.00 \quad 0.00$

$\begin{array}{lll}0.00 & 0.00 & 0.00\end{array}$

$\begin{array}{lll}0.00 & 0.00 & 0.00\end{array}$

$\begin{array}{llll}0.00 & 0.00 & 0.00\end{array}$

$\begin{array}{lll}0.00 & 0.00 & 0.00\end{array}$

$\begin{array}{llll}0.00 & 0.00 & 0.00\end{array}$

$\begin{array}{llll}0.00 & 0.00 & 0.00\end{array}$

$\begin{array}{llll}0.00 & 0.00 & 0.00\end{array}$

$\begin{array}{llll}0.00 & 0.00 & 0.00\end{array}$

$\begin{array}{lll}0.00 & 0.00 & 0.00\end{array}$

$\begin{array}{llll}0.00 & 0.00 & 0.00\end{array}$

$\begin{array}{llll}0.00 & 0.00 & 0.00\end{array}$

$\begin{array}{llll}0.00 & 0.00 & 0.00\end{array}$

$\begin{array}{llll}0.00 & 0.00 & 0.00\end{array}$

$\begin{array}{llll}0.00 & 0.00 & 0.00\end{array}$

$\begin{array}{llll}0.00 & 0.00 & 0.00\end{array}$

$\begin{array}{llll}0.00 & 0.00 & 0.00\end{array}$

$\begin{array}{llll}0.00 & 0.00 & 0.00\end{array}$

$\begin{array}{llll}0.00 & 0.00 & 0.00\end{array}$

$\begin{array}{llll}0.00 & 0.00 & 0.00\end{array}$

$\begin{array}{llll}0.00 & 0.00 & 0.00\end{array}$

$\begin{array}{llll}0.00 & 0.00 & 0.00\end{array}$

$\begin{array}{llll}0.00 & 0.00 & 0.00\end{array}$

$\begin{array}{llll}0.00 & 0.00 & 0.00\end{array}$

$\begin{array}{llll}0.00 & 0.00 & 0.00\end{array}$

$0.00 \quad 0.00 \quad 0.00$

$\begin{array}{llll}0.00 & 0.00 & 0.00\end{array}$

$\begin{array}{lll}0.00 & 0.00 & 0.00\end{array}$

$\begin{array}{llll}0.00 & 0.00 & 0.00\end{array}$

$\begin{array}{llll}0.00 & 0.00 & 0.00\end{array}$

$\begin{array}{lll}0.00 & 0.00 & 0.00\end{array}$

$\begin{array}{lll}0.00 & 0.00 & 0.00\end{array}$

$0.00 \quad 0.00$

$0.00 \quad 0.00 \quad 0.00$

$0.00 \quad 0.00 \quad 0.00$

$\begin{array}{llll}0.00 & 0.00 & 0.00\end{array}$

$\begin{array}{llll}0.00 & 0.00 & 0.00\end{array}$

$\begin{array}{lll}0.00 & 0.00 & 0.00\end{array}$

$\begin{array}{lll}0.00 & 0.00 & 0.00\end{array}$

$0.00 \quad 0.00$

$\begin{array}{lll}0.00 & 0.00 & 0.00\end{array}$

$\begin{array}{lll}0.00 & 0.00 & 0.00\end{array}$

$\begin{array}{llll}0.00 & 0.00 & 0.00\end{array}$

$\begin{array}{llll}-59.92 & 0.76 & -0.42\end{array}$

$\begin{array}{lll}0.00 & 0.00 & 0.00\end{array}$

$\begin{array}{lll}0.00 & 0.00 & 0.00\end{array}$

$\begin{array}{llll}0.00 & 0.00 & 0.00\end{array}$

$\begin{array}{llll}0.00 & 0.00 & 0.00\end{array}$

$0.00 \quad 0.00$

$\begin{array}{lll}2.99 & 0.24\end{array}$

$3.04 \quad 0.29$

$2.81 \quad 0.11$

$2.85 \quad 0.26$

$\begin{array}{ll}2.49 & 0.07\end{array}$ 
$\begin{array}{llllll}54 & 70 & 124 & -5.4 & -8.9-1042.30\end{array}$

$\begin{array}{lllll}54 & 71 & 125 & -5.8 & -8.6-1051.07\end{array}$

$\begin{array}{llllll}54 & 72 & 126 & -6.1 & -8.4-1059.57\end{array}$

$\begin{array}{llllll}54 & 73 & 127 & -6.5 & -8.1-1067.81\end{array}$

$\begin{array}{llllll}54 & 74 & 128 & -6.9 & -7.9-1075.79\end{array}$

$\begin{array}{llllll}54 & 75 & 129 & -7.2 & -7.6-1083.52\end{array}$

$\begin{array}{llllll}54 & 76 & 130 & -7.6 & -7.4-1091.01\end{array}$

$\begin{array}{llllll}54 & 77 & 131 & -7.9 & -7.1-1098.26\end{array}$

$\begin{array}{llllll}54 & 78 & 132 & -8.3 & -6.9-1105.27\end{array}$

$\begin{array}{llllll}54 & 79 & 133 & -8.6 & -6.7-1112.05\end{array}$

$\begin{array}{llllll}54 & 80 & 134 & -8.9 & -6.4-1118.61\end{array}$

$\begin{array}{llllll}54 & 81 & 135 & -9.3 & -6.2-1124.95\end{array}$

$\begin{array}{llllll}54 & 82 & 136 & -9.6 & -6.0-1131.08\end{array}$

$\begin{array}{llllll}54 & 83 & 137 & -9.9 & -5.8-1136.99\end{array}$

$5484 \quad 138-10.3-5.6-1142.71$

$5485 \quad 139-10.6-5.4-1148.22$

$5486 \quad 140-10.9-5.2-1153.54$

$5487141-11.2-5.0-1158.66$

$5488 \quad 142-11.5 \quad-4.8-1163.60$

$5489 \quad 143-11.8-4.7-1168.35$

$5490 \quad 144-12.1-4.5-1172.93$

$5491 \quad 145-12.4 \quad-4.3-1177.33$

$5492 \quad 146-12.7-4.1-1181.56$

$5493 \quad 147-13.0-4.0-1185.62$

$5494 \quad 148-13.3-3.8-1189.52$

$5495 \quad 149-13.6-3.7-1193.25$

$5496 \quad 150-13.9-3.5-1196.83$

$5497 \quad 151-14.2-3.3-1200.26$

$\begin{array}{llll}54 & 98 & 152-14.4 & -3.2-1203.54\end{array}$

$\begin{array}{lllll}54 & 99 & 153-14.7 & -3.1-1206.66\end{array}$

$54100154-15.0-2.9-1209.65$

$54101 \quad 155-15.2-2.8-1212.49$

$\begin{array}{llll}54 & 102 & 156-15.5 & -2.6-1215.20\end{array}$

$54 \quad 103 \quad 157-15.8-2.5-1217.77$

$54104158-16.0-2.4-1220.20$

$54105 \quad 159-16.3-2.2-1222.51$

$54106 \quad 160-16.5 \quad-2.1-1224.69$

$54107 \quad 161-16.8-2.0-1226.75$

$54108 \quad 162-17.0-1.9-1228.69$

$54109 \quad 163-17.2-1.8-1230.51$

$54110164-17.5-1.6-1232.21$

$54111 \quad 165-17.7-1.5-1233.80$

$54112166-17.9-1.4-1235.28$

$54113167-18.2-1.3-1236.65$

$54114168-18.4-1.2-1237.91$

$\begin{array}{llll}54 & 115 & 169-18.6 & -1.1-1239.07\end{array}$

$54116170-18.8-1.0-1240.13$

$54117171-19.0-0.9-1241.09$

$54118 \quad 172-19.2-0.8-1241.95$

$\begin{array}{llll}54 & 119 & 173-19.5 & -0.7-1242.72\end{array}$

$\begin{array}{lllll}54 & 120 & 174-19.7 & -0.6-1243.39\end{array}$

$54121 \quad 175-19.9-0.5-1243.98$

$54122176-20.0-0.5-1244.48$

$\begin{array}{llll}54 & 123 & 177-20.2 & -0.4-1244.89\end{array}$

$54 \quad 124 \quad 178-20.4 \quad-0.3-1245.22$

$54125 \quad 179-20.6-0.2-1245.46$

$54126180-20.8-0.1-1245.63$

$\begin{array}{lll}54 & 127 & 181\end{array}$

$\begin{array}{lll}54 & 128 & 182\end{array}$

$\begin{array}{lll}55 & 51 & 106\end{array}$

$\begin{array}{lll}55 & 52 & 107\end{array}$

$\begin{array}{lll}55 & 53 & 108\end{array}$

$\begin{array}{lll}55 & 54 & 109\end{array}$

$\begin{array}{lll}55 & 55 & 110\end{array}$

$55 \quad 56111$

$\begin{array}{lll}55 & 57 & 112\end{array}$ $0.0 \quad 0.0 \quad 0.00$

$2.9-15.9-812.53$

$2.5-15.5-828.23$

$2.1-15.1-843.51$

$1.7-14.7-858.38$

$1.2-14.3-872.85$

$0.8-13.9-886.93$

$0.4-13.5-900.63$
$2.34 \quad 0.00 \quad-5.82-1045.78-87.37 \quad-87.66$ $2.061 .16-5.65-1053.50-87.02-87.19$ $\begin{array}{llllll}1.86 & 0.00 & -5.49-1063.20 & -88.65 & -89.17\end{array}$ $\begin{array}{lllllll}1.51 & 1.15 & -5.33-1070.49 & -87.86 & -88.32\end{array}$ $\begin{array}{lllllll}1.01 & 0.00 & -5.19-1079.97 & -89.27 & -89.86\end{array}$ $\begin{array}{llllll}0.48 & 1.14 & -5.05-1086.95 & -88.19 & -88.70\end{array}$ $\begin{array}{llll}-0.31 & 0.00 & -4.91-1096.23 & -89.39\end{array}$ $-1.111 .13-4.78-1103.02-88.11$ $-2.360 .00-4.66-1112.29$ $-3.591 .12-4.54-1119.06$ $-4.920 .00-4.43-1127.96$ $-6.101 .11$

$-7.200 .00-4.21-1142.49$ $-6.621 .10-4.11-1146.62$

$-5.350 .00-4.01-1152.07$

$-4.021 .09-3.92-1155.07$

$-3.010 .00-3.83-1160.37$

$-89.31$

$-88.01$

$-2.371 .08-3.74-1163.69$

$\begin{array}{llll}-1.76 & 0.00 & -3.66-1169.02\end{array}$

$-1.231 .08-3.58-1172.09$

$-0.620 .00-3.50-1177.05$

$\begin{array}{llllll}18 & 1.07 & -3.42-1179.87 & -51.96\end{array}$

$0.02 \quad 0.00-3.35-1184.89-48.91$

$0.091 .06-3.28-1187.75-43.70$

$\begin{array}{llllll}0.44 & 0.00 & -3.21-1192.29 & -40.17\end{array}$

$0.551 .05-3.15-1194.80-34.61$

$0.88 \quad 0.00-3.09-1199.04-30.77$

$0.841 .04-3.02-1201.40-25.06$

$1.090 .00-2.96-1205.41-21.00$

$0.92 \quad 1.04-2.91-1207.61-15.13$

$\begin{array}{lllll}1.05 & 0.00 & -2.85-1211.45 & -10.90\end{array}$

$0.741 .03-2.80-1213.52$

$0.880 .00-2.75-1217.06$

$0.701 .02-2.70-1218.74$

$1.07 \quad 0.00-2.65-1221.78$

$1.06 .1 .02-2.60-1223.04$

$1.370 .00-2.55-1225.88$

$1.261 .01-2.51-1226.99$

$1.44 \quad 0.00-2.47-1229.72$

$1.221 .00-2.42-1230.71$

$\begin{array}{llll}1.29 & 0.00 & -2.38-1233.30\end{array}$

$1.011 .00-2.34-1234.14$

$0.960 .00-2.31-1236.62$

$0.58 \quad 0.99-2.27-1237.34$

$0.560 .00-2.23-1239.58$

$\begin{array}{lll}0.02 & 0.99 & -2.20-1240.30\end{array}$

$-0.380 .00-2.16-1242.67$

$-0.90 \quad 0.98-2.13-1243.13$

$-1.310 .00-2.10-1245.36$

$-2.090 .98-2.06-1245.90108 .01$

$\begin{array}{lllll}-2.49 & 0.00 & -2.03-1247.92 & 114.07\end{array}$

$\begin{array}{lllll}-3.42 & 0.97 & -2.00-1248.43 & 121.62\end{array}$

$\begin{array}{llllll}-4.24 & 0.00 & -1.97-1250.69 & 127.43\end{array}$

$\begin{array}{lllll}-5.64 & 0.97 & -1.95-1251.51 & 134.69\end{array}$

$\begin{array}{lllll}-6.49 & 0.00 & -1.92-1253.62 & 140.64\end{array}$

$\begin{array}{lllll}-8.19 & 0.96 & -1.89-1254.58 & 147.76\end{array}$

$\begin{array}{lllll}-8.73 & 0.00 & -1.86-1256.22 & 154.19\end{array}$

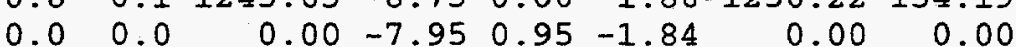

$-6.62$

0.00

0.00

$-821.11$

$-8.78$ $\begin{array}{lllll}-1.80 & 1.26 & -8.89 & -837.66 & -17.25\end{array}$

$\begin{array}{llllll}-1.10 & 2.25 & -9.25 & -851.61 & -23.14\end{array}$

$\begin{array}{lllll}-0.51 & 1.26 & -9.62 & -867.25 & -30.70\end{array}$

$-0.272 .51-10.00-880.61-36.00$ $\begin{array}{llllll}0.16 & 1.26 & -9.63 & -895.14 & -42.45\end{array}$ $\begin{array}{llllll}0.84 & 2.23 & -9.28 & -906.84 & -46.08\end{array}$
$2.05-0.29$

$1.89-0.17$

$1.34-0.52$

$1.05-0.46$

$0.42-0.59$

$-0.03-0.51$

$-0.80-0.49$

$-88.43-1.43-0.32$

$\begin{array}{lll}-89.29 & -2.34 & 0.02\end{array}$

$\begin{array}{lll}-87.66 & -3.24 & 0.35\end{array}$

$\begin{array}{lll}-88.13 & -4.22 & 0.70\end{array}$

$\begin{array}{llll}-86.50 & -5.54 & 0.56\end{array}$

$\begin{array}{llll}-86.43 & -6.41 & 0.79\end{array}$

$\begin{array}{llll}-82.38 & -5.72 & 0.90\end{array}$

$\begin{array}{llll}-80.11 & -4.80 & 0.55\end{array}$

$-75.69-4.13-0.11$

$-72.99-3.18-0.17$

$-68.32-2.63-0.26$

$-65.50-1.94-0.18$

$\begin{array}{llll}0.00 & 0.00 & 0.00\end{array}$

$0.00 \quad 0.00 \quad 0.00$

$0.00 \quad 0.00 \quad 0.00$

$\begin{array}{llll}0.00 & 0.00 & 0.00\end{array}$

$\begin{array}{llll}0.00 & 0.00 & 0.00\end{array}$

$0.00 \quad 0.00 \quad 0.00$

$\begin{array}{llll}0.00 & 0.00 & 0.00\end{array}$

$0.00 \quad 0.00 \quad 0.00$

$0.00 \quad 0.00 \quad 0.00$

$0.00 \quad 0.00 \quad 0.00$

$0.00 \quad 0.00 \quad 0.00$

$\begin{array}{llll}0.00 & 0.00 & 0.00\end{array}$

$\begin{array}{llll}0.00 & 0.00 & 0.00\end{array}$

$\begin{array}{llll}0.00 & 0.00 & 0.00\end{array}$

$0.00 \quad 0.00 \quad 0.00$

$0.00 \quad 0.00 \quad 0.00$

$0.00 \quad 0.00 \quad 0.00$

$\begin{array}{llll}0.00 & 0.00 & 0.00\end{array}$

$0.00 \quad 0.00 \quad 0.00$

$0.00 \quad 0.00 \quad 0.00$

$0.00 \quad 0.00 \quad 0.00$

$0.00 \quad 0.00 \quad 0.00$

$0.00 \quad 0.00 \quad 0.00$

$0.00 \quad 0.00 \quad 0.00$

$\begin{array}{llll}0.00 & 0.00 & 0.00\end{array}$

$\begin{array}{llll}0.00 & 0.00 & 0.00\end{array}$

$0.00 \quad 0.00 \quad 0.00$

$\begin{array}{llll}0.00 & 0.00 & 0.00\end{array}$

$\begin{array}{llll}0.00 & 0.00 & 0.00\end{array}$

$0.00 \quad 0.00 \quad 0.00$

$0.00 \quad 0.00 \quad 0.00$

$0.00 \quad 0.00 \quad 0.00$

$0.00 \quad 0.00 \quad 0.00$

$\begin{array}{llll}0.00 & 0.00 & 0.00\end{array}$

$0.00 \quad 0.00 \quad 0.00$

$0.00 \quad 0.00 \quad 0.00$

$0.00 \quad 0.00 \quad 0.00$

$0.00 \quad 0.00 \quad 0.00$

$0.00 \quad 0.00 \quad 0.00$

$\begin{array}{llll}0.00 & 0.00 & 0.00\end{array}$

$0.00 \quad 0.00 \quad 0.00$

$0.00 \quad 0.00 \quad 0.00$

$\begin{array}{lll}0.00 & 0.00 & 0.00\end{array}$

$0.00 \quad 0.00 \quad 0.00$

$0.00 \quad 0.00 \quad 0.00$

$0.00 \quad 0.00 \quad 0.00$

$0.00 \quad 0.00 \quad 0.00$ 
$55 \quad 102 \quad 157-14.9-2.9-1230.39$

$\begin{array}{llll}55 & 103 & 158-15.1 & -2.8-1233.22\end{array}$ $55 \quad 104 \quad 159-15.4 \quad-2.6-1235.92$ $55105 \quad 160-15.7 \quad-2.5-1238.48$ $55106 \quad 161-15.9-2.4-1240.91$ $55107 \quad 162-16.2-2.2-1243.21$ $55108 \quad 163-16.4 \quad-2.1-1245.39$ $55109164-16.6-2.0-1247.45$ $55110165-16.9-1.9-1249.39$ $55111 \quad 166-17.1-1.8-1251.22$ $\begin{array}{llll}55 & 112 & 167-17.3 & -1.6-1252.92\end{array}$ $55113 \quad 168-17.6-1.5-1254.52$ $55114 \quad 169-17.8-1.4-1256.01$ $55115 \quad 170-18.0-1.3-1257.39$ $55116 \quad 171-18.2-1.2-1258.67$ $\begin{array}{llll}55 & 117 & 172-18.5 & -1.1-1259.84\end{array}$ $55 \quad 118 \quad 173-18.7-1.0-1260.91$ $55119 \quad 174-18.9-0.9-1261.89$ $\begin{array}{llll}55 & 120 & 175-19.1 & -0.8-1262.77\end{array}$ $\begin{array}{llll}55 & 121 & 176-19.3 & -0.7-1263.55\end{array}$ $55 \quad 122 \quad 177-19.5-0.6-1264.25$ $\begin{array}{lllll}55 & 123 & 178-19.7 & -0.6-1264.86\end{array}$

$$
2
$$

\section{(1)}$$
2
$$

$2.56 \quad 1.26-6.42-1030.84-81.29-81.06$

$2.36 \quad 2.17-6.22-1039.65-82.03-81.73$

$2.451 .26-6.04-1049.70-84.01-84.11$

$2.202 .16-5.87-1058.02-84.26-84.35$

$2.051 .26-5.70-1067.77-85.94-86.25$

$\begin{array}{llllll}1.76 & 2.15 & -5.54-1075.62 & -85.71 & -85.93\end{array}$

$\begin{array}{llllll}1.38 & 1.26 & -5.39-1085.08 & -87.10 & -87.51\end{array}$

$\begin{array}{llllll}0.89 & 2.14 & -5.24-1092.63 & -86.58 & -86.85\end{array}$

$\begin{array}{llllll}0.37 & 1.26 & -5.10-1101.73 & -87.61 & -88.07\end{array}$ (1)
$2-1$
$4-2$

$-3$ $7-6$

$1-1$

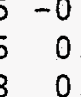$$
\text { 0.8. }
$$

$$
0.98
$$

\subsection{8}

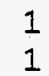

. 
$\begin{array}{llllllllllll}55 & 124 & 179 & -19.9 & -0.5-1265.37 & -5.97 & 1.26 & -1.98-1272.06 & 129.48\end{array}$ $\begin{array}{lllllllll}55 & 125 & 180-20.1 & -0.4-1265.81 & -7.59 & 2.02 & -1.95-1273.34 & 136.28\end{array}$ $\begin{array}{lllllllll}55 & 126 & 181-20.3 & -0.3-1266.16 & -8.24 & 1.26 & -1.93-1275.05 & 142.63\end{array}$ $\begin{array}{lllllllll}55 & 127 & 182-20.4 & -0.2-1266.43 & -7.40 & 2.01 & -1.90-1273.72 & 152.04\end{array}$ $\begin{array}{lllllllll}55 & 128 & 183-20.6 & -0.2-1266.62 & -6.22 & 1.26 & -1.87-1273.45 & 160.38\end{array}$ $\begin{array}{lllllllll}55 & 129 & 184-20.8 & -0.1-1266.74 & -5.24 & 2.01 & -1.85-1271.82 & 170.09\end{array}$

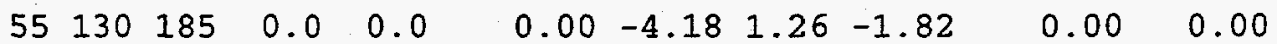
$\begin{array}{lllllllllll}56 & 52 & 108 & 3.1-15.9 & -825.44 & -1.04 & 0.00 & -8.56 & -835.04 & -7.36\end{array}$ $\begin{array}{lllllllllll}56 & 53 & 109 & 2.7 & -15.5 & -841.15 & -0.38 & 1.28 & -8.91 & -849.16 & -13.40\end{array}$ $\begin{array}{lllllllllll}56 & 54 & 110 & 2.2 & -15.1 & -856.44 & -0.14 & 0.00 & -9.26 & -865.84 & -22.02\end{array}$

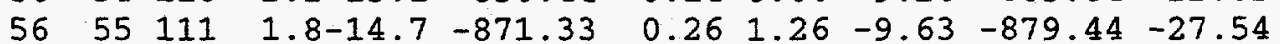

$56 \quad 56 \quad 112 \quad 1.4-14.3-885.83$

$\begin{array}{llllll}56 & 57 & 113 & 1.0-13.9 & -899.94\end{array}$

$\begin{array}{llllll}56 & 58 & 114 & 0.6-13.5 & -913.67\end{array}$

$\begin{array}{lllll}56 & 59 & 115 & 0.2-13.2 & -927.03\end{array}$

$\begin{array}{llllll}56 & 60 & 116 & -0.2 & -12.8 & -940.03\end{array}$

$\begin{array}{llllll}56 & 61 & 117 & -0.6-12.5 & -952.68\end{array}$

$\begin{array}{lllll}56 & 62 & 118 & -1.0-12.1 & -964.98\end{array}$

$\begin{array}{llllll}56 & 63 & 119 & -1.4-11.8 & -976.94\end{array}$

$\begin{array}{llllll}56 & 64 & 120 & -1.8-11.5 & -988.57\end{array}$

$\begin{array}{llllll}56 & 65 & 121 & -2.2-11.1 & -999.88\end{array}$

$\begin{array}{llll}56 & 66 & 122 & -2.6-10.8-1010.87\end{array}$

$\begin{array}{llll}56 & 67 & 123 & -3.0-10.5-1021.56\end{array}$

$\begin{array}{llll}56 & 68 & 124 & -3.4-10.2-1031.94\end{array}$

$\begin{array}{llllll}56 & 69 & 125 & -3.8 & -9.9-1042.02\end{array}$

$\begin{array}{llllll}56 & 70 & 126 & -4.1 & -9.7-1051.82\end{array}$

$\begin{array}{llllll}56 & 71 & 127 & -4.5 & -9.4-1061.33\end{array}$

$\begin{array}{llllll}56 & 72 & 128 & -4.9 & -9.1-1070.57\end{array}$

$\begin{array}{llllll}56 & 73 & 129 & -5.2 & -8.8-1079.54\end{array}$

$\begin{array}{llllll}56 & 74 & 130 & -5.6 & -8.6-1088.24\end{array}$

$\begin{array}{llllll}56 & 75 & 131 & -6.0 & -8.3-1096.69\end{array}$

$\begin{array}{llllll}56 & 76 & 132 & -6.3 & -8.1-1104.88\end{array}$

$\begin{array}{llllll}56 & 77 & 133 & -6.7 & -7.8-1112.83\end{array}$

$\begin{array}{llllll}56 & 78 & 134 & -7.0 & -7.6-1120.53\end{array}$

$\begin{array}{llllll}56 & 79 & 135 & -7.3 & -7.3-1128.00\end{array}$

$\begin{array}{llllll}56 & 80 & 136 & -7.7 & -7.1-1135.24\end{array}$

$\begin{array}{llllll}56 & 81 & 137 & -8.0 & -6.9-1142.25\end{array}$

$\begin{array}{llllll}56 & 82 & 138 & -8.3 & -6.7-1149.04\end{array}$

$\begin{array}{llllll}56 . & 83 & 139 & -8.7 & -6.5-1155.61\end{array}$

$\begin{array}{llllll}56 & 84 & 140 & -9.0 & -6.3-1161.97\end{array}$

56

56

56

56

56
56

56

56

56

56

56

$5696 \quad 152-12.6-4.1-1223.35$

$\begin{array}{llll}56 & 97 & 153-12.9 & -3.9-1227.34\end{array}$

$5698 \quad 154-13.2 \quad-3.7-1231.17$

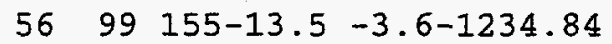

$\begin{array}{lllll}56 & 100 & 156-13.7 & -3.4-1238.36\end{array}$

$\begin{array}{lllll}56 & 101 & 157-14.0 & -3.3-1241.74\end{array}$

$\begin{array}{llll}56 & 102 & 158-14.3 & -3.2-1244.97\end{array}$

$\begin{array}{lllll}56 & 103 & 159-14.5 & -3.0-1248.06\end{array}$

$56 \quad 104 \quad 160-14.8-2.9-1251.02$

$56105161-15.1-2.7-1253.83$

$\begin{array}{llll}56 & 106 & 162-15.3 & -2.6-1256.52\end{array}$

$\begin{array}{llll}56 & 107 & 163-15.6 & -2.5-1259.07\end{array}$

$56 \quad 108 \quad 164-15.8-2.4-1261.50$

$56109165-16.0-2.2-1263.80$

$\begin{array}{llll}56 & 110 & 166-16.3 & -2.1-1265.98\end{array}$ $\begin{array}{llllll}0.93 & 0.00-10.00 & -894.90 & -34.93\end{array}$

$\begin{array}{llllll}1.47 & 1.25 & -9.64 & -906.86 & -38.81\end{array}$

$\begin{array}{llllll}1.65 & 0.00 & -9.29 & -921.31 & -45.20\end{array}$

$\begin{array}{llllll}1.82 & 1.23 & -8.96 & -932.94 & -48.76\end{array}$

$\begin{array}{llllll}1.77 & 0.00 & -8.65 & -946.91 & -54.66\end{array}$

$\begin{array}{llllll}1.97 & 1.22 & -8.36 & -957.85 & -57.52\end{array}$

$\begin{array}{llllll}2.22 & 0.00 & -8.08 & -970.84 & -62.44\end{array}$

$\begin{array}{lllllll}2.41 & 1.21 & -7.81 & -981.14 & -64.67\end{array}$

$\begin{array}{lllllll}2.60 & 0.00 & -7.56 & -993.53 & -68.99\end{array}$

$\begin{array}{llllll}2.66 & 1.19 & -7.32-1003.35 & -70.73\end{array}$

$2.650 .00-7.09-1015.31^{\prime}-74.63$

$2.57 \quad 1.18-6.87-1024.67-75.92$

$2.510 .00-6.66-1036.09-79.26$

$2.351 .17-6.46-1044.96-80.07$

$2.33 \quad 0.00-6.27-1055.76-82.79$

$2.181 .16-6.09-1064.08-83.04$

$2.20 \quad 0.00 \quad-5.92-1074.29-85.17$

$1.951 .15-5.75-1082.19-85.01$

$\begin{array}{llllll}1.76 & 0.00 & -5.59-1092.07 & -86.82\end{array}$

$1.36 \quad 1.14-5.44-1099.63-86.30$

$\begin{array}{llllll}0.93 & 0.00 & -5.29-1109.24 & -87.85\end{array}$

$0.381 .13-5.15-1116.47-87.00$

$-0.550 .00-5.02-1126.10-88.56$

$\begin{array}{lllll}-1.45 & 1.12 & -4.89-1133.22 & -87.61\end{array}$

$\begin{array}{llllll}-3.01 & 0.00 & -4.77-1143.01 & -89.33\end{array}$

$\begin{array}{lllll}-4.18 & 1.11 & -4.65-1149.97 & -88.21\end{array}$

$\begin{array}{lllll}-5.29 & 0.00 & -4.53-1158.86 & -89.03\end{array}$

$\begin{array}{lllll}-4.66 & 1.10 & -4.42-1163.59 & -85.70\end{array}$

$\begin{array}{lllll}-3.53 & 0.00 & -4.32-1169.82 & -83.85\end{array}$

$\begin{array}{lllll}-2.16 & 1.09 & -4.22-1173.41 & -79.37\end{array}$

$\begin{array}{llllll}-1.60 & 0.00 & -4.12-1179.80 & -77.68\end{array}$

$-1.331 .08-4.02-1184.10-73.92$

$\begin{array}{lllll}-0.94 & 0.00 & -3.93-1190.27 & -72.01\end{array}$

$-0.571 .08-3.84-1194.10-67.78$

$-0.020 .00-3.76-1199.73-65.33$

$0.201 .07-3.68-1203.36-60.89$

$\begin{array}{lllll}0.35 & 0.00 & -3.60-1209.02 & -58.48\end{array}$

$0.271 .06-3.52-1212.61-54.00$

$\begin{array}{llllll}0.58 & 0.00 & -3.45-1217.77 & -51.08\end{array}$

$0.531 .05-3.38-1221.00-46.25$

$0.870 .00-3.31-1225.79-42.96$

$0.76 \quad 1.04-3.24-1228.78-37.88$

$\begin{array}{llllll}0.96 & 0.00 & -3.18-1233.39 & -34.42\end{array}$

$0.77 \quad 1.04-3.12-1236.15-29.11$

$0.870 .00-3.06-1240.55-25.44$

$0.51 \quad 1.03-3.00-1243.20-20.02$

$\begin{array}{llllll}0.58 & 0.00 & -2.94-1247.34 & -16.08\end{array}$

$\begin{array}{lllll}0.46 & 1.02 & -2.89-1249.47 & -10.14\end{array}$

$0.820 .00-2.84-1253.03$

$0.861 .02-2.79-1254.74$

$1.160 .00-2.74-1258.09$

$1.121 .01-2.69-1259.63$

$1.37 \quad 0.00-2.64-1262.77$

$1.191 .00-2.59-1264.20$

$1.380 .00-2.55-1267.15$ 45 \begin{tabular}{lll}
0.00 & 0.00 & 0.00 \\
0.00 & 0.00 & 0.00 \\
0.00 & 0.00 & 0.00 \\
0.00 & 0.00 & 0.00 \\
0.00 & 0.00 & 0.00 \\
0.00 & 0.00 & 0.00 \\
0.00 & 0.00 & 0.00 \\
0.00 & 0.00 & 0.00 \\
0.00 & 0.00 & 0.00 \\
0.00 & 0.00 & 0.00 \\
0.00 & 0.00 & 0.00 \\
0.00 & 0.00 & 0.00 \\
0.00 & 0.00 & 0.00 \\
0.00 & 0.00 & 0.00 \\
0.00 & 0.00 & 0.00 \\
0.00 & 0.00 & 0.00 \\
0.00 & 0.00 & 0.00 \\
0.00 & 0.00 & 0.00 \\
0.00 & 0.00 & 0.00 \\
0.00 & 0.00 & 0.00 \\
0.00 & 0.00 & 0.00 \\
0.00 & 0.00 & 0.00 \\
0.00 & 0.00 & 0.00 \\
0.00 & 0.00 & 0.00 \\
\hline 9.55 & 2.87 & 0.52 \\
0.00 & 0.00 & 0.00 \\
0.80 & 2.42 & 0.24
\end{tabular}

$\begin{array}{lll}-82.80 & 2.42 & 0.24\end{array}$

$\begin{array}{llll}-85.47 & 1.90 & -0.30\end{array}$

$\begin{array}{llll}-85.08 & 1.88 & -0.07\end{array}$

$\begin{array}{llll}-87.29 & 1.29 & -0.47\end{array}$

$\begin{array}{llll}-86.71 & 0.95 & -0.41\end{array}$

$\begin{array}{llll}-88.45 & 0.33-0.60\end{array}$

$-87.57-0.19-0.57$

$-88.96-0.95-0.40$

$-87.87-1.71-0.26$

$\begin{array}{lll}-88.90 & -2.58 & 0.43\end{array}$

$\begin{array}{llll}-87.73 & -3.70 & 0.48\end{array}$

$\begin{array}{lll}-88.27 & -4.53 & 0.76\end{array}$

$\begin{array}{llll}-84.92 & -3.88 & 0.78\end{array}$

$-83.27-2.95 \cdot 0.58$

$\begin{array}{llll}-79.73 & -2.52 & -0.36\end{array}$

$-77.85-1.77-0.17$

$\begin{array}{llll}-73.98 & -1.39 & -0.06\end{array}$

$\begin{array}{llll}-71.84 & -0.77 & 0.17\end{array}$

$-68.13-0.92-0.35$

$\begin{array}{lll}-65.08 & 0.23 & 0.25\end{array}$

$-61.49-0.40-0.60$

$0.00 \quad 0.00 \quad 0.00$

$0.00 \quad 0.00 \quad 0.00$

$0.00 \quad 0.00 \quad 0.00$

$0.00 \quad 0.00 \quad 0.00$

$0.00 \quad 0.00 \quad 0.00$

$0.00 \quad 0.00 \quad 0.00$

$0.00 \quad 0.00 \quad 0.00$

$\begin{array}{llll}0.00 & 0.00 & 0.00\end{array}$

$0.00 \quad 0.00 \quad 0.00$

$0.00 \quad 0.00 \quad 0.00$

$\begin{array}{llll}0.00 & 0.00 & 0.00\end{array}$

$\begin{array}{llll}0.00 & 0.00 & 0.00\end{array}$

$0.00 \quad 0.00 \quad 0.00$

0.73

5.45

11.99

16.92

23.56

28.68

0.00

0.00

0.00

$0.00 \quad 0.00 \quad 0.00$

$0.00 \quad 0.00 \quad 0.00$

$0.00 \quad 0.00 \quad 0.00$

$0.00 \quad 0.00 \quad 0.00$

$0.00 \quad 0.00 \quad 0.00$ 
$\begin{array}{llll}56 & 111 & 167-16.5 & -2.0-1268.04\end{array}$ $56 \quad 112168-16.8-1.9-1269.98$ $56 \quad 113 \quad 169-17.0-1.8-1271.81$ $\begin{array}{llll}56 & 114 & 170-17.2 & -1.7-1273.52\end{array}$ $56 \quad 115 \quad 171-17.4-1.5-1275.13$

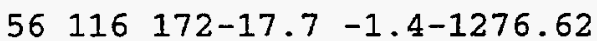
$\begin{array}{llll}56 & 117 & 173-17.9 & -1.3-1278.01\end{array}$ $56118174-18.1-1.2-1279.30$ $\begin{array}{llll}56 & 119 & 175-18.3 & -1.1-1280.49\end{array}$ $56 \quad 120176-18.5-1.0-1281.58$ $\begin{array}{llll}56 & 121 & 177-18.7 & -0.9-1282.57\end{array}$ $\begin{array}{lllll}56 & 122 & 178-18.9 & -0.8-1283.47\end{array}$ $\begin{array}{llll}56 & 123 & 179-19.1 & -0.8-1284.27\end{array}$ $\begin{array}{lllll}56 & 124 & 180-19.3 & -0.7-1284.99\end{array}$ $\begin{array}{llll}56 & 125 & 181-19.5 & -0.6-1285.61\end{array}$ $\begin{array}{lllll}56 & 126 & 182-19.7 & -0.5-1286.15\end{array}$ $\begin{array}{lllll}56 & 127 & 183-19.9 & -0.4-1286.61\end{array}$ $\begin{array}{lllll}56 & 128 & 184-20.1 & -0.3-1286.98\end{array}$ $\begin{array}{llll}56 & 129 & 185-20.3 & -0.3-1287.28\end{array}$ $\begin{array}{lllll}56 & 130 & 186-20.5 & -0.2-1287.50\end{array}$ $\begin{array}{lllll}56 & 131 & 187-20.6 & -0.1-1287.64\end{array}$ $\begin{array}{rrrrrr}56 & 132 & 188 & 0.0 & 0.0 & 0.00 \\ 56 & 133 & 189 & 0.0 & 0.0 & 0.00\end{array}$

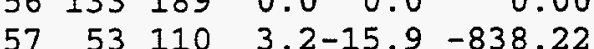
$\begin{array}{lllll}57 & 54 & 111 & 2.8-15.5 & -853.93\end{array}$ $\begin{array}{llllll}57 & 55 & 112 & 2.4-15.1 & -869.24\end{array}$ $\begin{array}{llllll}57 & 56 & 113 & 2.0 & -14.7 & -884.15\end{array}$ $\begin{array}{llllll}57 & 57 & 114 & 1.6-14.3 & -898.67\end{array}$ $\begin{array}{llllll}57 & 58 & 115 & 1.2-14.0 & -912.81\end{array}$ $\begin{array}{llllll}57 & 59 & 116 & 0.7-13.6 & -926.58\end{array}$ $\begin{array}{llllll}57 & 60 & 117 & 0.3-13.2 & -939.98\end{array}$ $\begin{array}{lllll}57 & 61 & 118 & -0.1-12.9 & -953.03\end{array}$ $\begin{array}{lllll}57 & 62 & 119 & -0.5-12.5 & -965.73\end{array}$ $\begin{array}{lllll}57 & 63 & 120 & -0.8-12.2 & -978.09\end{array}$ $\begin{array}{llllll}57 & 64 & 121 & -1.2 & -11.9 & -990.11\end{array}$ $57.65122-1.6-11.5-1001.81$ $57 \quad 66 \quad 123-2.0-11.2-1013.19$ $\begin{array}{llll}57 & 67 & 124 & -2.4-10.9-1024.26\end{array}$ $57 \quad 68 \quad 125-2.8-10.6-1035.02$ $\begin{array}{llll}57 & 69 & 126 & -3.1-10.3-1045.48\end{array}$ $\begin{array}{lllll}57 & 70 & 127 & -3.5-10.0-1055.65\end{array}$ $\begin{array}{lllll}57 & 71 & 128 & -3.9 & -9.7-1065.53\end{array}$ $\begin{array}{lllll}57 & 72 & 129 & -4.3 & -9.5-1075.14\end{array}$ $\begin{array}{llllll}57 & 73 & 130 & -4.6 & -9.2-1084.47\end{array}$ $\begin{array}{lllll}57 & 74 & 131 & -5.0 & -8.9-1093.53\end{array}$

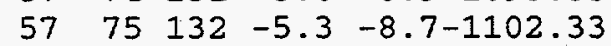
$\begin{array}{llllll}57 & 76 & 133 & -5.7 & -8.4-1110.88\end{array}$

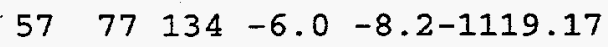
$\begin{array}{llllll}57 & 78 & 135 & -6.4 & -7.9-1127.22\end{array}$

$\begin{array}{lllll}57 & 79 & 136 & -6.7 & -7.7-1135.03\end{array}$ $\begin{array}{llllll}57 & 80 & 137 & -7.1 & -7.5 & -1142.61\end{array}$ $\begin{array}{llllll}57 & 81 & 138 & -7.4 & -7.2-1149.96\end{array}$ $\begin{array}{lllll}57 & 82 & 139 & -7.7 & -7.0-1157.08\end{array}$ $\begin{array}{lllll}83 & 140 & -8.1 & -6.8-1163.98\end{array}$ $84 \quad 141-8.4-6.6-1170.67$ $\begin{array}{lllll}85 & 142 & -8.7 & -6.4-1177.14\end{array}$ $\begin{array}{lllll}86 & 143 & -9.0 & -6.2-1183.41\end{array}$ $\begin{array}{llll}87 & 144 & -9.3 & -6.0-1189.48\end{array}$ $88 \quad 145-9.6-5.8-1195.35$ $89146-10.0-5.6-1201.03$ $90 \quad 147-10.3-5.4-1206.52$ 91 148-10.6 -5.2-1211.82 $92 \quad 149-10.9-5.0-1216.94$ $93150-11.2-4.9-1221.88$ $94151-11.4-4.7-1226.65$ $\begin{array}{llll}57 & 95 & 152-11.7 & -4.5-1231.25\end{array}$
$1.141 .00-2.51-1268.41$ $1.17 \quad 0.00-2.47-1271.27$ $0.72 \quad 0.99-2.43-1272.52$ $0.800 .00-2.39-1275.11$ $0.34 \quad 0.99-2.35-1276.15$ $0.190 .00-2.31-1278.74$ $\begin{array}{lll}-0.59 & 0.98 & -2.27-1279.90\end{array}$ $\begin{array}{llll}-0.72 & 0.00 & -2.24-1282.26\end{array}$ $\begin{array}{lll}-1.55 & 0.98 & -2.20-1283.27\end{array}$ $\begin{array}{llll}-1.47 & 0.00 & -2.17-1285.22\end{array}$ $\begin{array}{llll}-2.52 & 0.97 & -2.14-1286.26\end{array}$

$\begin{array}{llll}-3.07 & 0.00 & -2.11-1288.65 & 104.04\end{array}$ $\begin{array}{lllll}-4.45 & 0.97 & -2.08 & -1289.83 & 110.92\end{array}$ $\begin{array}{lllll}-5.26 & 0.00 & -2.05-1292.29 & 116.53\end{array}$ $\begin{array}{lllll}-6.97 & 0.96 & -2.02-1293.64 & 123.26\end{array}$ $\begin{array}{lllll}-7.39 & 0.00 & -1.99-1295.53 & 129.44\end{array}$ $\begin{array}{lllll}-6.59 & 0.95 & -1.96-1294.20 & 138.84\end{array}$ $\begin{array}{lllll}-5.59 & 0.00 & -1.93-1294.51 & 146.61\end{array}$ $\begin{array}{llllll}-4.57 & 0.95 & -1.91-1292.81 & 156.38\end{array}$ $\begin{array}{llll}-3.37 & 0.00 & -1.88-1292.75 & 164.51\end{array}$ $\begin{array}{llll}-3.32 & 0.95 & -1.86-1291.87 & 173.46\end{array}$ $\begin{array}{lllll}-2.75 & 0.00 & -1.83 & 0.00 & 0.00\end{array}$ $\begin{array}{lllll}3.04 & 0.94 & -1.81 & 0.00 & 0.00\end{array}$ $\begin{array}{lllll}0.06 & 2.24 & -8.58 & -844.50 & -1.47\end{array}$ $\begin{array}{llllll}0.31 & 1.25 & -8.93 & -861.30 & -10.19\end{array}$ $\begin{array}{llllll}0.86 & 2.23 & -9.28 & -875.43 & -16.25\end{array}$ $\begin{array}{llllll}0.94 & 1.25 & -9.64 & -891.59 & -24.34\end{array}$ $\begin{array}{lllll}1.25 & 2.48-10.00 & -904.94 & -29.62\end{array}$ $\begin{array}{llllll}1.43 & 1.25 & -9.64 & -919.77 & -36.38\end{array}$ $\begin{array}{llllll}1.44 & 2.20 & -9.30 & -932.24 & -40.77\end{array}$ $\begin{array}{llllll}1.34 & 1.25 & -8.98 & -946.37 & -46.84\end{array}$ $\begin{array}{llllll}1.54 & 2.19 & -8.67 & -957.97 & -50.37\end{array}$ $\begin{array}{lllll}1.76 & 1.25 & -8.38 & -971.11 & -55.43\end{array}$ $\begin{array}{llllll}2.06 & 2.18 & -8.11 & -981.95 & -58.20\end{array}$ $\begin{array}{llllll}2.28 & 1.25 & -7.84 & -994.43 & -62.61\end{array}$ $\begin{array}{lllll}2.35 & 2.17 & -7.59-1004.88 & -64.99\end{array}$ $2.38 \quad 1.25-7.35-1016.92-68.95$ $2.37 \quad 2.16-7.13-1026.85-70.81$ $2.36 \quad 1.25-6.91-1038.32-74.21$ $2.26 \quad 2.15-6.70-1047.77-75.59$ $2.331 .25-6.51-1058.58-78.33$ $2.24 \quad 2.15-6.32-1067.46-79.14$ $2.341 .25-6.14-1077.69-81.29$ $2.17 \quad 2.14 \quad-5.96-1086.12-81.66$ $2.081 .25-5.80-1096.00-83.47-8$ $1.742 .13-5.64-1104.10-83.49-83$ $\begin{array}{lllll}1.36 & 1.25 & -5.49-1113.76 & -85.08\end{array}$ $0.86 \quad 2.12 \quad-5.34-1121.53-84.78-8$ $0.181 .25-5.20-1131.00-86.18-8$ $\begin{array}{llll}-0.67 & 2.12 & -5.07-1138.66 & -85.76\end{array}$ $\begin{array}{llll}-2.22 & 1.25 & -4.94-1148.52 & -87.56\end{array}$ $\begin{array}{llll}-3.37 & 2.11 & -4.82-1156.03 & -87.00\end{array}$ $\begin{array}{lllll}-4.50 & 1.25 & -4.70-1165.03 & -87.92 & -87\end{array}$ $\begin{array}{lllll}-3.76 & 2.10 & -4.58-1170.22 & -85.04 & -84\end{array}$ $\begin{array}{lllll}-2.73 & 1.25 & -4.47-1176.62 & -83.37\end{array}$ $\begin{array}{lllll}-1.32 & 2.10 & -4.37-1180.73 & -79.41\end{array}$ $\begin{array}{lllll}-0.93 & 1.25 & -4.27-1187.36 & -77.97 & -78\end{array}$ $\begin{array}{lllll}-0.78 & 2.09 & -4.17-1192.34 & -74.87 & -74\end{array}$ $-0.501 .25-4.07-1198.68-73.14-7$ $-0.022 .08-3.98-1202.95-69.34-69$ $0.381 .25-3.90-1208.78-67.11-67$ $\begin{array}{lllll}0.26 & 2.08 & -3.81-1213.29 & -63.54 & -63\end{array}$ $0.321 .25-3.73-1219.10-61.28$ $0.152 .07-3.65-1223.31-57.42$ $0.431 .25-3.57-1228.55-54.58$ $0.372 .07-3.50-1232.31-50.28$ 46 $\begin{array}{lll}0.00 & 0.00 & 0.00 \\ 0.00 & 0.00 & 0.00 \\ 0.00 & 0.00 & 0.00 \\ 0.00 & 0.00 & 0.00 \\ 0.00 & 0.00 & 0.00 \\ 0.00 & 0.00 & 0.00 \\ 0.00 & 0.00 & 0.00 \\ 0.00 & 0.00 & 0.00 \\ 0.00 & 0.00 & 0.00 \\ 0.00 & 0.00 & 0.00 \\ 0.00 & 0.00 & 0.00 \\ 0.00 & 0.00 & 0.00 \\ 0.00 & 0.00 & 0.00 \\ 0.00 & 0.00 & 0.00 \\ 0.00 & 0.00 & 0.00 \\ 0.00 & 0.00 & 0.00 \\ 0.00 & 0.00 & 0.00 \\ 0.00 & 0.00 & 0.00 \\ 0.00 & 0.00 & 0.00 \\ 0.00 & 0.00 & 0.00 \\ 0.00 & 0.00 & 0.00 \\ 0.00 & 0.00 & 0.00 \\ 0.00 & 0.00 & 0.00 \\ 0.00 & 0.00 & 0.00 \\ 0.00 & 0.00 & 0.00 \\ 0.00 & 0.00 & 0.00 \\ 0.00 & 0.00 & 0.00 \\ 0.00 & 0.00 & 0.00 \\ 0.00 & 0.00 & 0.00 \\ 0.00 & 0.00 & 0.00 \\ 0.00 & 0.00 & 0.00 \\ 0.00 & 0.00 & 0.00 \\ 0.00 & 0.00 & 0.00 \\ 0.00 & 0.00 & 0.00 \\ 0.00 & 0.00 & 0.00 \\ 0.00 & 0.00 & 0.00 \\ 0.00 & 0.00 & 0.00 \\ 0.00 & 0.00 & 0.00 \\ 0.00 & 0.00 & 0.00 \\ 0.00 & 0.00 & 0.00 \\ 0.00 & 0.00 & 0.00 \\ 8.82 & 2.56 & 0.32\end{array}$ $\begin{array}{lll}-78.82 & 2.56 & 0.32\end{array}$ $\begin{array}{lll}-81.36 & 2.27 & -0.07\end{array}$ $\begin{array}{lll}0.00 & 0.00 & 0.00\end{array}$ $\begin{array}{llll}-83.75 & 1.80 & -0.28\end{array}$ $-83.74 \quad 1.49-0.25$ $0.00 \cdot 0.00 \quad 0.00$ $\begin{array}{llll}-85.25 & 0.39 & -0.47\end{array}$ $-86.67-0.31-0.49$ $-86.03-0.94-0.27$ $\begin{array}{lll}-87.13 & -1.79 & 0.43\end{array}$ $\begin{array}{lll}-86.53 & -2.90 & 0.47\end{array}$ $\begin{array}{lll}-87.24 & -3.82 & 0.68\end{array}$ $\begin{array}{lll}-84.33 & -3.05 & 0.71\end{array}$ $\begin{array}{lll}-82.98 & -2.34 & 0.39\end{array}$ $\begin{array}{llll}-80.03 & -1.94 & -0.62\end{array}$ $-78.20-1.16-0.23$ $\begin{array}{llll}-74.94 & -0.85 & -0.07\end{array}$ $\begin{array}{lll}-73.03 & -0.39 & 0.11\end{array}$ $\begin{array}{lll}-69.18 & 0.14 & 0.16\end{array}$ $\begin{array}{lll}-67.24 & 0.25 & -0.13\end{array}$ $-63.81-0.01-0.27$ $\begin{array}{lll}0.00 & 0.00 & 0.00\end{array}$ $\begin{array}{lll}0.00 & 0.00 & 0.00\end{array}$ $\begin{array}{lll}0.00 & 0.00 & 0.00\end{array}$ $\begin{array}{llll}0.00 & 0.00 & 0.00\end{array}$ 
$5796 \quad 153-12.0-4.3-1235.68$

$5797 \quad 154-12.3-4.2-1239.95$

$5798 \quad 155-12.6-4.0-1244.06$

$5799 \quad 156-12.9-3.9-1248.01$

$57100 \quad 157-13.1-3.7-1251.80$

$57101158-13.4-3.6-1255.45$

$\begin{array}{lllll}57 & 102 & 159-13.7 & -3.4-1258.95\end{array}$

$\begin{array}{llll}57 & 103 & 160-13.9 & -3.3-1262.30\end{array}$

$57104 \quad 161-14.2-3.1-1265.51$

$57105162-14.5-3.0-1268.59$

$57106 \quad 163-14.7-2.9-1271.52$

$57107164-15.0-2.7-1274.33$

$57108 \quad 165-15.2-2.6-1277.00$

$57109 \quad 166-15.5-2.5-1279.55$

$57 \quad 110 \quad 167-15.7 \quad-2.4-1281.97$

$57111168-15.9-2.2-1284.27$

$57 \quad 112 \quad 169-16.2 \quad-2.1-1286.45$

$57 \quad 113 \quad 170-16.4 \quad-2.0-1288.51$

$57 \quad 114 \quad 171-16.6-1.9-1290.45$

$\begin{array}{lllll}57 & 115 & 172-16.9 & -1.8-1292.28\end{array}$

$57116 \quad 173-17.1-1.7-1294.00$

$57 \quad 117 \quad 174-17.3-1.6-1295.62$

$57118 \quad 175-17.5-1.5-1297.12$

$57 \quad 119 \quad 176-17.7-1.3-1298.52$

$57 \quad 120 \quad 177-18.0-1.2-1299.82$

$57121178-18.2-1.1-1301.03$

$57 \quad 122 \quad 179-18.4-1.1-1302.13$

$57123180-18.6-1.0-1303.14$

$57124181-18.8-0.9-1304.05$

$57125182-19.0-0.8-1304.87$

$57126183-19.2-0.7-1305.61$

$57127 \quad 184-19.4-0.6-1306.26$

$57128 \quad 185-19.6-0.5-1306.82$

$57129186-19.7-0.4-1307.29$

$\begin{array}{llll}57 & 130 & 187-19.9 & -0.4-1307.69\end{array}$

$57131188-20.1-0.3-1308.01$

$57 \quad 132189-20.3-0.2-1308.25$

$57 \quad 133 \quad 190-20.5-0.1-1308.41$

$\begin{array}{llllll}57 & 134 & 191 & 0.0 & 0.0 & 0.00 \\ 57 & 135 & 192 & 0.0 & 0.0 & 0.00\end{array}$

$\begin{array}{lll}58 & 55 & 113\end{array}$

$2.9-15.5-866.58$

$2.5-15.1-881.90$

$\begin{array}{lll}58 & 56 & 114\end{array}$

$58 \quad 57 \quad 115$

$\begin{array}{lll}58 & 58 & 116\end{array}$

$2.1-14.7-896.83$

1. $7-14.4-911.37$

$59 \quad 117$

$1.3-14.0-925.55$

$58 \quad 60 \quad 118$

$0.9-13.6-939.35$

$58 \quad 61119 \quad 0.5-13.3-952.80$

$\begin{array}{llllll}58 & 62 & 120 & 0.1-12.9 & -965.89\end{array}$

$\begin{array}{llllll}58 & 63 & 121 & -0.3-12.6 & -978.64\end{array}$

$\begin{array}{lllll}58 & 64 & 122 & -0.6-12.2 & -991.06\end{array}$

$5865123-1.0-11.9-1003.14$

$58 \quad 66 \quad 124-1.4-11.6-1014.91$

$58.67 \quad 125-1.8-11.3-1026.35$

$\begin{array}{llll}58 & 68 & 126 & -2.2-11.0-1037.49\end{array}$

$\begin{array}{llll}58 & 69 & 127 & -2.5-10.7-1048.33\end{array}$

$\begin{array}{lllll}58 & 70 & 128 & -2.9-10.4-1058.87\end{array}$

$58 \quad 71 \quad 129-3.3-10.1-1069.12$

$\begin{array}{llllll}58 & 72 & 130 & -3.6 & -9.8-1079.09\end{array}$

$\begin{array}{llllll}58 & 73 & 131 & -4.0 & -9.6-1088.78\end{array}$

58. $\quad \begin{array}{lllll}74 & 132 & -4.4 & -9.3-1098.21\end{array}$

$\begin{array}{llllll}58 & 75 & 133 & -4.7 & -9.0 & -1107.36\end{array}$

$\begin{array}{llllll}58 & 76 & 134 & -5.1 & -8.8-1116.26\end{array}$

$\begin{array}{llllll}58 & 77 & 135 & -5.4 & -8.5 & -1124.90\end{array}$

$\begin{array}{llllll}58 & 78 & 136 & -5.8 & -8.3-1133.30\end{array}$

$\begin{array}{lllll}58 & 79 & 137 & -6.1 & -8.0-1141.45\end{array}$

$\begin{array}{llllll}58 & 80 & 138 & -6.4 & -7.8-1149.36\end{array}$ $\begin{array}{lllllll}0.67 & 1.25 & -3.43-1237.19 & -47.08\end{array}$ $0.562 .06-3.36-1240.69-42.51$

$\begin{array}{lllll}0.79 & 1.25 & -3.29-1245.31 & -39.06\end{array}$

$0.632 .06-3.23-1248.55-34.23$

$\begin{array}{llllll}0.74 & 1.25 & -3.17-1252.98 & -30.59\end{array}$

$0.382 .05-3.10-1256.12-25.66$

$0.421 .25-3.05-1260.33-21.79$

$\begin{array}{llllll}0.38 & 2.05 & -2.99-1262.86 & -16.26\end{array}$

$\begin{array}{lllll}0.75 & 1.25 & -2.93-1266.45 & -11.77\end{array}$

$0.842 .04-2.88-1268.59$

$-5.84$

$1.191 .25-2.83-1271.92$

$1.152 .04-2.78-1273.92$

$-1.10$

4.97

$1.42 \quad 1.25-2.73-1277.07$

9.90

$1.362 .03-2.68-1278.84$

$1.52 \quad 1.25-2.64-1281.84$

$1.262 .03-2.59-1283.57$

$1.291 .25-2.55-1286.46$

$0.842 .03-2.51-1288.15$

$0.921 .25-2.47-1290.75$

$0.502 .02-2.43-1292.19$

$0.371 .25 \cdot-2.39-1294.77$

$-0.38 \quad 2.02-2.35-1296.33$

$-0.501 .25-2.31-1298.69$

$-1.262 .01-2.28-1300.05$

$-0.951 .25-2.24-1301.77$

$-2.112 .01-2.21-1303.34$

16.20

21.27

27.61

32.79

39.17

44.64

51.28

5.5 .76

63.28

68.99

75.70

82.05

88.56

$-2.651 .25-2.18-1305.71 \quad 94.26$

$\begin{array}{llll}-4.07 & 2.01 & -2.14-1307.34 & 100.69\end{array}$

$\begin{array}{lllll}-4.82 & 1.25 & -2.11-1309.74 & 106.37\end{array}$

$\begin{array}{lllll}-6.41 & 2.00 & -2.08-1311.36 & 112.81\end{array}$

$\begin{array}{lllll}-6.92 & 1.25 & -2.05-1313.33 & 118.92\end{array}$

$\begin{array}{lllll}-6.32 & 2.00 & -2.02-1312.60 & 127.72\end{array}$

$\begin{array}{lllll}-5.13 & 1.25 & -2.00-1312.69 & 135.70\end{array}$

$\begin{array}{lllll}-4.07 & 1.99 & -1.97-1311.34 & 145.13\end{array}$

$\begin{array}{lllll}-2.98 & 1.25 & -1.94-1311.36 & 153.17\end{array}$

$\begin{array}{lllll}-2.99 & 1.99 & -1.91-1310.92 & 161.69\end{array}$

$\begin{array}{lllll}-2.37 & 1.25 & -1.89-1311.26 & 169.42\end{array}$

$\begin{array}{llllll}-2.55 & 1.99 & -1.86-1310.84 & 177.91\end{array}$

$\begin{array}{lll}-1.95 & 1.25 & -1.84\end{array}$

0.00

0.00

$-2.08 \quad 1.98-1.82$

0.00

0.00

$\begin{array}{llllll}1.04 & 1.26 & -8.94 & -873.22 & -6.76\end{array}$

$\begin{array}{llllll}1.32 & 0.00 & -9.29 & -889.87 & -15.34\end{array}$

$\begin{array}{llllll}1.40 & 1.25 & -9.64 & -903.82 & -21.22\end{array}$

$1.28 \quad 0.00-10.00 \quad-920.09 \quad-29.42$

$\begin{array}{llllll}1.19 & 1.23 & -9.65 & -932.77 & -34.03\end{array}$

$\begin{array}{llllll}1.05 & 0.00 & -9.31 & -947.61 & -40.80\end{array}$

$\begin{array}{llllll}1.17 & 1.22 & -9.00 & -959.40 & -44.52\end{array}$

$\begin{array}{lllllll}1.36 & 0.00 & -8.69 & -973.23 & -50.27\end{array}$

$\begin{array}{llllll}1.60 & 1.21 & -8.41 & -984.24 & -53.22\end{array}$

$\begin{array}{lllllll}1.83 & 0.00 & -8.13 & -997.36 & -58.26\end{array}$

$1.95 \quad 1.19-7.87-1007.87-60.70$

$2.06 \quad 0.00-7.63-1020.47-65.23$

$2.07 \quad 1.18-7.39-1030.49-67.18$

$2.14 \quad 0.00-7.17-1042.52-71.13$

$2.101 .17-6.95-1052.01-72.55$

$2.24 \cdot 0.00-6.75-1063.38-75.85$

$2.221 .16-6.55-1072.29-76.69$

$2.320 .00-6.36-1083.13-79.46$

$2.361 .15-6.18-1091.46-79.71$

$2.240 .00-6.01-1101.98-82.16$

$1.961 .14-5.85-1110.11-82.22$

$1.72 \quad 0.00 \quad-5.69-1120.23-84.27$

$1.291 .13-5.54-1128.02-83.99$

$0.70 \quad 0.00-5.39-1137.99-85.89-8$

$-0.12 \quad 1.12-5.25-1145.70-85.53$

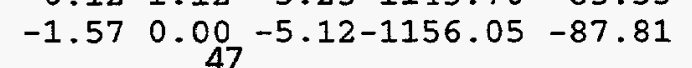

\begin{tabular}{|c|c|c|}
\hline 00 & 00 & \\
\hline 0.00 & .00 & \\
\hline .00 & .00 & \\
\hline & .00 & \\
\hline . & .00 & \\
\hline & 0.00 & \\
\hline 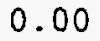 & 0.00 & \\
\hline & 0.00 & \\
\hline 0 & 0.00 & \\
\hline .0 & 0.00 & \\
\hline & 0.00 & \\
\hline & 0.00 & \\
\hline .0 & 0.00 & \\
\hline ( & 0.00 & \\
\hline & 0.00 & \\
\hline • & 0.00 & \\
\hline .0 & 0.00 & \\
\hline 0.0 & 0.00 & \\
\hline .0 & 0.00 & 0 \\
\hline .0 & 0.00 & \\
\hline. & 0.00 & 0 \\
\hline .0 & 0.00 & 0 \\
\hline .0 & 0.00 & 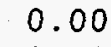 \\
\hline .0 & 0.00 & 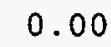 \\
\hline C & 0.00 & 0. \\
\hline .0 & 0.00 & 0 \\
\hline .0 & 0.00 & . \\
\hline. & 0.00 & \\
\hline .0 & 0.00 & 0 \\
\hline 0 & 0.00 & \\
\hline .0 & 0.00 & 0 \\
\hline .0 & 0.00 & 0 \\
\hline 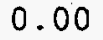 & 0.00 & 0 \\
\hline .0 & 0.00 & 0 \\
\hline 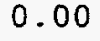 & 0.00 & . \\
\hline 0.0 & 0.00 & 0 . \\
\hline . 0 & 0.00 & 0. \\
\hline 0 & 0.00 & 0 \\
\hline . & 0.00 & 0 . \\
\hline .0 & 0.00 & 0 . \\
\hline 0 & 0.00 & 0. \\
\hline 0 & 0.00 & 0. \\
\hline 0 & 0.00 & 0 \\
\hline .0 & 0.00 & 0 . \\
\hline 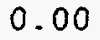 & 0.00 & 0. \\
\hline 0 & 0.00 & 0. \\
\hline 0 & 0.00 & 0. \\
\hline 0 & 0.00 & 0 \\
\hline 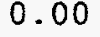 & 0.00 & 0. \\
\hline 0 & 0.00 & 0. \\
\hline 0 & 0.00 & 0. \\
\hline 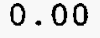 & 0.00 & 0. \\
\hline 0 & 0.00 & 0. \\
\hline 0.0 & 0.00 & 0 . \\
\hline 0. & 0.00 & 0. \\
\hline & 0.00 & 0 \\
\hline 0.0 & 0.00 & 0. \\
\hline & 0.00 & 0 . \\
\hline & 2.34 & -0. \\
\hline 0.0 & 0.00 & 0 . \\
\hline & 0.00 & 0. \\
\hline & 1.24 & -0 \\
\hline & 0.64 & -0 \\
\hline & 0.09 & 0. \\
\hline & -0.49 & -0 \\
\hline t & 1.33 & 0.2 \\
\hline
\end{tabular}

0.00 0.00 0.00 .00 .00 .00 0.00 .00 .00 .00 .00 .00 .00 .00
.00 .00 .00 .00
.00 .00 .00 .00
.00 .00 .00 .00
.00 .00 .00 .00
.00 .00 .00 .00
.00 .00 .00 .00
.00 .00 .00
.00 .00 .00 .00 .00 .00 .00
.00 .00 .00 .00
.00 .00 .02
.00 00 .65 .37 24 
58

58

58

58

58

58

58

58

58

58

58

58

$58 \quad 95 \quad 153-11.1-4.8-1242.68$

$58 \quad 96 \quad 154-11.4 \quad-4.6-1247.40$

$5897 \quad 155-11.7-4.5-1251.95$

$58 \quad 98 \quad 156-12.0 \quad-4.3-1256.34$

$\begin{array}{lllll}58 & 99 & 157-12.3 & -4.1-1260.56\end{array}$

$\begin{array}{lllll}58 & 100 & 158-12.5 & -4.0-1264.63\end{array}$

$\begin{array}{llll}58 & 101 & 159-12.8 & -3.8-1268.55\end{array}$

$\begin{array}{lllll}58 & 102 & 160-13.1 & -3.7-1272.32\end{array}$

$\begin{array}{llll}58 & 103 & 161-13.3 & -3.5-1275.94\end{array}$

$\begin{array}{lllll}58 & 104 & 162-13.6 & -3.4-1279.41\end{array}$

$\begin{array}{llll}58 & 105 & 163-13.9 & -3.3-1282.74\end{array}$

$58106 \quad 164-14.1-3.1-1285.93$

$\begin{array}{lllll}58 & 107 & 165-14.4 & -3.0-1288.99\end{array}$

$58108 \quad 166-14.6 \quad-2.9-1291.91$

$\begin{array}{lllll}58 & 109 & 167-14.9 & -2.7-1294.71\end{array}$

$\begin{array}{lllll}58 & 110 & 168-15.1 & -2.6-1297.37\end{array}$

$58 \quad 111 \quad 169-15.3 \quad-2.5-1299.91$

$\begin{array}{llll}58 & 112 & 170-15.6 & -2.4-1302.33\end{array}$

$\begin{array}{llll}58 & 113 & 171-15.8 & -2.2-1304.62\end{array}$

$\begin{array}{lllll}58 & 114 & 172-16.1 & -2.1-1306.80\end{array}$

$\begin{array}{lllll}58 & 115 & 173-16.3 & -2.0-1308.86\end{array}$

$\begin{array}{llll}58 & 116 & 174-16.5 & -1.9-1310.81\end{array}$

$\begin{array}{lllll}58 & 117 & 175-16.7 & -1.8-1312.64\end{array}$

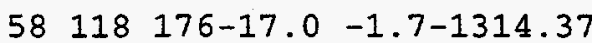

$58 \quad 119 \quad 177-17.2 \quad-1.6-1315.99$

$58 \quad 120 \quad 178-17.4 \quad-1.5-1317.51$

$\begin{array}{llll}58 & 121 & 179-17.6 & -1.4-1318.92\end{array}$

58122 180-17.8 $-1.3-1320.23$

$\begin{array}{llll}58 & 123 & 181-18.0 & -1.2-1321.44\end{array}$

$\begin{array}{llll}58 & 124 & 182-18.2 & -1.1-1322.56\end{array}$

$\begin{array}{llll}58 & 125 & 183-18.4 & -1.0-1323.59\end{array}$

$58 \quad 126 \quad 184-18.6-0.9-1324.52$

$\begin{array}{lllll}58 & 127 & 185-18.8 & -0.8-1325.36\end{array}$

$\begin{array}{lllll}58 & 128 & 186-19.0 & -0.7-1326.11\end{array}$

$\begin{array}{lllll}58 & 129 & 187-19.2 & -0.6-1326.78\end{array}$

$58 \quad 130188-19.4-0.5-1327.36$

$\begin{array}{llll}58 & 131 & 189-19.6 & -0.5-1327.86\end{array}$

$\begin{array}{lllll}58 & 132 & 190-19.8 & -0.4-1328.28\end{array}$

$\begin{array}{llll}58 & 133 & 191-19.9 & -0.3-1328.62\end{array}$

$58 \quad 134 \quad 192-20.1-0.2-1328.88$

$58 \quad 135 \quad 193-20.3-0.1-1329.07$

$58136 \quad 194-20.5 \quad-0.1-1329.19$

$\begin{array}{rrrrrr}58 & 137 & 195 & 0.0 & 0.0 & 0.00\end{array}$

$\begin{array}{lllll}59 & 56 & 115 & 3.1-15.5 & -879.10\end{array}$

$5957 \quad 116 \quad 2.7-15.1-894.43$

$\begin{array}{lllll}59 & 58 & 117 & 2.3-14.8 & -909.37\end{array}$

$\begin{array}{lllll}59 & 59 & 118 & 1.9-14.4 & -923.95\end{array}$

$\begin{array}{lllll}59 & 60 & 119 & 1.5-14.0 & -938.15\end{array}$

$59 \quad 61 \quad 120 \quad 1.1-13.7-951.99$

$\begin{array}{lllll}59 & 62 & 121 & 0.7-13.3 & -965.48\end{array}$

$\begin{array}{lllll}59 & 63 & 122 & 0.3-13.0 & -978.62\end{array}$

$\begin{array}{lllll}59 & 64 & 123 & -0.1-12.6 & -991.42\end{array}$ $\begin{array}{lllllll}-2.72 & 1.11 & -4.99-1163.65 & -87.33 & -86.97 & -2.36\end{array}$

$\begin{array}{lllllll}-3.86 & 0.00 & -4.87-1173.23 & -88.84 & -88.09 & -3.11\end{array}$

$\begin{array}{lllllll}-3.11 & 1.10 & -4.75-1178.49 & -86.03 & -85.44 & -2.52\end{array}$

$\begin{array}{lllllll}-2.06 & 0.00 & -4.63-1185.43 & -84.91 & -84.54 & -1.69\end{array}$

$\begin{array}{lllllll}-0.79 & 1.09 & -4.52 & -1189.76 & -81.16 & -81.62 & -1.25\end{array}$

$\begin{array}{lllllll}-0.47 & 0.00 & -4.42-1197.01 & -80.34 & -80.44 & -0.57\end{array}$

$\begin{array}{lllllll}-0.30 & 1.08 & -4.32 & -1202.04 & -77.30 & -77.11 & -0.11\end{array}$

$\begin{array}{lllllll}-0.01 & 0.00 & -4.22 & -1208.92 & -76.11 & -75.72 & 0.38\end{array}$

$0.401 .08-4.12-1213.33-72.44-72.18$

$\begin{array}{llllll}0.60 & 0.00 & -4.03-1219.90 & -70.95 & -70.43\end{array}$

$0.311 .07-3.94-1224.64-67.61-66.80$

$0.310 .00-3.86-1231.04-65.94-64.99$

$0.06 \quad 1.06-3.78-1235.39-62.22$

$0.28 \quad 0.00-3.70-1241.21-59.97$

$0.16 \quad 1.05-3.62-1245.09-55.78$

$0.400 .00-3.55-1250.55-53.16$

$0.24 \quad 1.04-3.48-1254.14-48.68$

$0.45 \quad 0.00-3.41-1259.29-45.76$

$0.291 .04-3.34-1262.58-40.98$

$\begin{array}{lllll}0.46 & 0.00 & -3.27-1267.45 & -37.78\end{array}$

$\begin{array}{lllll}0.11 & 1.03 & -3.21-1270.62 & -32.88\end{array}$

$\begin{array}{lllll}0.30 & 0.00 & -3.15-1275.17 & -29.35\end{array}$

$0.14 \quad 1.02-3.09-1277.86-23.98$

$\begin{array}{llll}0.62 & 0.00 & -3.03-1281.82 & -19.87\end{array}$

$0.67 \quad 1.02-2.98-1284.03-14.00$

$\begin{array}{llll}1.04 & 0.00 & -2.93-1287.82 & -9.72\end{array}$

$1.02 \quad 1.01-2.87-1289.83$

$1.34 \quad 0.00-2.82-1293.40$

$1.231 .00-2.77-1295.25$

$1.43 \quad 0.00-2.73-1298.67$

$1.271 .00-2.68-1300.32$

$1.34 \quad 0.00-2.63-1303.62$

$\begin{array}{llll}0.87 & 0.99 & -2.59-1305.35\end{array}$

$0.960 .00-2.55-1308.39$

$0.580 .99-2.51-1309.80$

$0.510 .00-2.47-1312.76$

$\begin{array}{lll}-0.16 & 0.98 & -2.43-1314.25\end{array}$

$-0.23 \quad 0.00-2.39-1316.99$

$\begin{array}{lll}-0.62 & 0.98 & -2.35-1317.99\end{array}$

$\begin{array}{llll}-0.51 & 0.00 & -2.32-1320.33\end{array}$

$\begin{array}{llll}-1.57 & 0.97 & -2.28-1321.80\end{array}$

$\begin{array}{llll}-2.13 & 0.00 & -2.25-1324.61\end{array}$

$\begin{array}{llll}-3.44 & 0.97 & -2.21-1326.13\end{array}$

$\begin{array}{llll}-4.29 & 0.00 & -2.18-1329.03\end{array}$

$-3.66$

0.85

7.07

11.72

18.14

22.91

29.25

34.29

40.94

46.05

52.64

57.97

65.04

70.77

77.37

82.64

89.18

94.35

$\begin{array}{lllll}-5.85 & 0.96 & -2.15-1330.62 & 100.83\end{array}$

$\begin{array}{llll}-6.39 & 0.00 & -2.12-1333.03 & 106.50\end{array}$

$\begin{array}{lllll}-5.61 & 0.95 & -2.09-1332.10 & 115.50\end{array}$

$\begin{array}{llll}-4.65 & 0.00 & -2.06-1332.82 & 122.85\end{array}$

$\begin{array}{llll}-3.40 & 0.95 & -2.03-1331.26 & 132.49\end{array}$

$\begin{array}{lllll}-2.42 & 0.00 & -2.00-1331.78 & 140.03\end{array}$

$\begin{array}{lllll}-2.48 & 0.95 & -1.97-1331.37 & 148.52\end{array}$

$\begin{array}{lllll}-1.87 & 0.00 & -1.95-1332.09 & 155.86\end{array}$

$\begin{array}{lllll}-1.97 & 0.94 & -1.92-1331.57 & 164.46\end{array}$

$\begin{array}{lllll}-0.94 & 0.00 & -1.90-1331.72 & 172.38\end{array}$

$\begin{array}{lllll}-1.50 & 0.94 & -1.87-1331.50 & 180.67\end{array}$

$\begin{array}{llll}-1.52 & 0.00 & -1.85-1332.55 & 187.69\end{array}$

$\begin{array}{lllll}-1.70 & 0.93 & -1.82 & 0.00 & 0.00\end{array}$

$\begin{array}{lllll}1.03 & 1.23 & -8.96 & -885.79 & -3.99\end{array}$

$\begin{array}{lllll}1.00 & 2.20 & -9.30 & -900.53 & -10.65\end{array}$

$\begin{array}{lllll}0.74 & 1.23 & -9.65 & -917.05 & -19.10\end{array}$

$0.592 .45-10.00-930.91-24.89$

$\begin{array}{llllll}0.40 & 1.23 & -9.65 & -946.17 & -32.07\end{array}$

$\begin{array}{llllll}0.42 & 2.18 & -9.32 & -958.71 & -36.55\end{array}$

$\begin{array}{lllll}0.61 & 1.23 & -9.01 & -972.65 & -42.41\end{array}$

$\begin{array}{lllllll}0.86 & 2.17 & -8.71 & -984.30 & -45.99\end{array}$

$\begin{array}{llllll}1.15 & 1.23 & -8.43 & -997.47 & -51.09\end{array}$ 48
0.66

1.12

1.12

0.00

1.26

0.36

0.75

0.59

0.37

$-0.46$

$-0.10$

0.39

0.26

0.52

0.81

0.95

$\begin{array}{lll}0.00 & 0.00 & 0.00\end{array}$

$\begin{array}{lll}0.00 & 0.00 & 0.00\end{array}$

$\begin{array}{lll}0.00 & 0.00 & 0.00\end{array}$

$\begin{array}{llll}0.00 & 0.00 & 0.00\end{array}$

$\begin{array}{llll}0.00 & 0.00 & 0.00\end{array}$

$\begin{array}{lll}0.00 & 0.00 & 0.00\end{array}$

$\begin{array}{lll}0.00 & 0.00 & 0.00\end{array}$

$\begin{array}{llll}0.00 & 0.00 & 0.00\end{array}$

$\begin{array}{lll}0.00 & 0.00 & 0.00\end{array}$

$\begin{array}{llll}0.00 & 0.00 & 0.00\end{array}$

$\begin{array}{lll}0.00 & 0.00 & 0.00\end{array}$

$\begin{array}{lll}0.00 & 0.00 & 0.00\end{array}$

$\begin{array}{llll}0.00 & 0.00 & 0.00\end{array}$

$\begin{array}{llll}0.00 & 0.00 & 0.00\end{array}$

$\begin{array}{lll}0.00 & 0.00 & 0.00\end{array}$

$\begin{array}{llll}0.00 & 0.00 & 0.00\end{array}$

$\begin{array}{llll}0.00 & 0.00 & 0.00\end{array}$

$\begin{array}{llll}0.00 & 0.00 & 0.00\end{array}$

$\begin{array}{llll}0.00 & 0.00 & 0.00\end{array}$

$\begin{array}{llll}0.00 & 0.00 & 0.00\end{array}$

$\begin{array}{llll}0.00 & 0.00 & 0.00\end{array}$

$\begin{array}{llll}0.00 & 0.00 & 0.00\end{array}$

$\begin{array}{llll}0.00 & 0.00 & 0.00\end{array}$

$\begin{array}{lll}0.00 & 0.00 & 0.00\end{array}$

$\begin{array}{llll}0.00 & 0.00 & 0.00\end{array}$

$\begin{array}{llll}0.00 & 0.00 & 0.00\end{array}$

0.00

0.00

0.00

0.00

0.00

0.00

0.00

0.00

0.00

0.00

0.00

0.00

0.00

0.00

0.00

0.00

0.00

0.00

0.00

0.00

0.00

0.00

0.00

0.00

0.00

0.00

0.00

0.00

0.00

0.00

0.00

0.00

0.00

0.00

0.00

0.00

0.00

0.00

0.00

0.00

0.00

0.00

0.00

0.00

0.00

0.00

0.00

0.00

0.00

0.00

0.00

0.00

0.00

0.00

0.00

0.00

0.00

0.00

0.00

0.00

0.00

0.00

0.00

0.00

0.00

0.00

0.00

0.00

0.00

0.00

0.00

0.00 
$\begin{array}{llll}59 & 65 & 124 & -0.5-12.3-1003.89\end{array}$

$5966 \quad 125-0.8-12.0-1016.03$

$5967 \quad 126-1.2-11.7-1027.86$

$5968 \quad 127-1.6-11.4-1039.37$

$\begin{array}{llll}59 & 69 & 128 & -2.0-11.1-1050.58\end{array}$

$5970 \quad 129-2.3-10.8-1061.49$

$\begin{array}{lllll}59 & 71 & 130 & -2.7-10.5-1072.11\end{array}$

$59 \quad 72 \quad 131-3.1-10.2-1082.45$

$\begin{array}{lllll}59 & 73 & 132 & -3.4 & -9.9-1092.50\end{array}$

$\begin{array}{llllll}59 & 74 & 133 & -3.8 & -9.6-1102.28\end{array}$

$\begin{array}{llllll}59 & 75 & 134 & -4.1 & -9.4-1111.79\end{array}$

$\begin{array}{llllll}59 & 76 & 135 & -4.5 & -9.1-1121.04\end{array}$

$\begin{array}{llllll}59 & 77 & 136 & -4.8 & -8.9 & -1130.03\end{array}$

$\begin{array}{llllll}59 & 78 & 137 & -5.2 & -8.6-1138.76\end{array}$

$\begin{array}{llllll}59 & 79 & 138 & -5.5 & -8.4-1147.26\end{array}$

$\begin{array}{llllll}59 & 80 & 139 & -5.8 & -8.1-1155.51\end{array}$

$\begin{array}{llllll}59 & 81 & 140 & -6.2 & -7.9-1163.53\end{array}$

$\begin{array}{llllll}59 & 82 & 141 & -6.5 & -7.7-1171.31\end{array}$

$\begin{array}{llllll}59 & 83 & 142 & -6.8 & -7.4-1178.87\end{array}$

$\begin{array}{llllll}59 & 84 & 143 & -7.2 & -7.2-1186.20\end{array}$

$\begin{array}{llllll}59 & 85 & 144 & -7.5 & -7.0-1193.32\end{array}$

$\begin{array}{llllll}59 & 86 & 145 & -7.8 & -6.8 & -1200.23\end{array}$

$\begin{array}{llllll}59 & 87 & 146 & -8.1 & -6.6-1206.93\end{array}$

$\begin{array}{llllll}59 & 88 & 147 & -8.4 & -6.4-1213.42\end{array}$

$\begin{array}{llllll}59 & 89 & 148 & -8.7 & -6.2-1219.72\end{array}$

$5990 \quad 149-9.0 \quad-6.0-1225.81$

$5991 \quad 150 \quad-9.3 \quad-5.8-1231.72$

$\begin{array}{llllll}59 & 92 & 151 & -9.6 & -5.6-1237.44\end{array}$

$\begin{array}{llllll}59 & 93 & 152 & -9.9 & -5.4-1242.98\end{array}$

$5994 \quad 153-10.2-5.3-1248.33$

$5995 \quad 154-10.5 \quad-5.1-1253.51$

$5996 \quad 155-10.8-4.9-1258.51$

$5997 \quad 156-11.1-4.7-1263.35$

$5998 \quad 157-11.4-4.6-1268.01$

$5999 \quad 158-11.7-4.4-1272.52$

$59 \quad 100 \quad 159-11.9-4.3-1276.86$

$59101160-12.2-4.1-1281.05$

$59102 \quad 161-12.5-4.0-1285.09$

$59103 \quad 162-12.7-3.8-1288.97$

$59104 \quad 163-13.0-3.7-1292.71$

$59105164-13.3-3.5-1296.30$

$59106 \quad 165-13.5-3.4-1299.75$

$59 \quad 107 \quad 166-13.8-3.2-1303.06$

$59108 \quad 167-14.0-3.1-1306.24$

$59109168-14.3-3.0-1309.28$

$59110 \quad 169-14.5-2.8-1312.19$

$\begin{array}{llll}59 & 111 & 170-14.8 & -2.7-1314.97\end{array}$

$59 \quad 112171-15.0-2.6-1317.62$

$\begin{array}{llll}59 & 113 & 172-15.2 & -2.5-1320.16\end{array}$

$\begin{array}{lllll}59 & 114 & 173-15.5 & -2.3-1322.57\end{array}$

$\begin{array}{lllll}59 & 115 & 174-15.7 & -2.2-1324.86\end{array}$

$59 \quad 116 \quad 175-15.9-2.1-1327.04$

$59117 \quad 176-16.2-2.0-1329.10$

$59118 \quad 177-16.4-1.9-1331.05$

$59119178-16.6-1.8-1332.89$

$59120179-16.8-1.7-1334.62$

$59121180-17.0-1.6-1336.25$

$\begin{array}{llll}59 & 122 & 181-17.3 & -1.5-1337.77\end{array}$

$59 \quad 123 \quad 182-17.5-1.4-1339.20$

$59 \quad 124 \quad 183-17.7-1.3-1340.52$

$59125184-17.9-1.2-1341.75$

$59126185-18.1-1.1-1342.88$

$59127186-18.3-1.0-1343.92$

$59128 \quad 187-18.5-0.9-1344.87$

$59129188-18.7-0.8-1345.73$

$59130189-18.9-0.7-1346.50$
$1.32 \quad 2.16-8.16-1008.57-54.12$

$1.491 .23-7.90-1021.22-58.69$

$1.562 .15-7.66-1031.81-61.21$

$1.66 \quad 1.23-7.43-1043.91-65.24$

$1.712 .14-7.20-1053.93-67.20$

$1.871 .23-6.99-1065.38-70.57$

$1.972 .13-6.79-1074.79-71.91$

$2.201 .23-6.59-1085.60-74.65$

$2.212 .13-6.41-1094.57-75.54$

$2.261 .23-6.23-1105.01-77.92$

$2.192 .12-6.06-1113.53-78.37$

$2.07 \quad 1.23 \quad-5.89-1123.62 \quad-80.39$

$1.632 .11-5.74-1132.02-80.71$

$1.161 .23-5.59-1141.96-82.58$

$0.392 .10-5.44-1150.20-82.75$

$\begin{array}{lllll}-0.98 & 1.23 & -5.30-1160.56 & -85.04\end{array}$

$\begin{array}{llllll}-2.07 & 2.10 & -5.17-1168.67 & -85.07 \\ -3.26 & 1.23 & -5.04-1178.38 & -86.71\end{array}$

$\begin{array}{llllll}-2.51 & 2.09 & -4.92-1184.20 & -84.47\end{array}$

$\begin{array}{llllll}-1.47 & 1.23 & -4.80-1191.24 & -83.43\end{array}$

$-0.342 .08-4.68-1196.26-80.38$

$0.202 .08-4.47-1209.12-77.10$

$0.55 \quad 1.23-4.37-1216.01-75.91$

$0.652 .07-4.27-1221.26-73.10$

$0.541 .23-4.17-1228.22-71.98$

$\begin{array}{lllll}0.22 & 2.07 & -4.08-1233.52 & -69.21\end{array}$

$\begin{array}{lllll}0.22 & 1.23 & -3.99-1239.98 & -67.60\end{array}$

$\begin{array}{lllll}-0.08 & 2.06 & -3.91-1244.90 & -64.45\end{array}$

$0.061 .23-3.83-1250.86-62.34$

$-0.092 .06-3.75-1255.29-58.69$

$\begin{array}{lllll}0.13 & 1.23 & -3.67-1260.82 & -56.15\end{array}$

$\begin{array}{lllll}-0.02 & 2.05 & -3.59-1264.91 & -52.17\end{array}$

$0.201 .23-3.52-1270.10-49.29$

$0.062 .04-3.45-1273.87-44.99$

$\begin{array}{lllll}0.12 & 1.23 & -3.39-1278.90 & -41.94\end{array}$

$\begin{array}{lllll}-0.10 & 2.04 & -3.32-1282.43 & -37.41\end{array}$

$0.121 .23-3.26-1286.99-33.90$

$\begin{array}{llll}-0.02 & 2.03 & -3.20-1290.15 & -28.99\end{array}$

$\begin{array}{llllll}0.35 & 1.23 & -3.14-1294.26 & -25.02\end{array}$

$0.532 .03-3.08-1296.82-19.51$

$\begin{array}{lllll}0.88 & 1.23 & -3.02-1300.66 & -15.28\end{array}$

$\begin{array}{lllll}0.91 & 2.03 & -2.97-1303.09 & -9.64\end{array}$

$1.261 .23-2.92-1306.66$

$1.232 .02-2.87-1308.89$

$-5.14$

$1.471 .23-2.82-1312.30$

0.70

$1.342 .02-2.77-1314.38$

$1.441 .23-2.72-1317.67$

$1.112 .01-2.68-1319.71$

$1.031 .23-2.63-1322.94$

$0.692 .01-2.59-1324.75$

$0.651 .23-2.55-1327.70$

$0.042 .00-2.51-1329.56$

$-0.041 .23-2.47-1332.32$

$\begin{array}{lll}-0.40 & 2.00 & -2.43-1333.72\end{array}$

$-0.261 .23-2.39-1336.04$

$-1.252 .00-2.35-1337.86$

$-1.711 .23-2.32-1340.57$

$-3.101 .99-2.28-1342.59$

$-3.911 .23-2.25-1345.45$

5.37

11.36

16.14

22.17

27.02

33.27

38.40

44.61

49.92

56.59

62.34

68.59

73.95

80.01

85.22

$-5.401 .99-2.22-1347.38$

91.36

$\begin{array}{llll}-5.96 & 1.23 & -2.18-1349.79 & 97.02\end{array}$

$\begin{array}{lllll}-5.20 & 1.99 & -2.15-1349.29 & 105.59\end{array}$

$\begin{array}{llll}-4.13 & 1.23 & -2.12-1349.89 & 113.06\end{array}$

$\begin{array}{lllll}-2.95 & 1.98 & -2.09-1348.79 & 122.24\end{array}$

$-2.141 .23-2.06-1349.47129 .63$ $\begin{array}{lll}0.00 & 0.00 & 0.00 \\ 0.00 & 0.00 & 0.00 \\ 0.00 & 0.00 & 0.00 \\ 0.00 & 0.00 & 0.00 \\ 0.00 & 0.00 & 0.00 \\ 0.00 & 0.00 & 0.00 \\ 0.00 & 0.00 & 0.00 \\ 0.00 & 0.00 & 0.00 \\ 0.00 & 0.00 & 0.00 \\ 0.00 & 0.00 & 0.00 \\ 0.00 & 0.00 & 0.00\end{array}$

$0.00 \quad 0.00 \quad 0.00$

$-80.92$

$1.54-0.53$

$0.97-0.66$

$0.54-0.62$

$0.00-0.39$

$\begin{array}{lll}-84.84 & -0.78 & 0.20 \\ -84.70 & -1.70 & 0.37\end{array}$

$-86.03-2.58$

0.68

0.67

$\begin{array}{rrr}-83.08 & -1.12 & 0.35 \\ -80.76 & -0.72 & -0.38\end{array}$

$-79.64-0.02-0.10$

$\begin{array}{lll}-76.75 & 0.55 & 0.35\end{array}$

$\begin{array}{lll}-75.47 & 0.99 & 0.44\end{array}$

$\begin{array}{lll}-72.49 & 1.26 & 0.61\end{array}$

$\begin{array}{lll}-70.99 & 1.53 & 0.99\end{array}$

$\begin{array}{rrr}-68.00 & 1.43 & 1.21\end{array}$

$\begin{array}{llll}0.00 & 0.00 & 0.00\end{array}$

$\begin{array}{llll}0.00 & 0.00 & 0.00\end{array}$

$\begin{array}{llll}0.00 & 0.00 & 0.00\end{array}$

$\begin{array}{llll}0.00 & 0.00 & 0.00\end{array}$

$\begin{array}{llll}0.00 & 0.00 & 0.00\end{array}$

$\begin{array}{llll}0.00 & 0.00 & 0.00\end{array}$

$0.00 \quad 0.00 \quad 0.00$

$0.00 \quad 0.00 \quad 0.00$

$0.00 \quad 0.00 \quad 0.00$

$0.00 \quad 0.00 \quad 0.00$

$0.00 \quad 0.00 \quad 0.00$

$0.00 \quad 0.00 \quad 0.00$

$0.00 \quad 0.00 \quad 0.00$

$\begin{array}{llll}0.00 & 0.00 & 0.00\end{array}$

$0.00 \quad 0.00 \quad 0.00$

$\begin{array}{llll}0.00 & 0.00 & 0.00\end{array}$

$0.00 \quad 0.00 \quad 0.00$

$\begin{array}{llll}0.00 & 0.00 & 0.00\end{array}$

$0.00 \quad 0.00 \quad 0.00$

$0.00 \quad 0.00 \quad 0.00$

$\begin{array}{llll}0.00 & 0.00 & 0.00\end{array}$

$0.00 \cdot 0.00 \quad 0.00$

$0.00 \quad 0.00 \quad 0.00$

$0.00 \quad 0.00 \quad 0.00$

$0.00 \quad 0.00 \quad 0.00$

$0.00 \quad 0.00 \quad 0.00$

$0.00 \quad 0.00 \quad 0.00$

$0.00 \cdot 0.00 \quad 0.00$

$0.00 \quad 0.00 \quad 0.00$

$0.00 \quad 0.00 \quad 0.00$

$0.00 \quad 0.00 \quad 0.00$

$\begin{array}{llll}0.00 & 0.00 & 0.00\end{array}$

$0.00 \quad 0.00 \quad 0.00$

$0.00 \quad 0.00 \quad 0.00$

$0.00 \quad 0.00 \quad 0.00$

$\begin{array}{llll}0.00 & 0.00 & 0.00\end{array}$

$\begin{array}{llll}0.00 & 0.00 & 0.00\end{array}$

$0.00 \quad 0.00 \quad 0.00$

$\begin{array}{lll}0.00 & 0.00 & 0.00\end{array}$ 
$\begin{array}{lllllllll}59 & 131 & 190-19.1 & -0.6-1347.18 & -2.26 & 1.98 & -2.04-1349.50 & 137.67\end{array}$ $\begin{array}{llllllll}59 & 132 & 191-19.2 & -0.6-1347.78 & -1.62 & 1.23 & -2.01-1350.18 & 145.06\end{array}$ $\begin{array}{llllllll}59 & 133 & 192-19.4 & -0.5-1348.30 & -1.20 & 1.98 & -1.98-1349.51 & 153.80\end{array}$ $\begin{array}{llllllll}59 & 134 & 193-19.6 & -0.4-1348.74 & -0.65 & 1.23 & -1.96-1350.12 & 161.26\end{array}$ $\begin{array}{llllllll}59 & 135 & 194-19.8 & -0.3-1349.11 & -1.21 & 1.97 & -1.93-1350.27 & 169.18\end{array}$ $\begin{array}{llllllll}59 & 136 & 195-20.0 & -0.2-1349.39 & -1.15 & 1.23 & -1.90-1351.21 & 176.31\end{array}$ $\begin{array}{llllllll}59 & 137 & 196-20.1 & -0.2-1349.60 & -1.48 & 1.97 & -1.88-1351.00 & 184.60\end{array}$ $\begin{array}{llllllll}59 & 138 & 197-20.3 & -0.1-1349.75 & -1.31 & 1.23 & -1.86-1351.68 & 191.99\end{array}$ $\begin{array}{lllllllllll}59 & 139 & 198 & 0.0 & 0.0 & 0.00 & -1.58 & 1.97 & -1.83 & 0.00 & 0.00\end{array}$ $\begin{array}{lllll}60 & 58 & 118 & 2.8-15.2 & -906.82\end{array}$

$\begin{array}{lllll}60 & 59 & 119 & 2.4-14.8 & -921.79\end{array}$

$\begin{array}{llllll}60 & 60 & 120 & 2.0 & -14.4 & -936.38\end{array}$

$\begin{array}{lllll}60 & 61 & 121 & 1.7-14.1 & -950.62\end{array}$

$\begin{array}{lllll}60 & 62 & 122 & 1.3-13.7 & -964.49\end{array}$

$\begin{array}{llllll}60 & 63 & 123 & 0.9-13.4 & -978.02\end{array}$

$\begin{array}{lllll}60 & 64 & 124 & 0.5-13.0 & -991.21\end{array}$

$\begin{array}{llll}60 & 65 & 125 & 0.1-12.7-1004.06\end{array}$

$\begin{array}{llll}60 & 66 & 126 & -0.3-12.4-1016.58\end{array}$

$\begin{array}{llll}60 & 67 & 127 & -0.6-12.0-1028.78\end{array}$

$\begin{array}{llll}60 & 68 & 128 & -1.0-11.7-1040.67\end{array}$

$\begin{array}{llll}60 & 69 & 129 & -1.4-11.4-1052.25\end{array}$

$60 \quad 70 \quad 130 \quad-1.7-11.1-1063.53$

$6071 \quad 131-2.1-10.8-1074.51$

$\begin{array}{llll}60 & 72 & 132 & -2.5-10.5-1085.21\end{array}$

$\begin{array}{llll}60 & 73 & 133 & -2.8-10.3-1095.62\end{array}$

$\begin{array}{llll}60 & 74 & 134 & -3.2-10.0-1105.75\end{array}$

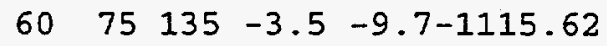

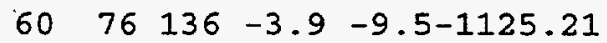

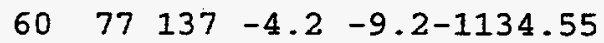

$\begin{array}{llllll}60 & 78 & 138 & -4.6 & -9.0 & -1143.63\end{array}$

$\begin{array}{lllll}60 & 79 & 139 & -4.9 & -8.7-1152.47\end{array}$

$\begin{array}{llllll}60 & 80 & 140 & -5.2 & -8.5-1161.05\end{array}$

$\begin{array}{lllll}60 & 81 & 141 & -5.6 & -8.2-1169.40\end{array}$

$\begin{array}{llllll}60 & 82 & 142 & -5.9 & -8.0 & -1177.52\end{array}$

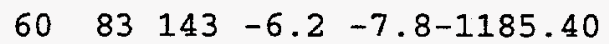

$\begin{array}{llllll}60 & 84 & 144 & -6.6 & -7.5 & -1193.07\end{array}$

$\begin{array}{llllll}60 & 85 & 145 & -6.9 & -7.3 & -1200.51\end{array}$

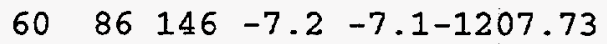

$\begin{array}{lllll}60 & 87 & 147 & -7.5 & -6.9-1214.74\end{array}$

$\begin{array}{lllll}60 & 88 & 148 & -7.8 & -6.7-1221.55\end{array}$

$\begin{array}{lllll}60 & 89 & 149 & -8.1 & -6.5-1228.15\end{array}$

$\begin{array}{lllll}60 & 90 & 150 & -8.4 & -6.3-1234.56\end{array}$

$\begin{array}{lllll}60 & 91 & 151 & -8.7 & -6.1-1240.77\end{array}$

$\begin{array}{lllll}60 & 92 & 152 & -9.0 & -5.9-1246.78\end{array}$

$\begin{array}{lllll}60 & 93 & 153 & -9.3 & -5.7-1252.62\end{array}$

$\begin{array}{llllll}60 & 94 & 154 & -9.6 & -5.6 & -1258.26\end{array}$

$\begin{array}{lllll}60 & 95 & 155 & -9.9 & -5.4-1263.73\end{array}$

$6096 \quad 156-10.2-5.2-1269.02$

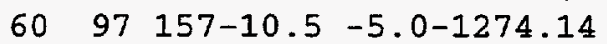

$6098 \quad 158-10.8-4.9-1279.09$

$\begin{array}{llll}60 & 99 & 159-11.1 & -4.7-1283.88\end{array}$

$60100160-11.3-4.5-1288.50$

$60101 \quad 161-11.6-4.4-1292.96$

$60102 \quad 162-11.9-4.2-1297.26$

$60103 \quad 163-12.1-4.1-1301.41$

$60104 \quad 164-12.4-3.9-1305.41$

$\begin{array}{lllll}60 & 105 & 165-12.7 & -3.8-1309.27\end{array}$

$\begin{array}{llll}60 & 106 & 166-12.9 & -3.6-1312.98\end{array}$

$60107 \quad 167-13.2 \quad-3.5-1316.54$

$60108 \quad 168-13.4 \quad-3.4-1319.97$

$60109 \quad 169-13.7-3.2-1323.26$

$\begin{array}{llll}60 & 110 & 170-13.9 & -3.1-1326.42\end{array}$

$60111 \quad 171-14.2-3.0-1329.44$

$60 \quad 112 \quad 172-14.4 \quad-2.8-1332.34$

$\begin{array}{lllll}60 & 113 & 173-14.7 & -2.7-1335.11\end{array}$

$60 \quad 114 \quad 174-14.9-2.6-1337.76$ $\begin{array}{llllll}0.45 & 0.00 & -9.31 & -915.69 & -10.46\end{array}$

$\begin{array}{lllll}0.27 & 1.23 & -9.65 & -929.94 & -16.64\end{array}$

$\begin{array}{lllll}0.00 & 0.00-10.00 & -946.38 & -25.01\end{array}$

$\begin{array}{llllll}0.03 & 1.22 & -9.66 & -959.03 & -29.58\end{array}$

$\begin{array}{llllll}0.22 & 0.00 & -9.33 & -973.61 & -36.09\end{array}$

$\begin{array}{llllll}0.48 & 1.21 & -9.03 & -985.36 & -39.77\end{array}$

$\begin{array}{llllll}0.76 & 0.00 & -8.73 & -999.18 & -45.52\end{array}$

$0.991 .19-8.45-1010.33-48.60$

$\begin{array}{lllll}1.18 & 0.00 & -8.19-1023.59 & -53.79\end{array}$

$\begin{array}{lllll}1.27 & 1.18 & -7.93-1034.26 & -56.39\end{array}$

$\begin{array}{lllll}1.40 & 0.00 & -7.69-1046.96 & -61.02\end{array}$

$\begin{array}{lllll}1.44 & 1.17 & -7.46-1057.10 & -63.09\end{array}$

$\begin{array}{lllll}1.64 & 0.00 & -7.24-1069.13 & -67.04\end{array}$

$\begin{array}{lllll}1.77 & 1.16 & -7.03-1078.61 & -68.45\end{array}$

$\begin{array}{lllll}2.03 & 0.00 & -6.83-1090.00 & -71.77\end{array}$

$2.32 \quad 1.15-6.63-1098.78-72.48$

$2.41 \quad 0.00-6.45-1109.79-75.42$

$2.301 .14-6.27-1118.45-76.00$

$2.210 .00-6.10-1129.10-78.59$

$1.901 .13-5.94-1137.46-78.87-79$

$1.50 \quad 0.00-5.78-1147.92-81.26$

$0.72 \quad 1.12-5.63-1156.26-81.53$

$\begin{array}{lllll}-0.58 & 0.00 & -5.49-1167.12 & -84.32\end{array}$

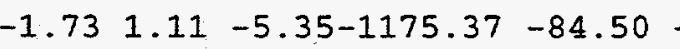

$\begin{array}{lllll}-2.88 & 0.00 & -5.22-1185.62 & -86.67 & -85\end{array}$

$-2.141 .10-5.09-1191.53-84.52$

$\begin{array}{rrrrr}-1.04 & 0.00 & -4.97-1199.07 & -83.98 \\ 0.10 & 1.09 & -4.85-1204.16 & -81.00\end{array}$

$\begin{array}{llllll}0.56 & 0.00 & -4.73-1211.90 & -80.67 & -80\end{array}$

$\begin{array}{llllll}0.63 & 1.08 & -4.62-1217.65 & -78.35 & -78.16\end{array}$

$\begin{array}{llllll}0.85 & 0.00 & -4.52-1225.22 & -77.84 & -77.42\end{array}$

$\begin{array}{llllll}0.76 & 1.08 & -4.42 & -1230.73 & -75.29 & -74.39\end{array}$

$\begin{array}{llllll}0.54 & 0.00 & -4.32-1238.33 & -74.82 & -73.69\end{array}$

$\begin{array}{llllll}0.19 & 1.07 & -4.22-1243.73 & -72.14 & -70.96\end{array}$

$0.110 .00-4.13-1250.81-71.15-70.16$

$\begin{array}{llll}-0.17 & 1.06 & -4.04-1255.77 & -68.04\end{array}$

$-0.130 .00-3.96-1262.35-66.55$

$\begin{array}{lllll}-0.30 & 1.05 & -3.87-1266.85 & -62.98\end{array}$

$\begin{array}{llll}-0.15 & 0.00 & -3.79-1272.97 & -61.02\end{array}$

$-0.331 .04-3.72-1277.14-57.13$

$\begin{array}{lllll}-0.16 & 0.00 & -3.64-1282.89 & -54.81\end{array}$

$-0.331 .04-3.57-1286.74-50.58$

$-0.240 .00-3.50-1292.24-48.00$

$-0.531 .03-3.43-1295.89-43.59$

$\begin{array}{lllll}-0.41 & 0.00 & -3.37-1301.04 & -40.67\end{array}$

$\begin{array}{lllll}-0.49 & 1.02 & -3.30-1304.18 & -35.74\end{array}$

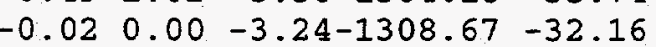

$\begin{array}{lllll}0.20 & 1.02 & -3.18-1311.23 & -26.64\end{array}$

$\begin{array}{lllll}0.71 & 0.00 & -3.12 & -1315.39 & -22.73\end{array}$

$\begin{array}{lllll}0.72 & 1.01 & -3.07-1317.88 & -17.15\end{array}$

$\begin{array}{lllll}1.08 & 0.00 & -3.01-1321.90 & -13.10\end{array}$

$\begin{array}{llll}1.07 & 1.00 & -2.96-1324.15 & -7.27\end{array}$

$1.350 .00-2.91-1327.98$

$1.201 .00-2.86-1330.10$

$1.320 .00-2.81-1333.83$

$1.010 .99-2.76-1335.87$

$0.990 .00-2.72-1339.49$ 50

2.91

7.26

13.29

17.74

\begin{tabular}{|c|c|c|}
\hline 00 & 0 & 0 \\
\hline 0.0 & .00 & \\
\hline .00 & 0.00 & .0 \\
\hline 0.00 & 0.00 & .00 \\
\hline 0 & 000 & \\
\hline 0 & & \\
\hline 0.00 & 0.00 & 0.00 \\
\hline .00 & 0.00 & 0.00 \\
\hline 0 & & 0.00 \\
\hline 0.0 & & \\
\hline 0.0 & 0.00 & 0.00 \\
\hline 0.00 & 0.00 & 0.00 \\
\hline 0.0 & 0.00 & 0.00 \\
\hline 0.0 & 0.00 & \\
\hline 0.0 & . & \\
\hline 0.0 & 0.00 & 0.00 \\
\hline 0.0 & 0.00 & 0.00 \\
\hline 0.0 & & \\
\hline 0.0 & $\cdot$ & 0.00 \\
\hline 0.00 & 0.00 & 0.00 \\
\hline 0.00 & 0.00 & 0.00 \\
\hline 0.0 & & 0.00 \\
\hline 0.0 & 0. & 0.00 \\
\hline 0.0 & 0.00 & 0.00 \\
\hline 0.0 & 0.1 & 0.00 \\
\hline 0.00 & 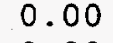 & 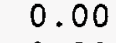 \\
\hline 0.00 & 0.0 & 0.00 \\
\hline 9.16 & 1.64 & -0.57 \\
\hline 9.51 & 126 & -0 \\
\hline 0.00 & 0.00 & 0 . \\
\hline .0 & 0.19 & -0.53 \\
\hline 1.4 & -0.73 & -0.15 \\
\hline .2 & -1. & \\
\hline .9 & -2. & 0.11 \\
\hline .0 & -1.63 & 0.51 \\
\hline 3.7 & -0.82 & 0.22 \\
\hline 1.4 & & 0 \\
\hline .9 & 0.29 & \\
\hline 8.1 & 0.8 & 0.1 \\
\hline 7.42 & 1.2 & .42 \\
\hline 4.3 & 1.6 & 0 \\
\hline 73.6 & 1 & \\
\hline 70.9 & 1.3 & 1.18 \\
\hline 70.1 & 1.1 & . \\
\hline 0.0 & 0 & \\
\hline 0.0 & 0 & \\
\hline 0.00 & 0.0 & 0.00 \\
\hline 0.06 & 0.0 &. \\
\hline 0.0 & & \\
\hline 0.0 & 0 & \\
\hline 0.06 & 0.0 & 0. \\
\hline 0.0 & 0.0 & 0.0 \\
\hline 0.0 & & \\
\hline 0.0 & 0 & \\
\hline 0.00 & 0.00 & 0 \\
\hline .00 & 0.00 & .1 \\
\hline 0.0 & & \\
\hline 0.00 & 0.0 & \\
\hline 0.00 & 0 & 0 \\
\hline 0.0 & & \\
\hline 0.0 & 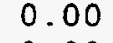 & 0.00 \\
\hline 0.00 & 0 & 0 . \\
\hline .00 & $c$ & \\
\hline & & \\
\hline. & & 0 . \\
\hline 0 & 1 & 0 \\
\hline
\end{tabular}

0.00

0.00

$0.29-0.27$

1.27

$\begin{array}{ll}1.66 & 0.90\end{array}$

$1.37 \quad 1.18$

$1.10 \quad 0.99$

$0.00 \quad 0.00$

$\begin{array}{llll}0.00 & 0.00 & 0.00\end{array}$

$\begin{array}{lll}0.00 & 0.00 & 0.00\end{array}$

$\begin{array}{llll}0.00 & 0.00 & 0.00\end{array}$

$0.00 \quad 0.00 \quad 0.00$

$\begin{array}{lll}0.00 & 0.00 & 0.00\end{array}$

$\begin{array}{lll}0.00 & 0.00 & 0.00\end{array}$

$\begin{array}{llll}0.00 & 0.00 & 0.00\end{array}$

$0.00 \quad 0.00 \quad 0.00$

$\begin{array}{llll}0.00 & 0.00 & 0.00\end{array}$

$\begin{array}{llll}0.00 & 0.00 & 0.00\end{array}$

$0.00 \quad 0.00 \quad 0.00$

$\begin{array}{llll}0.00 & 0.00 & 0.00\end{array}$

$\begin{array}{lll}0.00 & 0.00 & 0.00\end{array}$

$\begin{array}{llll}0.00 & 0.00 & 0.00\end{array}$

0.00 
$60 \quad 115 \quad 175-15.1-2.5-1340.29$ $60 \quad 116 \quad 176-15.4-2.3-1342.69$ $60 \quad 117 \quad 177-15.6 \quad-2.2-1344.98$ $60 \quad 118 \quad 178-15.8 \quad-2.1-1347.16$ $60 \quad 119 \quad 179-16.0-2.0-1349.22$ $60 \quad 120180-16.3-1.9-1351.18$ $60121 \quad 181-16.5-1.8-1353.02$ $60122 \quad 182-16.7-1.7-1354.76$ $60123183-16.9-1.6-1356.40$ $60124 \quad 184-17.1-1.5-1357.93-3.5000 .00-2.32-1363.75$ $60125185-17.3 \quad-1.4-1359.36-5.000 .96-2.29-1365.69$ $60126 \quad 186-17.5 \quad-1.3-1360.70 \quad-5.48 \quad 0.00-2.25-1368.43$

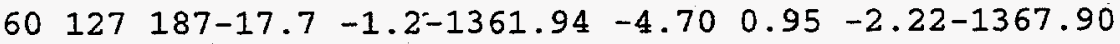
$60128 \quad 188-17.9-1.1-1363.08-3.740 .00-2.19-1369.01 \quad 101.22$ $60129189-18.1-1.0-1364.14-2.56 \quad 0.95-2.16-1367.91 \quad 110.40$ $\begin{array}{llllllll}60 & 130 & 190-18.3 & -0.9-1365.10 & -1.79 & 0.00 & -2.13-1369.02 & 117.35\end{array}$ $\begin{array}{llllllll}60 & 131 & 191-18.5 & -0.8-1365.98 & -1.72 & 0.95 & -2.10-1368.85 & 125.59\end{array}$ $\begin{array}{llllllll}60 & 132 & 192-18.7 & -0.7-1366.77 & -1.03 & 0.00 & -2.07-1369.87 & 132.65\end{array}$ $\begin{array}{lllllllll}60 & 133 & 193-18.9 & -0.7-1367.47 & -0.67 & 0.94 & -2.04-1369.24 & 141.35\end{array}$ $\begin{array}{llllllll}60 & 134 & 194-19.1 & -0.6-1368.09 & -0.21 & 0.00 & -2.01-1370.32 & 148.34\end{array}$ $\begin{array}{llllllll}60 & 135 & 195-19.3 & -0.5-1368.63 & -0.56 & 0.94 & -1.99-1370.25 & 156.49\end{array}$ $60136 \quad 196-19.4-0.4-1369.09-0.59 \quad 0.00-1.96-1371.65163 .16$ $\begin{array}{llllllll}60 & 137 & 197-19.6 & -0.3-1369.48 & -1.05 & 0.93 & -1.94-1371.53 & 171.34\end{array}$ $60138198-19.8 \quad-0.3-1369.79-0.90 \quad 0.00 \quad-1.91-1372.60178 .35$ $\begin{array}{llllllll}60 & 139 & 199-20.0 & -0.2-1370.02 & -1.22 & 0.93 & -1.89-1372.20 & 186.81\end{array}$ $60140 \quad 200-20.1-0.1-1370.19-1.100 .00-1.86-1373.15193 .94$

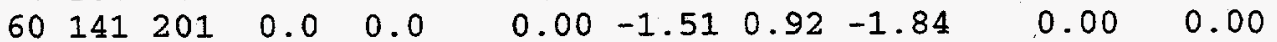
$\begin{array}{llllllllll}61 & 59 & 120 & 3.0-15.2 & -919.09 & -0.02 & 2.18 & -9.32 & -926.25 & -5.67\end{array}$

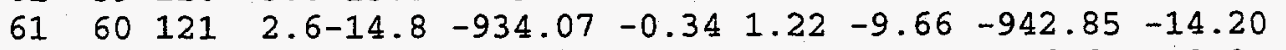

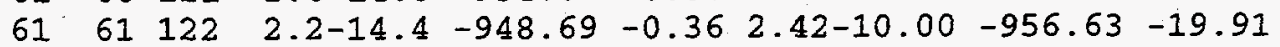
$\begin{array}{lll}61 & 62 & 123\end{array}$

$\begin{array}{lll}61 & 63 & 124\end{array}$

$\begin{array}{lll}61 & 64 & 125\end{array}$ $1.8-14.1-962.95$ $1.4-13.7-976.86$ $1.1-13.4-990.43$ 65126 $0.7-13.1-1003.66$ $\begin{array}{lll}61 & 66 & 127\end{array}$ $0.3-12.7-1016.56$ $\begin{array}{llll}61 & 67 & 128 & -0.1-12.4-1029.13\end{array}$ $\begin{array}{lllll}61 & 68 & 129 & -0.4-12.1-1041.39\end{array}$ $\begin{array}{llll}61 & 69 & 130 & -0.8-11.8-1053.34\end{array}$ $6170 \quad 131-1.2-11.5-1064.99$ $61 \quad 71 \quad 132-1.5-11.2-1076.33$ $61 \quad 72 \quad 133-1.9-10.9-1087.39$ $61 \quad 73 \quad 134-2.2-10.6-1098.15$ $61 \quad 74 \quad 135-2.6-10.3-1108.64$ $6175 \quad 136-2.9-10.1-1118.86$ $\begin{array}{llllll}61 & 76 & 137 & -3.3 & -9.8-1128.80\end{array}$ $\begin{array}{lllll}61 & 77 & 138 & -3.6 & -9.5-1138.48\end{array}$ $\begin{array}{lllll}61 & 78 & 139 & -4.0 & -9.3-1147.91\end{array}$ $\begin{array}{lllll}61 & 79 & 140 & -4.3 & -9.0-1157.08\end{array}$ $\begin{array}{llllll}61 & 80 & 141 & -4.7 & -8.8-1166.01\end{array}$ $\begin{array}{llllll}61 & 81 & 142 & -5.0 & -8.6-1174.69\end{array}$ $\begin{array}{llllll}61 & 82 & 143 & -5.3 & -8.3-1183.13\end{array}$ $\begin{array}{llllll}61 & 83 & 144 & -5.6 & -8.1-1191.35\end{array}$

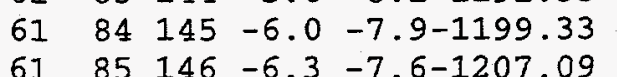
$\begin{array}{lllll}61 & 85 & 146 & -6.3 & -7.6-1207.09 \\ 61 & 86 & 147 & -6.6 & -7.4-1214.64\end{array}$ $\begin{array}{lllll}61 & 87 & 148 & -6.9 & -7.2-1221.96\end{array}$ $\begin{array}{lllll}61 & 88 & 149 & -7.2 & -7.0-1229.08\end{array}$ $\begin{array}{llllll}61 & 89 & 150 & -7.5 & -6.8-1235.99\end{array}$ $6190 \quad 151 \quad-7.8 \quad-6.6-1242.70$ $6191 \quad 152-8.1-6.4-1249.21$ $\begin{array}{lllll}61 & 92 & 153 & -8.5 & -6.2-1255.53\end{array}$ $\begin{array}{lllll}61 & 93 & 154 & -8.7 & -6.0-1261.66\end{array}$ $61 \quad 94 \quad 155-9.0-5.8-1267.60$ $\begin{array}{lllll}61 & 95 & 156 & -9.3 & -5.7-1273.36\end{array}$

$\begin{array}{llllllllll}61 & 96 & 157 & -9.6 & -5.5-1278.94 & -0.36 & 1.22 & -3.92-1282.00 & -62.78 \\ 61 & 97 & 158 & -9.9 & -5.3-1284.35 & -0.59 & 2.04 & -3.84-1286.74 & -59.44\end{array}$ 61 $\begin{array}{lllll}-0.19 & 1.22 & -9.66 & -971.59 & -26.79\end{array}$

$\begin{array}{lllll}0.07 & 2.16 & -9.35 & -983.98 & -31.11\end{array}$ $\begin{array}{llllll}0.35 & 1.22 & -9.04 & -997.90 & -36.96\end{array}$ $0.562 .15-8.75-1009.70-40.69$ $0.791 .22-8.48-1023.03-45.94$ $0.92 \quad 2.14-8.21-1034.29-49.13$ $\begin{array}{lllll}1.07 & 1.22 & -7.96-1047.07 & -53.84\end{array}$ $\begin{array}{lllll}1.12 & 2.13 & -7.72-1057.81 & -56.52\end{array}$ $1.331 .22-7.49-1069.93-60.56$ $\begin{array}{lllll}1.41 & 2.12 & -7.27-1080.07 & -62.63\end{array}$ $\begin{array}{lllll}1.73 & 1.22 & -7.07-1091.50 & -65.99\end{array}$ $\begin{array}{lllll}1.99 & 2.12 & -6.87-1100.91 & -67.33\end{array}$ $2.301 .22-6.67-1111.80-70.14$ $\begin{array}{lllll}2.17 & 2.11 & -6.49-1121.07 & -71.35\end{array}$ $2.231 .22-6.31-1131.67-73.87$ $2.12 \quad 2.10-6.14-1140.41-74.54$ $\begin{array}{lllll}1.77 & 1.22 & -5.98-1150.90 & -76.97\end{array}$ $\begin{array}{llll}1.09 & 2.09 & -5.83-1159.73 & -77.72\end{array}$ $\begin{array}{llll}1.0 .15 & 1.22 & -5.68-1170.62 & -80.53\end{array}$ $-1.242 .09-5.53-1179.38-81.23-8$ $\begin{array}{lllll}-2.45 & 1.22 & -5.40-1189.76 & -83.54\end{array}$ $-1.702 .08-5.26-1196.23-81.94$ $\begin{array}{lllll}-0.61 & 1.22 & -5.14-1203.86 & -81.49\end{array}$ $0.512 .07-5.01-1209.52-79.09$ $0.861 .22-4.90-1217.45-78.94$ $\begin{array}{lllll}0.85 & 2.07 & -4.78-1223.83 & -77.25 & -76.88 \\ 0.98 & 1.22 & -4.67-1231.55 & -76.90 & -76.07\end{array}$ $\begin{array}{llllll}0.86 & 2.06 & -4.57-1237.64 & -74.91 & -73.61\end{array}$ $0.601 .22-4.46-1245.35-74.55-73.40$ $\begin{array}{llllll}0.24 & 2.05 & -4.37-1251.29 & -72.42 & -71.27\end{array}$ $\begin{array}{llllll}0.12 & 1.22 & -4.27-1258.46 & -71.53 & -70.67\end{array}$ $-0.222 .05-4.18-1264.01-69.00$

$-68.41$ 0.00 0.00 0.00 0.00

0.00

0.00

0.00

0.00

0.00

0.00

0.00

0.00

0.00

0.00

0.00

0.00

0.00

0.00

$-77.53$

$-78.38$

$-81.09$

-81.43
-81.28

$-79.46$

$-79.05$

$\begin{array}{llll}-0.43 & 2.04 & -4.00-1275.75 & -64.60\end{array}$
0.00 0.00 0.00

0.00

0.00 0.00 0.00 0.00 0.00 0.00 0.00 0.00 0.00 0.00 0.00 0.00 0.00 0.00

0.00

0.00

0.00

0.00 0.00 0.00 0.00 0.00 0.00

0.00

0.00 0.00 0.00 0.00 0.00 0.00

0.00

0.00

0.00

0.00

0.00

0.00

0.00

0.00

0.00

0.00

$-82.97$

0.00

0.00

0.00

0.00

0.00

0.00

0.00

0.00

0.00

0.00

0.00

0.00

0.00

0.00

0.00

0.00

0.00

0.00

0.00

0.00

0.00

0.00

0.00

0.00

0.00

0.00

2.27

0.00

$1.21-0.56$

$0.43-0.66$

$-0.09$

$-1.10$

0.06

0.14

0.57

$\begin{array}{lll}-1.19 & 0.51\end{array}$

$\begin{array}{ll}-0.40 & 0.21\end{array}$

$0.14-0.37$

$0.75-0.11$

$\begin{array}{lll}1.22 & 0.37\end{array}$

$\begin{array}{ll}1.81 & 0.83\end{array}$

$2.16 \quad 1.30$

$1.75 \quad 1.15$

$1.39 \quad 1.15$

$\begin{array}{lll}0.98 & 0.86\end{array}$

$\begin{array}{lll}0.37 & 0.59\end{array}$

$0.00 \quad 0.00$

$0.00 \quad 0.00$

51
$0.00 \cdot 0.00$

$0.00 \quad 0.00$ 
$61 \quad 98 \quad 159-10.2 \quad-5.1-1289.58-0.49 \quad 1.22-3.76-1292.61-57.25$ $6199 \quad 160-10.5 \quad-5.0-1294.64-0.71 \quad 2.03 \quad-3.69-1297.01 \quad-53.57$ $61 \quad 100 \quad 161-10.7 \quad-4.8-1299.54-0.641 .22-3.62-1302.57 \quad-51.06$ $\begin{array}{llllllll}61 & 101 & 162-11.0 & -4.7-1304.27 & -0.92 & 2.03 & -3.55-1306.71 & -47.13\end{array}$ $61 \quad 102 \quad 163-11.3 \quad-4.5-1308.85-0.801 .22-3.48-1311.91-44.25$ $\begin{array}{llllllll}61 & 103 & 164-11.6 & -4.3-1313.27 & -0.86 & 2.02 & -3.41-1315.52 & -39.79\end{array}$ $\begin{array}{llllllll}61 & 104 & 165-11.8 & -4.2-1317.53 & -0.42 & 1.22 & -3.35-1320.08 & -36.29\end{array}$ $61105 \quad 166-12.1 \quad-4.0-1321.65-0.172 .02-3.28-1323.09-31.22$ $61106 \quad 167-12.3 \quad-3.9-1325.62$ $61 \quad 107168-12.6-3.7-1329.44$ $61 \quad 108 \quad 169-12.9-3.6-1333.12$ $61 \quad 109 \quad 170-13.1-3.5-1336.66$ $61 \quad 110 \quad 171-13.4 \quad-3.3-1340.07$ $61 \quad 111.172-13.6 \quad-3.2-1343.34$ $\begin{array}{llll}61 & 112 & 173-13.9 & -3.1-1346.48\end{array}$ $\begin{array}{lllll}61 & 113 & 174-14.1 & -2.9-1349.49\end{array}$ $\begin{array}{lllll}61 & 114 & 175-14.3 & -2.8-1352.38\end{array}$ $\begin{array}{llll}61 & 115 & 176-14.6 & -2.7-1355.14\end{array}$ $61116 \quad 177-14.8-2.6-1357.78$ $\begin{array}{llll}61 & 117 & 178-15.0 & -2.5-1360.30\end{array}$ $\begin{array}{llll}61 & 118 & 179-15.3 & -2.3-1362.70\end{array}$

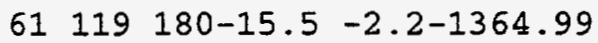
$\begin{array}{llll}61 & 120 & 181-15.7 & -2.1-1367.17\end{array}$ $\begin{array}{lllll}61 & 121 & 182-15.9 & -2.0-1369.23\end{array}$ $\begin{array}{lll}-0.71 & 1.98 & -2.50-1370.46\end{array}$ $\begin{array}{llllllll}61 & 122 & 183-16.1 & -1.9-1371.19 & -1.08 & 1.22 & -2.47-1373.52\end{array}$ $\begin{array}{lllllll}61 & 123 & 184-16.4 & -1.8-1373.04 & -2.32 & 1.98 & -2.43-1375.81\end{array}$ $\begin{array}{lllllll}61 & 124 & 185-16.6 & -1.7-1374.78 & -3.21 & 1.22 & -2.39-1379.17\end{array}$ $61125186-16.8-1.6-1376.43-4.681 .98-2.36-1381.49$ $61126 \quad 187-17.0-1.5-1377.97-5.16 \quad 1.22 \quad-2.32-1384.23$ $\begin{array}{lllllll}61 & 127 & 188-17.2 & -1.4-1379.41 & -4.36 & 1.97 & -2.29-1384.09\end{array}$ $\begin{array}{lllllll}61 & 128 & 189-17.4 & -1.3-1380.76 & -3.38 & 1.22 & -2.26-1385.18\end{array}$ $\begin{array}{lllllll}61 & 129 & 190-17.6 & -1.2-1382.01 & -2.45 & 1.97 & -2.22-1384.72\end{array}$ $61130191-17.8-1.1-1383.17-1.74 \quad 1.22-2.19-1385.89107 .77$ $\begin{array}{llllllll}61 & 131 & 192-18.0 & -1.0-1384.24 & -1.40 & 1.97 & -2.16-1385.84 & 115.89\end{array}$ $\begin{array}{llllllll}61 & 132 & 193-18.2 & -0.9-1385.22 & -0.75 & 1.22 & -2.13-1386.88 & 122.91\end{array}$ $\begin{array}{llllllll}61 & 133 & 194-18.4 & -0.8-1386.11 & -0.30 & 1.96 & -2.10-1386.55 & 131.31\end{array}$ $\begin{array}{llllllll}61 & 134 & 195-18.6 & -0.8-1386.92 & 0.03 & 1.22 & -2.08-1387.74 & 138.19\end{array}$ $\begin{array}{llllllll}61 & 135 & 196-18.7 & -0.7-1387.64 & -0.46 & 1.96 & -2.05-1388.19 & 145.82\end{array}$

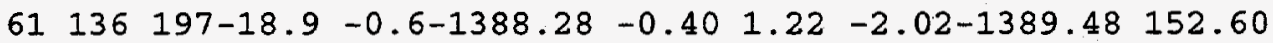
$\begin{array}{lllllllll}61 & 137 & 198-19.1 & -0.5-1388.84 & -0.84 & 1.96 & -1.99-1389.72 & 160.43\end{array}$ $\begin{array}{llllllll}61 & 138 & 199-19.3 & -0.4-1389.33 & -0.70 & 1.22 & -1.97-1390.78 & 167.45\end{array}$ $\begin{array}{llllllll}61 & 139 & 200-19.5 & -0.4-1389.73 & -1.03 & 1.95 & -1.94-1390.75 & 175.54\end{array}$ $\begin{array}{llllllll}61 & 140 & 201-19.6 & -0.3-1390.06 & -0.95 & 1.22 & -1.92-1391.71 & 182.65\end{array}$ $\begin{array}{llllllll}61 & 141 & 202-19.8 & -0.2-1390.32 & -1.31 & 1.95 & -1.89-1391.58 & 190.86\end{array}$ $\begin{array}{lllllllll}61 & 142 & 203-20.0 & -0.1-1390.51 & -1.56 & 1.22 & -1.87-1392.72 & 197.79\end{array}$ $\begin{array}{lllllllll}61 & 143 & 204-20.1 & -0.1-1390.62 & -2.11 & 1.95 & -1.85-1392.63 & 205.95\end{array}$

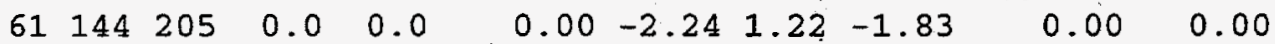
$\begin{array}{llllllllll}62 & 61 & 123 & 2.7-14.8 & -946.22 & -0.37 & 1.22 & -9.66 & -955.03 & -11.03\end{array}$

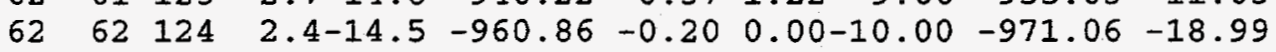
$\begin{array}{lllll}62 & 63 & 125 & 2.0-14.1 & -975.15\end{array}$ $\begin{array}{lllll}62 & 64 & 126 & 1.6-13.8 & -989.10\end{array}$ $\begin{array}{llll}62 & 65 & 127 & 1.2-13.4-1002.70\end{array}$ $\begin{array}{llll}62 & 66 & 128 & 0.9-13.1-1015.98\end{array}$ $\begin{array}{llll}62 & 67 & 129 & 0.5-12.8-1028.92\end{array}$ $\begin{array}{llll}62 & 68 & 130 & 0.1-12.5-1041.55\end{array}$ $\begin{array}{llll}62 & 69 & 131 & -0.2-12.2-1053.86\end{array}$ $\begin{array}{llll}62 & 70 & 132 & -0.6-11.9-1065.87\end{array}$ $\begin{array}{llll}62 & 71 & 133 & -1.0-11.6-1077.58\end{array}$ $\begin{array}{llll}62 & 72 & 134 & -1.3-11.3-1088.99\end{array}$ $6273 \quad 135-1.7-11.0-1100.11$ $\begin{array}{lllll}62 & 74 & 136 & -2.0-10.7-1110.96\end{array}$ $\begin{array}{llll}62 & 75 & 137 & -2.4-10.4-1121.52\end{array}$ $\begin{array}{llll}62 & 76 & 138 & -2.7-10.2-1131.81\end{array}$ $\begin{array}{lllll}62 & 77 & 139 & -3.1 & -9.9-1141.84\end{array}$ $\begin{array}{lllll}62 & 78 & 140 & -3.4 & -9.6-1151.60\end{array}$ $\begin{array}{lllll}62 & 79 & 141 & -3.7 & -9.4-1161.11\end{array}$ $0.351 .22-3.22-1327.27-27.34$ $\begin{array}{llll}0.52 & 2.01 & -3.17-1330.07 & -22.06\end{array}$ $0.871 .22-3.11-1334.14-18.06$ $0.872 .01-3.05-1336.84-12.69$ $\begin{array}{llll}1.20 & 1.22 & -3.00-1340.65 & -8.43\end{array}$ $1.152 .00-2.95-1343.14$ $1.311 .22-2.90-1346.85$ $1.042 .00-2.85-1349.30$ $1.041 .22-2.80-1352.92$ $0.792 .00-2.76-1355.11$ $0.791 .22-2.71-1358.48$ $0.491 .99-2.67-1360.49$ $0.331 .22-2.63-1363.78$ $0.541 .99-2.58-1365.05$ $0.18 \quad 1.22-2.54-1368.31$

\begin{tabular}{|c|c|c|}
\hline 0 & U & 0.00 \\
\hline م0 & 0.00 & 00 \\
\hline 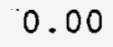 & & \\
\hline .00 & .00 & .00 \\
\hline 0 & .00 & .00 \\
\hline ח & 00 & 00 \\
\hline & 0 & .00 \\
\hline .00 & .00 & .00 \\
\hline 0 & .00 & 00 \\
\hline & & \\
\hline 0 & 00 & 00 \\
\hline .0 & .00 & 0.00 \\
\hline חת & & \\
\hline & & \\
\hline & & \\
\hline .0 & .00 & 0.00 \\
\hline 0 & 0.00 & 0.00 \\
\hline$\therefore$ & & .00 \\
\hline & & \\
\hline 0.0 & & 0.00 \\
\hline 0 & 0.00 & 0.00 \\
\hline & & 00 \\
\hline & & \\
\hline & & 0.00 \\
\hline 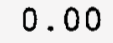 & & 0.00 \\
\hline & & \\
\hline & & \\
\hline & & \\
\hline & & \\
\hline & & \\
\hline & & \\
\hline 8 & & 00 \\
\hline & & \\
\hline & & \\
\hline 0. & 0. & 00 \\
\hline & & \\
\hline & & \\
\hline. & & \\
\hline 0.0 & 0. & 0 \\
\hline & & \\
\hline & & \\
\hline & & \\
\hline 0. & & \\
\hline & & \\
\hline & & \\
\hline & & \\
\hline 0. & & \\
\hline & & \\
\hline & & \\
\hline & & \\
\hline & & \\
\hline & & \\
\hline & & 0. \\
\hline & & 00 \\
\hline & & \\
\hline & & \\
\hline & & 0 \\
\hline 0.0 & & 0.00 \\
\hline & & \\
\hline & & \\
\hline & & 0.00 \\
\hline n & & 0. \\
\hline & & \\
\hline & & 07 \\
\hline 0.0 & 0.00 & .00 \\
\hline & 0.79 & 0 \\
\hline
\end{tabular}

$\begin{array}{llllll}0.04 & 1.21 & -9.67 & -983.58 & -23.43\end{array}$ $\begin{array}{llllll}0.39 & 0.00 & -9.36 & -998.06 & -29.85\end{array}$ $\begin{array}{llllll}0.57 & 1.19 & -9.06-1009.99 & -33.71\end{array}$ $\begin{array}{lllll}0.76 & 0.00 & -8.77-1023.99 & -39.63\end{array}$ $\begin{array}{lllll}0.93 & 1.18 & -8.50-1035.31 & -42.88\end{array}$ $\begin{array}{llllll}1.03 & 0.00 & -8.24-1048.76 & -48.26\end{array}$ $\begin{array}{lllll}1.12 & 1.17 & -7.99-1059.56 & -50.99\end{array}$ $\begin{array}{lllll}1.19 & 0.00 & -7.75-1072.44 & -55.79\end{array}$ $\begin{array}{lllll}1.38 & 1.16 & -7.53-1082.57 & -57.85\end{array}$ $\begin{array}{lllll}1.68 & 0.00 & -7.31-1094.62 & -61.83\end{array}$ $\begin{array}{lllll}1.89 & 1.15 & -7.10-1104.18 & -63.32\end{array}$ $\begin{array}{llllll}2.15 & 0.00 & -6.90-1115.71 & -66.78\end{array}$ $2.101 .14-6.71-1124.99-67.99$ $\begin{array}{llllll}2.18 & 0.00 & -6.53-1136.16 & -71.09\end{array}$ $2.12 \quad 1.13-6.36-1144.95-71.80$ $\begin{array}{lllll}1.82 & 0.00 & -6.19-1155.97 & -74.76\end{array}$ $1.16 \quad 1.12-6.03-1164.86-75.57-75.94$ 


\begin{tabular}{|c|c|c|c|c|c|c|c|c|c|c|c|}
\hline 62 & 80 & 42 & -4.1 & $-9.1-1170.37$ & .00 & .00 & $-5.87-1176.25$ & -78.89 & -78.99 & -0.10 & 0.10 \\
\hline 62 & 1 & 143 & -4.4 & $-8.9-1179.39$ & -1.09 & 11 & $-5.72-1185.09$ & -79.66 & -79.53 & -0.96 & 0.13 \\
\hline 62 & 2 & 14 & .7 & 1188.16 & -2.28 & 00 & 02 & 52 & 7 & & \\
\hline 62 & 83 & 45 & .5 .1 & 70 & -1.51 & 10 & 55 & .98 & & & \\
\hline 52 & 84 & 46 & -5.4 & 1205.01 & -0.41 & 0.00 & $-5.31-1210.73$ & -81.09 & -81.00 & -0.32 & \\
\hline 02 & 85 & 47 & & 1213.09 & 0.67 & 1.09 & $-5.18-1216.51$ & -78.80 & -79.28 & & \\
\hline 62 & 6 & 18 & & 20.95 & & & & & & & \\
\hline 62 & 87 & 19 & -6.3 & -7.5 & 1.22 & .08 & 31.23 & -77.37 & -77.15 & 1.44 & \\
\hline 52 & 88 & 50 & -6.6 & 236.02 & 1.31 & .00 & 9.54 & -77.61 & -77.06 & 86 & \\
\hline 52 & 89 & 51 & -7.0 & 243.24 & 1.24 & 1.08 & $-4.72-$ & 65 & 59 & 30 & \\
\hline 2 & 90 & 52 & $-7,3$ & 0.26 & 0.90 & 0 & 53.97 & & & & \\
\hline 2 & & 53 & - & 57.08 & 2 & 7 & 60.00 & -73.85 & -72.57 & 1.80 & \\
\hline 2 & 9 & 54 & & 63.69 & 0.38 & 0.00 & -1267.73 & -73.51 & 46 & 1.43 & \\
\hline 2 & 93 & 55 & -8.2 & 270.12 & 0.06 & 1.06 & $-4.32-1273.32$ & -71.03 & -70.20 & 0.89 & \\
\hline 2 & 94 & 56 & -8.5 & -6 & -0.01 & 0.00 & 0.59 & -70 & 37 & 85 & \\
\hline 62 & & 57 & -8 & -6 & -6 & 1 & -4 & 8 & 66.77 & 0.29 & \\
\hline 62 & 96 & 58 & -9.0 & 88.27 & -0.16 & 0.00 & 92.48 & -65.98 & 0.00 & 0.00 & \\
\hline 2 & 97 & 59 & 9.3 & 93.96 & -0.48 & 1.04 & 7.36 & -62 & 0.00 & 0.00 & \\
\hline 52 & 98 & 60 & & & & 0.00 & .79 & -6 & 0 & 0 & \\
\hline 2 & 99 & 61 & & -5 & -6 & 1 & 2 & & 0.00 & 0.00 & 0.00 \\
\hline 62 & 100 & $2<-$ & -10.2 & 09.99 & -0.67 & 0.00 & -3.73 & -55.61 & .00 & 0.00 & \\
\hline 2 & 01 & & 10.4 & -4.9 & -0.97 & 1.03 & 3.60 & -51.74 & .00 & 00 & \\
\hline 2 & & & & -4.8 & & 0.00 & .31 & -49 & 10 & & \\
\hline 2 & 3 & & & -4 & $-c$ & 1. & 7 & - & & 0 & 0.00 \\
\hline 62 & 04 & 06 & -11.2 & $-4.5-1329.07$ & -0.37 & 0.00 & $-3.46-$ & -41.82 & 0 & Do & 0 \\
\hline 62 & 05 & & 11.5 & 3.45 & -0.15 & 1.02 & .97 & -36 & 0 & 00 & \\
\hline 2 & & & 11.8 & -4.2 & & 0 & .78 & & & & \\
\hline 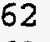 & 07 & & & -4 & & 1.01 & 2 & & 10 & 0 & \\
\hline 62 & 08 & $170-$ & -12.3 & $-3.9-$ & 0.6 & 0.00 & $-3.21-$ & -24.93 & .00 & 00 & 0.00 \\
\hline 62 & 109 & $\perp$ & 12.5 & -3.7 & 0. & 1.00 & .01 & -1 & 0 & & \\
\hline 2 & & & & -3.6 & & & 29 & & & & \\
\hline ? & 1 & & & -3 & & .00 & 33 & & 0 & 0.00 & 0 \\
\hline 2 & 12 & 14. & 13.3 & $-3 \cdot 3-1$ & 1.05 & 0.00 & -2.99 & -6.35 & .00 & 0.00 & 0 \\
\hline 62 & 13 & J- & 13.5 & 3.31 & 0.7 & 0.99 & 4.46 & -0.75 & 10 & 00 & 00 \\
\hline 2 & 14 & & & -3 & 0 . & & -2 & & & & \\
\hline & 5 & & & & & & & & & & \\
\hline 62 & 16 & 78. & 14.2 & $-2.8-1$ & 0.70 & 0.00 & 4.40 & 13.53 & .00 & .00 & 0 \\
\hline 52 & 117 & $19-$ & 14.5 & $-2 \cdot 7-1$ & 0.50 & 0.98 & -2.75 & 19.68 & 0.00 & 0.00 & 0.00 \\
\hline 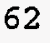 & 18 & & 7 & -2.8 & 0 . & 0 & -2 & 2 & 0 & 0 & \\
\hline & 9 & & & & 0 & & & & & & \\
\hline 2 & 20 & $82 \quad-2$ & 2 & $-2.3-1$ & 0.23 & 0.00 & 4.99 & 35.22 & 0.00 & .00 & 0 \\
\hline 2 & 21 & 83. & 5.4 & $-2 \cdot 2-1$ & -0.53 & 0.97 & 7.03 & 41. & 0 & 10 & 0.00 \\
\hline 2 & 2 & & & -2 & -5 & & -2 & & & & \\
\hline & 3 & & & -2 & & & 9 & & & & 0 \\
\hline & 24 & 8 & 0 & -1.9 & -2. & 0.00 & 5.50 & 56. & 0 & 10 & 0 \\
\hline 2 & 25 & & 5.2 & -1.8 & -4 & 0. & 3.76 & 61. & 0 & 10 & 0.00 \\
\hline 2 & 126 & & 4 & -1 & & & & & & & \\
\hline & & & & -1.6 & & & 1.74 & & & & 0 \\
\hline . & 128 & 190 & 9 & -1.5 & -3.02 & 0. & 3.24 & 81.55 & 0 & 10 & 0.00 \\
\hline 2 & 129 & & .1 & -1.4 & -2.1 & 0.95 & -2 . & 0. & 0 & & 00 \\
\hline & 30 & & & 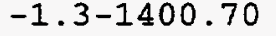 & & & & & & & \\
\hline & 131 & 93 & .5 & -1.2 & -0.93 & 0.95 & .18 & 2 & 0 & 10 & 00 \\
\hline 2 & 132 & 94 & .7 & $-1.1-$ & -0.22 & 0.00 & 5.56 & 1 & 0 & 0 & 0.00 \\
\hline & 133 & & & 1.0 & 0.1 & & -2 & & & & \\
\hline & & & & & & & & & & & \\
\hline 2 & 135 & 19 & 8.2 & -0.9 & -0.02 & 0.94 & 7.32 & & 0 & & 0.00 \\
\hline & 6 & 198 & & -0.8 & -0.03 & . & -2 . & 140 & 0. & & 0 \\
\hline 2 & 137 & & & 0.7 & -0.3 & & & 8 & 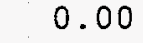 & & 0 \\
\hline & & & & & & & & & & & \\
\hline 2 & 139 & 0 & .9 & .94 & -0.60 & 0.93 & .61 & & & & 0 . \\
\hline & 40 & 02 & & -0. & & $\theta^{\circ}$ & -1 & & & & 0.00 \\
\hline 2 & 141 & 93 & & 19.8 & 0.87 & .92 & $1.95-$ & 7 & 0.0 & 0 & 0 . \\
\hline & 42 & & & & & & & & & & \\
\hline & 143 & 95 & .6 & 0.50 & 1.71 & & 3.19 & & & & 0.00 \\
\hline & & & & 1 & & & 4.44 & 199. & 0. & 0 . & 0.00 \\
\hline & & & & & & & & & & & \\
\hline
\end{tabular}




\begin{tabular}{|c|c|c|c|c|c|c|c|c|c|c|c|}
\hline & 146 & 08 & 0.0 & 0.0 & -2.77 & 0.00 & -1.83 & 1 & 0 & 0.00 & 0.00 \\
\hline & 62 & 25 & & 8.24 & 0.16 & 21 & -9.67 & & 0.00 & 0.00 & 0.00 \\
\hline & 63 & 6 & & 72.90 & .17 & 35 & $10.00-980.35$ & -12.92 & 0.00 & 0.00 & 0.00 \\
\hline & 64 & 27 & 2 . & -987.22 & 0.37 & 1.21 & $-9.67-995.32$ & -19.82 & 00 & .00 & 0.00 \\
\hline & 6 & 28 & & 1001.20 & 0.49 & 14 & $36-1007$ & 37 & & & 10 \\
\hline & 66 & 29 & & & & & & & 0.00 & 0.00 & .00 \\
\hline & 67 & 30 & 1. & 1028.16 & 0.73 & .13 & $-8.79-1034.08$ & -34.38 & 0.00 & 0.00 & 0.00 \\
\hline & 68 & 131 & 0.7 & $12.8-1041.15$ & 0.76 & 1.21 & $-8.52-1047.70$ & -39.92 & 0.00 & .00 & 0.00 \\
\hline & 69 & 32 & & $2.5-1053.83$ & 0.78 & 12 & 1059.19 & -4 & 00 & & 0.00 \\
\hline & 70 & 133 & & $2-1066.20$ & 0.86 & 21 & & & 0.00 & 0.00 & 0.00 \\
\hline & 71 & 34 & -0.4 & $11.9-1078.26$ & 1.07 & 2.11 & 1082.86 & -50.87 & 0.00 & 0.00 & 0.00 \\
\hline & 72 & 35 & 0.8 & $11.6-1090.03$ & 1.37 & 1.21 & $-7.56-1095.01$ & -54.95 & 0.00 & 00 & 0.00 \\
\hline & 73 & 36 & 1.1 & 101.51 & 1.65 & 2.11 & 10 & 6 & 0 & & 0.00 \\
\hline & 74 & 37 & -1.5 & $11.0-1112.70$ & 1.97 & 1.21 & $-7.14-1116.66$ & -60.45 & 0.00 & 0.00 & 0.00 \\
\hline & 75 & 38 & -1.8 & $10.8-1123.61$ & 1.93 & 2.10 & -1126.52 & -62.24 & 0.00 & 0.00 & 0.00 \\
\hline & 76 & 39 & $-2.2-$ & $10.5-1134.25$ & 1.94 & 1.21 & $-6.75-1137.85$ & -65.50 & 0.00 & 00 & 0.00 \\
\hline & 77 & 40 & $-2.5-$ & $10.2-1144.62$ & 2.04 & 2.09 & 7.06 & -66 & 0 & & 0.00 \\
\hline & 78 & 41 & -2.8 & $10.0-1154.72$ & 1.89 & 1.21 & 1158.02 & -69.53 & -69.91 & 1.51 & -0.38 \\
\hline & 79 & 12 & -3.2 & 1164.57 & 1.27 & .08 & .45 & -70.88 & -71.59 & 0.56 & -0.71 \\
\hline & 80 & 43 & -3.5 & $-9.5-1174.16$ & 0.21 & 1.21 & $-6.07-1178.82$ & -74.18 & -74.38 & 0.01 & -0.20 \\
\hline & 81 & 44 & -3.8 & 3.51 & -0.77 & 2.08 & 88.12 & -75.41 & 5.65 & -1.01 & -0.24 \\
\hline & 82 & 45 & -4.2 & -1192.61 & -2.00 & 1.21 & $7-1199.17$ & -78.39 & -78.00 & -1.61 & 0.39 \\
\hline & 83 & 6 & -4.5 & $-8.7-1201.48$ & -1.13 & 2.07 & $-5.63-1206.16$ & -77.31 & -77.13 & -0.95 & 0.18 \\
\hline & 84 & 47 & -4.8 & $-8.5-1210.11$ & -0.13 & 1.21 & $-5.49-1214.52$ & -77.60 & .56 & -0.09 & 0.04 \\
\hline & 85 & 48 & -5 & 3.51 & 0.8 & & 5 & -75.96 & 24 & 7 & .28 \\
\hline & 86 & 49 & -5.4 & $-8.1-1226.69$ & 1.18 & 1.21 & $-5.23-1229.53$ & -76.46 & -76.45 & 1.19 & 0.01 \\
\hline & 87 & 50 & -5.8 & $-7.8-1234.64$ & 1.32 & 2.06 & $-5.11-1236.37$ & -75.24 & -74.80 & 1.76 & 0.44 \\
\hline & 88 & 51 & -6.1 & 2.38 & 1.39 & 1.21 & -4 & -75.57 & .66 & 2.30 & 0.91 \\
\hline & 89 & 52 & -6 & 9.91 & 1.28 & 2.05 & $-4.88-1251.46$ & -74.18 & -72.90 & 2.56 & 1.28 \\
\hline & 90 & 53 & -6.7 & $-7.2-1257.23$ & 1.02 & 1.21 & $-4.77-1259.77$ & -74.42 & -73.38 & 2.06 & 1.04 \\
\hline & 91 & 54 & -7.0 & $-7.0-1264.35$ & 0.70 & 2.04 & 6.27 & -72.85 & & 1.80 & 1.10 \\
\hline & 92 & 5 & -7.3 & L.27 & 0.54 & 1.21 & 4.08 & -72 & & 30 & 0.76 \\
\hline & 93 & 56 & -7.6 & $-6.6-1$. & 0.25 & 2.04 & $-4.46-1280.16$ & -70.60 & -70.10 & 0 . & 0.50 \\
\hline & 94 & 57 & -7.9 & $-6.4-1284.52$ & 0.21 & 1.21 & $-4.36-1287.47$ & -69.83 & -6 & 0 . & 0.36 \\
\hline & 95 & 58 & -8.2 & 0.86 & 0.08 & 2.03 & .18 & -67 & & 8 & .26 \\
\hline & 96 & 9 & -8 & .02 & & & & & 6 & 0.16 & 0.24 \\
\hline & 97 & 60 & -8.7 & $-5.9-1$ & -0.42 & 2.03 & $-4 \cdot 10-1$ & -63.64 & 0.00 & 0.00 & 0.00 \\
\hline & 98 & 61 & -9.0 & $-5.7-1308.80$ & -0.45 & 1.21 & $-4.01-1312.05$ & -62.13 & 0 . & 0.00 & 0.00 \\
\hline & 99 & 52 & -9.3 & -5.5 & -0 & 2.02 & 5 & & & & 00 \\
\hline & 0 & 63 & -9 & -5 & & & 4 & -57 & 10 & 0.00 & 0.00 \\
\hline & 1 & 64 & -9.9 & 5.16 & -1.03 & 2.02 & $-3 \cdot 78-1$ & -53.81 & 0 . & 0 . & 0.00 \\
\hline & 02 & 55 & -10.1 & $-5 \cdot 0-1$ & -0.98 & 1.21 & $-3.71-1333.75$ & -51.54 & 0.00 & 0 . & 0.00 \\
\hline & 03 & 0 & 10 & -4 & -1 & 2.01 & -3 & & & & \\
\hline & 4 & & & -4 & & & $\$ 3.10$ & -44 & ( & 0.00 & 0.00 \\
\hline & 5 & & & 4.68 & -0 . & 2.01 & $-3.50-1$ & -40.30 & 0.00 & 0.00 & 0.00 \\
\hline & 106 & 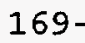 & -11.2 & 9.16 & -0.06 & 1.21 & $-3.43-1351.45$ & -36.96 & 0.00 & 0.00 & 0.00 \\
\hline & 07 & & 11 & -4 & & 2.00 & & & & & \\
\hline & 08 & & 11 & .70 & & 1.21 & 9.48 & -28 & .00 & 0 & 0.00 \\
\hline & 09 & & -12 & 1.75 & 0.34 & 2.00 & $-3.25-1$ & -23.95 & 0.00 & 0 . & 0.00 \\
\hline & 10 & & -12.2 & -3.8 & 0 & 1.21 & -3 & & 00 & 0 & 00 \\
\hline & 11 & & & -3 & & 1.99 & & & & & \\
\hline & 12 & & & -3 & & 1.21 & $-3.09-1$ & -11 & 0 & 0 . & 0.00 \\
\hline & 3 & & 13.0 & -3.4 & 0 . & 1.99 & $-3.03-1376.88$ & -5 . & 0.00 & 0.00 & 0.00 \\
\hline & 114 & {$[\%$} & 13.2 & -3.3 & 0 . & 1.21 & & -1 & & & 00 \\
\hline & 115 & & & -3 & & 98 & -2 . & & & & 0.00 \\
\hline & 116 & & 13 & -3. & 0.67 & 1.21 & $-2.88-1387.27$ & 7. & 0.00 & 0.00 & 0.00 \\
\hline & 117 & 180 & 13 & -2.5 & 0. & 1.98 & $-2.84-1389.63$ & 13. & 0 & 0. & 0.00 \\
\hline & & & & & & 21 & 25 & & & & 0.00 \\
\hline & 119 & 18. & 4 & -2.7 & 0. & 1.98 & $-2.75-1$ & 24 & & 0. & 0.00 \\
\hline & 120 & 18 & 14.6 & -2.6 & 0 . & 1.21 & $-2.70-1$ & 28 & 0.00 & 0.00 & 0.00 \\
\hline & 121 & 18 & & -2 & -0 . & 1.97 & -2 & & & & 0.00 \\
\hline & 122 & 18 & & 2.38 & 0 . & 1.21 & $-2.62-1$ & 39. & 0 & 0.00 & 0.00 \\
\hline & 123 & 18 & 5.3 & $-2.2-$ & -1.5 & 1.97 & $-2.58-1407.26$ & 44.45 & 0.00 & 0.00 & 0.00 \\
\hline & 124 & 18 & & 6.84 & & 1.21 & $-2.54-1410.84$ & 48 . & 0.00 & 0.00 & 0.00 \\
\hline & 125 & . & & -2.0 & -1 & 1.97 & -2.500 & & 0 & 0.00 & 0.00 \\
\hline & & & 5.9 & 0.87 & & 1.21 & $-2.47-1416.71$ & 59.21 & 0.00 & 0.00 & 0.00 \\
\hline
\end{tabular}


$\begin{array}{lllllllll}63 & 127 & 190-16.1 & -1.8-1412.73 & -3.75 & 1.96 & -2.43-1416.95\end{array}$

$63128191-16.3-1.7-1414.48-2.761 .21-2.39-1418.43$

$63129192-16.5-1.6-1416.14-2.001 .96-2.36-1418.55$

$63130193-16.7-1.5-1417.70-1.30 \quad 1.21-2.33-1420.12$

$63131194-16.9-1.4-1419.17$

$63 \quad 132195-17.1-1.3-1420.54$

$63 \quad 133196-17.3-1.2-1421.81$

$63134197-17.5-1.1-1423.00$

$63 \quad 135198-17.7-1.1-1424.10$

$63136199-17.9-1.0-1425.11$

$\begin{array}{llll}63 & 137 & 200-18.1 & -0.9-1426.03\end{array}$

$63 \quad 138 \quad 201-18.3 \quad-0.8-1426.88$

$63139202-18.4-0.7-1427.63$

$63140203-18.6-0.6-1428.31$

$63141204-18.8-0.6-1428.91$

$63142205-19.0-0.5-1429.44$

$63143 \quad 206-19.1-0.4-1429.88$

$\begin{array}{llll}63 & 144 & 207-19.3 & -0.3-1430.26\end{array}$

$63145208-19.5-0.3-1430.56$

$63146209-19.6-0.2-1430.79$

$63147210-19.8-0.1-1430.95$

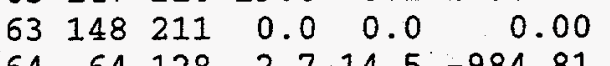

$\begin{array}{lllll}64 & 64 & 128 & 2.7-14.5 & -984.81 \\ 64 & 65 & 129 & 2.3-14.2 & -999.16\end{array}$

$\begin{array}{lllll}64 & 65 & 129 & 2.3-14.2 & -999.16 \\ 64 & 66 & 130 & 1.9-13.8-1013.17\end{array}$

$\begin{array}{llll}64 & 67 & 131 & 1.6-13.5-1026.85\end{array}$

$64 \quad 68 \quad 132,1.2-13.2-1040.20$

$64.69133 \quad 0.9-12.9-1053.24$

$64 \quad 70 \quad 134 \quad 0.5-12.6-1065.97$

$64 \quad 71 \quad 135 \quad 0.1-12.3-1078.39$

$64 \quad 72 \quad 136-0.2-12.0-1090.51$

$64 \quad 73 \quad 137-0.6-11.7-1102.34$

$6474 \quad 138-0.9-11.4-1113.88$

$6475 \quad 139-1.2-11.1-1125.14$

$6476140-1.6-10.8-1136.12$

$6477 \quad 141-1.9-10.6-1146.83$

$64 \quad 78 \quad 142-2.3-10.3-1157.28$

$\begin{array}{llll}64 & 79 & 143 & -2.6-10.1-1167.46\end{array}$

$\begin{array}{llllll}64 & 80 & 144 & -2.9 & -9.8 & -1177.39\end{array}$

$\begin{array}{llllll}64 & 81 & 145 & -3.3 & -9.5-1187.06\end{array}$

$\begin{array}{llllll}64 & 82 & 146 & -3.6 & -9.3-1196.49\end{array}$

$\begin{array}{llllll}64 & 83 & 147 & -3.9 & -9.1-1205.68\end{array}$

$\begin{array}{llllll}64 & 84 & 148 & -4.2 & -8.8 & -1214.63\end{array}$

$\begin{array}{llllll}64 & 85 & 149 & -4.6 & -8.6 & -1223.35\end{array}$

$\begin{array}{llllll}64 & 86 & 150 & -4.9 & -8.4-1231.85\end{array}$

$\begin{array}{llllll}64 & 87 & 151 & -5.2 & -8.2-1240.12\end{array}$

$\begin{array}{llllll}64 & 88 & 152 & -5.5 & -7.9 & -1248.17\end{array}$

$\begin{array}{lllll}64 & 89 & 153 & -5.8 & -7.7-1256.01\end{array}$

$\begin{array}{llllll}64 & 90 & 154 & -6.1 & -7.5-1263.63\end{array}$

$\begin{array}{llllll}64 & 91 & 155 & -6.4 & -7.3-1271.05\end{array}$

$\begin{array}{llllll}64 & 92 & 156 & -6.7 & -7.1-1278.27\end{array}$

$6493.157-7.0-6.9-1285.29$

$\begin{array}{llllll}64 & 94 & 158 & -7.3 & -6.7-1292.12\end{array}$

$\begin{array}{llllll}64 & 95 & 159 & -7.6 & -6.5 & -1298.75\end{array}$

$\begin{array}{llllll}64 & 96 & 160 & -7.9 & -6.3-1305.20\end{array}$

$\begin{array}{llllll}64 & 97 & 161 & -8.2 & -6.2-1311.46\end{array}$

$\begin{array}{lllll}64 & 98 & 162 & -8.5 & -6.0-1317.54\end{array}$

$\begin{array}{llllll}64 & 99 & 163 & -8.7 & -5.8-1323.44\end{array}$

$\begin{array}{llllll}64 & 100 & 164 & -9.0 & -5.6-1329.17\end{array}$

$\begin{array}{lllll}64 & 101 & 165 & -9.3 & -5.5-1334.73\end{array}$

$64102166-9.6-5.3-1340.13$

$\begin{array}{llllll}64 & 103 & 167 & -9.8 & -5.1-1345.35\end{array}$

$64104168-10.1-5.0-1350.42$

$64105 \quad 169-10.4-4.8-1355.33$

$64106170-10.6-4.7-1360.08$

$64107 \quad 171-10.9-4.5-1364.68$
$-0.601 .95-2.29-1420.11 \quad 96.17$

$0.031 .21-2.26-1421.56 \quad 102.79$

$0.401 .95-2.23-1421.69110 .73$

$0.681 .21-2.20-1423.32117 .18$

$0.231 .95-2.17-1424.09124 .47$

$0.291 .21-2.14-1425.75 \quad 130.88$

$-0.08 \quad 1.94-2.11-1426.28 \quad 138.42$

$0.07 \quad 1.21-2.09-1427.69 \quad 145.10$

$-0.271 .94-2.06-1428.02 \quad 152.83$

$\begin{array}{llllll}-0.19 & 1.21 & -2.03-1429.33 & 159.59\end{array}$

$\begin{array}{llllll}0.64 & 1.94 & -2.01-1429.62 & 167.37\end{array}$

$\begin{array}{llll}-0.92 & 1.21 & -1.98-1431.13 & 173.93\end{array}$

$-1.481 .93-1.96-1431.39181 .75$

$-1.621 .21-1.93-1432.60 \quad 188.60$

$-2.511 .93-1.91-1433.05 \quad 196.23$

$\begin{array}{lllll}-2.69 & 1.21 & -1.89-1434.16 & 203.19\end{array}$

$-3.091 .93-1.86-1433.98 \quad 211.44$

$\begin{array}{lllll}-2.98 & 1.21 & -1.84 & 0.00 & 0.00\end{array}$

$0.600 .00-10.00-994.21-11.44$

$0.651 .19-9.68-1006.99-16.15$

$\begin{array}{lllll}0.71 & 0.00 & -9.37-1021.83 & -22.92\end{array}$

$0.771 .18-9.08-1033.98-26.99$

$0.67 \cdot 0.00-8.80-1048.34-33.28$

$\begin{array}{llllll}0.65 & 1.17 & -8.54-1059.96 & -36.83\end{array}$

$0.70 \quad 0.00-8.29-1073.56-42.35$

$0.891 .16-8.04-1084.39-45.11$

$1.20 \quad 0.00-7.81-1097.12-49.78$

$1.47 \quad 1.15-7.59-1107.31-51.90$

$1.58 \quad 0.00-7.38-1119.68-56.19$

$\begin{array}{llllll}1.73 & 1.14 & -7.17-1129.45 & -57.89\end{array}$

$1.70 \quad 0.00-6.98-1141.40-61.77$

$2.551 .13-6.79-1149.94-62.24$

$1.690 .00-6.61-1162.20-66.42$

$1.08 \quad 1.12-6.44-1171.70-67.85$

$0.200 .00-6.27-1183.46-71.54$

$-0.901 .11-6.11-1192.96-72.98$

$-2.06 \quad 0.00-5.96-1204.51-76.45$

$-1.171 .10-5.81-1211.56-75.43$

$\begin{array}{lllll}-0.15 & 0.00 & -5.67-1220.45 & -76.25\end{array}$

$0.851 .09-5.53-1226.95-74.67$

$1.30 \quad 0.00-5.40-1235.95-75.60$

$1.511 .08-5.27-1242.80-74.38$

$1.590 .00 \quad-5.15-1251.73-75.24$

$1.58 \quad 1.08-5.03-1258.39-73.83$

$1.33 \quad 0.00-4.92-1267.23-74.60$

$1.051 .07-4.81-1273.75-73.05$

$0.890 .00-4.71-1282.09-73.32$

$0.621 .06-4.60-1288.22-71.37$

$0.56 \quad 0.00-4.50-1296.06-71.15$

$0.261 .05-4.41-1301.85-68.86$

$0.210 .00-4.32-1309.30-68.24$

$\begin{array}{lllll}-0.13 & 1.04 & -4.23-1314.77 & -65.64\end{array}$

$\begin{array}{lllll}-0.19 & 0.00 & -4.14-1321.87 & -64.67\end{array}$

$\begin{array}{lllll}-0.52 & 1.04 & -4.06-1326.98 & -61.71\end{array}$

$\begin{array}{lllll}-0.56 & 0.00 & -3.98-1333.71 & -60.37\end{array}$

$-0.90 .1 .03-3.90-1338.50-57.08$

$\begin{array}{lllll}-0.82 & 0.00 & -3.82-1344.77 & -55.28\end{array}$

$\begin{array}{lllll}-1.00 & 1.02 & -3.75-1349.08 & -51.52\end{array}$

$\begin{array}{lllll}-0.65 & 0.00 & -3.68-1354.75 & -49.12\end{array}$

$\begin{array}{llllll}-0.55 & 1.02 & -3.61-1358.47 & -44.77\end{array}$

$-0.280 .00-3.54-1363.90-42.13$

$-0.301 .01-3.48-1367.45-37.60$ $\begin{array}{lll}0.00 & 0.00 \quad 0.00\end{array}$

$0.00 \quad 0.00 \quad 0.00$

$0.00 \quad 0.00 \quad 0.00$

$0.00 \quad 0.00 \quad 0.00$

$0.00 \quad 0.00 \quad 0.00$

$0.00 \quad 0.00 \quad 0.00$

$0.00 \quad 0.00 \quad 0.00$

$0.00 \quad 0.00 \quad 0.00$

$\begin{array}{llll}0.00 & 0.00 & 0.00\end{array}$

$\begin{array}{llll}0.00 & 0.00 & 0.00\end{array}$

$0.00 \quad 0.00 \quad 0.00$

$0.00 \quad 0.00 \quad 0.00$

$0.00 \quad 0.00 \quad 0.00$

$\begin{array}{llll}0.00 & 0.00 & 0.00\end{array}$

$\begin{array}{llll}0.00 & 0.00 & 0.00\end{array}$

$\begin{array}{llll}0.00 & 0.00 & 0.00\end{array}$

$0.00 \quad 0.00 \quad 0.00$

$0.00 \quad 0.00 \quad 0.00$

$0.00 \quad 0.00 \quad 0.00$

$0.00 \quad 0.00 \quad 0.00$

$0.00 \quad 0.00 \quad 0.00$

$\begin{array}{llll}0.00 & 0.00 & 0.00\end{array}$

$\begin{array}{llll}0.00 & 0.00 & 0.00\end{array}$

$0.00 \quad 0.00 \quad 0.00$

$0.00 \quad 0.00 \quad 0.00$

$0.00 \quad 0.00 \quad 0.00$

$\begin{array}{llll}0.00 & 0.00 & 0.00\end{array}$

$\begin{array}{llll}0.00 & 0.00 & 0.00\end{array}$

$0.00 \quad 0.00 \quad 0.00$

$0.00 \quad 0.00 \quad 0.00$

$0.00 \quad 0.00 \quad 0.00$

$\begin{array}{llll}0.00 & 0.00 & 0.00\end{array}$

$\begin{array}{llll}0.00 & 0.00 & 0.00\end{array}$

$\begin{array}{llll}0.00 & 0.00 & 0.00\end{array}$

$0.00 \quad 0.00 \quad 0.00$

$\begin{array}{llll}0.00 & 0.00 & 0.00\end{array}$

$\begin{array}{llll}0.00 & 0.00 & 0.00\end{array}$

$0.00 \quad 0.00 \quad 0.00$

$\begin{array}{llll}0.00 & 0.00 & 0.00\end{array}$

$\begin{array}{lll}-72.95 & -0.87 & 0.03\end{array}$

$\begin{array}{lll}-76.10 & -1.71 & 0.35\end{array}$

$\begin{array}{lll}-75.37 & -1.11 & 0.06\end{array}$

$-76.28-0.18-0.03$

$\begin{array}{llll}-75.14 & 0.38 & -0.47\end{array}$

$\begin{array}{llll}-75.77 & 1.13 & -0.17\end{array}$

$\begin{array}{lll}-74.20 & 1.69 & 0.18\end{array}$

$\begin{array}{llll}-74.72 & 2.11 & 0.52\end{array}$

$\begin{array}{lll}-72.89 & 2.52 & 0.94\end{array}$

$\begin{array}{lll}-73.72 & 2.21 & 0.88\end{array}$

$\begin{array}{lll}-72.08 & 2.02 & 0.97\end{array}$

$\begin{array}{lll}-72.54 & 1.67 & 0.78\end{array}$

$\begin{array}{lll}-70.83 & 1.16 & 0.54\end{array}$

$\begin{array}{lll}-70.70 & 1.01 & 0.45\end{array}$

$\begin{array}{lll}-68.57 & 0.55 & 0.29\end{array}$

$\begin{array}{llll}-67.95 & 0.50 & 0.29\end{array}$

$\begin{array}{llll}-65.52 & -0.01 & 0.12\end{array}$

$\begin{array}{lll}-64.29 & 0.19 & 0.38\end{array}$

$0.00 \quad 0.00 \quad 0.00$

$0.00 \quad 0.00 \quad 0.00$

$0.00 \quad 0.00 \quad 0.00$

$0.00 \quad 0.00 \quad 0.00$

$\begin{array}{llll}0.00 & 0.00 & 0.00\end{array}$

$0.00 \quad 0.00 \quad 0.00$

$0.00 \quad 0.00 \quad 0.00$

$0.00 \quad 0.00 \quad 0.00$

$\begin{array}{lll}0.00 & 0.00 & 0.00\end{array}$ 
$\begin{array}{lllllllll}64 & 108 & 172-11.1 & -4.4-1369.13 & -0.02 & 0.00 & -3.41-1372.57 & -34.65\end{array}$ $64109173-11.4-4.2-1373.43$ $64110 \quad 174-11.7-4.1-1377.59$ $64111 \quad 175-11.9-3.9-1381.61$ $64112 \quad 176-12.2-3.8-1385.49$ $64 \quad 113 \quad 177-12.4 \quad-3.7-1389.23$ $64114 \quad 178-12.6 \quad-3.5-1392.84$ $64115179-12.9-3.4-1396.31$ $64116180-13.1-3.3-1399.66$ $64117181-13.4 \quad-3.2-1402.88$ $64118 \quad 182-13.6-3.0-1405.97$ $\begin{array}{llll}64 & 119 & 183-13.8 & -2.9-1408.95\end{array}$ $\begin{array}{llll}64 & 120 & 184-14.0 & -2.8-1411.80\end{array}$ $\begin{array}{llll}64 & 121 & 185-14.3 & -2.7-1414.53\end{array}$ $64122186-14.5-2.6-1417.15$ $64123 \quad 187-14.7-2.4-1419.66$ $64124 \quad 188-14.9-2.3-1422.05$ $64 \quad 125 \quad 189-15.1-2.2-1424.33$ $64126190-15.4-2.1-1426.51$ $64 \quad 127 \quad 191-15.6-2.0-1428.57$ $\begin{array}{llll}64 & 128 & 192-15.8 & -1.9-1430.54\end{array}$ $64129193-16.0-1.8-1432.40$ $64130194-16.2-1.7-1434.17$ $64131195-16.4-1.6-1435.83$ $64132 \quad 196-16.6-1.5-1437.40$ $64133 \quad 197-16.8-1.4-1438.87$ $64134198-17.0-1.3-1440.25$

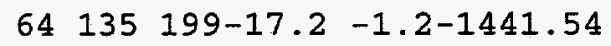
$\begin{array}{llll}64 & 136 & 200-17.4 & -1.2-1442.74\end{array}$ $\begin{array}{llll}64 & 137 & 201-17.6 & -1.1-1443.85\end{array}$ $64 \quad 138 \quad 202-17.7 \quad-1.0-1444.88$ $\begin{array}{llll}64 & 139 & 203-17.9 & -0.9-1445.82\end{array}$ $\begin{array}{llll}64 & 140 & 204-18.1 & -0.8-1446.68\end{array}$ $\begin{array}{llll}64 & 141 & 205-18.3 & -0.7-1447.46\end{array}$ $64142206-18.5-0.7-1448.15$ $64143207-18.6-0.6-1448.77$ $64 \quad 144 \quad 208-18.8 \quad-0.5-1449.32$ $64145209-19.0-0.4-1449.78$ $64 \quad 146 \quad 210-19.1 \quad-0.4-1450.18$ $\begin{array}{llll}64 & 147 & 211-19.3 & -0.3-1450.50\end{array}$ $64148 \quad 212-19.5 \quad-0.2-1450.76$ $\begin{array}{llll}64 & 149 & 213-19.6 & -0.1-1450.94\end{array}$ $\begin{array}{llllll}64 & 150 & 214 & 0.0 & 0.0 & 0.00\end{array}$ $\begin{array}{lllll}65 & 65 & 130 & 2.8-14.5 & -996.59\end{array}$

65. $66131 \quad 2.5-14.2-1010.96$

$\begin{array}{llll}65 & 67 & 132 & 2.1-13.9-1025.00\end{array}$

$\begin{array}{llll}65 & 68 & 133 & 1.8-13.6-1038.72\end{array}$

$\begin{array}{llll}65 & 69 & 134 & 1.4-13.2-1052.12\end{array}$

$6570 \quad 135 \quad 1.0-12.9-1065.20$

$\begin{array}{llll}65 & 71 & 136 & 0.7-12.6-1077.98\end{array}$

$\begin{array}{llll}65 & 72 & 137 & 0.3-12.3-1090.45\end{array}$

$\begin{array}{llll}65 & 73 & 138 & 0.0-12.0-1102.63\end{array}$

$\begin{array}{llll}65 & 74 & 139 & -0.4-11.7-1114.51\end{array}$

$\begin{array}{llll}65 & 75 & 140 & -0.7-11.5-1126.12\end{array}$

$\begin{array}{llll}65 & 76 & 141 & -1.0-11.2-1137.44\end{array}$

$\begin{array}{llll}65 & 77 & 142 & -1.4-10.9-1148.49\end{array}$

$\begin{array}{llll}65 & 78 & 143 & -1.7-10.6-1159.27\end{array}$

$\begin{array}{llll}65 & 79 & 144 & -2.0-10.4-1169.79\end{array}$

$\begin{array}{llll}65 & 80 & 145 & -2.4-10.1-1180.05\end{array}$

$\begin{array}{lllll}65 & 81 & 146 & -2.7 & -9.9-1190.05\end{array}$

$\begin{array}{lllll}65 & 82 & 147 & -3.0 & -9.6-1199.81\end{array}$

$\begin{array}{lllll}65 & 83 & 148 & -3.4 & -9.4-1209.32\end{array}$

$\begin{array}{lllll}65 & 84 & 149 & -3.7 & -9.2-1218.59\end{array}$

$\begin{array}{lllll}65 & 85 & 150 & -4.0 & -8.9-1227.63\end{array}$

$\begin{array}{llllll}65 & 86 & 151 & -4.3 & -8.7-1236.44\end{array}$

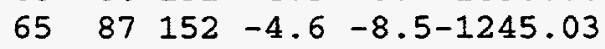

$\begin{array}{lllll}-0.02 & 1.00 & -3.35-1375.80 & -29.82\end{array}$ $0.290 .00-3.29-1380.60-26.54$ $0.28 \quad 1.00-3.24-1383.57-21.44$ $\begin{array}{lllll}0.52 & 0.00 & -3.18-1388.15 & -17.95\end{array}$ $\begin{array}{llll}0.33 & 0.99 & -3.13-1391.03 & -12.76\end{array}$ $\begin{array}{llll}0.45 & 0.00 & -3.07-1395.46 & -9.12\end{array}$ $\begin{array}{llll}0.23 & 0.99 & -3.02-1398.12 & -3.70\end{array}$ $\begin{array}{llll}0.44 & 0.00 & -2.97-1402.19 & 0.29\end{array}$ $\begin{array}{llll}0.23 & 0.98 & -2.92-1404.59 & 5.97\end{array}$ $\begin{array}{llll}0.34 & 0.00 & -2.88-1408.51 & 10.12\end{array}$ $\begin{array}{llll}0.39 & 0.98 & -2.83-1410.41 & 16.29\end{array}$ $\begin{array}{llll}0.07 & 0.00 & -2.79-1414.51 & 20.26\end{array}$ $\begin{array}{llll}-0.59 & 0.97 & -2.74-1416.89\end{array}$ $\begin{array}{llll}-0.86 & 0.00 & -2.70-1420.71\end{array}$ $\begin{array}{lll}-1.93 & 0.97 & -2.66-1423.28\end{array}$ $\begin{array}{llll}-2.55 & 0.00 & -2.62-1427.22\end{array}$ $-3.98 .0 .96-2.58-1429.93$ $\begin{array}{llll}-4.38 & 0.00 & -2.54-1433.43\end{array}$ $-3.540 .95-2.50-1433.66$ $\begin{array}{lll}-2.55 & 0.00 & -2.47-1435.56\end{array}$ $\begin{array}{lll}-1.71 & 0.95 & -2.43-1435.59\end{array}$ $\begin{array}{llll}-0.95 & 0.00 & -2.40-1437.51\end{array}$ $\begin{array}{lll}-0.29 & 0.95 & -2.36-1437.54\end{array}$ $0.360 .00-2.33-1439.37$ $\begin{array}{lllll}0.62 & 0.94 & -2.30-1439.61 & 100.09\end{array}$ $\begin{array}{llllll}1.05 & 0.00 & -2.27-1441.47 & 106.30\end{array}$ $\begin{array}{lllll}0.65 & 0.94 & -2.23-1442.19 & 113.65\end{array}$ $\begin{array}{lllll}0.66 & 0.00 & -2.20-1444.29 & 119.63\end{array}$ $\begin{array}{lllll}0.36 & 0.93 & -2.18-1444.74 & 127.25\end{array}$ $\begin{array}{lllll}0.50 & 0.00 & -2.15-1446.53 & 133.53\end{array}$ $\begin{array}{lllll}0.14 & 0.93 & -2.12-1446.87 & 141.26\end{array}$ $\begin{array}{lllll}0.19 & 0.00 & -2.09-1448.58 & 147.62\end{array}$ $\begin{array}{llll}-0.27 & 0.92 & -2.06-1448.87 & 155.40\end{array}$ $\begin{array}{lllll}-0.47 & 0.00 & -2.04-1450.66 & 161.68\end{array}$ $\begin{array}{lllll}-1.05 & 0.92 & -2.01-1450.92 & 169.50\end{array}$ $\begin{array}{lllll}-1.29 & 0.00 & -1.99-1452.59 & 175.89\end{array}$ $\begin{array}{lllll}-2.02 & 0.91 & -1.96-1452.85 & 183.71\end{array}$ $\begin{array}{llll}-2.12 & 0.00 & -1.94-1454.24 & 190.39\end{array}$ $\begin{array}{lllll}-2.70 & 0.91 & -1.92-1454.21 & 198.49\end{array}$ $\begin{array}{lllll}-2.61 & 0.00 & -1.89-1455.26 & 205.51\end{array}$ $\begin{array}{llll}-2.84 & 0.91 & -1.87-1454.74 & 214.10\end{array}$ $\begin{array}{lllll}-2.63 & 0.00 & -1.85 & 0.00 & 0.00\end{array}$ $0.452 .36-10.00-1003.78-5.65$ $0.441 .19-9.68-1019.01-12.82$ $0.412 .12-9.38-1031.86-17.59$ $0.301 .19-9.10-1046.32-23.99$ $0.202 .11-8.82-1058.63-28.22$ $0.211 .19-8.56-1072.36-33.88$ $\begin{array}{lllll}0.39 & 2.10 & -8.31-1083.79 & -37.24\end{array}$ $\begin{array}{lllll}0.76 & 1.19 & -8.07-1096.56 & -41.94\end{array}$ $\begin{array}{llll}1.05 & 2.10 & -7.84-1107.32 & -44.63\end{array}$ $1.361 .19-7.62-1119.58-48.81$ $1.292 .09-7.41-1130.15-51.31$ $\begin{array}{llll}1.37 & 1.19 & -7.21-1142.08 & -55.18\end{array}$ $2.302 .08-7.01-1151.12-56.14$ $1.57 \quad 1.19-6.83-1163.33-60.28$ $1.002 .07-6.65-1173.36-62.24$ $\begin{array}{lllll}0.21 & 1.19 & -6.48-1185.12 & -65.92\end{array}$ $\begin{array}{llll}0.65 & 2.07 & -6.31-1194.95 & -67.68\end{array}$ $\begin{array}{lll}-1.94 & 1.19 & -6.15-1206.71-71.37\end{array}$ $\begin{array}{rrrr}-0.88 & 2.06 & -6.00-1214.14 & -70.73 \\ 0.01 & 1.19 & -5.85-1223.24 & -71.77\end{array}$

$0.912 .05-5.71-1230.38-70.83$

$\begin{array}{lllll}1.28 & 1.19 & -5.58-1239.54 & -71.92\end{array}$

1.452 .05

\begin{tabular}{|c|c|c|}
\hline 00 & & \\
\hline .00 & 0.00 & \\
\hline 00 & 00 & \\
\hline .00 & & \\
\hline 0 & & .00 \\
\hline 0 & .00 & 0.00 \\
\hline & & \\
\hline & & \\
\hline & & 0 \\
\hline 0.00 & 0.00 & .00 \\
\hline & & \\
\hline & & \\
\hline & & (n) \\
\hline .0 & .00 & 00 \\
\hline 0 & 0.00 & .00 \\
\hline & & \\
\hline 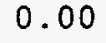 & & \\
\hline & & \\
\hline 0 & 0. & 0 \\
\hline & & \\
\hline & & \\
\hline 0.6 & & \\
\hline .00 & 0.00 & .00 \\
\hline & & \\
\hline & & \\
\hline 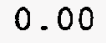 & & \\
\hline 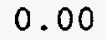 & & \\
\hline & & \\
\hline & & \\
\hline 0.4 & & \\
\hline 0.0 & & \\
\hline & & \\
\hline & & \\
\hline 0 & & 0 \\
\hline . & & \\
\hline & & \\
\hline 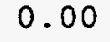 & & \\
\hline 0. & 0 & 0 \\
\hline 0. & & \\
\hline & & \\
\hline 0. & & \\
\hline 0. & 0. & \\
\hline 0.1 & & \\
\hline & & \\
\hline 0.5 & & \\
\hline 0. & 0. & \\
\hline 0.1 & & \\
\hline & & \\
\hline & & \\
\hline 0.0 & 0. & \\
\hline 0. & & \\
\hline & & \\
\hline & & \\
\hline 0.0 & 0. & \\
\hline 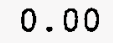 & 0 . & \\
\hline$\cdots$ & & \\
\hline & & \\
\hline & 0.0 & \\
\hline . & -0.12 & -0 . \\
\hline & & \\
\hline & & \\
\hline & -0 & \\
\hline 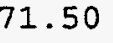 & & \\
\hline & & ( \\
\hline & & \\
\hline & & \\
\hline
\end{tabular}
$\overline{56}$ 
65

$65.89154-5.2-8.0-1261.53$

$\begin{array}{lllll}65 & 90 & 155 & -5.5 & -7.8-1269.46\end{array}$

$\begin{array}{llllll}65 & 91 & 156 & -5.8 & -7.6-1277.19\end{array}$

$\begin{array}{llllll}65 & 92 & 157 & -6.1 & -7.4-1284.70\end{array}$

$\begin{array}{lllll}65 & 93 & 158 & -6.4 & -7.2-1292.02\end{array}$

$\begin{array}{llllll}65 & 94 & 159 & -6.7 & -7.0-1299.14\end{array}$

$\begin{array}{lllll}65 & 95 & 160 & -7.0 & -6.8-1306.06\end{array}$

$\begin{array}{llllll}65 & 96 & 161 & -7.3 & -6.6 & -1312.80\end{array}$

$\begin{array}{lllll}65 & 97 & 162 & -7.6 & -6.5-1319.35\end{array}$

$\begin{array}{lllll}65 & 98 & 163 & -7.9 & -6.3-1325.71\end{array}$

$\begin{array}{lllll}65 & 99 & 164 & -8.2 & -6.1-1331.90\end{array}$

$\begin{array}{lllll}65 & 100 & 165 & -8.4 & -5.9-1337.91\end{array}$

$\begin{array}{llllll}65 & 101 & 166 & -8.7 & -5.7-1343.74\end{array}$

$\begin{array}{lllll}65 & 102 & 167 & -9.0 & -5.6-1349.41\end{array}$

$\begin{array}{lllll}65 & 103 & 168 & -9.3 & -5.4-1354.91\end{array}$

$\begin{array}{llllll}65 & 104 & 169 & -9.5 & -5.3 & -1360.24\end{array}$

$\begin{array}{llllll}65 & 105 & 170 & -9.8 & -5.1-1365.41\end{array}$

$65106 \quad 171-10.1-4.9-1370.43$

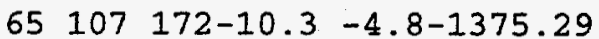

$\begin{array}{lllll}65 & 108 & 173-10.6 & -4.6-1380.00\end{array}$

$65109 \quad 174-10.8-4.5-1384.56$

$65110 \quad 175-11.1-4.3-1388.97$

$65111 \quad 176-11.3-4.2-1393.24$

$65 \quad 112 \quad 177-11.6 \quad-4.1-1397.36$

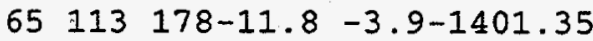

$65 \quad 114 \quad 179-12.1-3.8-1405.20$

$65115 \quad 180-12.3-3.6-1408.92$

$\begin{array}{lllll}65 & 116 & 181-12.6 & -3.5-1412.50\end{array}$

$\begin{array}{llll}65 & 117 & 182-12.8 & -3.4-1415.96\end{array}$

$65118183-13.0-3.3-1419.29$

$\begin{array}{llll}65 & 119 & 184-13.3 & -3.1-1422.49\end{array}$

$65 \quad 120185-13.5-3.0-1425.57$

$65121186-13.7-2.9-1428.53$

$\begin{array}{llll}65 & 122 & 187-13.9 & -2.8-1431.37\end{array}$

$65123 \quad 188-14.2-2.7-1434.10$

$65124 \quad 189-14.4-2.6-1436.71$

$65125190-14.6-2.4-1439.21$

65126 191-14.8 $-2.3-1441.60$

$\begin{array}{lllll}65 & 127 & 192-15.0 & -2.2-1443.88\end{array}$

$\begin{array}{lllll}65 & 128 & 193-15.2 & -2.1-1446.06\end{array}$

$65129194-15.5 \quad-2.0-1448.13$

$65130195-15.7-1.9-1450.10$

$65 \quad 131 \quad 196-15.9-1.8-1451.96$

$65 \quad 132 \quad 197-16.1-1.7-1453.73$

$65133198-16.3-1.6-1455.41$

65 134 199-16.5 -1.5-1456.98

$\begin{array}{llll}65 & 135 & 200-16.7 & -1.4-1458.47\end{array}$

$65 \quad 136 \quad 201-16.9-1.3-1459.86$

$65 \quad 137 \quad 202-17.0 \quad-1.3-1461.16$

$\begin{array}{llll}65 & 138 & 203-17.2 & -1.2-1462.37\end{array}$

$65139204-17.4-1.1-1463.50$

$65 \quad 140 \quad 205-17.6-1.0-1464.54$

$\begin{array}{llll}65 & 141 & 206-17.8 & -0.9-1465.49\end{array}$

$\begin{array}{lllll}65 & 142 & 207-18.0 & -0.8-1466.37\end{array}$

$\begin{array}{llll}65 & 143 & 208-18.1 & -0.8-1467.16\end{array}$

$\begin{array}{lllll}65 & 144 & 209-18.3 & -0.7-1467.88\end{array}$

$\begin{array}{llll}65 & 145 & 210-18.5 & -0.6-1468.52\end{array}$

$\begin{array}{lllll}65 & 146 & 211-18.7 & -0.5-1469.08\end{array}$

$\begin{array}{lllll}65 & 147 & 212-18.8 & -0.4-1469.57\end{array}$

$\begin{array}{llll}65 & 148 & 213-19.0 & -0.4-1469.98\end{array}$

$\begin{array}{llll}65 & 149 & 214-19.1 & -0.3-1470.33\end{array}$

$\begin{array}{llllllll}65 & 150 & 215-19.3 & -0.2-1470.60 & -2.40 & 1.19 & -1.90-1473.71 & 210.48\end{array}$

$\begin{array}{llllllll}65 & 151 & 216-19.5 & -0.2-1470.81 & -2.92 & 1.91 & -1.88-1473.70 & 218.57\end{array}$

$\begin{array}{lllllllll}65 & 152 & 217-19.6 & -0.1-1470.95 & -2.85 & 1.19 & -1.86-1474.46 & 225.87\end{array}$

$65153218=0.0 \quad 0.0$
$1.541 .19-5.32-1255.97-72.21-71.32$

$1.562 .04-5.20-1263.13-71.29-70.15$

$1.341 .19-5.08-1272.01-72.10-71.26$

$1.102 .03-4.97-1279.02-71.04-70.10$

$0.991 .19-4.86-1287.38-71.33-70.77$

$0.732 .03-4.75-1294.01-69.89-69.48$

$\begin{array}{llllll}0.64 & 1.19 & -4.65-1301.95 & -69.76 & -69.54\end{array}$

$0.382 .02-4.55-1308.21-67.95-67.85$

$\begin{array}{llllll}0.25 & 1.19 & -4.45-1315.81 & -67.47 & -67.47\end{array}$

$-0.052 .02-4.36-1321.74-65.34-65.68$

$\begin{array}{llllll}-0.22 & 1.19 & -4.27-1329.01 & -64.53 & -64.70\end{array}$

$-0.552 .01-4.19-1334.62-62.07$

$-0.942 .01-4.02-1346.70-58.01$

$\begin{array}{lllll}-0.95 & 1.19 & -3.94-1353.11 & -56.34\end{array}$

$-1.142 .00-3.87-1357.91-53.08$

$\begin{array}{llll}-0.87 & 1.19 & -3.79-1363.71 & -50.80\end{array}$

$\begin{array}{lllll}-0.88 & 2.00 & -3.72-1368.02 & -47.04\end{array}$

$\begin{array}{lllll}-0.65 & 1.19 & -3.65-1373.54 & -44.49\end{array}$

$.671 .99-3.59-1377.56-40.43$

$-0.431 .99-3.46-1386.46-33.19$

$-0.091 .19-3.40-1391.26-29.93$

$\begin{array}{llll}-0.06 & 1.98 & -3.34-1394.65 & -25.24\end{array}$

$\begin{array}{lllll}0.28 & 1.19 & -3.28-1399.17 & -21.69\end{array}$

$0.011 .98-3.22-1402.59-17.04$

$0.191 .19-3.17-1406.99-13.36$

$\begin{array}{llll}-0.04 & 1.97 & -3.11-1410.10 & -8.41\end{array}$

$0.221 .19-3.06-1414.15$

$0.091 .97-3.01-1416.91$

$0.261 .19-2.96-1420.80$

$0.251 .97-2.92-1423.19$

$-4.39$

0.93

5.11

$-0.061 .19-2.87-1427.31$

$-0.711 .96-2.82-1430.10$

14.74

20.02

24.29

29.53

33.68

38.84

43.22

50.78

57.02

64.57

70.77

78.28

$101.94-2.43-1452$.

$0.521 .19-2.40-1454.42$

84.49

$0.801 .94-2.36-1455.03 \quad 91.95$

$3.381 .19-2.33-1454.74 \quad 100.31$

$0.821 .94-2.30-1458.01105 .11$

$0.841 .19-2.27-1460.09111 .10$

$0.561 .93-2.24-1460.90118 .36$

$0.651 .19-2.21-1462.74124 .60$

$0.35 \quad 1.93-2.18-1463.40 \quad 132.01$

$0.351 .19-2.15-1465.14138 .34$

$-0.081 .93-2.12-1465.77 \quad 145.78$

$\begin{array}{llll}-0.05 & 1.19 & -2.10-1467.32 & 152.30\end{array}$

$\begin{array}{lllll}-0.85 & 1.92 & -2.07-1468.16 & 159.53\end{array}$

$\begin{array}{lllll}-1.15 & 1.19 & -2.04-1469.88 & 165.89\end{array}$

$\begin{array}{lllll}-1.81 & 1.92 & -2.02-1470.42 & 173.41\end{array}$

$\begin{array}{lllll}-1.89 & 1.19 & -1.99-1471.77 & 180.14\end{array}$

$\begin{array}{lllll}-2.35 & 1.92 & -1.97-1471.97 & 188.01\end{array}$

$\begin{array}{lllll}-2.28 & 1.19 & -1.95-1473.01 & 195.04\end{array}$

$-2.541 .91-1.92-1472.88 \quad 203.25$

0.00

$-3.161 .91$

57
0.00

0.00

0.00

0.00

0.00

0.00

0.00

0.00

0.00

0.00

0.00

0.00

0.00

0.00

0.00

0.00

0.00

0.00

0.00

0.00

0.00

0.00

0.00

0.00

0.00

0.00

0.00

0.00

0.00

0.00

0.00

0.00

0.00

0.00

0.00

0.00

0.00

0.00

0.00

0.00

0.00

0.00

0.00

0.00

0.00

0.00

0.00

0.00

0.00

0.00

0.00

0.00

0.00

0.00
2.43

2.70

2.18

2.04

1.55

1.14

0.86

0.48

0.25

$-0.39$

0.89

1.14

0.84

0.94

0.56

0.41

0.22

0.10

0.00

$-0.34$

$0.00 \quad 0.00$

$0.00 \quad 0.00$

$0.00 \quad 0.00$

$0.00 \quad 0.00$

$0.00 \quad 0.00$

$0.00 \quad 0.00$

$0.00 \quad 0.00$

$0.00 \cdot 0.00$

$0.00 \quad 0.00$

$0.00 \quad 0.00$

$0.00 \quad 0.00$

$0.00 \quad 0.00$

$0.00 \quad 0.00$

$0.00 \quad 0.00$

$0.00 \quad 0.00$

$0.00 \quad 0.00$

$0.00 \quad 0.00$

$0.00 \quad 0.00$

$0.00 \quad 0.00$

$0.00 \quad 0.00$

$0.00 \quad 0.00$

$0.00 \quad 0.00$

$0.00 \quad 0.00$

$0.00 \quad 0.00$

$0.00 \quad 0.00$

$0.00 \quad 0.00$

$0.00 \quad 0.00$

$0.00 \quad 0.00$

$0.00 \quad 0.00$

$0.00 \quad 0.00$

$0.00 \quad 0.00$

$0.00 \quad 0.00$

$0.00 \quad 0.00$

$0.00 \quad 0.00$

$0.00 \quad 0.00$

$0.00 \quad 0.00$

$0.00 \quad 0.00$

$0.00 \quad 0.00$

$0.00 \quad 0.00$

$0.00 \quad 0.00$

$0.00 \quad 0.00$

$0.00 \quad 0.00$

$0.00 \quad 0.00$

$0.00 \quad 0.00$

$0.00 \quad 0.00$

$0.00 \quad 0.00$

$0.00 \quad 0.00$

$0.00 \quad 0.00$

$0.00 \quad 0.00$

$0.00 \quad 0.00$

$0.00 \quad 0.00$

$0.00 \quad 0.00$

$0.00 \quad 0.00$

$0.00 \quad 0.00$ 


\section{6}

$\begin{array}{llll}66 & 68 & 134 & 2.3-13.9-1036.71\end{array}$

$\begin{array}{llll}66 & 69 & 135 & 1.9-13.6-1050.46\end{array}$

$\begin{array}{lll}66 & 70 & 136\end{array}$

$\begin{array}{lll}66 & 71 & 137\end{array}$

1. $6-13.3-1063.89$

$1.2-13.0-1077.02$

$0.9-12.7-1089.85$

$\begin{array}{lll}66 & 72 & 138\end{array}$

73139

$0.5-12.4-1102.37$

$\begin{array}{lll}66 & 74 & 140\end{array}$

$0.2-12.1-1114.60$

$66 \quad 75 \quad 141-0.2-11.8-1126.55$

$\begin{array}{llll}66 & 76 & 142 & -0.5-11.5-1138.21\end{array}$

$\begin{array}{llll}66 & 77 & 143 & -0.8-11.2-1149.60\end{array}$

$6678 \quad 144-1.2-11.0-1160.71$

$6679145-1.5-10.7-1171.56$

$6680146-1.8-10.5-1182.15$

$\begin{array}{lllll}66 & 81 & 147 & -2.2-10.2-1192.49\end{array}$

$6682148-2.5-10.0-1202.57$

$\begin{array}{llllll}66 & 83 & 149 & -2.8 & -9.7-1212.40\end{array}$

$\begin{array}{llllll}66 & 84 & 150 & -3.1 & -9.5-1222.00\end{array}$

$\begin{array}{llllll}66 & 85 & 151 & -3.4 & -9.2-1231.35\end{array}$

$\begin{array}{llllll}66 & 86 & 152 & -3.8 & -9.0-1240.48\end{array}$

$\begin{array}{llllll}66 & 87 & 153 & -4.1 & -8.8-1249.37\end{array}$

$\begin{array}{llllll}66 & 88 & 154 & -4.4 & -8.6-1258.04\end{array}$

$\begin{array}{llllll}66 & 89 & 155 & -4.7 & -8.3-1266.50\end{array}$

$\begin{array}{llllll}66 & 90 & 156 & -5.0 & -8.1-1274.73\end{array}$

$\begin{array}{lllll}66 & 91 & 157 & -5.3 & -7.9-1282.76\end{array}$

$\begin{array}{llllll}66 & 92 & 158 & -5.6 & -7.7-1290.57\end{array}$

$\begin{array}{lllll}66 & 93 & 159 & -5.9 & -7.5-1298.19\end{array}$

$\begin{array}{lllll}66 & 94 & 160 & -6.2 & -7.3-1305.60\end{array}$

$\begin{array}{llllll}66 & 95 & 161 & -6.5 & -7.1-1312.82\end{array}$

$\begin{array}{llllll}66 & 96 & 162 & -6.8 & -6.9-1319.84\end{array}$

$\begin{array}{llllll}66 & 97 & 163 & -7.0 & -6.7-1326.67\end{array}$

$\begin{array}{llllll}66 & 98 & 164 & -7.3 & -6.6-1333.32\end{array}$

$0.181 .18-9.69-1030.96 \quad-9.42$

$0.020 .00-9.39-1046.08-16.46$

$\begin{array}{lllll}-0.05 & 1.17 & -9.11-1058.45 & -20.76\end{array}$

$\begin{array}{lllll}-0.12 & 0.00 & -8.84-1072.85 & -27.10\end{array}$

$0.051 .16-8.58-1084.39-30.56$

$\begin{array}{llllll}0.39 & 0.00 & -8.33-1097.79 & -35.89\end{array}$

$0.611 .15-8.09-1108.71-38.73$

$0.84 \quad 0.00-7.87-1121.63-43.59$

$0.97 \quad 1.14-7.65-1132.09-45.97$

$1.04 \quad 0.00-7.44-1144.61-50.43$

$2.03 \quad 1.13-7.24-1153.68-51.42$

$1.250 .00-7.05-1166.51-56.18$

$0.68 \quad 1.12-6.86-1176.63-58.23$

$\begin{array}{lllll}-0.01 & 0.00 & -6.68-1188.85 & -62.38\end{array}$

$\begin{array}{lllll}-1.07 & 1.11 & -6.51-1198.96 & -64.42\end{array}$

$-2.170 .00-6.35-1211.09-68.47$

$-1.321 .10-6.19-1218.82-68.13$

$-0.310 .00-6.04-1228.35-69.59$

$0.771 .09-5.90-1235.39-68.56$

$1.250 .00-5.75-1244.98-70.08$

$1.521 .08-5.62-1252.39-69.42$

$1.630 .00-5.49-1261.90-70.86$

$1.701 .08-5.36-1269.08-69.97$

$1.56 \quad 0.00-5.24-1278.41-71.23$

$1.351 .07-5.12-1285.46-70.21$

$1.240 .00-5.01-1294.34-71.02$

$1.031 .06-4.90-1301.00-69.60-6$

$0.920 .00-4.80-1309.48-70.00-6$

$0.641 .05-4.69-1315.82-68.28-6$

$0.470 .00-4.59-1323.96-68.35$

$0.161 .04-4.50-1329.97-66.28$

$\begin{array}{llllll}66 & 99 & 165 & -7.6 & -6.4-1339.79\end{array}$

$-0.06 \quad 0.00-4.41-1337.79-66.03$

$\begin{array}{llllll}66 & 100 & 166 & -7.9 & -6.2-1346.08\end{array}$

$-0.41 \quad 1.04-4.32-1343.48-63.65$

$\begin{array}{llllllllll}66 & 101 & 167 & -8.2 & -6.0 & -1352.19 & -0.89 & 1.03 & -4.15-1356.19 & -60.22\end{array}$

$\begin{array}{llllllllll}66 & 102 & 168 & -8.4 & -5.9-1358.13 & -0.93 & 0.00 & -4.07-1363.12 & -59.08\end{array}$

$\begin{array}{llllllllll}66 & 103 & 169 & -8.7 & -5.7-1363.90 & -1.22 & 1.02 & -3.99-1368.08 & -55.97\end{array}$

$\begin{array}{llllllllll}66 & 104 & 170 & -9.0 & -5.5-1369.50 & -0.96 & 0.00 & -3.91-1374.37 & -54.19\end{array}$

$\begin{array}{llllllllll}66 & 105 & 171 & -9.2 & -5.4-1374.94 & -1.04 & 1.02 & -3.84-1378.80 & -50.54\end{array}$

$\begin{array}{llllllllll}66 & 106 & 172 & -9.5 & -5.2-1380.22 & -0.86 & 0.00 & -3.77-1384.85 & -48.52\end{array}$

$\begin{array}{llllllllll}66 & 107 & 173 & -9.8 & -5.0-1385.34 & -0.96 & 1.01 & -3.70-1388.99 & -44.59\end{array}$

$\begin{array}{lllllllll}66 & 108 & 174-10.0 & -4.9-1390.31 & -0.78 & 0.00 & -3.63-1394.72 & -42.25\end{array}$

$\begin{array}{lllllllll}66 & 109 & 175-10.3 & -4.7-1395.12 & -0.80 & 1.00 & -3.56-1398.48 & -37.94\end{array}$

$66 \quad 110 \quad 176-10.5-4.6-1399.79-0.51 \quad 0.00-3.50-1403.80-35.18$

$\begin{array}{lllllllll}66 & 111 & 177-10.8 & -4.4-1404.31 & -0.53 & 1.00 & -3.44-1407.28 & -30.59\end{array}$

$\begin{array}{lllllllll}66 & 112 & 178-11.0 & -4.3-1408.68 & -0.27 & 0.00 & -3.38-1412.33 & -27.57\end{array}$

$\begin{array}{llllllll}66 & 113 & 179-11.3 & -4.2-1412.92 & -0.41 & 0.99 & -3.32-1415.65 & -22.83\end{array}$

$66 \quad 114 \quad 180-11.5-4.0-1417.01-0.23 \quad 0.00-3.26-1420.51-19.61$

$66 \quad 115181-11.8-3.9-1420.97-0.41 \quad 0.99-3.21-1423.60-14.63$

$66 \quad 116 \quad 182-12.0-3.8-1424.79-0.14 \quad 0.00-3.15-1428.09-11.05$

$\begin{array}{lllllll}66 & 117 & 183-12.3 & -3.6-1428.49 & -0.22 & 0.98 & -3.10-1430.83\end{array}$

$66 \quad 118184-12.5-3.5-1432.05-0.01 \quad 0.00-3.05-1435.11$

$\begin{array}{lllllll}66 & 119 & 185-12.7 & -3.4-1435.49 & -0.04 & 0.98 & -3.00-1437.55\end{array}$

$\begin{array}{llllllll}66 & 120 & 186-12.9 & -3.2-1438.80 & -0.36 & 0.00 & -2.95-1442.11\end{array}$

$\begin{array}{llllllll}66 & 121 & 187-13.2 & -3.1-1441.98 & -0.93 & 0.97 & -2.91-1444.85\end{array}$

$\begin{array}{llllllll}66 & 122 & 188-13.4 & -3.0-1445.05 & -1.11 & 0.00 & -2.86-1449.02\end{array}$

$\begin{array}{llllllll}66 & 123 & 189-13.6 & -2.9-1448.00 & -2.02 & 0.97 & -2.82-1451.87\end{array}$

$\begin{array}{lllllll}66 & 124 & 190-13.8 & -2.8-1450.83 & -2.55 & 0.00 & -2.77-1456.16\end{array}$

$\begin{array}{llllllll}66 & 125 & 191-14.1 & -2.7-1453.55 & -3.82 & 0.96 & -2.73-1459.14\end{array}$

$\begin{array}{llllllll}66 & 126 & 192-14.3 & -2.5-1456.16 & -4.25 & 0.00 & -2.69-1463.10\end{array}$

$\begin{array}{llllllll}66 & 127 & 193-14.5 & -2.4-1458.65 & -3.26 & 0.95 & -2.65-1463.61\end{array}$

$\begin{array}{llllllll}66 & 128 & 194-14.7 & -2.3-1461.04 & -2.39 & 0.00 & -2.61-1466.04\end{array}$

$\begin{array}{llllllll}66 & 129 & 195-14.9 & -2.2-1463.32 & -1.42 & 0.95 & -2.57-1466.37\end{array}$

$\begin{array}{llllllll}66 & 130 & 196-15.1 & -2.1-1465.50 & -0.41 & 0.00 & -2.54-1468.44\end{array}$

$66 \quad 131 \quad 197-15.3-2.0-1467.57$

$0.170 .95-2.50-1468.95$

$0.790 .00-2.47-1471.22$

\subsection{0}

0.00

0.00

0.00

0.00

0.00

0.00

0.00

0.00

0.00

0.00

0.00

0.00

0.00

0.00

0.00

0.00

0.00

0.00

0.00

0.00

0.00

0.00

0.00

0.00

0.00

0.00

0.00

0.00

0.00

0.00

0.00

0.00

0.00

0.00

0.00

0.00

$-64.46$

$-67.98-1.68$

0.00

$-1.11$

0.00

$-69.32$

0.00

$-0.04$

0.49

$-68.76$

$-0.04$

0.27

$-70.13$

0.57

$-0.20$

$-69.15$

20

$-0.05$

70.40

2.09

0.27

$-69.16$

2. 51

0.46

$-70.53$

2. 26

0.81

$-69.43$

2.13

0.70

$-70.42$

1.84

$-69.18$

1.45

0.60

$-69.68$

1.24

0.42

$-68.06$

0.86

0.32

68.19

0.63

0.22

$-66.39$

0.05

0.16

65.98

$-0.01$

$-0.11$

0.05

0.03

0.34

0.28

$.94-0.61$

0.00

0.00

0.00

$\begin{array}{lll}0.00 & 0.00 & 0.00\end{array}$

$0.00 \quad 0.00 \quad 0.00$

$0.00 \quad 0.00 \quad 0.00$

0.00

0.00

0.00

0.00

0.00

0.00

$0.00 \quad 0.00 \quad 0.00$

$0.00 \quad 0.00 \quad 0.00$

0.00

0.00

0.00

0.00

0.00

0.00

$0.00 \quad 0.00 \quad 0.00$

$0.00 \quad 0.00 \quad 0.00$

0.00

0.00

0.00

0.00

0.00

0.00

$\begin{array}{llll}0.00 & 0.00 & 0.00\end{array}$

$0.00 \quad 0.00 \quad 0.00$

$-1.93$

3.70

7.22

12.55

0.00

0.00

0.00

0.00

0.00

0.00

$\begin{array}{llll}0.00 & 0.00 & 0.00\end{array}$

16.45

21.67

25.46

30.54

34.66

42.22

47.86

55.61

0.00

0.00

0.00

$\begin{array}{llll}0.00 & 0.00 & 0.00\end{array}$

0.00

0.00

0.00

$\begin{array}{llll}0.00 & 0.00 & 0.00\end{array}$

$0.00 \quad 0.00 \quad 0.00$

$\begin{array}{llll}0.00 & 0.00 & 0.00\end{array}$

0.00

0.00

0.00

0.00

0.00

0.00

61.60

69.16

0.00

0.00

0.00

0.00

0.00

0.00

0.00

0.00

0.00

74.97

0.00

0.00

0.00 
$66 \quad 133199-15.7-1.8-1471.41$ $\begin{array}{llll}66 & 134 & 200-15.9 & -1.7-1473.19\end{array}$ $66 \quad 135 \quad 201-16.1-1.6-1474.87$ $66 \quad 136202-16.3-1.5-1476.45$ $66 \quad 137203-16.5-1.4-1477.95$ $66 \quad 138 \quad 204-16.7-1.4-1479.35$ $66 \quad 139205-16.9-1.3-1480.66$ $66 \quad 140 \quad 206-17.1-1.2-1481.88$ $\begin{array}{lll}66 & 141 & 207-17.3-1.1-1483.03\end{array}$ $66 \quad 142 \quad 208-17.5-1.0-1484.08$ $\begin{array}{lllll}66 & 143 & 209-17.6 & -0.9-1485.05\end{array}$ $\begin{array}{llll}66 & 144 & 210-17.8 & -0.8-1485.94\end{array}$ $66 \quad 145 \quad 211-18.0-0.8-1486.76$ $\begin{array}{llll}66 & 146 & 212-18.2 & -0.7-1487.49\end{array}$ $\begin{array}{llll}66 & 147 & 213-18.3 & -0.6-1488.14\end{array}$ $\begin{array}{llll}66 & 148 & 214-18.5 & -0.5-1488.73\end{array}$ $\begin{array}{llll}66 & 149 & 215-18.7 & -0.5-1489.23\end{array}$ $\begin{array}{llll}66 & 150 & 216-18.8 & -0.4-1489.67\end{array}$ $66 \quad 151217-19.0-0.3-1490.03$ $66 \quad 152 \quad 218-19.1-0.3-1490.33$ $66.153 \quad 219-19.3-0.2-1490.56$ $\begin{array}{llll}66 & 154 & 220-19.4 & -0.1-1490.72\end{array}$

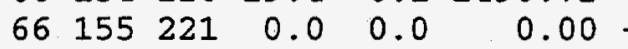
$\begin{array}{llll}67 & 69 & 136 & 2.4-13.9-1048.28\end{array}$ $\begin{array}{llll}67 & 70 & 137 & 2.1-13.6-1062.06\end{array}$ $\begin{array}{llll}67 & 71 & 138 & 1.7-13.3-1075.54\end{array}$ $\begin{array}{llll}67 & 72 & 139 & 1.4-13.0-1088.71\end{array}$ $67 \quad 73 \quad 140 \quad 1.1-12.7-1101.58$ $67 \quad 74 \quad 141 \cdot 0.7-12.4-1114.15$ $\begin{array}{llll}67 & 75 & 142 & 0.4-12.1-1126.44\end{array}$ $67 \quad 76 \quad 143 \quad 0.0-11.9-1138.44$ $67 \quad 77 \quad 144-0.3-11.6-1150.16$ $6778145-0.6-11.3-1161.61$ $67 \quad 79146-1.0-11.0-1172.80$ $67 \quad 80 \quad 147-1.3-10.8-1183.71$ $6781148-1.6-10.5-1194.37$ $\begin{array}{llll}67 & 82 & 149 & -1.9-10.3-1204.78\end{array}$ $67 \quad 83 \quad 150-2.3-10.0-1214.94$

$\begin{array}{llllll}67 & 84 & 151 & -2.6 & -9.8-1224.85\end{array}$ $\begin{array}{llllll}67 & 85 & 152 & -2.9 & -9.5-1234.52\end{array}$

$\begin{array}{llllll}67 & 86 & 153 & -3.2 & -9.3-1243.96\end{array}$

$\begin{array}{llllll}67 & 87 & 154 & -3.5 & -9.1 & -1253.17\end{array}$

$\begin{array}{llllll}67 & 88 & 155 & -3.8 & -8.9 & -1262.15\end{array}$

$\begin{array}{lllll}67 & 89 & 156 & -4.1 & -8.6-1270.91\end{array}$

$\begin{array}{llllll}67 & 90 & 157 & -4.4 & -8.4-1279.45\end{array}$

$\begin{array}{lllll}67 & 91 & 158 & -4.7 & -8.2-1287.77\end{array}$

$\begin{array}{llllll}67 & 92 & 159 & -5.0 & -8.0 & -1295.89\end{array}$

$\begin{array}{lllll}67 & 93 & 160 & -5.3 & -7.8-1303.80\end{array}$

$\begin{array}{llllll}67 & 94 & 161 & -5.6 & -7.6-1311.50\end{array}$

$\begin{array}{llllll}67 & 95 & 162 & -5.9 & -7.4-1319.01\end{array}$

$\begin{array}{llllll}67 & 96 & 163 & -6.2 & -7.2-1326.32\end{array}$

$\begin{array}{llllll}67 & 97 & 164 & -6.5 & -7.0-1333.44\end{array}$

$\begin{array}{llllll}67 & 98 & 165 & -6.8 & -6.8-1340.38\end{array}$

$\begin{array}{lllll}67 & 99 & 166 & -7.1 & -6.7-1347.12\end{array}$

$\begin{array}{llllll}67 & 100 & 167 & -7.3 & -6.5-1353.69\end{array}$

$\begin{array}{llllll}67 & 101 & 168 & -7.6 & -6.3-1360.08\end{array}$

$\begin{array}{llllll}67 & 102 & 169 & -7.9 & -6.1-1366.29\end{array}$

$\begin{array}{llllll}67 & 103 & 170 & -8.2 & -6.0-1372.33\end{array}$

$\begin{array}{llllll}67 & 104 & 171 & -8.4 & -5.8-1378.20 \\ 67 & 105 & 172 & -8.7 & -5.6-1383.91\end{array}$

$\begin{array}{llllll}67 & 106 & 173 & -9.0 & -5.5-1389.45\end{array}$

$\begin{array}{llllll}67 & 107 & 174 & -9.2 & -5.3-1394.84\end{array}$

$\begin{array}{llllll}67 & 108 & 175 & -9.5 & -5.1-1400.06\end{array}$

$\begin{array}{llllll}67 & 109 & 176 & -9.7 & -5.0-1405.14\end{array}$

$67110 \quad 177-10.0-4.8-1410.06$

$67111178-10.2-4.7-1414.83$
$1.08 \quad 0.94-2.43-1471.82$

$1.460 .00-2.40-1474.13$

$\begin{array}{lllll}1.16 & 0.94 & -2.37-1475.14 & 95.26\end{array}$

$1.18 \quad 0.00-2.33-1477.60 \quad 100.87$

$0.92 \quad 0.93-2.30-1478.40 \quad 108.15$

$1.04 \quad 0.00-2.27-1480.58 \quad 114.04$

$0.690 .93-2.24-1481.28121 .40$

$\begin{array}{llllll}0.69 & 0.00 & -2.21-1483.41 & 127.35\end{array}$

$0.31 \quad 0.92-2.18-1483.98 \quad 134.85$

$0.290 .00-2.16-1485.94 \quad 140.95$

$\begin{array}{lllll}-0.22 & 0.92 & -2.13-1486.48 & 148.49\end{array}$

$\begin{array}{lllll}-0.62 & 0.00 & -2.10-1488.66 & 154.38\end{array}$

$\begin{array}{llllll}-1.29 & 0.91 & -2.08-1489.21 & 161.91\end{array}$

$\begin{array}{lllll}-1.49 & 0.00 & -2.05-1491.03 & 168.16\end{array}$

$\begin{array}{lllll}-1.96 & 0.91 & -2.02-1491.22 & 176.04\end{array}$

$\begin{array}{llllll}-1.86 & 0.00 & -2.00-1492.59 & 182.74\end{array}$

$\begin{array}{llllll}-2.21 & 0.91 & -1.98-1492.52 & 190.88\end{array}$

$\begin{array}{llllll}-2.09 & 0.00 & -1.95-1493.71 & 197.76\end{array}$

$\begin{array}{lllll}-2.65 & 0.90 & -1.93-1493.71 & 205.83\end{array}$

$\begin{array}{llllll}-2.62 & 0.00 & -1.91-1494.86 & 212.75\end{array}$

$\begin{array}{llllll}-2.81 & 0.90 & -1.89-1494.36 & 221.33\end{array}$

$\begin{array}{lllll}-2.59 & 0.00 & -1.86-1495.18 & 228.58\end{array}$ $\begin{array}{lllll}-2.75 & 0.89 & -1.84 & 0.00 & 0.00\end{array}$ $\begin{array}{llll}-0.67 & 2.10 & -9.40-1056.25 & -11.28\end{array}$

$\begin{array}{lllll}-0.76 & 1.18 & -9.12-1070.76 & -17.73\end{array}$ $\begin{array}{lllll}-0.59 & 2.09 & -8.85-1082.89 & -21.78\end{array}$ $\begin{array}{lllll}-0.27 & 1.18 & -8.60-1096.40 & -27.22\end{array}$ $0.012 .09-8.35-1107.84-30.59$ $0.411 .18-8.12-1120.68-35.36$ $0.342 .08-7.89-1131.92-38.52$ $0.521 .18-7.68-1144.42-42.95$ $\begin{array}{llllll}1.67 & 2.07 & -7.47-1153.89 & -44.36\end{array}$ $0.98 \quad 1.18-7.27-1166.72-49.12$ $0.442 .06-7.08-1177.37-51.70$ $-0.221 .18-6.90-1189.65-55.90$ $\begin{array}{lllll}-0.92 & 2.06 & -6.72-1199.96 & -58.14\end{array}$ $-2.061 .18-6.55-1212.21-62.32$ $-1.072 .05-6.39-1220.35-62.38$ $-0.231 .18-6.23-1230.13-64.10$ $0.68 \quad 2.04-6.08-1237.88-63.78$ $1.161 .18-5.94-1247.55-65.38$ $\begin{array}{lllll}1.47 & 2.04 & -5.80-1255.46 & -65.21\end{array}$ $1.541 .18-5.66-1265.09-66.77$ $\begin{array}{lllll}1.61 & 2.03 & -5.53-1272.80 & -66.41\end{array}$ $\begin{array}{lllll}1.52 & 1.18 & -5.40-1282.15 & -67.69\end{array}$ $\begin{array}{lllll}1.36 & 2.02 & -5.28-1289.67 & -67.14\end{array}$ $1.311 .18-5.17-1298.56-67.96$ $\begin{array}{lllll}1.08 & 2.02 & -5.05-1305.75 & -67.08\end{array}$ $0.951 .18-4.94-1314.32-67.57$ $0.662 .01-4.84-1321.18-66.36$ $0.46 \quad 1.18-4.74-1329.42-66.53$ $0.132 .01-4.64-1335.95-64.98$ $-0.121 .18-4.54-1343.86-64.82$ $\begin{array}{lllll}-0.52 & 2.00 & -4.45-1350.09 & -62.99\end{array}$ $-0.631 .18-4.36-1357.50-62.32$ $\begin{array}{lllll}-1.04 & 2.00 & -4.27-1363.40 & -60.15\end{array}$ $\begin{array}{lllll}-1.17 & 1.18 & -4.19-1370.47 & -59.15\end{array}$ $\begin{array}{lllll}-1.44 & 1.99 & -4.11-1375.89 & -56.50 \\ -1.26 & 1.18 & -4.03-1382.31 & -54.85\end{array}$ $\begin{array}{lllll}-1.41 & 1.99 & -3.95-1387.29 & -51.76\end{array}$ $\begin{array}{lllll}-1.31 & 1.18 & -3.88-1393.46 & -49.86\end{array}$ $\begin{array}{lllll}-1.49 & 1.98 & -3.81-1398.15 & -46.48\end{array}$ $-1.301 .18-3.74-1403.92-44.17$ $\begin{array}{lllll}-1.32 & 1.98 & -3.67-1408.15 & -40.33\end{array}$ $-1.021 .18-3.60-1413.50-37.61$ $-1.04 \quad 1.97-3.54-1417.44 \quad-33.47$ 59

\begin{tabular}{|c|c|c|}
\hline & & \\
\hline 0.00 & 0.00 & \\
\hline 0 & 00 & .06 \\
\hline 00 & .00 & .0 \\
\hline .00 & 0.00 & 0.0 \\
\hline 0.00 & & 00 \\
\hline 0 & & 0 \\
\hline 0.00 & 10 & .0 \\
\hline 0.00 & 0.00 & 0.0 \\
\hline 0.00 & 0.00 & 0.0 \\
\hline 0.00 & & \\
\hline 0.00 & & 0.0 \\
\hline 0.00 & 0.00 & 0.0 \\
\hline 0.00 & 0.00 & 0.0 \\
\hline 00 & & \\
\hline 0.0 & & \\
\hline 0.00 & 0.00 & 0.06 \\
\hline 0.00 & & \\
\hline no & 0. & 000 \\
\hline .0 & 0.1 & 0.0 \\
\hline 00 & 0.00 & 0.00 \\
\hline 0.0 & 0.8 & 0.00 \\
\hline .00 & 0.00 & 0.00 \\
\hline 0.00 & 0.00 & 0.06 \\
\hline 0.00 & 0.00 & 0.00 \\
\hline 0.00 & 0.00 & 0.00 \\
\hline .0 & 0 . & 0.0 \\
\hline 0.00 & 0.00 & 0.0 \\
\hline 0.00 & 0.00 & 0.00 \\
\hline 00 & 0.1 & 0.00 \\
\hline 0.0 & 0. & 0.00 \\
\hline 0.00 & 0.00 & 0.00 \\
\hline 0.00 & .00 & 0.00 \\
\hline 0 & 0. & .00 \\
\hline 0.00 & 0.00 & 0.00 \\
\hline 0.0 & 0.00 & 0.00 \\
\hline 0.0 & 0.00 & .00 \\
\hline .06 & -0.75 & .32 \\
\hline 3.7 & 0.16 & 0. \\
\hline .7 & 0.73 & 0.05 \\
\hline .0 & 1.52 & \\
\hline .6 & 2. & 0. \\
\hline 6.0 & 2.2 & 0. \\
\hline 0.0 & 0. & 0.00 \\
\hline 6.8 & 2 & \\
\hline 2 & 2 . & \\
\hline .3 & 1.5 & 0.62 \\
\hline .3 & 1.77 & 0.69 \\
\hline .2 & 1.31 & \\
\hline .0 & 0.5 & 0.1 \\
\hline .3 & 0.60 & 0.14 \\
\hline 0 & & \\
\hline 0 & -0 & \\
\hline 0 & & \\
\hline .2 & & \\
\hline 2 & -1.15 & \\
\hline .8 & 1083 & \\
\hline 5.25 & -1.19 & \\
\hline 0.00 & & \\
\hline 0.0 & & \\
\hline U. & & \\
\hline 0.00 & 0 & 0. \\
\hline 0.00 & & 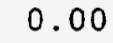 \\
\hline & & \\
\hline - & & \\
\hline & 8 & \\
\hline
\end{tabular}


$67 \begin{array}{lllllllll}612 & 179-10.5 & -4.5-1419.45 & -0.71 & 1.18 & -3.48-1422.46 & -30.42\end{array}$

$67113180-10.7-4.4-1423.93-0.86 \quad 1.97-3.42-1426.24 \quad-26.14$

$67114181-11.0 \quad-4.3-1428.27-0.641 .18-3.36-1431.09-22.91$

$67115182-11.2-4.1-1432.47-0.801 .96-3.30-1434.61-18.36$

$67116183-11.5-4.0-1436.54-0.51 \quad 1.18-3.25-1439.11-14.79$

$67117184-11.7-3.9-1440.47-0.601 .96-3.19-1442.30 \quad-9.91$

$67118185-11.9-3.7-1444.27-0.22 \quad 1.18-3.14-1446.45$

$67119186-12.2-3.6-1447.94-0.31 \quad 1.96-3.09-1449.38$

$67120187-12.4 \quad-3.5-1451.48-0.621 .18-3.04-1453.96$

$-5.98$

$-0.85$

2.65

$67121188-12.6-3.4-1454.89-1.10 \quad 1.95-2.99-1457.04$

7.64

$\begin{array}{lllllll}67 & 122 & 189-12.9 & -3.2-1458.19 & -1.30 & 1.18 & -2.95-1461.25\end{array}$

11.50

$67123190-13.1-3.1-1461.36-2.191 .95-2.90-1464.50$

16.32

$67124 \quad 191-13.3-3.0-1464.42-2.691 .18-2.86-1468.78$

20.11

$\begin{array}{llllllll}67 & 125 & 192-13.5 & -2.9-1467.35 & -3.78 & 1.94 & -2.81-1472.00\end{array}$

24.96

$\begin{array}{llllllll}67 & 126 & 193-13.8 & -2.8-1470.18 & -4.27 & 1.18 & -2.77-1476.03\end{array}$

$\begin{array}{llllllll}67 & 127 & 194-14.0 & -2.7-1472.89 & -3.28 & 1.94 & -2.73-1476.96\end{array}$

$67128 \quad 195-14.2-2.5-1475.49-2.32 \quad 1.18-2.69-1479.32$

$67129196-14.4-2.4-1477.98-1.16 \quad 1.94-2.65-1479.85$

$67130197-14.6-2.3-1480.36-0.241 .18-2.61-1482.03$

$67131.198-14.8-2.2-1482.64$

$67132199-15.0-2.1-1484.82$

$67133200-15.2-2.0-1486.90$

$0.281 .93-2.57-1483.00$

$0.871 .18-2.54-1485.31$

29.00

36.15

41.86

49.40

55.29

62.39

$1.171 .93-2.50-1486.30$

68.16

$\begin{array}{lllll}67 & 134 & 201-15.4 & -1.9-1488.87\end{array}$

$67 \quad 135202-15.6-1.8-1490.75$

$67136203-15.8-1.7-1492.53$

$67137204-16.0-1.6-1494.21$

$67138205-16.2-1.5-1495.81$

$1.491 .18-2.47-1488.66$

75.24

$1.231 .93-2.43-1490.02$

80.94

$1.291 .18-2.40-1492.46 \quad 93.29$

$1.021 .92-2.37-1493.64 \quad 100.18$

$1.121 .18-2.33-1495.84106 .05$

$0.801 .92-2.30-1496.90113 .07$

$67139206-16.4-1.5-1497.31$

$67 \quad 140207-16.6-1.4-1498.72$

$67141208-16.8-1.3-1500.05$

$0.801 .18-2.27-1499.01 \quad 119.02$

$0.411 .92-2.24-1499.97 \quad 126.14$

$67142 \quad 209-17.0-1.2-1501.28$

$0.491 .18-2.22-1501.83 \quad 132.35$

67 143. 210-17.1 -1.1-1502.44

$-0.101 .91-2.19-1502.81 \quad 139.44$

$67144211-17.3-1.0-1503.51-0.471 .18-2.16-1504.96 \quad 145.36$

$\begin{array}{llllllll}67 & 145 & 212-17.5 & -0.9-1504.49 & -1.13 & 1.91 & -2.13-1505.85 & 152.54\end{array}$

$\begin{array}{lllllllll}67 & 146 & 213-17.7 & -0.9-1505.40 & -1.19 & 1.18 & -2.11-1507.52 & 158.95\end{array}$

$\begin{array}{lllllllll}67 & 147 & 214-17.8 & -0.8-1506.23 & -1.73 & 1.91 & -2.08-1508.13 & 166.40\end{array}$

$67148 \quad 215-18.0-0.7-1506.98-1.74 \quad 1.18-2.05-1509.59173 .01$

$67149216-18.2 \quad-0.6-1507.66-2.14 \quad 1.90-2.03-1509.92180 .75$

$67 \quad 150 \quad 217-18.3 \quad-0.6-1508.26-2.10 \quad 1.18-2.01-1511.18187 .57$

$67151218-18.5-0.5-1508.78-2.68 \quad 1.90-1.98-1511.55 \quad 195.27$

$67152219-18.7-0.4-1509.24-2.71 \quad 1.18-1.96-1512.73202 .16$

$67153220-18.8-0.3-1509.63-3.03 \quad 1.90-1.94-1512.69210 .27$

$67154221-19.0-0.3-1509.94-2.73 \quad 1.18-1.91-1513.41217 .63$

$67155 \quad 222-19.1 \quad-0.2-1510.19-2.95 \quad 1.90-1.89-1513.14225 .96$

$67156 \quad 223-19.3 \quad-0.1-1510.38-2.75 \quad 1.18-1.87-1513.82 \quad 233.36$

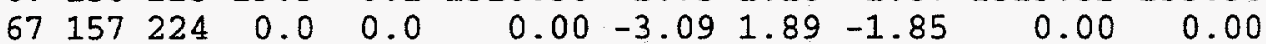

$\begin{array}{llllllllll}68 & 70 & 138 & 2.6-14.0-1059.72 & -1.04 & 0.00 & -9.41-1070.17 & -9.85\end{array}$

$6871139 \quad 2.3-13.7-1073.54-0.93 \quad 1.16-9.13-1082.44-14.06$

$\begin{array}{llllllllll}68 & 72 & 140 & 1.9-13.4-1087.05 & -0.52 & 0.00 & -8.87-1096.44 & -19.99\end{array}$

$\begin{array}{lllllllll}68 & 73 & 141 & 1.6-13.1-1100.26 & -0.31 & 1.15 & -8.62-1108.04 & -23.52\end{array}$

$\begin{array}{llll}68 & 74 & 142 & 1.2-12.8-1113.18\end{array}$

$\begin{array}{llll}68 & 75 & 143 & 0.9-12.5-1125.80\end{array}$

$\begin{array}{lllll}0.05 & 0.00 & -8.37-1121.50 & -28.91\end{array}$

0.00

0.00

0.00

0.00

0.00

0.00

0.00

0.00

0.00

0.00

0.00

0.00

0.00

0.00

0.00

0.00

0.00

0.00

0.00

0.00

0.00

0.00

0.00

0.00

0.00

0.00

0.00

0.00

0.00

0.00

0.00

0.00

0.00

0.00

0.00

0.00

0.00

0.00

0.00

0.00

0.00

0.00

0.00

0.00

0.00

0.00

0.00

0.00

0.00

0.00

0.00

0.00

0.00

0.00

0.00

0.00

0.00

0.00

0.00

0.00

0.00

0.00

0.00

0.00

0.00

0.00

0.00

0.00

0.00

0.00

0.00

0.00

0.00

0.00

0.00

0.00

0.00

0.00

0.00

0.00

0.00

0.00

0.00

0.00

0.00

0.00

0.00

0.00

0.00

0.00

0.00

0.00

0.00

0.00

0.00

0.00

0.00

0.00

0.00

0.00

0.00

0.00

0.00

0.00

0.00

0.00

0.00

0.00

0.00

0.00

0.00

0.00

0.00

0.00

0.00

0.00

0.00

0.00

0.00

0.00

0.00

0.00

0.00

0.00

0.00

0.00

0.00

0.00

0.00

0.00

0.00

0.00

0.00

0.00

0.00

0.00

0.00

0.00

0.00

0.00

0.00

0.00

0.00

0.00

0.00

0.00

0.00

0.00

0.00

0.00

0.00

0.00

0.00

$0.141 .14-8.14-1132.67-32.00$

$0.340 .00-7.92-1145.72-36.98$

$\begin{array}{lllll}68 & 76 & 144 & 0.6-12.2-1138.14 \\ 68 & 77 & 145 & 0.2-11.9-1150.20\end{array}$

$6878 \quad 146-0.1-11.6-1161.98$

$1.38 \quad 1.13-7.71-1155.39-38.58$

0.00

0.00

0.00

$0.670 .00-7.50-1168.81-43.93$

0.00

0.00

0.00

0.00

0.00

0.00

0.00

0.00

0.00

$6879.147-0.4-11.4-1173.49$

$0.141 .12-7.30-1179.53-46.58$

0.00

0.00

0.00

$\begin{array}{lllll}68 & 80 & 148 & -0.8-11.1-1184.73\end{array}$

$\begin{array}{lllll}-0.51 & 0.00 & -7.11-1192.36 & -51.33\end{array}$

$81149-1.1-10.9-1195.72$

$68 \quad 82150-1.4-10.6-1206.45$

$\begin{array}{lllll}-1.23 & 1.11 & -6.93-1202.77 & -53.68\end{array}$

0.00

0.00

0.00

$\begin{array}{llll}68 & 83 & 151 & -1.7-10.4-1216.92\end{array}$

$\begin{array}{llll}68 & 84 & 152 & -2.0-10.1-1227.16\end{array}$

$\begin{array}{llllll}68 & 85 & 153 & -2.3 & -9.9-1237.15\end{array}$

$\begin{array}{lllll}68 & 86 & 154 & -2.7 & -9.6-1246.90\end{array}$

$\begin{array}{llllll}68 & 87 & 155 & -3.0 & -9.4-1256.41\end{array}$

$\begin{array}{lllll}68 & 88 & 156 & -3.3 & -9.2-1265.70\end{array}$

$\begin{array}{llllll}68 & 89 & 157 & -3.6 & -8.9 & -1274.77\end{array}$

$\begin{array}{lllll}.34 & 0.00 & -6.76-1215.55 & -58.38\end{array}$

$-1.16$

0.07

$0.00 \quad 0.00$

0.00

$\begin{array}{llllll}-1.35 & 1.10 & -6.59-1223.76 & -58.52\end{array}$

$\begin{array}{rrrrr}-0.51 & 0.00 & -6.43-1234.09 & -60.78 \\ 0.35 & 1.09 & -6.27-1241.97 & -60.59\end{array}$

0.00

0.00

0.00

$-0.35$

0.16

$0.990 .00-6.12-1252.03-62.57-62.62$

$1.431 .08-5.98-1259.88-62.35-62.22$

0.00

$\begin{array}{lllll}1.62 & 0.00 & -5.84-1269.92 & -64.32\end{array}$

0.00

1.56

$-0.05$

1.761 .08 
$\begin{array}{lllllll}68 & 90 & 158 & -3.9 & -8.7 & -1283.61\end{array}$

$\begin{array}{llllll}68 & 91 & 159 & -4.2 & -8.5-1292.24\end{array}$

$\begin{array}{llllll}68 & 92 & 160 & -4.5 & -8.3-1300.65\end{array}$

$\begin{array}{llllll}68 & 93 & 161 & -4.8 & -8.1-1308.86\end{array}$

$\begin{array}{llllll}68 & 94 & 162 & -5.1 & -7.9-1316.86\end{array}$

$\begin{array}{llllll}68 & 95 & 163 & -5.4 & -7.7 & -1324.66\end{array}$

68. $96 \quad 164 \quad-5.7 \quad-7.5-1332.26$

$\begin{array}{llllll}68 & 97 & 165 & -5.9 & -7.3-1339.66\end{array}$

$\begin{array}{llllll}68 & 98 & 166 & -6.2 & -7.1-1346.88\end{array}$

$\begin{array}{llllll}68 & 99 & 167 & -6.5 & -6.9-1353.91\end{array}$

$\begin{array}{lllll}68 & 100 & 168 & -6.8 & -6.8-1360.75\end{array}$

$\begin{array}{llllll}68 & 101 & 169 & -7.1 & -6.6-1367.42\end{array}$

$\begin{array}{llllll}68 & 102 & 170 & -7.3 & -6.4-1373.90\end{array}$

$\begin{array}{llllll}68 & 103 & 171 & -7.6 & -6.2-1380.22\end{array}$

$\begin{array}{llllll}68 & 104 & 172 & -7.9 & -6.1-1386.36\end{array}$

$\begin{array}{llllll}68 & 105 & 173 & -8.1 & -5.9-1392.33\end{array}$

$\begin{array}{llllll}68 & 106 & 174 & -8.4 & -5.7-1398.14\end{array}$

$\begin{array}{llllll}68 & 107 & 175 & -8.7 & -5.6-1403.78\end{array}$

$\begin{array}{lllll}68 & 108 & 176 & -8.9 & -5.4-1409.27\end{array}$

$\begin{array}{llllll}68 & 109 & 177 & -9.2 & -5.2-1414.60\end{array}$

$\begin{array}{lllllllllll}68 & 110 & 178 & -9.4 & -5.1-1419.77 & -1.36 & 0.00 & -3.71-1424.85 & -41.68 \\ 68 & 111 & 179 & -9.7 & -4.9-1424.80 & -1.44 & 1.00 & -3.65-1428.89 & -37.65\end{array}$

$\begin{array}{lllllllllll}68 & 112 & 180 & -9.9 & -4.8 & -1429.67 & -1.16 & 0.00 & -3.58-1434.41 & -35.10\end{array}$

$\begin{array}{lllllllll}68 & 113 & 181-10.2 & -4.7-1434.40 & -1.33 & 0.99 & -3.52-1438.26 & -30.88\end{array}$

$\begin{array}{lllllllll}68 & 114 & 182-10.4 & -4.5-1438.99 & -1.11 & 0.00 & -3.46-1443.56 & -28.10\end{array}$

$\begin{array}{lllllllll}68 & 115 & 183-10.7 & -4.4-1443.43 & -1.24 & 0.99 & -3.40-1447.08 & -23.56\end{array}$

$\begin{array}{llllllllll}68 & 116 & 184-10.9 & -4.2-1447.74 & -0.93 & 0.00 & -3.34-1452.01 & -20.41\end{array}$

$\begin{array}{lllllllll}68 & 117 & 185-11.2 & -4.1-1451.90 & -1.01 & 0.98 & -3.29-1455.22 & -15.55\end{array}$

$68118186-11.4-4.0-1455.94-0.59 \quad 0.00-3.23-1459.76-12.02$

$\begin{array}{llllllll}68 & 119 & 187-11.6 & -3.8-1459.84 & -0.73 & 0.98 & -3.18-1462.78 & -6.97\end{array}$

$\begin{array}{lllllllll}68 & 120 & 188-11.9 & -3.7-1463.62 & -1.01 & 0.00 & -3.13-1467.76\end{array}$

$\begin{array}{llllllll}68 & 121 & 189-12.1 & -3.6-1467.26 & -1.46 & 0.97 & -3.08-1470.83\end{array}$

$\begin{array}{llllllll}68 & 122 & 190-12.3 & -3.5-1470.79 & -1.65 & 0.00 & -3.03-1475.47\end{array}$

$\begin{array}{llllllll}68 & 123 & 191-12.6 & -3.3-1474.19 & -2.52 & 0.97 & -2.98-1478.72\end{array}$

$\begin{array}{lllllllll}68 & 124 & 192-12.8 & -3.2-1477.46 & -2.99 & 0.00 & -2.94-1483.39\end{array}$

$\begin{array}{lllllllll}68 & 125 & 193-13.0 & -3.1-1480.62 & -4.17 & 0.96 & -2.89 & -1486.73\end{array}$

$68 \quad 126194-13.2-3.0-1483.66-4.510 .00-2.85-1491.02$

$\begin{array}{llllllll}68 & 127 & 195-13.4 & -2.9-1486.59 & -3.49 & 0.95 & -2.81-1491.93\end{array}$

$\begin{array}{llllllll}68 & 128 & 196-13.7 & -2.8-1489.41 & -2.61 & 0.00 & -2.76-1494.78\end{array}$

$\begin{array}{llllllll}68 & 129 & 197-13.9 & -2.6-1492.11 & -1.36 & 0.95 & -2.72-1495.25\end{array}$

$\begin{array}{llllllll}68 & 130 & 198-14.1 & -2.5-1494.71 & -0.38 & 0.00 & -2.68-1497.77\end{array}$

$68 \quad 131 \quad 199-14.3-2.4-1497.19$

$68 \quad 132 \quad 200-14.5 \quad-2.3-1499.58$

$68 \quad 133 \quad 201-14.7-2.2-1501.85$

$\begin{array}{lllll}68 & 134 & 202-14.9 & -2.1-1504.03\end{array}$

$68 \quad 135 \quad 203-15.1-2.0-1506.11$

$68 \quad 136204-15.3-1.9-1508.09$

$\begin{array}{llll}68 & 137 & 205-15.5 & -1.8-1509.97\end{array}$

$\begin{array}{llll}68 & 138 & 206-15.7 & -1.7-1511.76\end{array}$

$68 \quad 139207-15.9-1.6-1513.45$

$68 \quad 140 \quad 208-16.1-1.6-1515.05$

$68 \quad 141209-16.3-1.5-1516.56$

$68 \quad 142 \quad 210-16.4-1.4-1517.99$

$68 \quad 143 \quad 211-16.6-1.3-1519.32$

$\begin{array}{llll}68 & 144 & 212-16.8 & -1.2-1520.57\end{array}$

$\begin{array}{llll}68 & 145 & 213-17.0 & -1.1-1521.74\end{array}$

$0.290 .95-2.65-1498.60$

$0.90 \quad 0.00-2.61-1501.28$

$1.210 .94-2.57-1502.28$

$1.590 .00-2.54-1504.98$

$1.440 .94-2.50-1506.23$

$1.540 .00-2.47-1509.01$

$1.290 .93-2.43-1510.18$

$-3.87$

1.12

4.56

9.37

12.78

17.51

21.29

28.45

33.67

41.28

46.83

54.06

59.46

66.54

71.90

78.72

84.01

$1.410 .00-2.40-1512.75$

90.92

$\begin{array}{llllll}1.13 & 0.93 & -2.37-1513.76 & 103.48\end{array}$

$1.15 \cdot 0.00-2.34-1516.24 \quad 109.07$

$0.740 .92-2.31-1517.21 \quad 116.18$

$0.79 \quad 0.00-2.28-1519.47 \quad 121.98$

$\begin{array}{lllll}0.39 & 0.92 & -2.25 & -1520.26 & 129.27\end{array}$

$\begin{array}{lllll}0.20 & 0.00 & -2.22-1522.59 & 135.01\end{array}$

$68146214-17.2-1.0-1522.82-0.82 \quad 0.00-2.16-1525.81147 .93$

$\begin{array}{lllllllll}68 & 147 & 215-17.3 & -1.0-1523.82 & -1.33 & 0.91 & -2.14-1526.38 & 155.43\end{array}$

$\begin{array}{lllllllll}68 & 148 & 216-17.5 & -0.9-1524.75 & -1.37 & 0.00 & -2.11-1528.23 & 161.65\end{array}$

$\begin{array}{lllllllll}68 & 149 & 217-17.7 & -0.8-1525.59 & -1.83 & 0.91 & -2.09-1528.60 & 169.35\end{array}$

$\begin{array}{lllllllll}68 & 150 & 218-17.9 & -0.7-1526.36 & -1.85 & 0.00 & -2.06-1530.27 & 175.76\end{array}$

$\begin{array}{lllllllll}68 & 151 & 219-18.0 & -0.7-1527.05 & -2.46 & 0.90 & -2.04-1530.65 & 183.45\end{array}$

$\begin{array}{lllllllll}68 & 152 & 220-18.2 & -0.6-1527.67 & -2.51 & 0.00 & -2.01-1532.19 & 189.97\end{array}$

$\begin{array}{lllllllll}68 & 153 & 221-18.4 & -0.5-1528.22 & -2.79 & 0.90 & -1.99-1532.10 & 198.14\end{array}$

$\begin{array}{llllllll}68 & 154 & 222-18.5 & -0.4-1528.69 & -2.62 & 0.00 & -1.97-1533.28 & 205.03\end{array}$

$\begin{array}{lllllllll}68 & 155 & 223-18.7 & -0.4-1529.10 & -2.89 & 0.89 & -1.94-1533.04 & 213.34\end{array}$
0

2.24

0.00

$-64.57$

2.03

0.60

$1.85-0.50$

$1.58 \quad 0.38$

1.310 .39

$1.02 \quad 0.32$

0.630 .31

0.320 .25

$\begin{array}{ll}-0.23 & 0.14\end{array}$

$\begin{array}{ll}-0.21 & 0.33\end{array}$

0.32

0.58

0.47

0.59

0.00

0.00

0.00

0.00

0.00

0.00

0.00

0.00

0.00

0.00

0.00

0.00

0.00

0.00

0.00

0.00

0.00

0.00

0.00

0.00

0.00

0.00

0.00

$0.00 \quad 0.00$

0.00

0.00

0.00

0.00

0.00

0.00

0.00

0.00

0.00

0.00

0.00

0.00

0.00

0.00

0.00

0.00

0.00

0.00

0.00

0.00

0.00

0.00

0.00

0.00

0.00

0.00

0.00

0.00

0.00

0.00

0.00

0.00

0.00

0.00

0.00

0.00

0.00

0.00

0.00

0.00

0.00

0.00

0.00

0.00

0.00

0.00

0.00

0.00

0.00

0.00

0.00

0.00

0.00

0.00

0.00

0.00

0.00

0.00

0.00

0.00

0.00

0.00

0.00

0.00

0.00

0.00

0.00

0.00

0.00

0.00

0.00

0.00

0.00

0.00

0.00

0.00

0.00

0.00

0.00

$\begin{array}{llll}0.00 & 0.00 & 0.00\end{array}$

$0.00 \quad 0.00$

0.00

0.00

0.00

0.00

0.00

0.00

0.00

0.00

0.00

0.00

0.00

0.00

0.00

0.00

0.00

0.00

0.00

0.00

0.00

0.00

0.00

0.00

0.00

0.00

0.00

0.00

0.00

0.00

0.00

0.00

0.00

0.00

0.00

0.00

0.00

0.00

0.00

0.00

0.00

0.00

0.00

0.00 
$\begin{array}{lllllllll}68 & 156 & 224-18.8 & -0.3-1529.44 & -2.76 & 0.00 & -1.92-1534.12 & 220.34\end{array}$ $\begin{array}{lllllllll}68 & 157 & 225-19.0 & -0.2-1529.71 & -3.08 & 0.89 & -1.90-1533.80 & 228.73\end{array}$ $\begin{array}{llllllll}68 & 158 & 226-19.1 & -0.2-1529.92 & -3.03 & 0.00 & -1.88-1534.82 & 235.77\end{array}$ $\begin{array}{llllllll}68 & 159 & 227-19.3 & -0.1-1530.06 & -3.53 & 0.89 & -1.86-1534.56 & 244.11\end{array}$ $\begin{array}{lllllllll}69 & 72 & 141 & 2.4-13.7-1084.88 & -1.20 & 1.17 & -9.15-1094.05 & -10.32\end{array}$ $\begin{array}{lllllllll}69 & 73 & 142 & 2.1-13.4-1098.43 & -0.91 & 2.08 & -8.88-1106.15 & -14.35\end{array}$ $\begin{array}{lllllllll}69 & 74 & 143 & 1.8-13.1-1111.68 & -0.49 & 1.17 & -8.63-1119.64 & -19.76\end{array}$ $\begin{array}{llllllll}69 & 75 & 144 & 1.4-12.8-1124.64 & -0.57 & 2.07 & -8.39-1131.54 & -23.60\end{array}$ $\begin{array}{lll}69 & 76 & 145\end{array}$

69. $77 \quad 146$

$\begin{array}{lll}69 & 78 & 147\end{array}$

$\begin{array}{lll}69 & 79 & 148\end{array}$ $1.1-12.5-1137.32$ $0.8-12.2-1149.71$ $0.4-12.0-1161.82$

$6981 \quad 150-0.5-11.2-1196.53$

$\begin{array}{llll}69 & 82 & 151 & -0.9-10.9-1207.58\end{array}$

69

69

69

69

69

69

69

69

\section{9}

69

69

69

69

69

$6999168-6.0-7.2-1360.15$

$\begin{array}{llllll}69 & 100 & 169 & -6.2 & -7.0 & -1367.27\end{array}$

$\begin{array}{lllll}69 & 101 & 170 & -6.5 & -6.8-1374.21\end{array}$

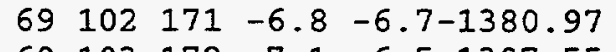

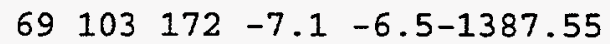

$\begin{array}{llllll}69 & 104 & 173 & -7.3 & -6.3-1393.97\end{array}$

$\begin{array}{lllll}69 & 105 & 174 & -7.6 & -6.2-1400.20\end{array}$

$\begin{array}{llllll}69 & 106 & 175 & -7.9 & -6.0-1406.28\end{array}$

$\begin{array}{llllll}69 & 107 & 176 & -8.1 & -5.8-1412.18\end{array}$

$\begin{array}{llllll}69 & 108 & 177 & -8.4 & -5.7-1417.93 \\ 69 & 109 & 178 & -8.6 & -5.5-1423.52\end{array}$

$\begin{array}{lllll}69 & 109 & 178 & -8.6 & -5.5-1423.52 \\ 69 & 110 & 179 & -8.9 & -5.3-1428.95\end{array}$

$\begin{array}{llllll}69 & 111 & 180 & -9.2 & -5.2-1434.22\end{array}$

$\begin{array}{llllll}69 & 112 & 181 & -9.4 & -5.0-1439.35 \\ 69 & 113 & 182 & -9.7 & -4.9-1444.33\end{array}$

$\begin{array}{lllll}69 & 113 & 182 & -9.7 & -4.9 \\ 69 & 114 & 183 & -9.9 & -4.844 .33 \\ 6 & 115 & 184 & -10.1 & -449.16\end{array}$

$69115 \quad 184-10.1-4.6-1453.85$

$69116 \quad 185-10.4-4.5-1458.40$

$\begin{array}{lllll}69 & 117 & 186-10.6 & -4.3-1462.80\end{array}$

$\begin{array}{llll}69 & 118 & 187-10.9 & -4.2-1467.08\end{array}$

69119 188-11.1 -4.1-1471.22

$69 \quad 120189-11.3-3.9-1475.22$

$\begin{array}{llll}69 & 121 & 190-11.6 & -3.8-1479.10 \\ 69 & 122 & 191-11.8 & -3.7-1482.85\end{array}$

$\begin{array}{llll}69 & 122 & 191-11.8 & -3.7-1482.85 \\ 69 & 123 & 192-12.0 & -3.6-1486.48\end{array}$

$69124193-12.2-3.4-1489.98$

$69125 \quad 194-12.5-3.3-1493.36$

$69126 \quad 195-12.7 \quad-3.2-1496.62$

$\begin{array}{lllll}69 & 127 & 196-12.9 & -3.1-1499.77\end{array}$

$69128 \quad 197-13.1-3.0-1502.80$

$69129198-13.3-2.9-1505.72$

$69130 \quad 199-13.6 \quad-2.7-1508.52$

$69131200-13.8-2.6-1511.22$

$\begin{array}{llll}69 & 132 & 201-14.0 & -2.5-1513.81\end{array}$

$69133 \quad 202-14.2 \quad-2.4-1516.30$ $-0.341 .17-8.16-1144.65-28.63$ $0.862 .06-7.94-1154.73-30.64$ $\begin{array}{lllll}0.19 & 1.17 & -7.73-1168.19 & -36.03\end{array}$

$\begin{array}{llll}-0.37 & 2.05 & -7.53-1179.50 & -39.27\end{array}$

$\begin{array}{llll}-0.99 & 1.17 & -7.33-1192.38 & -44.08\end{array}$

$\begin{array}{llll}-1.42 & 2.05 & -7.15-1203.05 & -46.68\end{array}$

$\begin{array}{llll}-2.43 & 1.17 & -6.97-1215.81 & -51.36\end{array}$

$\begin{array}{llll}-1.48 & 2.04 & -6.79-1224.61 & -52.10\end{array}$

$\begin{array}{lllll}-0.60 & 1.17 & -6.62-1234.98 & -54.39\end{array}$

$0.172 .03-6.46-1243.49-54.83$

$0.801 .17-6.31-1253.63-56.90$

$\begin{array}{lllll}1.21 & 2.03 & -6.16-1262.05 & -57.24 & -56\end{array}$

$\begin{array}{llll}1.43 & 1.17 & -6.02-1272.13 & -59.26\end{array}$

$\begin{array}{lllll}1.62 & 2.02 & -5.88 & -1280.32 & -59.38\end{array}$

$\begin{array}{lllll}1.67 & 1.17 & -5.74-1290.14 & -61.12\end{array}$

$\begin{array}{lllll}1.69 & 2.01 & -5.61-1298.07 & -60.98\end{array}$

$\begin{array}{lllll}1.55 & 1.17 & -5.49-1307.64 & -62.48 & -62\end{array}$

$1.37 \quad 2.01-5.37-1315.36-62.13$

$\begin{array}{llll}1.26 & 1.17 & -5.25-1324.49 & -63\end{array}$

$-63.18$

$0.92 \quad 2.00-5.14-1331.97-62$

$0.73 \quad 1.17-5.03-1340.77-63$

$0.332 .00-4.92-1347.93$

$0.041 .17-4.82-1356.45$

$-0.431 .99-4.72-1363.31-61$

$\begin{array}{llll}-0.60 & 1.17 & -4.63-1371.33-61 \\ -1.07 & 1.99 & -4.54-1377.83-60\end{array}$

$\begin{array}{lll}-1.21 & 1.17 & -4.45-1385.46\end{array}$

$-1.561 .98-4.36-1391.49-5$

$-62.60-61$

0.00

0.00

0.00

0.00

0.00

0.00

0.00

0.00

0.00

0.00

0.00

0.00

0.00

0.00

0.00

0.00

0.00

0.00

0.00

0.00

0.00

0.00

0.00

0.00

0.00

0.00

0.00

0.00

0.00

0.00

0.00

0.00

0.00

0.00

0.00

0.00

0.00

0.00

0.00

0.00

0.00

0.00

0.00

$-54.55$

0.00

0.00

0.45

1.02

0.28

1.49

0.22

56.96

0.00

0.00

0.28

0.00

0.00

0.00

0.00

0.00

2.21

1.93

1.95

0.52

$-62.10$

$-61.55$

1.70

.38

1.53

1.12

0.86

0.35

$-62.86-62.55$

$-61.65-61.32$

$-0.10$

$-61.28-0.28$

$-60.03-59.80$

$-59.22$

$-0.84$

$-0.85$

$-57.55$

$-57.38$

$-1.39$

$\begin{array}{lllllll}-1.52 & 1.17 & -4.28 & -1398.59 & -56.57 & -56.27 & -1.22\end{array}$

$\begin{array}{llll}-1.76 & 1.98 & -4.19-1404.18 & -54.09\end{array}$

$-53.87$

$-1.54$

$\begin{array}{lllll}-1.80 & 1.17 & -4.11-1411.02 & -52.86\end{array}$

0.30

$-1.24$

$-2.01 \quad 1.17-3.96-1422.73-48.43$

$\begin{array}{llll}-2.12 & 1.97 & -3.89-1427.56 & -45.19\end{array}$

0.00

0.00

0.00

0.00

0.00

0.00

0.00

0.00

0.00

$\begin{array}{llll}-1.93 & 1.96 & -3.75-1437.95 & -39.43\end{array}$

$\begin{array}{llll}-1.64 & 1.17 & -3.69-1443.51 & -36.92\end{array}$

$\begin{array}{llll}-1.80 & 1.96 & -3.62-1447.79 & -33.13\end{array}$

0.00

0.00

0.00

0.00

0.00

0.00

0.00

0.00

$\begin{array}{lllll}-1.68 & 1.95 & -3.50-1457.07 & -26.27\end{array}$

$\begin{array}{lllll}-1.40 & 1.17 & -3.44-1462.07 & -23.19\end{array}$

$-1.441 .95-3.38-1465.68-18.73$

$-0.991 .17-3.33-1470.22-15.21$

$-1.061 .95-3.27-1473.60$

$-10.51$

$-1.371 .17-3.22-1478.64$

$-1.791 .94-3.17-1482.12$

$-2.821 .94-3.07-1490.43$

$-3.251 .17-3.02-1495.08$

$-4.401 .93-2.97-1498.80$

$-4.781 .17-2.93-1503.16$

$-3.681 .93-2.89-1504.40$

$-2.811 .17 .2 .84-1507.28$

$-1.491 .93-2.80-1508.08$

$-0.331 .17-2.76-1510.44$

$0.081 .92-2.72-1511.94$

$0.761 .17-2.68-1514.56$

$1.131 .92-2.64-1515.89$ $\overline{62}$
$-7.48$

$-2.89$

0.56

4.95

8.37

0.00

0.00

0.00

0.00

0.00

0.00

0.00

0.00

0.00

0.00

0.00

0.00

0.00

0.00

0.00

0.00

0.00

12.72

16.43

23.26

28.45

35.72

41.43

48.01

53.46

60.20

0.00

0.00

0.00

0.00

0.00

0.00

0.00
0.58

0.44

0.61

0.39

0.53

0.31

0.33

0.32

0.23

0.36

0.17

0.30

0.22

0.56

0.00

0.00

0.00

0.00

0.00

0.00

0.00

0.00

0.00 
$\begin{array}{lllll}69 & 134 & 203-14.4 & -2.3-1518.67\end{array}$ $\begin{array}{llll}69 & 135 & 204-14.6 & -2.2-1520.95\end{array}$

$69136205-14.8-2.1-1523.13$ $69137206-15.0-2.0-1525.21$ $69138 \quad 207-15.2-1.9-1527.19$ $\begin{array}{llll}69 & 139 & 208-15.4 & -1.8-1529.08\end{array}$ $69140 \quad 209-15.6-1.7-1530.87$ $\begin{array}{llll}69 & 141 & 210-15.8 & -1.7-1532.57\end{array}$ $\begin{array}{llll}69 & 142 & 211-15.9 & -1.6-1534.18\end{array}$ $69143 \quad 212-16.1-1.5-1535.70$

$69 \quad 144 \quad 213-16.3-1.4-1537.14$

$69 \quad 145 \quad 214-16.5-1.3-1538.48$

$69146215-16.7-1.2-1539.74$

$69147216-16.9-1.1-1540.92$

$69148217-17.0-1.1-1542.02$

$69149218-17.2-1.0-1543.04$

$69 \quad 150 \quad 219-17.4 \quad-0.9-1543.98$

$69 \quad 151220-17.5-0.8-1544.84$

$69 \quad 152 \quad 221-17.7-0.7-1545.62$

$\begin{array}{lllll}69 & 153 & 222-17.9 & -0.7-1546.33\end{array}$

$69 \quad 154 \quad 223-18.0 \quad-0.6-1546.97$

$69155 \quad 224-18.2-0.5-1547.54$

$69156225-18.4-0.5-1548.03$

$69 \quad 157226-18.5-0.4-1548.46$

$\begin{array}{lllll}69 & 158 & 227-18.7 & -0.3-1548.82\end{array}$

$69159228-18.8-0.3-1549.11$

$69160 \quad 229-19.0-0.2-1549.34$

$69161230-19.1-0.1-1549.50$

$70 \quad 73 \quad 143 \quad 2.6-13.7-1096.09$

$70 \quad 74 \quad 144$

$70 \quad 75145$

$\begin{array}{lll}70 & 76 & 146\end{array}$

$\begin{array}{lll}70 & 77 & 147\end{array}$

2. 3-13.4-1109.68

$1.9-13.1-1122.97$

$1.6-12 \cdot 9-1135 \cdot 98$

$1.3-12 \cdot 6-1148.69$

$0.9-12 \cdot 3-1161 \cdot 13$

$78 \quad 148$

$\begin{array}{lll}70 & 79 & 149\end{array}$

$70 \quad 80 \quad 150$

$0.6-12.0-1173.30$

$0.3-11.8-1185.19$

$0.0-11.5-1196.82$

81151

$0.0-11.5-1196.82$
$-0.3-11.2-1208.19$

83153

$\begin{array}{llll}70 & 83 & 153 & -0.7-11.0-1219.31 \\ 70 & 84 & 154 & -1.0-10.7-1230.17\end{array}$

$70 \quad 85155-1.3-10.5-1240.79$

$7086 \quad 156-1.6-10.3-1251.16$

$70 \quad 87 \quad 157-1.9-10.0-1261.30$

$70 \quad 88 \quad 158-2.2-9.8-1271.20$

$\begin{array}{llllll}70 & 89 & 159 & -2.5 & -9.6-1280.87\end{array}$

$70 \quad 90 \quad 160-2.8 \quad-9.3-1290.32$

$\begin{array}{llllll}70 & 91 & 161 & -3.1 & -9.1-1299.54\end{array}$

$\begin{array}{llllll}70 & 92 & 162 & -3.4 & -8.9-1308.55\end{array}$

$7093 \quad 163-3.7-8.7-1317.35$

$7094 \quad 164-4.0 \quad-8.5-1325.94$

$7095 \quad 165-4.3-8.3-1334.31$

$7096 \quad 166-4.6 \quad-8.1-1342.49$

$\begin{array}{llllll}70 & 97 & 167 & -4.9 & -7.9-1350.47\end{array}$

$\begin{array}{llllll}70 & 98 & 168 & -5.1 & -7.7-1358.25\end{array}$

$70 \quad 99 \quad 169-5.4 \quad-7.5-1365.84$

$70 \begin{array}{llllll}700 & 170 & -5.7 & -7.3-1373.25\end{array}$

$\begin{array}{llllll}70 & 101 & 171 & -6.0 & -7.1-1380.46\end{array}$

$\begin{array}{lllll}70 & 102 & 172 & -6.3 & -6.9-1387.50\end{array}$

$\begin{array}{llllll}70 & 103 & 173 & -6.5 & -6.8-1394.35\end{array}$

$\begin{array}{llllll}70 & 104 & 174 & -6.8 & -6.6-1401.03 & -1\end{array}$

$70 \begin{array}{llllll}705 & 175 & -7.1 & -6.4-1407.54\end{array}$

$\begin{array}{llllll}70 & 106 & 176 & -7.3 & -6.2-1413.88 & -1\end{array}$

$\begin{array}{llllll}70 & 107 & 177 & -7.6 & -6.1-1420.05\end{array}$

$\begin{array}{llllll}70 & 108 & 178 & -7.9 & -5.9-1426.05\end{array}$

$70109179-8.1-5.8-1431.90$

$\begin{array}{llllll}70 & 110 & 180 & -8.4 & -5.6-1437.58 & -2.06\end{array}$

$1.541 .17-2.61-1518.57$

$1.441 .92-2.57-1520.17$

65.59

72.06

$1.58 \quad 1.17-2.53-1522.91$

$1.331 .91-2.50-1524.47$

77.39

83.91

$1.451 .17-2.47-1527.04 \quad 89.41$

$1.171 .91-2.43-1528.43 \quad 96.08$

$1.191 .17-2.40-1530.91101 .68$

$0.78 \quad 1.91-2.37-1532.26 \quad 108.40$

$0.841 .17-2.34-1534.51 \quad 114.22$

$0.37 .1 .90-2.31-1535.74 \quad 121.06$

$0.18 \quad 1.17-2.28-1538.06 \quad 126.81$

$\begin{array}{lllll}-0.29 & 1.90 & -2.25-1539.12 & 133.82\end{array}$

$\begin{array}{lllll}-0.68 & 1.17 & -2.22-1541.48 & 139.54\end{array}$

$\begin{array}{lllll}-1.32 & 1.90 & -2.19-1542.54 & 146.55\end{array}$

$\begin{array}{lllll}-1.31 & 1.17 & -2.17-1544.33 & 152.83\end{array}$

$\begin{array}{lllll}-1.78 & 1.89 & -2.14-1545.07 & 160.16\end{array}$

$\begin{array}{lllll}-1.81 & 1.17 & -2.12-1546.73 & 166.57\end{array}$

$\begin{array}{lllll}-2.42 & 1.89 & -2.09-1547.46 & 173.92\end{array}$

$\begin{array}{lllll}-2.47 & 1.17 & -2.07-1548.99 & 180.46\end{array}$

$\begin{array}{lllll}-2.80 & 1.89 & -2.04-1549.29 & 188.23\end{array}$

$\begin{array}{llllll}-2.65 & 1.17 & -2.02-1550.47 .195 .12\end{array}$

$\begin{array}{lllll}-2.98 & 1.88 & -1.99-1550.63 & 203.03\end{array}$

$\begin{array}{llllll}-2.86 & 1.17 & -1.97-1551.69 & 210.04\end{array}$

$\begin{array}{lllll}-3.25 & 1.88 & -1.95-1551.77 & 218.03\end{array}$

$\begin{array}{llllll}-3.23 & 1.17 & -1.93-1552.80 & 225.07\end{array}$

$\begin{array}{llllll}-3.73 & 1.88 & -1.91-1552.86 & 233.08\end{array}$

$\begin{array}{lllll}-3.82 & 1.17 & -1.88-1553.87 & 240.15\end{array}$

$\begin{array}{llll}-4.38 & 1.88-1.86-1553.87 & 248.22\end{array}$

$-1.191 .15$

$-9.16-1105.29$

$-6.21$

$-0.790 .00-8.90-1119.37-12.22$

$-0.801 .14-8.65-1131.28-16.06$

$0.96 \quad 0.00-8.41-1143.43-20.14$

$0.471 .13-8.19-1155.28-23.92$

$\begin{array}{lllll}-0.25 & 0.00 & -7.97-1169.35 & -29.92\end{array}$

$\begin{array}{lllll}-0.78 & 1.12 & -7.76-1180.72 & -33.21\end{array}$

$\begin{array}{lllll}-1.46 & 0.00 & -7.56-1194.21 & -38.63\end{array}$

$\begin{array}{llllll}-1.87 & 1.11 & -7.36-1204.95 & -41.30\end{array}$

$\begin{array}{lllll}-2.92 & 0.00 & -7.18-1218.29 & -46.57\end{array}$

$\begin{array}{lllll}-1.92 & 1.10 & -7.00-1227.13 & -47.33\end{array}$

$-1.040 .00$

$-6.83-1238.04-5$

$-50.17$

0.121 .09

$-6.66-1246.23$

$-50.30$

0.560 .00

$-6.50-1257.10-53.09$

$0.981 .08-6.35-1265.58-53.50$

1.380 .00

$-6.20-1276.02-$

$-55.87$

1.651 .08

$-6.05-1284.20-55.98$

$1.75 \quad 0.00$

$-5.92-1294.49-58.19$

$\begin{array}{lllll}1.84 & 1.07 & -5.78-1302.42 & -58.06\end{array}$

$1.820 .00-5.65-1312.39-59.95$

$1.631 .06 \quad-5.53-1320.19-59.68$

$1.470 .00-5.41-1329.87-61.29$

$1.211 .05-5.29-1337.34-60.69$

$0.960 .00-5.18-1346.71-61.99$

$0.63 \quad 1.04-5.07-1353.87-61.07$

$0.320 .00-4.97-1362.90-62.03$

$\begin{array}{llllll}-0.11 & 1.04 & -4.86-1369.78 & -60.84\end{array}$

$-0.340 .00$

$-4.77-1378.35-61.34$

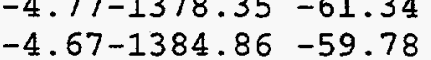

0.761 .03

$-4.58-1393.01-59$

$1.321 .02-4$

$-59.86$

$4.49-1399.14-57.91$

$-4.40-1406.73$

$-57.44$

691.02

$-4.32-1412.53$

$-55.16$

$-4.24-1419.85-54.42$

$-4.16-1425.31-51.80$

$-4.08-1432.23-50.65$

$-4.00-1437.16-47.51$

63
0.00

0.00

$0.00 \quad 0.00$

0.00

$0.00 \quad 0.00 \quad 0.00$

$0.00 \quad 0.00 \quad 0.00$

$0.00 \quad 0.00 \quad 0.00$

$0.00 \quad 0.00 \quad 0.00$

$\begin{array}{llll}0.00 & 0.00 & 0.00\end{array}$

$0.00 \quad 0.00 \quad 0.00$

$\begin{array}{llll}0.00 & 0.00 & 0.00\end{array}$

$0.00 \quad 0.00 \quad 0.00$

$0.00 \quad 0.00 \quad 0.00$

$\begin{array}{llll}0.00 & 0.00 & 0.00\end{array}$

$0.00 \quad 0.00 \quad 0.00$

$0.00 \quad 0.00 \quad 0.00$

$0.00 \quad 0.00 \quad 0.00$

$0.00 \cdot 0.00 \quad 0.00$

$0.00 \quad 0.00 \quad 0.00$

$\begin{array}{llll}0.00 & 0.00 & 0.00\end{array}$

$0.00 \quad 0.00 \quad 0.00$

$\begin{array}{llll}0.00 & 0.00 & 0.00\end{array}$

$\begin{array}{llll}0.00 & 0.00 & 0.00\end{array}$

$\begin{array}{llll}0.00 & 0.00 & 0.00\end{array}$

$\begin{array}{llll}0.00 & 0.00 & 0.00\end{array}$

$\begin{array}{llll}0.00 & 0.00 & 0.00\end{array}$

$\begin{array}{llll}0.00 & 0.00 & 0.00\end{array}$

$0.00 \quad 0.00 \quad 0.00$

$0.00 \quad 0.00 \quad 0.00$

$\begin{array}{llll}0.00 & 0.00 & 0.00\end{array}$

$\begin{array}{llll}0.00 & 0.00 & 0.00\end{array}$

$\begin{array}{llll}0.00 & 0.00 & 0.00\end{array}$

$0.00 \quad 0.00 \quad 0.00$

$0.00 \quad 0.00 \quad 0.00$

$\begin{array}{llll}0.00 & 0.00 & 0.00\end{array}$

$\begin{array}{llll}0.00 & 0.00 & 0.00\end{array}$

$\begin{array}{llll}0.00 & 0.00 & 0.00\end{array}$

$\begin{array}{llll}0.00 & 0.00 & 0.00\end{array}$

$0.00 \quad 0.00 \quad 0.00$

$0.00 \quad 0.00 \quad 0.00$

$0.00 \quad 0.00 \quad 0.00$

$\begin{array}{llll}0.00 & 0.00 & 0.00\end{array}$

$0.00 \quad 0.00 \quad 0.00$

$-53.39$

0.00

$0.26-0.30$

$0.00 \quad 0.00$

$\begin{array}{llll}-56.02 & 1.23-0.15\end{array}$

$0.00 \quad 0.00 \quad 0.00$

$\begin{array}{llll}0.00 & 0.00 & 0.00\end{array}$

$\begin{array}{llll}0.00 & 0.00 \quad 0.00\end{array}$

$0.00 \quad 0.00 \quad 0.00$

$-59.37$

1. 94

0.31

$\begin{array}{lll}0.00 & 0.00 & 0.00\end{array}$

$\begin{array}{lll}-60.18 & 1.72 & 0.51\end{array}$

$\begin{array}{lll}-61.59 & 1.36 & 0.40\end{array}$

$-60.60$

1.10

0.47

$\begin{array}{rrrr}-61.58 & 0.77 & 0.45\end{array}$

$\begin{array}{lll}-60.37 & 0.36 & 0.47\end{array}$

$\begin{array}{lll}-60.77 & 0.23 & 0.57\end{array}$

$-59.31-0.29$

0.47

0.60

0.35

0.49

0.46

0.92

0.80

0.94

$9.71-1.16$

$0.00 \quad 0.00$

0.00

$0.00 \quad 0.00$

0.00 
$\begin{array}{lllllllllll}70 & 111 & 181 & -8.6 & -5.4-1443.11 & -2.18 & 1.00 & -3.86-1448.16 & -42.36\end{array}$ $\begin{array}{llllllllll}70 & 112 & 182 & -8.9 & -5.3 & -1448.49 & -2.01 & 0.00 & -3.79-1454.30 & -40.43\end{array}$ $\begin{array}{llllllllll}70 & 113 & 183 & -9.1 & -5.1-1453.72 & -2.06 & 0.99 & -3.73-1458.51 & -36.58\end{array}$ $\begin{array}{llllllllll}70 & 114 & 184 & -9.4 & -5.0-1458.80 & -1.95 & 0.00 & -3.66-1464.41 & -34.40\end{array}$ $\begin{array}{llllllllll}70 & 115 & 185 & -9.6 & -4.9-1463.73 & -2.13 & 0.99 & -3.60-1468.47 & -30.39\end{array}$ $\begin{array}{llllllllll}70 & 116 & 186 & -9.9 & -4.7-1468.52 & -1.78 & 0.00 & -3.54-1473.84 & -27.69\end{array}$ $\begin{array}{lllllllll}70 & 117 & 187-10.1 & -4.6-1473.17 & -1.85 & 0.98 & -3.48-1477.52 & -23.29\end{array}$ $\begin{array}{llllllllll}70 & 118 & 188-10.3 & -4.4-1477.68 & -1.46 & 0.00 & -3.42-1482.56 & -20.27\end{array}$

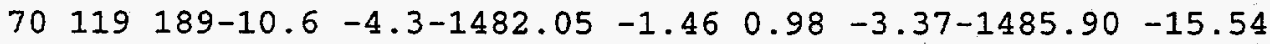
$\begin{array}{lllllllll}70 & 120 & 190-10.8 & -4.2-1486.29 & -1.78 & 0.00 & -3.31-1491.39 & -12.95\end{array}$ $70121 \quad 191-11.0-4.0-1490.40-2.220 .97-3.26-1494.91$ $\begin{array}{llllllll}70 & 122 & 192-11.3 & -3.9-1494.38 & -2.39 & 0.00 & -3.21-1499.98\end{array}$ $\begin{array}{lllllll}70 & 123 & 193-11.5 & -3.8-1498.24 & -3.30 & 0.97 & -3.16-1503.73\end{array}$ $\begin{array}{llllllll}70 & 124 & 194-11.7 & -3.7-1501.96 & -3.73 & 0.00 & -3.11-1508.80\end{array}$ $70125195-11.9-3.5-1505.57-4.88 \quad 0.96-3.06-1512.55$ $70126196-12.2-3.4-1509.05-5.190 .00-3.01-1517.25$ $\begin{array}{llllllll}70 & 127 & 197-12.4 & -3.3-1512.42 & -4.26 & 0.95 & -2.97-1518.69\end{array}$ $\begin{array}{llllllll}70 & 128 & 198-12.6 & -3.2-1515.67 & -3.25 & 0.00 & -2.92-1521.84\end{array}$ $\begin{array}{llllllll}70 & 129 & 199-12.8 & -3.1-1518.80 & -1.80 & 0.95 & -2.88-1522.53\end{array}$ $70130200-13.0-3.0-1521.82 \quad-0.68 \quad 0.00-2.84-1525.33$ $\begin{array}{llllllll}70 & 131 & 201-13.2 & -2.8-1524.73 & -0.14 & 0.95 & -2.80-1526.72\end{array}$ $\begin{array}{lllll}70 & 132 & 202-13.5 & -2.7-1527.52\end{array}$ $\begin{array}{lllll}70 & 133 & 203-13.7 & -2.6-1530.22\end{array}$ $\begin{array}{lllll}70 & 134 & 204-13.9 & -2.5-1532.80\end{array}$ $70 \quad 135 \quad 205-14.1-2.4-1535.28$ $70136206-14.3-2.3-1537.66$ $70 \quad 137 \quad 207-14.5 \quad-2.2-1539.94$ $70138 \quad 208-14.7-2.1-1542.12$ $70 \quad 139 \quad 209-14.9-2.0-1544.20$ $70 \quad 140 \quad 210-15.1-1.9-1546.18$ $70141211-15.2-1.8-1548.07$ $70 \quad 142 \quad 212-15.4 \quad-1.7-1549.87$ $70 \quad 143 \quad 213-15.6-1.7-1551.58$ $70 \quad 144 \quad 214-15.8-1.6-1553.20$ $70145 \quad 215-16.0-1.5-1554.73$ $70146 \quad 216-16.2-1.4-1556.17$ $0.610 .00-2.76-1529.67$ $0.890 .94-2.72-1531.10$ $1.520 .00-2.68-1533.96$ $1.54 \quad 0.94-2.64-1535.45$ $1.74 \quad 0.00-2.60-1538.52$ $1.520 .93-2.57-1540.06$ $1.650 .00-2.53-1543.00$ $1.390 .93-2.50-1544.38$ $1.42 \quad 0.00-2.47-1547.23$ $1.080 .92-2.43-1548.51$ $\begin{array}{lllll}1.11 & 0.00 & -2.40-1551.17 & 104.84\end{array}$ $0.65 \quad 0.92-2.37-1552.38111 .69$ $0.620 .00-2.34-1554.92 \quad 117.23$ $\begin{array}{lllll}0.02 & 0.91 & -2.31-1556.11 & 124.12\end{array}$ $70147217-16.4-1.3-1557.53-0.84 \quad 0.91-2.25-1559.72 \quad 136.65$ $\begin{array}{lllllllll}70 & 148 & 218-16.5 & -1.2-1558.80 & -0.92 & 0.00 & -2.23-1561.95 & 142.49\end{array}$ $\begin{array}{lllllllll}70 & 149 & 219-16.7 & -1.1-1560.00 & -1.45 & 0.91 & -2.20-1562.74 & 149.77\end{array}$ $70150220-16.9-1.1-1561.11-1.490 .00-2.17-1564.77 \quad 155.81$ $\begin{array}{lllllllll}70 & 151 & 221-17.1 & -1.0-1562.14 & -2.11 & 0.90 & -2.15-1565.49 & 163.16\end{array}$ $\begin{array}{lllllllll}70 & 152 & 222-17.2 & -0.9-1563.09 & -2.15 & 0.00 & -2.12-1567.36 & 169.36\end{array}$ $\begin{array}{lllllllll}70 & 153 & 223-17.4 & -0.8-1563.97 & -2.47 & 0.90 & -2.09-1567.64 & 177.15\end{array}$ $\begin{array}{lllllllll}70 & 154 & 224-17.6 & -0.8-1564.77 & -2.32 & 0.00 & -2.07-1569.16 & 183.70\end{array}$

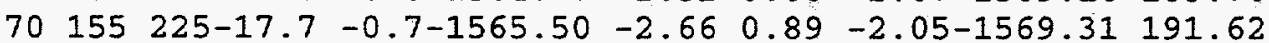
$70156226-17.9-0.6-1566.16-2.60 \quad 0.00-2.02-1570.78 \quad 198.23$ $70157227-18.0-0.5-1566.74-3.05 \quad 0.89-2.00-1570.90 \quad 206.18$ $\begin{array}{llllllll}70 & 158 & 228-18.2 & -0.5-1567.25 & -3.05 & 0.00 & -1.98-1572.28 & 212.87\end{array}$ $70 \begin{array}{llllllll}759 & 229-18.4 & -0.4-1567.70 & -3.67 & 0.89 & -1.95-1572.44 & 220.78\end{array}$ $\begin{array}{lllllllll}70 & 160 & 230-18.5 & -0.3-1568.08 & -3.83 & 0.00 & -1.93-1573.84 & 227.45\end{array}$ $\begin{array}{lllllllll}70 & 161 & 231-18.7 & -0.3-1568.39 & -4.45 & 0.88 & -1.91-1573.87 & 235.49\end{array}$ $\begin{array}{lllllllll}70 & 162 & 232-18.8 & -0.2-1568.64 & -4.58 & 0.00 & -1.89-1575.11 & 242.33\end{array}$ $\begin{array}{lllllllll}70 & 163 & 233-19.0 & -0.2-1568.83 & -4.76 & 0.88 & -1.87-1574.58 & 250.93\end{array}$ $\begin{array}{lllllllllll}70 & 164 & 234 & 0.0 & 0.0 & 0.00 & -4.40 & 0.00 & -1.85 & 0.00 & 0.00\end{array}$ $\begin{array}{lllllllll}71 & 75 & 146 & 2.4-13.5-1120.79 & -1.17 & 2.06 & -8.91-1128.82 & -6.32\end{array}$ $\begin{array}{lllllllll}71 & 76 & 147 & 2.1-13.2-1134.13 & 0.36 & 1.16 & -8.67-1141.27 & -10.71\end{array}$ $\begin{array}{lllllllll}71 & 77 & 148 & 1.8-12.9-1147.17 & -0.08 & 2.05 & -8.43-1153.64 & -14.99\end{array}$ $7178 \quad 149 \quad 1.5-12.6-1159.94-0.80 \quad 1.16-8.21-1167.79-21.08$ $7179150 \quad 1.1-12.3-1172.43-1.27 \quad 2.04-7.99-1179.65-24.86$ $7180151 \quad 0.8-12.1-1184.64-1.88 \quad 1.16-7.79-1193.15-30.30$ $\begin{array}{lllllllll}71 & 81 & 152 & 0.5-11.8-1196.59 & -2.28 & 2.04 & -7.59-1204.42 & -33.50\end{array}$ $\begin{array}{lllllllll}71 & 82 & 153 & 0.2-11.6-1208.28 & -3.30 & 1.16 & -7.39-1217.82 & -38.82\end{array}$ $7183154-0.1-11.3-1219.71-2.32 \quad 2.03-7.21-1227.21-40.14$ $7184155-0.5-11.0-1230.89-1.42 \quad 1.16-7.03-1238.18-43.04$ $\begin{array}{lllllllll}71 & 85 & 156 & -0.8-10.8-1241.82 & -0.35 & 2.02 & -6.86-1247.00 & -43.79\end{array}$ $7186 \quad 157-1.1-10.6-1252.50 \quad 0.16 \quad 1.16-6.69-1257.88-46.59$ 64
0.00

0.00

0.00

0.00

0.00

0.00

0.00

0.00

0.00

0.00

0.00

0.00

0.00

0.00

0.00

0.00

0.00

0.00

0.00

0.00

0.00

0.00

0.00

0.00

0.00

0.00

0.00

0.00

0.00

0.00

0.00

0.00

0.00

0.00

0.00

0.00

0.00

0.00

0.00

0.00

0.00

0.00

0.00

0.00

0.00

0.00

0.00

0.00

0.00

0.00

0.00

0.00

0.00

0.00

0.00

0.00

0.00

0.00

0.00

0.00

0.00

0.00

0.00

0.00

0.00

0.00

0.00

0.00

0.00

0.00

0.00

0.00

0.00

0.00

0.00

.00

0.00

$\begin{array}{llll}0.00 & 0.00 & 0.00\end{array}$

$\begin{array}{llll}0.00 & 0.00 & 0.00\end{array}$

$\begin{array}{llll}0.00 & 0.00 & 0.00\end{array}$

0.00

0.00

0.00

0.00

0.00

0.00

$0.00 \quad 0.00 \quad 0.00$

$0.00 \quad 0.00 \quad 0.00$

0.00

0.00

0.00

0.00

0.00

0.00

0.00

0.00

0.00

0.00

0.00

0.00

0.00

0.00

0.00

0.00

0.00

0.00

$\begin{array}{llll}0.00 & 0.00 & 0.00\end{array}$

$\begin{array}{llll}0.00 & 0.00 & 0.00\end{array}$

$\begin{array}{llll}0.00 & 0.00 & 0.00\end{array}$

$\begin{array}{llll}0.00 & 0.00 & 0.00\end{array}$

$\begin{array}{llll}0.00 & 0.00 & 0.00\end{array}$

$\begin{array}{llll}0.00 & 0.00 & 0.00\end{array}$

$\begin{array}{llll}0.00 & 0.00 & 0.00\end{array}$

$\begin{array}{llll}0.00 & 0.00 & 0.00\end{array}$

$\begin{array}{llll}0.00 & 0.00 & 0.00\end{array}$

$\begin{array}{llll}0.00 & 0.00 & 0.00\end{array}$

$\begin{array}{llll}0.00 & 0.00 & 0.00\end{array}$

$\begin{array}{llll}0.00 & 0.00 & 0.00\end{array}$

$\begin{array}{llll}0.00 & 0.00 & 0.00\end{array}$

$\begin{array}{llll}0.00 & 0.00 & 0.00\end{array}$

$0.00 \quad 0.00 \quad 0.00$

$\begin{array}{llll}0.00 & 0.00 & 0.00\end{array}$

$\begin{array}{llll}0.00 & 0.00 & 0.00\end{array}$

$\begin{array}{llll}0.00 & 0.00 & 0.00\end{array}$

$0.00 \quad 0.00 \quad 0.00$

$\begin{array}{llll}0.00 & 0.00 & 0.00\end{array}$

$\begin{array}{llll}0.00 & 0.00 & 0.00\end{array}$

$\begin{array}{llll}0.00 & 0.00 & 0.00\end{array}$

$0.00 \quad 0.00 \quad 0.00$

$\begin{array}{llll}0.00 & 0.00 & 0.00\end{array}$

$0.00 \quad 0.00 \quad 0.00$ 
$7187 \quad 158-1.4-10.3-1262.94$

71. $88159-1.7-10.1-1273.15$

$\begin{array}{lllll}71 & 89 & 160 & -2.0 & -9.9-1283.13\end{array}$

$\begin{array}{llllll}71 & 90 & 161 & -2.3 & -9.6-1292.88\end{array}$

$\begin{array}{llllll}71 & 91 & 162 & -2.6 & -9.4-1302.40\end{array}$

$\begin{array}{llllll}71 & 92 & 163 & -2.9 & -9.2-1311.71\end{array}$

$\begin{array}{lllll}71 & 93 & 164 & -3.2 & -9.0-1320.80\end{array}$

$\begin{array}{llllll}71 & 94 & 165 & -3.5 & -8.8-1329.67\end{array}$

$7195 \quad 166-3.8-8.6-1338.34$

$\begin{array}{llllll}71 & 96 & 167 & -4.0 & -8.4-1346.81\end{array}$

$\begin{array}{llllll}71 & 97 & 168 & -4.3 & -8.2-1355.07\end{array}$

$\begin{array}{llllll}71 & 98 & 169 & -4.6 & -8.0-1363.14\end{array}$

$\begin{array}{llllll}71 & 99 & 170 & -4.9 & -7.8-1371.01\end{array}$

$\begin{array}{llllll}71 & 100 & 171 & -5.2 & -7.6-1378.69\end{array}$

$\begin{array}{llllll}71 & 101 & 172 & -5.4 & -7.4-1386.18\end{array}$

$\begin{array}{llllll}71 & 102 & 173 & -5.7 & -7.2-1393.49\end{array}$

71. $103 \quad 174-6.0-7.0-1400.61$

$\begin{array}{llllll}71 & 104 & 175 & -6.3 & -6.9-1407.56\end{array}$

$71 \quad 105176-6.5-6.7-1414.34$

$\begin{array}{llllll}71 & 106 & 177 & -6.8 & -6.5-1420.94\end{array}$

$\begin{array}{llllll}71 & 107 & 178 & -7.1 & -6.3-1427.37\end{array}$

$\begin{array}{llllll}71 & 108 & 179 & -7.3 & -6.2-1433.64\end{array}$

$71.109 \quad 180-7.6-6.0-1439.74$

$\begin{array}{llllll}71 & 110 & 181 & -7.8 & -5.9-1445.68\end{array}$

$\begin{array}{llllll}71 & 111 & 182 & -8.1 & -5.7-1451.47\end{array}$

$\begin{array}{llllll}71 & 112 & 183 & -8.3 & -5.5-1457.10\end{array}$

$\begin{array}{llllll}71 & 113 & 184 & -8.6 & -5.4-1462.57\end{array}$

$\begin{array}{llllll}71 & 114 & 185 & -8.8 & -5.2-1467.90\end{array}$

$\begin{array}{llllll}71 & 115 & 186 & -9.1 & -5.1-1473.08\end{array}$

$\begin{array}{llllll}71 & 116 & 187 & -9.3 & -5.0-1478.11\end{array}$

$\begin{array}{lllll}71 & 117 & 188 & -9.6 & -4.8-1483.00\end{array}$

$\begin{array}{llllll}71 & 118 & 189 & -9.8 & -4.7-1487.75\end{array}$

$71119190-10.0-4.5-1492.36$

$71 \quad 120191-10.3-4.4-1496.84$

$71121192-10.5-4.3-1501.18$

$71 \quad 122 \quad 193-10.7-4.1-1505.39$

$71123194-11.0-4.0-1509.47$

$71 \quad 124195-11.2-3.9-1513.42$

$71125196-11.4-3.8-1517.25$

$71 \quad 126 \quad 197-11.6 \quad-3.6-1520.96$

$\begin{array}{lllll}71 & 127 & 198-11.9 & -3.5-1524.54\end{array}$

$71 \quad 128 \quad 199-12.1-3.4-1528.01$

$71129200-12.3-3.3-1531.36$

$71130 \quad 201-12.5 \quad-3.2-1534.59$

$\begin{array}{lllll}71 & 131 & 202-12.7 & -3.1-1537.71\end{array}$

$\begin{array}{lllll}71 & 132 & 203-12.9 & -3.0-1540.72\end{array}$

$71 \quad 133204-13.1 \quad-2.8-1543.62$

$71 \quad 134 \quad 205-13.4 \quad-2.7-1546.41$

$71 \quad 135206-13.6-2.6-1549.10$

$71 \quad 136 \quad 207-13.8 \quad-2.5-1551.68$

$71 \quad 137208-14.0 \quad-2.4-1554.16$

$71 \quad 138 \quad 209-14.2 \quad-2.3-1556.53$

$71 \quad 139210-14.4 \quad-2.2-1558.81$

$71 \quad 140 \quad 211-14.6-2.1-1560.99$

$\begin{array}{llll}71 & 141 & 212-14.7 & -2.0-1563.08\end{array}$

$71 \quad 142 \quad 213-14.9-1.9-1565.06$

$\begin{array}{llll}71 & 143 & 214-15.1 & -1.8-1566.96\end{array}$

$71 \quad 144 \quad 215-15.3-1.8-1568.76$

$\begin{array}{llll}71 & 145 & 216-15.5 & -1.7-1570.48\end{array}$

$71 \quad 146 \quad 217-15.7-1.6-1572.10$

$71 \quad 147218-15.9-1.5-1573.64$

$71 \quad 148219-16.0-1.4-1575.10$

$71 \quad 149220-16.2-1.3-1576.47$

$71 \quad 150 \quad 221-16.4-1.2-1577.75$

$71 \quad 151 \quad 222-16.6-1.2-1578.96$

$71 \quad 152 \quad 223-16.7-1.1-1580.08$

$\begin{array}{lllllll}0.61 & 2.02 & -6.54-1266.85 & -47.50 & -47.34\end{array}$

$\begin{array}{llllll}1.02 & 1.16 & -6.38-1277.35 & -49.93 & -49.72\end{array}$

$1.322 .01-6.23-1286.03-50.53 \quad 0.00$

$1.501 .16-6.09-1296.31-52.74$

$1.652 .00-5.95-1304.70-53.06$

$1.73 \quad 1.16-5.82-1314.64-54.93-54.77$

$\begin{array}{llllll}1.65 & 2.00 & -5.69-1322.84 & -55.06 & 0.00\end{array}$

$\begin{array}{llllll}1.43 & 1.16 & -5.57-1332.65 & -56.80 & -56.26\end{array}$

$1.211 .99-5.45-1340.59-56.66-56.11$

$1.06 \quad 1.16-5.33-1349.92 \quad-57.92-57.47$

$0.751 .99-5.22-1357.55-57.48-57.09$

$0.441 .16-5.11-1366.65-58.51-58.08$

$0.071 .98-5.01-1373.96-57.75-57.31$

$\begin{array}{llllll}-0.17 & 1.16 & -4.91-1382.60 & -58.32 & -57.83\end{array}$

$\begin{array}{llllll}-0.60 & 1.98 & -4.81-1389.61 & -57.25 & -56.74\end{array}$

$-0.741 .16-4$

$-4.71-1397.78-57.35$

$-56$

$-56.89$

$\begin{array}{llll}-4.62-1404.44 & -55.94 & -55.58 \\ -4.53-1412.17 & -55.60 & -55.17\end{array}$

$-53.39$

$-52.40$

$-50.36$

$-49.12$

$-46.69$

$-8.48$

$-2.811 .16-3.30-1510.34$

$-3.741 .93-3.24-1514.53$

$-4.60$

$\begin{array}{rll}-4.14 & 1.16 & -3.19-1519.60 \\ -5.22 & 1.92 & -3.14-1523.69\end{array}$

$-1.60$

2.38

5.75

$-5.491 .16-3.10-1528.39$

$-4.661 .92-3.05-1530.33$

11.88

$-3.641 .16-3.00-1533.49$

16.79

23.71

29.07

$-1.011 .16-2.91-1537.36$

$-0.451 .91-2.87-1539.12$

$0.331 .16-2.83-1542.06$

$0.671 .91-2.79-1543.83$

$1.301 .16-2.75-1546.70$

$1.361 .91-2.71-1548.54$

$1.631 .16-2.67-1551.56$

$1.501 .90-2.64-1553.39$

35.38

40.51

46.81

52.01

58.24

63.29

$1.591 .16-2.60-1556.39$

69.53

$1.331 .90-2.57-1558.15$

$1.38 \quad 1.16-2.53-1560.98$

74.61

80.92

$1.061 .90-2.50-1562.62$

86.16

$\begin{array}{llll}1.05 & 1.16 & -2.47-1565.32 & 97.96\end{array}$

$0.641 .89-2.43-1566.86 \quad 104.49$

$0.601 .16-2.40-1569.41 \quad 110.02$

$0.041 .89-2.37-1570.92 \quad 116.58$

$\begin{array}{llll}-0.02 & 1.16 & -2.34-1573.31 & 122.26\end{array}$

$\begin{array}{lllll}-0.52 & 1.89 & -2.31-1574.59 & 129.05\end{array}$

$\begin{array}{lllll}-0.94 & 1.16 & -2.28-1577.16 & 134.55\end{array}$

$\begin{array}{lllll}-1.42 & 1.88 & -2.26-1578.26 & 141.52\end{array}$

$\begin{array}{lllll}-1.49 & 1.16 & -2.23-1580.31 & 147.54\end{array}$

$-2.111 .88-2.20-1581.39154 .54$

$-2.181 .16-2.17-1583.28160 .72$
0.00

0.00

0.00

0.00

0.00

0.00

0.00

0.00

0.00

0.00

0.00

0.00

0.00

0.00

0.00

0.00

0.00

0.77

1. 23

0.00

0.00

0.00

1.89

0.00

1. 97

1.76

1.51

1.14

0.87

0.51

0.32

$-0.09$

$-0.28$

$-0.82$

$-0.80$

1.22

1.46

$-1.30$

$-1.71$

0.00

0.00

0.00

0.00

0.00

0.00

0.00

0.00

0.00

0.00

0.00

0.00

0.00

0.00

0.00

0.00

0.00

0.00

0.00

0.00

0.00

0.00

0.00

0.00

0.00

0.00

0.00

0.00

0.00

0.00

0.00

0.00

0.00

0.00

0.00

0.00

0.00

0.00

0.00

0.00

0.00

0.00

0.00

0.00

0.00

0.00

0.00

0.00

0.00

0.00

0.00

0.00

0.00

0.00

0.00

0.00

0.00

0.00

0.00

0.00

0.00

0.00

0.00

0.00

0.00

0.00

0.00

0.00

0.00

0.00

0.00

0.16

0.21

0.00

0.00

0.00

0.16

0.00

0.54

0.55

0.45

0.39

0.43

0.44

0.49

0.51

0.46

0.36

0.43

0.40

0.84

0.76

0.95

0.71

0.00

0.00

0.00

0.00

0.00

0.00

0.00

0.00

0.00

0.00

0.00

0.00

0.00

0.00

0.00

0.00

0.00 
$\begin{array}{llllllllll}71 & 153 & 224-16.9 & -1.0-1581.13 & -2.49 & 1.88 & -2.15-1583.89 & 168.18\end{array}$ $\begin{array}{lllllllll}71 & 154 & 225-17.1 & -0.9-1582.10 & -2.39 & 1.16 & -2.12-1585.45 & 174.69\end{array}$

$\begin{array}{lllllllll}71 & 155 & 226-17.2 & -0.9-1582.99 & -2.72 & 1.87 & -2.10-1585.93 & 182.28\end{array}$ $\begin{array}{lllllllll}71 & 156 & 227-17.4 & -0.8-1583.81 & -2.65 & 1.16 & -2.07-1587.37 & 188.91\end{array}$ $\begin{array}{lllllllll}71 & 157 & 228-17.6 & -0.7-1584.55 & -3.11 & 1.87 & -2.05-1587.84 & 196.51\end{array}$ $\begin{array}{lllllllll}71 & 158 & 229-17.7 & -0.6-1585.23 & -3.17 & 1.16 & -2.03-1589.27 & 203.16\end{array}$ $\begin{array}{llllllll}71 & 159 & 230-17.9 & -0.6-1585.83 & -3.81 & 1.87 & -2.00-1589.77 & 210.72\end{array}$

$\begin{array}{lllllllll}71 & 160 & 231-18.0 & -0.5-1586.36 & -4.02 & 1.16 & -1.98-1591.21 & 217.36\end{array}$ $\begin{array}{lllllllll}71 & 161 & 232-18.2 & -0.4-1586.83 & -4.69 & 1.87 & -1.96-1591.61 & 225.03\end{array}$ $\begin{array}{lllllllll}71 & 162 & 233-18.4 & -0.4-1587.23 & -4.85 & 1.16 & -1.94-1592.86 & 231.86\end{array}$ $\begin{array}{lllllllll}71 & 163 & 234-18.5 & -0.3-1587.56 & -4.99 & 1.86 & -1.92-1592.60 & 240.18\end{array}$ $\begin{array}{lllllllll}71 & 164 & 235-18.7 & -0.2-1587.83 & -4.81 & 1.16 & -1.90-1593.38 & 247.48\end{array}$ $\begin{array}{lllllllll}71 & 165 & 236-18.8 & -0.2-1588.03 & -4.94 & 1.86 & -1.88-1592.99 & 255.94\end{array}$ $\begin{array}{lllllllll}71 & 166 & 237-18.9 & -0.1-1588.18 & -4.73 & 1.16 & -1.86-1593.61 & 263.39\end{array}$

$\begin{array}{lllllllll}72 & 77 & 149 & 2.3-13.2-1145.15 & -0.33 & 1.13 & -8.69-1153.03 & -7.12\end{array}$

$7278150 \quad 2.0-12.9-1158.24-1.140 .00-8.45-1167.83-13.84$

$\begin{array}{lllllllll}72 & 79 & 151 & 1.6-12.7-1171.05 & -1.64 & 1.12 & -8.23-1179.80 & -17.74\end{array}$

$\begin{array}{lllllllll}72 & 80 & 152 & 1.3-12.4-1183.59 & -2.33 & 0.00 & -8.02-1193.93 & -23.80\end{array}$

$\begin{array}{lllllllll}72 & 81 & 153 & 1.0-12.1-1195.85 & -2.82 & 1.11 & -7.81-1205.38 & -27.17\end{array}$

$\begin{array}{lllllllll}72 & 82 & 154 & 0.7-11.9-1207.86 & -3.98 & 0.00 & -7.61-1219.45 & -33.18\end{array}$

$\begin{array}{lllllllll}72 & 83 & 155 & 0.4-11.6-1219.60 & -2.88 & 1.10 & -7.42-1228.80 & -34.46\end{array}$

$\begin{array}{lllllllll}72 & 84 & 156 & 0.1-11.4-1231.09 & -2.02 & 0.00 & -7.24-1240.35 & -37.93\end{array}$

$\begin{array}{llllllllll}72 & 85 & 157 & -0.3-11 & 1-1242.33 & -0.96 & 1.09 & -7.06-1249.26 & -38.77\end{array}$

$7286158-0.6-10.9-1253.32$

$7287159-0.9-10.6-1264.07$

$7288 \quad 160-1.2-10.4-1274.58$

$72 \quad 89161-1.5-10.2-1284.86$

$\begin{array}{llllll}72 & 90 & 162 & -1.8 & -9.9-1294.91\end{array}$

$\begin{array}{lllll}72 & 91 & 163 & -2.1 & -9.7-1304.73\end{array}$

$\begin{array}{llllll}72 & 92 & 164 & -2.4 & -9.5 & -1314.33\end{array}$

$\begin{array}{llllll}72 & 93 & 165 & -2.7 & -9.3-1323.72\end{array}$

$7294 \quad 166-2.9-9.1-1332.89$

$\begin{array}{llllll}72 & 95 & 167 & -3.2 & -8.9-1341.84\end{array}$

$\begin{array}{llllll}72 & 96 & 168 & -3.5 & -8.6-1350.59\end{array}$

$\begin{array}{llllll}72 & 97 & 169 & -3.8 & -8.4-1359.14\end{array}$

$\begin{array}{llllll}72 & 98 & 170 & -4.1 & -8.2-1367.49\end{array}$

$\begin{array}{llllll}72 & 99 & 171 & -4.4 & -8.1-1375.64\end{array}$

$\begin{array}{llllll}72 & 100 & 172 & -4.6 & -7.9 & -1383.60\end{array}$ $\begin{array}{lllll}-0.10 & 0.00 & -6.89-1260.31 & -41.76\end{array}$ $0.28 \quad 1.08-6.73-1269.44-42.81$ $\begin{array}{llllll}0.74 & 0.00 & -6.57-1280.42 & -45.71\end{array}$ $1.131 .08-6.42-1289.08-46.30$ $1.38 \quad 0.00-6.27-1299.80-48.96$ $\begin{array}{lllll}1.58 & 1.07 & -6.13-1308.21 & -49.30\end{array}$ $\begin{array}{lllll}1.72 & 0.00 & -5.99-1318.61 & -51.62\end{array}$ $\begin{array}{lllll}1.74 & 1.06 & -5.86-1326.78 & -51.72\end{array}$ $\begin{array}{llllll}1.68 & 0.00 & -5.73-1336.94 & -53.81\end{array}$ $1.471 .05-5.61-1344.93-53.73$ $\begin{array}{llllll}1.27 & 0.00 & -5.49-1354.81 & -55.54\end{array}$ $\begin{array}{lllll}1.02 & 1.04 & -5.37-1362.45 & -55.10\end{array}$ $0.76 \quad 0.00-5.26-1371.99-56.57$ $0.42 \quad 1.04-5.15-1379.34-55.85$ $0.20 \quad 0.00-5.05-1388.45-56.89$

$\begin{array}{llllll}72 & 101 & 173 & -4.9 & -7.7-1391.37\end{array}$ $-0.171 .03-4.95-1395.45-55.82$

0.00

0.00

0.00

0.00

0.00

0.00

$0.00 \quad 0.00 \quad 0.00$

$0.00 \quad 0.00 \quad 0.00$

0.00

0.00

0.00

0.00

0.00

0.00

$0.00 \quad 0.00 \quad 0.00$

$\begin{array}{llll}0.00 & 0.00 & 0.00\end{array}$

$0.00 \quad 0.00 \quad 0.00$

0.00

0.00

0.00

0.00

0.00

0.00

$0.00 .0 .00 \quad 0.00$

0.00

0.00

0.00

0.00

0.00

0.00

0.00

0.00

0.00

0.00

0.00

0.00

0.00

0.00

0.00

0.00

0.00

0.00

0.00

0.00

0.00

0.00

0.00

0.00

0.00

0.00

0.00

0.00

0.00

0.00

0.00

0.00

0.00

0.00

0.00

$-46.06$

0.39

0.00

0.00

0.00

$-0.35$

49.18

1.16

0.00

0.00

0.00

$-0.22$

0.00

0.00

0.00

0.00

0.00

0.00

0.00

0.00

0.00

0.00

0.00

0.00

0.00

0.00

$\begin{array}{llllll}72 & 102 & 174 & -5.2 & -7.5 & -1398.95\end{array}$

$-0.380 .00-4.85-1404.18$

$-56.48$

54.81

1.31

0.29

0.00

0.00

0.00

$\begin{array}{lllll}103 & 175 & -5.5 & -7.3-1406.35\end{array}$

$72104176-5.7-7.1-1413.57$

$-0.801 .02-4.75-1410.88$

6.39

0.00

0.00

0.00

0.50

0.00

0.00

$72105177-6.0-6.9-1420.61$

$-0.90 \quad 0.00-4.66-1419.13-5$

$55.10-54.49$

0.25

0.63

0.61

0.70

$72106178-6.3-6.8-1427.47$

$-1.331 .02-4.57-1425.49-5$

$5.28-54.58$

$-0.20$

0.68

$\begin{array}{lllll}72 & 107 & 179 & -6.5 & -6.6-1434.17\end{array}$

$-1.971 .01-4.40-1439.53-5$

$-53.50$

$-52.89$

$-0.65$

1.05

$72108180-6.8-6.4-1440.70$

$72109181-7.0-6.3-1447.06-$

$\begin{array}{llllll}-1.99 & 0.00 & -4.32-1447.00 & -50.87\end{array}$

$-50.47$

$-0.48$

1.00

1.08

$\begin{array}{llllllllll}72 & 110 & 182 & -7.3 & -6.1-1453.26 & -2.16 & 0.00 & -4.16-1459.58 & -4\end{array}$

$\begin{array}{lll}-49.79 & -0.91\end{array}$

0.86

$\begin{array}{llllllllll}72 & 111 & 183 & -7.6 & -6.0-1459.30 & -2.36 & 1.00 & -4.09-1464.74 & -4\end{array}$

$-47.30$

$-47.42-1.33$

$\begin{array}{llllllllll}72 & 112 & 184 & -7.8 & -5.8-1465.18 & -2.25 & 0.00 & -4.01-1471.44 & -4\end{array}$

$-44.40-43.29-1.25$

1.11

$\begin{array}{llllllllll}72 & 113 & 185 & -8.1 & -5.6-1470.90 & -2.38 & 0.99 & -3.94-1476.23 & -39.74\end{array}$

$-41.50-0.73$

1.52

$\begin{array}{llllllllll}72 & 114 & 186 & -8.3 & -5.5-1476.48 & -2.42 & 0.00 & -3.87-1482.77 & -38.21\end{array}$

0.00

0.00

0.00

$\begin{array}{llllllllll}72 & 115 & 187 & -8.6 & -5.3-1481.90 & -2.67 & 0.99 & -3.81-1487.39 & -34.76\end{array}$

$\begin{array}{llllllllll}72 & 116 & 188 & -8.8 & -5.2-1487.18 & -2.47 & 0.00 & -3.74-1493.39 & -32.69\end{array}$

0.00

0.00

0.00

0.00

0.00

0.00

$\begin{array}{llllllllll}72 & 117 & 189 & -9.0 & -5.1-1492.31 & -2.52 & 0.98 & -3.68-1497.53 & -28.75\end{array}$

0.00

0.00

0.00

$\begin{array}{lllllllllll}72 & 118 & 190 & -9.3 & -4.9-1497.30 & -2.46 & 0.00 & -3.62-1503.37 & -26.53\end{array}$

0.00

0.00

0.00

0.00

0.00

0.00

$\begin{array}{llllllllll}72 & 119 & 191 & -9.5 & -4.8-1502.14 & -2.25 & 0.98 & -3.56-1506.98 & -22.06\end{array}$

$\begin{array}{llllllllll}72 & 120 & 192 & -9.8 & -4.6-1506.86 & -2.60 & 0.00 & -3.50-1512.96 & -19.97\end{array}$

$72121193-10.0-4.5-1511.43-3.120 .97-3.44-1517.02-15.96$

0.00

0.00

0.00

$\begin{array}{lllllllll}72 & 122 & 194-10.2 & -4.4-1515.87 & -3.48 & 0.00 & -3.39-1522.74 & -13.61\end{array}$

$\begin{array}{llllllll}72 & 123 & 195-10.4 & -4.2-1520.18 & -4.33 & 0.97 & -3.33-1526.88\end{array}$

$-9.68$

0.00

0.00

0.00

0.00

0.00

0.00

0.00

0.00

0.00

$\begin{array}{llllllll}72 & 124 & 196-10.7 & -4.1-1524.36 & -4.76 & 0.00 & -3.28-1532.41\end{array}$

$-7.13$

0.00

0.00

0.00

$72125197-10.9-4.0-1528.42-5.810 .96-3.23-1536.50$

$-3.15$

0.00

0.00

0.00

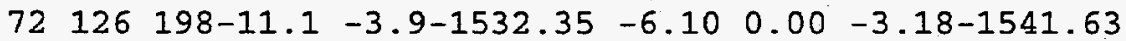

$72127199-11.3-3.7-1536.15$

$-5.260 .95-3.13-1543.59$

$-0.21$

0.00

0.00

0.00

5.90

0.00

0.00

0.00

$72128200-11.6-3.6-1539.84$

0.00

0.00

0.00

$0.00 \quad 0.00 \quad 0.00$ 
$72129201-11.8 \quad-3.5-1543.40-3.03 \quad 0.95-3.04-1548.52$

$72 \quad 130 \quad 202-12.0-3.4-1546.85-1.71 \quad 0.00-2.99-1551.56$

$\begin{array}{llllllll}72 & 131 & 203-12.2 & -3.3-1550.19 & -0.81 & 0.95 & -2.95-1553.00\end{array}$

$72 \quad 132 \quad 204-12.4-3.2-1553.41$

$72133205-12.6-3.0-1556.51$

$0.010 .00-2.91-1556.30$

$0.51 \quad 0.94-2.87-1557.93$

$72134206-12.8-2.9-1559.51$

$72135207-13.0-2.8-1562.40$

$72136208-13.3 \quad-2.7-1565.19$

$\begin{array}{lllll}72 & 137 & 209-13.5 & -2.6-1567.87\end{array}$

$72138 \quad 210-13.7 \quad-2.5-1570.44$

$\begin{array}{lllll}72 & 139 & 211-13.9 & -2.4-1572.92\end{array}$

$72140 \quad 212-14.0 \quad-2.3-1575.30$

$72141213-14.2-2.2-1577.57$

$72142 \quad 214-14.4-2.1-1579.75$

$\begin{array}{lllll}72 & 143 & 215-14.6 & -2.0-1581.84\end{array}$

$72144 \quad 216-14.8-1.9-1583.83$

$72 \quad 145 \quad 217-15.0-1.9-1585.73$

$0.990 .00-2.82-1561.35$

$1.35 \quad 0.94-2.79-1562.90$

$1.660 .00-2.75-1566.27$

$1.54 \quad 0.93-2.71-1568.10$

$1.74 \quad 0.00-2.67-1571.37$

$1.490 .93-2.64-1573.14$

$1.54 \quad 0.00-2.60-1576.35$

$1.23 \quad 0.92-2.57-1577.98$

17.11

22.15

0.00

0.00

0.00

28.77

0.00

0.00

0.00

33.54

0.00

0.00

0.00

39.99

0.00

0.00

0.00

44.64

0.00

0.00

0.00

51.16

0.00

0.00

0.00

55.86

0.00

0.00

0.00

62.10

0.00

0.00

0.00

66.90

0.00

0.00

0.00

73.21

0.00

0.00

0.00

78.06

0.00

0.00

$1.28 \quad 0.00-2.53-1581.00$

84.50

0.00

0.00

0.00

$0.87 \quad 0.92-2.50-1582.55$

89.56

0.00

0.00

0.00

$\begin{array}{lllll}0.82 & 0.00 & -2.47-1585.48 & 101.22\end{array}$

0.00

0.00

0.00

$\begin{array}{llllll}0.32 & 0.91 & -2.43-1586.93 & 107.84\end{array}$

0.00

0.00

0.00

0.00

0.00

$72146218-15.2-1.8-1587.54$

$0.260 .00 .-2.40-1589.69 \quad 113.16$

$\begin{array}{llllllll}72 & 147 & 219-15.4 & -1.7-1589.26 & -0.22 & 0.91 & -2.37-1590.95 & 119.97\end{array}$

$\begin{array}{llllllll}72 & 148 & 220-15.6 & -1.6-1590.90 & -0.33 & 0.00 & -2.34-1593.57 & 125.42\end{array}$

$\begin{array}{lllllllll}72 & 149 & 221-15.7 & -1.5-1592.45 & -1.06 & 0.91 & -2.31-1594.92 & 132.14\end{array}$

$\begin{array}{llllllllll}72 & 150 & 222-15.9 & -1.4-1593.91 & -1.17 & 0.00 & -2.29-1597.37 & 137.77\end{array}$

$\begin{array}{llllllll}72 & 151 & 223-16.1 & -1.3-1595.29 & -1.71 & 0.90 & -2.26-1598.36 & 144.85\end{array}$

$\begin{array}{llllllll}72 & 152 & 224-16.3 & -1.3-1596.59 & -1.85 & 0.00 & -2.23-1600.67 & 150.60\end{array}$

$72153225-16.4-1.2-1597.81-2.05 \quad 0.90-2.20-1601.16158 .18$

$72154226-16.6-1.1-1598.94-2.01 \quad 0.00-2.18-1603.13 \quad 164.29$

$\begin{array}{llllllll}72 & 155 & 227-16.8 & -1.0-1600.00 & -2.07 & 0.89 & -2.15-1603.33 & 172.15\end{array}$

$72156228-16.9-0.9-1600.99-2.090 .00-2.13-1605.21178 .35$

$\begin{array}{lllllllll}72 & 157 & 229-17.1 & -0.9-1601.90 & -2.61 & 0.89 & -2.10-1605.72 & 185.91\end{array}$

$72158 \quad 230-17.3-0.8-1602.73-2.93 \quad 0.00-2.08-1607.74191 .96$

$\begin{array}{llllllll}72 & 159 & 231-17.4 & -0.7-1603.49 & -3.62 & 0.89 & -2.06-1608.28 & 199.49\end{array}$

$\begin{array}{lllllllll}72 & 160 & 232-17.6 & -0.7-1604.18 & -3.86 & 0.00 & -2.03-1610.08 & 205.77\end{array}$

$\begin{array}{lllllllll}72 & 161 & 233-17.7 & -0.6-1604.80 & -4.56 & 0.88 & -2.01-1610.49 & 213.43\end{array}$

$\begin{array}{llllllll}72 & 162 & 234-17.9 & -0.5-1605.36 & -4.77 & 0.00 & -1.99-1612.11 & 219.88\end{array}$

$\begin{array}{lllllllll}72 & 163 & 235-18.1 & -0.4-1605.84 & -4.98 & 0.88 & -1.97-1611.91 & 228.15\end{array}$

$72164236-18.2-0.4-1606.26-4.70 \quad 0.00-1.95-1612.90 \quad 235.23$

$\begin{array}{lllllllll}72 & 165 & 237-18.3 & -0.3-1606.61 & -5.02 & 0.88 & -1.92-1612.68 & 243.52\end{array}$

$\begin{array}{lllllllll}72 & 166 & 238-18.5 & -0.3-1606.90 & -4.81 & 0.00 & -1.90-1613.61 & 250.66\end{array}$

$\begin{array}{lllllllll}72 & 167 & 239-18.6 & -0.2-1607.13 & -5.15 & 0.87 & -1.88-1613.29 & 259.06\end{array}$

$\begin{array}{llllllll}72 & 168 & 240-18.8 & -0.1-1607.29 & -3.26 & 0.00 & -1.86-1612.42 & 268.00\end{array}$

$\begin{array}{lllllllll}73 & 78 & 151 & 2.5-13.3-1156.04 & -1.81 & 1.15 & -8.70-1165.40 & -4.14\end{array}$

$\begin{array}{lllllllll}73 & 79 & 152 & 2.1-13.0-1169.17 & -2.33 & 2.04 & -8.47-1177.94 & -8.60\end{array}$

$\begin{array}{lllllllll}73 & 80 & 153 & 1.8-12.7-1182.03 & -3.03 & 1.15 & -8.25-1192.16 & -14.75\end{array}$

$\begin{array}{lllllllll}73 & 81 & 154 & 1.5-12.4-1194.61 & -3.49 & 2.03 & -8.04-1204.11 & -18.63\end{array}$

$\begin{array}{llllllllll}73 & 82 & 155 & 1.2-12.2-1206.93 & -4.67 & 1.15 & -7.84-1218.29 & -24.74\end{array}$

$\begin{array}{lllllllll}73 & 83 & 156 & 0.9-11.9-1218.99 & -3.53 & 2.02 & -7.64-1228.13 & -26.51\end{array}$

$\begin{array}{llllllllll}73 & 84 & 157 & 0.6-11.7-1230.79 & -2.71 & 1.15 & -7.45-1239.80 & -30.11\end{array}$

$7385158 \quad 0.3-11.4-1242.33$

$-1.522 .01-7.27-1249.11-31.34$

$7386 \quad 159-0.1-11.2-1253.63$

$\begin{array}{lllll}-0.79 & 1.15 & -7.09-1260.37 & -34.53\end{array}$

$\begin{array}{lllll}73 & 87 & 160 & -0.4-10.9-1264.69\end{array}$

$73 \quad 88 \quad 161-0.7-10.7-1275.50$

$-0.312 .01-6.92-1269.92-36.01$

$89162-1.0-10.5-1286.08$

$0.241 .15-6.76-1280.88-38.90$

$0.622 .00-6.60-1290.06-40.01$

$90163-1.3-10.2-1296.43$

$0.931 .15-6.45-1300.80-42.68$

$\begin{array}{lllll}1.18 & 2.00 & -6.31-130.9 .68 & -43.49\end{array}$

$1.38 \quad 1.15-6.17-1320.08-45.82$

0.00

0.00

0.00

0.00

0.00

0.00

0.00

0.00

0.00

0.00

0.00

0.00

0.00

0.00

0.00

0.00

0.00

0.00

0.00

0.00

0.00

0.00

0.00

0.00

0.00

0.00

0.00

0.00

0.00

0.00

0.00

0.00

0.00

0.00

0.00

0.00

0.00

0.00

0.00

0.00

0.00

0.00

0.00

0.00

0.00

0.00

0.00

0.00

0.00

0.00

0.00

0.00

0.00

0.00

0.00

0.00

0.00

0.00

0.00

0.00

0.00

0.00

0.00

0.00

0.00

0.00
0.00

0.00

0.00

0.00

0.00

0.00

0.00

0.00

0.00

0.00

0.00

0.00

0.00

0.00

0.00

0.00

0.00

0.00

0.00

0.00

0.00

0.00

0.00

0.00

0.00

0.00

0.00

0.00

0.00

0.00

0.00

0.00

0.00

0.00

0.00

$-39.90$

0.73

0.00

$91164-1.6-10.0-1306.55$

$\begin{array}{lllll}73 & 92 & 165 & -1.8 & -9.8-1316.44\end{array}$

$\begin{array}{llllll}73 & 93 & 166 & -2.1 & -9.6-1326.12\end{array}$

$\begin{array}{llllll}73 & 94 & 167 & -2.4 & -9.3-1335.58\end{array}$

$\begin{array}{lllll}73 & 95 & 168 & -2.7 & -9.1-1344.82\end{array}$

$\begin{array}{llllll}73 & 96 & 169 & -3.0 & -8.9-1353.86\end{array}$

$\begin{array}{lllll}1.46 & 1.99 & -6.03-1328.70 & -46.36\end{array}$

$\begin{array}{llllll}1.45 & 1.15 & -5.90-1338.88 & -48.47\end{array}$

$1.38 \quad 1.98-5.77-1347.23-48.75$

$1.18 \quad 1.15-5.65-1357.18-50.63$

42.54

1.07

0.11

0.00

0.00

0.14

0.00

0.00

0.00

0.00

0.00

0.00

0.00

0.00

0.00

0.00

0.00

0.00

$97170-3.3-8.7-1362.69$

$\begin{array}{llllll}73 & 98 & 171 & -3.6 & -8.5-1371.32\end{array}$

$\begin{array}{llllll}73 & 99 & 172 & -3.8 & -8.3-1379.75\end{array}$

$0.971 .98-5.53-1365.27-50.65$

0.00

0.00

0.00

$0.741 .15-5.41-1374.85-52.15$

$0.471 .97-5.30-1382.61-51.85$

0.00

0.00

0.00

0.00

0.00

0.00

$\begin{array}{lllll}0.26 & 1.15 & -5.19-1391.77 & -52.94\end{array}$

$-51.47$

0.85

0.00

0.00

0.00

.38

$\begin{array}{llllll}73 & 101 & 174 & -4.4 & -7.9-1396.03\end{array}$

$-0.041 .97-5.09-1399.19-52.29$

0.00

0.00

0.00

0.00

0.00 
$\begin{array}{llllllllllllllll}73 & 104 & 177 & -5.2 & -7.4-1419.05 & -0.81 & 1.15 & -4.79-1423.50 & -52.38 & -51.73 & -0.16\end{array}$

$\begin{array}{llllllllllll}73 & 105 & 178 & -5.5 & -7.2 & -1426.35 & -1.23 & 1.96 & -4.70-1430.33 & -51.13 & -50.54 & -0.64\end{array}$

$\begin{array}{lllllllllllll}73 & 106 & 179 & -5.7 & -7.0 & -1433.49 & -1.53 & 1.15 & -4.61-1438.48 & -51.21 & -50.37 & -0.69\end{array}$

$\begin{array}{lllllllllllll}73 & 107 & 180 & -6.0 & -6.9-1440.44 & -1.93 & 1.95 & -4.52-1444.94 & -49.61 & -48.94 & -1.26\end{array}$

$\begin{array}{lllllllllllll}73 & 108 & 181 & -6.3 & -6.7-1447.23 & -2.02 & 1.15 & -4.44-1452.54 & -49.13 & -48.44 & -1.33\end{array}$

$\begin{array}{lllllllllllll}73 & 109 & 182 & -6.5 & -6.5-1453.85 & -2.29 & 1.95 & -4.36-1458.55 & -47.07 & -46.44 & -1.66\end{array}$

$\begin{array}{llllllllllll}73 & 110 & 183 & -6.8 & -6.4-1460.31 & -2.21 & 1.15 & -4.28-1465.64 & -46.09 & -45.30 & -1.42\end{array}$

$\begin{array}{llllllllllllll}73 & 111 & 184 & -7.0 & -6.2-1466.60 & -2.52 & 1.94 & -4.20 & -1471.38 & -43.75 & -42.84 & -1.61\end{array}$

$\begin{array}{llllllllllllll}73 & 112 & 185 & -7.3 & -6.1-1472.73 & -2.46 & 1.15 & -4.13-1478.17 & -42.47 & -41.40 & -1.39\end{array}$

$\begin{array}{lllllllllllllllll}73 & 113 & 186 & -7 & 5 & -5 & 9-1478.71 & -2.76 & 1 & 94 & -4.05-1483 & 58 & -39.82 & -38.62 & -1.56\end{array}$

$\begin{array}{llllllllll}73 & 114 & 187 & -7.8 & -5.7-1484.53 & -2.84 & 1.15 & -3.98-1490.20 & -38.37\end{array}$

$\begin{array}{llllllllll}73 & 115 & 188 & -8.0 & -5.6-1490.20 & -3.03 & 1.93 & -3.91-1495.21 & -35.30\end{array}$

$\begin{array}{llllllllll}73 & 116 & 189 & -8.3 & -5.4-1495.72 & -2.91 & 1.15 & -3.85-1501.33 & -33.35\end{array}$

$\begin{array}{lllllllllll}73 & 117 & 190 & -8.5 & -5.3-1501.09 & -3.00 & 1.93 & -3.78-1505.94 & -29.89\end{array}$

$\begin{array}{llllllllll}73 & 118 & 191 & -8.8 & -5.2-1506.32 & -3.12 & 1.15 & -3.72-1512.01 & -27.89\end{array}$

$\begin{array}{llllllllll}73 & 119 & 192 & -9.0 & -5.0 & -1511.41 & -2.69 & 1.93 & -3.66-1515.83 & -23.63\end{array}$

$\begin{array}{llllllllll}73 & 120 & 193 & -9.2 & -4.9-1516.35 & -3.07 & 1.15 & -3.60-1521.87 & -21.61\end{array}$

$\begin{array}{llllllllll}73 & 121 & 194 & -9.5 & -4.7-1521.16 & -3.63 & 1.92 & -3.54-1526.41 & -18.07\end{array}$

$\begin{array}{lllllllllll}73 & 122 & 195 & -9.7 & -4.6-1525.83 & -4.03 & 1.15 & -3.48-1532.20 & -15.79\end{array}$

$\begin{array}{lllllllllll}73 & 123 & 196 & -9.9 & -4.5-1530.38 & -4.87 & 1.92 & -3.43-1536.75 & -12.27\end{array}$

$\begin{array}{llllllll}73 & 124 & 197-10.2 & -4.3-1534.78 & -5.30 & 1.15 & -3.37-1542.31 & -9.76\end{array}$

$\begin{array}{llllllll}73 & 125 & 198-10.4 & -4.2-1539.06 & -6.32 & 1.91 & -3.32-1546.79\end{array}$

$73126 \quad 199-10.6-4.1-1543.22-6.59 \quad 1.15-3.27-1551.93$

$\begin{array}{llllllll}73 & 127 & 200-10.8 & -4.0-1547.25 & -5.75 & 1.91 & -3.22-1554.30\end{array}$

$-6.17$

$-3.23$

$73128 \quad 201-11.1-3.8-1551.15-4.741 .15-3.17-1557.91$

2.46

6.93

$\begin{array}{lllllll}73 & 129 & 202-11.3 & -3.7-1554.93 & -3.43 & 1.91 & -3.12-1559.58\end{array}$

13.33

$\begin{array}{llllllll}73 & 130 & 203-11.5 & -3.6-1558.60 & -2.20 & 1.15 & -3.07-1562.72\end{array}$

$\begin{array}{llllllll}73 & 131 & 204-11.7 & -3.5-1562.15 & -1.34 & 1.90 & -3.03-1564.61\end{array}$

$73 \quad 132205-11.9-3.4-1565.58-0.431 .15-2.99-1567.84$

$73133206-12.1-3.3-1568.90$

$0.051 .90-2.94-1569.89$

18.25

24.44

29.28

$\begin{array}{rrr}0.00 & 0.00\end{array}$

$0.00 \quad 0.00$

$0.00 \quad 0.00$

$0.00 \quad 0.00$

$0.00 \quad 0.00$

$0.00 \quad 0.00$

$0.00 \quad 0.00$

$0.00 \quad 0.00$

$0.00 \quad 0.00$

$0.00 \quad 0.00$

$0.00 \quad 0.00$

$0.00 \quad 0.00$

$0.00 \quad 0.00$

$0.00 \quad 0.00$

$0.00 \quad 0.00$

$0.00 \quad 0.00$

$0.00 \quad 0.00$

$0.00 \quad 0.00$

35.30

0.00

0.00

$0.00 \quad 0.00$

40.02

$\begin{array}{llll}73 & 135 & 208-12.5 & -3.0-1575.20\end{array}$

$0.611 .15-2.90-1573.25$

46.20

$\begin{array}{lllll}73 & 136 & 209-12.7 & -2.9-1578.19\end{array}$

$1.031 .90-2.86-1575.13$

50.93

0.00

0.00

$1.381 .15-2.82-1578.48$

56.85

0.00

0.00

$1.331 .89-2.78-1580.63$

$\begin{array}{lllll}73 & 138 & 211-13.2 & -2.7-1583.85\end{array}$

$1.601 .15-2.74-1583.84$

61.71

0.00

0.00

$0.00 \quad 0.00$

67.60

$1.321 .89-2.70-1586.02$

0.00

0.00

$0.00 \quad 0.00$

72.47

$73 \quad 140 \quad 213-13.5 \quad-2.5-1589.10$

$73 \quad 141214-13.7-2.4-1591.57$

$1.391 .15-2.67-1589.23$

78.56

0.00

0.00

$1.111 .89-2.63-1591.20$

83.60

0.00

0.00

$0.00 \quad 0.00$

$0.82 \quad 1.88-2.56-1596.08 \quad 89.83$

$73143216-14.1-2.2-1596.22$

94.96

$73 \quad 144 \quad 217-14.3-2.1-1598.40$

$0.401 .88-2.50-1600.71 \quad 101.34$

0.00

0.00

0.00

0.00

0.00

0.00

$0.341 .15-2.47-1603.47 \quad 106.66$

0.00

0.00

0.65

0.59

0.84

0.67

0.69

0.63

0.79

0.91

1.07

1.20

0.00

0.00

0.00

0.00

0.00

0.00

0.00

0.00

0.00

0.00

0.00

0.00

0.00

0.00

0.00

0.00

0.00

0.00

0.00

0.00

0.00

0.00

0.00

0.00

0.00

0.00

0.00

0.00

0.00

0.00

$73 \quad 146 \quad 219-14.7-1.9-1602.49$

$\begin{array}{lllllllll}73 & 147 & 220-14.9 & -1.9-1604.39 & -0.18 & 1.88 & -2.43-1605.13 & 113.06 \\ 73 & 148 & 221-15.1 & -1.8-1606.21 & -0.28 & 1.15 & -2.40-1607.75 & 118.52\end{array}$

$73149222-15.2-1.7-1607.94-0.81 \quad 1.87-2.37-1609.25125 .09$

0.00

0.00

0.00

0.00

0.00

0.00

0.00

0.00

0.00

$73150 \quad 223-15.4-1.6-1609.58-0.91 \quad 1.15-2.35-1611.69 \quad 130.72$

0.00

0.00

0.00

$\begin{array}{lllllllll}73 & 151 & 224-15.6 & -1.5-1611.14 & -1.41 & 1.87 & -2.32-1612.99 & 137.48\end{array}$

$\begin{array}{lllllllll}73 & 152 & 225-15.8 & -1.4-1612.61 & -1.80 & 1.15 & -2.29-1615.55 & 143.00\end{array}$

$\begin{array}{llllllll}73 & 153 & 226-16.0 & -1.3-1614.00 & -1.76 & 1.87 & -2.26-1616.15 & 150.47\end{array}$

$\begin{array}{lllllllll}73 & 154 & 227-16.1 & -1.3-1615.31 & -1.78 & 1.15 & -2.23-1618.18 & 156.52\end{array}$

0.00

0.00

0.00

0.00

0.00

0.00

0.00

0.00

0.00

0.00

0.00

0.00

$\begin{array}{llllllll}73 & 155 & 228-16.3 & -1.2-1616.54 & -1.98 & 1.87 & -2.21-1618.86 & 163.90\end{array}$

$\begin{array}{llllllll}73 & 156 & 229-16.5 & -1.1-1617.69 & -2.03 & 1.15 & -2.18-1620.76 & 170.08\end{array}$

$73157230-16.6-1.0-1618.77-2.58 \quad 1.86-2.16-1621.64177 .27$

0.00

0.00

0.00

0.00

0.00

0.00

0.00

0.00

0.00

$\begin{array}{lllllllll}73 & 158 & 231-16.8 & -1.0-1619.77 & -2.77 & 1.15 & -2.13-1623.52 & 183.46\end{array}$

0.00

0.00

0.00

$\begin{array}{llllllll}73 & 159 & 232-17.0 & -0.9-1620.69 & -3.63 & 1.86 & -2.11-1624.57 & 190.48\end{array}$

0.00

0.00

0.00

$\begin{array}{lllllllll}73 & 160 & 233-17.1 & -0.8-1621.54 & -3.91 & 1.15 & -2.08-1626.38 & 196.74\end{array}$

$\begin{array}{lllllllll}73 & 161 & 234-17.3 & -0.7-1622.32 & -4.61 & 1.86 & -2.06-1627.13 & 204.06\end{array}$

$\begin{array}{lllllllll}73 & 162 & 235-17.4 & -0.7-1623.03 & -4.86 & 1.15 & -2.04-1628.78 & 210.49\end{array}$

$\begin{array}{lllllllll}73 & 163 & 236-17.6 & -0.6-1623.66 & -5.10 & 1.85 & -2.02-1628.92 & 218.41\end{array}$

0.00

0.00

0.00

0.00

0.00

0.00

0.00

0.00

0.00

0.00

0.00

$\begin{array}{lllllllll}73 & 164 & 237-17.7 & -0.5-1624.23 & -4.99 & 1.15 & -1.99-1630.07 & 225.34\end{array}$

$\begin{array}{lllllllll}73 & 165 & 238-17.9 & -0.5-1624.74 & -5.24 & 1.85 & -1.97-1630.10 & 233.38\end{array}$

$73166239-18.0-0.4-1625.17-5.07 \quad 1.15-1.95-1631.05 \quad 240.50$

$\begin{array}{lllllllll}73 & 167 & 240-18.2 & -0.3-1625.55 & -4.81 & 1.85 & -1.93-1630.44 & 249.18\end{array}$

$\begin{array}{lllllllll}73 & 168 & 241-18.3 & -0.3-1625.86 & -3.82 & 1.15 & -1.91-1630.44 & 257.26\end{array}$

$73169242-18.5-0.2-1626.10-4.061 .85$

0.00

0.00

0.00

0.00

0.00

0.00

0.00

0.00

0.00

0.00

0.00

0.00

0.00

0.00

0.00

0.00

0.00

0.00 
$\begin{array}{lllllllllll}73 & 170 & 243-18.6 & -0.2-1626.29 & -4.21 & 1.15 & -1.87-1631.22 & 272.61\end{array}$ $\begin{array}{lllllllll}74 & 80 & 154 & 2.3-13.0-1179.97 & -3.65 & 0.00 & -8.49-1192.11 & -7.43\end{array}$

$\begin{array}{lllllllll}74 & 81 & 155 & 2.0-12.8-1192.87 & -4.21 & 1.11 & -8.27-1204.24 & -11.49\end{array}$

$\begin{array}{lllllllll}74 & 82 & 156 & 1.7-12.5-1205.50 & -5.24 & 0.00 & -8.06-1218.80 & -17.98\end{array}$

$\begin{array}{lllllllll}74 & 83 & 157 & 1.4-12.2-1217.87 & -4.25 & 1.10 & -7.86-1228.88 & -19.98\end{array}$

$\begin{array}{lllllllll}74 & 84 & 158 & 1.1-12.0-1229.98 & -3.44 & 0.00 & -7.67-1241.08 & -24.12\end{array}$

$\begin{array}{lllllllll}74 & 85 & 159 & 0.8-11.7-1241.83 & -2.08 & 1.09 & -7.48-1250.30 & -25.26\end{array}$

$\begin{array}{lllllllll}74 & 86 & 160 & 0.4-11.5-1253.44 & -1.27 & 0.00 & -7.30-1262.01 & -28.89\end{array}$

$\begin{array}{lllllllll}74 & 87 & 161 & 0.1-11.2-1264.80 & -0.64 & 1.08 & -7.12-1271.48 & -30.29\end{array}$

$\begin{array}{lllllllll}74 & 88 & 162 & -0.2-11.0-1275.91 & -0.11 & 0.00 & -6.96-1282.98 & -33.73\end{array}$

$74 \quad 89163-0.5-10.8-1286.79$

$7490 \quad 164-0.8-10.5-1297.44$

$0.311 .08-6.79-1292.20-34.88$

$7491165-1.0-10.3-1307.85$

$0.68 \quad 0.00-6.64-1303.40-38.00$

$7492 \quad 166-1.3-10.1-1318.04$

$\begin{array}{llllll}74 & 93 & 167 & -1.6 & -9.9-1328.01\end{array}$

$7494 \quad 168-1.9-9.6-1337.76$

$\begin{array}{llllll}74 & 95 & 169 & -2.2 & -9.4-1347.29\end{array}$

$\begin{array}{llllll}74 & 96 & 170 & -2.5 & -9.2-1356.61\end{array}$

$\begin{array}{llllll}74 & 97 & 171 & -2.8 & -9.0-1365.73\end{array}$

$\begin{array}{llllll}74 & 98 & 172 & -3.1 & -8.8 & -1374.64\end{array}$

$\begin{array}{llllll}74 & 99 & 173 & -3.3 & -8.6-1383.35\end{array}$

$\begin{array}{llllll}74 & 100 & 174 & -3.6 & -8.4-1391.86\end{array}$

$\begin{array}{llllll}74 & 101 & 175 & -3.9 & -8.2-1400.18\end{array}$

$\begin{array}{llllll}74 & 102 & 176 & -4.2 & -8.0-1408.31\end{array}$

$0.96 \quad 1.07-6.49-1312.31-38.84$

$1.20 \quad 0.00-6.34-1323.18-41.64$

$1.331 .06-6.20-1331.82-42.21$

$\begin{array}{lllll}1.40 & 0.00 & -6.07-1342.42 & -44.74\end{array}$

$\begin{array}{lllll}1.39 & 1.05 & -5.93-1350.78 & -45.03\end{array}$

$1.28 \quad 0.00-5.81-1361.14-47.31$

$1.15 \quad 1.04 \quad-5.68-1369.22 \quad-47.32$

$1.00 \quad 0.00-5.57-1379.20-49.24$

$0.771 .04-5.45-1386.99-48.95$

$0.610 .00-5.34-1396.59-50.48$

$0.371 .03-5.23-1404.01-49.83$

$74103177-4.4-7.8-1416.25$

$0.100 .00-5.13-1413.34-51.08$

$74 \begin{array}{lllll}104 & 178 & -4.7 & -7.7-1424.01\end{array}$

$-0.241 .02-5.03-1420.49-50.17$

$74 \begin{array}{lllllllll}105 & 179 & -5.0 & -7.5-1431.58 & -0.86 & 1.02 & -4.83-1436.25 & -49.79\end{array}$

$\begin{array}{llllllllll}74 & 106 & 180 & -5.2 & -7.3-1438.98 & -1.21 & 0.00 & -4.74-1444.93 & -50.39\end{array}$

$74107181-5.5-7.1-1446.20-1.61 \quad 1.01-4.65-1451.45-4$

$74 \begin{array}{llllllll}708 & 182 & -5.8 & -7.0-1453.25 & -1.71 & 0.00 & -4.56-1459.52 & -48\end{array}$

$\begin{array}{lllllllll}74 & 109 & 183 & -6.0 & -6.8-1460.12 & -2.00 & 1.00 & -4.48-1465.60 & -46.84\end{array}$

$74110184-6.3-6.6-1466.83-2.02 \quad 0.00-4.40-1473.25-4$

$\begin{array}{lllllllll}74 & 111 & 185 & -6.5 & -6.5-1473.38 & -2.29 & 1.00 & -4.32-1478.99 & -4\end{array}$

$74 \begin{array}{llllllll}712 & 186 & -6.8 & -6.3-1479.77 & -2.38 & 0.00 & -4.24-1486.39 & -43.42\end{array}$

$\begin{array}{llllllllll}74 & 113 & 187 & -7.0 & -6.1-1485.99 & -2.72 & 0.99 & -4.16-1491.88 & -40.84\end{array}$

$\begin{array}{llllllllll}74 & 114 & 188 & -7.3 & -6.0-1492.06 & -2.71 & 0.00 & -4.09-1498.86 & -39.75\end{array}$

$\begin{array}{llllllllll}74 & 115 & 189 & -7.5 & -5.8-1497.98 & -3.04 & 0.99 & -4.02-1504.05 & -36.87\end{array}$

$\begin{array}{llllllllll}74 & 116 & 190 & -7.8 & -5.7 & -1503.74 & -2.90 & 0.00 & -3.95-1510.59 & -35.34\end{array}$

$\begin{array}{llllllllll}74 & 117 & 191 & -8.0 & -5.5-1509.36 & -3.52 & 0.98 & -3.88-1515.78 & -32.46\end{array}$

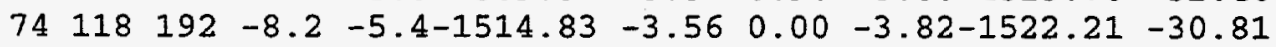

$\begin{array}{llllllllll}74 & 119 & 193 & -8.5 & -5.2 & -1520.15 & -3.10 & 0.98 & -3.76-1526.03 & -26.56\end{array}$

$\begin{array}{llllllllll}74 & 120 & 194 & -8.7 & -5.1 & -1525.33 & -3.52 & 0.00 & -3.69-1532.55 & -25.01\end{array}$

$\begin{array}{llllllllll}74 & 121 & 195 & -9.0 & -5.0-1530.38 & -4.14 & 0.97 & -3.63-1537.18 & -21.57\end{array}$

$\begin{array}{llllllllll}74 & 122 & 196 & -9.2 & -4.8-1535.28 & -4.65 & 0.00 & -3.58-1543.51 & -19.83\end{array}$

$\begin{array}{lllllllllll}74 & 123 & 197 & -9.4 & -4.7 & -1540.05 & -5.51 & 0.97 & -3.52-1548.12 & -16.36\end{array}$

$\begin{array}{llllllllll}74 & 124 & 198 & -9.6 & -4.6-1544.69 & -6.05 & 0.00 & -3.46-1554.20 & -14.38\end{array}$

$\begin{array}{llllllllll}74 & 125 & 199 & -9.9 & -4.4-1549.20 & -7.12 & 0.96 & -3.41-1558.77 & -10.87\end{array}$

$\begin{array}{llllllll}74 & 126 & 200-10.1 & -4.3-1553.57 & -7.34 & 0.00 & -3.36-1564.27\end{array}$

$\begin{array}{llllllll}74 & 127 & 201-10.3 & -4.2-1557.82 & -6.47 & 0.95 & -3.30-1566.64\end{array}$

$74 \begin{array}{lllllll}128 & 202-10.5 & -4.1-1561.95 & -5.53 & 0.00 & -3.25-1570.73\end{array}$

$\begin{array}{llllllll}74 & 129 & 203-10.8 & -3.9-1565.95 & -4.34 & 0.95 & -3.20-1572.55\end{array}$

$\begin{array}{llllllll}74 & 130 & 204-11.0 & -3.8-1569.83 & -3.08 & 0.00 & -3.16-1576.07\end{array}$

$74131205-11.2-3.7-1573.60-1.920 .95-3.11-1577.68$

$74132206-11.4 \quad-3.6-1577.24-0.97 \quad 0.00-3.07-1581.28$

$74133207-11.6 \quad-3.5-1580.77-0.24 \quad 0.94-3.02-1583.09$

$74 \quad 134208-11.8-3.4-1584.19$

$74135209-12.0-3.2-1587.49$

$74136 \quad 210-12.2-3.1-1590.69$

$74137211-12.4 \quad-3.0-1593.77$

$\begin{array}{lllll}74 & 138 & 212 & -12.7 & -2.9-1596.75\end{array}$

$74 \quad 139213-12.9-2.8-1599.63$

$74 \quad 140 \quad 214-13.1 \quad-2.7-1602.40$

$74 \quad 141215-13.2-2.6-1605.07$

$74 \quad 142 \quad 216-13.4-2.5-1607.64$

$\begin{array}{llll}74 & 143 & 217-13.6 & -2.4-1610.11\end{array}$

$74144218-13.8-2.3-1612.48$

$0.300 .00-2.98-1586.86$

$0.640 .94-2.94-1588.85$

$1.230 .00-2.89-1592.35$

$1.290 .93-2.85-1594.41$

$1.550 .00-2.81-1598.02$

$1.410 .93-2.78-1600.06$

$\begin{array}{llll}1.51 & 0.00 & -2.74-1603.63\end{array}$

$1.260 .92-2.70-1605.59$

$1.330 .00-2.67-1608.97$

$1.010 .92-2.63-1610.81$

$0.970 .00-2.60-1614.11$

$-8.30$

$-2.60$

1. 38

7.64

12.18

18.64

23.12

29.38

33.67

39.76

44.33

50.35

54.81

60.83

65.34

71.45

76.14

82.37

87.15

0.00

0.00

0.00

0.00

$\begin{array}{lll}0.00 & 0.00 & 0.00\end{array}$

$\begin{array}{llll}0.00 & 0.00 & 0.00\end{array}$

0.00

0.00

0.00

0.00

0.00

0.00

0.00

0.00

0.00

0.00

0.00

0.00

0.00

0.00

0.00

0.00

0.00

$-38.36$

0.00

0.00

0.00

0.32

0.00

$-41.90$

0.00

0.00

$-0.36$

0.00

0.94

0.00

0.00

0.00

$-0.26$

0.00

0.00

.00

0.00

0.00

0.00

0.00

0.00

0.00

0.00

0.00

0.00

0.00

0.00

0.00

0.00

0.00

0.00

0.00

0.00

0.00

0.00

0.00

0.00

0.00

$-50.44$

0.00

0.00

$-49.31$

0.10

$-0.38$

0.57

0.48

0.74

0.57

0.59

0.47

0.72

0.70

0.91

0.93

1.08

1. 39

1.03

$-35.48-1.65$

$-34.31-1.87$

$0.00 \quad 0.00$

0.00

0.00

0.00

0.00

0.00

0.00

0.00

0.00

0.00

0.00

0.00

0.00

0.00

0.00

0.00

0.00

0.00

0.00

0.00

0.00

0.00

0.00

0.00

0.00

0.00

0.00

0.00

0.00

0.00

0.00

0.00

0.00

0.00

0.00

0.00

0.00
0.00

0.00

0.00

0.00

0.00

0.00

0.00

0.00

0.00

0.00

0.00

0.00

0.00

0.00

0.00

0.00

0.00

0.00

0.00

0.00

0.00

0.00

0.00

0.00

0.00

0.00

0.00

0.00

0.00

0.00

0.00

0.00

0.00

0.00

0.00

$0.00 \quad 0.00 \quad 0.00$

0.00

0.00

0.00

0.00

0.00

0.00 
$74 \quad 145 \quad 219-14.0 \quad-2.2-1614.76$ $74146 \quad 220-14.2-2.1-1616.94$ $74 \quad 147 \quad 221-14.4 \quad-2.0-1619.03$ $74 \quad 148 \quad 222-14.6-2.0-1621.03$ $74 \quad 149223-14.8-1.9-1622.94$ $74 \quad 150 \quad 224-14.9-1.8-1624.77$ $74 \quad 151225-15.1-1.7-1626.50$

$74 \quad 152 \quad 226-15.3-1.6-1628.15$

$74 \quad 153 \quad 227-15.5-1.5-1629.72$

$74 \quad 154228-15.6-1.4-1631.20$

$74 \quad 155 \quad 229-15.8-1.4-1632.60$

$74156230-16.0-1.3-1633.92$

$\begin{array}{llll}74 & 157 & 231-16.2 & -1.2-1635.16\end{array}$

$74 \quad 158 \quad 232-16.3-1.1-1636.33$

$74159233-16.5-1.0-1637.41$

$74160 \quad 234-16.7-1.0-1638.43$

$74 \quad 161235-16.8-0.9-1639.37$

$74 \quad 162 \quad 236-17.0-0.8-1640.23$

$\begin{array}{llll}74 & 163 & 237-17.1 & -0.8-1641.03\end{array}$

$\begin{array}{llll}74 & 164 & 238-17.3 & -0.7-1641.75\end{array}$

$74 \quad 165 \quad 239-17.4 \quad-0.6-1642.41$

$\begin{array}{lllll}74 & 166 & 240-17.6 & -0.6-1643.00\end{array}$

$\begin{array}{lllll}74 & 167 & 241-17.7 & -0.5-1643.52\end{array}$

$74 \quad 168 \quad 242-17.9-0.4-1643.98$

$74169243-18.0-0.4-1644.37$

$74 \quad 170 \quad 244-18.2-0.3-1644.70$

$74171245-18.3-0.2-1644.96$

$74 \quad 172 \quad 246-18.5-0.2-1645.17$

$\begin{array}{lllll}74 & 173 & 247-18.6 & -0.1-1645.32\end{array}$

$\begin{array}{lll}75 & 81 & 156\end{array}$

$\begin{array}{lll}75 & 82 & 157\end{array}$

$$
2.5-13.1-1190.64
$$

$2.2-12.8-1203.58$

$1.9-12.5-1216.26$

83158

$1.6-12.3-1228.67$

84159

$1.2-12.0-1240.84$

85160

$0.9-11.8-1252.74$

$0.6-11.5-1264.40$

87162

88163

$0.3-11.3-1275.82$

$0.0-11.1-1287.00$

89164

$\begin{array}{lll}90 & 165 & -0.2-10.8-1297.94 \\ 91 & 166 & -0.5-10.6-1308.65\end{array}$

$92167-0.8-10.4-1319.13$

$93168-1.1-10.1-1329.39$

$94 \quad 169-1.4-9.9-1339.43$

$95 \quad 170-1.7-9.7-1349.25$

$96171-2.0-9.5-1358.85$

$97 \begin{array}{llll}972 & -2.3 & -9.3-1368.25\end{array}$

$98 \quad 173-2.5 \quad-9.1-1377.44$

$\begin{array}{lllll}75 & 99 & 174 & -2.8 & -8.9-1386.43\end{array}$

$\begin{array}{llllll}75 & 100 & 175 & -3.1 & -8.7-1395.22\end{array}$

$\begin{array}{lllll}75 & 101 & 176 & -3.4 & -8.5-1403.81\end{array}$

$\begin{array}{lllll}75 & 102 & 177 & -3.6 & -8.3-1412.22\end{array}$

$\begin{array}{lllll}75 & 103 & 178 & -3.9 & -8.1-1420.43\end{array}$

$\begin{array}{llllll}75 & 104 & 179 & -4.2 & -7.9-1428.45\end{array}$

$\begin{array}{lllll}75 & 105 & 180 & -4.5 & -7.7-1436.29\end{array}$

$\begin{array}{lllll}75 & 106 & 181 & -4.7 & -7.6-1443.95\end{array}$

$\begin{array}{lllll}75 & 107 & 182 & -5.0 & -7.4-1451.44\end{array}$

$\begin{array}{llllll}75 & 108 & 183 & -5.2 & -7.2-1458.75\end{array}$

$\begin{array}{llllll}75 & 109 & 184 & -5.5 & -7.0-1465.88\end{array}$

$\begin{array}{llllll}75 & 110 & 185 & -5.8 & -6.9-1472.85\end{array}$

$\begin{array}{llllll}75 & 111 & 186 & -6.0 & -6.7-1479.65\end{array}$

$\begin{array}{llllll}75 & 112 & 187 & -6.3 & -6.6-1486.29\end{array}$

$\begin{array}{lllll}75 & 113 & 188 & -6.5 & -6.4-1492.76\end{array}$

$\begin{array}{llllllll}75 & 114 & 189 & -6.8 & -6.2-1499.08 & -2.85 & 1.14\end{array}$

$\begin{array}{llllllll}75 & 115 & 190 & -7.0 & -6.1-1505.24 & -3.27 & 1.93\end{array}$

$\begin{array}{llllllll}75 & 116 & 191 & -7.3 & -5.9-1511.25 & -3.48 & 1.14\end{array}$

$\begin{array}{llllllll}75 & 117 & 192 & -7.5 & -5.8 & -1517.11 & -4.12 & 1.92\end{array}$ $\begin{array}{llll}0.55 & 0.91 & -2.56-1615.86 & 93.47\end{array}$ $\begin{array}{lllll}0.55 & 0.00 & -2.53-1618.92 & 98.48\end{array}$ $0.04 \quad 0.91-2.50-1620.58 \quad 104.89$ $-0.010 .00-2.47-1623.51110 .03$ $-0.540 .91-2.44-1625.01116 .60$ $0.620 .00-2.41-1627.79121 .89$ $-1.10 \quad 0.90-2.38-1629.07 \quad 128.68$ $\begin{array}{lllll}-1.16 & 0.00 & -2.35-1631.66 & 134.17\end{array}$ $-1.420 .90-2.32-1632.56 \quad 141.34$ $1.320 .00-2.29-1634.81147 .16$ $\begin{array}{lllll}.71 & 0.89 & -2.26-1635.68 & 154.36\end{array}$ .00-2.24-1637.94 160.17 $0.00-2.01-1638.81167 .37$ $\begin{array}{lllll}-3.26 & 0.89 & -2.16-1641.95 & 180.38\end{array}$ $\begin{array}{lllll}-3.54 & 0.00 & -2.14-1644.10 & 186.29\end{array}$ $\begin{array}{llllll}-4.26 & 0.88 & -2.11-1644.86 & 193.61\end{array}$ $\begin{array}{lllll}-4.54 & 0.00 & -2.09-1646.86 & 199.68\end{array}$ $\begin{array}{lllll}4.92 & 0.88 & -2.07-1647.14 & 207.48\end{array}$ $\begin{array}{lllll}-4.72 & 0.00 & -2.04-1648.52 & 214.17\end{array}$ $\begin{array}{lllll}89 & 0.88 & -2.02-1648.45 & 222.31\end{array}$ $0.00-2.00-1649.53229 .30$ $\begin{array}{lllll}76 & 0.00 & -1.96-1649.69 & 245.28\end{array}$ $0.87-1.94-1649.75253 .29$

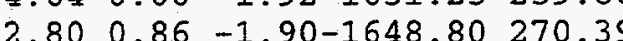
$\begin{array}{lllll}-3.19 & 0.00 & -1.88-1650.24 & 277.02\end{array}$ $\begin{array}{lllll}17 & 0.86 & -1.86-1649.49 & 285.84\end{array}$ $2.02-8.51-1202.22-2.19$ $\begin{array}{lllll}5.09 & 2.01 & -8.08-1227.42 & -11.25\end{array}$ $\begin{array}{llllll}-4.30 & 1.14 & -7.88-1239.72 & -15.48\end{array}$ $.01-7.69-1249.57-17.26$ $\begin{array}{llll}1.14 & -7.51-1261.21 & -20.82\end{array}$ $-0.831 .14-7.15-1282.67-26.14$ $-0.331 .99-6.99-1292.32-27.72$ $0.09 \quad 1.14-6.83-1303.54-30.87$ $\begin{array}{lllll}0.45 & 1.99 & -6.67-1312.88 & -32.14\end{array}$ $0.811 .14-6.52-1323.70-34.89$ $\begin{array}{lllll}0.93 & 1.98 & -6.38-1332.85 & -35.97\end{array}$ $1.08 \quad 1.14-6.24-1343.44-38.49$ $1.121 .98-6.10-1352.25-39.22$ $\begin{array}{lllll}1.07 & 1.14 & -5.97-1362.62 & -41.52\end{array}$ $0.96 \quad 1.97-5.84-1371.17-41.99$ $\begin{array}{llllll}0.89 & 1.14 & -5.72-1381.14 & -43.89\end{array}$ $0.641 .96-5.60-1389.43-44.12$ $0.491 .14-5.49-1399.08-45.70$ $\begin{array}{lllll}0.19 & 1.96 & -5.38-1407.04 & -45.59\end{array}$ $0.00 \quad 1.14-5.27-1416.35-46.82$ $-0.341 .95-5.17-1423.98-46.38$ $-0.611 .14-5.06-1432.99 \cdot-47.31$ $-0.971 .95-4.97-1440.28-46.54$ $1.301 .14-4.87-1448.99-47.17$ $\begin{array}{lllll}1.73 & 1.94 & -4.78-1456.00 & -46.11\end{array}$ $\begin{array}{lllll}1.95 & 1.14 & -4.69-1464.25 & -46.29\end{array}$

$.191 .94-4.60-1470.73-44.70$

$1.14-4.52-1478.42-44.32$ $-4.44-1484.63-42.46$ $-4.36-1491.98-41.74$ $41.22-1.96$ $\begin{array}{lllll}4.28-1497.99 & -39.68 & -39.02 & -2.22\end{array}$ $-4.20-1505.00-38.61-37.99-2.23$ $-4.13-1510.72-36.26-35.58-2.59$ $-4.06-1517.65-35.13$ 70

\begin{tabular}{|c|c|c|}
\hline 0.00 & 0.00 & 0.00 \\
\hline 0.00 & 0.00 & 0.00 \\
\hline 0.00 & 0.00 & 0.00 \\
\hline 0.00 & 0.00 & 0.00 \\
\hline 0.00 & 0.00 & 0.00 \\
\hline 0.00 & 0.00 & 0.00 \\
\hline 0.00 & 0.00 & 0.00 \\
\hline 0.00 & 0.00 & 0.00 \\
\hline 0.00 & 0.00 & 0.00 \\
\hline 0.00 & 0.00 & 0.00 \\
\hline 0.00 & 0.00 & 0.00 \\
\hline 0.00 & 0.00 & 0.00 \\
\hline 0.00 & 0.00 & 0.00 \\
\hline 0.00 & 0.00 & 0.00 \\
\hline 0.00 & 0.00 & 0.00 \\
\hline 0.00 & 0.00 & 0.00 \\
\hline 0.00 & 0.00 & 0.00 \\
\hline 0.00 & 0.00 & 0.00 \\
\hline 0.00 & 0.00 & 0.00 \\
\hline 0.00 & 0.00 & 0.00 \\
\hline 0.00 & 0.00 & 0.00 \\
\hline 0.00 & 0.00 & 0.00 \\
\hline 0.00 & 0.00 & 0.00 \\
\hline 0.00 & 0.00 & 0.00 \\
\hline 0.00 & 0.00 & 0.00 \\
\hline 0.00 & 0.00 & 0.00 \\
\hline 0.00 & 0.00 & 0.00 \\
\hline 0.00 & 0.00 & 0.00 \\
\hline 0.00 & 0.00 & 0.00 \\
\hline 0.00 & 0.00 & 0.00 \\
\hline 0.00 & 0.00 & 0.00 \\
\hline 0.00 & 0.00 & 0.00 \\
\hline 0.00 & 0.00 & 0.00 \\
\hline 0.00 & 0.00 & 0.00 \\
\hline 0.00 & 0.00 & 0.00 \\
\hline 0.00 & 0.00 & 0.00 \\
\hline 0.00 & 0.00 & 0.00 \\
\hline 0.00 & 0.00 & 0.00 \\
\hline 0.00 & 0.00 & 0.00 \\
\hline 1.97 & 0.62 & 0.17 \\
\hline 4.86 & 0.84 & 0.03 \\
\hline 0.00 & 0.00 & 0.00 \\
\hline 0.00 & 0.00 & 0.00 \\
\hline 0.00 & 0.00 & 0.00 \\
\hline 0.00 & 0.00 & 0.00 \\
\hline 0.00 & 0.00 & 0.00 \\
\hline 0.00 & 0.00 & 0.00 \\
\hline 0.00 & 0.00 & 0.00 \\
\hline 0.00 & 0.00 & 0.00 \\
\hline 0.00 & 0.00 & 0.00 \\
\hline 0.00 & 0.00 & 0.00 \\
\hline 5.78 & 0.26 & 0.60 \\
\hline 16.60 & 0.10 & 0.71 \\
\hline 5.84 & -0.27 & 0.70 \\
\hline 0.00 & 0.00 & 0.00 \\
\hline 15.45 & -1.07 & 0.66 \\
\hline 5.81 & -1.47 & 0.48 \\
\hline 4.22 & -1.71 & 0.48 \\
\hline 3.83 & -1.70 & 0.49 \\
\hline 41.93 & -1.95 & 0.53 \\
\hline 11.22 & -1.96 & 0.52 \\
\hline 39.02 & -2.22 & 0.66 \\
\hline 7.99 & -2.23 & 0.62 \\
\hline .58 & -2.59 & 0.68 \\
\hline 36 & -2.71 & 0.77 \\
\hline & 0.00 & 0.0 \\
\hline
\end{tabular}


$\begin{array}{lllllllllll}75 & 118 & 193 & -7.7 & -5.6 & -1522.82 & -4.22 & 1.14 & -3.92-1529.82 & -31.15\end{array}$

$\begin{array}{lllllllll}75 & 119 & 194 & -8.0 & -5.5-1528.38 & -4.33 & 1.92 & -3.86-1534.65 & -27.91\end{array}$

$\begin{array}{llllllllll}75 & 120 & 195 & -8.2 & -5.3-1533.80 & -4.14 & 1.14 & -3.79-1540.60 & -25.78\end{array}$

$\begin{array}{llllllllll}75 & 121 & 196 & -8.4 & -5.2 & -1539.08 & -4.75 & 1.91 & -3.73-1545.65 & -22.76\end{array}$

$\begin{array}{llllllllll}75 & 122 & 197 & -8.7 & -5.1-1544.22 & -5.36 & 1.14 & -3.67-1552.11 & -21.15\end{array}$

$\begin{array}{lllllllll}75 & 123 & 198 & -8.9 & -4.9-1549.22 & -6.20 & 1.91 & -3.61-1557.12 & -18.09\end{array}$

$\begin{array}{llllllllll}75 & 124 & 199 & -9.1 & -4.8-1554.09 & -6.74 & 1.14 & -3.56-1563.24 & -16.14\end{array}$

$\begin{array}{lllllllllll}75 & 125 & 200 & -9.4 & -4.7-1558.82 & -7.62 & 1.91 & -3.50-1568.03 & -12.86\end{array}$

$\begin{array}{llllllllll}75 & 126 & 201 & -9.6 & -4.5 & -1563.42 & -7.86 & 1.14 & -3.44-1573.59 & -10.34\end{array}$

$\begin{array}{llllllllll}75 & 127 & 202 & -9.8 & -4.4-1567.90 & -7.04 & 1.90 & -3.39-1576.43 & -5.11\end{array}$

$\begin{array}{lllllllll}75 & 128 & 203-10.0 & -4.3-1572.24 & -5.97 & 1.14 & -3.34-1580.41 & -1.03\end{array}$

$\begin{array}{llllllll}75 & 129 & 204-10.3 & -4.2-1576.46 & -4.69 & 1.90 & -3.29-1582.55\end{array}$

$75 \quad 130205-10.5-4.0-1580.56-3.56 \quad 1.14-3.24-1586.23$

$\begin{array}{lllllll}75 & 131 & 206-10.7 & -3.9-1584.54 & -2.58 & 1.89 & -3.19-1588.42\end{array}$

4.91

9.30

$75 \quad 132 \quad 207-10.9-3.8-1588.40-1.60 \quad 1.14-3.15-1592.01$

15.18

$\begin{array}{llllllll}75 & 133 & 208-11.1 & -3.7-1592.14 & -0.89 & 1.89 & -3.10-1594.24\end{array}$

19.66

$75 \quad 134 \quad 209-11.3-3.6-1595.77$

$-0.221 .14-3.06-1597.91$

25.50

$75 \quad 135 \quad 210-11.5 \quad-3.5-1599.28$

$0.171 .89-3.01-1600.24$

$75 \quad 136 \quad 211-11.7-3.3-1602.68$

$0.821 .14-2.97-1603.69$

$\begin{array}{lllll}75 & 137 & 212-11.9 & -3.2-1605.97\end{array}$

$0.931 .88-2.93-1606.09$

$75 \quad 138 \quad 213-12.2-3.1-1609.16$

$1.261 .14-2.89-1609.64$

$1.171 .88-2.85-1612.03$

75 140 $215-12.6 \quad-2.9-1615.20$

$1.301 .14-2.81-1615.57$

$1.06 \quad 1.88-2.77-1617.90$

29.91

35.65

40.26

45.94

50.46

56.14

$\begin{array}{llll}75 & 141 & 216-12.8 & -2.8-1618.07\end{array}$

$\begin{array}{llll}75 & 142 & 217-12.9 & -2.7-1620.83\end{array}$

$1.161 .14-2.73-1621.27$

60.67

0.00

0.00

0.00

0.00

0.00

0.00

0.00

0.00

0.00

0.00

0.00

0.00

0.00

0.00

0.00

0.00

0.00

0.00

0.00

0.00

0.00

0.00

0.00

0.00

0.00

0.00

0.00

0.00

0.00

0.00

0.00

0.00

0.00

0.00

0.00

0.00

0.00

0.00

0.00

0.00

0.00

0.00

0.00

0.00

0.00

0.00

0.00

0.00

0.00

0.00

0.00

0.00

0.00

0.00

0.00

0.00

0.00

0.00

0.00

0.00

0.00

0.00

0.00

0.00

0.00

0.00

0.00

0.00

66.41

0.00

0.00

0.00

71.12

0.00

0.00

0.00

$75 \quad 143218-13.1-2.6-1623.50$

$0.86 \quad 1.87-2.70-1623.46$

81.7 .9

0.00

0.00

0.00

$0.851 .14-2.66-1626.74$

$\begin{array}{llll}75 & 145 & 220-13.5 & -2.4-1628.53\end{array}$

$0.471 .87-2.63-1628.82$

87.78

0.00

0.00

0.00

0.00

0.00

0.00

$0.41 \quad 1.14-2.59-1631.95 \quad 92.72$

$\begin{array}{llll}75 & 146 & 221-13.7 & -2.3-1630.90\end{array}$

$0.001 .87-2.56-1633.88 \quad 98.87$

$\begin{array}{llll}75 & 148 & 223-14.1 & -2.1-1635.37\end{array}$

$\begin{array}{llll}-0.08 & 1.14 & -2.53-1636.84 & 103.98\end{array}$

0.00

0.00

0.00

0.00

0.00

0.00

0.00

0.00

0.00

0.00

0.00

0.00

0.00

0.00

0.00

$\begin{array}{llllllll}75 & 150 & 225-14.5 & -2.0-1639.47 & -0.64 & 1.14 & -2.47-1641.43 & 115.52\end{array}$

$\begin{array}{llllllll}75 & 151 & 226-14.6 & -1.9-1641.38 & -1.17 & 1.86 & -2.44-1643.13 & 121.90\end{array}$

$\begin{array}{llllllll}75 & 152 & 227-14.8 & -1.8-1643.21 & -1.22 & 1.14 & -2.41-1645.70 & 127.40\end{array}$

$\begin{array}{llllllll}75 & 153 & 228-15.0 & -1.7-1644.95 & -1.44 & 1.86 & -2.38-1646.91 & 134.26\end{array}$

$\begin{array}{llllllll}75 & 154 & 229-15.2 & -1.6-1646.61 & -1.45 & 1.14 & -2.35-1649.27 & 139.97\end{array}$

$\begin{array}{llllllll}75 & 155 & 230-15.3 & -1.5-1648.18 & -1.83 & 1.86 & -2.32-1650.48 & 146.84\end{array}$

$\begin{array}{lllllllll}75 & 156 & 231-15.5 & -1.4-1649.68 & -1.89 & 1.14 & -2.29-1652.72 & 152.67\end{array}$

$\begin{array}{lllllllll}75 & 157 & 232 & -15.7 & -1.4-1651.09 & -2.35 & 1.85 & -2.27-1653.85 & 159.61\end{array}$

$\begin{array}{llllllll}75 & 158 & 233-15.9 & -1.3-1652.42 & -2.56 & 1.14 & -2.24-1656.08 & 165.45\end{array}$

$\begin{array}{lllllllll}75 & 159 & 234-16.0 & -1.2-1653.68 & -3.25 & 1.85 & -2.21-1657.29 & 172.31\end{array}$

$75 \quad 160235-16.2-1.1-1654.85-3.621 .14-2.19-1659.52178 .15$

$\begin{array}{llllllll}75 & 161 & 236-16.4 & -1.1-1655.95 & -4.25 & 1.85 & -2.16-1660.52 & 185.22\end{array}$

$75 \quad 162237-16.5-1.0-1656.98-4.591 .14-2.14-1662.57 \quad 191.24$

$\begin{array}{lllllllll}75 & 163 & 238-16.7 & -0.9-1657.93 & -4.95 & 1.85 & -2.12 & -1663.16 & 198.73\end{array}$

$\begin{array}{lllllllll}75 & 164 & 239-16.8 & -0.8-1658.82 & -4.71 & 1.14 & -2.09-1664.48 & 205.48\end{array}$

$\begin{array}{lllllllll}75 & 165 & 240-17.0 & -0.8-1659.63 & -4.94 & 1.84 & -2.07-1664.80 & 213.23\end{array}$

$\begin{array}{llllllll}75 & 166 & 241-17.1 & -0.7-1660.37 & -4.21 & 1.14 & -2.05-1665.49 & 220.61\end{array}$

$\begin{array}{lllllllll}75 & 167 & 242-17.3 & -0.6-1661.04 & -4.18 & 1.84 & -2.03-1665.41 & 228.76\end{array}$

$\begin{array}{lllllllll}75 & 168 & 243-17.4 & -0.6-1661.65 & -4.21 & 1.14 & -2.00-1666.72 & 235.52\end{array}$

$\begin{array}{lllllllll}75 & 169 & 244-17.6 & -0.5-1662.19 & -4.74 & 1.84 & -1.98-1667.07 & 243.24\end{array}$

$\begin{array}{lllllllll}75 & 170 & 245-17.7 & -0.4-1662.66 & -4.19 & 1.14 & -1.96-1667.68 & 250.71\end{array}$

$\begin{array}{llllllll}75 & 171 & 246-17.9 & -0.4-1663.07 & -3.29 & 1.83 & -1.94-1666.47 & 259.99\end{array}$

$\begin{array}{lllllllll}75 & 172 & 247-18.0 & -0.3-1663.42 & -3.60 & 1.14 & -1.92-1667.81 & 266.72\end{array}$

$\begin{array}{lllllllll}75 & 173 & 248-18.2 & -0.3-1663.71 & -3.78 & 1.83 & -1.90-1667.56 & 275.04\end{array}$

$\begin{array}{llllllll}75 & 174 & 249-18.3 & -0.2-1663.94 & -4.22 & 1.14 & -1.88-1668.90 & 281.77\end{array}$

$\begin{array}{llllllll}75 & 175 & 250-18.4 & -0.1-1664.11 & -5.01 & 1.83 & -1.86-1669.15 & 289.59\end{array}$

$\begin{array}{llllllll}76 & 83 & 159 & 2.3-12.9-1214.16 & -6.09 & 1.10 & -8.31-1227.46 & -4.01\end{array}$

$\begin{array}{llllllll}76 & 84 & 160 & 2.0-12.6-1226.88 & -5.30 & 0.00 & -8.11-1240.29 & -8.77\end{array}$

$\begin{array}{lllllllll}76 & 85 & 161 & 1.7-12.3-1239.35 & -3.85 & 1.09 & -7.91-1250.01 & -10.42\end{array}$

$\begin{array}{llllllllll}76 & 86 & 162 & 1.4-12.1-1251.56 & -2.81 & 0.00 & -7.72-1262.09 & -14.42\end{array}$

$\begin{array}{lllllllll}76 & 87 & 163 & 1.1-11.8-1263.52 & -1.91 & 1.08 & -7.53-1271.88 & -16.15\end{array}$

$\begin{array}{lllllllll}76 & 88 & 164 & 0.8-11.6-1275.24 & -1.21 & 0.00 & -7.35-1283.80 & -20.00\end{array}$

$\begin{array}{lllllllll}76 & 89 & 165 & 0.5-11.4-1286.71 & -0.67 & 1.08 & -7.18-1293.49 & -21.61\end{array}$

7690166

$0.2-11.1-1297.95-0.190 .00-7.02-1305.15-25.21$

0.00

0.00

0.00

0.00

0.00

0.00

0.00

0.00

0.00

0.00

0.00

0.00

0.00

0.00

0.00

0.00

0.00

0.00

0.00

0.00

0.00

0.00

0.00

0.00

0.00

0.00

0.00

0.00

0.00

0.00

0.00

0.00

0.00

0.00

0.00

0.00

0.00

0.00

0.00

0.00

0.00

0.00

0.00

0.00

0.00

0.00

0.00

0.00

0.00

0.00

0.00

0.00

0.00

0.00

0.00

0.00

0.00

0.00

0.00

0.00

0.00

0.00

0.00

0.00

0.00

0.00

0.00

0.00

0.00

0.00

0.00

0.00

0.00

0.00

0.00

0.00

0.00

0.00

0.00

0.00

0.00

0.00

0.00

0.00

0.00

0.00

0.00

0.00

0.00

0.00

0.00

0.00

0.00

0.00

0.00 
$\begin{array}{llll}76 & 91 & 167 & 0.0-10.9-1308.95\end{array}$

$76 \quad 92 \quad 168-0.3-10.7-1319.72$

$7693 \quad 169-0.6-10.4-1330.27$

$7694 \quad 170-0.9-10.2-1340.59$

$7695 \quad 171-1.2-10.0-1350.70$

$\begin{array}{llllll}76 & 96 & 172 & -1.5 & -9.8-1360.59\end{array}$

$\begin{array}{llllll}76 & 97 & 173 & -1.8 & -9.6-1370.27\end{array}$

$\begin{array}{llllll}76 & 98 & 174 & -2.0 & -9.4-1379.74\end{array}$

$\begin{array}{lllll}76 & 99 & 175 & -2.3 & -9.2-1389.01\end{array}$

$\begin{array}{llllll}76 & 100 & 176 & -2.6 & -9.0-1398.08\end{array}$

$\begin{array}{llllll}76 & 101 & 177 & -2.9 & -8.8-1406.94\end{array}$

$\begin{array}{llllll}76 & 102 & 178 & -3.1 & -8.6-1415.62\end{array}$

$\begin{array}{lllll}76 & 103 & 179 & -3.4 & -8.4-1424.10\end{array}$

$\begin{array}{llllll}76 & 104 & 180 & -3.7 & -8.2-1432.39\end{array}$

$\begin{array}{llllll}76 & 105 & 181 & -3.9 & -8.0-1440.50\end{array}$

$\begin{array}{llllll}76 & 106 & 182 & -4.2 & -7.8-1448.42\end{array}$

$\begin{array}{llllll}76 & 107 & 183 & -4.5 & -7.7-1456.17\end{array}$

$\begin{array}{llllll}76 & 108 & 184 & -4.7 & -7.5-1463.73\end{array}$

$\begin{array}{llllll}76 & 109 & 185 & -5.0 & -7.3-1471.13\end{array}$

$\begin{array}{llllll}76 & 110 & 186 & -5.2 & -7.1-1478.35\end{array}$

$\begin{array}{llllll}76 & 111 & 187 & -5.5 & -7.0-1485.41\end{array}$

$\begin{array}{lllll}76 & 112 & 188 & -5.8 & -6.8-1492.30\end{array}$

$\begin{array}{llllll}76 & 113 & 189 & -6.0 & -6.6-1499.03\end{array}$

$76 \quad 114 \quad 190-6.3-6.5-1505.59$

$\begin{array}{llllll}76 & 115 & 191 & -6.5 & -6.3-1512.00 \\ 76 & 116 & 192 & -6.7 & -6.2-1518.25\end{array}$

$\begin{array}{llllll}76 & 116 & 192 & -6.7 & -6.2-1518.25 \\ 76 & 117 & 193 & -7.0 & -6.0-1524.36\end{array}$

$\begin{array}{llllll}76 & 118 & 194 & -7.2 & -5.9-1530.30\end{array}$

$\begin{array}{llllll}76 & 119 & 195 & -7.5 & -5.7-1536.11\end{array}$

$\begin{array}{llllll}76 & 120 & 196 & -7.7 & -5.6-1541.76\end{array}$

$\begin{array}{llllll}76 & 121 & 197 & -7.9 & -5.4-1547.28\end{array}$

$\begin{array}{llllll}76 & 122 & 198 & -8.2 & -5.3-1552.65\end{array}$

$\begin{array}{llllll}76 & 123 & 199 & -8.4 & -5.2-1557.88\end{array}$

$\begin{array}{llllll}76 & 124 & 200 & -8.6 & -5.0 & -1562.97\end{array}$

$\begin{array}{llllll}76 & 125 & 201 & -8.9 & -4.9-1567.94\end{array}$

$\begin{array}{llllll}76 & 126 & 202 & -9.1 & -4.8-1572.76\end{array}$

$\begin{array}{llllll}76 & 127 & 203 & -9.3 & -4.6-1577.46 \\ 76 & 128 & 204 & -9.5 & -4.5-1582.03\end{array}$

$\begin{array}{llllll}76 & 129 & 205 & -9.8 & -4.4-1586.47\end{array}$

$76130206-10.0-4.3-1590.79$

$76131207-10.2-4.1-1594.98$

$\begin{array}{llll}76 & 132 & 208-10.4 & -4.0-1599.06\end{array}$

$\begin{array}{llll}76 & 133 & 209-10.6 & -3.9-1603.01\end{array}$

$\begin{array}{llll}76 & 134 & 210-10.8 & -3.8-1606.85\end{array}$

$\begin{array}{llll}76 & 135 & 211-11.0 & -3.7-1610.57\end{array}$

$\begin{array}{llll}76 & 136 & 212-11.2 & -3.5-1614.18\end{array}$

$\begin{array}{lllll}76 & 137 & 213-11.5 & -3.4-1617.68\end{array}$

$\begin{array}{llll}76 & 138 & 214-11.7 & -3.3-1621.06\end{array}$

$\begin{array}{llll}76 & 139 & 215-11.9 & -3.2-1624.34\end{array}$

$\begin{array}{llll}76 & 140 & 216-12.1 & -3.1-1627.51\end{array}$

$\begin{array}{llll}76 & 141 & 217-12.3 & -3.0-1630.58\end{array}$

$\begin{array}{llll}76 & 142 & 218-12.5 & -2.9-1633.54\end{array}$

$\begin{array}{llll}76 & 143 & 219-12.7 & -2.8-1636.40\end{array}$

$\begin{array}{llll}76 & 144 & 220-12.8 & -2.7-1639.16\end{array}$

$\begin{array}{llll}76 & 145 & 221-13.0 & -2.6-1641.82\end{array}$

$\begin{array}{llll}76 & 146 & 222-13.2 & -2.5-1644.38\end{array}$

$\begin{array}{lllll}76 & 147 & 223-13.4 & -2.4-1646.85\end{array}$

$\begin{array}{llll}76 & 148 & 224-13.6 & -2.3-1649.22\end{array}$

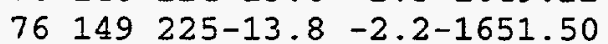

$76 \quad 150 \quad 226-14.0-2.1-1653.68$

$\begin{array}{lllllllll}76 & 152 & 228-14.3 & -2.0-1657.79 & -0.91 & 0.00 & -2.47-1661.17 & 119.21\end{array}$

$\begin{array}{llllllll}76 & 153 & 229-14.5 & -1.9-1659.71 & -1.13 & 0.90 & -2.44-1662.38 & 126.07\end{array}$

$\begin{array}{llllllll}76 & 154 & 230-14.7 & -1.8-1661.54 & -1.12 & 0.00 & -2.41-1665.07 & 131.45\end{array}$

$\begin{array}{lllllllll}76 & 155 & 231-14.9 & -1.7-1663.29 & -1.47 & 0.89 & -2.38-1666.25 & 138.34\end{array}$

$76 \quad 156232-15.0-1.6-1664.96$ $\begin{array}{llll}.42 & 0.00-2.53-1656.63 & 107.60\end{array}$

$0.161 .07-6.86-1314.58-26.56$

$0.520 .00-6.70-1325.91-29.82$

$.831 .06-6.55-1334.93-30.77$

$1.031 .05-6.27-1354.89-34.59$

$1.090 .00-6.14-1365.64-37.26$

$1.041 .04-6.01-1374.19-37.75$

$0.93 \quad 0.00-5.88-1384.69-40.17$

$0.95 \quad 1.04 \quad-5.76-1392.78-40.19$

$\begin{array}{llllll}0.76 & 0.00 & -5.64-1402.96 & -42.29\end{array}$

$0.561 .03-5.53-1410.88-42.14$

$0.33 \quad 0.00-5.41-1420.70-43.90$

$0.081 .02-5.31-1428.30-43.42$

$\begin{array}{lllll}0.28 & 0.00 & -5.20-1437.87 & -44.93\end{array}$

$0.940 .00-5.00-1454.37-45.27$

$\begin{array}{lllll}-1.32 & 1.01 & -4.91-1461.38 & -44.22\end{array}$

1.82

$-4.73-1476.67$

$-44.93$

$-43.37$

$-41.68$

2.43

3.07

4.170 .98

1.68

$-7.00$

$-7.58$

$-8.82$

$-8.02$

$.970 .00-3.43-1592.43$

$\begin{array}{llll}-3.38 & 0.95 & -3.28-1600.69\end{array}$

$\begin{array}{llll}-2.33 & 0.00 & -3.23-1604.62\end{array}$

$\begin{array}{lll}.41 & 0.94 & -3.18-1606.66\end{array}$

$0.24 \quad 0.94-3.09-1612.97$

$0.350 .00-3.05-1616.88$

$0.710 .93-3.00-1619.04$

$1.090 .00-2.96-1622.94$

$1.07 \quad 0.93-2.92-1625.27$

$.270 .00-2.88-1629.12$

$\begin{array}{lll}14 & 0.92 & -2.84-1631.36\end{array}$

$0.990 .00-2.73-1640.90$

$0.650 .91-2.69-1642.95$

$0.640 .00-2.66-1646.40$

$0.190 .91-2.63-1648.37$

$0.190 .91-2.63-1648.37$

91.65

96.38

$\begin{array}{llll}0.35 & 0.91 & -2.56-1653.50 & 102.66\end{array}$ $-1.480 .00$

$72.35-1668.79 \quad 143.87$
0.00

$-30.12$

0.00

$-33.93$

0.00

0.00

0.00

0.00

0.00

0.00

0.00

0.00

0.00

0.00

0.00

$-44.54$

0.00

0.0

$0.22-0.30$

$0.00 \quad 0.00$

$0.84-0.14$

$0.00 \quad 0.00$

0.00

0.00

$0.00 \quad 0.00$

$0.00 \quad 0.00$

$0.00 \quad 0.00$

$0.00 \quad 0.00$

$0.00 \quad 0.00$

$0.00 \quad 0.00$

$0.00 \quad 0.00$

$0.00 \quad 0.00$

0.00

0.00

0.73

0.00

0.67

0.56

0.50

0.46

0.19

0.28

0.01

0.19

0.23

0.37

0.37

0.14

$-0.05$

. $30-4$.

$0.00 \quad 0.00$

0.00

0.00

0.00

0.00

0.00

0.00

0.00

$0.00 \quad 0.00$

0.00

$0.00 \quad 0.00$

0.00

0.00

0.00

0.00

0.00

0.00

0.00

0.00

0.00

0.00

$0.00 \quad 0.00 \quad 0.00$

0.00

0.00

0.00

0.00

0.00

0.00

0.00

0.00

0.00

$\begin{array}{llll}0.00 & 0.00 & 0.00\end{array}$

$0.00 \quad 0.00 \quad 0.00$

$0.00 \quad 0.00$

0.00

$\begin{array}{llll}0.00 & 0.00 & 0.00\end{array}$

$\begin{array}{llll}0.00 & 0.00 & 0.00\end{array}$

$\begin{array}{llll}0.00 & 0.00 & 0.00\end{array}$

$0.00 \quad 0.00$

0.00

$\begin{array}{llll}0.00 & 0.00 & 0.00\end{array}$

$0.00 \quad 0.00 \quad 0.00$

$\begin{array}{llll}0.00 & 0.00 & 0.00\end{array}$

$0.00 \quad 0.00$

0.00

$\begin{array}{llll}0.00 & 0.00 & 0.00\end{array}$

$0.00 \quad 0.00 \quad 0.00$

$0.00 \quad 0.00 \quad 0.00$

$\begin{array}{llll}0.00 & 0.00 & 0.00\end{array}$

$\begin{array}{llll}0.00 & 0.00 & 0.00\end{array}$

$0.00 \quad 0.00 \quad 0.00$

$0.00 \quad 0.00 \quad 0.00$

$\begin{array}{llll}0.00 & 0.00 & 0.00\end{array}$

$\begin{array}{llll}0.00 & 0.00 & 0.00\end{array}$

$\begin{array}{llll}0.00 & 0.00 & 0.00\end{array}$

$0.00 \quad 0.00 \quad 0.00$

$0.00 \quad 0.00 \quad 0.00$

$\begin{array}{llll}0.00 & 0.00 & 0.00\end{array}$ 
$\begin{array}{llllllllll}76 & 157 & 233-15.2 & -1.5-1666.54 & -1.85 & 0.89 & -2.32-1669.82 & 150.91\end{array}$ $\begin{array}{lllllllll}76 & 158 & 234-15.4 & -1.5-1668.04 & -2.05 & 0.00 & -2.30-1672.39 & 156.42\end{array}$ $\begin{array}{llllllll}76 & 159 & 235-15.6 & -1.4-1669.46 & -2.71 & 0.89 & -2.27-1673.56 & 163.32\end{array}$ $\begin{array}{lllllllll}76 & 160 & 236-15.7 & -1.3-1670.81 & -3.06 & 0.00 & -2.24-1676.11 & 168.84\end{array}$ $\begin{array}{lllllllll}76 & 161 & 237-15.9 & -1.2-1672.07 & -3.72 & 0.88 & -2.22-1677.13 & 175.89\end{array}$ $\begin{array}{llllllll}76 & 162 & 238-16.0 & -1.1-1673.26 & -4.09 & 0.00 & -2.19-1679.55 & 181.54\end{array}$ $76163239-16.2-1.1-1674.38-4.38 \quad 0.88-2.17-1680.05189 .11$ $\begin{array}{lllllllll}76 & 164 & 240-16.4 & -1.0-1675.42 & -4.05 & 0.00 & -2.14-1681.61 & 195.62\end{array}$ $\begin{array}{lllllllll}76 & 165 & 241-16.5 & -0.9-1676.39 & -4.06 & 0.88 & -2.12-1681.69 & 203.61\end{array}$ $\begin{array}{lllllllll}76 & 166 & 242-16.7 & -0.9-1677.29 & -3.41 & 0.00 & -2.10-1682.79 & 210.58\end{array}$ $\begin{array}{lllllllll}76 & 167 & 243-16.8 & -0.8-1678.11 & -3.79 & 0.87 & -2.07-1683.11 & 218.34\end{array}$ $\begin{array}{lllllllll}76 & 168 & 244-17.0 & -0.7-1678.87 & -3.60 & 0.00 & -2.05-1684.52 & 225.00\end{array}$ 76 169. $245-17.1-0.7-1679.56-2.62 \quad 0.87-2.03-1683.34234 .25$ $\begin{array}{lllllllll}76 & 170 & 246-17.3 & -0.6-1680.18 & -1.99 & 0.00 & -2.01-1684.18 & 241.48\end{array}$ $\begin{array}{lllllllll}76 & 171 & 247-17.4 & -0.5-1680.74 & -3.54 & 0.86 & -1.99-1685.41 & 248.33\end{array}$ $\begin{array}{lllllllll}76 & 172 & 248-17.6 & -0.5-1681.24 & -3.89 & 0.00 & -1.97-1687.09 & 254.71\end{array}$ $\begin{array}{lllllllll}76 & 173 & 249-17.7 & -0.4-1681.67 & -3.69 & 0.86 & -1.95-1686.44 & 263.43\end{array}$ $\begin{array}{llllllllll}76 & 174 & 250-17.9 & -0.3-1682.03 & -4.12 & 0.00 & -1.93-1688.08 & 269.87\end{array}$ $\begin{array}{lllllllll}76 & 175 & 251-18.0 & -0.3-1682.34 & -4.97 & 0.86 & -1.91-1688.36 & 277.66\end{array}$ $\begin{array}{lllllllll}76 & 176 & 252-18.2 & -0.2-1682.59 & -5.19 & 0.00 & -1.89-1689.67 & 284.42\end{array}$ $\begin{array}{lllllllll}76 & 177 & 253-18.3 & -0.2-1682.78 & -6.32 & 0.85 & -1.87-1690.11 & 292.05\end{array}$ $\begin{array}{lllllllll}77 & 85 & 162 & 2.2-12.6 & -1237.37 & -5.21 & 2.00 & -8.13-1248.71 & -1.85\end{array}$

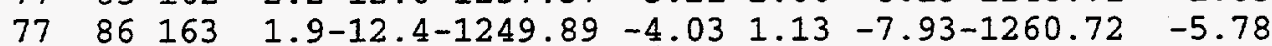
$\begin{array}{lll}77 & 87 & 164\end{array}$

$\begin{array}{lll}77 & 88 & 165\end{array}$

$\begin{array}{lll}77 & 89 & 166\end{array}$

7790167

$\begin{array}{lll}77 & 91 & 168\end{array}$

$\begin{array}{lll}77 & 92 & 169\end{array}$ 1. 6-12.1-1262.15 $1.3-11.9-1274.16$ 1. 0-11.6-1285.93 $0.7-11.4-1297.46$ $0.4-11.2-1308.76$ $0.2-10.9-1319.82$ $93170-0.1-10.7-1330.66$ $94171-0.4-10.5-1341.27$

$77 \quad 95 \quad 172-0.7-10.3-1351.66$

$7796 \quad 173-1.0-10.1-1361.83$

$\begin{array}{llllll}77 & 97 & 174 & -1.3 & -9.9-1371.79\end{array}$

$7798 \quad 175-1.5-9.6-1381.54$

$77 \quad 99176-1.8-9.4-1391.09$

$\begin{array}{llllll}77 & 100 & 177 & -2.1 & -9.2-1400.43\end{array}$

$\begin{array}{llllll}77 & 101 & 178 & -2.4 & -9.0-1409.57\end{array}$

$77 \begin{array}{lllll}702 & 179 & -2.6 & -8.8-1418.51\end{array}$

$\begin{array}{llllll}77 & 103 & 180 & -2.9 & -8.7-1427.26\end{array}$

$\begin{array}{llllll}77 & 104 & 181 & -3.2 & -8.5-1435.82\end{array}$

$\begin{array}{llllll}77 & 105 & 182 & -3.4 & -8.3-1444.20\end{array}$

$\begin{array}{llllll}77 & 106 & 183 & -3.7 & -8.1-1452.39\end{array}$

$\begin{array}{llllll}77 & 107 & 184 & -4.0 & -7.9-1460.39\end{array}$

$\begin{array}{lllll}77 & 108 & 185 & -4.2 & -7.7-1468.22\end{array}$

$\begin{array}{lllll}77 & 109 & 186 & -4.5 & -7.6-1475.87\end{array}$

$\begin{array}{lllll}77 & 110 & 187 & -4.7 & -7.4-1483.35\end{array}$

$\begin{array}{llllll}77 & 111 & 188 & -5.0 & -7.2-1490.66\end{array}$

$77 \begin{array}{lllll}712 & 189 & -5.3 & -7.1-1497.80\end{array}$

$\begin{array}{llllll}77 & 113 & 190 & -5.5 & -6.9-1504.78\end{array}$

$\begin{array}{llllll}77 & 114 & 191 & -5.8 & -6.7-1511.60\end{array}$

$\begin{array}{llllll}77 & 115 & 192 & -6.0 & -6.6-1518.25\end{array}$

$\begin{array}{lllll}77 & 116 & 193 & -6.2 & -6.4-1524.75\end{array}$

$\begin{array}{lllll}77 & 117 & 194 & -6.5 & -6.3-1531.09\end{array}$

$77 \begin{array}{lllll}718 & 195 & -6.7 & -6.1-1537.28\end{array}$

$\begin{array}{llllll}77 & 119 & 196 & -7.0 & -6.0-1543.33\end{array}$

$\begin{array}{llllll}77 & 120 & 197 & -7.2 & -5.8-1549.22\end{array}$

$\begin{array}{llllll}77 & 121 & 198 & -7.4 & -5.7-1554.97\end{array}$

$\begin{array}{llllll}77 & 122 & 199 & -7.7 & -5.5-1560.57\end{array}$

$\begin{array}{llllll}77 & 123 & 200 & -7.9 & -5.4-1566.04\end{array}$

$\begin{array}{lllll}77 & 124 & 201 & -8.1 & -5.3-1571.36\end{array}$

$\begin{array}{llllll}77 & 125 & 202 & -8.4 & -5.1-1576.55\end{array}$

$-4.031 .13$

$-2.051 .13$

$-7.74-1270.85$

$-7.84$

$\begin{array}{lllll}-1.35 & 1.98 & -7.38-1292.68 & -13.53\end{array}$

$-0.761 .13-7.21-1304.30-17.08$

$\begin{array}{lllll}-0.34 & 1.98 & -7.05 & -1314.16 & -18.87\end{array}$

$0.141 .13-6.89-1325.44-22.07$

$\begin{array}{llllll}0.47 & 1.97 & -6.73-1334.95 & -23.51\end{array}$

$0.771 .13-6.59-1345.96-26.45$

$0.991 .97-6.44-1355.14-27.57$

$1.191 .13-6.30-1365.82-30.17$

$1.221 .96-6.17-1374.78-31.06$

$1.221 .13-6.04-1385.24-33.44$

$0.891 .96-5.92-1394.16-34.29$

$1.031 .13-5.79-1404.06-36.13$

$0.681 .95-5.68-1412.61-36.61$

$0.361 .13-5.56-1422.59-38.51$

$0.181 .95-5.45-1430.59-38.44$

$-0.171 .13-5.34-1440.21-39.99$

$\begin{array}{llllll}-0.29 & 1.94 & -5.24-1447.79 & -39.49\end{array}$

$\begin{array}{lllll}-0.72 & 1.13 & -5.14-1457.12 & -40.75\end{array}$

$\begin{array}{llll}-1.04 & 1.94 & -5.04-1464.54 & -40.10\end{array}$

$-1.421 .13-4.95-1473.46-40.95$

$\begin{array}{llll}-1.63 & 1.93 & -4.85-1480.43 & -39.85\end{array}$

$-1.471 .13$

$-1.631 .93$

$-1.731 .13$

$-2.261 .92$

$-4.77-1488.46$

$-39.81$

$-2.541 .13$

$-3.121 .92$

$-3.621 .13$

$-4.221 .91$

$-4.721 .13$

$-5.181 .91$

$-5.391 .13$

$-6.461 .90$

$\begin{array}{lllll}-7.01 & 1.13 & -3.87-1570.32 & -24.81\end{array}$

$\begin{array}{lllll}-7.89 & 1.90 & -3.81-1575.83 & -22.25\end{array}$

$\begin{array}{lllll}-8.48 & 1.13 & -3.75-1582.46 & -20.81\end{array}$

$\begin{array}{lllll}-9.50 & 1.90 & -3.69-1587.84 & -18.12\end{array}$

$\begin{array}{llllll}77 & 126 & 203 & -8.6 & -5.0-1581.60\end{array}$

$-9.691 .13-3.63-1593.79-16.00$

$\begin{array}{llllll}77 & 127 & 204 & -8.8 & -4.9-1586.52\end{array}$

$\begin{array}{llllll}77 & 128 & 205 & -9.0 & -4.7-1591.32\end{array}$

$\begin{array}{llll}-8.75 & 1.89 & -3.57-1596.95\end{array}$

$-7.861 .13-3.52-1601.57$

$\begin{array}{llllll}77 & 129 & 206 & -9.3 & -4.6-1595.98\end{array}$
$-7.63$ $-2.09$ $-6.541 .89-3.46-1604.09$

\begin{tabular}{|c|c|c|}
\hline 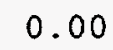 & 0.00 & \\
\hline 0 & 0.00 & 0.00 \\
\hline & & \\
\hline .00 & & \\
\hline 00 & 10 & \\
\hline 00 & & \\
\hline & & \\
\hline .00 & .00 & \\
\hline .00 & .00 & \\
\hline & & \\
\hline & & \\
\hline 00 & .00 & \\
\hline & & \\
\hline & & \\
\hline & & \\
\hline 0 & 0 & \\
\hline & & \\
\hline & & \\
\hline 0 & & \\
\hline 0.0 & & \\
\hline & & \\
\hline & & \\
\hline 0 & 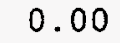 & \\
\hline . & & \\
\hline .00 & & \\
\hline & & \\
\hline U. & 0 & \\
\hline 0.6 & 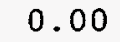 & \\
\hline 0.00 & & \\
\hline & & \\
\hline .3 & 6 & \\
\hline 0 & & \\
\hline 0 & & \\
\hline & & \\
\hline 0 & 0 & \\
\hline & & \\
\hline & & \\
\hline & & \\
\hline .0 & 0 & \\
\hline 0 & 0 & \\
\hline 0 & & \\
\hline 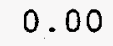 & 0 & \\
\hline 0.0 & & \\
\hline 9.5 & 0 & \\
\hline & & \\
\hline$n_{0}$ & $-c$ & \\
\hline 0.0 & & \\
\hline 38.3 & -1 & \\
\hline & 7 & \\
\hline & -2 & \\
\hline & -2.75 & -0 . \\
\hline & -3 & \\
\hline & -3.72 & \\
\hline & -4 & \\
\hline .70 & -4.63 & \\
\hline 29.46 & -5 & \\
\hline & -5 & \\
\hline & & \\
\hline & 0 & \\
\hline & & \\
\hline & & \\
\hline & & \\
\hline & 0 & \\
\hline & & \\
\hline & 0. & \\
\hline & 0.00 & \\
\hline
\end{tabular}

$\begin{array}{lll}0.00 & 0.00 & 0.00\end{array}$ $-38.33-1.64-0.01$ $\begin{array}{lll}-38.46 & -1.98 & -0.25\end{array}$ $\begin{array}{lll}-36.71 & -2.20 & 0.06\end{array}$ $-36.72-2.75-0.21$ $-34.84-3.16-0.04$ $-34.54-3.72-0.10$ $-32.54-4.30-0.08$ $\begin{array}{lll}-31.70 & -4.63 & 0.09\end{array}$ $\begin{array}{lll}-29.46 & -5.27 & -0.09\end{array}$ $\begin{array}{llll}-28.29 & -5.57-0.18\end{array}$ $\begin{array}{llll}0.00 & 0.00 & 0.00\end{array}$ $\begin{array}{llll}0.00 & 0.00 & 0.00\end{array}$ $\begin{array}{lll}0.00 & 0.00 & 0.00\end{array}$ $\begin{array}{llll}0.00 & 0.00 & 0.00\end{array}$ $\begin{array}{llll}0.00 & 0.00 & 0.00\end{array}$ $\begin{array}{llll}0.00 & 0.00 & 0.00\end{array}$ $0.00 \quad 0.00 \quad 0.00$ $0.00 \quad 0.00 \quad 0.00$ $0.00 \quad 0.00 \quad 0.00$ 
$\begin{array}{llllllllll}77 & 130 & 207 & -9.5 & -4.5 & -1600.52 & -5.42 & 1.13 & -3.41-1608.22\end{array}$ $\begin{array}{llllllll}77 & 131 & 208 & -9.7 & -4.3-1604.93 & -4.16 & 1.89 & -3.36-1610.56\end{array}$ $\begin{array}{llllllll}77 & 132 & 209 & -9.9 & -4.2-1609.22 & -3.09 & 1.13 & -3.31-1614.49\end{array}$ $77 \quad 133 \quad 210-10.1-4.1-1613.38-2.10 \quad 1.88-3.26-1616.87$ $77 \quad 134211-10.3-4.0-1617.43-1.32 \quad 1.13-3.22-1620.84$ $\begin{array}{lllllll}77 & 135 & 212-10.5 & -3.9-1621.37 & -0.82 & 1.88 & -3.17-1623.48\end{array}$ $77136213-10.8-3.8-1625.18-0.211 .13-3.12-1627.39$

$77 \quad 137214-11.0-3.6-1628.89$

$77138 \quad 215-11.2-3.5-1632.48$

$77139216-11.4-3.4-1635.96$

$77 \quad 140 \quad 217-11.6 \quad-3.3-1639.33$

$77 \quad 141 \quad 218-11.8-3.2-1642.59$

$77 \quad 142 \quad 219-12.0 \quad-3.1-1645.75$

$\begin{array}{llll}77 & 143 & 220-12.2 & -3.0-1648.81\end{array}$

$77144 \quad 221-12.4 \quad-2.9-1651.76$

$\begin{array}{llll}77 & 145 & 222-12.6 & -2.8-1654.62\end{array}$

$\begin{array}{lllll}77 & 146 & 223-12.7 & -2.7-1657.37\end{array}$

$\begin{array}{lllll}77 & 147 & 224-12.9 & -2.6-1660.03\end{array}$

$77 \begin{array}{llll}748 & 225-13.1 & -2.5-1662.59\end{array}$

$77149226-13.3-2.4-1665.05$

$\begin{array}{llll}77 & 150 & 227-13.5 & -2.3-1667.42\end{array}$

$77 \quad 151228-13.7-2.2-1669.70$

$\begin{array}{llll}77 & 152 & 229-13.9 & -2.1-1671.89\end{array}$ $0.28 \quad 1.87-3.08-1629.81$ $0.671 .13-3.04-1633.71$ $0.801 .87-3.00-1636.28$ $1.001 .13-2.95-1640.16$ $0.871 .87-2.91-1642.77$ $1.011 .13-2.87-1646.49$ $0.811 .87-2.84-1648.97$ $0.90 \quad 1.13-2.80-1652.53$ $0.581 .86-2.76-1654.94$ $0.601 .13-2.73-1658.37$ $0.151 .86-2.69-1660.71$ $0.101 .13-2.66-1664.02$ $-0.391 .86-2.62-1666.21$ $-0.451 .13-2.59-1669.33 \quad 102.17$ $-0.881 .85-2.56-1671.29108 .29$ $-0.881 .13-2.53-1674.17 \quad 113.48$ $77153230-14.0-2.1-1673.99-1.07 \quad 1.85-2.50-1675.71 \quad 120.02$ $\begin{array}{llllllll}77 & 154 & 231-14.2 & -2.0-1676.00 & -0.95 & 1.13 & -2.47-1678.29 & 125.50\end{array}$ $77 \begin{array}{llllllll}755 & 232-14.4 & -1.9-1677.93 & -1.14 & 1.85 & -2.44-1679.66 & 132.21\end{array}$ $\begin{array}{llllllllll}77 & 156 & 233-14.6 & -1.8-1679.77 & -1.16 & 1.13 & -2.41-1682.21 & 137.73\end{array}$ $\begin{array}{lllllllll}77 & 157 & 234-14.7 & -1.7-1681.52 & -1.42 & 1.84 & -2.38-1683.48 & 144.53\end{array}$ $77158 \quad 235-14.9-1.6-1683.20-1.631 .13-2.35-1686.05150 .03$ $\begin{array}{lllllllll}77 & 159 & 236-15.1 & -1.5-1684.79 & -2.23 & 1.84 & -2.32-1687.50 & 156.65\end{array}$ $\begin{array}{lllllllll}77 & 160 & 237-15.3 & -1.5-1686.30 & -2.58 & 1.13 & -2.30-1690.05 & 162.18\end{array}$ $77161238-15.4-1.4-1687.73-3.35 .1 .84-2.27-1691.51168 .78$ $77 \begin{array}{llllllll}762 & 239-15.6 & -1.3-1689.08 & -3.65 & 1.13 & -2.25-1693.85 & 174.51\end{array}$ $\begin{array}{lllllllll}77 & 163 & 240-15.8 & -1.2-1690.36 & -3.91 & 1.84 & -2.22-1694.66 & 181.78\end{array}$ $\begin{array}{llllllll}77 & 164 & 241-15.9 & -1.2-1691.56 & -3.63 & 1.13 & -2.20-1696.26 & 188.25\end{array}$ $\begin{array}{llllllll}77 & 165 & 242-16.1 & -1.1-1692.69 & -3.42 & 1.83 & -2.17-1696.45 & 196.13\end{array}$ $77166243-16.2-1.0-1693.75-3.281 .13-2.15-1698.05202 .60$ $\begin{array}{llllllll}77 & 167 & 244-16.4 & -0.9-1694.73 & -3.61 & 1.83 & -2.12-1698.63 & 210.09\end{array}$ $\begin{array}{llllllll}77 & 168 & 245-16.5 & -0.9-1695.64 & -2.31 & 1.13 & -2.10-1698.93 & 217.87\end{array}$ $\begin{array}{llllllll}77 & 169 & 246-16.7 & -0.8-1696.49 & -2.58 & 1.83 & -2.08-1699.32 & 225.55\end{array}$ $\begin{array}{lllllllll}77 & 170 & 247-16.8 & -0.7-1697.26 & -2.28 & 1.13 & -2.06-1700.47 & 232.47\end{array}$ $\begin{array}{lllllllll}77 & 171 & 248-17.0 & -0.7-1697.97 & -4.11 & 1.83 & -2.04-1702.29 & 238.72\end{array}$ 77172 249-17.1 -0.6-1698.61-4.44 1.13-2.01-1703.93 245.15 $\begin{array}{llllllll}77 & 173 & 250-17.3 & -0.5-1699.19 & -4.27 & 1.82 & -1.99-1703.63 & 253.53\end{array}$ $\begin{array}{lllllllll}77 & 174 & 251-17.4 & -0.5-1699.70 & -4.43 & 1.13 & -1.97-1704.97 & 260.25\end{array}$ $\begin{array}{llllllll}77 & 175 & 252-17.6 & -0.4-1700.15 & -5.28 & 1.82 & -1.95-1705.56 & 267.74\end{array}$ $\begin{array}{llllllll}77 & 176 & 253-17.7 & -0.4-1700.53 & -5.68 & 1.13 & -1.93-1707.02 & 274.35\end{array}$ $\begin{array}{llllllll}77 & 177 & 254-17.9 & -0.3-1700.86 & -6.87 & 1.82 & -1.91-1707.82 & 281.61\end{array}$ $\begin{array}{lllllllll}77 & 178 & 255-18.0 & -0.2-1701.13 & -6.97 & 1.13 & -1.89-1708.86 & 288.64\end{array}$ $77179256-18.1-0.2-1701.34-6.941 .82-1.88-1708.33297 .24$ $78179256-18.1-0.2-1701.34-6.941 .82-1.88-1708.33297 .24$ $\begin{array}{lllllllll}78 & 87 & 165 & 2.1-12.4-1260.29 & -3.53 & 1.08 & -7.95-1270.69 & -0.41\end{array}$ $\begin{array}{lllllllll}78 & 88 & 166 & 1.8-12.2-1272.60 & -2.61 & 0.00 & -7.76-1282.98 & -4.62\end{array}$ $\begin{array}{lllllllll}78 & 89 & 167 & 1.5 & -11.9-1284.67 & -1.65 & 1.08 & -7.58-1292.82 & -6.40\end{array}$ $1.2-11.7-1296.49-0.880 .00-7.41-1304.78-10.28$ 78.91169

91169

$1.2-11.7-1296.49$ $0.9-11.5-1308.08$ 92170 $0.6-11.2-1319.43$

$\begin{array}{lll}78 & 93 & 171\end{array}$ $0.4-11.0-1330.55$

$\begin{array}{llll}78 & 94 & 172 & 0.1-10.8-1341.45\end{array}$

$\begin{array}{llll}78 & 95 & 173 & -0.2-10.6-1352.12\end{array}$

$7896 \quad 174-0.5-10.3-1362.58$

$7897 \quad 175-0.8-10.1-1372.82$

$\begin{array}{lllll}78 & 98 & 176 & -1.1 & -9.9-1382.85\end{array}$

$\begin{array}{llllll}78 & 99 & 177 & -1.3 & -9.7-1392.67\end{array}$

$\begin{array}{llllll}78 & 100 & 178 & -1.6 & -9.5-1402.29\end{array}$

$\begin{array}{lllll}78 & 101 & 179 & -1.9 & -9.3-1411.70\end{array}$

$\begin{array}{llllll}78 & 102 & 180 & -2.2 & -9.1-1420.91\end{array}$
$-0.880 .00-7.41-1304.78-10.28$

$\begin{array}{llllll}0.50 & 1.07 & -7.24-1314.75 & -12.18\end{array}$ $0.08 \quad 0.00-7.08-1326.42-15.78$ $0.491 .06-6.92-1335.92-17.21$ $0.820 .00-6.77-1347.39-20.61$ $\begin{array}{lllll}0.96 & 1.05 & -6.62-1356.73 & -21.88\end{array}$ $\begin{array}{llllll}1.17 & 0.00 & -6.48-1367.88 & -24.96\end{array}$ $\begin{array}{lllll}1.15 & 1.04 & -6.34-1376.96 & -25.97\end{array}$ $1.260 .00-6.20-1387.79-28.73$ $1.261 .04-6.08-1396.45-29.31$ $1.100 .00-5.95-1407.14-31.93$ $1.06 \quad 1.03-5.83-1415.44-32.16$ $0.940 .00 \quad-5.71-1425.69-34.33$
0.00

0.00

0.00

0.00

0.00

0.00

0.00

0.00

0.00

0.00

0.00

0.00

0.00

0.00

0.00

0.00

0.00

0.00

0.00

0.00

0.00

0.00

0.00

0.00

0.00

0.00

0.00

0.00

0.00

0.00

0.00

0.00

0.00

0.00

0.00

0.00

0.00

0.00

0.00

0.00

0.00

0.00

0.00

0.00

0.00

0.00

0.00

0.00

0.00

0.00

0.00

0.00

0.00

0.00

0.00

0.00

0.00

$-21.23$

0.00

$-25.32$

0.00

0.00

0.00

0.00

0.00

0.00
0.00

0.00

0.00

0.00

0.00

0.00

0.00

0.00

0.00

0.00

0.00

0.00

0.00

0.00

0.00

0.00

0.00

0.00

0.00

0.00

0.00

0.00

0.00

0.00

0.00

0.00

0.00

0.00

0.00

0.00

0.00

0.00

0.00

0.00

0.00

0.00

0.00

0.00

0.00

0.00

0.00

0.00

0.00

0.00

0.00

0.00

0.00

0.00

0.00

0.00

0.00

0.00

0.00

0.00

0.00

0.00

0.00

$0.20-0.62$

$0.00 \quad 0.00$

$0.81-0.36$

$0.00 \quad 0.00$

$0.00 \quad 0.00$

0.00

0.00

0.00

$0.00 \quad 0.00$

0.00

0.00

0.00

0.00

0.00

.00
0.00

0.00

0.00

0.00

0.00

0.00

.00
0.00

0.00

.00

0.00

0.00

0.00

.00

0.00

0.00

0.00

0.00

0.00

0.00

0.00

0.00

0.00 0.00 0.00 0.00 .00 0.00

.00
.00 .00

.00
.00 .00

.00
.00 .00 -.62
0.00 
$\begin{array}{lllllll}78 & 103 & 181 & -2.4 & -8.9 & -1429.93\end{array}$

$\begin{array}{llllll}78 & 104 & 182 & -2.7 & -8.7-1438.76\end{array}$

$\begin{array}{llllll}78 & 105 & 183 & -3.0 & -8.5-1447.40\end{array}$

$\begin{array}{llllll}78 & 106 & 184 & -3.2 & -8.4-1455.85\end{array}$

$\begin{array}{llllll}78 & 107 & 185 & -3.5 & -8.2-1464.12\end{array}$

$\begin{array}{llllll}78 & 108 & 186 & -3.7 & -8.0 & -1472.21\end{array}$

$\begin{array}{llllll}78 & 109 & 187 & -4.0 & -7.8-1480.12\end{array}$

$78 \quad 110 \quad 188,-4.2-7.6-1487.85$

$\begin{array}{llllll}78 & 111 & 189 & -4.5 & -7.5-1495.42\end{array}$

$\begin{array}{llllll}78 & 112 & 190 & -4.8 & -7.3-1502.81\end{array}$

$\begin{array}{llllll}78 & 113 & 191 & -5.0 & -7.1-1510.04\end{array}$

$\begin{array}{llllll}78 & 114 & 192 & -5.3 & -7.0-1517.10\end{array}$

$\begin{array}{lllll}78 & 115 & 193 & -5.5 & -6.8-1524.00\end{array}$

$\begin{array}{llllll}78 & 116 & 194 & -5.7 & -6.7-1530.75\end{array}$

$\begin{array}{llllll}78 & 117 & 195 & -6.0 & -6.5-1537.33\end{array}$

$\begin{array}{lllll}78 & 118 & 196 & -6.2 & -6.4-1543.76\end{array}$

$\begin{array}{lllll}78 & 119 & 197 & -6.5 & -6.2-1550.05\end{array}$

$\begin{array}{lllll}78 & 120 & 198 & -6.7 & -6.1-1556.18\end{array}$

$\begin{array}{llllll}78 & 121 & 199 & -6.9 & -5.9-1562.16\end{array}$

$\begin{array}{lllll}78 & 122 & 200 & -7.2 & -5.8-1568.00\end{array}$

$\begin{array}{llllll}78 & 123 & 201 & -7.4 & -5.6-1573.69\end{array}$

$\begin{array}{llllll}78 & 124 & 202 & -7.6 & -5.5-1579.25\end{array}$

$\begin{array}{llllll}78 & 126 & 204 & -8.1 & -5.2-1589.95-10.40\end{array}$

$\begin{array}{llllll}78 & 127 & 205 & -8.3 & -5.1-1595.09\end{array}$

$\begin{array}{lllll}78 & 128 & 206 & -8.5 & -4.9-1600.11\end{array}$

$\begin{array}{lllll}78 & 129 & 207 & -8.8 & -4.8-1604.99\end{array}$

$\begin{array}{llllll}78 & 130 & 208 & -9.0 & -4.7-1609.75\end{array}$

$\begin{array}{lllll}78 & 131 & 209 & -9.2 & -4.6-1614.37\end{array}$

$\begin{array}{llllll}78 & 132 & 210 & -9.4 & -4.4-1618.88\end{array}$

$\begin{array}{lllll}78 & 133 & 211 & -9.6 & -4.3-1623.26\end{array}$

$\begin{array}{lllll}78 & 134 & 212 & -9.8 & -4.2-1627.52\end{array}$

$78 \quad 135 \quad 213-10.1-4.1-1631.67$

$78 \quad 136 \quad 214-10.3-4.0-1635.69$

$\begin{array}{llll}78 & 137 & 215-10.5 & -3.8-1639.60\end{array}$

$\begin{array}{llll}78 & 138 & 216-10.7 & -3.7-1643.40\end{array}$

$\begin{array}{lllll}78 & 139 & 217-10.9 & -3.6-1647.08\end{array}$

$78 \quad 140 \quad 218-11.1-3.5-1650.66$

$78 \quad 141 \quad 219-11.3-3.4-1654.12$

$\begin{array}{llll}78 & 142 & 220-11.5 & -3.3-1657.48\end{array}$

$\begin{array}{lllll}78 & 143 & 221-11.7 & -3.2-1660.74\end{array}$

$\begin{array}{lllll}78 & 144 & 222-11.9 & -3.1-1663.88\end{array}$

$78 \quad 145 \quad 223-12.1-3.0-1666.93$

$\begin{array}{llll}78 & 146 & 224-12.3 & -2.9-1669.88\end{array}$

$\begin{array}{lllll}78 & 147 & 225-12.5 & -2.8-1672.72\end{array}$

$78 \quad 148 \quad 226-12.6 \quad-2.7-1675.47$

$\begin{array}{llll}78 & 149 & 227-12.8 & -2.6-1678.12\end{array}$

$\begin{array}{lllll}78 & 150 & 228-13.0 & -2.5-1680.68\end{array}$

$\begin{array}{llll}78 & 151 & 229-13.2 & -2.4-1683.14\end{array}$

$\begin{array}{llll}78 & 152 & 230-13.4 & -2.3-1685.52\end{array}$

$\begin{array}{lllll}78 & 153 & 231-13.6 & -2.2-1687.79\end{array}$

$\begin{array}{lllll}78 & 154 & 232-13.7 & -2.1-1689.98\end{array}$

$\begin{array}{lllll}78 & 155 & 233-13.9 & -2.1-1692.09\end{array}$

$78 \quad 156 \quad 234-14.1-2.0-1694.10$

$78 \quad 157235-14.3-1.9-1696.03$

$78158,236-14.4-1.8-1697.88$

$78 \quad 159 \quad 237-14.6-1.7-1699.64$

$78 \quad 160 \quad 238-14.8-1.6-1701.32$

$\begin{array}{llll}78 & 161 & 239-15.0 & -1.6-1702.92\end{array}$

$\begin{array}{llll}78 & 162 & 240-15.1 & -1.5-1704.44\end{array}$

$\begin{array}{llll}78 & 163 & 241-15.3 & -1.4-1705.88\end{array}$

$-9.58$

$-8.54$

$-6.02$

$-4.91$

$-3.78$

$-2.78$

$-1.84 \quad 0.00-3.30-1632.66$

$-1.240 .94-3.25-1635.22$

$\begin{array}{llll}-0.52 & 0.00 & -3.20-1639.42\end{array}$

$\begin{array}{llll}-0.11 & 0.93 & -3.16-1641.94\end{array}$

$0.350 .00-3.11-1646.16$

$0.590 .93-3.07-1648.64$

$0.880 .00-3.03-1652.81$

$0.84 \quad 0.92-2.99-1655.35$

$1.04 \quad 0.00-2.95-1659.39$

$0.890 .92-2.91-1661.83$

$0.950 .00-2.87-1665.80$

$0.650 .91-2.83-1668.20$

$0.710 .00-2.79-1671.96$

$0.350 .91-2.76-1674.22$

$0.320 .00-2.72-1677.87$

$-0.08 \cdot 0.91-2.69-1679.99$

$-0.110 .00-2.65-1683.44$

$-0.540 .90-2.62-1685.40$

$-0.500 .00-2.59-1688.60$

$-0.660 .90-2.56-1690.11112 .88$

$-0.540 .00-2.53-1693.05118 .02$

$\begin{array}{lllll}-0.85 & 0.89 & -2.50-1694.54 & 124.60\end{array}$

$\begin{array}{lllll}-0.71 & 0.00 & -2.47-1697.28 & 129.93\end{array}$

$\begin{array}{lllll}-1.03 & 0.89 & -2.44-1698.61 & 136.67\end{array}$

$\begin{array}{lllll}-1.12 & 0.00 & -2.41-1701.41 & 141.95\end{array}$

$\begin{array}{lllll}-1.70 & 0.89 & -2.38-1702.83 & 148.59\end{array}$

$\begin{array}{lllll}-2.00 & 0.00 & -2.35-1705.67 & 153.82\end{array}$

$\begin{array}{llll}-2.75 & 0.88 & -2.33-1707.11 & 160.45\end{array}$

$\begin{array}{llll}-3.02 & 0.00 & -2.30-1709.76 & 165.88\end{array}$

$\begin{array}{lllll}-3.28 & 0.88 & -2.27-1710.56 & 173.15\end{array}$

$\begin{array}{lllllllll}78 & 164 & 242-15.5 & -1.3-1707.25 & -3.08 & 0.00 & -2.25-1712.58 & 179.20\end{array}$

$\begin{array}{lllllllll}78 & 165 & 243-15.6 & -1.2-1708.54 & -2.86 & 0.88 & -2.22-1712.75 & 187.11\end{array}$

$\begin{array}{lllllllll}78 & 166 & 244-15.8 & -1.2-1709.75 & -2.68 & 0.00 & -2.20-1714.63 & 193.29\end{array}$

$\begin{array}{lllllllll}78 & 167 & 245-15.9 & -1.1-1710.90 & -0.92 & 0.87 & -2.17-1713.12 & 202.88\end{array}$

$78 \quad 168 \quad 246-16.1-1.0-1711.96$

$-1.900 .00-2.15-1716.02 \quad 208.05$

0.00

0.00

0.00

0.00

0.00

$0.00 \quad 0.00$

0.00

$0.00 \quad 0.00$

0.00

0.00

0.00

0.00

0.00

$-37.79$

0.00

$-0.29$

0.23

0.00

$-37.83-1.01$

$-0.85$

$-36.49$

$-1.27$

$-0.76$

$-37.33$

$-1.87$

$-0.90$

$-36.30$

$-2.16$

$-0.72$

$-34.49$

$-3.29$

$-0.67$

$-34.79$

$-4.00$

$-0.69$

$-32.67$

$-4.58$

$-0.54$

$-30.44$

$-5.77$

$-0.36$

$-29.93$

$-6.29$

$-0.36$

$-27.42-6.91$

0.23

$-26.63-7.45$

0.31

$-23.75$

$-7.97$

0.67

0.00

0.00

0.00

0.00

0.00

0.00

0.00

0.00

0.00

0.00

0.00

0.00

0.00

0.00

0.00

0.00

0.00

0.00

0.00

0.00

0.00

0.00

0.00

0.00

0.00

0.00

0.00

0.00

0.00

0.00

0.00

0.00

0.00

0.00

0.00

0.00

0.00

0.00

0.00

0.00

0.00

0.00

0.00

0.00

0.00

0.00

0.00

0.00

0.00

0.00

0.00

0.00

0.00

0.00

0.00

0.00

0.00

0.00

0.00

0.00

0.00

0.00

0.00

0.00

0.00

0.00

0.00

0.00

0.00

0.00

0.00

0.00

0.00

0.00

0.00

0.00

0.00

0.00

0.00

0.00

0.00

0.00

0.00

0.00

0.00

0.00

0.00

0.00

0.00

0.00

0.00

0.00

0.00

0.00

0.00

0.00

0.00

0.00

0.00

0.00

0.00

0.00

0.00

0.00

0.00

0.00

0.00

0.00

0.00

0.00

0.00

0.00

0.00

0.00

0.00

0.00

0.00

0.00

0.00

0.00

0.00

0.00

0.00

.00

0.00

0.00

0.00

0.00

0.00

0.00 
$\begin{array}{llllllllll}78 & 169 & 247-16.2 & -1.0-1712.96 & -2.11 & 0.87 & -2.13-1716.33 & 215.81\end{array}$ $\begin{array}{lllllllll}78 & 170 & 248-16.4 & -0.9-1713.89 & -2.09 & 0.00 & -2.11-1718.08 & 222.13\end{array}$ $\begin{array}{lllllllll}78 & 171 & 249-16.6 & -0.8-1714.75 & -2.79 & 0.86 & -2.08-1718.76 & 229.53\end{array}$ $\begin{array}{lllllllll}78 & 172 & 250-16.7 & -0.8-1715.54 & -3.37 & 0.00 & -2.06-1720.97 & 235.38\end{array}$ $\begin{array}{lllllllll}78 & 173 & 251-16.9 & -0.7-1716.26 & -4.10 & 0.86 & -2.04-1721.54 & 242.88\end{array}$ $\begin{array}{lllllllll}78 & 174 & 252-17.0 & -0.6-1716.92 & -4.52 & 0.00 & -2.02-1723.46 & 249.04\end{array}$ $\begin{array}{lllllllll}78 & 175 & 253-17.1 & -0.6-1717.51 & -5.27 & 0.86 & -2.00-1723.92 & 256.64\end{array}$ $\begin{array}{llllllllll}78 & 176 & 254-17.3 & -0.5-1718.04 & -5.69 & 0.00 & -1.98-1725.71 & 262.93\end{array}$ $\begin{array}{lllllllll}78 & 177 & 255-17.4 & -0.4-1718.51 & -7.35 & 0.85 & -1.96-1726.96 & 269.75\end{array}$ $\begin{array}{lllllllll}78 & 178 & 256-17.6 & -0.4-1718.92 & -7.47 & 0.00 & -1.94-1728.33 & 276.46\end{array}$ $\begin{array}{lllllllll}78 & 179 & 257-17.7 & -0.3-1719.26 & -7.45 & 0.85 & -1.92-1727.78 & 285.07\end{array}$ $\begin{array}{lllllllll}78 & 180 & 258-17.8 & -0.3-1719.55 & -7.77 & 0.00 & -1.90-1729.22 & 291.71\end{array}$ $\begin{array}{lllllllll}78 & 181 & 259-18.0 & -0.2-1719.78 & -8.40 & 0.85 & -1.88-1729.21 & 299.79\end{array}$ $\begin{array}{llllllll}78 & 182 & 260-18.1 & -0.1-1719.95 & -8.52 & 0.00 & -1.86-1730.33 & 306.74\end{array}$

$\begin{array}{lrl}79 & 88 & 167\end{array}$

$\begin{array}{lll}79 & 89 & 168\end{array}$

$7990 \quad 169$ $2.3-12.5-1270.57-4.021 .12-7.97-1281.44$ $2.0-12.2-1282.92-3.02 \quad 1.98-7.79-1291.76$

$\begin{array}{lll}79 & 91 & 170\end{array}$ $1.7-12.0-1295.04-2.17 \quad 1.12-7.61-1303.70$ $1.4-11.8-1306.91-1.34 \quad 1.97-7.43-1313.72$

$\begin{array}{lll}79 & 92 & 171\end{array}$

$\begin{array}{lll}79 & 93 & 172\end{array}$

$\begin{array}{lll}79 & 94 & 173\end{array}$

$\begin{array}{lll}79 & 95 & 174\end{array}$

$1.1-11 \cdot 5-1318.55$

$\begin{array}{lll}-1.34 & 1.97 & -7.43-1313.72 \\ -0.70 & 1.12 & -7.27-1325.40\end{array}$ $0.8-11.3-1329.96$ $0.5-11.1-1341.15$

$\begin{array}{lll}79 & 96 & 175\end{array}$ $0.3-10.8-1352.10$

$7997176-0.3-10.4-1373.36$

$7998177-0.6-10.2-1383.67$

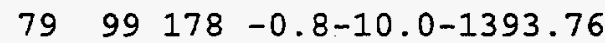

$\begin{array}{llllll}79 & 100 & 179 & -1.1 & -9.8-1403.65\end{array}$

$\begin{array}{llllll}79 & 101 & 180 & -1.4 & -9.6-1413.34\end{array}$

$\begin{array}{llllll}79 & 102 & 181 & -1.7 & -9.4-1422.82\end{array}$

$\begin{array}{llllll}79 & 103 & 182 & -1.9 & -9.2-1432.11\end{array}$

$\begin{array}{llllll}79 & 104 & 183 & -2.2 & -9.0-1441.21\end{array}$

$\begin{array}{llllll}79 & 105 & 184 & -2.5 & -8.8-1450.11\end{array}$

$\begin{array}{llllll}79 & 106 & 185 & -2.7 & -8.6 & -1458.82\end{array}$

$\begin{array}{llllll}79 & 107 & 186 & -3.0 & -8.4-1467.35\end{array}$

$\begin{array}{llllll}79 & 108 & 187 & -3.2 & -8.3-1475.70\end{array}$

$\begin{array}{llll}-0.05 & 1.96 & -7.10-1335.15 & -9.17\end{array}$

$0.431 .12-6.95-1346.54-12.49$

$0.881 .96-6.80-1356.06-13.93$

$1.191 .12-6.65-1367.18-16.98$

$1.471 .95-6.51-1376.44-18.17$

$1.601 .12-6.37-1387.32-20.98$

$1.691 .95-6.24-1396.36-21.95$

$1.661 .12-6.11-1406.98-24.50$

$1.601 .94-5.98-1415.78-25.22$

$1.431 .12-5.86-1426.14-27.51$

$1.271 .94 \quad-5.75-1434.65-27.95$

$0.991 .12-5.63-1444.73-29.96$

$0.761 .93-5.52-1452.94-30.10$

$0.471 .12-5.42-1462.65-31.74$

$0.191 .93-5.31-1470.55-31.56$

$\begin{array}{lllll}79 & 109 & 188 & -3.5 & -8.1-1483.87\end{array}$

$-0.141 .12-5.21-1479.93-32.88$

$\begin{array}{llllllllll}79 & 110 & 189 & -3.8 & -7.9-1491.86 & -0.79 & 1.12 & -5.02 & -1496.55 & -33.35\end{array}$

$\begin{array}{llllllllllll}79 & 111 & 190 & -4.0 & -7.7 & -1499.68 & -1.18 & 1.92 & -4.93-1503.87 & -32.60 & -3\end{array}$

$\begin{array}{lllllllllll}79 & 112 & 191 & -4.3 & -7.6-1507.32 & -1.67 & 1.12 & -4.84-1512.71 & -33.37 & -3\end{array}$

$\begin{array}{llllllllll}79 & 113 & 192 & -4.5 & -7.4-1514.80 & -2.15 & 1.91 & -4.75-1519.79 & -32.37\end{array}$

$\begin{array}{lllllllllll}79 & 114 & 193 & -4.8 & -7.2 & -1522.11 & -2.78 & 1.12 & -4.67-1528.44 & -32.96 & -31\end{array}$

$\begin{array}{lllllllllll}79 & 115 & 194 & -5.0 & -7.1 & -1529.26 & -3.35 & 1.91 & -4.59-1535.29 & -31.73 & -3\end{array}$

$\begin{array}{llllllllll}79 & 116 & 195 & -5.2 & -6.9-1536.25 & -4.08 & 1.12 & -4.51-1543.71 & -32.09 & -3\end{array}$

$\begin{array}{llllllllll}79 & 117 & 196 & -5.5 & -6.7-1543.08 & -4.79 & 1.90 & -4.43-1550.39 & -30.69 & -3\end{array}$

$\begin{array}{llllllllll}79 & 118 & 197 & -5.7 & -6.6-1549.75 & -5.56 & 1.12 & -4.35-1558.54 & -30.77 & -3\end{array}$

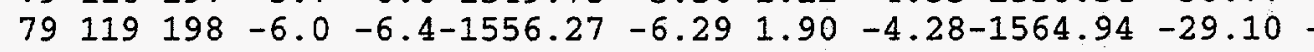

$79120199-6.2-6.3-1562.64-6.941 .12-4.21-1572.67-28.75$

$\begin{array}{llllllllll}79 & 121 & 200 & -6.4 & -6.1-1568.86 & -8.00 & 1.90 & -4.14-1579.10 & -27.11 & -2\end{array}$

$79 \begin{array}{llllllllll}122 & 201 & -6.7 & -6.0 & -1574.93 & -8.62 & 1.12 & -4.07-1586.50 & -26.44 & -2.26\end{array}$

$\begin{array}{llllllllll}79 & 123 & 202 & -6.9 & -5.9-1580.86 & -9.48 & 1.89 & -4.01-1592.45 & -24.32 & -2.2\end{array}$

$\begin{array}{llllllllll}79 & 124 & 203 & -7.1 & -5.7-1586.64-10.10 & 1.12 & -3.94-1599.56 & -23.36\end{array}$

$\begin{array}{llllllllll}79 & 125 & 204 & -7.4 & -5.6-1592.29-11.14 & 1.89 & -3.88-1605.42 & -21.15\end{array}$

$\begin{array}{lllllllll}79 & 126 & 205 & -7.6 & -5.4-1597.79-11.39 & 1.12 & -3.82-1611.88 & -19.54\end{array}$

$\begin{array}{llllllllll}79 & 127 & 206 & -7.8 & -5.3-1603.16-10.41 & 1.88 & -3.76-1615.45 & -15.03\end{array}$

$\begin{array}{llllllllll}79 & 128 & 207 & -8.0 & -5.2-1608.40 & -9.47 & 1.12 & -3.70-1620.45 & -11.97\end{array}$

$\begin{array}{lllllllll}79 & 129 & 208 & -8.3 & -5.0-1613.51 & -8.25 & 1.88 & -3.64-1623.52\end{array}$

$\begin{array}{lllllllll}79 & 130 & 209 & -8.5 & -4.9-1618.48 & -6.93 & 1.12 & -3.59-1627.88\end{array}$

$\begin{array}{lllllllll}79 & 131 & 210 & -8.7 & -4.8-1623.33 & -5.77 & 1.88 & -3.53-1630.76\end{array}$

$\begin{array}{lllllllll}79 & 132 & 211 & -8.9 & -4.7-1628.05 & -4.65 & 1.12 & -3.48-1635.07\end{array}$

$\begin{array}{lllllllll}79 & 133 & 212 & -9.1 & -4.5-1632.65 & -3.76 & 1.87 & -3.43-1637.97\end{array}$

$\begin{array}{lllllllll}79 & 134 & 213 & -9.4 & -4.4-1637.12 & -2.80 & 1.12 & -3.38-1642.19\end{array}$

$\begin{array}{lllllllll}79 & 135 & 214 & -9.6 & -4.3-1641.48 & -1.99 & 1.87 & -3.33-1644.93\end{array}$

$\begin{array}{lllllllll}79 & 136 & 215 & -9.8 & -4.2-1645.71 & -1.25 & 1.12 & -3.28-1649.13\end{array}$

$\begin{array}{lllllll}79 & 137 & 216-10.0 & -4.1-1649.83 & -0.65 & 1.87 & -3.24-1651.85\end{array}$

$79138217-10.2-3.9-1653.83-0.151 .12-3.19-1656.06$

$79139218-10.4-3.8-1657.72$

$0.191 .86-3.15-1658.82$ $7 \overline{6}$ \begin{tabular}{rrr}
0.00 & 0.00 & 0.00 \\
0.00 & 0.00 & 0.00 \\
0.00 & 0.00 & 0.00 \\
0.00 & 0.00 & 0.00 \\
0.00 & 0.00 & 0.00 \\
0.00 & 0.00 & 0.00 \\
0.00 & 0.00 & 0.00 \\
0.00 & 0.00 & 0.00 \\
0.00 & 0.00 & 0.00 \\
0.00 & 0.00 & 0.00 \\
0.00 & 0.00 & 0.00 \\
0.00 & 0.00 & 0.00 \\
0.00 & 0.00 & 0.00 \\
0.00 & 0.00 & 0.00 \\
0.00 & 0.00 & 0.00 \\
0.00 & 0.00 & 0.00 \\
0.00 & 0.00 & 0.00 \\
0.00 & 0.00 & 0.00 \\
0.00 & 0.00 & 0.00 \\
0.00 & 0.00 & 0.00 \\
0.00 & 0.00 & 0.00 \\
4.16 & 0.65 & -0.23 \\
17.16 & 1.01 & -0.18 \\
0.00 & 0.00 & 0.00 \\
0.00 & 0.00 & 0.00 \\
0.00 & 0.00 & 0.00 \\
0.00 & 0.00 & 0.00 \\
0.00 & 0.00 & 0.00 \\
0.00 & 0.00 & 0.00 \\
0.00 & 0.00 & 0.00 \\
0.00 & 0.00 & 0.00 \\
0.00 & 0.00 & 0.00 \\
0.00 & 0.00 & 0.00 \\
0.00 & 0.00 & 0.00 \\
0.00 & 0.00 & 0.00 \\
0.00 & 0.00 & 0.00 \\
0.00 & 0.00 & 0.00 \\
\hline 2.89 & -1.47 & -0.29
\end{tabular}

$\begin{array}{rrr}0.00 & 0.00 & 0.00 \\ 32.89 & -1.47 & -0.29\end{array}$

$-33.87-2.17-0.50$

$-32.78-2.56-0.41$

$-33.42-3.24-0.46$

$-32.29-3.91-0.56$

$-32.59-4.58-0.50$

$-31.17-5.27-0.48$

$-31.16-5.95-0.39$

$-29.60-6.79-0.50$

$-29.12-7.31-0.37$

$\begin{array}{lll}-27.28 & -8.17 & -0.17\end{array}$

$\begin{array}{lll}-26.41 & -8.59 & 0.03\end{array}$

$-24.42-9.58-0.10$

$\begin{array}{lll}-23.15 & -9.89 & 0.21\end{array}$

$\begin{array}{lll}0.00 & 0.00 & 0.00\end{array}$

$\begin{array}{llll}0.00 & 0.00 & 0.00\end{array}$

$\begin{array}{lll}0.00 & 0.00 & 0.00\end{array}$

$\begin{array}{llll}0.00 & 0.00 & 0.00\end{array}$

$\begin{array}{llll}0.00 & 0.00 & 0.00\end{array}$

$\begin{array}{llll}-6.96 & 0.00 & 0.00 & 0.00 \\ -3.25 & 0.00 & 0.00 & 0.00\end{array}$

$\begin{array}{lllll}1.94 & 0.00 & 0.00 & 0.00\end{array}$

$\begin{array}{llll}5.71 & 0.00 & 0.00 & 0.00\end{array}$

$\begin{array}{llll}10.88 & 0.00 & 0.00 & 0.00\end{array}$

$\begin{array}{llll}14.73 & 0.00 & 0.00 & 0.00\end{array}$

$\begin{array}{llll}20.06 & 0.00 & 0.00 & 0.00\end{array}$

$\begin{array}{lllll}23.93 & 0.00 & 0.00 & 0.00\end{array}$

$\begin{array}{llll}29.28 & 0.00 & 0.00 & 0.00\end{array}$

$\begin{array}{llll}33.14 & 0.00 & 0.00 & 0.00\end{array}$

$\begin{array}{llll}38.45 & 0.00 & 0.00 & 0.00\end{array}$ 
$79140 \quad 219-10.6-3.7-1661.50$ $\begin{array}{llll}79 & 141 & 220-10.8 & -3.6-1665.17\end{array}$ $\begin{array}{lllll}79 & 142 & 221-11.0 & -3.5-1668.72\end{array}$ $\begin{array}{llll}79 & 143 & 222-11.2 & -3.4-1672.17\end{array}$ $79 \begin{array}{llll}744 & 223-11.4 & -3.3-1675.52\end{array}$ $\begin{array}{lllll}79 & 145 & 224-11.6 & -3.2-1678.76\end{array}$ $79146225-11.8-3.1-1681.90$ $\begin{array}{llll}79 & 147 & 226-12.0 & -3.0-1684.94\end{array}$ $\begin{array}{llll}79 & 148 & 227-12.2 & -2.9-1687.88\end{array}$ $\begin{array}{lllll}79 & 149 & 228-12.4 & -2.8-1690.72\end{array}$ $\begin{array}{lllll}79 & 150 & 229-12.5 & -2.7-1693.46\end{array}$ $79 \quad 151 \quad 230-12.7 \quad-2.6-1696.11$ $\begin{array}{llll}79 & 152 & 231-12.9 & -2.5-1698.66\end{array}$ $79 \begin{array}{llll}153 & 232-13.1 & -2.4-1701.13\end{array}$ $79 \quad 154233-13.3-2.3-1703.50$ $\begin{array}{llll}79 & 155 & 234-13.5 & -2.2-1705.78\end{array}$ $\begin{array}{llll}79 & 156 & 235-13.6 & -2.1-1707.97\end{array}$ $79157236-13.8-2.1-1710.07$ $79 \quad 158 \quad 237-14.0-2.0-1712.10$ $7915.9238-14.2-1.9-1714.03$ $79 \quad 160 \quad 239-14.3-1.8-1715.88$ $79 \quad 161240-14.5-1.7-1717.65$ $79162 \quad 241-14.7-1.6-1719.34$ $79163242-14.8-1.6-1720.95$ $79164243-15.0-1.5-1722.48$ $79165244-15.2-1.4-1723.93$

$79166245-15.3-1.3-1725.31$

$79167246-15.5-1.3-1726.61$

$79168 \quad 247-15.6-1.2-1727.83$

$79169248-15.8-1.1-1728.99$

$79170249-16.0-1.0-1730.07$

$79 \begin{array}{llll}771 & 250-16.1 & -1.0-1731.08\end{array}$

$\begin{array}{llll}79 & 172 & 251-16.3 & -0.9-1732.02\end{array}$

$\begin{array}{llll}79 & 173 & 252-16.4 & -0.8-1732.90\end{array}$

$\begin{array}{llll}79 & 174 & 253-16.6 & -0.8-1733.70\end{array}$

$\begin{array}{lllll}79 & 175 & 254-16.7 & -0.7-1734.44 \\ 79 & 176 & 255-16.9 & -0.6-1735.12\end{array}$

$\begin{array}{lllll}79 & 176 & 255-16.9 & -0.6-1735.12 \\ 79 & 177 & 256-17.0 & -0.6-1735.73\end{array}$

$\begin{array}{llll}79 & 178 & 257-17.1 & -0.5-1736.28\end{array}$

$79 \begin{array}{llll}779 & 258-17.3 & -0.5-1736.76\end{array}$

$\begin{array}{llll}79 & 180 & 259-17.4 & -0.4-1737.19\end{array}$

$\begin{array}{llll}79 & 181 & 260-17.6 & -0.3-1737.55\end{array}$

$\begin{array}{llll}79 & 182 & 261-17.7 & -0.3-1737.86\end{array}$

$\begin{array}{llll}79 & 183 & 262-17.8 & -0.2-1738.11\end{array}$

$\begin{array}{llll}79 & 184 & 263-18.0 & -0.2-1738.30\end{array}$

$8090 \quad 170 \quad 2.2-12.3-1293.11$

$8091171 \quad 1.9-12.0-1305.28$

$80 \quad 92$ 172 $1.6-11.8-1317.20$

$\begin{array}{llll}80 & 93 & 173 & 1.3-11 \cdot 6-1328.90 \\ 80 & 94 & 174 & 1.0-11 \cdot 3-1340.36\end{array}$

$8095175 \quad 0.7-11.1-1351.60$

$8096 \quad 176 \quad 0.5-10.9-1362.62$

$80 \quad 97 \quad 177 \quad 0.2-10.7-1373.42$

$8098 \quad 178-0.1-10.5-1384.00$

$80 \quad 99 \quad 179-0.4-10.3-1394.37$

$80100180-0.6-10.1-1404.53$

$\begin{array}{llllll}80 & 101 & 181 & -0.9 & -9.9-1414.49\end{array}$

$\begin{array}{lllll}80 & 102 & 182 & -1.2 & -9.7-1424.25\end{array}$

$\begin{array}{llllll}80 & 103 & 183 & -1.4 & -9.5-1433.80\end{array}$

$80 \quad 104 \quad 184-1.7-9.3-1443.16$

$\begin{array}{llllll}80 & 105 & 185 & -2.0 & -9.1-1452.33\end{array}$

$80 \begin{array}{lllll}806 & 186 & -2.2 & -8.9-1461.31\end{array}$

$\begin{array}{llllll}80 & 107 & 187 & -2.5 & -8.7 & -1470.10\end{array}$

$\begin{array}{llllll}80 & 108 & 188 & -2.8 & -8.5 & -1478.70\end{array}$

$\begin{array}{llllll}80 & 109 & 189 & -3.0 & -8.3-1487.13\end{array}$

$80110 \quad 190-3.3-8.2-1495.37$
$0.51 \quad 1.12-3.10-1662.97$ $0.531 .86-3.06-1665.84$ $0.811 .12-3.02-1669.82$ $0.711 .86-2.98-1672.59$ $0.78 \quad 1.12-2.94-1676.56$ $0.551 .85-2.90-1679.26$ $0.621 .12-2.86-1683.03$ $0.311 .85-2.83-1685.60$ $0.311 .12-2.79-1689.24$ $\begin{array}{lll}-0.06 & 1.85 & -2.75-1691.68\end{array}$ $-0.081 .12-2.72-1695.14$ $-0.441 .84-2.69-1697.39$

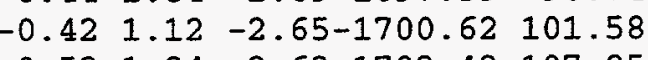
$-0.521 .84-2.62-1702.42107 .85$ $\begin{array}{llll}-0.40 & 1.12 & -2.59-1705.36 & 112.98\end{array}$ $\begin{array}{lllll}-0.67 & 1.84 & -2.56-1707.17 & 119.25\end{array}$ $-0.561 .12-2.53-1709.94 \quad 124.55$ $\begin{array}{lllll}-0.87 & 1.84 & -2.50-1711.60 & 130.95\end{array}$ $\begin{array}{llll}-1.01 & 1.12 & -2.47-1714.45 & 136.18\end{array}$ $\begin{array}{lllll}-1.31 & 1.83 & -2.44-1715.94 & 142.76\end{array}$ $\begin{array}{llll}-1.78 & 1.12 & -2.41-1718.95 & 147.82\end{array}$ $\begin{array}{lllll}-2.40 & 1.83 & -2.38-1720.60 & 154.24\end{array}$ $\begin{array}{lllll}-2.70 & 1.12 & -2.35-1723.27 & 159.64\end{array}$ $\begin{array}{lllll}-2.90 & 1.83 & -2.33-1724.35 & 166.64\end{array}$ $-2.401 .12-2.30-1726.06 \quad 173.00$

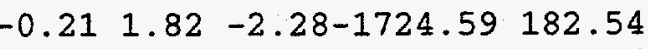
$\begin{array}{lllll}-0.50 & 1.12 & -2.25-1726.94 & 188.26\end{array}$ $\begin{array}{llll}-1.14 & 1.82 & -2.23-1728.15 & 195.12\end{array}$ $\begin{array}{llll}-1.88 & 1.12 & -2.20-1730.80 & 200.55\end{array}$ $\begin{array}{lllll}-2.33 & 1.82 & -2.18-1731.67 & 207.74\end{array}$ $\begin{array}{lllll}-2.77 & 1.12 & -2.15-1733.88 & 213.61\end{array}$ $\begin{array}{lllll}-4.76 & 1.82 & -2.13-1736.16 & 219.40\end{array}$ $\begin{array}{lllll}-5.08 & 1.12 & -2.11-1738.10 & 225.53\end{array}$ $\begin{array}{llllll}-5.79 & 1.81 & -2.09-1738.96 & 232.74\end{array}$ $\begin{array}{lllll}-5.04 & 1.12 & -2.07-1739.69 & 240.08\end{array}$ $\begin{array}{lllll}-5.80 & 1.81 & -2.04-1740.48 & 247.37\end{array}$ $\begin{array}{lllll}-7.16 & 1.12 & -2.02-1743.19 & 252.73\end{array}$ $\begin{array}{llll}-7.94 & 1.81 & -2.00-1743.86 & 260.12\end{array}$ $\begin{array}{lllll}-8.15 & 1.12 & -1.98-1745.29 & 266.76\end{array}$ $\begin{array}{llll}-8.17 & 1.81 & -1.96-1745.09 & 275.04\end{array}$ $\begin{array}{llll}-8.46 & 1.12 & -1.94-1746.47 & 281.73\end{array}$ $\begin{array}{lllll}-9.09 & 1.81 & -1.92-1746.76 & 289.51\end{array}$ $\begin{array}{lllll}-9.22 & 1.12 & -1.91-1747.87 & 296.48\end{array}$ $\begin{array}{lllll}-9.77 & 1.80 & -1.89-1747.96 & 304.45\end{array}$

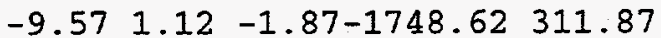
$\begin{array}{llll}-2.78 & 0.00 & -7.81-1303.70 & 5.34\end{array}$ $\begin{array}{llll}-1.90 & 1.07 & -7.63-1313.74 & 3.37\end{array}$ $\begin{array}{llll}-1.22 & 0.00 & -7.46-1325.88 & -0.70\end{array}$ $\begin{array}{lllll}-0.55 & 1.06 & -7.29-1335.68 & -2.42\end{array}$ $\begin{array}{llll}-0.02 & 0.00 & -7.13-1347.52 & -6.18\end{array}$ $0.451 .05-6.98-1357.08-7.68$ $0.78 \quad 0.00-6.83-1368.67-11.19$ $1.131 .04-6.68-1377.92-12.38$ $1.320 .00-6.54-1389.22-15.60$ $1.451 .04-6.40-1398.29-16.60$ $1.48 \quad 0.00-6.27-1409.32-19.57$ $1.461 .03-6.14-1418.14-20.31$ $1.320 .00-6.02-1428.95-23.04$ $1.191 .02-5.90-1437.49-23.51$ $0.910 .00-5.78-1448.03-25.99$ $0.671 .02-5.67-1456.31-26.19$ $0.420 .00-5.56-1466.44-28.26$ $0.121 .01-5.45-1474.42-28.16$ $\begin{array}{llllll}-0.25 & 0.00 & -5.35-1484.30 & -29.97\end{array}$ $\begin{array}{lllll}-0.55 & 1.00 & -5.25-1491.92 & -29.52\end{array}$ $-1.000 .00-5.15-1501.53-31.05$
0.00

0.00 0.00

0.00

0.00

0.00

0.00

0.00

0.00

0.00

0.00

0.00

0.00

0.00

0.00

0.00

0.00

0.00

$0.00^{\circ}$

0.00

0.00

0.00

0.00

0.00

0.00

0.00

0.00

0.00

0.00

0.00

0.00

0.00

0.00

0.00

0.00

0.00

0.00

0.00

0.00

0.00

0.00

0.00

0.00

0.00

0.00

0.00

0.00

0.00

0.00

0.00

0.00

0.00

0.00

0.00

0.00

0.00

0.00

0.00

0.00

0.00

0.00

0.00

0.00

0.00

0.00

0.00

0.00

0.00

0.00

0.00

0.00

0.00

0.00

0.00

0.00

0.00

0.00

0.00

0.00

0.00

0.00

0.00

0.00

0.00

0.00

0.00

0.00

0.00

0.00

0.00

0.00

0.00

0.00

0.00

0.00

0.00

0.00

0.00

0.00

0.00

0.00

0.00

0.00

0.00

0.00

0.00

0.00

0.00

0.00

0.00

0.00

0.09

0.00

0.60

0.00

0.00

0.00

0.00

0.00

0.00

0.00

0.00

0.00

0.00

0.00

0.00

0.00

0.00

0.00

0.00

0.00

0.00

0.00

0.00

0.00

0.00

0.00

0.00

0.00

0.00

0.00

0.00

0.00

0.00

0.00

0.00

0.00

0.00

0.00

0.00

0.00

0.00

0.00

0.00

0.00

0.00

0.00

0.00

0.00

0.00

0.00

0.00 
$\begin{array}{llllllllllllll}80 & 111 & 191 & -3.5 & -8.0 & -1503.44 & -1.42 & 1.00 & -5.06 & -1508.92 & -30.38 & -30.69 & -1.73 & -0.31\end{array}$

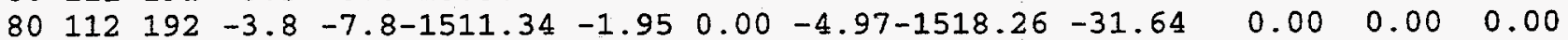

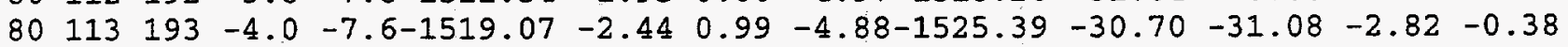
$\begin{array}{llllllllllll}80 & 114 & 194 & -4.3 & -7.5-1526.63 & -3.07 & 0.00 & -4.79-1534.49 & -31.73 & -32.25 & -3.59 & -0.52\end{array}$

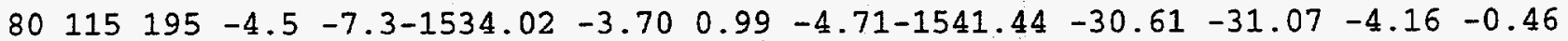

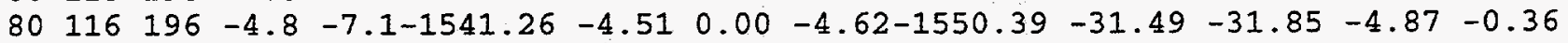
$\begin{array}{lllllllllllll}80 & 117 & 197 & -5.0 & -7.0 & -1548.33 & -5.12 & 0.98 & -4.54-1557.01 & -30.04 & -30.57 & -5.65 & -0.53\end{array}$

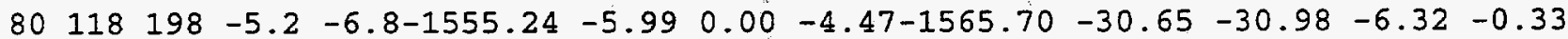
$\begin{array}{lllllllllllll}80 & 119 & 199 & -5.5 & -6.7-1562.00 & -6.75 & 0.98 & -4.39 & -1572.16 & -29.05 & -29.57 & -7.27 & -0.52\end{array}$ $\begin{array}{lllllllllllll}80 & 120 & 200 & -5.7 & -6.5-1568.61 & -7.52 & 0.00 & -4.32 & -1580.44 & -29.25 & -29.53 & -7.80 & -0.28\end{array}$ $\begin{array}{lllllllllllll}80 & 121 & 201 & -6.0 & -6.4-1575.06 & -8.37 & 0.97 & -4.25 & -1586.70 & -27.45 & -27.69 & -8.61 & -0.24\end{array}$

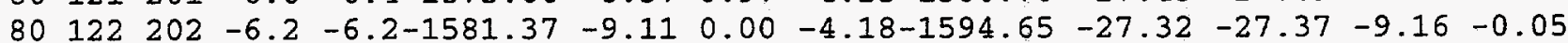
$\begin{array}{lllllllllll}80 & 123 & 203 & -6.4 & -6.1-1587.53-10.01 & 0.97 & -4.11-1600.68 & -25.28 & -25.29-10.02 & -0.01\end{array}$ $\begin{array}{llllllllll}80 & 124 & 204 & -6.7 & -5.9-1593.54-10.69 & 0.00 & -4.04-1608.27 & -24.80 & -24\end{array}$

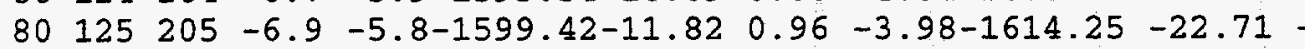
$80126 \quad 206-7.1-5.7-1605.15-12.000 .00-3.91-1621.07-21.45-26$ $\begin{array}{lllllllll}80 & 127 & 207 & -7.3 & -5.5-1610.75-11.13 & 0.95 & -3.85-1624.78 & -17.09\end{array}$ $\begin{array}{lllllllll}80 & 128 & 208 & -7.6 & -5.4-1616.21-10.13 & 0.00 & -3.79-1630.13 & -14.37\end{array}$ $\begin{array}{lllllllll}80 & 129 & 209 & -7.8 & -5.3-1621.54 & -8.91 & 0.95 & -3.74-1633.23 & -9.40\end{array}$ $\begin{array}{lllllllll}80 & 130 & 210 & -8.0 & -5.1-1626.73 & -7.60 & 0.00 & -3.68-1638.01\end{array}$ $\begin{array}{lllllllll}80 & 131 & 211 & -8.2 & -5.0-1631.80 & -6.44 & 0.95 & -3.62 & -1640.92\end{array}$ $\begin{array}{lllllllll}80 & 132 & 212 & -8.4 & -4.9-1636.74 & -5.31 & 0.00 & -3.57-1645.62\end{array}$ $\begin{array}{lllllllll}80 & 133 & 213 & -8.7 & -4.7-1641.55 & -4.29 & 0.94 & -3.52-1648.41\end{array}$ $\begin{array}{lllllllll}80 & 134 & 214 & -8.9 & -4.6-1646.24 & -3.37 & 0.00 & -3.47-1653.07\end{array}$ $\begin{array}{llllllll}80 & 135 & 215 & -9.1 & -4.5-1650.80^{\prime} & -2.35 & 0.94 & -3.41-1655.63\end{array}$ $\begin{array}{lllllllll}80 & 136 & 216 & -9.3 & -4.4-1655.25 & -1.49 & 0.00 & -3.37-1660.10\end{array}$ $\begin{array}{lllllllll}80 & 137 & 217 & -9.5 & -4.3-1659.57 & -0.68 & 0.93 & -3.32-1662.64\end{array}$ $\begin{array}{lllllllll}80 & 138 & 218 & -9.7 & -4.1-1663.78 & -0.08 & 0.00 & -3.27-1667.13\end{array}$ $\begin{array}{lllllllll}80 & 139 & 219 & -9.9 & -4.0-1667.88 & 0.24 & 0.93 & -3.23-1669.94\end{array}$ $80 \quad 140 \quad 220-10.1 \quad-3.9-1671.86$ $80141221-10.3-3.8-1675.73$ $0.58 \quad 0.00-3.18-1674.46$ $\begin{array}{llll}0.53 & 0.92 & -3.14-1677.41\end{array}$ $80 \quad 142 \quad 222-10.5 \quad-3.7-1679.48$ $\begin{array}{llll}80 & 143 & 223-10.7 & -3.6-1683.13\end{array}$ $\begin{array}{lllll}80 & 144 & 224-10.9 & -3.5-1686.68\end{array}$ $80 \quad 145 \quad 225-11.1-3.4-1690.11$ $80146 \quad 226-11.3 \quad-3.3-1693.45$ $\begin{array}{llll}80 & 147 & 227-11.5 & -3.2-1696.68\end{array}$ $\begin{array}{lllll}80 & 148 & 228-11.7 & -3.1-1699.81\end{array}$ $\begin{array}{lllll}80 & 149 & 229-11.9 & -3.0-1702.84\end{array}$ $\begin{array}{llll}0.85 & 0.00 & -3.09-1681.73\end{array}$ $\begin{array}{llll}0.78 & 0.92 & -3.05-1684.49\end{array}$ $0.88 \quad 0.00-3.01-1688.81$ $\begin{array}{llll}0.65 & 0.91 & -2.97-1691.52\end{array}$ $0.760 .00-2.93-1695.62$ $0.45 \quad 0.91-2.89-1698.21$ $0.48 \quad 0.00-2.86-1702.18$ $-6.11$ $-0.94$ 2.43 7.70 11.12 16.63 20.23 25.76 29.34 34.61 38.16 43.28 47.03 52.34 56.09 61.45 65.43 70.90 $80 \quad 150 \quad 230-12.1 \quad-2.9-1705.77$ $0.13 \quad 0.91-2.82-1704.62$ 80.64 $\begin{array}{llll}0.17 & 0.00 & -2.79-1708.38 & 84.95\end{array}$ $\begin{array}{llllllll}80 & 151 & 231-12.3 & -2.8-1708.60 & -0.17 & 0.90 & -2.75-1710.62 & 90.78\end{array}$ $\begin{array}{llllllll}80 & 152 & 232-12.4 & -2.7-1711.34 & -0.11 & 0.00 & -2.7 .2-1714.17 & 95.31\end{array}$ $\begin{array}{llllllll}80 & 153 & 233-12.6 & -2.6-1713.98 & -0.19 & 0.90 & -2.68-1715.96 & 101.59\end{array}$ $80 \quad 154 \quad 234-12.8 \quad-2.5-1716.54 \quad-0.02 \quad 0.00-2.65-1719.21 \quad 106.41$

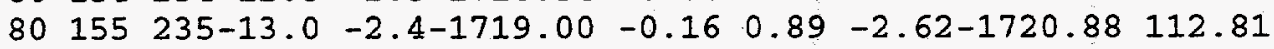
$\begin{array}{lllllllll}80 & 156 & 236-13.2 & -2.3-1721.37 & -0.13 & 0.00 & -2.59-1724.08 & 117.68\end{array}$ $\begin{array}{llllllll}80 & 157 & 237-13.3 & -2.2-1723.65 & -0.42 & 0.89 & -2.55-1725.74 & 124.10\end{array}$ $80158 \quad 238-13.5 \quad-2.1-1725.84 \quad-0.51 \quad 0.00-2.52-1728.88 \quad 129.02$ $\begin{array}{llllllll}80 & 159 & 239-13.7 & -2.1-1727.95 & -0.94 & 0.89 & -2.50-1730.50 & 135.47\end{array}$ $\begin{array}{llllllll}80 & 160 & 240-13.9 & -2.0-1729.98 & -1.27 & 0.00 & -2.47-1733.71 & 140.33\end{array}$ $80 \quad 161 \quad 241-14.0 \quad-1.9-1731.92 \quad-1.80 \quad 0.88 \quad-2.44-1735.27 \quad 146.85$ $\begin{array}{lllllllll}80 & 162 & 242-14.2 & -1.8 & -1733.77 & -1.99 & 0.00 & -2.41-1738.17 & 152.02\end{array}$

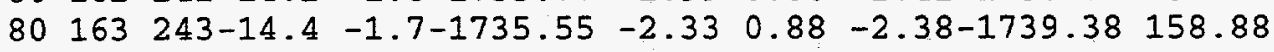
$\begin{array}{llllllll}80 & 164 & 244-14.5 & -1.7-1737.24 & -0.13 & 0.00 & -2.36-1739.73 & 166.60\end{array}$ $\begin{array}{llllllll}80 & 165 & 245-14.7 & -1.6-1738.86 & -0.63 & 0.88 & -2.33-1740.94 & 173.46\end{array}$ $80166 \quad 246-14.9-1.5-1740.40-1.24 \quad 0.00-2.30-1743.94 \quad 178.53$ $\begin{array}{lllllllll}80 & 167 & 247-15.0 & -1.4-1741.86 & -2.12 & 0.87 & -2.28 & -1745.39 & 185.16\end{array}$ $\begin{array}{llllllll}80 & 168 & 248-15.2 & -1.3-1743.25 & -2.86 & 0.00 & -2.25-1748.36 & 190.25\end{array}$ $80 \quad 169 \quad 249-15.3 \quad-1.3-1744.56 \quad-3.72 \quad 0.87 \quad-2.23-1749.64 \quad 197.04$ $80170 \quad 250-15.5 \quad-1.2-1745.80-4.13 \quad 0.00-2.20-1752.14 \quad 202.62$ $80171 \quad 251-15.7 \quad-1.1-1746.97 \quad-4.83 \quad 0.86-2.18-1753.11209 .72$

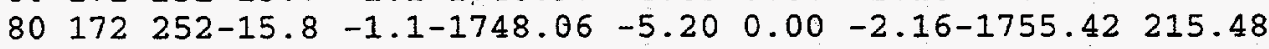
$\begin{array}{lllllllll}80 & 173 & 253-16.0 & -1.0 & -1749.09 & -5.88 & 0.86 & -2.14-1756.24 & 222.73\end{array}$ $\begin{array}{lllllllll}80 & 174 & 254-16.1 & -0.9-1750.05 & -6.14 & 0.00 & -2.11-1758.30 & 228.74\end{array}$ $80 \quad 175 \quad 255-16.3 \quad-0.9-1750.94 \quad-7.00 \quad 0.86-2.09-1759.17 \quad 235.95$ $\begin{array}{llllllll}80 & 176 & 256-16.4 & -0.8-1751.76 & -7.35 & 0.00 & -2.07-1761.18 & 242.01\end{array}$

$-24.72-10.61$

$-22.31-11.42$

$-20.97-11.52$

$-16.27-10.31$

$0.00 \quad 0.00$

0.00

0.00

0.00

0.000 .00

0.00

0.00

0.00

0.00

0.00

0.00

0.00

0.00

0.00

0.00

0.00

0.00

0.00

0.00

0.00

0.00

0.00

0.00

0.00

0.00

0.00

0.00

0.00

0.00

0.00

0.00

0.00

0.00

0.00

0.00

0.00

0.00

0.00

0.00

0.00

0.00

0.00

0.00

0.00

0.00

0.00

0.00

0.00

0.00

0.00

0.00

0.00

0.00

0.00

0.00

0.00

0.00

0.00

0.00

0.00

0.00

0.00

0.00

0.00

0.00

0.00

0.00

0.00

0.00

0.00

0.00

0.00

0.00

0.00

0.00

0.00

0.00

0.00

0.00

0.00

0.00

0.00

0.00

0.00

0.00

0.00

0.00

0.00

0.00

0.00

0.00

0.00

0.00

0.00

0.00
0.08

0.40

0.48

0.82

0.00

0.00

0.00

0.00

0.00

0.00

0.00

0.00

0.00

0.00

0.00

0.00

0.00

0.00

0.00

0.00

0.00

0.00

0.00

0.00

0.00

0.00

0.00

0.00

0.00

0.00

0.00

0.00

0.00

0.00

0.00

0.00

0.00

0.00

0.00

0.00

0.00

0.00

0.00

0.00

0.00

0.00

0.00

0.00

0.00

0.00

0.00

0.00

0.00 
$\begin{array}{llllllllll}80 & 177 & 257-16.6 & -0.7-1752.52 & -8.06 & 0.85 & -2.05-1761.77 & 249.49\end{array}$

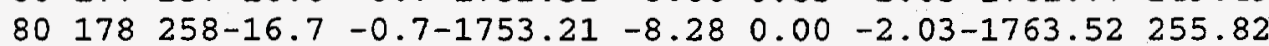
$\begin{array}{llllllll}80 & 179 & 259-16.9 & -0.6-1753.83 & -8.46 & 0.85 & -2.01-1763.45 & 263.95\end{array}$ $80180 \quad 260-17.0 \quad-0.5-1754.40-8.72 \quad 0.00-1.99-1765.11270 .37$

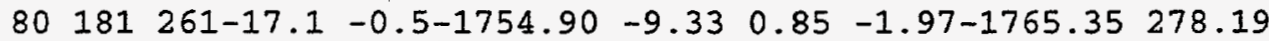
$\begin{array}{lllllllll}80 & 182 & 262-17.3 & -0.4-1755.35 & -9.48 & 0.00 & -1.95-1766.78 & 284.84\end{array}$ $80183 \quad 263-17.4-0.4-1755.73-10.07 \quad 0.85-1.93-1766.88 \quad 292.80$ $80 \quad 184 \quad 264-17.5 \quad-0.3-1756.05 \quad-9.86 \quad 0.00-1.91-1767.83 \quad 299.93$ $80 \quad 185 \quad 265-17.7-0.2-1756.32-9.07 \quad 0.84-1.89-1766.44 \quad 309.39$ $80186266-17.8-0.2-1756.53-8.34 \quad 0.00-1.88-1766.75 \quad 317.15$ $\begin{array}{llllllll}81 & 92 & 173 & 2.1-12.1-1315.38 & -1.98 & 1.11 & -7.66-1323.91 & 8.55\end{array}$ $8193 \quad 174 \quad 1.8-11.9-1327.36-1.19 \quad 1.96-7.49-1334.08$

8194175

8195176

$8196 \quad 177$

8197178

$1.5-11.6-1339.11-0.641 .11-7.32-1345.96$ $1.2-11.4-1350.63$

$-0.031 .95-7.16-1355.87$

$0.9-11.2-1361.92$

$0.7-11.0-1373.00$

$0.331 .11-7.01-1367.49$

$0.701 .95-6.86-1377.21$

$0.881 .11-6.71-1388.58$

$1.131 .94-6.57-1398.00$

$1.111 .11-6.43-1409.15$

$1.221 .93-6.30-1418.31$

$1.061 .11-6.18-1429.19$

$1.041 .93-6.05-1438.09$

$0.811 .11-5.93-1448.65$

$0.601 .92-5.82-1457.36$

$0.311 .11-5.70-1467.59$

$0.001 .92-5.59-1476.03$

6.45

2.65

0.81

$-2.74$

$-4.39$

$-7.69$

$-9.04$

$-12.12$

$-13.21$

$-16.02$

$81103 \quad 184-1.0 \quad-9.7-1435.01$

$81104 \quad 185-1.2-9.5-1444.64$

$81105186-1.5 \quad-9.3-1454.07$

$81 \quad 106187-1.8-9.1-1463.30$

$81107188-2.0-9.0-1472.35$

$81108 \quad 189-2.3-8.8-1481.22$

$-0.401 .11-5.49-1486.00$

$81109190-2.5-8.6-1489.90$

$\begin{array}{llllll}81 & 110 & 191 & -2.8 & -8.4-1498.40\end{array}$

$81111192-3.0 \quad-8.2-1506.73$

$\begin{array}{llllll}81 & 112 & 193 & -3.3 & -8.1-1514.88 \\ 81 & 113 & 194 & -3.5 & -7.9-1522.85\end{array}$

$\begin{array}{llllll}81 & 113 & 194 & -3.5 & -7.9-1522.85 \\ 81 & 114 & 195 & -3.8 & -7.7-1530.66\end{array}$

$-0.791 .91-5.39-1494.16$

$-1.321 .11-5.29-1503.90$

$-1.791 .91-5.19-1511.79$

$-2.431 .11-5.09-1521.29$

$\begin{array}{llllll}81 & 114 & 195 & -3.8 & -7.7-1530.66 \\ 81 & 115 & 196 & -4.0 & -7.6-1538.30\end{array}$

$-2.911 .91-5.00-1528.86$

$81116 \quad 197-4.3-7.4-1545.78$

$-3.721 .11-4.91-1538.18$

$\begin{array}{llllll}81 & 117 & 198 & -4.5 & -7.2-1553.09\end{array}$

$\begin{array}{lllll}81 & 118 & 199 & -4.8 & -7.1-1560.25\end{array}$

$-5.131 .11-4.74-1554.54$

$81119200-5.0-6.9-1567.24$

$-5.871 .90-4.66-1561.72$

$81120201-5.2-6.8-1574.09-8.27 \quad 1.11-4.43-1585.67$

$\begin{array}{lllllllll}81 & 121 & 202 & -5.5 & -6.6 & -1580.78 & -9.25 & 1.89 & -4.35-1592.49\end{array}$

$81122 \quad 203-5.7-6.5-1587.32-9.97 \quad 1.11-4.28-1600.46$

$81123204-5.9-6.3-1593.71-10.83 \quad 1.88-4.21-1606.87$

$81124205-6.2-6.2-1599.96-11.581 .11-4.14-1614.57$

$81125206-6.4-6.0-1606.06-12.581 .88-4.08-1620.84$

$81126207-6.6-5.9-1612.02-12.82 \quad 1.11-4.01-1627.75$

$81127208-6.9-5.7-1617.84-11.891 .88-3.95-1631.81$

$81128 \quad 209-7.1-5.6-1623.53-10.891 .11-3.89-1637.20$

$\begin{array}{lllllllll}81 & 129 & 210 & -7.3 & -5.5-1629.08 & -9.60 & 1.87 & -3.83-1640.63\end{array}$

$\begin{array}{lllllllll}81 & 130 & 211 & -7.5 & -5.3-1634.50 & -8.40 & 1.11 & -3.77-1645.56\end{array}$

$\begin{array}{lllllllll}81 & 131 & 212 & -7.7 & -5.2 & -1639.78 & -7.34 & 1.87 & -3.71-1648.96\end{array}$

$81 \quad 132213-8.0 \quad-5.1-1644.94-6.16 \quad 1.11-3.66-1653.64$

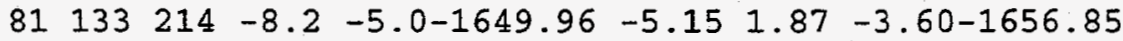

$\begin{array}{lllllllll}81 & 134 & 215 & -8.4 & -4.8-1654.86 & -4.12 & 1.11 & -3.55-1661.43\end{array}$

$81135216-8.6 \quad-4.7-1659.64-3.121 .86-3.50-1664.40$

$\begin{array}{lllllllll}81 & 136 & 217 & -8.8 & -4.6-1664.30 & -2.33 & 1.11 & -3.45-1668.97\end{array}$

$\begin{array}{lllllllll}81 & 137 & 218 & -9.0 & -4.5-1668.83 & -1.88 & 1.86 & -3.40-1672.26\end{array}$

$\begin{array}{lllllllll}81 & 138 & 219 & -9.2 & -4.4-1673.25 & -1.17 & 1.11 & -3.35-1676.66\end{array}$

$\begin{array}{llllll}81 & 139 & 220 & -9.4 & -4.2-1677.55\end{array}$

$\begin{array}{llllll}81 & 140 & 221 & -9.6 & -4.1-1681.74\end{array}$

$81 \quad 141222-9.8-4.0-1685.81$

$81 \quad 142223-10.0-3.9-1689.77$

$81 \quad 143 \quad 224-10.2-3.8-1693.62$

$81144225-10.4-3.7-1697.36$

$81145 \quad 226-10.6-3.6-1700.99$

$81146 \quad 227-10.8 \quad-3.5-1704.52$

$81147228-11.0-3.4-1707.94$

$-0.471 .85-3.30-1679.47$

$0.231 .11-3.26-1683.66$

$0.251 .85-3.21-1686.92$

$0.601 .11-3.17-1691.23$

$0.571 .85-3.13-1694.32$

$0.701 .11-3.09-1698.63$

$0.571 .85-3.04-1701.62$

$0.701 .11-3.00-1705.71$

$0.401 .84-2.96-1708.66$

79

$-19.85$

$-19.97$

$-22.13$

$-22.50$

$-24.39$

$-24.49$

$-26.15$

$-25.98$

-27.40
-26.90

$-28.15$

$-27.50$

$-28.36$

-27.48 .

$-28.04$

$-26.95$

$-27.21$

$-25.96$

$-25.86$

$-24.19$

$-23.82$

$-22.02$

$-20.86$

$-16.85$

-14.17
-9.53

$-6.38$

$-1.72$

1.67

6.54

10.04

15.13

18.64

23.42

27.08

32.35

36.23

41.04

44.81

49.78

53.54

58.63

62.61

67.73

0.00

0.00

0.00

0.00

0.00

0.00

0.00

0.00

0.00

0.00

0.00

0.00

0.00

0.00

0.00

0.00

0.00

$\begin{array}{llll}0.00 & 0.00 & 0.00\end{array}$

$\begin{array}{llll}0.00 & 0.00 & 0.00\end{array}$

0.00

0.00

0.00

0.00

0.00

0.00

$0.00 \quad 0.00 \quad 0.00$

0.00

0.00

0.00

0.00

0.00

0.00

0.00

0.00

$\begin{array}{llll}0.00 & 0.00 & 0.00\end{array}$

$0.00 \quad 0.00 \quad 0.00$

$-7.97$

0.60

$-0.28$

0.00

0.00

0.00

0.00

0.00

0.00

0.00

0.00

0.00

0.00

0.00

0.00

0.00

0.00

0.00

$0.00 \quad 0.00 \quad 0.00$

$0.00 \quad 0.00 \quad 0.00$

0.00

0.00

$0.00 \quad 0.00$

0.00

0.00

0.00

0.00

0.00

0.00

0.00

$-27.44$

0.00

0.00

$-2.47$

0.00

$-28.27$

3.84

$-0.04$

0.00

0.00

0.00

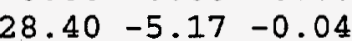

$-27.52-5.91-0.04$

$-28.14-6.74-0.10$

$-27.07-7.61-0.12$

$\begin{array}{llll}-27.20 & -8.26 & 0.01\end{array}$

$-26.00-9.29-0.04$

$\begin{array}{lll}-25.78 & -9.89 & 0.08\end{array}$

$-24.37-11.01-0.18$

$-23.84-11.60-0.02$

$-22.28-12.84-0.26$

$-21.05-13.01-0.19$

$\begin{array}{lll}-16.77-11.81 & 0.08\end{array}$

$-13.65-10.37$

0.52

0.27

0.00

0.00

0.00

0.00

0.00

0.00

0.00

0.00

0.00

0.00

0.00

0.00

0.00

0.00

0.00

0.00

0.00

0.00

0.00

0.00

0.00

0.00

0.00

0.00

0.00

0.00

0.00

0.00

0.00

0.00

0.00

0.00

0.00

0.00

0.00

0.00

0.00

0.00

0.00

0.00

0.00

0.00

0.00

0.00

0.00

0.00

0.00

0.00 
$81 \quad 148 \quad 229-11.2-3.3-1711.26$

$81 \quad 149230-11.4-3.2-1714: 48$

$81 \quad 150 \quad 231-11.6 \quad-3.1-1717.60$

$81151232-11.8-3.0-1720.62$

$81 \quad 152 \quad 233-12.0-2.9-1723.54$

$81 \quad 153 \quad 234-12.2-2.8-1726.37$

$81 \quad 154 \quad 235-12.3-2.7-1729.11$

$81155236-12.5-2.6-1731.75$

$81156 \quad 237-12.7 \quad-2.5-1734.30$

$81157 \quad 238-12.9-2.4-1736.76$

$81158239-13.1-2.3-1739.13$

$81159240-13.2 \quad-2.2-1741.41$

$81 \quad 160 \quad 241-13.4 \quad-2.1-1743.61$

$81 \quad 161242-13.6-2.1-1745.72$

$81162 \quad 243-13.7-2.0-1747.75$

$81163244-13.9-1.9-1749.69$

$81164245-14.1-1.8-1751.56$

$.691 .11-2.41-1753.55160 .06$

$81166247-14.4-1.7-1755.04-111.82-2.38-1755.02166 .66$

$81167248-14.6-1.6-1756.67-2.10 \quad 1.81-2.33-1759.28178 .54$

$81168 \quad 249-14.7-1.5-1758.21-2.58 \quad 1.11-2.31-1761.99183 .90$

$81169250-14.9-1.4-1759.69-3.15 \quad 1.81-2.28-1763.31190 .66$

$81170 \quad 251-15.1-1.4-1761.08-3.51 \quad 1.11-2.26-1765.74196 .29$

$81171252-15.2-1.3-1762.41-4.12 \quad 1.81-2.23-1766.95 \quad 203.15$

$81172253-15.4-1.2-1763.66-4.62 \quad 1.11-2.21-1769.38208 .80$

$\begin{array}{lllllllll}81 & 173 & 254-15.5 & -1.1-1764.84 & -5.24 & 1.81 & -2.18-1770.46 & 215.79\end{array}$

$81174 \quad 255-15.7-1.1-1765.95-5.64 \quad 1.11-2.16-1772.64 \quad 221.68$

$81175256-15.8-1.0-1766.99-6.40 \quad 1.80-2.14-1773.72 \quad 228.67$

$81176 \quad 257-16.0 \quad-0.9-1767.96-7.24 \quad 1.11-2.12-1776.21234 .25$

$81177258-16.1-0.9-1768.86-7.78 \quad 1.80-2.10-1776.94241 .60$

$81178 \quad 259-16.3 \quad-0.8-1769.70-8.11 \quad 1.11-2.07-1778.78 \quad 247.83$

$81 \quad 179260-16.4-0.7-1770.47-8.94 \quad 1.80-2.05-1779.67 \quad 255.01$

$81180261-16.6-0.7-1771.18-9.20 \quad 1.11-2.03-1781.30 \quad 261.44$

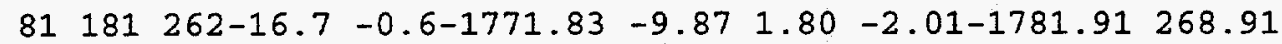

$81182263-16.8-0.5-1772.41-10.051 .11-1.99-1783.34275 .55$

$81 \quad 183264-17.0-0.5-1772.93-10.52 \quad 1.79-1.97-1783.63 \quad 283.33$

$81184265-17.1-0.4-1773.39-10.301 .11-1.95-1784.54290 .50$

$81185266-17.3-0.4-1773.79-9.49 \quad 1.79-1.94-1783.43 \quad 299.68$

$81186 \quad 267-17.4 \quad-0.3-1774.14 \quad-8.77 \quad 1.11-1.92-1783.72 \quad 307.46$

$\begin{array}{llllllllll}81 & 187 & 268-17.5 & -0.3-1774.42 & -7.70 & 1.79 & -1.90-1782.23 & 317.01\end{array}$

$\begin{array}{lllllllll}81 & 188 & 269-17.7 & -0.2-1774.65 & -6.76 & 1.11 & -1.88-1782.19 & 325.13\end{array}$

$\begin{array}{lllllllll}82 & 93 & 175 & 2.2-12.1-1325.36 & -1.50 & 1.06 & -7.68-1333.48 & 14.33\end{array}$

$2.0-11.9-1337.39-0.96 \quad 0.00-7.51-1345.85 \quad 10.02$

$\begin{array}{llllllll}82 & 95 & 177 & 1.7-11.7-1349.18 & -0.44 & 1.05 & -7.35-1355.92\end{array}$

$8296 \quad 178 \quad 1.4-11.5-1360.76-0.07 \quad 0.00-7.19-1368.01$

8297179 1.1-11.2-1372.11

$8298180 \quad 0.9-11.0-1383.24$

$8299181 \quad 0.6-10.8-1394.16$

$82100182 \quad 0.3-10.6-1404.87$

$82101183 \quad 0.0-10.4-1415.36$

$82102184-0.2-10.2-1425.65$

$82103185-0.5-10.0-1435.74$

$82.104186-0.8-9.8-1445.63$

$82105187-1.0 \quad-9.6-1455.32$

$82106 \quad 188-1.3 \quad-9.4-1464.82$

$0.341 .04-7.03-1377.76$

10.02

8.03

4.01

$0.48 \quad 0.00-6.88-1389.65$

2.34

$0.731 .04-6.74-1399.13$

$0.750 .00-6.60-1410.72$

$0.841 .03-6.47-1419.96$

$0.800 .00-6.33-1431.19-10$.

$.800 .00-6.33-1431.19-10.74$

$0.610 .00-6.08-1451.11-14.51$

$0.561 .02-5.97-1459.71-15.05$

$\begin{array}{lllll}0.21 & 0.00 & -5.85-1470.46 & -17.73\end{array}$

$82107189-1.5 \quad-9.2-1474.13-0.02 \quad 1.01-5.74-1478.88-18.07$

$\begin{array}{llllllllll}82 & 108 & 190 & -1.8 & -9.0-1483.26 & -0.43 & 0.00 & -5.63-1489.32 & -20.44\end{array}$

$\begin{array}{llllllllll}82 & 109 & 191 & -2.1 & -8.8-1492.19 & -0.70 & 1.00 & -5.52-1497.41 & -20.46\end{array}$

$\begin{array}{llllllllll}82 & 110 & 192 & -2.3 & -8.7-1500.95 & -1.33 & 0.00 & -5.42-1507.70 & -22.68\end{array}$

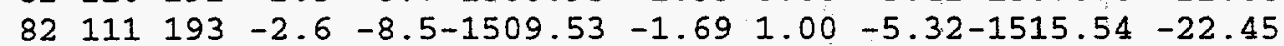

$\begin{array}{llllllllll}82 & 112 & 194 & -2.8 & -8.3-1517.93 & -2.42 & 0.00 & -5.22-1525.57 & -24.41\end{array}$

$\begin{array}{llllllllll}82 & 113 & 195 & -3.1 & -8.1-1526.15 & -2.88 & 0.99 & -5.13-1533.17 & -23.93\end{array}$

$\begin{array}{llllllllll}82 & 114 & 196 & -3.3 & -8.0-1534.21 & -3.68 & 0.00 & -5.04-1542.93 & -25.62\end{array}$

$\begin{array}{llllllllll}82 & 115 & 197 & -3.6 & -7.8-1542.10 & -4.26 & 0.99 & -4.95-1550.32 & -24.94\end{array}$

$\begin{array}{llllllllll}82 & 116 & 198 & -3.8 & -7.6-1549.82 & -5.08 & 0.00 & -4.86-1559.76 & -26.31\end{array}$

82

82
0.00

0.00

0.00

0.00

0.00

0.00

0.00

0.00

0.00

0.00

0.00

0.00

0.00

0.00

0.00

0.00

0.00

0.00

0.00

0.00

0.00

0.00

0.00

0.00

0.00

0.00

0.00

0.00

0.00

0.00

0.00

0.00

0.00

0.00

0.00

0.00

0.00

0.00

0.00

0.00

0.00

0.00

0.00

0.00

0.00

0.00

0.00

0.00

0.00

0.00

0.00

0.00

0.00

0.00

0.00

0.00

0.00

0.00

0.00

0.00

0.00

0.00

0.00

0.00

0.00

0.00

0.00

0.00

0.00

0.00

0.00

0.00

0.00

0.00

0.00

0.00

0.00

0.00

0.00

0.00

0.00

0.00

0.00

0.00

0.00

0.00

0.00

0.00

0.00

0.00

0.00

0.00

0.00

0.00

0.00

0.00

0.00

0.00

0.00

0.00

0.00

0.00

0.00

0.00

0.00

0.00

0.00

0.00

0.00

0.00

0.00

0.00

0.00

0.00

0.00

0.00

0.00

0.00

0.00

0.00

0.00

0.00

0.00

0.00

0.00

0.00

0.00

0.00

.00

0.00

0.00

0.00

0.00

$-6.82$

0.00

0.00

0.00

0.34

0.00

0.00

0.00

$-0.41$

0.00

0.00

0.00

0.00

0.00

0.00

0.00

0.00

0.00

0.00

0.00

0.00

0.00

0.00

0.00

0.00

0.00

0.00

0.00

0.00

0.00

0.00

0.00

0.00

0.00

0.00

0.00

0.00

0.00

0.00

0.00

0.00

0.00

0.00

0.00

0.00

0.00

0.00

0.00

0.00

$-25.27-5.62$

0.00

0.13 
$\begin{array}{llllllllllll}82 & 118 & 200 & -4.3 & -7.3-1564.77 & -6.59 & 0.00 & -4.70-1576.05 & -26.46 & 0.00 & 0.00\end{array}$

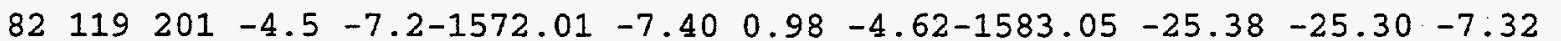

$\begin{array}{llllllllllll}82 & 120 & 202 & -4.8 & -7.0 & -1579.09 & -8.22 & 0.00 & -4.54-1591.84 & -26.11 & -25.96 & -8.07\end{array}$

$\begin{array}{llllllllllll}82 & 121 & 203 & -5.0 & -6.8 & -1586.01 & -9.13 & 0.97 & -4.46-1598.63 & -24.83 & -24.81 & -9.11\end{array}$

$\begin{array}{lllllllllll}82 & 122 & 204 & -5.2 & -6.7-1592.79-10.02 & 0.00 & -4.39-1607.20 & -25.32 & -25.13 & -9.83\end{array}$

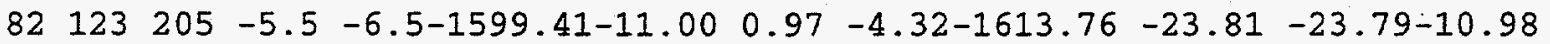

$\begin{array}{llllllllll}82 & 124 & 206 & -5.7 & -6.4-1605.89-11.82 & 0.00 & -4.25-1621.96 & -23.94 & -23.81-11.69\end{array}$

$\begin{array}{llllllllll}82 & 125 & 207 & -5.9 & -6.3-1612.22-12.68 & 0.96 & -4.18-1628.12 & -22.03 & -22.48-13.13 & -0\end{array}$

$-21.03-21.48-13.13-0.45$

$\begin{array}{lllllllllll}82 & 126 & 208 & -6.1 & -6.1-1618.41-12.84 & 0.00 & -4.11-1635.36 & -21.20 & -21.77-13.41 & -0.57 \\ 82 & 127 & 209 & -6.4 & -6.0-1624.46-11.96 & 0.95 & -4.05-1639.51 & -17.28 & -17.64-12.32 & -0.36\end{array}$

$\begin{array}{llllllllll}82 & 128 & 210 & -6.6 & -5.8-1630.37-10.92 & 0.00 & -3.99-1645.27 & -14.97 & -14.75-10.70 & 0.22\end{array}$

$\begin{array}{lllllllll}82 & 129 & 211 & -6.8 & -5.7-1636.14 & -9.65 & 0.95 & -3.92-1648.76\end{array}$

$\begin{array}{lllllllll}82 & 130 & 212 & -7.0 & -5.6-1641.78 & -8.50 & 0.00 & -3.86-1654.14\end{array}$

$\begin{array}{lllllllll}82 & 131 & 213 & -7.3 & -5.4-1647.28 & -7.26 & 0.95 & -3.81-1657.40\end{array}$

$\begin{array}{lllllllll}82 & 132 & 214 & -7.5 & -5.3-1652.65 & -6.22 & 0.00 & -3.75-1662.62\end{array}$

$\begin{array}{lllllllll}82 & 133 & 215 & -7.7 & -5.2 & -1657.90 & -5.23 & 0.94 & -3.69-1665.88\end{array}$

$\begin{array}{lllllllll}82 & 134 & 216 & -7.9 & -5.0 & -1663.01 & -4.36 & 0.00 & -3.64-1671.01\end{array}$

$\begin{array}{lllllllll}82 & 135 & 217 & -8.1 & -4.9-1668.00 & -3.59 & 0.94 & -3.59-1674.24\end{array}$

$\begin{array}{lllllllll}82 & 136 & 218 & -8.3 & -4.8-1672.87 & -2.80 & 0.00 & -3.53-1679.20\end{array}$

$\begin{array}{llllllll}82 & 137 & 219 & -8.5 & -4.7-1677.62 & -2.09 & 0.93 & -3.48-1682.26\end{array}$

$\begin{array}{lllllllll}82 & 138 & 220 & -8.7 & -4.6-1682.24 & -1.36 & 0.00 & -3.43-1687.03\end{array}$

$\begin{array}{lllllllll}82 & 139 & 221 & -9.0 & -4.4-1686.75 & -0.05 & 0.93 & -3.38-1689.25\end{array}$

$82 \quad 140222-9.2-4.3-1691.14$

$\begin{array}{lllll}82 & 141 & 223 & -9.4 & -4.2-1695.41\end{array}$

$0.370 .00-3.34-1694.10$

$\begin{array}{llllll}82 & 142 & 224 & -9.6 & -4.1-1699.57\end{array}$

$0.38 \quad 0.92-3.29-1697.40$

$\begin{array}{lllll}82 & 143 & 225 & -9.8 & -4.0-1703.62\end{array}$

$82 \quad 144 \quad 226-10.0 \quad-3.9-1707.56$

$\begin{array}{lllll}82 & 145 & 227-10.2 & -3.8-1711.39\end{array}$

$82 \quad 146 \quad 228-1.0 .4 \quad-3.7-1715.11$

$\begin{array}{lllll}82 & 147 & 229-10.6 & -3.6-1718.73\end{array}$

$\begin{array}{lllll}82 & 148 & 230-10.7 & -3.5-1722.24\end{array}$

$82 \quad 149 \quad 231-10.9 \quad-3.4-1725.65$

$0.65 \quad 0.00-3.25-1702.17$

$0.720 .92-3.20-1705.18$

$0.820 .00-3.16-1709.90$

$0.640 .91-3.12-1712.95$

$0.76 \quad 0.00-3.08-1717.43$

$0.46 \quad 0.91-3.04-1720.39$

$0.550 .00-3.00-1724.69$

$0.22 \quad 0.91-2.96-1727.48$

$82 \quad 150 \quad 232-11.1 \quad-3.3-1728.96$

$0.30 \quad 0.00-2.92-1731.58$

$\begin{array}{llll}82 & 151 & 233-11.3 & -3.2-1732.17\end{array}$

$\begin{array}{llll}-0.04 & 0.90 & -2.88-1734.19\end{array}$

10.39

$-10.49-9.75$

$-0.10$

$-2.88$

$-0.03$

7.72

12.56

15.67

20.69

23.99

29.84

33.06

$-7.57$

$-8.38$

0.12

37.83

0.00

0.00

0.00

0.00

0.00

0.00

0.00

0.00

0.00

0.00

0.00

41.14

46.19

49.55

0.00

0.00

0.00

0.00

54.57

0.00

0.00

0.00

0.00

0.00

0.00

0.00

0.00

0.00

0.00

0.00

0.00

$\begin{array}{lll}0.00 & 0.00 & 0.00\end{array}$

$82 \quad 152 \quad 234-11.5-3.1-1735.28$

$82153 \quad 235-11.7-3.0-1738.29$

$82 \quad 154 \quad 236-11.9-2.9-1741.21$

$82155 \quad 237-12.1-2.8-1744.03$

$82156 \quad 238-12.2-2.7-1746.76$

$82 \quad 157 \quad 239-12.4 \quad-2.6-1749.40$

$\begin{array}{llll}0.12 & 0.00 & -2.85-1738.01\end{array}$

$0.090 .90-2.81-1740.12$

$0.32 \quad 0.00-2.78-1743.67$

58.16

63.27

67.05

72.32

0.00

0.00

0.00

$0.00 \quad 0.00 \quad 0.00$

$0.210 .89-2.74-1745.67102 .56$

$0.360 .00-2.71-1749.11 \quad 107.19$

$0.090 .89-2.68-1751.10 \quad 113.28$

$82158 \quad 240-12.6-2.5-1751.95$

$0.07 \quad 0.00-2.64-1754.53 \quad 117.92$

$82159241-12.8-2.4-1754.41$

$82 \quad 160 \quad 242-12.9-2.3-1756.78$

$82161243-13.1-2.2-1759.07$

$\begin{array}{llllll}-0.35 & 0.89 & -2.61-1756.49 & 124.03\end{array}$

$\begin{array}{lllll}0.91 & 0.00 & -2.58-1758.45 & 130.14\end{array}$

$82162 \quad 244-13.3-2.2-1761.26$

$\begin{array}{lllll}0.52 & 0.88 & -2.55-1760.22 & 136.45\end{array}$

$82163 \quad 245-13.5-2.1-1763.38$

$0.16 \quad 0.00-2.52-1763.63 \quad 141.11$

$82164246-13.6-2.0-1765.41-0.810 .00-2.47-1768.69152 .19$

$82165 \quad 247-13.8-1.9-1767.36-1.08 \quad 0.88-2.44-1770.00158 .95$

$82166248-14.0-1.8-1769.23-1.390 .00-2.41-1773.03 \quad 163.99$

$\begin{array}{llllllllll}82 & 167 & 249-14.1 & -1.7-1771.02 & -2.06 & 0.87 & -2.38-1774.59 & 170.50\end{array}$

$82168 \quad 250-14.3-1.7-1772.73-2.51 \quad 0.00-2.36-1777.60175 .57$

$82 \quad 169251-14.4 \quad-1.6-1774.36-3.08 \quad 0.87-2.33-1778.91 \quad 182.33$

$82170 \quad 252-14.6-1.5-1775.92 \quad-3.47 \quad 0.00-2.31-1781.70 \quad 187.61$

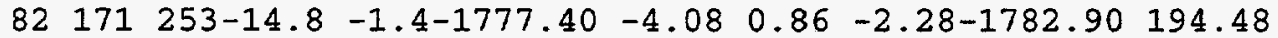

$\begin{array}{lllllllll}82 & 172 & 254-14.9 & -1.4-1778.81 & -4.43 & 0.00 & -2.26-1785.50 & 199.95\end{array}$

$\begin{array}{lllllllll}82 & 173 & 255-15.1 & -1.3-1780.14 & -5.06 & 0.86 & -2.23-1786.58 & 206.94\end{array}$

$82 \quad 174256-15.2-1.2-1781.41-5.42 \quad 0.00-2.21-1789.04 \quad 212.55$

$82 \quad 175 \quad 257-15.4-1.2-1782.60-6.13 \quad 0.86-2.19-1790.06 \quad 219.60$

$82176258-15.5 \quad-1.1-1783.72 \quad-6.60 \quad 0.00-2.16-1792.49225 .25$

$\begin{array}{lllllllll}82 & 177 & 259-15.7 & -1.0-1784.77 & -7.47 & 0.85 & -2.14-1793.53 & 232.27\end{array}$

$\begin{array}{lllllllll}82 & 178 & 260-15.8 & -0.9-1785.76 & -7.93 & 0.00 & -2.12-1795.81 & 238.07\end{array}$

$82 \cdot 179261-16.0 \quad-0.9-1786.68 \quad-8.73 \quad 0.85 \quad-2.10-1796.66 \quad 245.29$

$82 \quad 180 \quad 262-16.1-0.8-1787.53-8.95 \quad 0.00-2.08-1798.56 \quad 251.46$

$82181263-16.3 \quad-0.8-1788.32 \quad-9.66 \quad 0.85-2.06-1799.19258 .90$

$82182 \quad 264-16.4 \quad-0.7-1789.04 \quad-9.79 \quad 0.00-2.04-1800.87 \quad 265.29$

$82183265-16.6-0.6-1789.71-10.24 \quad 0.85-2.02-1801.12 \quad 273.12$

$\begin{array}{llll}0.00 & 0.00 & 0.00 \\ 0.00 & 0.00 & 0.00\end{array}$

$0.00 \quad 0.00 \quad 0.00$

$0.00 \quad 0.00 \quad 0.00$

$0.00 \quad 0.00 \quad 0.00$

$0.00 \quad 0.00 \quad 0.00$

$0.00 \quad 0.00 \quad 0.00$

$0.00 \quad 0.00 \quad 0.00$

$0.00 \quad 0.00 \quad 0.00$

$\begin{array}{llll}0.00 & 0.00 & 0.00\end{array}$

$0.00 \quad 0.00 \quad 0.00$

$\begin{array}{lll}0.00 & 0.00 & 0.00\end{array}$

$\begin{array}{lll}0.00 & 0.00 & 0.00\end{array}$

$\begin{array}{llll}0.00 & 0.00 & 0.00\end{array}$

$\begin{array}{lll}0.00 & 0.00 & 0.00\end{array}$

$\begin{array}{lll}0.00 & 0.00 & 0.00\end{array}$

$\begin{array}{lll}0.00 & 0.00 & 0.00\end{array}$

$\begin{array}{llll}0.00 & 0.00 & 0.00\end{array}$

$\begin{array}{llll}0.00 & 0.00 & 0.00\end{array}$

$\begin{array}{llll}0.00 & 0.00 & 0.00\end{array}$

$\begin{array}{lll}0.00 & 0.00 & 0.00\end{array}$

$\begin{array}{llll}0.00 & 0.00 & 0.00\end{array}$

$\begin{array}{llll}0.00 & 0.00 & 0.00\end{array}$

$\begin{array}{lll}0.00 & 0.00 & 0.00\end{array}$

$\begin{array}{llll}0.00 & 0.00 & 0.00\end{array}$

$\begin{array}{llll}0.00 & 0.00 & 0.00\end{array}$

$\begin{array}{llll}0.00 & 0.00 & 0.00\end{array}$

$\begin{array}{lll}0.00 & 0.00 & 0.00\end{array}$

$\begin{array}{lll}0.00 & 0.00 & 0.00\end{array}$

$\begin{array}{llll}0.00 & 0.00 & 0.00\end{array}$

$\begin{array}{lll}0.00 & 0.00 & 0.00\end{array}$

$\begin{array}{llll}0.00 & 0.00 & 0.00\end{array}$

$\begin{array}{lll}0.00 & 0.00 & 0.00\end{array}$

$\begin{array}{lll}0.00 & 0.00 & 0.00\end{array}$

$\begin{array}{llll}0.00 & 0.00 & 0.00\end{array}$

$\begin{array}{llll}0.00 & 0.00 & 0.00\end{array}$

$\begin{array}{llll}0.00 & 0.00 & 0.00\end{array}$

$\begin{array}{lll}0.00 & 0.00 & 0.00\end{array}$ 
$82184266-16.7 \quad-0.6-1790.31-10.01 \quad 0.00-2.00-1802.32 \quad 279.99$

$\begin{array}{lllllllll}82 & 185 & 267-16.8 & -0.5-1790.85 & -9.18 & 0.84 & -1.98-1801.16 & 289.22\end{array}$

$82186268-17.0 \quad-0.4-1791.32 \quad-8.47 \quad 0.00-1.96-1801.75 \quad 296.70$

$\begin{array}{lllllllll}82 & 187 & 269-17.1 & -0.4-1791.75 & -7.45 & 0.84 & -1.94-1800.30 & 306.22\end{array}$

$\begin{array}{lllllllll}82 & 188 & 270 & -17.2 & -0.3-1792.11 & -6.57 & 0.00 & -1.92-1800.60 & 313.99\end{array}$

$\begin{array}{lllllllll}82 & 189 & 271-17.4 & -0.3-1792.41 & -6.06 & 0.84 & -1.90-1799.54 & 323.12\end{array}$

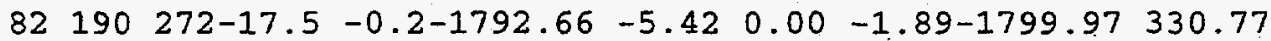

$\begin{array}{llllllll}82 & 191 & 273-17.6 & -0.2-1792.86 & -5.19 & 0.83 & -1.87-1799.08 & 339.72\end{array}$

$\begin{array}{lllllllll}83 & 95 & 178 & 2.1-12.0-1347.28 & 0.62 & 1.94 & -7.53-1352.25 & 18.98\end{array}$

$83 \quad 96 \quad 179 \quad 1.9-11.7-1359.13$

$1.02 \quad 1.10-7.37-1364.38 \quad 14.92$

$83 \quad 97 \quad 180 \quad 1.6-11.5-1370.75$

$\begin{array}{llll}83 & 98 & 181 & 1.3-11.3-1382.16\end{array}$

$\begin{array}{llll}83 & 99 & 182 & 1.0-11.1-1393.35\end{array}$

$\begin{array}{llll}83 & 100 & 183 & 0.8-10.9-1404.32\end{array}$

$\begin{array}{llll}83 & 101 & 184 & 0.5-10.7-1415.09\end{array}$

$\begin{array}{llll}83 & 102 & 185 & 0.2-10.5-1425.65\end{array}$

$83103186 \quad 0.0-10.2-1436.00$

$83104187-0.3-10.1-1446.16$

$\begin{array}{lllll}83 & 105 & 188 & -0.5 & -9.9-1456.11\end{array}$

$\begin{array}{llllll}83 & 106 & 189 & -0.8 & -9.7-1465.87\end{array}$

$\begin{array}{lllll}83 & 107 & 190 & -1.1 & -9.5-1475.44\end{array}$

$\begin{array}{lllll}83 & 108 & 191 & -1.3 & -9.3-1484.82\end{array}$

$\begin{array}{lllll}83 & 109 & 192 & -1.6 & -9.1-1494.01\end{array}$

$1.401 .94-7.21-1374.63$

12.74

$1.84 \quad 1.10-7.06-1386.28$

9.16

$1.851 .93-6.91-1396.48$

7.03

$2.031 .10-6.77-1407.96$

3.62

$2.121 .93-6.63-1417.67$

1.98

$1.961 .10-6.50-1429.08$

$-1.36$

$1.921 .92-6.37-1438.53$

$1.691 .10-6.24-1449.61$

$1.561 .92-6.12-1458.75$

$-2.73$

$-5.74$

$1.241 .10-6.00-1469.53$

$-6.81$

$-9.52$

$0.961 .91-5.88-1478.45-10.37$

$0.531 .10-5.77-1488.96-12.81$

$83 \quad 110 \quad 193-1.8-8.9-1503.02$

$0.171 .91-5.66-1497.60-13.38$

$83 \quad 111 \quad 194-2.1-8.7-1511.85$

$\begin{array}{lllll}-0.39 & 1.10 & -5.56-1507.87 & -15.57 \\ -0.82 & 1.90 & -5.45-1516.22 & -15.86\end{array}$

$83112195-2.3-8.6-1520.50-1.49 \quad 1.10-5.35-1526.25$

$-17.81$

$\begin{array}{llllllllll}83 & 113 & 196 & -2.6 & -8.4-1528.98 & -1.98 & 1.90 & -5.26-1534.32 & -17.81\end{array}$

$\begin{array}{lllllllll}83 & 114 & 197 & -2.8 & -8.2-1537.28 & -2.76 & 1.10 & -5.16-1544.10 & -19.52\end{array}$

$\begin{array}{llllllllll}83 & 115 & 198 & -3.1 & -8.0-1545.41 & -3.42 & 1.89 & -5.07-1552.01 & -19.36\end{array}$

$83116199-3.3-7.9-1553.38-4.24 \quad 1.10-4.98-1561.50-2$

$83117200-3.6-7.7-1561.18-4.94 \quad 1.89-4.90-1569.12$

$-20.78$

$\begin{array}{lllllllll}83 & 118 & 201 & -3.8 & -7.6-1568.81 & -5.75 & 1.10 & -4.81-1578.27\end{array}$

$83119202-4.0-7.4-1576.29-6.57 \quad 1.88-4.73-1585.70$

$83120203 \cdot-4.3-7.2-1583.61-7.391 .10-4.65-1594.55$

$83 \quad 121204-4.5-7.1-1590.77-8.281 .88-4.57-1601.74$

$\begin{array}{lllllllll}83 & 122 & 205 & -4.7 & -6.9-1597.78 & -9.08 & 1.10 & -4.50-1610.25 & -2 .\end{array}$

$\begin{array}{lllllllll}83 & 123 & 206 & -5.0 & -6.8 & -1604.63 & -9.99 & 1.88 & -4.42-1617.17\end{array}$

$83124207-5.2-6.6-1611.34-10.77 \quad 1.10-4.35-1625.36$

$83125208-5.4-6.5-1617.90-11.70 \quad 1.87-4.28-1632.01$

20.33

$-21.41$

0.00

0.00

0.00

0.00

0.00

0.00

0.00

0.00

0.00

$\begin{array}{llll}0.00 & 0.00 & 0.00\end{array}$

$\begin{array}{llll}0.00 & 0.00 & 0.00\end{array}$

$\begin{array}{llll}0.00 & 0.00 & 0.00\end{array}$

$\begin{array}{llll}0.00 & 0.00 & 0.00\end{array}$

$0.00 \quad 0.00 \quad 0.00$

$\begin{array}{llll}0.00 & 0.00 \quad 0.00\end{array}$

$\begin{array}{llll}0.00 & 0.00 & 0.00\end{array}$

$\begin{array}{llll}0.00 & 0.00 & 0.00\end{array}$

$\begin{array}{llll}0.00 & 0.00 & 0.00\end{array}$

$\begin{array}{llll}0.00 & 0.00 & 0.00\end{array}$

$0.00 \quad 0.00 \quad 0.00$

$\begin{array}{llll}0.00 & 0.00 & 0.00\end{array}$

$0.00 \quad 0.00 \quad 0.00$

$0.00 \quad 0.00 \quad 0.00$

$\begin{array}{llll}0.00 & 0.00 & 0.00\end{array}$

$\begin{array}{llll}0.00 & 0.00 & 0.00\end{array}$

$0.00 \quad 0.00 \quad 0.00$

$0.00 \quad 0.00 \quad 0.00$

$0.00 \quad 0.00 \quad 0.00$

$0.00 \quad 0.00 \quad 0.00$

$0.00 \quad 0.00 \quad 0.00$

$0.00 \quad 0.00 \quad 0.00$

$0.00 \quad 0.00 \quad 0.00$

$-17.96-2.13-0.15$

$-19.64-2.88-0.12$

$-19.54-3.60-0.18$

$-20.92-4.38-0.14$

$-20.40-5.01-0.07$

$20.77-20.81-6.61-0.04$

$\begin{array}{lllll}-21.54 & -21.58 & -7.43 & -0.04\end{array}$

$-20.66-20.73-8.35-0.07$

$-21.10-21.08-9.06 \quad 0.02$

$-19.95-20.05-10.09-0.10$

$-20.07-20.08-10.78-0.01$

$-18.65-18.89-11.94-0.24$

$-17.95-18.28-12.28-0.33$

$83127210-5.9-6.2-1630.59-11.07 .1 .87-4.15-1643.94$

$83128211-6.1-6.1-1636.73-9.99 \quad 1.10-4.08-1649.70$

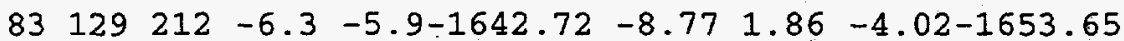

$-14.43$

$-12.12$

$-8.00$

$-14.81-11.45$

$-0.38$

$\begin{array}{lllllllll}83 & 130 & 213 & -6.6 & -5.8-1648.58 & -7.64 & 1.10 & -3.96-1659.08\end{array}$

$\begin{array}{llllllllll}83 & 131 & 214 & -6.8 & -5.7-1654.30 & -6.37 & 1.86 & -3.90-1662.71\end{array}$

$\begin{array}{llllllllll}83 & 132 & 215 & -7.0 & -5.5-1659.89 & -5.41 & 1.10 & -3.84-1668.04\end{array}$

$\begin{array}{llllllllll}83 & 133 & 216 & -7.2 & -5.4-1665.35 & -4.51 & 1.86 & -3.78-1671.79\end{array}$

$\begin{array}{llllllllll}83 & 134 & 217 & -7.4 & -5.3-1670.68 & -3.70 & 1.10 & -3.73-1677.01\end{array}$

$\begin{array}{lllllllll}83 & 135 & 218 & -7.6 & -5.1-1675.89 & -2.99 & 1.85 & -3.67-1680.70\end{array}$

$\begin{array}{llllllllll}83 & 136 & 219 & -7.9 & -5.0 & -1680.97 & -2.15 & 1.10 & -3.62-1685.63\end{array}$

$\begin{array}{lllllllll}83 & 137 & 220 & -8.1 & -4.9-1685.92 & -1.52 & 1.85 & -3.57-1689.16\end{array}$

$\begin{array}{lllllllll}83 & 138 & 221 & -8.3 & -4.8-1690.75 & -0.90 & 1.10 & -3.52-1694.07\end{array}$

$\begin{array}{lllllllll}83 & 139 & 222 & -8.5 & -4.7-1695.47 & -0.35 & 1.85 & -3.47-1697.44\end{array}$

$83 \quad 140223-8.7-4.5-1700.06$

$0.051 .10-3.42-1702.33$

$83 \quad 141224 \quad-8.9-4.4-1704.54$

$83142 \quad 225-9.1-4.3-1708.90$

$\begin{array}{llllll}83 & 143 & 226 & -9.3 & -4.2-1713.15\end{array}$

$\begin{array}{llllll}83 & 144 & 227 & -9.5 & -4.1-1717.29\end{array}$

$0.101 .84-3.37-1705.97$

$0.511 .10-3.32-1710.62$

$0.511 .84-3.28-1714.08$

$0.641 .10-3.23-1718.79$

$0.471 .84-3.19-1722.20$

$\begin{array}{llllll}83 & 145 & 228 & -9.7 & -4.0-1721.32\end{array}$

$\begin{array}{llllll}83 & 146 & 229 & -9.9 & -3.9 & -1725.24\end{array}$

$83 \quad 147 \quad 230-10.1-3.8-1729.05$

$83 \quad 148 \quad 231-10.3 \quad-3.6-1732.75$

$0.641 .10-3.15-1726.64$

$0.361 .83-3.11-1729.96$

$0.461 .10-3.07-1734.26$

$0.151 .83-3.03-1737.40$

$83 \quad 149232-10.5-3.5-1736.36$

$83 \quad 150 \quad 233-10.7 \quad-3.4-1739.85$

$0.261 .10-2.99-1741.48$

$83 \quad 151 \quad 234-10.8-3.3-1743.25$

$-0.031 .83-2.95-1744.40$

$83152 \quad 235-11.0-3.2-1746.55$

$0.111 .10 \quad \frac{2}{82} .91-1748.25$

$-5.36$

$-8.14$

$-9.74$

0.25

$-0.92$

$-5.24$

$-8.91$

$-0.14$

1.82

$-1.22$

$-7.52$

0.12

6.15

1. 71

$-6.67$

$-0.30$

9.00

0.00

0.00

$-0.11$

13.38

0.00

0.00

0.00

16.52

0.00

0.00

0.00

21.06

24.22

0.00

0.00

0.00

0.00

0.00

0.00

28.93

0.00

0.00

0.00

32.11

0.00

0.00

0.00

36.54

39.96

0.00

0.00

0.00

0.00

0.00

0.00

44.57

0.00

0.00

0.00

47.94

0.00

0.00

0.00

52.59

56.22

0.00

0.00

0.00

0.00

0.00

0.00

60.97

0.00

0.00

0.00

64.75

69.68

0.00

0.00

0.00

0.00

0.00

0.00

73.67

0.00

0.00

0.00

78.82

0.00

0.00

0.00

83.04

0.00

0.00

0.00

0.00

0.00

0.00 
$83153236-11.2-3.1-1749.75$ $83 \quad 154 \quad 237-11.4 \quad-3.0-1752.85$

$83.155 \quad 238-11.6-3.0-1755.86$

$\begin{array}{lllll}83 & 156 & 239-11.8 & -2.9-1758.77\end{array}$

$\begin{array}{llll}83 & 157 & 240-11.9 & -2.8-1761.59\end{array}$

$83 \quad 158 \quad 241-12.1-2.7-1764.31$

$83 \quad 159 \quad 242-12.3-2.6-1766.95$

$83 \quad 160 \quad 243-12.5-2.5-1769.49$

$\begin{array}{lllll}83 & 161 & 244-12.7 & -2.4-1771.95\end{array}$

$83 \quad 162 \quad 245-12.8-2.3-1774.32$

$83163246-13.0-2.2-1776.61$

$83 \quad 164 \quad 247-13.2 \quad-2.2-1778.81$

$83 \quad 165 \quad 248-13.3-2.1-1780.93$

$83166249-13.5-2.0-1782.96$

$83167250-13.7-1.9-1784.92$

$83168 \quad 251-13.8-1.8-1786.79$

$83169252-14.0-1.8-1788.59$

$83 \quad 170 \quad 253-14.2-1.7-1790.31$

$83171254-14.3-1.6-1791.95$

$83 \quad 172 \quad 255-14.5-1.5-1793.51$

$83173256-14.6-1.5-1795.01$

$83 \quad 174 \quad 257-14.8-1.4-1796.43$

$83175258-14.9-1.3-1797.77$

$83 \quad 176 \quad 259-15.1-1.2-1799.05$

$83177260-15.3-1.2-1800.25$

$83178261-15.4-1.1-1801.39$

$83179262-15.6-1.0-1802.45$

$83 \quad 180 \quad 263-15.7-1.0-1803.45$

$83181264-15.8-0.9-1804.38$

$83 \quad 182265-16.0-0.8-1805.25$

$83183266-16.1-0.8-1806.06$

$83 \quad 184267-16.3-0.7-1806.80$.

$83185268-16.4-0.6-1807.47$

$83 \quad 186269-16.6-0.6-1808.09$

$83187270-16.7-0.5-1808.65$

$83188 \quad 271-16.8-0.5-1809.15$

$83 \quad 189 \quad 272-17.0 \quad-0.4-1809.58$

$83 \quad 190 \quad 273-17.1-0.3-1809.96$

$83191274-17.2-0.3-1810.29$

$83 \quad 192 \quad 275-17.4 \quad-0.2-1810.56$

$83 \quad 193276-17.5-0.2-1810.77$

$8497 \quad 181 \quad 2.0-11.8-1368.94$

$8498 \quad 182 \quad 1.8-11.6-1380.61$

$8499183 \quad 1.5-11.3-1392.07$

$84100184 \quad 1.2-11.1-1403.32$

84101185 1.0-10.9-1414.35

$84102186 \quad 0.7-10.7-1425.18$

$84103187 \quad 0.4-10.5-1435.80$

$84104188 \quad 0.2-10.3-1446.21$

$84105189-0.1-10.1-1456.43$

$84106190-0.3-9.9-1466.45$

$\begin{array}{llllll}84 & 107 & 191 & -0.6 & -9.7-1476.28\end{array}$

$\begin{array}{llllll}84 & 108 & 192 & -0.9 & -9.5-1485.91\end{array}$

$\begin{array}{llllll}84 & 109 & 193 & -1.1 & -9.4-1495.36\end{array}$

$84110194-1.4 \quad-9.2-1504.62$

$84111195-1.6-9.0-1513.70$

$\begin{array}{llllll}84 & 112 & 196 & -1.9 & -8.8-1522.61\end{array}$

$84113197-2.1-8.6-1531.33$

$\begin{array}{llllll}84 & 114 & 198 & -2.4 & -8.5-1539.88\end{array}$

$84115199-2.6 \quad-8.3-1548.26$

$84116200-2.8-8.1-1556.46$

$84117201-3.1-8.0-1564.51$

$\begin{array}{llllll}84 & 118 & 202 & -3.3 & -7.8-1572.38\end{array}$

$\begin{array}{llllll}84 & 119 & 203 & -3.6 & -7.6-1580.10\end{array}$

$\begin{array}{llllll}84 & 120 & 204 & -3.8 & -7.5 & -1587.65\end{array}$

$84.121205-4.0-7.3-1595.05$
$0.111 .83-2.88-1750.69$

$0.351 .10-2.84-1754.24$

$0.281 .82-2.81-1756.56$

88.67

93.20

98.95

$0.411 .10-2.77-1760.03 \quad 103.55$

$0.181 .82-2.74-1762.33 \quad 109.32$

$0.181 .10=2.71-1765.74 \quad 113.99$

$-0.211 .82-2.67-1768.01119 .78$

$\begin{array}{lllll}1.05 & 1.10 & -2.64-1769.99 & 125.88\end{array}$

$0.771 .81-2.61-1771.98 \quad 131.96$

$0.411 .10-2.58-1775.39136 .61$

$-0.041 .81-2.55-1777.39142 .69$

$\begin{array}{llll}-0.33 & 1.10 & -2.52-1780.56 & 147.59\end{array}$

$\begin{array}{lllll}-0.89 & 1.81 & -2.49-1782.51 & 153.72\end{array}$

$\begin{array}{lllll}-1.15 & 1.10 & -2.47-1785.48 & 158.81\end{array}$

$\begin{array}{lllll}-1.87 & 1.81 & -2.44-1787.42 & 164.94\end{array}$

$\begin{array}{lllll}-2.27 & 1.10 & -2.41-1790.37 & 170.06\end{array}$

$\begin{array}{lllll}-2.83 & 1.80 & -2.39-1792.00 & 176.51\end{array}$

$\begin{array}{lllll}-3.14 & 1.10 & -2.36-1794.71 & 181.87\end{array}$

$\begin{array}{lllll}-3.76 & 1.80 & -2.33-1796.24 & 188.41\end{array}$

$\begin{array}{lllll}-4.16 & 1.10 & -2.31-1798.88 & 193.84\end{array}$

$\begin{array}{llll}-4.75 & 1.80 & -2.28-1800.24 & 200.55\end{array}$

$\begin{array}{llll}-5.09 & 1.10 & -2.26-1802.67 & 206.19\end{array}$

$\begin{array}{llllll}-5.78 & 1.80 & -2.24-1803.99 & 212.95\end{array}$

$\begin{array}{llll}-6.40 & 1.10 & -2.21-1806.56 & 218.45\end{array}$

$\begin{array}{llllll}-7.02 & 1.79 & -2.19-1807.67 & 225.41\end{array}$

$\begin{array}{lllll}-7.36 & 1.10 & -2.17-1809.81 & 231.34\end{array}$

$\begin{array}{lllll}-8.22 & 1.79 & -2.15-1811.03 & 238.19\end{array}$

$\begin{array}{llll}-8.48 & 1.10 & -2.12-1812.96 & 244.34\end{array}$

$\begin{array}{lllll}-9.11 & 1.79 & -2.10-1813.81 & 251.56\end{array}$

$\begin{array}{lllll}-9.23 & 1.10 & -2.08-1815.47 & 257.97\end{array}$

$\begin{array}{lllll}-9.67 & 1.79 & -2.06-1816.00 & 265.51\end{array}$

$\begin{array}{lllll}-9.40 & 1.10 & -2.04-1817.14 & 272.44\end{array}$

$\begin{array}{lllll}-8.56 & 1.78 & -2.02-1816.27 & 281.38\end{array}$

$\begin{array}{lllll}-7.86 & 1.10 & -2.00-1816.85 & 288.87\end{array}$

$\begin{array}{llllll}-6.82 & 1.78 & -1.98-1815.67 & 298.12\end{array}$

$\begin{array}{lllll}-5.98 & 1.10 & -1.96-1815.99 & 305.88\end{array}$

$\begin{array}{lllll}-5.45 & 1.78 & -1.95-1815.20 & 314.74\end{array}$

$\begin{array}{lllll}-4.78 & 1.10 & -1.93-1815.57 & 322.44\end{array}$

$\begin{array}{lllll}-4.57 & 1.78 & -1.91-1814.99 & 331.09\end{array}$

$\begin{array}{llll}-3.90 & 1.10 & -1.89-1815.25 & 338.90\end{array}$

$-1.241 .78-1.88-1812.11350 .11$

$1.431 .04-7.40-1373.86$

20.78

$1.40 \quad 0.00-7.24-1386.45$

$1.281 .04-7.09-1396.84$

$1.210 .00-6.94-1409.05$

$1.071 .03-6.80-1419.05$

$1.120 .00-6.66-1430.72$

$1.091 .02-6.53-1440.21$

$1.320 .00-6.40-1451.29$

$1.421 .02-6.27-1460.26$

$1.480 .00-6.15-1471.12$

$1.471 .01-6.03-1479.83$

$0.92 \quad 0.00-5.92-1490.91$

$0.651 .00-5.80-1499.51$

16.26

13.94

9.80

7.88

4.28

2.86

$-0.15$

$-1.05$

$-3.84$

$-4.47$

$-7.48$

$0.45 \quad 0.00-5.70-1509.87-10.30$

$0.001 .00-5.59-1518.30-10.66$

$-0.500 .00-5.49-1528.59-12.88$

$\begin{array}{lllll}-1.10 & 0.99 & -5.39-1536.82 & -13.04\end{array}$

$-1.73 \cdot 0.00-5.29-1546.90-15.05$

$-2.31 .0 .99-5.20-1554.78-14.85$

$\begin{array}{lllll}-3.15 & 0.00 & -5.11-1564.72 & -16.73\end{array}$

$\begin{array}{lllll}-3.80 & 0.98 & -5.02-1572.34 & -16.27\end{array}$

$\begin{array}{lllll}-4.64 & 0.00 & -4.93-1581.95 & -17.81\end{array}$

$\begin{array}{llll}-5.43 & 0.98 & -4.85-1589.40 & -17.19\end{array}$

$\begin{array}{lllll}-6.26 & 0.00 & -4.77-1598.68 & -18.40\end{array}$

$\begin{array}{rr}-7.13 & 0.97 \\ 83\end{array}$
0.00

0.00

0.00

0.00

0.00

$\begin{array}{llll}0.00 & 0.00 & 0.00\end{array}$

$\begin{array}{llll}0.00 & 0.00 & 0.00\end{array}$

0.00

0.00

0.00

$0.00 \quad 0.00 \quad 0.00$

$0.00 \quad 0.00 \quad 0.00$

$\begin{array}{llll}0.00 & 0.00 & 0.00\end{array}$

$\begin{array}{llll}0.00 & 0.00 & 0.00\end{array}$

$\begin{array}{llll}0.00 & 0.00 & 0.00\end{array}$

$\begin{array}{llll}0.00 & 0.00 & 0.00\end{array}$

$0.00 \quad 0.00 \quad 0.00$

$\begin{array}{llll}0.00 & 0.00 & 0.00\end{array}$

$0.00 \quad 0.00 \quad 0.00$

$0.00 \quad 0.00 \quad 0.00$

$0.00,0.00 \quad 0.00$

$\begin{array}{llll}0.00 & 0.00 & 0.00\end{array}$

$\begin{array}{llll}0.00 & 0.00 & 0.00\end{array}$

$0.00 \quad 0.00 \quad 0.00$

$0.00 \quad 0.00 \quad 0.00$

$0.00 \quad 0.00 \quad 0.00$

$\begin{array}{llll}0.00 & 0.00 & 0.00\end{array}$

$0.00 \quad 0.00 \quad 0.00$

$0.00 \quad 0.00 \quad 0.00$

$\begin{array}{llll}0.00 & 0.00 & 0.00\end{array}$

$\begin{array}{llll}0.00 & 0.00 & 0.00\end{array}$

$\begin{array}{llll}0.00 & 0.00 & 0.00\end{array}$

$0.00 \quad 0.00 \quad 0.00$

$\begin{array}{llll}0.00 & 0.00 & 0.00\end{array}$

$\begin{array}{llll}0.00 & 0.00 & 0.00\end{array}$

$\begin{array}{llll}0.00 & 0.00 & 0.00\end{array}$

$0.00 \quad 0.00 \quad 0.00$

$0.00 \quad 0.00 \quad 0.00$

$\begin{array}{llll}0.00 & 0.00 & 0.00\end{array}$

$0.00 \quad 0.00 \quad 0.00$

$\begin{array}{lll}0.00 & 0.00 & 0.00\end{array}$

$\begin{array}{llll}0.00 & 0.00 & 0.00\end{array}$

$\begin{array}{llll}0.00 & 0.00 & 0.00\end{array}$

$0.00 \quad 0.00 \quad 0.00$

$\begin{array}{llll}0.00 & 0.00 & 0.00\end{array}$

$0.00 \quad 0.00 \quad 0.00$

$0.00 \quad 0.00 \quad 0.00$

$\begin{array}{llll}0.00 & 0.00 & 0.00\end{array}$

$\begin{array}{lll}0.00 & 0.00 \quad 0.00\end{array}$

$\begin{array}{llll}0.00 & 0.00 & 0.00\end{array}$

$\begin{array}{llll}0.00 & 0.00 & 0.00\end{array}$

$\begin{array}{llll}0.00 & 0.00 & 0.00\end{array}$

$\begin{array}{llll}0.00 & 0.00 & 0.00\end{array}$

$\begin{array}{llll}0.00 & 0.00 & 0.00\end{array}$

$\begin{array}{llll}0.00 & 0.00 & 0.00\end{array}$

$\begin{array}{llll}0.00 & 0.00 & 0.00\end{array}$

$0.00 \quad 0.00 \quad 0.00$

$\begin{array}{llll}0.00 & 0.00 & 0.00\end{array}$

$\begin{array}{llll}0.00 & 0.00 & 0.00\end{array}$

$0.00 \quad 0.00 \quad 0.00$

$0.00 \quad 0.00 \quad 0.00$

$\begin{array}{llll}0.00 & 0.00 & 0.00\end{array}$

$0.00 \quad 0.00 \quad 0.00$

$0.00 \quad 0.00 \quad 0.00$

$0.00 \quad 0.00 \quad 0.00$

$0.00 \quad 0.00 \quad 0.00$

$0.00 \quad 0.00 \quad 0.00$

$\begin{array}{llll}0.00 & 0.00 & 0.00\end{array}$

$-17.35-5.59-0.16$

$\begin{array}{llll}0.00 & 0.00 & 0.00\end{array}$

$\begin{array}{llll}-17.55 & -7.14 & -0.01\end{array}$ 
$\begin{array}{lllllllllllllll}84 & 122 & 206 & -4.3 & -7.2-1602.29 & -8.07 & 0.00 & -4.61-1614.97 & -18.54 & -18.20 & -7.73\end{array}$ $\begin{array}{lllllllllllll}84 & 123 & 207 & -4.5 & -7.0-1609.38 & -9.01 & 0.97 & -4.53-1621.96 & -17.46 & -17.17 & -8.72\end{array}$ $\begin{array}{llllllllllll}84 & 124 & 208 & -4.7 & -6.9-1616.32 & -9.80 & 0.00 & -4.46-1630.58 & -18.01 & -17.49 & -9.28\end{array}$

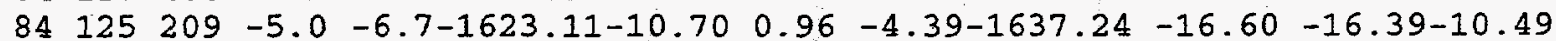
$84126210-5.2-6.6-1629.75-10.840 .00-4.32-1644.91-16.20-15.98-10.62$ $\begin{array}{lllllllllll}84 & 127 & 211 & -5.4 & -6.4-1636.25 & -9.99 & 0.95 & -4.25-1649.54 & -12.76 & -12.46 & -9.69\end{array}$ $\begin{array}{lllllllll}84 & 128 & 212 & -5.6 & -6.3-1642.61 & -8.97 & 0.00 & -4.18-1655.76 & -1\end{array}$ $84129213-5.9-6.1-1648.83-7.72 \quad 0.95-4.12-1659.72$ $84130214-6.1-6.0-1654.91-6.57 \quad 0.00-4.05-1665.53$ $\begin{array}{lllllllll}84 & 131 & 215 & -6.3 & -5.9-1660.85 & -5.40 & 0.95 & -3.99-1669.30\end{array}$ $\begin{array}{lllllllll}84 & 132 & 216 & -6.5 & -5.7-1666.66 & -4.46 & 0.00 & -3.93-1675.05\end{array}$ $\begin{array}{lllllllll}84 & 133 & 217 & -6.7 & -5.6-1672.34 & -3.82 & 0.94 & -3.87-1679.09\end{array}$ $\begin{array}{lllllllll}84 & 134 & 218 & -7.0 & -5.5-1677.88 & -3.05 & 0.00 & -3.82-1684.75\end{array}$ $\begin{array}{lllllllll}84 & 135 & 219 & -7.2 & -5.3-1683.30 & -2.19 & 0.94 & -3.76-1688.31\end{array}$ $\begin{array}{lllllllll}84 & 136 & 220 & -7.4 & -5.2-1688.59 & -1.41 & 0.00 & -3.71-1693.70\end{array}$ $\begin{array}{lllllllll}84 & 137 & 221 & -7.6 & -5.1-1693.75 & -0.86 & 0.93 & -3.65-1697.34\end{array}$ $\begin{array}{llllllllll}84 & 138 & 222 & -7.8 & -5.0 & -1698.80 & -0.26 & 0.00 & -3.60-1702.66\end{array}$ $\begin{array}{lllllllll}84 & 139 & 223 & -8.0 & -4.9-1703.72 & -0.11 & 0.93 & -3.55-1706.45\end{array}$ $84140 \quad 224-8.2-4.7-1708.52$ $84141225-8.4-4.6-1713.20$ $\begin{array}{lllll}84 & 142 & 226 & -8.6 & -4.5-1717.77\end{array}$ $\begin{array}{llllll}84 & 143 & 227 & -8.8 & -4.4-1722.22\end{array}$ $84144228-9.0-4.3-1726.55$ $\begin{array}{lllll}84 & 145 & 229 & -9.2 & -4.2-1730.78\end{array}$ $\begin{array}{llllll}84 & 146 & 230 & -9.4 & -4.1-1734.89\end{array}$ $\begin{array}{llllll}84 & 147 & 231 & -9.6 & -3.9-1738.90\end{array}$ $\begin{array}{llllll}84 & 148 & 232 & -9.8 & -3.8-1742.80\end{array}$ $84 \quad 149 \quad 233-10.0-3.7-1746.59$ $84150 \quad 234-10.2-3.6-1750.28$ $84151235-10.4-3.5-1753.87$ $84152 \quad 236-10.6-3.4-1757.36$ $84 \quad 153 \quad 237-10.8 \quad-3.3-1760.74$ $\begin{array}{lllll}84 & 154 & 238-10.9 & -3.2-1764.03\end{array}$ $84155 \quad 239-11.1-3.1-1767.22$ $84156240-11.3-3.0-1770.31$ $84157241-11.5 \quad-2.9-1773.31$ $84 \quad 158 \quad 242-11.7-2.9-1776.21$ $84159243-11.8-2.8-1779.03$ $84160 \quad 244-12.0 \quad-2.7-1781.75$ $84161245-12.2-2.6-1784.38$ $84 \quad 162 \quad 246-12.4 \quad-2.5-1786.93$ $84 \quad 163 \quad 247-12.5 \quad-2.4-1789.39$ $84 \quad 164 \quad 248-12.7 \quad-2.3-1791.76$ $84 \quad 165 \quad 249-12.9-2.2-1794.04$ $84166 \quad 250-13.1-2.2-1796.25$ $84 \quad 167 \quad 251-13.2-2.1-1798.37$ $84 \quad 168 \quad 252-13.4 \quad-2.0-1800.41$ $84 \quad 169 \quad 253-13.6-1.9-1802.37$ $84 \quad 170 \quad 254-13.7-1.8-1804.25$ $84171255-13.9-1.8-1806.05$ $84 \quad 172 \quad 256-14.0-1.7-1807.78$ $84173257-14.2-1.6-1809.43$ $84 \quad 174 \quad 258-14.4-1.5-1811.00$ $84175259-14.5-1.5-1812.50$ $84176 \quad 260-14.7-1.4-1813.93$ $84177261-14.8-1.3-1815.29$ $84178 \quad 262-15.0-1.2-1816.57$ $84179263-15.1-1.2-1817.79$ $0.290 .00-3.50-1711.73$ $0.280 .92-3.45-1715.45$ $0.63 \quad 0.00-3.40-1720.54$ $0.58 \quad 0.92-3.36-1724.07$ $0.71 \quad 0.00-3.31-1729.15$ $0.530 .91-3.27-1732.60$ $0.690 .00-3.22-1737.43$ $0.35 \quad 0.91-3.18-1740.82$ $0.470 .00-3.14-1745.47$ $0.18 \quad 0.91-3.10-1748.61$ $0.30 \quad 0.00-3.06-1753.04$ $-0.020 .90-3.02-1756.01$ $0.180 .00-2.98-1760.16$ $0.190 .90-2.94-1762.60$ $0.49 \quad 0.00-2.91-1766.44$ $0.45 \quad 0.89-2.87-1768.74$ $0.66 \quad 0.00-2.84-1772.49$

-10.91
-6.79

$-4.54$

$-0.23$

2. 09

6.12

8.53

13.04

15.72

20.16

22.91

27.19

29.98

34.33

37.31

41.85

44.84

49.47

52.71

57.39

60.81

65.75

69.38

74.49

78.41

84.04

88.27

94.04

$\begin{array}{lllll}47 & 0.89 & -2.80-1774.75 & 104.17\end{array}$ $\begin{array}{lllll}0.52 & 0.00 & -2.77-1778.46 & 108.53\end{array}$ $0.14 \quad 0.89-2.74-1780.74 \quad 114.33$ $0.05 \quad 0.00-2.70-1784.40 \quad 118.73$ $\begin{array}{llllll}-0.39 & 0.88 & -2.67-1786.56 & 124.65\end{array}$ $\begin{array}{llllll}-0.43 & 0.00 & -2.64-1790.00 & 129.28\end{array}$ $0.53 \quad 0.88-2.61-1790.59 \quad 136.77$ $0.090 .00-2.58-1794.25141 .18$ $\begin{array}{lllll}-0.34 & 0.88 & -2.55-1796.06 & 147.44\end{array}$ $\begin{array}{llllll}-0.58 & 0.00 & -2.52-1799.35 & 152.22\end{array}$ $\begin{array}{lllll}-1.29 & 0.87 & -2.49-1801.28 & 158.36\end{array}$ $\begin{array}{llllll}-1.68 & 0.00 & -2.47-1804.56 & 163.15\end{array}$ $\begin{array}{llllll}-2.20 & 0.87 & -2.44-1806.14 & 169.64\end{array}$ $\begin{array}{llllll}-2.52 & 0.00 & -2.41-1809.18 & 174.67\end{array}$ $\begin{array}{lllll}-3.12 & 0.86 & -2.39-1810.69 & 181.23\end{array}$ $\begin{array}{lllll}-4.10 & 0.00 & -2.36-1814.24 & 185.76\end{array}$ $\begin{array}{lllll}-4.08 & 0.86 & -2.34-1814.98 & 193.09\end{array}$ $\begin{array}{llllll}-4.40 & 0.00 & -2.31-1817.71 & 198.43\end{array}$ $\begin{array}{lllll}-5.15 & 0.86 & -2.29-1819.08 & 205.13\end{array}$ $\begin{array}{llll}-5.63 & 0.00 & -2.26-1821.82 & 210.46\end{array}$ $\begin{array}{llll}-6.27 & 0.85 & -2.24-1822.94 & 217.41\end{array}$ $\begin{array}{llllll}-6.59 & 0.00 & -2.22-1825.38 & 223.04\end{array}$ $\begin{array}{llllll}-7.43 & 0.85 & -2.19-1826.56 & 229.93\end{array}$ $84180 \quad 264-15.3-1.1-1818.94-7.75 \quad 0.00-2.17-1828.86 \quad 235.71$ $\begin{array}{lllllllll}84 & 181 & 265-15.4 & -1.0-1820.02 & -8.35 & 0.85 & -2.15-1829.67 & 242.97\end{array}$ $84182 \quad 266-15.6-1.0-1821.03 \quad-8.450 .00-2.13-1831.61249 .10$ $\begin{array}{lllllllll}84 & 183 & 267-15.7 & -0.9-1821.98 & -8.89 & 0.85 & -2.11-1832.13 & 256.65\end{array}$ $\begin{array}{llllllllll}84 & 184 & 268-15.9 & -0.8-1822.86 & -8.61 & 0.00 & -2.09-1833.56 & 263.29\end{array}$ $\begin{array}{lllllllll}84 & 185 & 269-16.0 & -0.8-1823.68 & -7.76 & 0.84 & -2.07-1832.67 & 272.26\end{array}$ $84186270-16.1 \quad-0.7-1824.44 \quad-7.06 \quad 0.00-2.05-1833.55 \quad 279.45$ $84187271-16.3 \quad-0.7-1825.13-6.13 \quad 0.84 \quad-2.03-1832.45 \quad 288.62$
0.34

0.29

0.52

0.21

0.22

0.30

0.52

$\begin{array}{rr}10.39 & -8.45 \\ -6.68 & -7.61\end{array}$

$-4.49-6.52$

0.11

$-0.54-5.71$

0.05

$\begin{array}{llll}1.76 & -4.79 & -0.33\end{array}$

$\begin{array}{lll}0.00 & 0.00 & 0.00\end{array}$

$\begin{array}{llll}8.35 & -3.23 & -0.18\end{array}$

$\begin{array}{lll}0.00 & 0.00 & 0.00\end{array}$

$\begin{array}{llll}0.00 & 0.00 & 0.00\end{array}$

$0.00 \quad 0.00 \quad 0.00$

$\begin{array}{llll}0.00 & 0.00 & 0.00\end{array}$

$0.00 \quad 0.00 \quad 0.00$

$\begin{array}{llll}0.00 & 0.00 & 0.00\end{array}$

$0.00 \quad 0.00 \quad 0.00$

$\begin{array}{llll}0.00 & 0.00 & 0.00\end{array}$

$0.00 \quad 0.00 \quad 0.00$

$0.00 \quad 0.00 \quad 0.00$

$0.00 \quad 0.00 \quad 0.00$

$\begin{array}{llll}0.00 & 0.00 & 0.00\end{array}$

$\begin{array}{llll}0.00 & 0.00 & 0.00\end{array}$

$\begin{array}{llll}0.00 & 0.00 & 0.00\end{array}$

$\begin{array}{llll}0.00 & 0.00 & 0.00\end{array}$

$0.00 \quad 0.00 \quad 0.00$

$\begin{array}{llll}0.00 & 0.00 & 0.00\end{array}$

$\begin{array}{llll}0.00 & 0.00 & 0.00\end{array}$

$0.00 \quad 0.00 \quad 0.00$

$0.00 \quad 0.00 \quad 0.00$

$0.00 \quad 0.00 \quad 0.00$

$0.00 \quad 0.00 \quad 0.00$

$0.00 \quad 0.00 \quad 0.00$

$\begin{array}{llll}0.00 & 0.00 & 0.00\end{array}$

$0.00 \quad 0.00 \quad 0.00$

$\begin{array}{llll}0.00 & 0.00 & 0.00\end{array}$

$0.00 \quad 0.00 \quad 0.00$

$\begin{array}{llll}0.00 & 0.00 & 0.00\end{array}$

$0.00 \quad 0.00 \quad 0.00$

$\begin{array}{llll}0.00 & 0.00 & 0.00\end{array}$

$\begin{array}{llll}0.00 & 0.00 & 0.00\end{array}$

$\begin{array}{llll}0.00 & 0.00 & 0.00\end{array}$

$\begin{array}{llll}0.00 & 0.00 & 0.00\end{array}$

$0.00 \quad 0.00 \quad 0.00$

$0.00 \quad 0.00 \quad 0.00$

$0.00 \quad 0.00 \quad 0.00$

$0.00 \quad 0.00 \quad 0.00$

$0.00 \quad 0.00 \quad 0.00$

$0.00 \quad 0.00 \quad 0.00$

$\begin{array}{llll}0.00 & 0.00 & 0.00\end{array}$

$0.00 \quad 0.00 \quad 0.00$

$0.00 \quad 0.00 \quad 0.00$

$0.00 \quad 0.00 \quad 0.00$

$0.00 \quad 0.00 \quad 0.00$

$\begin{array}{llll}0.00 & 0.00 & 0.00\end{array}$

$0.00 \quad 0.00 \quad 0.00$

$0.00 \quad 0.00 \quad 0.00$

$\begin{array}{llll}0.00 & 0.00 & 0.00\end{array}$

$\begin{array}{llll}0.00 & 0.00 & 0.00\end{array}$

$0.00 \quad 0.00 \quad 0.00$

$0.00 \quad 0.00 \quad 0.00$

$\begin{array}{llll}0.00 & 0.00 & 0.00\end{array}$

$0.00 \quad 0.00 \quad 0.00$ 
$\begin{array}{lllllllllll}84 & 188 & 272 & -16.4 & -0.6-1825.77 & -5.26 & 0.00 & -2.01-1833.03 & 296.10\end{array}$ $84189273-16.5-0.5-1826.34 \quad-4.88 \quad 0.84-1.99-1832.37 \quad 304.84$ $\begin{array}{lllllllll}84 & 190 & 274-16.7 & -0.5-1826.86 & -4.23 & 0.00 & -1.97-1833.05 & 312.23\end{array}$ $\begin{array}{llllllll}84 & 191 & 275-16.8 & -0.4-1827.31 & -4.06 & 0.83 & -1.95-1832.49 & 320.86\end{array}$ $\begin{array}{llllllllll}84 & 192 & 276-16.9 & -0.4-1827.71 & -1.00 & 0.00 & -1.93-1830.64 & 330.78\end{array}$ $84193277-17.1 \quad-0.3-1828.05-1.25 \quad 0.83-1.92-1830.39339 .11$ $\begin{array}{lllllllll}84 & 194 & 278-17.2 & -0.3-1828.34 & -1.15 & 0.00 & -1.90-1831.39 & 346.18\end{array}$ $84195 \quad 279-17.3-0.2-1828.58$ $8599184 \quad 2.0-11.6-1390.34$ $85100185 \cdot 1.7-11.4-1401.86$ $85101186 \quad 1.4-11.2-1413.15$ $85102187 \quad 1.2-11.0-1424.24$ $85103188 \quad 0.9-10.8-1435.13$ $851041890.6-10.6-1445.81$ $85105190 \quad 0.4-10.4-1456.28$ $85106191 \quad 0.1-10.2-1466.56$ $85107192-0.1-10.0-1476.65$ $\begin{array}{llllll}85 & 108 & 193 & -0.4 & -9.8-1486.54\end{array}$ $85109194-0.6 \quad-9.6-1496.24$ $85110 \quad 195 \quad-0.9-9.4-1505.76$ $85111196-1.1-9.2-1515.09$ $\begin{array}{llllll}85 & 112 & 197 & -1.4 & -9.1-1524.24\end{array}$ $\begin{array}{llllll}85 & 113 & 198 & -1.6 & -8.9-1533.21\end{array}$ $\begin{array}{llllll}85 & 114 & 199 & -1.9 & -8.7-1542.01\end{array}$ $\begin{array}{llllll}85 & 115 & 200 & -2.1 & -8.5-1550.63\end{array}$ $\begin{array}{llllll}85 & 116 & 201 & -2.4 & -8.4-1559.08\end{array}$ $\begin{array}{llllll}85 & 117 & 202 & -2.6 & -8.2-1567.36\end{array}$ $\begin{array}{llllll}85 & 118 & 203 & -2.9 & -8.0-1575.48\end{array}$ $\begin{array}{llllll}85 & 119 & 204 & -3.1 & -7.9-1583.43\end{array}$ $\begin{array}{llllll}85 & 120 & 205 & -3.3 & -7.7-1591.23\end{array}$ $\begin{array}{llllll}85 & 121 & 206 & -3.6 & -7.6-1598.86\end{array}$ $\begin{array}{llllll}85 & 122 & 207 & -3.8 & -7.4-1606.34\end{array}$ $\begin{array}{llllll}85 & 123 & 208 & -4.0 & -7.2-1613.66\end{array}$ $\begin{array}{llllll}85 & 124 & 209 & -4.3 & -7.1-1620.83\end{array}$ $\begin{array}{llllll}85 & 125 & 210 & -4.5 & -6.9-1627.85\end{array}$ $\begin{array}{llllll}85 & 126 & 211 & -4.7 & -6.8-1634.72\end{array}$ $\begin{array}{llllll}85 & 127 & 212 & -5.0 & -6.6-1641.44\end{array}$ $\begin{array}{lllll}85 & 128 & 213 & -5.2 & -6.5-1648.02\end{array}$ $\begin{array}{llllll}85 & 129 & 214 & -5.4 & -6.4-1654.47\end{array}$ $85 \quad 130 \quad 215-5.6-6.2-1660.77$ $85131216-5.8-6.1-1666.93$ $\begin{array}{lllll}85 & 132 & 217 & -6.1 & -6.0-1672.96\end{array}$ $85133218-6.3-5.8-1678.85$ $85134219-6.5-5.7-1684.61$ $\begin{array}{llllllll}135 & 220 & -6.7 & -5.6-1690.24 & -2.07 & 1.85 & -3.85-1694.31\end{array}$ $\begin{array}{lllllllll}85 & 136 & 221 & -6.9 & -5.4 & -1695.74 & -1.43 & 1.09 & -3.79-1699.87\end{array}$ $\begin{array}{llllllllll}85 & 137 & 222 & -7.1 & -5.3-1701.12 & -0.86 & 1.84 & -3.74-1703.87\end{array}$ $\begin{array}{lllllllll}85 & 138 & 223 & -7.3 & -5.2-1706.37 & -0.29 & 1.09 & -3.69-1709.25\end{array}$ $\begin{array}{lllllllll}85 & 139 & 224 & -7.5 & -5.1-1711.50 & -0.18 & 1.84 & -3.63-1713.47\end{array}$ $85 \quad 140 \quad 225 \quad-7.8-4.9-1716.50$ $\begin{array}{lllll}85 & 141 & 226 & -8.0 & -4.8-1721.39\end{array}$ $\begin{array}{lllll}85 & 142 & 227 & -8.2 & -4.7-1726.16\end{array}$ $\begin{array}{llllll}85 & 143 & 228 & -8.4 & -4.6-1730.81\end{array}$ $\begin{array}{llllll}85 & 144 & 229 & -8.6 & -4.5-1735.35\end{array}$ $\begin{array}{llllll}85 & 145 & 230 & -8.8 & -4.4-1739.77\end{array}$ $\begin{array}{llllll}85 & 146 & 231 & -9.0 & -4.3-1744.08\end{array}$ $\begin{array}{llllll}85 & 147 & 232 & -9.2 & -4.1-1748.29\end{array}$ $\begin{array}{llllll}85 & 148 & 233 & -9.3 & -4.0-1752.38\end{array}$ $\begin{array}{llllll}85 & 149 & 234 & -9.5 & -3.9-1756.37\end{array}$ $\begin{array}{llllll}85 & 150 & 235 & -9.7 & -3.8-1760.25\end{array}$ $\begin{array}{llllll}85 & 151 & 236 & -9.9 & -3.7-1764.02\end{array}$ $85 \quad 152 \quad 237-10.1-3.6-1767.70$ $\begin{array}{llll}85 & 153 & 238-10.3 & -3.5-1771.27\end{array}$ $\begin{array}{lllll}85 & 154 & 239-10.5 & -3.4-1774.74\end{array}$ $85 \quad 155 \quad 240-10.7-3.3-1778.12$ $85156 \quad 241-10.9-3.2-1781.39$

$1.031 .93-7.26-1394.65$ $0.831 .09-7.11-1407.05$ $0.721 .92-6.97-1417.48$ $0.781 .09-6.83-1429.20$ $0.871 .91-6.69-1439.03$ $1.001 .09-6.56-1450.27$ $1.151 .91-6.43-1459.65$ $1.491 .09-6.30-1470.28$ $1.241 .90-6.18-1479.68$ $0.991 .09-6.06-1490.52$ $0.731 .90-5.95-1499.56$ $0.501 .09-5.84-1510.00$ $0.321 .89-5.73-1518.60$ $0.011 .09-5.62-1528.76$ $-0.321 .89-5.52-1537.16$ $-0.871 .09-5.42-1547.21$ $-1.491 .89-5.33-1555.56$ $-2.141 .09-5.23-1565.36$ $\begin{array}{llll}-2.87 & 1.88 & -5.14-1573.49 \\ -3.59 & 1.09 & -5.05-1583.03\end{array}$ $-4.561 .88-4.97-1591.08$ $-5.071 .09-4.88-1600.09$ $\begin{array}{llll}-6.21 & 1.87 & -4.80-1608.00 \\ -6.94 & 1.09 & -4.72-1616.90\end{array}$ $-7.771 .87-4.64-1624.20$ $-8.521 .09-4.57-1632.82$ $-9.441 .86-4.49-1639.91$ $\begin{array}{llll}-9.58 & 1.09 & -4.42-1647.63 \\ -8.69 & 1.86 & -4.35-1652.62\end{array}$ $-7.761 .09-4.28-1658.98$ $-6.521 .86-4.22-1663.35$ $-5.371 .09-4.15-1669.20$ $-4.261 .85-4.09-1673.42$ $-3.721 .09-4.03-1679.61$ $-3.211 .85-3.97-1684.17$ $-2.411 .09-3.91-1689.83$ $\begin{array}{lll}0.18 & 1.84 & -3.63-1713.47 \\ 0.16 & 1.09 & -3.58-1718.83\end{array}$ $0.131 .84-3.53-1722.96$ $0.441 .09-3.48-1728.11$ $0.391 .83-3.44-1732.02$ $0.511 .09-3.39-1737.14$ $0.321 .83-3.34-1740.97$ $0.481 .09-3.30-1745.81$ $0.161 .83-3.25-1749.56$ $0.321 .09-3.21-1754.18$ $0.021 .82-3.17-1757.69$ $0.181 .09-3.13-1762.11$ $-0.131 .82-3.09-1765.42$ $-0.061 .09-3.05-1769.72$ $0.151 .82-3.01-1772.32$ $0.441 .09-2.97-1776.19$ $0.511 .81-2.94-1778.73$ $0.711 .09-2.90-1782.49$

$\begin{array}{lll}0.00 & 0.00 & 0.00 \\ 0.00 & 0.00 & 0.00 \\ 0.00 & 0.00 & 0.00 \\ 0.00 & 0.00 & 0.00 \\ 0.00 & 0.00 & 0.00 \\ 0.00 & 0.00 & 0.00 \\ 0.00 & 0.00 & 0.00 \\ 0.00 & 0.00 & 0.00 \\ 0.00 & 0.00 & 0.00 \\ 0.00 & 0.00 & 0.00 \\ 0.00 & 0.00 & 0.00 \\ 0.00 & 0.00 & 0.00 \\ 0.00 & 0.00 & 0.00 \\ 0.00 & 0.00 & 0.00 \\ 0.00 & 0.00 & 0.00 \\ 0.00 & 0.00 & 0.00 \\ 0.00 & 0.00 & 0.00 \\ 0.00 & 0.00 & 0.00 \\ 0.00 & 0.00 & 0.00 \\ 0.00 & 0.00 & 0.00 \\ 0.00 & 0.00 & 0.00 \\ 0.00 & 0.00 & 0.00 \\ 0.00 & 0.00 & 0.00 \\ 0.00 & 0.00 & 0.00\end{array}$

23.40

19.08 16.71 13.07

11.31

8.14 6.83

4.27

2.94

0.18

$-0.79$

$-3.16$

$-3.69$

$-5.78$

$-6.11$

$-8.08$

$-8.36$

$-10.09$

$-8.94$

$-2.07$

$-0.58$

$-10.15$

$-11.62$

$-11.60$

$-12.53$

$-12.37$

$-13.21$

$-12.43$

$-12.98$

$-12.00$

$-11.64$

$-8.57$

$-6.85$

$-3.15$

$-0.93$

2.92

4.80

8. 31

10.72

14.31

16.82

20.89

23.59

27.44

30.15

34.10

37.02

41.17

44.13

48.37

51.60

55.93

59.37

63.93

67.59

72.34

76.12

81.59

85.80

91.32

95.63
$-10.74$

$-10.76$

$-12.29$

$-11.90$

$-12.49$

$-12.56$

$-12.90$

$-11.99-9$

$-11.67-9$

$-8.64$

$-6.60-7.51$

$-3.40-6.77$

$-1.27-5.71$

2.23

4.38

$8.08-3$

10.53

0.00

0.00

0.00

0.00

0.00

0.00

0.00

0.00

0.00

0.00

0.00

0.00

0.00

0.00

0.00

0.00

0.00

0.00

0.00

0.00

0.00

0.00
$-13.03-5$
$-0.65$ $-0.61$ $-0.67$ $-0.30$ $-0.50$ $-0.12$ $-0.07$ $-0.13$ 0.08 0.01 $-0.03$ $-0.07$ 0.25 $-0.34$ $4.14-0.42$ $-3.44-0.23$ $2.60-0.19$ $0.00 \quad 0.00$ $0.00 \quad 0.00$ $0.00 \quad 0.00$ $0.00 \quad 0.00$ $0.00 \quad 0.00$ $0.00 \quad 0.00$ $0.00 \quad 0.00$ $0.00 \quad 0.00$ $0.00 \quad 0.00$ $0.00 \quad 0.00$ $0.00 \quad 0.00$ $0.00 \quad 0.00$ $0.00 \quad 0.00$ $0.00 \quad 0.00$ $0.00 \quad 0.00$ $0.00 \quad 0.00$ $0.00 \quad 0.00$ $0.00 \quad 0.00$ $0.00 \quad 0.00$ $0.00 \quad 0.00$ $0.00 \quad 0.00$ $0.00 \quad 0.00$ 
$85 \quad 157242-11.0-3.1-1784.57$ $85 \quad 158 \quad 243-11.2 \quad-3.0-1787.66$ $85159244-11.4 \quad-2.9-1790.65$ $85 \quad 160 \quad 245-11.6 \quad-2.8-1793.55$ $85 \quad 161246-11.7-2.8-1796.36$ $85 \quad 162 \quad 247-11.9-2.7-1799.08$ $85 \quad 163248-12.1-2.6-1801.71$ $85 \quad 164 \quad 249-12.3-2.5-1804.25$ $85165250-12.4-2.4-1806.71$ $85166251-12.6-2.3-1809.08$ $85167252-12.8-2.2-1811.37$ $85 \quad 168 \quad 253-12.9-2.2-1813.58-1.30 \quad 1.09-2.52-1816.31158 .68$ $\begin{array}{lllllllll}85 & 169 & 254-13.1 & -2.1-1815.70 & -1.85 & 1.80 & -2.49-1818.25 & 164.81\end{array}$ $\begin{array}{lllllllll}85 & 170 & 255-13.3 & -2.0-1817.74 & -2.12 & 1.09 & -2.47-1821.24 & 169.89\end{array}$ $\begin{array}{lllllllll}85 & 171 & 256-13.4 & -1.9-1819.71 & -2.72 & 1.79 & -2.44-1823.08 & 176.12\end{array}$ $85 \quad 172 \quad 257-13.6-1.8-1821.60-3.91 \quad 1.09-2.41-1826.83 \quad 180.44$ $\begin{array}{lllllllll}85 & 173 & 258-13.8 & -1.8-1823.40 & -3.62 & 1.79 & -2.39-1827.62 & 187.72\end{array}$ $85174 \quad 259-13.9-1.7-1825.14-3.94 \quad 1.09-2.36-1830.35193 .06$ $85175260-14.1-1.6-1826.80-4.61 \quad 1.79-2.34-1831.95199 .53$ $85176 \quad 261-14.2-1.5-1828.38-5.14 \quad 1.09-2.31-1834.74 \quad 204.81$ $85 \quad 177 \quad 262-14.4 \quad-1.5-1829.89-5.74 \quad 1.79-2.29-1836.13211 .49$ $85178263-14.5-1.4-1831.33-6.05 \quad 1.09-2.26-1838.55 \quad 217.15$ $85179264-14.7-1.3-1832.70-6.71 \quad 1.78-2.24-1839.87223 .90$ $\begin{array}{llllllllll}85 & 180 & 265-14.8 & -1.3-1833.99 & -6.99 & 1.09 & -2.22-1842.11 & 229.73\end{array}$ $85181266-15.0-1.2-1835.22-7.59 \quad 1.78-2.20-1843.23 \quad 236.68$ $\begin{array}{llllllllll}85 & 182 & 267-15.1 & -1.1-1836.38 & -7.66 & 1.09 & -2.17-1845.13 & 242.86\end{array}$ $\begin{array}{llllllll}85 & 183 & 268-15.3 & -1.1-1837.48 & -8.10 & 1.78 & -2.15-1845.95 & 250.10\end{array}$ $85184 \quad 269-15.4-1.0-1838.51 \quad-7.79 \quad 1.09-2.13-1847.33 \quad 256.79$ $85 \quad 185 \quad 270-15.6 \quad-0.9-1839.47-6.94 \quad 1.78-2.11-1846.74265 .46$ $85 \quad 186 \quad 271-15.7-0.9-1840.36-6.28 .1 .09-2.09-1847.64 \quad 272.63$ $\begin{array}{llllllllll}85 & 187 & 272-15.9 & -0.8-1841.20 & -5.26 & 1.77 & -2.07-1846.75 & 281.59\end{array}$ $85188 \quad 273-16.0 \quad-0.7-1841.97-4.42 \quad 1.09-2.05-1847.35 \quad 289.06$ $\begin{array}{llllllll}85 & 189 & 274-16.1 & -0.7-1842.68 & -3.92 & 1.77 & -2.03-1846.86 & 297.62\end{array}$ $85190275-16.3-0.6-1843.33-3.41 \quad 1.09-2.01-1847.66 \quad 304.89$ $85191276-16.4 \quad-0.6-1843.92-1.33 \quad 1.77-1.99-1845.47315 .15$ $85192 \quad 277-16.5-0.5-1844.45-1.19 \quad 1.09-1.97-1846.53 \quad 322.17$ $\begin{array}{llllllll}85 & 193 & 278-16.7 & -0.4-1844.93 & -1.41 & 1.77 & -1.96-1846.53 & 330.24\end{array}$ $85194279-16.8-0.4-1845.34-1.31 \quad 1.09-1.94-1847.50 \quad 337.34$ $85195 \quad 280-16.9-0.3-1845.70-1.59 \quad 1.77-1.92-1847.45 \quad 345.46$ $85196281-17.1-0.3-1846.01-1.311 .09-1.90-1848.13 \quad 352.85$ $85197282-17.2-0.2-1846.26-1.541 .76-1.89-1847.93 \quad 361.13$ $86100186 \quad 2.1-11.7-1399.94$ 86101187 $1.9-11 \cdot 5-1411.50$ 86102188 86103189 86104190 1. 6-11.2-1422.86 $1.4-11.0-1434.00$ $1.1-10.8-1444.94$ $86 \quad 105191$ 86106192 $0.8-10.6-1455.68$ $0.6-10.4-1466.22$ $0.3-10.2-1476.56$ 86107193 $86 \quad 108 \quad 194$ $0.1-10.0-1486.71$ $86109195-0.2-9.9-1496.66$ $86110196-0.4-9.7-1506.43$ $\begin{array}{llllll}86 & 111 & 197 & -0.7 & -9.5-1516.01\end{array}$ $\begin{array}{llllll}86 & 112 & 198 & -0.9 & -9.3-1525.41\end{array}$ $\begin{array}{lllll}86 & 113 & 199 & -1.2 & -9.1-1534.63\end{array}$ $\begin{array}{llllll}86 & 114 & 200 & -1.4 & -9.0-1543.67\end{array}$ $86 \quad 115201-1.7-8.8-1552.54$ $86116202-1.9-8.6-1561.23-1.450 .00-5.36-1568.04$ $\begin{array}{lllllllll}86 & 117 & 203 & -2.2 & -8.4-1569.76 & -2.13 & 0.98 & -5.27-1576.17\end{array}$ $\begin{array}{lllllllll}86 & 118 & 204 & -2.4 & -8.3-1578.11 & -2.90 & 0.00 & -5.17-1586.19\end{array}$ $\begin{array}{lllllllll}86 & 119 & 205 & -2.6 & -8.1-1586.31 & -3.72 & 0.98 & -5.09-1594.14\end{array}$ $\begin{array}{llllllllll}86 & 120 & 206 & -2.9 & -7.9-1594.34 & -4.26 & 0.00 & -5.00-1603.59\end{array}$ $\begin{array}{lllllllll}86 & 121 & 207 & -3.1 & -7.8-1602.20 & -5.19 & 0.97 & -4.92-1611.34\end{array}$ $\begin{array}{lllllllll}86 & 122 & 208 & -3.3 & -7.6-1609.91 & -5.83 & 0.00 & -4.83-1620.58\end{array}$ $\begin{array}{lllllllll}86 & 123 & 209 & -3.6 & -7.5-1617.47 & -6.66 & 0.97 & -4.75-1627.92\end{array}$ $\begin{array}{lllllllll}86 & 124 & 210 & -3.8 & -7.3-1624.87 & -7.45 & 0.00 & -4.68-1636.99\end{array}$ $8 \overline{6}$
26.76

24.33

20.25

18.37

14.82

13.56

10.44

9.44

6.47

5.45

2.62

1.95

$-0.47$

$-0.88$

$-3.02$

$-3.21$

$-5.50$

$-5.56$

$-7.51$

$-7.38$

$-8.77$

$-8.44$

$-9.61$

$-8.87$

$-9.88$

\begin{tabular}{|c|c|c|}
\hline .00 & .0 & 0.0 \\
\hline .00 & 0.00 & 0.00 \\
\hline 0.00 & 0.00 & 0.00 \\
\hline 0.00 & 0.00 & .00 \\
\hline 0.00 & 0.00 & 0.00 \\
\hline 0.00 & 0.00 & 0.00 \\
\hline 0.00 & 0.00 & 0.00 \\
\hline 0.00 & 0.00 & 0.00 \\
\hline 0.00 & 0.00 & 0.00 \\
\hline 0.00 & 0.00 & 0.00 \\
\hline 0.00 & 0.00 & 0.00 \\
\hline 0.00 & 0.00 & 0.00 \\
\hline 0.0 & 0 & .00 \\
\hline 0.00 & 0.00 & 0.00 \\
\hline 0.00 & 0.00 & 0.00 \\
\hline 0.00 & 0.00 & 0.00 \\
\hline 0.00 & 0.00 & 0.00 \\
\hline 0.00 & 0.00 & 0.00 \\
\hline 0.00 & 0.00 & 0.00 \\
\hline 0.00 & 0.00 & 0.00 \\
\hline 0.00 & 0.00 & 0.00 \\
\hline 0.00 & 0.00 & 0.00 \\
\hline 0.00 & 0.00 & 0.00 \\
\hline 0.00 & 0.00 & 0.00 \\
\hline 0.00 & 0.00 & 0.00 \\
\hline 0.00 & 0.00 & 0.00 \\
\hline 0.00 & 0.00 & 0.00 \\
\hline 0.00 & 0.00 & 0.00 \\
\hline 0.0 & 0.00 & 0.00 \\
\hline 0.00 & 0.00 & 0.00 \\
\hline 0.00 & 0.00 & 0.00 \\
\hline 0.00 & 0.00 & 0.00 \\
\hline 0.0 & 0.00 & 0.00 \\
\hline 0.00 & 0.00 & 0.00 \\
\hline 0.00 & 0.00 & 0.00 \\
\hline 0.00 & 0.00 & 0.00 \\
\hline 0.00 & 0.00 & 0.00 \\
\hline 0.00 & 0.00 & 0.00 \\
\hline 0.00 & 0.00 & 0.00 \\
\hline 0.00 & 0.00 & 0.00 \\
\hline 0.00 & 0.00 & .00 \\
\hline 0.00 & 0.00 & 0.00 \\
\hline 0.00 & 0.00 & 0.00 \\
\hline 0.00 & 0.00 & .00 \\
\hline 0.00 & 0.00 & 0.00 \\
\hline 0.00 & 0.00 & 0.00 \\
\hline 0.00 & 0.00 & 0.00 \\
\hline 0.00 & 0.00 & .00 \\
\hline 0.00 & 0.00 & 0.00 \\
\hline 0.00 & 0.00 & 0.00 \\
\hline 0.00 & 0.00 & 0.00 \\
\hline 0.00 & 0.00 & .00 \\
\hline ח & 0.00 & 0.00 \\
\hline 0.00 & 0.00 & 0.00 \\
\hline 0.00 & 0.00 & 0.00 \\
\hline 0.00 & .00 & .00 \\
\hline 0.00 & 0.00 & 0.00 \\
\hline 0.00 & 0.00 & 0.00 \\
\hline 0.00 & 0.00 & .00 \\
\hline 0.00 & 0.00 & 0.00 \\
\hline 0.00 & 0.00 & 0.00 \\
\hline 0.00 & 0.00 & .00 \\
\hline .67 & -5.42 & -0.23 \\
\hline .00 & 0.00 & 0.00 \\
\hline 3.97 & 6.76 & -0.16 \\
\hline & .1 & \\
\hline
\end{tabular}


$\begin{array}{lllllllllll}86 & 125 & 211 & -4.0 & -7.2-1632.11 & -8.35 & 0.96 & -4.60-1644.11\end{array}$ $\begin{array}{lllllllll}86 & 126 & 212 & -4.3 & -7.0-1639.21 & -8.46 & 0.00 & -4.53-1652.20\end{array}$ $\begin{array}{lllllllll}86 & 127 & 213 & -4.5 & -6.9-1646.17 & -7.55 & 0.95 & -4.46-1657.22\end{array}$ $\begin{array}{lllllllll}86 & 128 & 214 & -4.7 & -6.7-1652.97 & -6.58 & 0.00 & -4.39-1663.94\end{array}$ $\begin{array}{lllllllll}86 & 129 & 215 & -4.9 & -6.6 & -1659.64 & -5.32 & 0.95 & -4.32-1668.32\end{array}$ $86130 \quad 216-5.2-6.4-1666.16-4.30 \quad 0.00-4.25-1674.71$ $\begin{array}{lllllllll}86 & 131 & 217 & -5.4 & -6.3-1672.54 & -3.61 & 0.95 & -4.19-1679.39\end{array}$ $\begin{array}{llllllllll}86 & 132 & 218 & -5.6 & -6.2-1678.78 & -2.84 & 0.00 & -4.12-1685.75\end{array}$ $\begin{array}{llllllllll}86 & 133 & 219 & -5.8 & -6.0-1684.89 & -2.58 & 0.94 & -4.06-1690.59\end{array}$ $\begin{array}{lllllllll}86 & 134 & 220 & -6.0 & -5.9-1690.87 & -1.98 & 0.00 & -4.00-1696.85\end{array}$ $\begin{array}{lllllllll}86 & 135 & 221 & -6.2 & -5.8-1696.72 & -1.75 & 0.94 & -3.94-1701.47\end{array}$ $\begin{array}{lllllllll}86 & 136 & 222 & -6.5 & -5.6-1702.43 & -0.98 & 0.00 & -3.88-1707.29\end{array}$ $\begin{array}{lllllllll}86 & 137 & 223 & -6.7 & -5.5-1708.02 & -0.52 & 0.93 & -3.83-1711.43\end{array}$ $\begin{array}{lllllllll}86 & 138 & 224 & -6.9 & -5.4-1713.48 & -0.04 & 0.00 & -3.77-1717.29\end{array}$ $86 \quad 139 \quad 225 \quad-7.1-5.3-1718.81$ $\begin{array}{llllll}86 & 140 & 226 & -7.3 & -5.1-1724.03\end{array}$ $\begin{array}{llllll}86 & 141 & 227 & -7.5 & -5.0 & -1729.12\end{array}$ $\begin{array}{llllll}86 & 142 & 228 & -7.7 & -4.9 & -1734.09\end{array}$ $\begin{array}{llllll}86 & 143 & 229 & -7.9 & -4.8-1738.94\end{array}$ $86 \quad 144 \quad 230-8.1-4.7-1743.68$ $\begin{array}{llllll}86 & 145 & 231 & -8.3 & -4.6-1748.30\end{array}$ $\begin{array}{llllll}86 & 146 & 232 & -8.5 & -4.5-1752.81\end{array}$ $\begin{array}{llllll}86 & 147 & 233 & -8.7 & -4.3-1.757 .21\end{array}$ $\begin{array}{llllll}86 & 148 & 234 & -8.9 & -4.2-1761.50\end{array}$ $\begin{array}{llllll}86 & 149 & 235 & -9.1 & -4.1-1765.68\end{array}$ $\begin{array}{llllll}86 & 150 & 236 & -9.3 & -4.0-1769.75\end{array}$ $\begin{array}{llllll}86 & 151 & 237 & -9.5 & -3.9-1773.72\end{array}$ $\begin{array}{llllll}86 & 152 & 238 & -9.7 & -3.8-1777.58\end{array}$ $\begin{array}{llllll}86 & 153 & 239 & -9.8 & -3.7-1781.34\end{array}$ $86 \quad 154240-10.0-3.6-1785.00$ $86 \quad 155 \quad 241-10.2-3.5-1788.56$ $86 \quad 156 \quad 242-10.4 \quad-3.4-1792.02$ $86157243-10.6-3.3-1795.38$ $\begin{array}{llll}86 & 158 & 244-10.8 & -3.2-1798.65\end{array}$ $86159 \quad 245-10.9-3.1-1801.82$ $86 \quad 160 \quad 246-11.1-3.0-1804.90$ $\begin{array}{lllll}86 & 161 & 247-11.3 & -2.9-1807.88\end{array}$

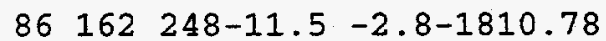
$\begin{array}{lllll}86 & 163 & 249-11.6 & -2.8-1813.58\end{array}$ $86164250-11.8 \quad-2.7-1816.30$ $86 \quad 165 \quad 251-12.0 \quad-2.6-1818.93$ $\begin{array}{lllll}86 & 166 & 252-12.2 & -2.5-1821.47\end{array}$ $86 \quad 167 \quad 253-12.3-2.4-1823.93$ $86168 \quad 254-12.5-2.3-1826.30$ $\begin{array}{lllllllll}86 & 169 & 255-12.7 & -2.2-1828.59 & -1.22 & 0.87 & -2.55-1831.49 & 158.84\end{array}$ $\begin{array}{llllllll}86 & 170 & 256-12.8 & -2.2-1830.80 & -1.48 & 0.00 & -2.52-1834.80 & 163.60\end{array}$ $\begin{array}{lllllllll}86 & 171 & 257-13.0 & -2.1-1832.92 & -2.06 & 0.86 & -2.49-1836.61 & 169.86\end{array}$ $\begin{array}{lllllllll}86 & 172 & 258-13.2 & -2.0-1834.97 & -3.25 & 0.00 & -2.47-1840.69 & 173.85\end{array}$ $\begin{array}{lllllllll}86 & 173 & 259-13.3 & -1.9-1836.94 & -2.89 & 0.86 & -2.44-1841.41 & 181.20\end{array}$ $\begin{array}{llllllll}86 & 174 & 260-13.5 & -1.8-1838.83 & -3.15 & 0.00 & -2.41-1844.40 & 186.29\end{array}$ $86175 \quad 261-13.6-1.8-1840.65-3.81 \quad 0.86-2.39-1845.99 \quad 192.77$ $\begin{array}{lllllllll}86 & 176 & 262-13.8 & -1.7-1842.39 & -4.50 & 0.00 & -2.36-1849.25 & 197.57\end{array}$ $\begin{array}{lllllllll}86 & 177 & 263-13.9 & -1.6-1844.06 & -5.11 & 0.85 & -2.34-1850.65 & 204.25\end{array}$ $\begin{array}{lllllllll}86 & 178 & 264-14.1 & -1.6-1845.65 & -5.20 & 0.00 & -2.31-1853.16 & 209.81\end{array}$ $\begin{array}{lllllllll}86 & 179 & 265-14.3 & -1.5-1847.17 & -5.87 & 0.85 & -2.29-1854.48 & 216.56\end{array}$ $\begin{array}{lllllllll}86 & 180 & 266-14.4 & -1.4-1848.62 & -6.08 & 0.00 & -2.27-1856.96 & 222.15\end{array}$ $\begin{array}{lllllllll}86 & 181 & 267-14.6 & -1.3-1850.00 & -6.60 & 0.85 & -2.24-1857.99 & 229.19\end{array}$ $86182 \quad 268-14.7-1.3-1851.31-6.63 \quad 0.00-2.22-1860.16 \quad 235.10$ $\begin{array}{lllllllll}86 & 183 & 269-14.9 & -1.2-1852.55 & -7.06 & 0.85 & -2.20-1860.96 & 242.37\end{array}$ $86184270-15.0-1.1-1853.72-6.73 \quad 0.00-2.18-1862.63 \quad 248.77$ $\begin{array}{lllllllll}86 & 185 & 271-15.1 & -1.1-1854.83 & -5.87 & 0.84 & -2.16-1862.01 & 257.46\end{array}$ $\begin{array}{lllllllll}86 & 186 & 272 & -15.3 & -1.0-1855.87 & -5.23 & 0.00 & -2.14-1863.23 & 264.31\end{array}$ $\begin{array}{lllllllll}86 & 187 & 273-15.4 & -0.9-1856.84 & -4.27 & 0.84 & -2.11-1862.39 & 273.22\end{array}$ $\begin{array}{lllllllll}86 & 188 & 274-15.6 & -0.9-1857.76 & -3.54 & 0.00 & -2.09-1863.39 & 280.29\end{array}$ $86 \quad 189275-15.7-0.8-1858.60-3.13 \quad 0.84-2.07-1862.97 \quad 288.78$ $\begin{array}{llllllll}86 & 190 & 276-15.8 & -0.8-1859.39 & -2.55 & 0.00 & -2.05-1864.00 & 295.83\end{array}$
$-8.78-8.21$

$-8.68-8.19$

0.14

$-5.72-7.38$

0.27

$-4.34-6.38$

0.17

$-1.19-5.66-0.34$

$-5.66-0.34$

$0.23-4.90-0.60$

$3.63-4.20-0.59$

$\begin{array}{llll}5.20 & -3.58 & -0.74\end{array}$

$8.83-2.91-0.33$

$10.59-2.37-0.39$

$\begin{array}{llll}0.00 & 0.00 & 0.00\end{array}$

$\begin{array}{llll}16.37 & -1.29 & -0.31\end{array}$

$0.00 \quad 0.00 \quad 0.00$

$0.00 \quad 0.00 \quad 0.00$

$0.00 \quad 0.00 \quad 0.00$

$\begin{array}{llll}0.00 & 0.00 & 0.00\end{array}$

$\begin{array}{llll}0.00 & 0.00 & 0.00\end{array}$

$0.00 \quad 0.00 \quad 0.00$

$0.00 \quad 0.00 \quad 0.00$

$\begin{array}{llll}0.00 & 0.00 & 0.00\end{array}$

$\begin{array}{llll}0.00 & 0.00 & 0.00\end{array}$

$\begin{array}{llll}0.00 & 0.00 & 0.00\end{array}$

$0.00 \quad 0.00 \quad 0.00$

$\begin{array}{llll}0.00 & 0.00 & 0.00\end{array}$

$0.00 \quad 0.00 \quad 0.00$

$\begin{array}{llll}0.00 & 0.00 & 0.00\end{array}$

$0.00 \quad 0.00 \quad 0.00$

$\begin{array}{llll}0.00 & 0.00 & 0.00\end{array}$

$\begin{array}{llll}0.00 & 0.00 & 0.00\end{array}$

$\begin{array}{llll}0.00 & 0.00 & 0.00\end{array}$

$0.00 \quad 0.00 \quad 0.00$

$\begin{array}{llll}0.00 & 0.00 & 0.00\end{array}$

$\begin{array}{llll}0.00 & 0.00 & 0.00\end{array}$

$\begin{array}{llll}0.00 & 0.00 & 0.00\end{array}$

$0.00 \quad 0.00 \quad 0.00$

$0.00 \quad 0.00 \quad 0.00$

$\begin{array}{llll}0.00 & 0.00 & 0.00\end{array}$

$\begin{array}{llll}0.00 & 0.00 & 0.00\end{array}$

$\begin{array}{llll}0.00 & 0.00 & 0.00\end{array}$

$0.00 \quad 0.00 \quad 0.00$

$\begin{array}{llll}0.00 & 0.00 & 0.00\end{array}$

$\begin{array}{llll}0.00 & 0.00 & 0.00\end{array}$

$\begin{array}{llll}0.00 & 0.00 & 0.00\end{array}$

$0.00 \quad 0.00 \quad 0.00$

$\begin{array}{llll}0.00 & 0.00 & 0.00\end{array}$

$\begin{array}{lll}0.00 & 0.00 & 0.00\end{array}$

$0.00 \quad 0.00 \quad 0.00$

$\begin{array}{llll}0.00 & 0.00 & 0.00\end{array}$

$\begin{array}{llll}0.00 & 0.00 & 0.00\end{array}$

$\begin{array}{lll}0.00 & 0.00 & 0.00\end{array}$

$\begin{array}{llll}0.00 & 0.00 & 0.00\end{array}$

$\begin{array}{llll}0.00 & 0.00 & 0.00\end{array}$

$\begin{array}{llll}0.00 & 0.00 & 0.00\end{array}$

$\begin{array}{llll}0.00 & 0.00 & 0.00\end{array}$

$\begin{array}{llll}0.00 & 0.00 & 0.00\end{array}$

$\begin{array}{llll}0.00 & 0.00 & 0.00\end{array}$

$\begin{array}{llll}0.00 & 0.00 & 0.00\end{array}$

$\begin{array}{llll}0.00 & 0.00 & 0.00\end{array}$

$\begin{array}{llll}0.00 & 0.00 & 0.00\end{array}$

$\begin{array}{llll}0.00 & 0.00 & 0.00\end{array}$

$0.00 \quad 0.00 \quad 0.00$

$\begin{array}{llll}0.00 & 0.00 & 0.00\end{array}$

$\begin{array}{llll}0.00 & 0.00 & 0.00\end{array}$

$\begin{array}{llll}0.00 & 0.00 & 0.00\end{array}$

$0.00 \quad 0.00 \quad 0.00$

$0.00 \quad 0.00 \quad 0.00$ 
$\begin{array}{llllllllll}86 & 191 & 277-16.0 & -0.7-1860.12 & -1.26 & 0.83 & -2.04-1862.58 & 305.32\end{array}$ $86192 \quad 278-16.1 \quad-0.6-1860.79-1.11 \quad 0.00-2.02-1863.91312 .06$ $\begin{array}{lllllllll}86 & 193 & 279-16.3 & -0.6-1861.39 & -1.37 & 0.83 & -2.00-1863.93 & 320.11\end{array}$ $86 \quad 194280-16.4 \quad-0.5-1861.94-1.26 \quad 0.00-1.98-1865.18 \quad 326.93$ $\begin{array}{lllllllll}86 & 195 & 281-16.5 & -0.5-1862.43 & -1.39 & 0.83 & -1.96-1864.96 & 335.23\end{array}$ $\begin{array}{lllllllll}86 & 196 & 282-16.7 & -0.4-1862.87 & -1.17 & 0.00 & -1.94-1865.98 & 342.28\end{array}$ $\begin{array}{lllllllll}86 & 197 & 283-16.8 & -0.3-1863.25 & -1.35 & 0.82 & -1.93-1865.70 & 350.63\end{array}$ $86198284-16.9-0.3-1863.57-1.52 \quad 0.00-1.91-1867.00 \quad 357.40$ $\begin{array}{lllllllll}86 & 199 & 285-17.0 & -0.2-1863.84 & -1.69 & 0.82 & -1.89-1866.60 & 365.87\end{array}$ $86200286-17.2-0.2-1864.06-1.570 .00-1.87-1867.50373 .04$ $87102189 \quad 2.1-11.5-1421.02$ $87103190 \quad 1.8-11.3-1432.43$ 87104191 1.5-11.1-1443.63 $871051921.3-10.9-1454.62$ $87106193 \quad 1.0-10.7-1465.42$ $87107194 \quad 0.8-10.5-1476.01$ 87108195 87109196 $0.5-10.3-1486.42$ $0.191 .08-7.17-1426.91$ $0.261 .91-7.02-1437.28$ $0.441 .08-6.88-1448.98$ $0.531 .90-6.75-1458.93$ $0.811 .08-6.61-1470.14$ $1.161 .90-6.49-1479.44$ $1.191 .08-6.36-1490.50$ $0.3-10.1-1496.62$ $1.471 .89-6.24-1499.50$ 87110197 $0.0-9.9-1506.64$ $\begin{array}{lllll}87 & 111 & 198 & -0.2 & -9.7-1516.48\end{array}$ $\begin{array}{llllll}87 & 112 & 199 & -0.5 & -9.6-1526.12\end{array}$ $\begin{array}{llllll}87 & 113 & 200 & -0.7 & -9.4-1535.59\end{array}$ $87114201-1.0 \quad-9.2-1544.88$ $\begin{array}{llllll}87 & 115 & 202 & -1.2 & -9.0-1553.99\end{array}$ $\begin{array}{lllllllll}87 & 116 & 203 & -1.5 & -8.8-1562.93 & -0.80 & 1.08 & -5.49-1568.13\end{array}$ $\begin{array}{llllllllll}87 & 117 & 204 & -1.7 & -8.7-1571.69 & -1.35 & 1.87 & -5.39-1576.56\end{array}$ $\begin{array}{lllllllll}87 & 118 & 205 & -1.9 & -8.5-1580.29 & -2.27 & 1.08 & -5.30-1586.77\end{array}$ $87 \begin{array}{llllllll}819 & 206 & -2.2 & -8.3-1588.72 & -3.05 & 1.87 & -5.21-1595.10\end{array}$ $\begin{array}{lllllllll}87 & 120 & 207 & -2.4 & -8.2-1596.98 & -3.66 & 1.08 & -5.12-1604.68\end{array}$ $87121208-2.6-8.0-1605.08-4.311 .87-5.03-1612.56$ $87 \begin{array}{llllllll}822 & 209 & -2.9 & -7.9-1613.03 & -4.90 & 1.08 & -4.95-1621.79\end{array}$ $\begin{array}{lllllllll}87 & 123 & 210 & -3.1 & -7.7-1620.81 & -5.65 & 1.86 & -4.87-1629.47\end{array}$ $87 \begin{array}{llllllll}824 & 211 & -3.3 & -7.5 & -1628.44 & -6.39 & 1.08 & -4.79-1638.54\end{array}$ $\begin{array}{lllllllll}87 & 125 & 212 & -3.6 & -7.4 & -1635.92 & -7.26 & 1.86 & -4.71-1646.03\end{array}$ $\begin{array}{lllllllll}87 & 126 & 213 & -3.8 & -7.2-1643.25 & -7.33 & 1.08 & -4.63-1654.13\end{array}$

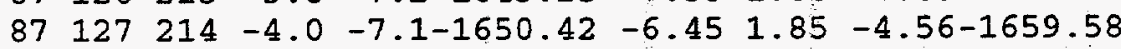
$87 \begin{array}{llllllll}128 & 215 & -4.2 & -7.0-1657.46 & -5.54 & 1.08 & -4.49-1666.40\end{array}$ $87 \begin{array}{llllllll}129 & 216 & -4.5 & -6.8-1664.34 & -4.31 & 1.85 & -4.42-1671.22\end{array}$ $87 \begin{array}{llllllll}830 & 217 & -4.7 & -6.7 & -1671.08 & -3.22 & 1.08 & -4.35-1677.57\end{array}$ $87 \quad 131218-4.9-6.5-1677.69-3.12 .1 .85-4.28-1683.24$ $87 \begin{array}{llllllll}832 & 219 & -5.1 & -6.4-1684.15 & -2.69 & 1.08 & -4.22-1689.98\end{array}$ $\begin{array}{lllllllll}87 & 133 & 220 & -5.3 & -6.3-1690.48 & -2.44 & 1.84 & -4.16-1695.23\end{array}$ $87134221-5.6 \quad-6.1-1696.67-2.00 \quad 1.08-4.09-1701.68$ $\begin{array}{lllllllll}87 & 135 & 222 & -5.8 & -6.0 & -1702.73 & -1.57 & 1.84 & -4.03-1706.49\end{array}$ $\begin{array}{lllllllll}87 & 136 & 223 & -6.0 & -5.9-1708.65 & -1.02 & 1.08 & -3.97-1712.56\end{array}$ $\begin{array}{lllllllll}87 & 137 & 224 & -6.2 & -5.7-1714.45 & -0.70 & 1.84 & -3.92-1717.23\end{array}$ $\begin{array}{lllllllll}87 & 138 & 225 & -6.4 & -5.6-1720.12 & -0.35 & 1.08 & -3.86-1723.25\end{array}$ $\begin{array}{lllllllll}87 & 139 & 226 & -6.6 & -5.5-1725.67 & -0.25 & 1.83 & -3.80-1727.89\end{array}$ $87 \begin{array}{llllllll}840 & 227 & -6.8 & -5.4-1731.08 & 0.06 & 1.08 & -3.75-1733.69\end{array}$ $87 \begin{array}{lllll}841 & 228 & -7.0 & -5.2-1736.38\end{array}$ $87 \begin{array}{llll}842 & 229 & -7.2 & -5.1-1741.56\end{array}$ $87 \begin{array}{lllll}843 & 230 & -7.4 & -5.0 & -1746.61\end{array}$ $87 \begin{array}{lllll}844 & 231 & -7.6 & -4.9 & -1751.55\end{array}$ $\begin{array}{llllll}87 & 145 & 232 & -7.8 & -4.8-1756.38\end{array}$ $\begin{array}{llllll}87 & 146 & 233 & -8.0 & -4.6-1761.08\end{array}$ $\begin{array}{lllll}87 & 147 & 234 & -8.2 & -4.5-1765.68\end{array}$ $\begin{array}{lllll}87 & 148 & 235 & -8.4 & -4.4-1770.16\end{array}$ $\begin{array}{llllll}87 & 149 & 236 & -8.6 & -4.3-1774.54\end{array}$ $\begin{array}{llllll}87 & 150 & 237 & -8.8 & -4.2-1778.80\end{array}$ $\begin{array}{llllll}87 & 151 & 238 & -9.0 & -4.1-1782.96\end{array}$ $\begin{array}{lllll}87 & 152 & 239 & -9.2 & -4.0-1787.01\end{array}$ $\begin{array}{llllll}87 & 153 & 240 & -9.4 & -3.9-1790.96\end{array}$ $87 \begin{array}{lllll}854 & 241 & -9.6 & -3.8-1794.81\end{array}$ $87 \begin{array}{lllll}155 & 242 & -9.8 & -3.7-1798.55\end{array}$ $\begin{array}{llllll}87 & 156 & 243 & -9.9 & -3.6-1802.19\end{array}$ $87157244-10.1-3.5-1805.74$ $0.011 .83-3.70-1738.24$ $0.191 .08-3.65-1743.93$ $0.041 .83-3.60-1748.34$ $0.181 .08-3.55-1753.84$ $-0.051 .82-3.50-1758.10$ $0.091 .08-3.45-1763.36$ $-0.171 .82-3.41-1767.44$ $0.011 .08-3.36-1772.43$ $-0.251 .82-3.32-1776.29$ $-0.051 .08-3.27-1781.04$ $-0.361 .81-3.23-1784.74$ $-0.291 .08-3.19-1789.41$ $\begin{array}{lll}-0.08 & 1.81 & -3.15-1792.38\end{array}$ $0.391 .08-3.11-1796.44$ $0.461 .81-3.07-1799.36$ $0.751 .08-3.03-1803.39$ $0.701 .80-3.00-1806.23$

29.90

27.61

23.97

22.09

18.96

17.73

14.74

13.81

10.94

9.81

7.28

6.51

4.27

3. 71

1. 68

1.33

$-0.82$

$-1.08$

$-2.58$

$-2.39$

$-3.55$

$-3.16$

$-4.15$

$-3.58$

$-3.60$

$-0.98$

0.27

3.52

5.24

7.64

8.98

11.80

13.42

16.68

18.68

22.08

24.14

27.57

29.84

33.36

35.74

39.40

41.98

45.78

48.59

52.59

55.67

59.88

63.20

67.57

70.97

0.00

0.00

0.00

0.00

0.00

0.00

0.00

0.00

0.00

0.00

0.00

0.00

0.00

0.00

0.00

0.00

0.00

0.00

0.00

0.00

0.00

0.00

0.00

0.00

0.00

0.00

0.00

0.00

0.00

0.00

0.00

0.00

0.00

0.00

0.00

0.00

0.00

0.00

0.00

0.00

0.00

0.00

0.00

0.00

0.00

0.00

0.00

0.00

0.00

0.00

0.00

0.00

0.00

0.00

0.00

0.00

0.00

0.00

0.00

0.00

0.00

0.00

0.00

0.00

$0.00 \quad 0.00$

0.00

$0.00 \quad 0.00$

$0.65-2.03-0.68$

$\begin{array}{llll}-1.26 & -2.71 & -0.44\end{array}$

$-1.41-3.38-0.33$

$-2.96-4.04-0.38$

$\begin{array}{llll}-2.70 & -4.62 & -0.31\end{array}$

$\begin{array}{llll}-3.83 & -5.18 & -0.28\end{array}$

$\begin{array}{llll}-3.40 & -5.89 & -0.24\end{array}$

$-4.20-6.44-0.05$

$\begin{array}{llll}-3.60 & -7.28 & -0.02\end{array}$

$-3.57-7.30 \cdot 0.03$

$\begin{array}{llll}-0.98 & -6.45 & 0.00\end{array}$

$\begin{array}{llll}0.29 & -5.52 & 0.02\end{array}$

$2.96-4.87-0.56$

$\begin{array}{llll}4.29 & -4.17 & -0.95\end{array}$

$\begin{array}{llll}7.04 & -3.72 & -0.60\end{array}$

$8.61-3.06-0.37$

$11.46-2.78-0.34$

$13.27-2.15-0.15$

$16.38-1.87-0.30$

$18.38-1.32-0.30$

$21.63-1.15-0.45$

$23.85-0.64-0.29$

$\begin{array}{llll}27.30 & -0.52 & -0.27\end{array}$

$29.59-0.19-0.25$

$\begin{array}{llll}0.00 & 0.00 & 0.00\end{array}$

$\begin{array}{llll}0.00 & 0.00 & 0.00\end{array}$

0.00

0.00

0.00

0.00

0.00

0.00

0.00

0.00

0.00

0.00

0.00

0.00

0.00

0.00

0.00

0.00

0.00

0.00

0.00

0.00

0.00

0.00

0.00

76.07

0.00

0.00

0.00

80.08

0.00

0.00

0.00

85.24

0.00

0.00

0.00

89.28

0.00

0.00

0.00

94.51

0.00

0.00

0.00

0.00

0.00 
$87 \quad 158 \quad 245-10.3 \quad-3.4-1809.19$ $87 \quad 159246-10.5-3.3-1812.54$ $\begin{array}{lllll}87 & 160 & 247-10.7 & -3.2-1815.79\end{array}$ $87161248-10.8-3.1-1818.96$ $87162 \quad 249-11.0-3.0-1822.03$ $87163250-11.2-2.9-1825.01$ $87164251-11.4 \quad-2.8-1827.90$ $87 \quad 165 \quad 252-11.5 \quad-2.8-1830.70$ $87166 \quad 253-11.7-2.7-1833.41$ $87167254-11.9-2.6-1836.03$ $87168 \quad 255-12.1-2.5-1838.58$ $87169256-12.2-2.4-1841.03$ $87 \quad 170 \quad 257-12.4-2.3-1843.41$ $87 \quad \begin{array}{llll}871 & 258-12.6 & -2.2-1845.70\end{array}$ $87172 \quad 259-12.7 \quad-2.2-1847.91$ $87173 \quad 260-12.9-2.1-1850.04$ $87 \quad 174261-13.0-2.0-1852.09$ $87 \begin{array}{lll}875 & 262-13.2 & -1.9-1854.07\end{array}$ $87176263-13.4-1.9-1855.96$ $87177264-13.5-1.8-1857.79$ $87 \quad 178 \quad 265-13.7-1.7-1859.53$ $87179266-13.8-1.6-1861.21$ $87180267-14.0-1.6-1862.81$ $87181268-14.1-1.5-1864.34$ $87182 \quad 269-14.3-1.4-1865.80$ $87183 \quad 270-14.4-1.4-1867.19$ $87184.271-14.6-1.3-1868.51$ $87 \quad 185 \quad 272-14.7-1.2-1869.76$ $87186273-14.9-1.1-1870.95$ $87 \quad 187274-15.0-1.1-1872.06$ $87188275-15.2-1.0-1873.12$ $87189276-15.3-1.0-1874.11$ $87190 \quad 277-15.4-0.9-1875.04$ $87191278-15.6-0.8-1875.90$ $87192 \quad 279-15.7-0.8-1876.70$ $87193280-15.8-0.7-1877.45$ $87194281-16.0-0.6-1878.13$ $87195282-16.1-0.6-1878.75$ $87196283-16.2-0.5-1879.32$ $87197284-16.4-0.5-1879.83$ $87198 \quad 285-16.5-0.4-1880.28$ $87199286-16.6-0.4-1880.68$ $87200287-16.8-0.3-1881.02$ $87201288-16.9-0.3-1881.31$ $87202 \quad 289-17.0-0.2-1881.54$ $88104192 \quad 2.0-11.4-1441.86$ $881051931.7-11.1-1453.12$ $88106 \quad 194 \quad 1.5-10.9-1464.17$ $88107 \quad 195 \quad 1.2-10.7-1475.02$ $88108196 \quad 1.0-10.6-1485.67$ $88109 \quad 197 \cdot 0.7-10.4-1496.13$ $88110198 \quad 0.5-10.2-1506.41$ $88111 \quad 199 \quad 0.2-10.0-1516.49$ $\begin{array}{llllll}88 & 112 & 200 & 0.0 & -9.8-1526.38\end{array}$ $\begin{array}{llllll}88 & 113 & 201 & -0.3 & -9.6-1536.10\end{array}$ $\begin{array}{llllll}88 & 114 & 202 & -0.5 & -9.4-1545.63\end{array}$ $\begin{array}{llllll}88 & 115 & 203 & -0.8 & -9.3-1554.98\end{array}$ $\begin{array}{llllll}88 & 116 & 204 & -1.0 & -9.1-1564.16\end{array}$ $\begin{array}{llllll}88 & 117 & 205 & -1.2 & -8.9-1573.17\end{array}$ $\begin{array}{llllll}88 & 118 & 206 & -1.5 & -8.7-1582.00\end{array}$ $\begin{array}{llllll}88 & 119 & 207 & -1.7 & -8.6-1590.67\end{array}$ $\begin{array}{llllllllll}88 & 120 & 208 & -2.0 & -8.4-1599.17 & -2.90 & 0.00 & -5.24-1607.31\end{array}$ $\begin{array}{lllllllll}88 & 121 & 209 & -2.2 & -8.3-1607.51 & -3.51 & 0.97 & -5.15-1615.20\end{array}$ $\begin{array}{lllllllll}88 & 122 & 210 & -2.4 & -8.1-1615.68 & -3.98 & 0.00 & -5.07-1624.73\end{array}$ $\begin{array}{lllllllll}88 & 123 & 211 & -2.7 & -7.9 & -1623.70 & -4.74 & 0.97 & -4.98-1632.46\end{array}$ $\begin{array}{lllllllll}88 & 124 & 212 & -2.9 & -7.8 & -1631.56 & -5.44 & 0.00 & -4.90-1641.90\end{array}$ 89
$0.851 .08-2.96-1810.2198 .60$ $0.521 .80-2.93-1813.14 \quad 103.74$ $0.491 .08-2.89-1817.11107 .85$ $0.101 .80-2.86-1819.91113 .11$ $\begin{array}{lllll}0.11 & 1.08 & -2.82-1823.66 & 117.44\end{array}$ $\begin{array}{llllll}-0.17 & 1.80 & -2.79-1826.17 & 123.00\end{array}$ $\begin{array}{lllll}1.23 & 1.08 & -2.76-1828.34 & 128.90\end{array}$ $0.931 .79-2.73-1830.70 \quad 134.62$ $0.671 .08-2.69-1834.35 \quad 139.04$ $0.071 .79-2.66-1836.84 \quad 144.62$ $\begin{array}{lllll}-0.36 & 1.08 & -2.63-1840.49 & 149.04\end{array}$ $\begin{array}{lllll}-0.89 & 1.79 & -2.60-1842.74 & 154.86\end{array}$ $\begin{array}{lllll}-1.20 & 1.08 & -2.58-1846.10 & 159.57\end{array}$ $\begin{array}{lllll}-1.77 & 1.79 & -2.55-1848.23 & 165.51\end{array}$ $\begin{array}{llllll}-2.85 & 1.08 & -2.52-1852.20 & 169.62\end{array}$ $\begin{array}{lllll}2.52 & 1.78 & -2.49-1853.27 & 176.61\end{array}$

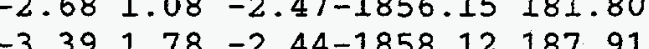
$\begin{array}{llll}-4.10 & 1.08 & -2.41-1861.40 & 192.70\end{array}$ $\begin{array}{llll}-4.67 & 1.78 & -2.39-1863.07 & 199.10\end{array}$ $\begin{array}{llll}-4.63 & 1.08 & -2.36-1865.45 & 204.80\end{array}$ $\begin{array}{llllll}-5.26 & 1.78 & -2.34-1867.03 & 211.28\end{array}$ $\begin{array}{lllll}.41 & 1.08 & -2.32-1869.45 & 216.93\end{array}$ $\begin{array}{llll}5.99 & 1.77-2.29-1870.85 & 223.61\end{array}$ $-6.421 .77-2.25-1874.08236 .52$ $\begin{array}{lllll}-6.07 & 1.08 & -2.22-1875.72 & 242.95\end{array}$ $\begin{array}{lllll}-5.20 & 1.77 & -2.20-1875.39 & 251.35\end{array}$ $\begin{array}{lllll}-4.57 & 1.08 & -2.18-1876.61 & 258.20\end{array}$ $\begin{array}{lllll}-3.56 & 1.77 & -2.16-1876.02 & 266.87\end{array}$ $\begin{array}{lllll}2.79 & 1.08 & -2.14-1876.96 & 273.99\end{array}$ $\begin{array}{lllll}1.45 & 1.76 & -2.12-1875.91 & 283.11\end{array}$ $-1.271 .08-2.10-1877.32 \quad 289.78$ $\begin{array}{llllll}-1.41 & 1.76 & -2.08-1877.63 & 297.54\end{array}$ $\begin{array}{lllll}1.27 & 1.08 & -2.06-1878.95 & 304.29\end{array}$ $-1.531 .76-2.04-1879.26 \quad 312.06$ $-1.441 .08-2.02-1880.51318 .88$ $-1.441 .76-2.00-1880.44327 .02$ $-1.351 .08-1.98-1881.57 \quad 333.96$ $\begin{array}{lllll}-1.12 & 1.76 & -1.97-1881.16 & 342.44\end{array}$ $-0.651 .08-1.95-1881.79 \quad 349.88$ $\begin{array}{lllll}-1.89 & 1.75 & -1.93-1882.74 & 357.00\end{array}$ $-1.721 .08-1.91-1883.57 \quad 364.24$ $-0.041 .75-1.90-1881.49374 .39$ $0.111 .08-1.88-1882.23 \quad 381.72$ $0.350 .00-7.05-1448.56 \quad 31.67$ $0.591 .02-6.91-1458.42$ $0.880 .00-6.77-1470.06$ $1.141 .01-6.64-1479.51$ $1.270 .00-6.51-1490.92$ $1.271 .00-6.39-1500.25$ $1.650 .00-6.27-1511.03$ $1.371 .00-6.15-1520.27$ $1.220 .00-6.04-1531.20$ $0.890 .99-5.93-1540.14$ $0.65 \quad 0.00-5.82-1550.80$ $\begin{array}{llll}0.25 & 0.99 & -5.72-1559.46\end{array}$ $-0.230 .00-5.62-1570.01$ $\begin{array}{llll}-0.78 & 0.98 & -5.52-1578.49\end{array}$ $\begin{array}{llll}-1.61 & 0.00 & -5.42-1589.04\end{array}$ $\begin{array}{lll}1.61 & 0.00 & -5.42-1589.04 \\ -31 & 0.98-5.33-1597.33\end{array}$ 29.88 26.31 24.93 21.60 20.33

17.63 16.45 13.60 12.73

10.14 9.55 7.08 6.67

4.19 3.97

2.06

2.24

0.78

1. 13 $-0.25$
0.00

0.00 0.00

0.00 0.00

0.00 0.00 0.00

0.00

0.00

0.00 0.00 0.00 0.00

0.00

0.00

0.00 0.00 0.00

0.00 0.00 0.00

0.00

0.00

0.00

0.00

0.00

0.00

0.00

0.00

0.00

0.00

0.00

0.00

0.00

0.00

0.00

0.00

0.00

0.00

0.00

0.00

0.00

0.00

0.00

0.00

0.00

0.00

0.00

0.00

0.00

0.00

0.00

0.00

0.00

0.00

0.00

0.00

0.00

0.00

0.00

0.00

0.00

0.00

0.00

0.00

0.00

0.00

0.00

0.00

0.00

0.00

0.00

0.00

0.00

0.00

0.00

0.00

0.00

0.00

0.00

0.00

0.00

0.00

0.00

0.00

0.00

0.00

0.00

0.00

0.00

0.00

0.00

0.00

0.00

0.00

0.00

0.00

0.00

0.00

0.00

0.00

0.00

0.00

0.00

0.00

0.00

0.00

0.00

0.00

0.00

0.00

0.00

0.00

0.00

0.00

0.00

0.00

0.00

0.00

0.00

0.00

0.00

0.00

0.00

0.00

$0.80-5.07$

0.00

0.00

0.00

0.00

0.00

0.00

0.00

0.00

0.00

0.00

0.00

0.00

0.00

0.00

0.00

0.00

0.00

0.00 
$\begin{array}{lllllllllll}88 & 125 & 213 & -3.1 & -7.6-1639.27 & -6.24 & 0.96 & -4.82-1649.37\end{array}$ $\begin{array}{llllllllll}88 & 126 & 214 & -3.3 & -7.5-1646.82 & -6.35 & 0.00 & -4.74-1657.91\end{array}$ $\begin{array}{lllllllll}88 & 127 & 215 & -3.6 & -7.3-1654.22 & -5.53 & 0.95 & -4.67-1663.47\end{array}$ $\begin{array}{llllllllll}88 & 128 & 216 & -3.8 & -7.2-1661.48 & -4.59 & 0.00 & -4.59-1670.66\end{array}$ $\begin{array}{llllllllll}88 & 129 & 217 & -4.0 & -7.0 & -1668.59 & -3.42 & 0.95 & -4.52-1675.58\end{array}$ $\begin{array}{llllllllll}88 & 130 & 218 & -4.2 & -6.9-1675.55 & -2.37 & 0.00 & -4.45-1682.37\end{array}$ $\begin{array}{lllllllll}88 & 131 & 219 & -4.5 & -6.7 & -1682.37 & -2.69 & 0.95 & -4.38-1688.50\end{array}$ $\begin{array}{lllllllll}88 & 132 & 220 & -4.7 & -6.6-1689.06 & -2.13 & 0.00 & -4.32-1695.50\end{array}$ $\begin{array}{llllllllll}88 & 133 & 221 & -4.9 & -6.5-1695.60 & -2.07 & 0.94 & -4.25-1700.98\end{array}$ $\begin{array}{llllllllll}88 & 134 & 222 & -5.1 & -6.3-1702.01 & -1.70 & 0.00 & -4.19-1707.90\end{array}$ $\begin{array}{lllllllll}88 & 135 & 223 & -5.3 & -6.2-1708.28 & -1.16 & 0.94 & -4.13-1712.63\end{array}$ $\begin{array}{lllllllll}88 & 136 & 224 & -5.5 & -6.1-1714.42 & -0.64 & 0.00 & -4.07-1719.13\end{array}$ $\begin{array}{lllllllll}88 & 137 & 225 & -5.7 & -5.9-1720.43 & -0.68 & 0.93 & -4.01-1724.18\end{array}$ $\begin{array}{lllllllllll}88 & 138 & 226 & -6.0 & -5.8-1726.31 & -0.30 & 0.00 & -3.95-1730.56\end{array}$ $\begin{array}{lllllllll}88 & 139 & 227 & -6.2 & -5.7-1732.06 & -0.23 & 0.93 & -3.89-1735.26\end{array}$ $\begin{array}{llllllll}88 & 140 & 228 & -6.4 & -5.6-1737.69 & 0.05 & 0.00 & -3.84-1741.47\end{array}$ $\begin{array}{llllll}88 & 141 & 229 & -6.6 & -5.4 & -1743.19\end{array}$ $\begin{array}{llllll}88 & 142 & 230 & -6.8 & -5.3-1748.57\end{array}$ $\begin{array}{llllll}88 & 143 & 231 & -7.0 & -5.2-1753.83\end{array}$ $\begin{array}{llllll}88 & 144 & 232 & -7.2 & -5.1 & -1758.97\end{array}$ $\begin{array}{llllll}88 & 145 & 233 & -7.4 & -5.0-1763.99\end{array}$ $\begin{array}{llllll}88 & 146 & 234 & -7.6 & -4.8-1768.90\end{array}$ $\begin{array}{lllllll}88 & 147 & 235 & -7.8 & -4.7-1773.69\end{array}$ $\begin{array}{llllll}88 & 148 & 236 & -8.0 & -4.6-1778.37\end{array}$ $\begin{array}{llllll}88 & 149 & 237 & -8.2 & -4.5-1782.94\end{array}$ $\begin{array}{llllll}88 & 150 & 238 & -8.4 & -4.4-1787.39\end{array}$ $\begin{array}{llllll}88 & 151 & 239 & -8.6 & -4.3-1791.74\end{array}$ $\begin{array}{llllll}88 & 152 & 240 & -8.7 & -4.2-1795.99\end{array}$ $\begin{array}{llllll}88 & 153 & 241 & -8.9 & -4.1-1800.12\end{array}$ $\begin{array}{llllll}88 & 154 & 242 & -9.1 & -4.0-1804.16\end{array}$ 88 155. $243-9.3-3.9-1808.09$ $\begin{array}{llllll}88 & 156 & 244 & -9.5 & -3.8-1811.91\end{array}$ $\begin{array}{llllll}88 & 157 & 245 & -9.7 & -3.7-1815.64\end{array}$ $\begin{array}{llllll}88 & 158 & 246 & -9.9 & -3.6-1819.27\end{array}$ $88 \quad 159 \quad 247-10.0 \quad-3.5-1822.81$ $88 \quad 160 \quad 248-10.2 \quad-3.4-1826.24$ $\begin{array}{lllll}88 & 161 & 249-10.4 & -3.3-1829.58\end{array}$ $\begin{array}{lllll}88 & 162 & 250-10.6 & -3.2-1832.83\end{array}$ $\begin{array}{llll}88 & 163 & 251-10.8 & -3.1-1835.98\end{array}$ $\begin{array}{lllll}88 & 164 & 252-10.9 & -3.0-1839.05\end{array}$ $88 \quad 165 \quad 253-11.1-2.9-1842.02$ $88 \quad 166 \quad 254-11.3 \quad-2.8-1844.90$ $88 \quad 167255-11.4 \quad-2.7-1847.70$ $88 \quad 168 \quad 256-11.6 \quad-2.7-1850.41$ $88 \quad 169257-11.8-2.6-1853.03$ $88170 \quad 258-11.9-2.5-1855.57$ $88 \quad 171 \quad 259-12.1-2.4-1858.03$ $\begin{array}{llll}88 & 172 & 260-12.3 & -2.3-1860.41\end{array}$ $88 \quad 173 \quad 261-12.4-2.2-1862.70-1.9200 .86-2.55-1866.31170 .85$ $\begin{array}{lllllllll}88 & 174 & 262-12.6 & -2.2-1864.91 & -2.10 & 0.00 & -2.52-1869.53 & 175.70\end{array}$ $88 \quad 175 \quad 263-12.8 \quad-2.1-1867.05-2.75 \quad 0.86 \quad-2.49-1871.43 \quad 181.87$ $\begin{array}{lllllllll}88 & 176 & 264-12.9 & -2.0-1869.10 & -3.55 & 0.00 & -2.47-1875.12 & 186.25\end{array}$ $\begin{array}{llllllllll}88 & 177 & 265-13.1 & -1.9-1871.08 & -3.99 & 0.85 & -2.44-1876.66 & 192.78\end{array}$ $88178266-13.2-1.9-1872.99-4.040 .00-2.41-1879.44198 .07$ $\begin{array}{lllllllll}88 & 179 & 267-13.4 & -1.8-1874.82 & -4.49 & 0.85 & -2.39-1880.84 & 204.74\end{array}$ $\begin{array}{lllllllll}88 & 180 & 268-13.5 & -1.7-1876.57 & -4.64 & 0.00 & -2.37-1883.58 & 210.08\end{array}$ $\begin{array}{lllllllll}88 & 181 & 269-13.7 & -1.6-1878.25 & -5.14 & 0.85 & -2.34-1884.89 & 216.84\end{array}$ $\begin{array}{lllllllll}88 & 182 & 270-13.8 & -1.6-1879.86 & -5.13 & 0.00 & -2.32-1887.31 & 222.49\end{array}$ $\begin{array}{lllllllll}88 & 183 & 271-14.0 & -1.5-1881.40 & -5.52 & 0.85 & -2.29-1888.37 & 229.50\end{array}$ $88 \quad 184 \quad 272-14.1 \quad-1.4-1882.87-5.150 .00-2.27-1890.29 \quad 235.65$ $88 \quad 185 \quad 273-14.3 \quad-1.4-1884.27-4.28 \quad 0.84-2.25-1889.96 \quad 244.06$ $88186274-14.4-1.3-1885.60-3.66 \quad 0.00-2.23-1891.49250 .60$ $\begin{array}{lllllllll}88 & 187 & 275-14.6 & -1.2-1886.86 & -2.71 & 0.84 & -2.20-1890.94 & 259.22\end{array}$ $88188276-14.7-1.2-1888.06-1.95 \quad 0.00-2.18-1892.20266 .03$ $\begin{array}{llllllllll}88 & 189 & 277-14.9 & -1.1-1889.20 & -1.33 & 0.84 & -2.16-1891.85 & 274.45\end{array}$ $88190278-15.0-1.0-1890.26-1.130 .00-2.14-1893.54280 .84$ $\begin{array}{rrr}0.31 & -6.29 & -0.05 \\ 0.08 & -6.15 & 0.20\end{array}$ $2.51-5.42 \quad 0.11$ $\begin{array}{llll}3.27 & -4.60 & -0.01\end{array}$ $\begin{array}{llll}5.86 & -3.99 & -0.57\end{array}$ $6.63-3.45-1.08$ $9.36-2.98-0.29$ $\begin{array}{llll}10.25 & -2.60 & -0.47\end{array}$ $\begin{array}{llll}12.94 & -2.45 & -0.38\end{array}$ $\begin{array}{llll}14.30 & -1.87 & -0.17\end{array}$ $17.23-1.74-0.58$ $\begin{array}{llll}18.80 & -1.23 & -0.59\end{array}$ $\begin{array}{llll}21.99 & -1.09 & -0.41\end{array}$ $23.66-0.74-0.44$ $27.17-0.53-0.30$ $28.94-0.34-0.39$ $\begin{array}{llll}32.66 & -0.16 & -0.07\end{array}$

$0.00 \quad 0.00 \quad 0.00$

$0.00 \quad 0.00 \quad 0.00$

$\begin{array}{llll}0.00 & 0.00 & 0.00\end{array}$

$\begin{array}{llll}0.00 & 0.00 & 0.00\end{array}$

$\begin{array}{llll}0.00 & 0.00 & 0.00\end{array}$

$0.00 \quad 0.00 \quad 0.00$

$\begin{array}{lll}0.00 & 0.00 & 0.00\end{array}$

$\begin{array}{llll}0.00 & 0.00 & 0.00\end{array}$

$\begin{array}{llll}0.00 & 0.00 & 0.00\end{array}$

$0.00 \quad 0.00 \quad 0.00$

$0.00 \quad 0.00 \quad 0.00$

$\begin{array}{llll}0.00 & 0.00 & 0.00\end{array}$

$\begin{array}{llll}0.00 & 0.00 & 0.00\end{array}$

$\begin{array}{llll}0.00 \quad 0.00 \quad 0.00 & 0.000\end{array}$

$0.00 \quad 0.00 \quad 0.00$

$\begin{array}{llll}0.00 & 0.00 & 0.00\end{array}$

$\begin{array}{llll}0.00 & 0.00 & 0.00\end{array}$

$\begin{array}{llll}0.00 & 0.00 & 0.00\end{array}$

$\begin{array}{llll}0.00 & 0.00 & 0.00\end{array}$

$0.00 \quad 0.00 \quad 0.00$

$\begin{array}{llll}0.00 & 0.00 & 0.00\end{array}$

$\begin{array}{llll}0.00 & 0.00 & 0.00\end{array}$

$\begin{array}{lll}0.00 & 0.00 & 0.00\end{array}$

$\begin{array}{llll}0.00 & 0.00 \quad 0.00\end{array}$

$\begin{array}{llll}0.00 & 0.00 & 0.00\end{array}$

$\begin{array}{llll}0.00 & 0.00 & 0.00\end{array}$

$\begin{array}{llll}0.00 & 0.00 & 0.00\end{array}$

$\begin{array}{llll}0.00 & 0.00 & 0.00\end{array}$

$\begin{array}{llll}0.00 & 0.00 & 0.00\end{array}$

$\begin{array}{llll}0.00 & 0.00 & 0.00\end{array}$

$0.00 \quad 0.00 \quad 0.00$

$\begin{array}{llll}0.00 & 0.00 & 0.00\end{array}$

$\begin{array}{llll}0.00 & 0.00 & 0.00\end{array}$

$\begin{array}{llll}0.00 & 0.00 & 0.00\end{array}$

$0.00 \quad 0.00 \quad 0.00$

$\begin{array}{llll}0.00 & 0.00 & 0.00\end{array}$

$\begin{array}{llll}0.00 & 0.00 & 0.00\end{array}$

$0.00 \quad 0.00 \quad 0.00$

$0.00 \quad 0.00 \quad 0.00$

$\begin{array}{llll}0.00 & 0.00 & 0.00\end{array}$

$0.00 \quad 0.00 \quad 0.00$

$0.00 \quad 0.00 \quad 0.00$

$\begin{array}{llll}0.00 & 0.00 & 0.00\end{array}$

$\begin{array}{llll}0.00 & 0.00 & 0.00\end{array}$

$\begin{array}{llll}0.00 & 0.00 & 0.00\end{array}$

$\begin{array}{llll}0.00 & 0.00 & 0.00\end{array}$

$0.00 \quad 0.00 \quad 0.00$

$0.00 \quad 0.00 \quad 0.00$

$0.00 \quad 0.00 \quad 0.00$ 
$\begin{array}{llllllllll}88 & 191 & 279-15.2 & -1.0-1891.27 & -1.34 & 0.83 & -2.12-1893.89 & 288.55\end{array}$ $\begin{array}{llllllll}88 & 192 & 280-15.3 & -0.9-1892.21 & -1.23 & 0.00 & -2.10-1895.54 & 294.97\end{array}$ $\begin{array}{lllllllll}88 & 193 & 281-15.4 & -0.8-1893.09 & -1.46 & 0.83 & -2.08-1895.80 & 302.79\end{array}$ $\begin{array}{lllllllll}88 & 194 & 282-15.6 & -0.8-1893.91 & -1.16 & 0.00 & -2.06-1897.13 & 309.53\end{array}$ $\begin{array}{lllllllll}88 & 195 & 283-15.7 & -0.7-1894.66 & -1.27 & 0.83 & -2.04-1897.15 & 317.58\end{array}$ $\begin{array}{lllllllll}88 & 196 & 284-15.8 & -0.7-1895.36 & -1.13 & 0.00 & -2.02-1898.52 & 324.28\end{array}$ $\begin{array}{lllllllll}88 & 197 & 285-16.0 & -0.6-1896.00 & -0.75 & 0.82 & -2.01-1897.93 & 332.94\end{array}$ $\begin{array}{lllllllll}88 & 198 & 286-16.1 & -0.6-1896.59 & -0.59 & 0.00 & -1.99-1899.16 & 339.78\end{array}$ $\begin{array}{lllllllll}88 & 199 & 287-16.2 & -0.5-1897.11 & -0.78 & 0.82 & -1.97-1899.04 & 347.97\end{array}$ $88200288-16.4-0.4-1897.58-1.65 \quad 0.00-1.95-1901.18 \quad 353.90$ $\begin{array}{llllllllll}88 & 201 & 289-16.5 & -0.4-1897.99 & -0.06 & 0.82 & -1.94-1899.17 & 363.99\end{array}$ $\begin{array}{llllllll}88 & 202 & 290-16.6 & -0.3-1898.36 & 0.07 & 0.00 & -1.92-1900.20 & 371.02\end{array}$ $\begin{array}{llllllll}88 & 203 & 291-16.7 & -0.3-1898.66 & -2.20 & 0.82 & -1.90-1901.95 & 377.35\end{array}$ $\begin{array}{lllllllll}88 & 204 & 292-16.9 & -0.2-1898.92 & -2.09 & 0.00 & -1.89-1902.89 & 384.48\end{array}$ $89106195 \quad 1.9-11.2-1462.47$

$89107196 \quad 1.7-11.0-1473.58$

$891081971.4-10.8-1484.48$

$89109198 \quad 1.2-10.6-1495.20$

$89110199 \quad 0.9-10.4-1505.72$

$89111200 \quad 0.7-10.2-1516.05$

$89112201 \quad 0.4-10.0-1526.19$

$\begin{array}{llllll}89 & 113 & 202 & 0.2 & -9.9-1536.15\end{array}$

$\begin{array}{llllll}89 & 114 & 203 & -0.1 & -9.7-1545.93\end{array}$

$\begin{array}{llllll}89 & 115 & 204 & -0.3 & -9.5-1555.52\end{array}$

$\begin{array}{llllll}89 & 116 & 205 & -0.6 & -9.3-1564.94\end{array}$

$\begin{array}{llllll}89 & 117 & 206 & -0.8 & -9.2-1574.19\end{array}$

$\begin{array}{llllll}89 & 118 & 207 & -1.0 & -9.0-1583.26\end{array}$

$\begin{array}{lllllllll}89 & 119 & 208 & -1.3 & -8.8-1592.17 & -1.52 & 1.86 & -5.46-1597.28\end{array}$

$-0.351 .87-5.65-1578.32$

$\begin{array}{lllllllll}89 & 120 & 209 & -1.5 & -8.6-1600.90 & -2.34 & 1.08 & -5.36-1607.53\end{array}$

$\begin{array}{llllllllll}89 & 121 & 210 & -1.7 & -8.5-1609.48 & -2.84 & 1.86 & -5.27-1615.73\end{array}$

$\begin{array}{lllllllll}89 & 122 & 211 & -2.0 & -8.3-1617.89 & -3.21 & 1.08 & -5.18-1625.20\end{array}$

$\begin{array}{lllllllll}89 & 123 & 212 & -2.2 & -8.2-1626.13 & -3.86 & 1.85 & -5.10-1633.24\end{array}$

$\begin{array}{lllllllll}89 & 124 & 213 & -2.4 & -8.0-1634.22 & -4.50 & 1.08 & -5.02-1642.66\end{array}$

$89 \quad 125 \cdot 214 \quad-2.7-7.9-1642.16-5.32 \quad 1.85-4.93-1650.56$

$\begin{array}{lllllllll}89 & 126 & 215 & -2.9 & -7.7-1649.94 & -5.47 & 1.08 & -4.85-1659.19\end{array}$

$\begin{array}{lllllllll}89 & 127 & 216 & -3.1 & -7.6-1657.57 & -4.60 & 1.85 & -4.78-1665.10\end{array}$

$\begin{array}{lllllllll}89 & 128 & 217 & -3.3 & -7.4-1665.05 & -3.67 & 1.08 & -4.70-1672.34\end{array}$

$\begin{array}{lllllllll}89 & 129 & 218 & -3.6 & -7.3-1672.38 & -2.74 & 1.84 & -4.63-1677.90\end{array}$

$\begin{array}{lllllllll}89 & 130 & 219 & -3.8 & -7.1-1679.56 & -1.63 & 1.08 & -4.56-1684.67\end{array}$

$\begin{array}{llllllllll}89 & 131 & 220 & -4.0 & -7.0-1686.61 & -2.34 & 1.84 & -4.49-1691.59\end{array}$

$\begin{array}{lllllllll}89 & 132 & 221 & -4.2 & -6.8-1693.51 & -1.96 & 1.08 & -4.42-1698.81\end{array}$

$\begin{array}{lllllllll}89 & 133 & 222 & -4.4 & -6.7-1700.27 & -1.96 & 1.84 & -4.35-1704.74\end{array}$

$\begin{array}{lllllllll}89 & 134 & 223 & -4.7 & -6.6-1706.89 & -1.38 & 1.08 & -4.28-1711.48\end{array}$

$\begin{array}{lllllllll}89 & 135 & 224 & -4.9 & -6.4-1713.38 & -1.06 & 1.83 & -4.22-1716.83\end{array}$

$\begin{array}{lllllllll}89 & 136 & 225 & -5.1 & -6.3-1719.73 & -0.79 & 1.08 & -4.16-1723.60\end{array}$

$\begin{array}{llllllll}89 & 137 & 226 & -5.3 & -6.2-1725.95 & -0.83 & 1.83 & -4.10-1729.05\end{array}$

$\begin{array}{llllllllll}89 & 138 & 227 & -5.5 & -6.0 & -1732.04 & -0.53 & 1.08 & -4.04-1735.53\end{array}$

$\begin{array}{lllllllll}89 & 139 & 228 & -5.7 & -5.9-1738.00 & -0.52 & 1.82 & -3.98-1740.67\end{array}$

$\begin{array}{lllllllll}89 & 140 & 229 & -5.9 & -5.8-1743.83 & -0.27 & 1.08 & -3.92-1746.95\end{array}$

$\begin{array}{lllllllll}89 & 141 & 230 & -6.1 & -5.6-1749.54 & -0.47 & 1.82 & -3.87-1752.06\end{array}$

$\begin{array}{lllllllll}89 & 142 & 231 & -6.3 & -5.5-1755.12 & -0.38 & 1.08 & -3.82 & -1758.24\end{array}$

$\begin{array}{lllllllll}89 & 143 & 232 & -6.5 & -5.4-1760.59 & -0.54 & 1.82 & -3.76-1763.07\end{array}$

$\begin{array}{lllllllll}89 & 144 & 233 & -6.7 & -5.3-1765.93 & -0.45 & 1.08 & -3.71-1769.01\end{array}$

$\begin{array}{lllllllll}89 & 145 & 234 & -6.9 & -5.2-1771.15 & -0.63 & 1.81 & -3.66-1773.63\end{array}$

$\begin{array}{lllllllll}89 & 146 & 235 & -7.1 & -5.0-1776.26 & -0.43 & 1.08 & -3.61-1779.22\end{array}$

$\begin{array}{llllllllll}89 & 147 & 236 & -7.3 & -4.9-1781.25 & -0.77 & 1.81 & -3.56-1783.77\end{array}$

$\begin{array}{lllllllll}89 & 148 & 237 & -7.5 & -4.8-1786.12 & -0.57 & 1.08 & -3.51-1789.13\end{array}$

$\begin{array}{llllllllll}89 & 149 & 238 & -7.7 & -4.7-1790.88 & -0.84 & 1.81 & -3.47-1793.38\end{array}$

$\begin{array}{llllllllll}89 & 150 & 239 & -7.9 & -4.6-1795.53 & -0.62 & 1.08 & -3.42-1798.50\end{array}$

$\begin{array}{lllllllllll}89 & 151 & 240 & -8.1 & -4.5-1800.08 & -0.97 & 1.81 & -3.38-1802.62\end{array}$

$\begin{array}{lllllllll}89 & 152 & 241 & -8.3 & -4.4-1804.51 & -0.70 & 1.08 & -3.34-1807.47\end{array}$

$\begin{array}{lllllllll}89 & 153 & 242 & -8.5 & -4.3-1808.84 & -0.43 & 1.80 & -3.29-1810.76\end{array}$

$\begin{array}{lllllllll}89 & 154 & 243 & -8.7 & -4.2-1813.06 & -0.04 & 1.08 & -3.25-1815.27\end{array}$

$89 \begin{array}{lllll}855 & 244 & -8.9 & -4.1-1817.17\end{array}$

$89 \begin{array}{llllll}856 & 245 & -9.0 & -4.0-1821.19\end{array}$

$\begin{array}{lllll}89 & 157 & 246 & -9.2 & -3.9-1825.10\end{array}$
$0.541 .08-6.93-1467.79$

$0.68 .1 .89-6.80-1477.81$

$0.861 .08-6.67-1489.22$

$1.031 .89-6.54-1498.82$

$1.471 .08-6.42-1509.59$

$1.741 .88-6.30-1518.73$

$1.571 .08-6.18-1529.73$

$1.281 .88-6.07-1539.07$

$1.021 .08-5.96-1549.79$

$0.621 .87-5.85-1558.89$

$0.161 .08-5.75-1569.46$

35.85

33.91

30.57

29.03

26.34

25.27

22.34

21.08

18.42

17.40

14.90

14.10

11.82

11. 29

9.11

8.98

7.58

7.62

6.26

6.44

5.88

8.04

8.87

11.38

12.68

13.84

14.69

16.83

18.16

20.89

22.18

24.81

26.40

29.33

31.12

34.09

35.97

39.21

41.34

44.80

47.28

50.81

53.51

57.33

60.28

64.24

67.46

72.24

75.80

80.79

$0.231 .80-3.21-1818.35$

$0.551 .80-3.13-1825.88$

91

84.48

89.40

\subsection{0}

0.00

0.00

0.00

0.00

0.00

0.00

0.00

0.00

0.00

0.00

0.00

0.00

0.00

0.00

0.00

0.00

0.00

0.00

0.00

0.00

$0.00 \quad 0.00$

0.00

0.00

0.00

0.00

0.00

0.00

0.00

0.00

0.00

0.00

0.00

0.00

0.00

0.00

0.00

0.00

0.00

0.00

0.00

0.00

0.00

0.00

0.00

0.00

0.00

0.00

0.00

0.00

0.00

0.00

0.00

0.00

$8.89-2.56$

$8.62-3.20$

$\begin{array}{llll}7.09 & -3.70 & -0.49\end{array}$

$7.24-4.24-0.38$

$6.10-4.66-0.16$

$\begin{array}{llll}6.38 & -5.38 & -0.06\end{array}$

$\begin{array}{llll}5.97 & -5.38 & 0.09\end{array}$

$8.06-4.58 \quad 0.02$

$8.69-3.85-0.18$

$10.82-3.30-0.56$

$11.54-2.77-1.14$

$13.73-2.45-0.11$

$14.50-2.15-0.19$

$16.60-2.19-0.23$

$17.82-1.72-0.34$

$20.20-1.75-0.69$

$21.63-1.34-0.55$

$24.30-1.34-0.51$

$25.85-1.08-0.55$

$\begin{array}{llll}28.89 & -0.96 & -0.44\end{array}$

$30.90-0.49-0.22$

$\begin{array}{llll}0.00 & 0.00 & 0.00\end{array}$

$\begin{array}{llll}35.91 & -0.44 & -0.06\end{array}$

$\begin{array}{llll}0.00 & 0.00 & 0.00\end{array}$

$0.00 \quad 0.00 \quad 0.00$

$0.00 \quad 0.00 \quad 0.00$

$0.00 \quad 0.00 \quad 0.00$

0.00

0.00

0.00

$0.00 \quad 0.00 \quad 0.00$

$0.00 \quad 0.00 \quad 0.00$

$0.00 \quad 0.00 \quad 0.00$

0.00

0.00

0.00

0.00

0.00

0.00

0.00

0.00

0.00

0.00

0.00

0.00

0.00

$0.00 \quad 0.00$

0.00

0.00

0.00

0.00

0.00 
$\begin{array}{llllll}89 & 158 & 247 & -9.4 & -3.8-1828.91\end{array}$ $\begin{array}{llllll}89 & 159 & 248 & -9.6 & -3.7-1832.62\end{array}$ $\begin{array}{llllll}89 & 160 & 249 & -9.8 & -3.6-1836.24\end{array}$ $89 \quad 161250-10.0-3.5-1839.76$ $89162 \quad 251-10.1-3.4-1843.18$ $89 \quad 163 \quad 252-10.3-3.3-1846.52$ $89 \quad 164 \quad 253-10.5 \quad-3.2-1849.75$ $89 \quad 165 \quad 254-10.7 \quad-3.1-1852.90$ $89166255-10.8-3.0-1855.96$ $89 \quad 167256-11.0-2.9-1858.92$ $89168257-11.2-2.8-1861.80$ $89 \begin{array}{llll}869 & 258-11.3 & -2.7-1864.60\end{array}$ $89170 \quad 259-11.5-2.7-1867.30$ $89 \quad 171 \quad 260-11.7 \quad-2.6-1869.93$ $89172261-11.8 \cdot-2.5-1872.46$ $89 \quad 173 \quad 262-12.0-2.4-1874.92$ $89 \quad 174 \quad 263-12.2 \quad-2.3-1877.30$ $89 \quad 175 \quad 264-12.3-2.3-1879.59$ $89176 \quad 265-12.5 \quad-2.2-1881.81$ $89 \quad 177 \quad 266-12.6-2.1-1883.95$ $\begin{array}{lllllllll}89 & 178 & 267-12.8 & -2.0-1886.01 & -3.62 & 1.08 & -2.47-1891.02 & 193.77\end{array}$ $89 \quad 179268-13.0-1.9-1887.99-3.98 \quad 1.77-2.44-1892.65 \quad 200.21$ $89 \quad 180 \quad 269-13.1-1.9-1889.90-4.11 \quad 1.08-2.42-1895.35 \quad 205.58$ $89181270-13.3-1.8-1891.74-4.60 \quad 1.77-2.39-1896.96 \quad 212.04$ $89182271-13.4-1.7-1893.50-4.541 .08-2.37-1899.33 \quad 217.74$ $\begin{array}{lllllllll}89 & 183 & 272-13.6 & -1.6-1895.19 & -4.93 & 1.76 & -2.34-1900.70 & 224.44\end{array}$ $89 \begin{array}{lllllllll}8 & 273-13.7 & -1.6-1896.81 & -4.55 & 1.08 & -2.32-1902.60 & 230.61\end{array}$ $89185274-13.9-1.5-1898.36-3.69 \quad 1.76-2.30-1902.58 \quad 238.70$ $89186275-14.0-1.4-1899.83-3.07 \quad 1.08-2.27-1904.10 \quad 245.25$ $89187276-14.2-1.4-1901.25-2.11 \quad 1.76-2.25-1903.85 \quad 253.58$ $89188 \quad 277-14.3 \quad-1.3-1902.59-1.36 \quad 1.08-2.23-1905.10 \quad 260.40$ $\begin{array}{lllllllll}89 & 189 & 278-14.5 & -1.2-1903.86 & -1.46 & 1.76 & -2.21-1905.77 & 267.80\end{array}$ $89190279-14.6-1.2-1905.07-1.31 \quad 1.08-2.19-1907.49 \quad 274.15$ $89191280-14.7-1.1-1906.22-1.44 \quad 1.75-2.17-1908.07281 .65$ $89192281-14.9-1.0-1907.30-1.36 \quad 1.08-2.14-1909.73 \quad 288.06$ $89193282-15.0-1.0-1908.32-1.63 \quad 1.75-2.12-1910.32 \quad 295.54$ $89194283-15.2 \quad-0.9-1909.27-1.44 \quad 1.08-2.10-1911.74 \quad 302.19$ $89195284-15.3 \quad-0.9-1910.17-1.63 \quad 1.75-2.09-1912.13 \quad 309.87$ $89196285-15.4 \quad-0.8-1911.00-1.36 \quad 1.08-2.07-1913.35 \quad 316.72$ $89197286-15.6-0.7-1911.77-1.31 \quad 1.75-2.05-1913.38 \quad 324.76$ $89 \begin{array}{llllllll}89 & 1987-15.7 & -0.7-1912.49 & -1.16 & 1.08 & -2.03-1914.60 & 331.61\end{array}$ $89199288-15.8-0.6-1913.14-1.98 \quad 1.75 \quad-2.01-1915.39338 .90$ $89200289-16.0 \quad-0.6-1913.74-1.83 \quad 1.08-1.99-1916.49 \quad 345.87$ $89201290-16.1 \quad-0.5-1914.29-0.301 .74-1.97-1914.82 \quad 355.61$ $89202291-16.2 \quad-0.5-1914.77-0.20 \quad 1.08-1.96-1915.85 \quad 362.65$ $89203292-16.3 \quad-0.4-1915.20-2.381 .74-1.94-1917.78 \quad 368.79$ $89204293-16.5 \quad-0.3-1915.58-2.23 \quad 1.08-1.92-1918.66 \quad 375.98$ $89205294-16.6-0.3-1915.91-2.49 \quad 1.74-1.91-1918.56 \quad 384.15$ $89206 \quad 295-16.7-0.2-1916.18$ $901081981.9-11.1-1482.85$ $901091991.6-10.9-1493.82$ $90110200 \quad 1.4-10.7-1504.59$ $90111201 \quad 1.1-10.5-1515.17$ $90 \quad 112.202 \quad 0.9-10.3-1525.55$ $90113203 \quad 0.6-10.1-1535.76$ $\begin{array}{llllll}90 & 114 & 204 & 0.4 & -9.9-1545.78\end{array}$ $90115205 \quad 0.1 \quad-9.7-1555.62$ $90116206-0.1-9.6-1565.28$ $90 \quad 117207-0.3 \quad-9.4-1574.76$ $90118208-0.6-9.2-1584.08-0.530 .00-5.68-1590.29$ $90119209-0.8-9.1-1593.22-1.12 \quad 0.98-5.58-1598.94$ $90 \quad 120 \quad 210-1.1 \quad-8.9-1602.19-1.71 \quad 0.00-5.49-1609.39$ $90 \quad 121 \quad 211-1.3-8.7-1610.99-2.230 .97-5.40-1617.65$ $\begin{array}{lllllllll}90 & 122 & 212 & -1.5 & -8.6-1619.64 & -2.55 & 0.00 & -5.30-1627.49\end{array}$ $\begin{array}{lllllllll}90 & 123 & 213 & -1.8 & -8.4-1628.12 & -3.22 & 0.97 & -5.22 & -1635.59\end{array}$ $90 \quad 124 \quad 214 \quad-2.0 \quad-8.2-1636.44-3.75 \quad 0.00 \quad-5.13-1645.32$ 92
391.50

36.61

33.29

32.40

29.42

28.14

25.09

24.03

21.11

20.24

17.48

16.90

14.53

14.34

12.57

12.54

10.88

\begin{tabular}{|c|c|c|}
\hline 00 & 00 & \\
\hline .00 & 00 & 0.00 \\
\hline 0.00 & 0.00 & 0.00 \\
\hline 0.00 & 0.00 & 00 \\
\hline 0.00 & 0.00 & .00 \\
\hline 0.00 & 0.00 & 0.00 \\
\hline 0.00 & 0.00 & 0.00 \\
\hline 0.00 & & \\
\hline 0.00 & 0.00 & \\
\hline 0.00 & 0.00 & 0.00 \\
\hline 0.00 & 0.00 & 0.00 \\
\hline 0.00 & 0.00 & 0.00 \\
\hline 0.00 & 0.00 & \\
\hline 0.00 & 0.00 & 0.00 \\
\hline 0.00 & 0.00 & 0.00 \\
\hline 0.00 & 0.00 & 0.00 \\
\hline 0.00 & 0.00 & \\
\hline 0.00 & 0.00 & 0.00 \\
\hline 0.00 & 0.00 & 0.00 \\
\hline 0.00 & 0.00 & 0.00 \\
\hline 0.00 & 0.00 & 0.00 \\
\hline 0.00 & 0.00 & 0.00 \\
\hline 0.00 & 0.00 & 0.00 \\
\hline 0.00 & 0.00 & 0.00 \\
\hline 0.00 & 0.00 & 0.00 \\
\hline 0.00 & 0.00 & 0.00 \\
\hline 0.00 & 0.00 & 0.00 \\
\hline 0.00 & 0.00 & 0.00 \\
\hline 0.00 & 0. & 0.00 \\
\hline 0.00 & 0.00 & 0.00 \\
\hline 0.00 & 0.00 & 0.00 \\
\hline 0.00 & 0.00 & 0.00 \\
\hline 0.00 & 0 & 0.00 \\
\hline 0.00 & 0.00 & 0.00 \\
\hline 0.00 & 0.00 & 0.00 \\
\hline 0.00 & 0 . & .00 \\
\hline 0.00 & 0.00 & 0.00 \\
\hline 0.00 & 0.00 & 0.00 \\
\hline 0.00 & 0.00 & 0.00 \\
\hline 0.00 & 0.00 & 0.00 \\
\hline 0.00 & 0 & 0.00 \\
\hline 0.00 & 0.00 & 0.00 \\
\hline 0.00 & 0.00 & 0.00 \\
\hline 0.00 & 0.0 & 0.00 \\
\hline 0.0 & 0 & 0.00 \\
\hline 0.00 & 0.00 & 0.00 \\
\hline 0.00 & 0.00 & 0.00 \\
\hline 0.00 & 0.0 & 0.00 \\
\hline 0.0 & & 0.00 \\
\hline 0.00 & 0.00 & 0.00 \\
\hline 0.00 & 0.00 & 0.00 \\
\hline 0.00 & 0.00 & 0.00 \\
\hline 0.0 & & 0.00 \\
\hline 0.0 & 0.00 & 0.00 \\
\hline 0.0 & 0.0 & 0.00 \\
\hline 0.00 & 0.0 & 0.00 \\
\hline 0.0 & & 0.00 \\
\hline 0.0 & 0.00 & 0.00 \\
\hline 0.00 & 0.00 & 0.00 \\
\hline 0.00 & 0.0 & 0.00 \\
\hline 0.08 & 0.00 & 0.00 \\
\hline 0.00 & 0.00 & 0.00 \\
\hline 0.00 & 0.00 & 0.00 \\
\hline 0 & 0,0 & \\
\hline & & 0.00 \\
\hline 0 & 0.00 & \\
\hline
\end{tabular}
0.00
0.00
0.00
0.00
0.00
0.00
0.00
0.00
0.00
.00
0.00
0.00
0.00
.00
.00
0.00
0.00
0.00
0.00
0.00
0.00
0.00
0.00
0.00
0.00
0.00
.00
0.00
0.00
0.00
.00
.00
0.00
0.00
0.00
0.00
0.00
0.00
0.00
0.00
0.00
0.00
0.00
0.00
0.00
0.00
0.00
0.00
0.00
0.00
0.00
0.00
0.00
0.00
0.00
0.00
0.00
0.00
0.00
0.00
0.00
0.00
0.00
0.00
.00
.00
0.00
.00
0.00
.00
.00
.00
.00
.00
.00
.00
.00
.00
.00 
$90 \quad 125 \quad 215 \quad-2.2 \quad-8.1-1644.60-4.56 \quad 0.96-5.05-1653.25$ $\begin{array}{lllllllll}90 & 126 & 216 & -2.4 & -7.9-1652.61 & -4.65 & 0.00 & -4.97-1662.22\end{array}$ $\begin{array}{lllllllll}90 & 127 & 217 & -2.7 & -7.8-1660.46 & -3.84 & 0.95 & -4.89-1668.23\end{array}$ $\begin{array}{lllllllll}90 & 128 & 218 & -2.9 & -7.6-1668.16 & -2.92 & 0.00 & -4.81-1675.89\end{array}$ $\begin{array}{lllllllll}90 & 129 & 219 & -3.1 & -7.5-1675.72 & -2.07 & 0.95 & -4.73-1681.57\end{array}$ $90 \quad 130 \quad 220-3.3 \quad-7.3-1683.12-1.190 .00-4.66-1688.97$ $\begin{array}{lllllllll}90 & 131 & 221 & -3.6 & -7.2-1690.39 & -1.79 & 0.95 & -4.59-1695.82\end{array}$ $\begin{array}{lllllllll}90 & 132 & 222 & -3.8 & -7.0-1697.50 & -1.44 & 0.00 & -4.52-1703.46\end{array}$ $\begin{array}{lllllllll}90 & 133 & 223 & -4.0 & -6.9-1704.48 & -1.43 & 0.94 & -4.45-1709.42\end{array}$ $90 \quad 134224-4.2-6.8-1711.32-0.650 .00-4.38-1716.35$ $\begin{array}{lllllllll}90 & 135 & 225 & -4.4 & -6.6-1718.02 & -0.76 & 0.94 & -4.32-1722.16\end{array}$ $\begin{array}{lllllllll}90 & 136 & 226 & -4.6 & -6.5-1724.59 & -0.19 & 0.00 & -4.25-1729.03\end{array}$ $90 \quad 137 \quad 227-4.8-6.4-1731.02-0.450 .93-4.19-1734.73$ $90 \quad 138 \quad 228-5.0-6.2-1737.32-0.30 \quad 0.00-4.13-1741.75$ $\begin{array}{lllllllll}90 & 139 & 229 & -5.3 & -6.1-1743.49 & -0.52 & 0.93 & -4.07-1747.15\end{array}$ $90 \quad 140 \quad 230-5.5 \quad-6.0-1749.53-0.43 \quad 0.00-4.01-1753.97$ $\begin{array}{lllllllll}90 & 141 & 231 & -5.7 & -5.8-1755.44 & -0.67 & 0.92 & -3.96-1759.14\end{array}$ $90 \quad 142 \quad 232 \quad-5.9-5.7-1761.23-0.600 .00-3.90-1765.73$ $90 \quad 143 \quad 233-6.1 \quad-5.6-1766.89-0.78 \quad 0.92-3.85-1770.60$ $90 \quad 144 \quad 234-6.3 \quad-5.5-1772.44-0.66 \quad 0.00-3.79-1776.89$ $90 \quad 145 \quad 235-6.5-5.4-1777.86-0.840 .91-3.74-1781.53$ $90146236-6.7-5.2-1783.16-0.67 \quad 0.00-3.69-1787.53$ $90 \quad 147 \quad 237-6.9-5.1-1788.35-1.02 \quad 0.91-3.64-1792.10$ $\begin{array}{lllllllll}90 & 148 & 238 & -7.1 & -5.0-1793.42 & -0.82 & 0.00 & -3.59-1797.84\end{array}$ $\begin{array}{lllllllll}90 & 149 & 239 & -7.3 & -4.9-1798.38 & -1.03 & 0.91 & -3.55-1802.05\end{array}$ $\begin{array}{lllllllll}90 & 150 & 240 & -7.5 & -4.8-1803.23 & -0.81 & 0.00 & -3.50-1807.54\end{array}$ $\begin{array}{lllllllll}90 & 151 & 241 & -7.7 & -4.7-1807.96 & -1.16 & 0.90 & -3.45-1811.67\end{array}$ $\begin{array}{lllllllll}90 & 152 & 242 & -7.8 & -4.6-1812.58 & -0.89 & 0.00 & -3.41-1816.88\end{array}$ $90 \quad 153243-8.0 \quad-4.5-1817.10-0.60 \quad 0.90-3.37-1820.17$ $90 \quad 154244-8.2-4.4-1821.51$ $90 \quad 155 \quad 245-8.4-4.2-1825.81$ $90 \quad 156 \quad 246-8.6 \quad-4.1-1830.01$ $\begin{array}{llllll}90 & 157 & 247 & -8.8 & -4.0 & -1834.11\end{array}$ $\begin{array}{llllll}90 & 158 & 248 & -9.0 & -3.9-1838.10\end{array}$ $90159249-9.2 \quad-3.8-1842.00$ $90 \quad 160 \quad 250 \quad-9.3 \quad-3.7-1845.80$ $90 \quad 161.251 .-9.5-3.6-1849.50$ $\begin{array}{llllll}90 & 162 & 252 & -9.7 & -3.6-1853.10\end{array}$ $90 \quad 163 \quad 253-9.9-3.5-1856.60$ $90 \quad 164254-10.0-3.4-1860.02$ $90165255-10.2-3.3-1863.34$ $90 \quad 166 \quad 256-10.4 \quad-3.2-1866.57$ $90167257-10.6-3.1-1869.71$ $90168 \quad 258-10.7-3.0-1872.76$ $90 \quad 169259-10.9-2.9-1875.72$ $90170 \quad 260-11.1-2.8-1878.60$ $90 \quad 171261-11.2-2.7-1881.38$ $90172262-11.4-2.7-1884.09-1.660 .00-2.69-1888.44$ 155.19 $90173263-11.6 \quad-2.6-1886.71-1.17 \quad 0.86-2.66-1889.68 \quad 162.02$ $90174264-11.7 \quad-2.5-1889.25 \quad-1.28 \quad 0.00-2.63-1893.16 \quad 166.61$ $90 \quad 175 \quad 265-11.9-2.4-1891.71 \quad-1.93 \quad 0.86-2.60-1895.38 \quad 172.46$ $90176 \quad 266-12.1-2.3-1894.08-2.50 \quad 0.00-2.57-1899.15 \quad 176.76$ $90177267-12.2 \quad-2.3-1896.38-3.04 \quad 0.85-2.54-1901.11182 .88$ $90178 \quad 268-12.4 \quad-2.2-1898.60-3.08 \quad 0.00-2.52-1904.20187 .86$

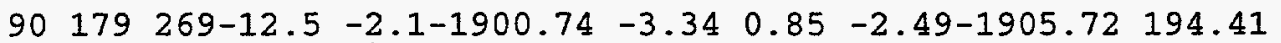
$90 \quad 180 \quad 270-12.7 \quad-2.0-1902.81-3.47 \quad 0.00-2.47-1908.74 \quad 199.46$

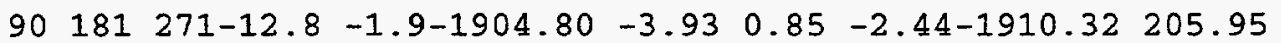
$90 \quad 182 \quad 272-13.0 \quad-1.9-1906.71 \quad-3.88 \quad 0.00-2.42-1913.01211 .34$ $90183273-13.1-1.8-1908.55-4.190 .85-2.39-1914.29218 .13$ $90184274-13.3-1.7-1910.32 \quad-3.80 \quad 0.00-2.37-1916.49 \quad 224.00$ $90 \quad 185 \quad 275-13.4-1.7-1912.02 \quad-2.92 \quad 0.84-2.34-1916.44232 .12$ $90186276-13.6-1.6-1913.65-2.32 \quad 0.00-2.32-1918.29 \quad 238.34$ $90 \quad 187 \quad 277-13.7-1.5-1915.20-1.43 \quad 0.84-2.30-1918.09 \quad 246.61$ $90188 \quad 278-13.9-1.5-1916.69-0.81 \quad 0.00-2.28-1919.78 \quad 252.99$ $90189279-14.0-1.4-1918.11-1.350 .84-2.25-1920.88 \quad 259.96$ $90190280-14.2-1.3-1919.47-1.190 .00-2.23-1922.89266 .03$
$10.89-4.70-0.14$

$0.00 \quad 0.00 \quad 0.00$

$12.16-3.86-0.02$

$12.35-3.16-0.24$

$14.45-2.61-0.54$

$14.65-2.19-1.00$

$\begin{array}{lll}16.92 & -1.75 & 0.04\end{array}$

$17.18-1.57-0.13$

$19.36-1.49-0.06$

$\begin{array}{llll}19.98 & -1.23 & -0.58\end{array}$

$\begin{array}{llll}22.28 & -1.30 & -0.54\end{array}$

$23.18-1.04-0.85$

$25.80-1.05-0.60$

$26.75-1.00-0.70$

$29.58-1.06-0.54$

$30.86-0.94-0.51$

$33.81-1.13-0.46$

$35.44-0.92-0.32$

$38.73-1.00-0.22$

$40.61-0.79-0.13$

$44.25-0.76 \quad 0.08$

$0.00 \quad 0.00 \quad 0.00$

$0.00 \quad 0.00 \quad 0.00$

$0.00 \quad 0.00 \quad 0.00$

$0.00 \quad 0.00 \quad 0.00$

$\begin{array}{llll}0.00 & 0.00 & 0.00\end{array}$

$0.00 \quad 0.00 \quad 0.00$

$\begin{array}{llll}0.00 & 0.00 & 0.00\end{array}$

$\begin{array}{llll}0.00 & 0.00 & 0.00\end{array}$

$\begin{array}{llll}0.00 & 0.00 & 0.00\end{array}$

$0.00 \quad 0.00 \quad 0.00$

$0.00 \quad 0.00 \quad 0.00$

$\begin{array}{llll}0.00 & 0.00 & 0.00\end{array}$

$\begin{array}{llll}0.00 & 0.00 & 0.00\end{array}$

$\begin{array}{llll}0.00 & 0.00 & 0.00\end{array}$

$\begin{array}{lll}0.00 & 0.00 & 0.00\end{array}$

$\begin{array}{llll}0.00 & 0.00 & 0.00\end{array}$

$\begin{array}{llll}0.00 & 0.00 & 0.00\end{array}$

$\begin{array}{llll}0.00 & 0.00 & 0.00\end{array}$

$\begin{array}{llll}0.00 & 0.00 & 0.00\end{array}$

$\begin{array}{llll}0.00 & 0.00 & 0.00\end{array}$

$\begin{array}{llll}0.00 & 0.00 & 0.00\end{array}$

$\begin{array}{llll}0.00 & 0.00 & 0.00\end{array}$

$0.00 \quad 0.00 \quad 0.00$

$\begin{array}{llll}0.00 & 0.00 & 0.00\end{array}$

$\begin{array}{llll}0.00 & 0.00 & 0.00\end{array}$

$\begin{array}{llll}0.00 & 0.00 & 0.00\end{array}$

$\begin{array}{llll}0.00 & 0.00 & 0.00\end{array}$

$\begin{array}{llll}0.00 & 0.00 & 0.00\end{array}$

$\begin{array}{llll}0.00 & 0.00 & 0.00\end{array}$

$0.00 \quad 0.00 \quad 0.00$

$0.00 \quad 0.00 \quad 0.00$

$0.00 \quad 0.00 \quad 0.00$

$\begin{array}{llll}0.00 & 0.00 & 0.00\end{array}$

$\begin{array}{llll}0.00 & 0.00 & 0.00\end{array}$

$0.00 \quad 0.00 \quad 0.00$

$0.00 \quad 0.00 \quad 0.00$

$0.00 \quad 0.00 \quad 0.00$

$\begin{array}{llll}0.00 & 0.00 & 0.00\end{array}$

$0.00 \quad 0.00 \quad 0.00$

$0.00 \quad 0.00 \quad 0.00$

$0.00 \quad 0.00 \quad 0.00$

$0.00 \quad 0.00 \quad 0.00$

$0.00 \quad 0.00 \quad 0.00$

$0.00 \quad 0.00 \quad 0.00$

$\begin{array}{llll}0.00 & 0.00 & 0.00\end{array}$ 


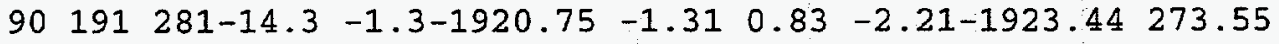

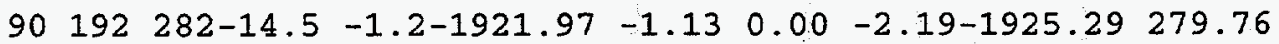
$90193 \quad 283-14.6-1.1-1923.13 \quad-1.44 \quad 0.83-2.17-1925.91287 .22$

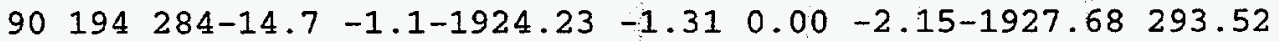
$90195 \quad 285-14.9 \quad-1.0-1925.26 \quad-1.50 \quad 0.83-2.13-1928.06 \quad 301.21$ $90196286-15.0 \quad-0.9-1926.23 \quad-1.24 \quad 0.00 \quad-2.11-1929.57 \quad 307.77$ $\begin{array}{llllllll}90 & 197 & 287-15.2 & -0.9-1927.13 & -1.21 & 0.82 & -2.09-1929.61 & 315.81\end{array}$ $90 \quad 198 \quad 288-15.3 \quad-0.8-1927.98 \quad-1.50 \quad 0.00 \quad-2.07-1931.55 \quad 321.93$ $\begin{array}{llllllll}90 & 199 & 289-15.4 & -0.8-1928.77 & -1.79 & 0.82 & -2.05-1931.79 & 329.77\end{array}$ $90200290-15.6 \quad-0.7-1929.50 \quad-0.06 \quad 0.00 \quad-2.03-1931.59338 .03$ $90201291-15.7 \quad-0.6-1930.17 \quad-0.38 \quad 0.82 \quad-2.01-1931.75 \quad 345.95$ $90202 \quad 292-15.8 \quad-0.6-1930.79-0.26 \quad 0.00-2.00-1933.05 \quad 352.72$ $90203 \quad 293-15.9 \quad-0.5-1931.35 \quad-2.26 \quad 0.82 \quad-1.98-1934.77 \quad 359.07$ $90204294-16.1 \quad-0.5-1931.85 \quad-2.11 \quad 0.00 \quad-1.96-1935.93 \quad 365.99$

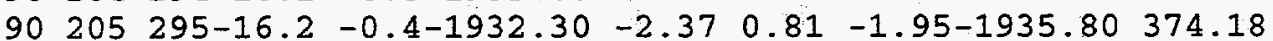
$90206296-16.3 \quad-0.4-1932.70-2.17 \quad 0.00-1.93-1936.80 \quad 381.26$ $90 \quad 207 \quad 297-16.4 \quad-0.3-1933.04 \quad-2.24 \quad 0.81 \quad-1.91-1936.38 \quad 389.75$

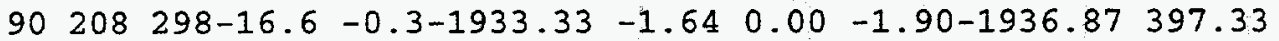
$90209299-16.7 \quad-0.2-1933.57 \quad-2.21 \quad 0.81 \quad-1.88-1936.85 \quad 405.42$ $91109200 \quad 2.0-11.1-1492.00$ $91110201 \quad 1.8-10.9-1503.02$ $91111202 \quad 1.5-10.7-1513.84$ 91112203 91113204 $91114 \quad 205$ 91115206 91116207 91117208 $1.3-10.5-1524.48$ $1.1-10.4-1534.92$ $0.8-10.2-1545.19$ $0.6-10.0-1555.27$ $0.3-9.8-1565.17$ $0.1-9.6-1574.90$ $\begin{array}{lllll}91 & 118 & 209 & -0.1 & -9.5-1584.44\end{array}$ $91 \quad 119210-0.4 \quad-9.3-1593.82$ $\begin{array}{lllll}91 & 120 & 211 & -0.6 & -9.1-1603.03\end{array}$ $\begin{array}{lllll}91 & 121 & 212 & -0.8 & -9.0-1612.07\end{array}$ $\begin{array}{llllll}91 & 122 & 213 & -1.1 & -8.8-1620.94\end{array}$ $\begin{array}{lllllllll}91 & 123 & 214 & -1.3 & -8.6-1629.65 & -2.55 & 1.85 & -5.34-1635.69\end{array}$

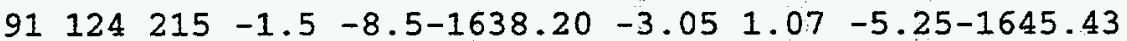
$91 \quad 125216-1.8 \quad-8.3-1646.59-3.791 .84-5.16-1653.70$

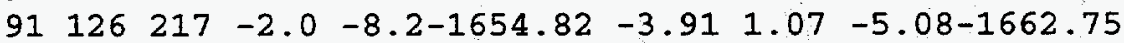
$\begin{array}{lllllllll}91 & 127 & 218 & -2.2 & -8.0 & -1662.90 & -3.03 & 1.84 & -5.00-1669.09\end{array}$ $\begin{array}{llllllll}91 & 128 & 219 & -2.4 & -7.8-1670.83 & -2.14 & 1.07 & -4.92-1676.82\end{array}$ $\begin{array}{lllllllll}91 & 129 & 220 & -2.7 & -7.7 & -1678.61 & -0.83 & 1.84 & -4.84-1682.44\end{array}$ $\begin{array}{lllllllll}91 & 130 & 221 & -2.9 & -7.6-1686.23 & 0.16 & 1.07 & -4.77-1689.77\end{array}$ $\begin{array}{llllllll}91 & 131 & 222 & -3.1 & -7.4-1693.72 & -1.53 & 1.83 & -4.69-1698.11\end{array}$ $\begin{array}{llllllll}91 & 132 & 223 & -3.3 & -7.3-1701.05 & -1.10 & 1.07 & -4.62-1705.71\end{array}$

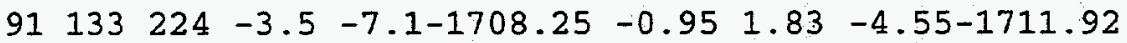

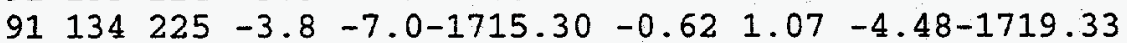
$\begin{array}{lllllllll}91 & 135 & 226 & -4.0 & -6.8 & -1722.22 & -0.39 & 1.82 & -4.41-1725.19\end{array}$

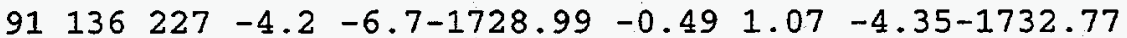
$\begin{array}{lllllllll}91 & 137 & 228 & -4.4 & -6.6-1735.64 & -0.74 & 1.82 & -4.29-1738.84\end{array}$

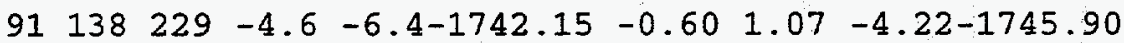

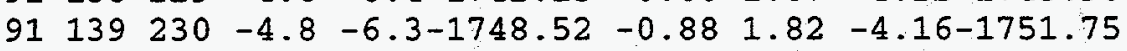

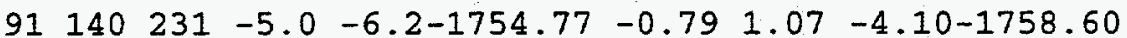
$91 \quad 141232 \quad-5.2 \quad-6.1-1760.89-1.03 \quad 1.81-4.04-1764.15$ $91 \quad 142 \quad 233 \quad-5.4 \quad-5.9-1766.88-0.981 .07 \quad-3.99-1770.78$

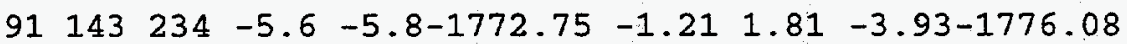

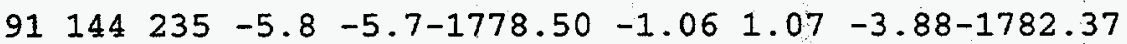
$91 \quad 145 \quad 236-6.0 \quad-5.6-1784.12 \quad-1.27 \quad 1.81 \quad-3.83-1787.41$ $\begin{array}{lllllllll}91 & 146 & 237 & -6.2 & -5.4-1789.63 & -1.04 & 1.07 & -3.77-1793.37\end{array}$

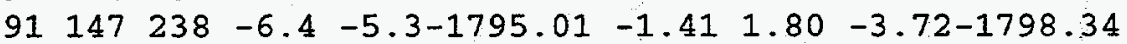

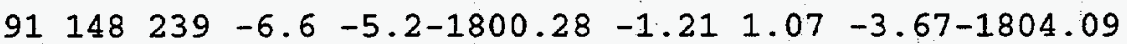
$\begin{array}{llllllll}91 & 149 & 240 & -6.8 & -5.1-1805.43 & -1.40 & 1.80 & -3.62-1808.65\end{array}$ $\begin{array}{lllllllll}91 & 150 & 241 & -7.0 & -5.0 & -1810.47 & -1.18 & 1.07 & -3.58-1814.16\end{array}$

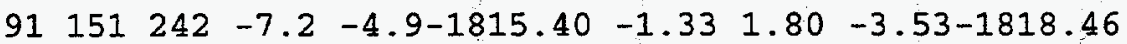
$\begin{array}{lllllllll}91 & 152 & 243 & -7.4 & -4.8-1820.21 & -1.29 & 1.07 & -3.48-1823.92\end{array}$

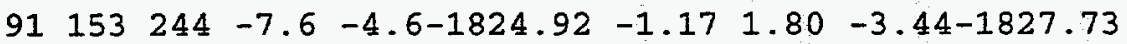
$91 \quad 154 \quad 245 \quad-7.8 \quad-4.5-1829.52 \quad-0.54 \quad 1.07 \quad-3.40-1832.39$

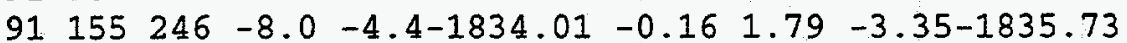
94

37.97

$\begin{array}{lll}0.00 & 0.00 & 0.00 \\ 0.00 & 0.00 & 0.00 \\ 0.00 & 0.00 & 0.00 \\ 0.00 & 0.00 & 0.00 \\ 0.00 & 0.00 & 0.00 \\ 0.00 & 0.00 & 0.00 \\ 0.00 & 0.00 & 0.00 \\ 0.00 & 0.00 & 0.00 \\ 0.00 & 0.00 & 0.00 \\ 0.00 & 0.00 & 0.00 \\ 0.00 & 0.00 & 0.00 \\ 0.00 & 0.00 & 0.00 \\ 0.00 & 0.00 & 0.00 \\ 0.00 & 0.00 & 0.00 \\ 0.00 & 0.00 & 0.00 \\ 0.00 & 0.00 & 0.00 \\ 0.00 & 0.00 & 0.00 \\ 0.00 & 0.00 & 0.00 \\ 0.00 & 0.00 & 0.00 \\ 0.00 & 0.00 & 0.00 \\ 0.00 & 0.00 & 0.00 \\ 0.00 & 0.00 & 0.00 \\ 0.00 & 0.00 & 0.00 \\ 0.00 & 0.00 & 0.00 \\ 0.00 & 0.00 & 0.00 \\ 0.00 & 0.00 & 0.00 \\ 0.00 & 0.00 & 0.00 \\ 0.00 & 0.00 & 0.00 \\ 0.00 & 0.00 & 0.00 \\ 0.00 & 0.00 & 0.00 \\ 0.00 & 0.00 & 0.00 \\ 0.00 & 0.00 & 0.00 \\ 0.00 & 0.00 & 0.00 \\ 0.00 & 0.00 & 0.00 \\ 1.68 & -3.41 & 0.36\end{array}$

46.11

42.76

41.53

38.30

36.75

33.86

32.40

29.72

28.43

25.67

24.71

22.32

21.86

20.08

19.71

18.04

17.84

16.87

18.59

18.93

21.39

22.13

21.86

22.34

24.19

24.85

27.06

27.56

29.56

30.57

32.79

34.02

36.53

40.74

42.53

45.56

47.67

50.78

53.09

56.60

59.17

62.94

65.55

69.81

73.23

77.96 $\begin{array}{llll}17.68 & -3.41 & -0.36\end{array}$

$\begin{array}{llll}17.68 & -3.95 & -0.16\end{array}$

$\begin{array}{lll}17.02 & -3.76 & 0.15\end{array}$

$\begin{array}{lll}18.60 & -3.02 & 0.01\end{array}$

$\begin{array}{llll}18.48 & -2.59 & -0.45\end{array}$

$20.32-1.90-1.07$

$\begin{array}{lll}0.00 & 0.00 & 0.00\end{array}$

$\begin{array}{lll}21.94 & -1.45 & 0.08\end{array}$

$22.31-1.13-0.03$

$\begin{array}{llll}23.78 & -1.36 & -0.41\end{array}$

$\begin{array}{llll}24.32 & -1.15 & -0.53\end{array}$

$26.01-1.44-1.05$

$\begin{array}{llll}26.83 & -1.22 & -0.73\end{array}$

$\begin{array}{llll}28.86 & -1.44 & -0.70\end{array}$

$\begin{array}{llll}29.89 & -1.28 & -0.68\end{array}$

$32.17-1.50-0.62$

$33.42-1.39-0.60$

$\begin{array}{llll}3.5 .92 & -1.64 & -0.61\end{array}$

$\begin{array}{llll}37.49 & -1.46 & -0.48\end{array}$

$\begin{array}{llll}40.33 & -1.62 & -0.41\end{array}$

$\begin{array}{llll}42.33 & -1.26 & -0.20\end{array}$

$\begin{array}{llll}45.34 & -1.49 & -0.22\end{array}$

$\begin{array}{llll}47.64 & -1.07 & -0.03\end{array}$

$\begin{array}{llll}50.77 & -1.42 & -0.01\end{array}$

$\begin{array}{lll}0.00 & 0.00 & 0.00\end{array}$

$\begin{array}{llll}0.00 & 0.00 & 0.00\end{array}$

$\begin{array}{llll}0.00 & 0.00 & 0.00\end{array}$

$\begin{array}{lll}0.00 & 0.00 & 0.00\end{array}$

$\begin{array}{llll}0.00 & 0.00 & 0.00\end{array}$

$\begin{array}{llll}0.00 & 0.00 & 0.00\end{array}$

$\begin{array}{llll}0.00 & 0.00 & 0.00\end{array}$

$\begin{array}{llll}0.00 & 0.00 & 0.00\end{array}$ 
$91 \quad 156 \quad 247-8.2 \quad-4.3-1838.39$ $91 \quad 157248-8.3-4.2-1842.68$ $\begin{array}{lllll}91 & 158 & 249 & -8.5 & -4.1-1846.85\end{array}$ $\begin{array}{lllll}91 & 159 & 250 & -8.7 & -4.0-1850.93\end{array}$ $\begin{array}{llllll}91 & 160 & 251 & -8.9 & -3.9-1854.91\end{array}$ $\begin{array}{llllll}91 & 161 & 252 & -9.1 & -3.8-1858.79\end{array}$ $91 \quad 162 \quad 253 *-9.2-3.7-1862.57$ $\begin{array}{lllll}91 & 163 & 254 & -9.4 & -3.6-1866.25\end{array}$ $\begin{array}{lllll}91 & 164 & 255 & -9.6 & -3.5-1869.84\end{array}$ $91165256-9.8 \quad-3.4-1873.34$ $91166257-10.0 \quad-3.4-1876.74$ $91167258-10.1-3.3-1880.06$ $91 \quad 168 \quad 259-10.3-3.2-1883.28$ $91169260-10.5-3.1-1886.41$ $91170 \quad 261-10.6 \quad-3.0-1889.45$ $91171262-10.8-2.9-1892.41$ $91 \quad 172 \quad 263-11.0 \quad-2.8-1895.28$ $91173 \quad 264-11.1 \quad-2.7-1898.06$ $91174265-11.3-2.7-1900.77$ $91175266-11.5 \quad-2.6-1903.39$ $91176 \quad 267-11.6 \quad-2.5-1905.93$ $91177268-11.8-2.4-1908.38$ 91178 269-11.9 $-2.3-1910.76-2.641 .07-2.57-1914.90 \quad 184.42$ $91 \quad 179270-12.1-2.3-1913.06-2.88 \quad 1.76-2.54-1916.72 \quad 190.68$ $91 \quad 180 \quad 271-12.3 \quad-2.2-1915.28-2.99 \quad 1.07-2.52-1919.72 \quad 195.75$ $91181272-12.4 \quad-2.1-1917.43 \quad-3.43 \quad 1.76-2.49-1921.59201 .95$ $91182273-12.6-2.0-1919.50-3.34 \quad 1.07-2.47-1924.23 \quad 207.38$ $91 \quad 183274-12.7-2.0-1921.49-3.641 .76-2.44-1925.81213 .87$ $91184 \quad 275-12.9-1.9-1923.41 \quad-3.22 \quad 1.07-2.42-1927.98 \quad 219.77$ $91185276-13.0-1.8-1925.26-2.34 \quad 1.75-2.39-1928.24227 .59$ $91186 \quad 277-13.2-1.7-1927.04 \quad-1.75 \quad 1.07-2.37-1930.09233 .81$ $91187278-13.3 \quad-1.7-1928.74-0.91 \quad 1.75-2.34-1930.24241 .73$ $91188 \quad 279-13.5 \quad-1.6-1930.38-1.24 \quad 1.07-2.32-1932.87247 .17$ $91189280-13.6-1.5-1931.94-1.421 .75-2.30-1933.91254 .20$ $91190 \quad 281-13.8-1.5-1933.44-1.18 \quad 1.07-2.28-1935.83 \quad 260.35$ $91191282-13.9-1.4-1934.87-1.341 .75-2.26-1936.72 \quad 267.54$ $91192283-14.1-1.3-1936.24-1.23 \quad 1.07-2.23-1938.63 \quad 273.70$ $91193 \quad 284-14.2 \quad-1.3-1937.53-1.56 \quad 1.74-2.21-1939.56 \quad 280.84$ $91194285-14.3-1.2-1938.77-1.41 \quad 1.07-2.19-1941.30287 .17$ $91195286-14.5-1.1-1939.94-1.67 \quad 1.74-2.17-1942.04294 .50$ $91196287-14.6-1.1-1941.04-1.48 \quad 1.07-2.15-1943.61301 .01$ $91197288-14.7-1.0-1942.09-1.691 .74-2.13-1944.17308 .51$ $91198 \quad 289-14.9-0.9-1943.07-1.59 \quad 1.07-2.11-1945.71315 .05$ $91199290-15.0-0.9-1944.00-1.81 \quad 1.74-2.09-1946.16 \quad 322.67$ $91200 \quad 291-15.2 \quad-0.8-1944.86-0.36 \quad 1.07-2.07-1946.22 \quad 330.67$ $91201292-15.3-0.8-1945.66-0.70 \quad 1.74-2.06-1946.68338 .29$ $91202 \quad 293-15.4 \quad-0.7-1946.41-0.60 \quad 1.07-2.04-1947.98 \quad 345.06$ $91203 \quad 294-15.5 \quad-0.7-1947.10-0.83 \quad 1.73-2.02-1948.21352 .90$ $91204295-15.7-0.6-1947.73-2.121 .07-2.00-1950.78358 .40$ $91205296-15.8-0.5-1948.30-2.42 \quad 1.73-1.98-1950.97 \quad 366.28$ $91206 \quad 297-15.9 \quad-0.5-1948.82 \quad-2.05 \quad 1.07-1.97-1951.77 \quad 373.55$ $91207298-16.1 \quad-0.4-1949.29-2.05 \quad 1.73-1.95-1951.56381 .84$ $91208299-16.2-0.4-1949.70-1.72 \quad 1.07-1.93-1952.29389 .18$ $91209300-16.3 \quad-0.3-1950.07-1.26 \quad 1.73-1.92-1951.51398 .03$ $91210 \quad 301-16.4 \quad-0.3-1950.37-2.42 \quad 1.07-1.90-1953.63 \quad 403.99$ $91211302-16.5-0.2-1950.63$ 92111203

$92 \quad 112 \quad 204$

92113205

92114206

$\begin{array}{lll}92 & 115 & 207\end{array}$

$\begin{array}{lll}92 & 116 & 208\end{array}$

92117209

$92118 \quad 210$

$\begin{array}{lll}92 & 119 & 211\end{array}$

$2.0-11.0-1512.08$

$1.7-10.8-1522.96$

$1.5-10.6-1533.65$

$1.3-10.4-1544.16$

1. 0-10.2-1554. 48

$0.8-10.0-1564.62$

$0.5-9.9-1574.59$

$0.3-9.7-1584.37$

$0.1-9.5-1593.98$

$\begin{array}{lllll}92 & 120 & 212 & -0.2 & -9.4-1603.42\end{array}$
$-2.541 .73-1.88-1953.33$

$1.321 .00-6.75-1516.51$

$1.50 \cdot 0.00-6.62-1528.09$

$1.460 .99-6.50-1537.70$

$1.570 .00-6.39-1548.97$

$1.200 .99-6.27-1558.56$

$1.080 .00-6.16-1569.70$

$0.790 .98-6.05-1578.86$

$0.180 .00-5.95-1590.14$

$\begin{array}{llll}-0.35 & 0.98 & -5.84-1599.20\end{array}$

$-0.880 .00-5.74-1610.05$ 95
0.00

0.00

$0.00 \quad 0.00$

0.00

0.00

0.00

0.00

0.00

0.00

0.00

0.00

0.00

0.00

0.00

0.00

0.00

0.00

0.00

0.00

0.00

0.00

0.00

0.00

0.00

0.00

0.00

0.00

0.00

0.00

0.00

0.00

0.00

0.00

0.00

0.00

0.00

0.00

0.00

0.00

0.00

0.00

0.00

0.00

0.00

0.00

0.00

0.00

0.00

0.00

0.00

0.00

0.00

0.00

0.00

0.00

0.00

0.00

0.00

0.00

0.00

0.00

0.00

0.00

0.00

0.00

0.00

0.00

0.00

0.00

0.00

0.00

0.00

0.00

0.00

0.00

0.00

0.00

0.00

0.00

0.00

0.00

0.00

0.00

0.00

0.00

0.00

0.00

0.00

0.00

0.00

0.00

0.00

0.00

0.00

0.00

0.00

0.00

0.00

0.00
0.00

0.00

0.00

0.00

0.00

0.00

0.00

0.00

0.00

0.00

0.00

0.00

0.00

0.00

0.00

0.00

0.00

0.00

0.00

0.00

0.00

0.00

0.00

0.00

0.00

$0.00^{\circ}$

0.00

0.00

0.00

0.00

0.00

0.00

0.00

0.00

0.00

0.00

0.00

0.00

0.00

0.00

0.00

0.00

0.00

0.00

0.00

0.00

0.00

0.00

0.00

0.00

0.00

0.00

0.00

0.00

$\begin{array}{llll}0.00 & 0.00 & 0.00\end{array}$

$\begin{array}{llll}0.00 & 0.00 & 0.00\end{array}$

$\begin{array}{llll}0.00 & 0.00 & 0.00\end{array}$

$\begin{array}{llll}0.00 & 0.00 & 0.00\end{array}$

49.30

45.80

44.25

41.05

39.53

36.47

35.38

32.18

31.18

28.41
0.00

0.00

0.00

0.00

0.00

0.00

0.00

0.00

0.00

0.00

0.00

0.00

0.00

0.00

0.00
0.00

0.00

0.00

0.00

0.00

0.00

0.00

0.00 
$\begin{array}{llllllllll}92 & 121 & 213 & -0.4 & -9.2-1612.70 & -1.30 & 0.97 & -5.64-1618.67\end{array}$ $\begin{array}{lllllllll}92 & 122 & 214 & -0.6 & -9.0-1621.80 & -1.49 & 0.00 & -5.55-1628.84\end{array}$ $\begin{array}{llllllllll}92 & 123 & 215 & -0.9 & -8.9-1630.74 & -2.07 & 0.97 & -5.46-1637.30\end{array}$ $\begin{array}{lllllllll}92 & 124 & 216 & -1.1 & -8.7-1639.52 & -2.52 & 0.00 & -5.37-1647.41\end{array}$ $\begin{array}{lllllllll}92 & 125 & 217 & -1.3 & -8.5-1648.14 & -3.26 & 0.96 & -5.28-1655.72\end{array}$ $92126218-1.6 \quad-8.4-1656.60-3.330 .00-5.19-1665.12$ $\begin{array}{lllllllll}92 & 127 & 219 & -1.8 & -8.2-1664.90 & -2.39 & 0.95 & -5.11-1671.45\end{array}$

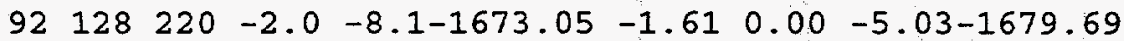
$\begin{array}{llllllllll}92 & 129 & 221 & -2.2 & -7.9-1681.05 & -1.27 & 0.95 & -4.95-1686.32\end{array}$ $\begin{array}{lllllllll}92 & 130 & 222 & -2.4 & -7.8-1688.90 & -0.51 & 0.00 & -4.87-1694.28\end{array}$ $\begin{array}{llllllllll}92 & 131 & 223 & -2.7 & -7.6-1696.60 & -0.95 & 0.95 & -4.80-1701.40\end{array}$ $92 \quad 132 \quad 224-2.9-7.5-1704.16-0.53 \quad 0.00-4.72-1709.41$ $\begin{array}{lllllllll}92 & 133 & 225 & -3.1 & -7.3-1711.57 & -0.33 & 0.94 & -4.65-1715.61\end{array}$ $\begin{array}{llllllll}92 & 134 & 226 & -3.3 & -7.2-1718.84 & 0.00 & 0.00 & -4.58-1723.42\end{array}$ $\begin{array}{lllllllll}92 & 135 & 227 & -3.5 & -7.1-1725.97 & -0.36 & 0.94 & -4.51-1729.90\end{array}$ $92136 \quad 228-3.7-6.9-1732.96-0.42 \quad 0.00-4.45-1737.82$ $\begin{array}{lllllllll}92 & 137 & 229 & -3.9 & -6.8-1739.81 & -0.77 & 0.93 & -4.38-1744.03\end{array}$ $92138230 \quad-4.2 \quad-6.6-1746.53 \quad-0.67 \quad 0.00-4.32-1751.52$ $\begin{array}{lllllllll}92 & 139 & 231 & -4.4 & -6.5-1753.12 & -0.98 & 0.93 & -4.25-1757.42\end{array}$

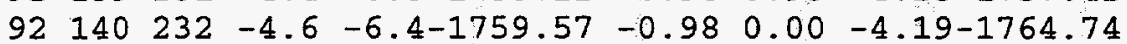
$\begin{array}{lllllllll}92 & 141 & 233 & -4.8 & -6.3-1765.90 & -1.27 & 0.92 & -4.13-1770.38\end{array}$ $92 \quad 142 \quad 234-5.0 \quad-6.1-1772.10-1.23 \quad 0.00-4.08-1777.40$

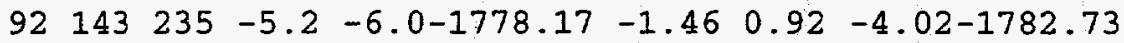
$92144236-5.4 \quad-5.9-1784.11-1.30 \quad 0.00-3.96-1789.38$ $92145 \quad 237-5.6 \quad-5.8-1789.94-1.51 \quad 0.91-3.91-1794.44$ $92146238-5.8 \quad-5.6-1795.64-1.27 \quad 0.00-3.86-1800.77$ $92147239-6.0-5.5-1801.23-1.630 .91-3.80-1805.75$ $92148 \quad 240-6.2 \quad-5.4-1806.69-1.43 \quad 0.00-3.75-1811.87$ $92149241-6.4 \quad-5.3-1812.04-1.62 \quad 0.91-3.70-1816.46$ $92 \quad 150 \quad 242-6.6 \quad-5.2-1817.27-1.41 \quad 0.00-3.65-1822.34$ $\begin{array}{lllllllll}92 & 151 & 243 & -6.8 & -5.1 & -1822.39 & -1.57 & 0.90 & -3.61-1826.67\end{array}$ $92152 \quad 244-7.0-4.9-1827.40-1.490 .00-3.56-1832.45$ $92153245-7.2-4.8-1832.30-1.41 \quad 0.90-3.51-1836.32$ $\begin{array}{llllllllll}92 & 154 & 246 & -7.3 & -4.7-1837.08 & -0.74 & 0.00 & -3.47-1841.29\end{array}$ $\begin{array}{llllllllll}92 & 155 & 247 & -7.5 & -4.6-1841.76 & -0.39 & 0.89 & -3.43-1844.68\end{array}$ $92156248-7.7-4.5-1846.33-0.08 \quad 0.00-3.38-1849.80$ $\begin{array}{llllllllll}92 & 157 & 249 & -7.9 & -4.4-1850.80 & -0.08 & 0.89 & -3.34-1853.33\end{array}$ $\begin{array}{lllllllll}92 & 158 & 250 & -8.1 & -4.3-1855.16 & 0.19 & 0.00 & -3.30-1858.27\end{array}$ $92 \quad \begin{array}{lllll}959 & 251 & -8.3 & -4.2-1859.42\end{array}$ $92 \quad 160 \quad 252-8.5 \quad-4.1-1863.58$ $92 \quad 161253 \quad-8.6 \quad-4.0-1867.64$ $92 \quad 162 \quad 254 \quad-8.8 \quad-3.9-1871.60$ $92 \quad 163255 \quad-9.0 \quad-3.8-1875.46$ $92 \quad 164256-9.2-3.7-1879.23$ $92 \quad 165257 \quad-9.3 \quad-3.6-1882.90$ $92 \quad 166 \quad 258-9.5-3.5-1886.48$ $\begin{array}{llllll}92 & 167 & 259 & -9.7 & -3.4-1889.96\end{array}$ $92168260-9.9-3.3-1893.36$ $92169261-10.0-3.3-1896.66$ $92 \quad 170 \quad 262-10.2 \quad-3.2-1899.87$

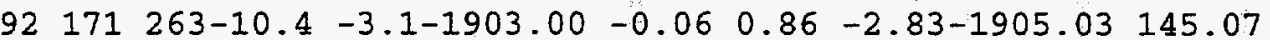
$92172264-10.5-3.0-1906.04-1.03 \quad 0.00-2.80-1909.87 \quad 148.30$ $92173 \quad 265-10.7-2.9-1908.99-0.66 \quad 0.86-2.77-1911.56 \quad 154.69$ $92174266-10.9-2.8-1911.86-0.72 \quad 0.00-2.74-1915.32 \quad 159.00$ $92175267-11.0-2.7-1914.64-1.29 \quad 0.86-2.71-1917.78 \quad 164.60$ $92176268-11.2 \quad-2.7-1917.34-1.75 \quad 0.00-2.68-1921.77 \quad 168.69$ $92177269-11.4 \quad-2.6-1919.96-2.23 \quad 0.85-2.65-1923.98 \quad 174.54$

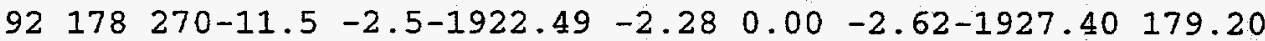

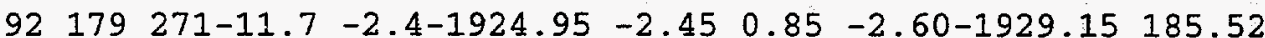
$92180272-11.8 \quad-2.3-1927.33-2.53 \quad 0.00-2.57-1932.43 \quad 190.31$

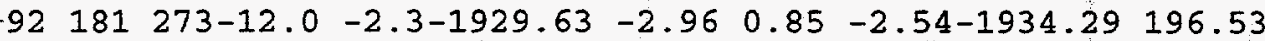
$92182 \quad 274-12.1-2.2-1931.86-2.84 \quad 0.00-2.52-1937.21201 .67$ $92183275-12.3 \quad-2.1-1934.01-3.13 \quad 0.85-2.49-1938.78 \quad 208.17$ $92184276-12.5 \quad-2.0-1936.08 \quad-2.71 \quad 0.00 \quad-2.47-1941.26213 .77$ $92185 \quad 277-12.6 \quad-2.0+1938.08-1.79 \quad 0.84-2.44-1941.47 \quad 221.63$ $92186278-12.8-1.9-1940.01-1.20 \quad 0.00-2.42-1943.62 \quad 227.55$

$\begin{array}{lll}0.00 & 0.00 & 0.00 \\ 0.00 & 0.00 & 0.00 \\ 0.00 & 0.00 & 0.00 \\ 0.00 & 0.00 & 0.00 \\ 0.00 & 0.00 & 0.00 \\ 0.00 & 0.00 & 0.00 \\ 0.00 & 0.00 & 0.00 \\ 0.00 & 0.00 & 0.00 \\ 0.00 & 0.00 & 0.00 \\ 0.00 & 0.00 & 0.00 \\ 0.00 & 0.00 & 0.00 \\ 0.00 & 0.00 & 0.00\end{array}$

$\begin{array}{llll}27.37 & -0.74 & -0.41\end{array}$

$27.17-0.87-0.87$

$0.00 \quad 0.00 \quad 0.00$

$\begin{array}{llll}29.21 & -0.99 & -0.57\end{array}$

$31.18-1.23-0.46$

$\begin{array}{llll}31.60 & -1.29 & -0.62\end{array}$

$33.78-1.59-0.61$

$34.59-1.53-0.55$

$36.92-1.93-0.66$

$38.14-1.72-0.49$

$\begin{array}{llll}40.92 & -1.91 & -0.45\end{array}$

$42.44-1.65-0.35$

$\begin{array}{llll}45.39 & -1.92 & -0.41\end{array}$

$\begin{array}{llll}47.31 & -1.50 & -0.23\end{array}$

$\begin{array}{llll}50.57 & -1.69 & -0.06\end{array}$

$52.71-1.30 \quad 0.13$

$0.00 \quad 0.00 \quad 0.00$

$0.00 \quad 0.00 \quad 0.00$

$0.00 \quad 0.00 \quad 0.00$

$0.00 \quad 0.00 \quad 0.00$

$0.00 \quad 0.00 \quad 0.00$

$0.00 \quad 0.00 \quad 0.00$

$\begin{array}{llll}0.00 & 0.00 & 0.00\end{array}$

$\begin{array}{llll}0.00 & 0.00 & 0.00\end{array}$

$\begin{array}{llll}0.00 & 0.00 & 0.00\end{array}$

$\begin{array}{llll}0.00 & 0.00 & 0.00\end{array}$

$\begin{array}{llll}0.00 & 0.00 & 0.00\end{array}$

$\begin{array}{llll}0.00 & 0.00 & 0.00\end{array}$

$0.00 \quad 0.00 \quad 0.00$

$\begin{array}{llll}0.00 & 0.00 & 0.00\end{array}$

$\begin{array}{llll}0.00 & 0.00 & 0.00\end{array}$

$\begin{array}{llll}0.00 & 0.00 & 0.00\end{array}$

$0.00 \quad 0.00 \quad 0.00$

$0.00 \quad 0.00 \quad 0.00$

$\begin{array}{llll}0.00 & 0.00 & 0.00\end{array}$

$0.00 \quad 0.00 \quad 0.00$

$\begin{array}{llll}0.00 & 0.00 & 0.00\end{array}$

$0.00 \quad 0.00 \quad 0.00$

$\begin{array}{llll}0.00 & 0.00 & 0.00\end{array}$

$\begin{array}{llll}0.00 & 0.00 & 0.00\end{array}$

$0.00 \quad 0.00 \quad 0.00$

$\begin{array}{llll}0.00 & 0.00 & 0.00\end{array}$

$\begin{array}{llll}0.00 & 0.00 & 0.00\end{array}$

$\begin{array}{llll}0.00 & 0.00 & 0.00\end{array}$

$0.00 \quad 0.00 \quad 0.00$

$0.00 \quad 0.00 \quad 0.00$

$0.00 \quad 0.00 \quad 0.00$

$0.00 \quad 0.00 \quad 0.00$

$0.00 \quad 0.00 \quad 0.00$

$\begin{array}{llll}0.00 & 0.00 & 0.00\end{array}$

$0.00 \quad 0.00 \quad 0.00$

$\begin{array}{llll}0.00 & 0.00 & 0.00\end{array}$

$0.00 \quad 0.00 \quad 0.00$

$0.00 \quad 0.00 \quad 0.00$ 
$92187279-12.9-1.8-1941.86 \quad-0.45 \quad 0.84-2.39-1943.86 \quad 235.38$ $92188280-13.1-1.7-1943.64-1.04 \quad 0.00-2.37-1947.05 \quad 240.26$ $92189281-13.2-1.7-1945.36-1.25 \quad 0.84-2.35-1948.12 \quad 247.27$

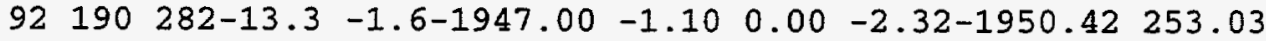
$92191283-13.5-1.5-1948.58-1.290 .83-2.30-1951.33 \quad 260.19$ $\begin{array}{lllllllll}92 & 192 & 284-13.6 & -1.5-1950.08 & -1.08 & 0.00 & -2.28-1953.44 & 266.16\end{array}$ $92193285-13.8-1.4-1951.53-1.33 \quad 0.83-2.26-1954.28 \quad 273.39$ $92194286-13.9-1.3-1952.90-1.21 \quad 0.00-2.24-1956.35279 .40$ $92195287-14.1-1.3-1954.21 \quad-1.46 \quad 0.83-2.22-1957.06 \quad 286.76$ $92196288-14.2-1.2-1955.46-1.20 \quad 0.00-2.19-1958.85293 .03$ $92197289-14.3-1.1-1956.64-1.380 .82-2.17-1959.37 \quad 300.59$ 92198 290-14.5 $-1.1-1957.76-1.290 .00-2.15-1961.20306 .83$ $92199291-14.6-1.0-1958.81-1.490 .82 \quad-2.13-1961.62 \quad 314.48$ $92200292-14.7-1.0-1959.81 \quad-0.35 \quad 0.00-2.12-1962.28 \quad 321.89$ $92201293-14.9-0.9-1960.75 \quad-0.66 \quad 0.82 \quad-2.10-1962.68 \quad 329.56$ $92202294-15.0 \quad-0.8-1961.63-0.71 \quad 0.00-2.08-1964.41335 .90$ $92203295-15.1 \quad-0.8-1962.44-1.01 \quad 0.82-2.06-1964.70343 .69$ $92204296-15.3 \quad-0.7-1963.20-0.83 \quad 0.00-2.04-1966.07 \quad 350.38$ $92205297-15.4 \quad-0.7-1963.91 \quad-0.83 \quad 0.81-2.02-1965.95 \quad 358.58$ $92206298-15.5 \quad-0.6-1964.56-1.62 \quad 0.00-2.01-1968.18 \quad 364.42$ $92207299-15.7 \quad-0.6-1965.15-1.77 \quad 0.81-1.99-1968.10 \quad 372.57$ $92208300-15.8-0.5-1965.69-1.28 \quad 0.00-1.97-1968.94 \quad 379.80$ $92209301-15.9-0.5-1966.17-2.42 \quad 0.81-1.95-1969.73 \quad 387.08$ $92210302-16.0 \quad-0.4-1966.60-2.44 \quad 0.00-1.94-1970.98 \quad 393.91$ $92211303-16.2 \quad-0.3-1966.98-2.57 \quad 0.81-1.92-1970.67402 .29$ $92212304-16.3 \quad-0.3-1967.31-0.44 \quad 0.00-1.91-1969.65 \quad 411.37$ $92213305-16.4-0.2-1967.58-2.430 .80-1.89-1971.10418 .00$ $93113206 \quad 1.9-10.8-1531.95$ $93 \quad 114207 \quad 1.7-10.7-1542.70$ 93115208 1.4-10.5-1553.26 93116209 93117210 $1.2-10.3-1563.64$ $1.0-10.1-1573.84$ $0.7-9.9-1583.86$ $1.071 .86-6.65-1535.67$ $1.191 .06-6.53-1546.98$ $1.121 .86-6.41-1556.69$ $0.991 .06-6.30-1567.89$ $0.731 .85-6.19-1577.44$ $0.421 .06-6.08-1588.46$ $\begin{array}{lll}93 & 118 & 211\end{array}$ $93 \quad 119 \quad 212$ $0.5-9.8-1593.71$ $-0.101 .85-5.97-1597.93$ $93 \begin{array}{lllllllll}120 & 213 & 0.3 & -9.6-1603.38 & -0.56 & 1.06 & -5.87-1608.76\end{array}$ $\begin{array}{lllllllll}93 & 121 & 214 & 0.0 & -9.4-1612.89 & -0.97 & 1.85 & -5.77-1617.79\end{array}$ $\begin{array}{lllllllll}93 & 122 & 215 & -0.2 & -9.2-1622.22 & -1.07 & 1.06 & -5.68-1627.91\end{array}$ $\begin{array}{lllllllll}93 & 123 & 216 & -0.4 & -9.1-1631.40 & -1.58 & 1.84 & -5.58-1636.71\end{array}$ $\begin{array}{lllllllll}93 & 124 & 217 & -0.7 & -8.9-1640.40 & -1.92 & 1.06 & -5.49-1646.75\end{array}$ $\begin{array}{lllllllll}93 & 125 & 218 & -0.9 & -8.8-1649.25 & -2.51 & 1.84 & -5.40-1655.32\end{array}$ $\begin{array}{lllllllll}93 & 126 & 219 & -1.1 & -8.6-1657.93 & -2.68 & 1.06 & -5.31-1664.86\end{array}$ $\begin{array}{lllllllll}93 & 127 & 220 & -1.3 & -8.4-1666.46 & -1.70 & 1.83 & -5.23-1671.55\end{array}$ $\begin{array}{lllllllll}93 & 128 & 221 & -1.6 & -8.3-1674.84 & -0.98 & 1.06 & -5.14-1679.90\end{array}$ $\begin{array}{lllllllll}93 & 129 & 222 & -1.8 & -8.1-1683.06 & 0.30 & 1.83 & -5.06-1685.99\end{array}$ $\begin{array}{lllllllll}93 & 130 & 223 & -2.0 & -8.0-1691.13 & -0.73 & 1.06 & -4.98-1695.78\end{array}$ $\begin{array}{llllllllll}93 & 131 & 224 & -2.2 & -7.8 & -1699.05 & -0.47 & 1.83 & -4.90-1702.59\end{array}$ $\begin{array}{lllllllll}93 & 132 & 225 & -2.4 & -7.7-1706.82 & 0.01 & 1.06 & -4.83-1710.58\end{array}$ $\begin{array}{lllllllll}93 & 133 & 226 & -2.7 & -7.6-1714.45 & -0.08 & 1.82 & -4.76-1717.46\end{array}$ $\begin{array}{llllllllll}93 & 134 & 227 & -2.9 \cdot & -7.4-1721.93 & -0.14 & 1.06 & -4.68-1725.69\end{array}$ $\begin{array}{lllllllll}93 & 135 & 228 & -3.1 & -7.3-1729.27 & -0.52 & 1.82 & -4.61-1732.59\end{array}$ $\begin{array}{lllllllll}93 & 136 & 229 & -3.3 & -7.1-1736.48 & -0.61 & 1.06 & -4.54-1740.57\end{array}$ $\begin{array}{llllllllll}93 & 137 & 230 & -3.5 & -7.0-1743.54 & -0.96 & 1.81 & -4.48-1747.16\end{array}$ $\begin{array}{lllllllll}93 & 138 & 231 & -3.7 & -6.9-1750: 47 & -0.93 & 1.06 & -4.41-1754.75\end{array}$ $93 \begin{array}{llllllll}139 & 232 & -3.9 & -6.7-1757.27 & -1.29 & 1.81 & -4.35-1761.09\end{array}$ $93 \quad 140 \quad 233-4.1 \quad-6.6-1763.93-1.351 .06-4.29-1768.51$ $\begin{array}{lllllllll}93 & 141 & 234 & -4.3 & -6.5-1770.46 & -1.65 & 1.81 & -4.23-1774.53\end{array}$ $\begin{array}{lllllllll}93 & 142 & 235 & -4.5 & -6.3-1776.86 & -1.66 & 1.06 & -4.17-1781.63\end{array}$ $\begin{array}{lllllllll}93 & 143 & 236 & -4.7 & -6.2-1783.14 & -1.85 & 1.80 & -4.11-1787.29\end{array}$ $\begin{array}{llllllllll}93 & 144 & 237 & -5.0 & -6.1-1789.29 & -1.74 & 1.06 & -4.05 & -1794.02\end{array}$ $\begin{array}{lllllllll}93 & 145 & 238 & -5.2 & -6.0-1795.31 & -1.92 & 1.80 & -3.99-1799.43\end{array}$ $\begin{array}{lllllllll}93 & 146 & 239 & -5.4 & -5.8 & -1801.22 & -1.87 & 1.06 & -3.94-1805.97\end{array}$ $\begin{array}{lllllllll}93 & 147 & 240 & -5.5 & -5.7-1807.00 & -2.08 & 1.80 & -3.89-1811.17\end{array}$ $\begin{array}{lllllllll}93 & 148 & 241 & -5.7 & -5.6-1812.66 & -1.87 & 1.06 & -3.83-1817.31\end{array}$ $\begin{array}{lllllllll}93 & 149 & 242 & -5.9 & -5.5-1818.21 & -2.06 & 1.79 & -3.78-1822.25\end{array}$ $\begin{array}{llllllllll}93 & 150 & 243 & -6.1 & -5.4-1823.63 & -1.87 & 1.06 & -3.73-1828.18\end{array}$ $93 \quad 151244-6.3 \quad-5.3-1828.95-2.00 \quad 1.79-3.68-1832.84$ 97

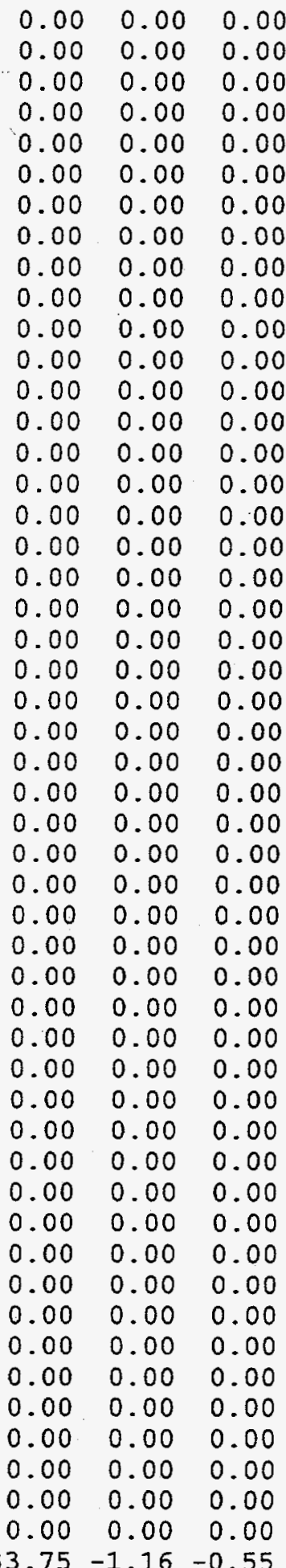
53.56 50.32 48.68 45.55 44.07 41.12 39.72 36.97 36.01 33.96 33.23 31.26 30.76 29.29 30.67 30.40 32.38 30.66 31.92 32.01 33.19 33.03 34.21 34.30 35.78 36.26 37.99 38.65 40.70 41.67 44.08 45.42 48.08 49.62 52.49 54.42 57.54 59.69 63.10 $35.22-1.52-0.56$ $35.62-1.57-0.64$ $\begin{array}{llll}0.00 & 0.00 & 0.00\end{array}$ $\begin{array}{llll}0.00 & 0.00 & 0.00\end{array}$ $\begin{array}{llll}39.95 & -2.40 & -0.75\end{array}$ $\begin{array}{llll}41.04 & -2.29 & -0.63\end{array}$ $\begin{array}{llll}43.37 & -2.56 & -0.71\end{array}$ $\begin{array}{llll}44.87 & -2.29 & -0.55\end{array}$ $\begin{array}{llll}47.45 & -2.55 & -0.63\end{array}$ $\begin{array}{llll}49.31 & -2.18 & -0.31\end{array}$ $\begin{array}{llll}52.32 & -2.25 & -0.17\end{array}$ $\begin{array}{llll}54.26 & -2.03 & -0.16\end{array}$ $\begin{array}{llll}57.41 & -2.19 & -0.13\end{array}$ $\begin{array}{llll}59.92 & -1.64 & 0.23\end{array}$ $\begin{array}{llll}0.00 & 0.00 \quad 0.00\end{array}$ 
$93152 \quad 245-6.5 \quad-5.1-1834.14-1.96 \quad 1.06-3.64-1838.68$ $\begin{array}{lllllllll}93 & 153 & 246 & -6.7 & -5.0-1839.23 & -1.83 & 1.79 & -3.59-1842.86\end{array}$ $\begin{array}{lllllllll}93 & 154 & 247 & -6.9 & -4.9-1844.21 & -1.19 & 1.06 & -3.54-1847.88\end{array}$ $93155248-7.1-4.8-1849.08-1.001 .79-3.50-1851.79$ $\begin{array}{lllllllll}93 & 156 & 249 & -7.3 & -4.7-1853.84 & -0.43 & 1.06 & -3.46-1856.66\end{array}$ $\begin{array}{lllllllll}93 & 157 & 250 & -7.5 & -4.6-1858.49 & -0.42 & 1.78 & -3.41-1860.54\end{array}$ $\begin{array}{lllllllll}93 & 158 & 251 & -7.7 & -4.5-1863.04 & -0.13 & 1.06 & -3.37-1865.48\end{array}$ $\begin{array}{lllllllll}93 & 159 & 252 & -7.8 & -4.4-1867.48 & -0.29 & 1.78 & -3.33-1869.32\end{array}$ $93160253-8.0 \quad-4.3-1871.82-0.17 \quad 1.06-3.29-1874.22$ $\begin{array}{lllllllll}93 & 161 & 254 & -8.2 & -4.2-1876.06 & -0.42 & 1.78 & -3.25-1877.95\end{array}$

$93162255-8.4-4.1-1880.20-0.301 .06-3.21-1882.65102 .08$ $93163256-8.6 \quad-4.0-1884.24-0.351 .77-3.17-1885.99 \quad 106.81$ $\begin{array}{llllllllll}93 & 164 & 257 & -8.7 & -3.9-1888.18 & 0.05 & 1.06 & -3.13-1890.21 & 110.66\end{array}$ $93165258-8.9-3.8-1892.03$ $\begin{array}{llllll}93 & 166 & 259 & -9.1 & -3.7-1895.78\end{array}$ $93167260-9.3-3.6-1899.44$ $93 \quad 168 \quad 261 \quad-9.4 \quad-3.5-1903.01$ $\begin{array}{llllll}93 & 169 & 262 & -9.6 & -3.4-1906.48\end{array}$ $93170 \quad 263-9.8-3.3-1909.86$ $\begin{array}{llllll}93 & 171 & 264 & -9.9 & -3.2-1913.16\end{array}$ $93172 \quad 265-10.1-3.2-1916.36$ $0.031 .77-3.10-1893.33115 .61$ $0.611 .06-3.06-1897.17 \quad 119.84$ $0.38 \quad 1.77-3.03-1900.32 \quad 124.76$ $0.581 .06-2.99-1904.36 \quad 128.80$ $0.341 .77-2.96-1907.33 \quad 133.89$ $0.48 \quad 1.06-2.92-1911.25 \quad 138.05$ $0.011 .76-2.89-1914.27143 .09$ $0.851 .06-2.86-1919.01146 .43$ $93173266-10.3 \quad-3.1-1919.48-0.451 .76-2.83-1921.00152 .51$ $93174267-10.4-3.0-1922.51-0.46 \quad 1.06-2.80-1924.71156 .87$ $93175268-10.6-2.9-1925.46-1.001 .76-2.77-1927.47 \quad 162.19$ $93176269-10.8-2.8-1928.32-1.46 \quad 1.06-2.74-1931.46 \quad 166.27$ $93177270-10.9-2.7-1931.10-1.93 \quad 1.76-2.71-1933.98171 .81$ $93 \quad 178 \quad 271-11.1-2.7-1933.80-1.95 \quad 1.06-2.68-1937.37 \quad 176.50$ $93179272-11.3 \quad-2.6-1936.42-2.02 \quad 1.75-2.65-1939.34182 .60$ $93 \quad 180 \quad 273-11.4 \quad-2.5-1938.96-2.02 \quad 1.06-2.62-1942.54 \quad 187.47$ $93181274-11.6-2.4-1941.41-2.401 .75-2.60-1944.66193 .43$ $93182 \quad 275-11.7 \quad-2.3-1943.80-2.22 \quad 1.06-2.57-1947.52 \quad 198.63$ $93183276-11.9-2.3-1946.10-2.57 \quad 1.75 \quad-2.54-1949.46 \quad 204.77$ $93184277-12.0 \quad-2.2-1948.33-2.13 \quad 1.06-2.52-1951.91210 .38$ $93185 \quad 278-12.2 \quad-2.1-1950.48-1.24 \quad 1.75 \quad-2.49-1952.46 \quad 217.91$ $93186279-12.3 \quad-2.0-1952.56-0.67 \quad 1.06-2.47-1954.63 \quad 223.81$ $93187280-12.5 \quad-2.0-1954.56-1.291 .74-2.44-1956.55 \quad 229.96$ $93188281-12.6-1.9-1956.49-1.11 \quad 1.06-2.42-1958.96 \quad 235.62$ $93189282-12.8-1.8-1958.35-1.38 \quad 1.74-2.39-1960.39242 .27$ $93 \quad 190283-12.9-1.8-1960.15-1.26 \quad 1.06-2.37-1962.72 \quad 248.01$ $\begin{array}{lllllllll}93 & 191 & 284-13.1 & -1.7-1961.87 & -1.36 & 1.74 & -2.35-1963.83 & 254.96\end{array}$ $93192285-13.2-1.6-1963.52-1.27 \quad 1.06-2.32-1966.05 \quad 260.81$ $93193286-13.4-1.5-1965.10-1.51 \quad 1.74-2.30-1967.18267 .76$ $93194287-13.5-1.5-1966.62-1.31 \quad 1.06-2.28-1969.15273 .86$ $\begin{array}{lllllllll}93 & 195 & 288-13.7 & -1.4-1968.07 & -1.52 & 1.74 & -2.26-1970.12 & 280.97\end{array}$ $93196289-13.8-1.3-1969.46-1.201 .06-2.24-1971.84287 .32$ $93197290-13.9-1.3-1970.78-1.01 \quad 1.73-2.22-1972.27294 .95$ $93198291-14.1-1.2-1972.03-0.87 \quad 1.06-2.20-1974.04301 .26$ $93199292-14.2-1.2-1973.23-0.441 .73-2.18-1974.11309 .26$ $93200293-14.3-1.1-1974.36-0.56 \quad 1.06-2.16-1976.02 \quad 315.42$ $93201294-14.5-1.0-1975.43-1.02 \quad 1.73-2.14-1976.86 \quad 322.65$ $93202295-14.6-1.0-1976.44-0.98 \quad 1.06-2.12-1978.48 \quad 329.10$ $93203296-14.7-0.9-1977.39-1.40 \quad 1.73-2.10-1979.16 \quad 336.49$ $93204 \quad 297-14.9-0.9-1978.28-1.24 \quad 1.06-2.08-1980.55 \quad 343.18$ $93205298-15.0-0.8-1979.12-1.52 \quad 1.73-2.06-1980.98 \quad 350.82$ $93206299-15.1 \quad-0.7-1979.89-1.28 \quad 1.06-2.04-1982.16 \quad 357.71$ $93207300-15.3 \quad-0.7-1980.61-2.23 \quad 1.72-2.03-1983.15 \quad 364.79$ $93208301-15.4-0.6-1981.28-2.31 \quad 1.06-2.01-1984.54371 .47$ $93209302-15.5-0.6-1981.89-2.73 \quad 1.72-1.99-1984.89379 .20$ $93210303-15.6-0.5-1982.44-2.74 \quad 1.06-1.98-1986.10386 .06$ $93211304-15.8-0.5-1982.94-2.84 \quad 1.72-1.96-1986.02 \quad 394.21$ $93212305-15.9-0.4-1983.39-0.76 \quad 1.06-1.94-1985.03 \quad 403.27$ $93213306-16.0-0.4-1983.78-2.68 \quad 1.72-1.93-1986.67409 .70$ $93214 \quad 307-16.1 \quad-0.3-1984.13-2.40 \quad 1.06-1.91-1987.38 \quad 417.06$ $93215308-16.2-0.3-1984.42-2.291 .72-1.89-1986.89425 .62$ $94115209 \quad 1.9-10.7-1551.61$ $94116210 \quad 1.6-10.5-1562.22$ $\begin{array}{llll}-2.29 & 1.72 & -1.89-1986.89 & 425.62\end{array}$ $\begin{array}{llll}0.96 & 0.99 & -6.56-1556.22 & 56.42\end{array}$ $0.950 .00-6.44-1567.71 \quad 53.00$ 98
0.00

0.00

0.00

0.00

0.00

0.00

0.00

$0.00 \quad 0.00$

0.00

0.00

0.00

0.00

0.00

0.00

0.00

0.00

0.00

0.00

0.00

0.00

0.00

0.00

0.00

0.00

0.00

0.00

0.00

0.00

0.00

0.00

0.00

0.00

0.00

0.00

0.00

0.00

0.00

0.00

0.00

0.00

0.00

0.00

0.00

0.00

0.00

0.00

0.00

0.00

0.00

0.00

0.00

0.00

0.00

0.00

0.00

0.00

0.00

0.00

0.00

0.00

0.00

0.00

0.00

0.00

0.00

0.00

0.00

0.00

0.00

0.00

0.00

0.00

0.00

0.00

0.00

0.00

0.00

.00

0.00

0.00

0.00

0.00

0.00

0.00

0.00

0.00

0.00

0.00

0.00

0.00

0.00

0.00

0.00

0.00

0.00

0.00

0.00

0.00

0.00

0.00

0.00

0.00

0.00

0.00

0.00

0.00

0.00

0.00

0.00

0.00

0.00

0.00

0.00

0.00

0.00

0.00

0.00

0.00

0.00

0.00

0.00

0.00

0.00

0.00

0.00

0.00

0.00

0.00

0.00

. 00

0.00

0.00

0.00

0.00

0.00

0.00

0.00

0.00

0.00

0.00

0.00

0.00

0.00

0.00

0.00

0.00

0.00

0.00

0.00

0.00

0.00

0.00

0.00

0.00

0.00

0.00

0.00

0.00

0.00

0.00

0.00

0.00

0.00

0.00

0.00

0.00

0.00

0.00

0.00

0.00

0.00

0.00

0.00

0.00

0.00

0.00

0.00

0.00

0.00 
$94 \quad 117211$

$\begin{array}{lll}94 & 118 & 212\end{array}$

94119213

94120214

94121215

$94122 \quad 216$

$94 \quad 123 \quad 217$

$94124218-0.2-9.1-1640.85$

$94 \quad 126220$

$94 \quad 127 \quad 221$

$\begin{array}{lll}94 & 128 & 222\end{array}$

$94 \quad 129223-1.3-8.4-1684.62-0$.

$94 \begin{array}{llllll}930 & 224 & -1.6 & -8.2-1692.91 & -0 \\ 94 & 131 & 225 & -1.8 & -8.1-1701.05 & -0 .\end{array}$

$\begin{array}{lllll}94 & 131 & 225 & -1.8 & -8.1-1701.05 \\ 94 & 132 & 226 & -2.0 & -7.9-1709.04\end{array}$

$\begin{array}{lllll}94 & 132 & 226 & -2.0 & -7.9-1709.04 \\ 94 & 133 & 227 & -2.2 & -7.8-1716.89\end{array}$

$\begin{array}{lllll}94 & 134 & 228 & -2.4 & -7.6-1724.59\end{array}$

$\begin{array}{lllll}94 & 135 & 229 & -2.6 & -7.5-1732.14\end{array}$

$94 \quad 136 \quad 230-2.9-7.3-1739.56$

$\begin{array}{llllll}94 & 137 & 231 & -3.1 & -7.2-1746.83\end{array}$

$\begin{array}{lllll}94 & 138 & 232 & -3.3 & -7.1-1753.97\end{array}$

$94 \begin{array}{llllllll}139 & 233 & -3.5 & -6.9-1760.98 & -1.17 & 0.93 & -4.44-1765.66\end{array}$

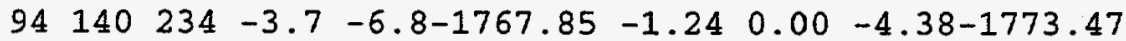

$\begin{array}{lllllllll}94 & 141 & 235 & -3.9 & -6.7-1774.58 & -1.59 & 0.92 & -4.32-1779.57\end{array}$

$\begin{array}{lllllllll}94 & 142 & 236 & -4.1 & -6.5-1781.19 & -1.64 & 0.00 & -4.26-1787.09\end{array}$

$\begin{array}{lllllllll}94 & 143 & 237 & -4.3 & -6.4-1787.67 & -1.87 & 0.92 & -4.20-1792.82\end{array}$

$\begin{array}{lllllllll}94 & 144 & 238 & -4.5 & -6.3-1794.02 & -1.78 & 0.00 & -4.14-1799.94\end{array}$

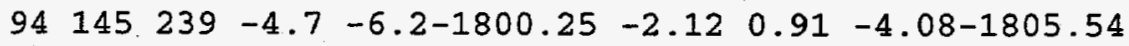

$\begin{array}{llllllll}94 & 146 & 240 & -4.9 & -6.0-1806.35 & -1.95 & 0.00 & -4.03-1812.33\end{array}$

$\begin{array}{lllllllll}94 & 147 & 241 & -5.1 & -5.9-1812.33 & -2.17 & 0.91 & -3.97-1817.56\end{array}$

$\begin{array}{lllllllll}94 & 148 & 242 & -5.3 & -5.8-1818.19 & -1.99 & 0.00 & -3.92-1824.10\end{array}$

$\begin{array}{lllllllll}94 & 149 & 243 & -5.5 & -5.7-1823.93 & -2.25 & 0.91 & -3.87-1829.14\end{array}$

$\begin{array}{llllllll}94 & 150 & 244 & -5.7 & -5.6-1829.55 & -2.08 & 0.00 & -3.81-1835.45\end{array}$

$\begin{array}{lllllllll}94 & 151 & 245 & -5.9 & -5.4-1835.06 & -2.48 & 0.90 & -3.76-1840.40\end{array}$

$\begin{array}{llllllll}94 & 152 & 246 & -6.1 & -5.3-1840.45 & -2.22 & 0.00 & -3.71-1846.39\end{array}$

$\begin{array}{lllllllll}94 & 153 & 247 & -6.3 & -5.2 & -1845.73 & -2.13 & 0.90 & -3.67-1850.63\end{array}$

$\begin{array}{llllllll}94 & 154 & 248 & -6.5 & -5.1-1850.90 & -1.45 & 0.00 & -3.62-1855.97\end{array}$

$\begin{array}{lllllllll}94 & 155 & 249 & -6.7 & -5.0 & -1855.95 & -1.25 & 0.89 & -3.57-1859.88\end{array}$

$\begin{array}{lllllllll}94 & 156 & 250 & -6.8 & -4.9-1860.90 & -0.73 & 0.00 & -3.53-1865.16\end{array}$

$\begin{array}{lllllllll}94 & 157 & 251 & -7.0 & -4.8-1865.74 & -0.74 & 0.89 & -3.48-1869.07\end{array}$

$\begin{array}{lllllllll}94 & 158 & 252 & -7.2 & -4.7-1870.47 & -0.46 & 0.00 & -3.44-1874.37\end{array}$

$\begin{array}{lllllllll}94 & 159 & 253 & -7.4 & -4.6-1875.10 & -0.59 & 0.89 & -3.40-1878.20\end{array}$

$94 \begin{array}{llllllll}160 & 254 & -7.6 & -4.5-1879.62 & -0.48 & 0.00 & -3.36-1883.46\end{array}$

$\begin{array}{lllllllll}94 & 161 & 255 & -7.8 & -4.4-1884.04 & -0.71 & 0.88 & -3.32-1887.19\end{array}$

$\begin{array}{lllllllll}94 & 162 & 256 & -7.9 & -4.3-1888.36 & -0.59 & 0.00 & -3.28-1892.23\end{array}$

$\begin{array}{llllllllll}94 & 163 & 257 & -8.1 & -4.2-1892.58 & -0.62 & 0.88 & -3.24-1895.56 & 104.51\end{array}$

$\begin{array}{llllllllll}94 & 164 & 258 & -8.3 & -4.1-1896.70 & -0.18 & 0.00 & -3.20-1900.08 & 108.06\end{array}$

$94 \quad 165 \quad 259-8.5 \quad-4.0-1900.73$

$\begin{array}{llllll}94 & 166 & 260 & -8.7 & -3.9-1904.65\end{array}$

$\begin{array}{llllll}94 & 167 & 261 & -8.8 & -3.8-1908.49\end{array}$

$\begin{array}{lllll}94 & 168 & 262 & -9.0 & -3.7-1912.22\end{array}$

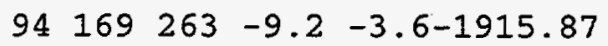

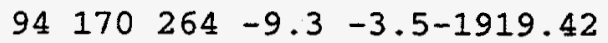

$\begin{array}{lllll}94 & 171 & 265 & -9.5 & -3.4-1922.89\end{array}$

$94 \quad 172 \quad 266-9.7 \quad-3.3-1926.26$

$\begin{array}{llll}-0.13 & 0.88 & -3.16-1903.14 & 113.07\end{array}$

$0.510 .00-3.13-1907.27 \quad 117.01$

$\begin{array}{lllll}0.32 & 0.87 & -3.09-1910.38 & 121.97\end{array}$

$0.350 .00-3.05-1914.93 \quad 125.50$

$\begin{array}{lllll}0.41 & 0.87 & -3.02-1917.61 & 130.89\end{array}$

$\begin{array}{llll}0.56 & 0.00 & -2.98-1921.85 & 134.72\end{array}$

$0.08 \quad 0.86-2.95-1924.89139 .75$

$\begin{array}{llllll}-3.3-1926.26 & -0.67 & 0.00 & -2.92-1929.85 & 142.86\end{array}$

$94174 \quad 268-10.0-3.2-1932.74-0.390 .86-2.89-1931.92148 .86$

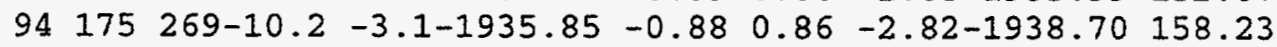

$\begin{array}{llllllll}94 & 176 & 270-10.3 & -3.0-1938.88 & -1.38 & 0.00 & -2.79-1943.06 & 161.94\end{array}$

$\begin{array}{llllllll}94 & 177 & 271-10.5 & -2.9-1941.82 & -1.73 & 0.85 & -2.76-1945.46 & 167.61\end{array}$

$\begin{array}{lllllllll}94 & 178 & 272-10.7 & -2.8-1944.68 & -1.74 & 0.00 & -2.73-1949.16 & 171.98\end{array}$

$\begin{array}{lllllllll}94 & 179 & 273-10.8 & -2.7-1947.46 & -1.81 & 0.85 & -2.70-1951.12 & 178.09\end{array}$

$\begin{array}{llllllll}94 & 180 & 274-11.0 & -2.7-1950.16 & -1.81 & 0.00 & -2.68-1954.64 & 182.64\end{array}$ $\begin{array}{lllllllll}94 & 181 & 275-11.1 & -2.6-1952.77 & -2.21 & 0.85 & -2.65-1956.78 & 188.57\end{array}$ $94182 \quad 276-11.3-2.5-1955.31-2.04 \quad 0.00-2.62-1959.97193 .45$
0.00

0.00

0.00

0.00

$\begin{array}{lll}0.00 & 0.00 & 0.00\end{array}$

$\begin{array}{llll}0.00 & 0.00 & 0.00\end{array}$

$\begin{array}{llll}0.00 & 0.00 & 0.00\end{array}$

$\begin{array}{llll}0.00 & 0.00 & 0.00\end{array}$

$\begin{array}{lll}0.00 & 0.00 & 0.00\end{array}$

$\begin{array}{llll}0.00 & 0.00 & 0.00\end{array}$

$\begin{array}{llll}0.00 & 0.00 & 0.00\end{array}$

$0.00 \quad 0.00 \quad 0.00$

$\begin{array}{llll}0.00 & 0.00 & 0.00\end{array}$

$\begin{array}{llll}0.00 & 0.00 & 0.00\end{array}$

$\begin{array}{llll}0.00 & 0.00 & 0.00\end{array}$

$\begin{array}{llll}0.00 & 0.00 & 0.00\end{array}$

$\begin{array}{llll}0.00 & 0.00 & 0.00\end{array}$

$\begin{array}{lll}0.00 & 0.00 & 0.00\end{array}$

$\begin{array}{lll}0.00 & 0.00 & 0.00\end{array}$

$\begin{array}{llll}0.00 & 0.00 & 0.00\end{array}$

$\begin{array}{llll}0.00 & 0.00 & 0.00\end{array}$

$\begin{array}{lll}0.00 & 0.00 & 0.00\end{array}$

$\begin{array}{llll}0.00 & 0.00 & 0.00\end{array}$

$\begin{array}{llll}38.35 & -1.45 & -0.65\end{array}$

$\begin{array}{llll}40.02 & -1.84 & -0.67\end{array}$

$\begin{array}{llll}40.34 & -1.86 & -0.62\end{array}$

$\begin{array}{llll}42.16 & -2.36 & -0.77\end{array}$

$\begin{array}{llll}42.88 & -2.24 & -0.60\end{array}$

$\begin{array}{llll}45.09 & -2.60 & -0.73\end{array}$

$\begin{array}{llll}46.16 & -2.39 & -0.61\end{array}$

$\begin{array}{llll}48.58 & -2.78 & -0.66\end{array}$

$\begin{array}{llll}50.12 & -2.36 & -0.41\end{array}$

$\begin{array}{llll}52.95 & -2.58 & -0.41\end{array}$

$\begin{array}{llll}54.71 & -2.18 & -0.19\end{array}$

$\begin{array}{llll}57.75 & -2.42 & -0.17\end{array}$

$\begin{array}{llll}59.80 & -1.97 & 0.11\end{array}$

$\begin{array}{lll}63.17 & -2.12 & 0.36\end{array}$

$\begin{array}{lll}65.39 & -1.72 & 0.50\end{array}$

$\begin{array}{llll}0.00 & 0.00 & 0.00\end{array}$

$\begin{array}{llll}0.00 & 0.00 & 0.00\end{array}$

$\begin{array}{llll}0.00 & 0.00 & 0.00\end{array}$

$\begin{array}{llll}0.00 & 0.00 & 0.00\end{array}$

$\begin{array}{llll}0.00 & 0.00 & 0.00\end{array}$

$\begin{array}{llll}0.00 & 0.00 & 0.00\end{array}$

$\begin{array}{llll}0.00 & 0.00 & 0.00\end{array}$

$\begin{array}{llll}0.00 & 0.00 & 0.00\end{array}$

$\begin{array}{llll}0.00 & 0.00 & 0.00\end{array}$

$\begin{array}{llll}0.00 & 0.00 & 0.00\end{array}$

$\begin{array}{llll}0.00 & 0.00 & 0.00\end{array}$

$\begin{array}{llll}0.00 & 0.00 & 0.00\end{array}$

$\begin{array}{llll}0.00 & 0.00 & 0.00\end{array}$

$\begin{array}{llll}0.00 & 0.00 & 0.00\end{array}$

$\begin{array}{lll}0.00 & 0.00 & 0.00\end{array}$

$\begin{array}{lll}0.00 & 0.00 & 0.00\end{array}$

$\begin{array}{llll}0.00 & 0.00 & 0.00\end{array}$

$\begin{array}{llll}0.00 & 0.00 & 0.00\end{array}$

$\begin{array}{llll}0.00 & 0.00 & 0.00\end{array}$

$\begin{array}{lll}0.00 & 0.00 & 0.00\end{array}$

$\begin{array}{llll}0.00 & 0.00 & 0.00\end{array}$

$\begin{array}{llll}0.00 & 0.00 & 0.00\end{array}$

$\begin{array}{lll}0.00 & 0.00 & 0.00\end{array}$

$\begin{array}{llll}0.00 & 0.00 & 0.00\end{array}$

$\begin{array}{llll}0.00 & 0.00 & 0.00\end{array}$

$\begin{array}{lll}0.00 & 0.00 & 0.00\end{array}$

$\begin{array}{lll}0.00 & 0.00 & 0.00\end{array}$

$\begin{array}{llll}0.00 & 0.00 & 0.00\end{array}$

$\begin{array}{llll}0.00 & 0.00 & 0.00\end{array}$

$\begin{array}{llll}0.00 & 0.00 & 0.00\end{array}$ 
$\begin{array}{lllllllll}94 & 183 & 277-11.5 & -2.4-1957.77 & -2.28 & 0.85 & -2.59-1961.80 & 199.70\end{array}$ $\begin{array}{llllllll}94 & 184 & 278-11.6 & -2.3-1960.15 & -1.83 & 0.00 & -2.57-1964.55 & 205.02\end{array}$ $\begin{array}{llllllll}94 & 185 & 279-11.8 & -2.3-1962.46 & -0.99 & 0.84 & -2.54-1965.15 & 212.49\end{array}$

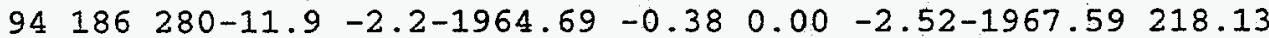
$\begin{array}{llllllll}94 & 187 & 281-12.1 & -2.1-1966.84 & 0.10 & 0.84 & -2.49-1968.40 & 225.39\end{array}$

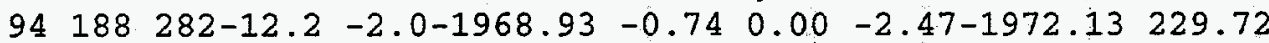
$\begin{array}{llllllll}94 & 189 & 283-12.4 & -2.0-1970.94 & -1.09 & 0.84 & -2.44-1973.63 & 236.29\end{array}$ $\begin{array}{lllllllll}94 & 190 & 284-12.5 & -1.9-1972.88 & -0.86 & 0.00 & -2.42-1976.15 & 241.84\end{array}$ $\begin{array}{lllllllll}94 & 191 & 285-12.7 & -1.8-1974.74 & -1.20 & 0.83 & -2.39-1977.50 & 248.56\end{array}$

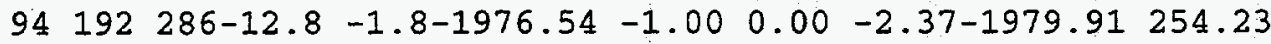
$94 \quad 193 \quad 287-13.0 \quad-1.7-1978.27-1.220 .83-2.35-1981.01261 .20$ $\begin{array}{llllllll}94 & 194 & 288-13.1 & -1.6-1979.93 & -0.90 & 0.00 & -2.33-1983.16 & 267.12\end{array}$ $\begin{array}{llllllll}94 & 195 & 289-13.2 & -1.6-1981.52 & -0.76 & 0.83 & -2.30-1983.76 & 274.59\end{array}$

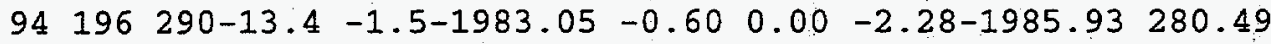
$\begin{array}{llllllll}94 & 197 & 291-13.5 & -1.4-1984.51 & -0.79 & 0.82 & -2.26-1986.74 & 287.76\end{array}$

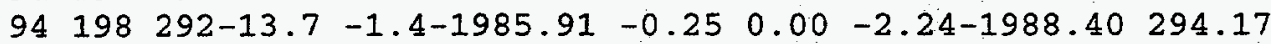
$\begin{array}{llllllll}94 & 199 & 293-13.8 & -1.3-1987.24 & -0.57 & 0.82 & -2.22-1989.21 & 301.43\end{array}$ $94200294-13.9 \quad-1.2-1988.51 \quad-0.540 .00 \quad-2.20-1991.25307 .46$ $94201295-14.1 \quad-1.2-1989.71 \quad-1.06 \quad 0.82 \quad-2.18-1992.13 \quad 314.65$ $94202 \quad 296-14.2-1.1-1990.86-1.120 .00-2.16-1994.14320 .71$ $\begin{array}{llllllll}94 & 203 & 297-14.3 & -1.0-1991.94 & -1.39 & 0.82 & -2.14-1994.66 & 328.27\end{array}$ $94204298-14.5 \quad-1.0-1992.97-1.410 .00-2.12-1996.50 \quad 334.50$ $94205299-14.6 \quad-0.9-1993.93 \quad-1.52 \quad 0.81 \quad-2.10-1996.74 \quad 342.33$ $94206 \quad 300-14.7 \quad-0.9-1994.84 \quad-1.820 .00 \quad-2.08-1998.74348 .40$ $\begin{array}{llllllll}94 & 207 & 301-14.9 & -0.8-1995.68 & -2.29 & 0.81 & -2.07-1999.23 & 355.98\end{array}$ $94208 \quad 302-15.0 \quad-0.8-1996.48 \quad-2.37 \quad 0.00 \quad-2.05-2000.89362 .39$ $94209303-15.1 \quad-0.7-1997.21 \quad-2.790 .81 \quad-2.03-2001.22 \quad 370.13$ $94210 \quad 304-15.3 \quad-0.6-1997.89-2.82 \quad 0.00-2.01-2002.72 \quad 376.70$ $94211305-15.4 \quad-0.6-1998.51-2.910 .81-2.00-2002.61384 .88$ $94212 \quad 306-15.5 \quad-0.5-1999.09-2.620 .00-1.98-2003.69391 .88$ $\begin{array}{lllllllll}94 & 213 & 307-15.6 & -0.5-1999.60 & -2.81 & 0.80 & -1.96-2003.57 & 400.07\end{array}$ $94 \quad 214 \quad 308-15.7 \quad-0.4-2000.07-2.42 \quad 0.00-1.95-2004.43 \quad 407.28$ $94215 \quad 309-15.9 \quad-0.4-2000.48 \quad-2.550 .80 \quad-1.93-2004.16 \quad 415.62$ $94216 \quad 310-16.0 \quad-0.3-2000.84 \quad-2.33 \quad 0.00-1.91-2005.09 \quad 422.77$ $94217 \quad 311-16.1 \quad-0.3-2001.15 \quad-2.52 \quad 0.80 \quad-1.90-2004.77 \quad 431.15$ $94 \quad 218 \quad 312-16.2 \quad-0.2-2001.42 \quad-2.52 \quad 0.00 \quad-1.88-2005.82 \quad 438.18$ $95117212 \quad 1.8-10.6-1571.05$

$95118 \quad 213$

95119214

$95 \quad 120 \quad 215$

95121216

$95122 \quad 217$

$95123 \quad 218$

95124219

95125220

95126221

$95 \quad 127 \quad 222$

$1.6-10.4-1581.55$

$1.4-10.2-1591.86$

$0.491 .85-6.47-1575.18$

$0.301 .05-6.35-1586.55$

$0.291 .84-6.24-1595.98$

1.1-10.1-1602.01 $-0.091 .05-6.14-1607.18$ $0.9-9.9-1611.97-0.391 .84-6.03-1616.56$ $\begin{array}{llllll}0.7 & -9.7-1621.77 & -0.51 & 1.05 & -5.93-1627.16\end{array}$ $0.4-9.5-1631.40-0.93 \quad 1.83-5.83-1636.33$ $0.2-9.4-1640.86-1.18 \quad 1.05-5.73-1646.72$ $0.0-9.2-1650.16-1.691 .83-5.64-1655.66$ $\begin{array}{lllll}-0.2 & -9.1-1659.30 & -1.88 & 1.05 & -5.55-1665.67\end{array}$ 95128223 $-0.5-8.9-1668.27$ $-0.791 .83-5.46-1672.70$ $-0.191 .05$ $\begin{array}{lll}1.05 & 1.82 & -5.29-1688.17\end{array}$ $0.221 .05-5.20-1698.20$ $0.38 \quad 1.82-5.12-1705.55$ $0.491 .05-5.04-1714.33$ $0.161 .82-4.97-1721.88$ $95 \quad 131 \quad 226-1.4 \quad-8.3-1702.62$ $\begin{array}{llllll}95 & 132 & 227 & -1.6 & -8.1 & -1710.83\end{array}$ $\begin{array}{lllll}95 & 133 & 228 & -1.8 & -8.0-1718.89\end{array}$

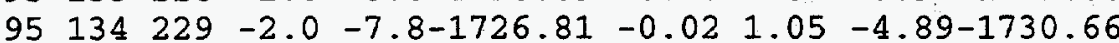

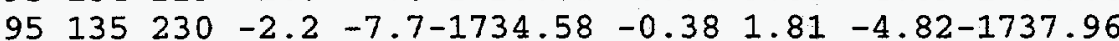
$\begin{array}{lllllllll}95 & 136 & 231 & -2.4 & -7.6-1742.20 & -0.51 & 1.05 & -4.75 & -1746.41\end{array}$ $\begin{array}{llllllll}95 & 137 & 232 & -2.6 & -7.4-1749.69 & -0.87 & 1.81 & -4.68-1753.43\end{array}$ $\begin{array}{lllllllll}95 & 138 & 233 & -2.8 & -7.3-1757.04 & -0.98 & 1.05 & -4.61-1761.58\end{array}$ $\begin{array}{lllllllll}95 & 139 & 234 & -3.1 & -7.1-1764.25 & -1.37 & 1.80 & -4.54 & -1768.36\end{array}$ $\begin{array}{lllllllll}95 & 140 & 235 & -3.3 & -7.0 & -1771.33 & -1.52 & 1.05 & -4.47-1776.27\end{array}$ $\begin{array}{lllllllll}95 & 141 & 236 & -3.5 & -6.9-1778.27 & -1.87 & 1.80 & -4.41-1782.75\end{array}$ $\begin{array}{lllllllll}95 & 142 & 237 & -3.7 & -6.7-1785.09 & -1.95 & 1.05 & -4.35-1790.33\end{array}$

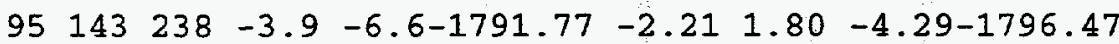
$\begin{array}{lllllllll}95 & 144 & 239 & -4.1 & -6.5 & -1798.32 & -2.12 & 1.05 & -4.23-1803.62\end{array}$ $\begin{array}{lllllllll}95 & 145 & 240 & -4.3 & -6.4-1804.75 & -2.51 & 1.79 & -4.17-1809.64\end{array}$ $95 \begin{array}{llllllll}146 & 241 & -4.5 & -6.2-1811.05 & -2.38 & 1.05 & -4.11-1816.49\end{array}$ 100

\begin{tabular}{|c|c|c|}
\hline 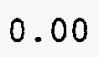 & 00 & - \\
\hline & 0 & 0 \\
\hline .00 & 00 & .00 \\
\hline 00 & 00 & .00 \\
\hline .00 & 0.00 & 0.08 \\
\hline .00 & 0.00 & 0.00 \\
\hline (20 & 00 & \\
\hline .00 & & \\
\hline .00 & 00 & .00 \\
\hline .00 & 0.00 & 0.00 \\
\hline 0.00 & .00 & \\
\hline 0 & & . \\
\hline 0.0 & 0 & \\
\hline 0.00 & 0.00 & 0.00 \\
\hline 0.00 & 0.00 & .00 \\
\hline 0.0 & 0 & 0.00 \\
\hline . & 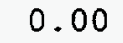 & 0. \\
\hline 0 & 0.00 & 0.00 \\
\hline 0.00 & 0.00 & 0.00 \\
\hline .0 & 0 & 0.00 \\
\hline .0 & 0 & 0.00 \\
\hline 0 & 0.00 & 0.00 \\
\hline 0.00 & 0.00 & 0.00 \\
\hline .0 & 0.0 & 0.00 \\
\hline 0.0 & 0.00 & 0. \\
\hline 0 & 0.00 & 0. \\
\hline 00 & 0.00 & 00 \\
\hline ) & & 0. \\
\hline 0.0 & 0 & 0. \\
\hline 0.0 & 0 . & 0. \\
\hline 0 & 00 & 00 \\
\hline 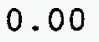 & & 0. \\
\hline 0.0 & 0.0 & 0. \\
\hline 0.0 & & 0.00 \\
\hline 0.0 & & 0 \\
\hline 0.0 & & 0. \\
\hline 0.0 & .00 & 0.00 \\
\hline 0.0 & 0 & 0. \\
\hline 0.00 & & a \\
\hline 0.6 & & 0 \\
\hline 0.0 & 0 & 0.00 \\
\hline 0.00 & 0 & 0.00 \\
\hline 0.0 & & \\
\hline 0.0 & & \\
\hline 0.0 & U & 0 . \\
\hline 0.00 & 0 & 0.00 \\
\hline 0.0 & & 100 \\
\hline 0.0 & - & \\
\hline 0.0 & 0 & 0.00 \\
\hline 0.0 & 0.00 & 0.00 \\
\hline 0.0 & & \\
\hline 0.0 & & \\
\hline 0.0 & 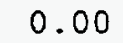 & 0 \\
\hline 0.0 & 0.00 & 0.00 \\
\hline 0.0 & & \\
\hline 0. & & \\
\hline 0 & 0.8 & \\
\hline 0.0 & 0.00 & 0.00 \\
\hline 0.0 & 0.00 & 0.00 \\
\hline .8 & & \\
\hline & 0 & \\
\hline 0.0 & 0.00 & 0.00 \\
\hline & & \\
\hline & & \\
\hline & & \\
\hline & & \\
\hline
\end{tabular}

57.57

56.22

53.09

51.78

49.25

48.16

45.83

44.96

43.02

44.07

43.24

44.74

42.78

43.51

42.79

43.31

42.60

43.38

43.00

44.05

43.98

45.27

45.42

47.01

47.51

49.44

50.36

52.42

53.63 
$\begin{array}{lllllllllll}95 & 147 & 242 & -4.7 & -6.1-1817.23 & -2.61 & 1.79 & -4.06-1822.11\end{array}$ $\begin{array}{lllllllll}95 & 148 & 243 & -4.9 & -6.0-1823.29 & -2.44 & 1.05 & -4.00-1828.68\end{array}$ $\begin{array}{lllllllll}95 & 149 & 244 & -5.1 & -5.9-1829.23 & -2.70 & 1.79 & -3.95-1834.08\end{array}$ $\begin{array}{lllllllll}95 & 150 & 245 & -5.3 & -5.8 & -1835.04 & -2.53 & 1.05 & -3.90-1840.42\end{array}$ $\begin{array}{lllllllll}95 & 151 & 246 & -5.5 & -5.6-1840.74 & -2.93 & 1.79 & -3.84-1845.73\end{array}$ $95 \quad 152 \quad 247-5.7 \quad-5.5-1846.33-2.66 \quad 1.05-3.79-1851.73$ $\begin{array}{lllllllll}95 & 153 & 248 & -5.8 & -5.4-1851.80 & -2.57 & 1.78 & -3.74-1856.33\end{array}$ $95 \quad 154 \quad 249-6.0 \quad-5.3-1857.15-1.88 \quad 1.05-3.70-1861.68$ $\begin{array}{lllllllll}95 & 155 & 250 & -6.2 & -5.2-1862.40 & -1.65 & 1.78 & -3.65 & -1865.92\end{array}$ $95156 \quad 251-6.4 \quad-5.1-1867.53-1.13 \quad 1.05-3.60-1871.21$ $\begin{array}{lllllllll}95 & 157 & 252 & -6.6 & -5.0 & -1872.56 & -1.13 & 1.78 & -3.56-1875.47\end{array}$ $\begin{array}{lllllllll}95 & 158 & 253 & -6.8 & -4.9 & -1877.47 & -0.85 & 1.05 & -3.51-1880.79\end{array}$ $\begin{array}{llllllllll}95 & 159 & 254 & -7.0 & -4.8 & -1882.28 & -1.00 & 1.77 & -3.47-1884.98\end{array}$ $\begin{array}{lllllllll}95 & 160 & 255 & -7.2 & -4.7-1886.99 & -0.86 & 1.05 & -3.43-1890.23\end{array}$ $\begin{array}{lllllllll}95 & 161 & 256 & -7.3 & -4.5 & -1891.59 & -1.05 & 1.77 & -3.39-1894.26\end{array}$ $\begin{array}{lllllllll}95 & 162 & 257 & -7.5 & -4.4-1896.09 & -0.92 & 1.05 & -3.35-1899.31\end{array}$ $\begin{array}{llllllllll}95 & 163 & 258 & -7.7 & -4.3-1900.49 & -0.93 & 1.77 & -3.31-1902.96 & 104.38\end{array}$ $\begin{array}{llllllllll}95 & 164 & 259 & -7.9 & -4.2-1904.79 & -0.47 & 1.05 & -3.27-1907.48 & 107.93\end{array}$ $\begin{array}{llllllllll}95 & 165 & 260 & -8.0 & -4.1-1908.99 & -0.39 & 1.77 & -3.23-1910.85 & 112.63\end{array}$ $\begin{array}{llllllllll}95 & 166 & 261 & -8.2 & -4.1-1913.10 & 0.30 & 1.05 & -3.19-1914.93 & 116.62\end{array}$ $\begin{array}{llllll}95 & 167 & 262 & -8.4 & -4.0-1917.10\end{array}$ $\begin{array}{llllll}95 & 168 & 263 & -8.6 & -3.9-1921.01\end{array}$ $\begin{array}{llllll}95 & 169 & 264 & -8.7 & -3.8-1924.83\end{array}$ $\begin{array}{llllll}95 & 170 & 265 & -8.9 & -3.7-1928.56\end{array}$ $\begin{array}{llllll}95 & 171 & 266 & -9.1 & -3.6-1932.19\end{array}$ $\begin{array}{lllll}95 & 172 & 267 & -9.3 & -3.5-1935.73\end{array}$ $\begin{array}{llllll}95 & 173 & 268 & -9.4 & -3.4-1939.18\end{array}$ $\begin{array}{llllll}95 & 174 & 269 & -9.6 & -3.3-1942.55\end{array}$ $\begin{array}{llllll}95 & 175 & 270 & -9.8 & -3.2-1945.82\end{array}$ $\begin{array}{lllll}95 & 176 & 271 & -9.9 & -3.1-1949.02\end{array}$ $95 \quad 177 \quad 272-10.1-3.1-1952.12$ $0.091 .76-3.15-1918.40 \quad 121.22$ $0.211 .05-3.12-1922.87 \quad 124.83$ $0.141 .76-3.08-1926.01 \quad 129.75$ $0.571 .05-3.05-1929.98 \quad 133.86$ $0.101 .76-3.01-1933.34138 .56$ $\begin{array}{lllll}-0.55 & 1.05 & -2.98-1938.21 & 141.77\end{array}$ $\begin{array}{llllll}-0.34 & 1.75 & -2.95-1940.71 & 147.34\end{array}$ $\begin{array}{llllll}-0.28 & 1.05 & -2.91-1944.69 & 151.43\end{array}$ $\begin{array}{llllll}-0.76 & 1.75 & -2.88-1947.71 & 156.48\end{array}$ $\begin{array}{lllll}-1.20 & 1.05 & -2.85-1952.01 & 160.25\end{array}$ $\begin{array}{llll}1.64 & 1.75 & -2.82-1954.83 & 165.51\end{array}$ $95178 \quad 273-10.2 \quad-3.0-1955.14-1.52 \quad 1.05-2.79-1958.40 \quad 170.01$ $\begin{array}{llllllllll}95 & 179 & 274-10.4 & -2.9-1958.08 & -1.53 & 1.75 & -2.76-1960.62 & 175.86\end{array}$ $95180 \quad 275-10.6 \quad-2.8-1960.94-1.49 \quad 1.05-2.73-1964.11180 .45$ $95181 \quad 276-10.7-2.7-1963.71-1.82 \quad 1.74-2.70-1966.49186 .13$ $95182 \quad 277-10.9-2.7-1966.41-1.62 \quad 1.05 \quad-2.67-1969.65191 .05$ $95183278-11.0 \quad-2.6-1969.02-1.82 \quad 1.74-2.65-1971.75 \quad 197.02$ $\begin{array}{lllllllll}95 & 184 & 279-11.2 & -2.5-1971.56 & -1.34 & 1.05 & -2.62-1974.47 & 202.37\end{array}$ $95185 \quad 280-11.4 \quad-2.4-1974.02 \quad-0.70 \quad 1.74 \quad-2.59-1975.57209 .34$

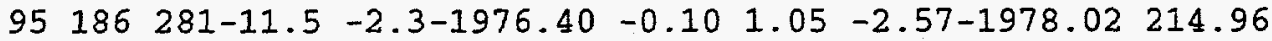
$95187282-11.7-2.3-1978.71 \quad-0.86 \quad 1.74-2.54-1980.37 \quad 220.68$ $95188 \quad 283-11.8 \quad-2.2-1980.95-0.71 \quad 1.05-2.52-1983.12226 .00$ $\begin{array}{llllllllll}95 & 189 & 284-12.0 & -2.1-1983.10 & -0.96 & 1.74 & -2.49-1984.82 & 232.38\end{array}$ $\begin{array}{lllllllll}95 & 190 & 285-12.1 & -2.0-1985.19 & -0.85 & 1.05 & -2.47-1987.46 & 237.81\end{array}$ $95191286-12.3 \quad-2.0-1987.21-1.13 \quad 1.73-2.44-1989.05 \quad 244.29$ $\begin{array}{llllllll}95 & 192 & 287-12.4 & -1.9-1989.15 & 0.20 & 1.05 & -2.42-1990.32 & 251.09\end{array}$ $95193 \quad 288-12.5 \quad-1.8-1991.03-1.11 \quad 1.73-2.40-1992.80 \quad 256.68$ $\begin{array}{lllllllll}95 & 194 & 289-12.7 & -1.8-1992.83 & -0.63 & 1.05 & -2.37-1994.78 & 262.77\end{array}$ $95195290-12.8-1.7-1994.57-0.84 \quad 1.73-2.35-1996.03 \quad 269.60$ $95196291-13.0-1.6-1996.24-0.381 .05-2.33-1997.89275 .80$ $95197292-13.1-1.6-1997.84-0.651 .73-2.31-1999.07 \quad 282.70$ $\begin{array}{llllllllll}95 & 198 & 293-13.3 & -1.5-1999.37 & -0.68 & 1.05 & -2.28-2001.29 & 288.55\end{array}$ $95199 \quad 294-13.4-1.4-2000.84-1.00 \quad 1.72-2.26-2002.38 \quad 295.53$ $95200295-13.5-1.4-2002.25 \quad-0.99 \quad 1.05-2.24-2004.43 \quad 301.55$ $95201296-13.7-1.3-2003.59-1.43 \quad 1.72-2.22-2005.52308 .53$ $95202 \quad 297-13.8-1.2-2004.87-1.57 \quad 1.05-2.20-2007.59 \quad 314.53$ $\begin{array}{lllllllll}95 & 203 & 298-13.9 & -1.2-2006.09 & -1.80 & 1.72 & -2.18-2008.35 & 321.84\end{array}$ $95204 \quad 299-14.1-1.1-2007.25 \quad-1.86 \quad 1.05-2.16-2010.22 \quad 328.05$ $95205 \quad 300-14.2-1.1-2008.35-2.20 \quad 1.72-2.14-2010.97335 .37$ $95206 \quad 301-14.3-1.0-2009.38-2.16 \quad 1.05-2.12-2012.61341 .79$ $95207302-14.5 \quad-0.9-2010.36-2.57 \quad 1.72 \quad-2.11-2013.32 \quad 349.16$ $95208 \quad 303-14.6-0.9-2011.28-2.64 \quad 1.05-2.09-2014.96 \quad 355.60$ $95209 \quad 304-14.7-0.8-2012.14-3.06 \quad 1.71-2.07-2015.56 \quad 363.07$ $95210 \quad 305-14.9-0.8-2012.95 \quad-3.08 \quad 1.05-2.05-2017.03 \quad 369.67$ $95211306-15.0-0.7-2013.70-3.16 \quad 1.71-2.03-2017.18 \quad 377.59$ $95212307-15.1-0.7-2014.39-2.86 \quad 1.05-2.02-2018.22 \quad 384.62$ 101 $\begin{array}{llll}55.47 & -3.23 & -0.62\end{array}$ $\begin{array}{llll}57.17 & -2.86 & -0.42\end{array}$ $\begin{array}{llll}59.88 & -3.07 & -0.37\end{array}$ $61.89-2.63-0.10$ $\begin{array}{llll}64.99 & -2.69 & 0.24\end{array}$ $0.00 \quad 0.00 \quad 0.00$ $\begin{array}{llll}0.00 & 0.00 & 0.00\end{array}$ $\begin{array}{llll}0.00 & 0.00 & 0.00\end{array}$ $\begin{array}{llll}0.00 & 0.00 & 0.00\end{array}$ $0.00 \quad 0.00 \quad 0.00$ $0.00 \quad 0.00 \quad 0.00$ $\begin{array}{llll}0.00 & 0.00 & 0.00\end{array}$ $\begin{array}{llll}0.00 & 0.00 & 0.00\end{array}$ $\begin{array}{llll}0.00 & 0.00 & 0.00\end{array}$ $\begin{array}{llll}0.00 & 0.00 & 0.00\end{array}$ $\begin{array}{llll}0.00 & 0.00 & 0.00\end{array}$ $0.00 \quad 0.00 \quad 0.00$ $\begin{array}{llll}0.00 & 0.00 & 0.00\end{array}$ $\begin{array}{llll}0.00 & 0.00 & 0.00\end{array}$ $\begin{array}{llll}0.00 & 0.00 & 0.00\end{array}$ $\begin{array}{llll}0.00 & 0.00 & 0.00\end{array}$ $\begin{array}{llll}0.00 & 0.00 & 0.00\end{array}$ $\begin{array}{llll}0.00 & 0.00 & 0.00\end{array}$ $\begin{array}{llll}0.00 & 0.00 & 0.00\end{array}$ $\begin{array}{llll}0.00 & 0.00 & 0.00\end{array}$ $\begin{array}{llll}0.00 & 0.00 & 0.00\end{array}$ $\begin{array}{llll}0.00 & 0.00 & 0.00\end{array}$ $\begin{array}{llll}0.00 & 0.00 & 0.00\end{array}$ $\begin{array}{llll}0.00 & 0.00 & 0.00\end{array}$ $\begin{array}{llll}0.00 & 0.00 & 0.00\end{array}$ $\begin{array}{llll}0.00 & 0.00 & 0.00\end{array}$ $\begin{array}{llll}0.00 & 0.00 & 0.00\end{array}$ $\begin{array}{llll}0.00 & 0.00 & 0.00\end{array}$ $\begin{array}{llll}0.00 & 0.00 & 0.00\end{array}$ $\begin{array}{llll}0.00 & 0.00 & 0.00\end{array}$ $\begin{array}{llll}0.00 & 0.00 & 0.00\end{array}$ $0.00 \quad 0.00 \quad 0.00$ $\begin{array}{llll}0.00 & 0.00 & 0.00\end{array}$ $\begin{array}{llll}0.00 & 0.00 & 0.00\end{array}$ $0.00 \quad 0.00 \quad 0.00$ $0.00 \quad 0.00 \quad 0.00$ $\begin{array}{llll}0.00 & 0.00 & 0.00\end{array}$ $\begin{array}{llll}0.00 & 0.00 & 0.00\end{array}$ $\begin{array}{llll}0.00 & 0.00 & 0.00\end{array}$ $0.00 \quad 0.00 \quad 0.00$ $\begin{array}{llll}0.00 & 0.00 & 0.00\end{array}$ $\begin{array}{llll}0.00 & 0.00 & 0.00\end{array}$ $\begin{array}{llll}0.00 & 0.00 & 0.00\end{array}$ $0.00 \quad 0.00 \quad 0.00$ $0.00 \quad 0.00 \quad 0.00$ $\begin{array}{llll}0.00 & 0.00 & 0.00\end{array}$ $0.00 \quad 0.00 \quad 0.00$ $0.00 \quad 0.00 \quad 0.00$ $\begin{array}{llll}0.00 & 0.00 & 0.00\end{array}$ $\begin{array}{llll}0.00 & 0.00 & 0.00\end{array}$ $0.00 \quad 0.00 \quad 0.00$ $0.00 \quad 0.00 \quad 0.00$ $\begin{array}{llll}0.00 & 0.00 & 0.00\end{array}$ $\begin{array}{llll}0.00 & 0.00 & 0.00\end{array}$ $\begin{array}{llll}0.00 & 0.00 & 0.00\end{array}$ $0.00 \quad 0.00 \quad 0.00$ $\begin{array}{llll}0.00 & 0.00 & 0.00\end{array}$ $\begin{array}{llll}0.00 & 0.00 & 0.00\end{array}$ $\begin{array}{llll}0.00 & 0.00 & 0.00\end{array}$ $\begin{array}{llll}0.00 & 0.00 & 0.00\end{array}$ $\begin{array}{llll}0.00 & 0.00 & 0.00\end{array}$ 
$95213 \quad 308-15.2 \quad-0.6-2015.04-3.03 \quad 1.71-2.00-2018.36 \quad 392.55$ $95 \quad 214 \quad 309-15.4 \quad-0.6-2015.62-2.70 \quad 1.05-1.98-2019.25 \quad 399.73$ $\begin{array}{llllllll}95 & 215 & 310-15.5 & -0.5-2016.15 & -2.92 & 1.71 & -1.97-2019.33 & 407.72\end{array}$ $95216 \quad 311-15.6 \quad-0.5-2016.64-2.57 \quad 1.05-1.95-2020.11415 .02$ $\begin{array}{lllllllll}95 & 217 & 312-15.7 & -0.4-2017.07 & -2.97 & 1.71 & -1.94-2020.27 & 422.93\end{array}$ $\begin{array}{llllllll}95 & 218 & 313-15.8 & -0.4-2017.45 & -2.95 & 1.05 & -1.92-2021.26 & 430.00\end{array}$ $95 \quad 219314-16.0 \quad-0.3-2017.78 \quad-3.21 \quad 1.71-1.90-2021.18 \quad 438.15$ $\begin{array}{llllllll}95 & 220 & 315-16.1 & -0.3-2018.06 & -3.19 & 1.05 & -1.89-2022.08 & 445.33\end{array}$ $\begin{array}{llllllll}96 & 119 & 215 & 1.8-10.5-1590.30 & 0.08 & 0.98 & -6.38-1595.63\end{array}$ $\begin{array}{lllllll}96 & 120 & 216 & 1.5-10.3-1600.68 & 0.02 & 0.00 & -6.27-1606.93\end{array}$ $\begin{array}{lllllll}96 & 121 & 217 & 1.3-10.1-1610.87 & -0.29 & 0.97 & -6.16-1616.36\end{array}$ $\begin{array}{lllllllll}96 & 122 & 218 & 1.1 & -9.9 & -1620.90 & -0.42 & 0.00 & -6.06-1627.38\end{array}$ $96 \quad 123 \quad 219$ 96124220 $96 \quad 125 \quad 221$ $96126 \quad 222$ $96 \quad 127 \quad 223$ $\begin{array}{lllll}0.9 & -9.8-1630.76 & -0.83 & 0.97 & -5.96-1636.58\end{array}$ $0.6-9.6-1640.45-1.09 \quad 0.00-5.86-1647.40$ $\begin{array}{llllll}0.4 & -9.4-1649.97 & -1.67 & 0.96 & -5.76-1656.45\end{array}$ $0.2-9.3-1659.33-1.76 \quad 0.00-5.67-1666.76$ $0.0-9.1-1668.53-0.78 \quad 0.95-5.58-1673.94$ $\begin{array}{lllllllll}96 & 128 & 224 & -0.3 & -9.0-1677.57 & -0.36 & 0.00 & -5.49-1683.42\end{array}$ $\begin{array}{llllllll}96 & 129 & 225 & -0.5 & -8.8-1686.46 & 1.18 & 0.95 & -5.40-1689.73\end{array}$

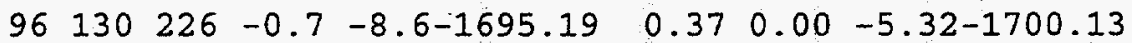

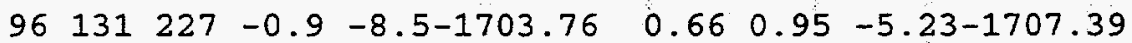
$\begin{array}{llllllll}96 & 132 & 228 & -1.1 & -8.3-1712.19 & 0.71 & 0.00 & -5.15-1716.63\end{array}$ $\begin{array}{llllllll}96 & 133 & 229 & -1.4 & -8.2-1720.46 & 0.42 & 0.94 & -5.07-1724.18\end{array}$ $\begin{array}{llllllll}96 & 134 & 230 & -1.6 & -8.1-1728.59 & 0.24 & 0.00 & -5.00-1733.35\end{array}$

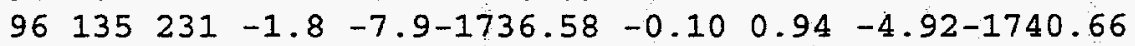
$\begin{array}{llllllll}96 & 136 & 232 & -2.0 & -7.8-1744.42 & -0.27 & 0.00 & -4.85-1749.54\end{array}$ $\begin{array}{lllllllll}96 & 137 & 233 & -2.2 & -7.6-1752.12 & -0.62 & 0.93 & -4.78-1756.58\end{array}$ $\begin{array}{lllllllll}96 & 138 & 234 & -2.4 & -7.5 & -1759.68 & -0.75 & 0.00 & -4.71-1765.13\end{array}$ $\begin{array}{llllllll}96 & 139 & 235 & -2.6 & -7.3-1767.10 & -1.15 & 0.93 & -4.64-1771.96\end{array}$ $\begin{array}{llllllll}96 & 140 & 236 & -2.8 & -7.2-1774.38 & -1.33 & 0.00 & -4.57-1780.28\end{array}$ $\begin{array}{lllllllll}96 & 141 & 237 & -3.0 & -7.1-1781.53 & -1.66 & 0.92 & -4.50-1786.77\end{array}$ $\begin{array}{lllllllll}96 & 142 & 238 & -3.2 & -6.9-1788.55 & -1.78 & 0.00 & -4.44-1794.77\end{array}$ $\begin{array}{lllllllll}96 & 143 & 239 & -3.4 & -6.8-1795.43 & -2.06 & 0.92 & -4.38-1800.96\end{array}$ $\begin{array}{lllllllll}96 & 144 & 240 & -3.6 & -6.7-1802.19 & -2.18 & 0.00 & -4.32 & -1808.69\end{array}$ $\begin{array}{lllllllll}96 & 145 & 241 & -3.8 & -6.6-1808.82 & -2.47 & 0.91 & -4.26-1814.63\end{array}$ $\begin{array}{lllllllll}96 & 146 & 242 & -4.0 & -6.4-1815.32 & -2.38 & 0.00 & -4.20-1821.90\end{array}$ $\begin{array}{lllllllll}96 & 147 & 243 & -4.2 & -6.3-1821.70 & -2.84 & 0.91 & -4.14-1827.77\end{array}$ $\begin{array}{lllllllll}96 & 148 & 244 & -4.4 & -6.2 & -1827.95 & -2.76 & 0.00 & -4.09-1834.80\end{array}$ $\begin{array}{lllllllll}96 & 149 & 245 & -4.6 & -6.1-1834.09 & -3.05 & 0.91 & -4.03-1840.26\end{array}$ $\begin{array}{lllllllll}96 & 150 & 246 & -4.8 & -5.9-1840.10 & -2.96 & 0.00 & -3.98-1847.04\end{array}$ $\begin{array}{lllllllll}96 & 151 & 247 & -5.0 & -5.8 & -1845.99 & -3.17 & 0.90 & -3.92-1852.19\end{array}$ $96 \begin{array}{llllllll}952 & 248 & -5.2 & -5.7-1851.77 & -3.00 & 0.00 & -3.87-1858.64\end{array}$

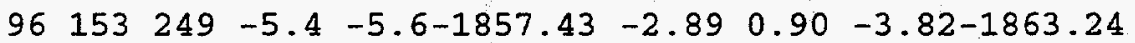
$\begin{array}{llllllll}96 & 154 & 250 & -5.6 & -5.5-1862.98 & -2.22 & 0.00 & -3.77-1868.97\end{array}$ $96 \quad 155 \quad 251-5.8 \quad-5.4-1868.41-2.02 \quad 0.89-3.73-1873.26$ $96 \quad 156 \quad 252-6.0 \quad-5.3-1873.73-1.62 \quad 0.00-3.68-1879.03$ $\begin{array}{lllllllll}96 & 157 & 253 & -6.2 & -5.2-1878.94 & -1.45 & 0.89 & -3.63-1883.14\end{array}$ $\begin{array}{llllllll}96 & 158 & 254 & -6.4 & -5.0-1884.05 & -1.19 & 0.00 & -3.59-1888.82\end{array}$ $\begin{array}{lllllllll}96 & 159 & 255 & -6.5 & -4.9-1889.04 & -1.33 & 0.89 & -3.54-1893.03\end{array}$ $\begin{array}{lllllllll}96 & 160 & 256 & -6.7 & -4.8-1893.93 & -1.20 & 0.00 & -3.50-1898.63\end{array}$ $\begin{array}{llllllll}96 & 161 & 257 & -6.9 & -4.7-1898.72 & -1.42 & 0.88 & -3.46-1902.71\end{array}$ $\begin{array}{lllllllll}96 & 162 & 258 & -7.1 & -4.6-1903.40 & -1.29 & 0.00 & -3.41-1908.10\end{array}$ $\begin{array}{llllllll}96 & 163 & 259 & -7.3 & -4.5-1907.98 & -1.30 & 0.88 & -3.37-1911.77\end{array}$ $96164260-7.4-4.4-1912.45-0.810 .00-3.33-1916.60106 .08$ $\begin{array}{llllllllll}96 & 165 & 261 & -7.6 & -4.3-1916.83 & -0.72 & 0.88 & -3.29-1919.97 & 110.78\end{array}$ $\begin{array}{lllllllll}96 & 166 & 262 & -7.8 & -4.2-1921.11 & -0.23 & 0.00 & -3.26-1924.60 & 114.23\end{array}$ $\begin{array}{llllllllll}96 & 167 & 263 & -8.0 & -4.1-1925.29 & -0.06 & 0.87 & -3.22-1927.70 & 119.20\end{array}$

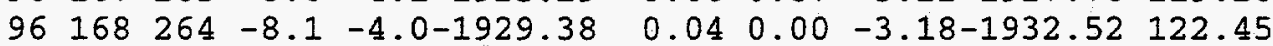

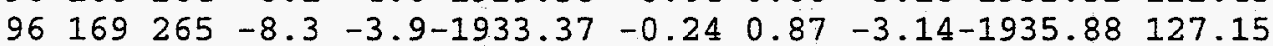
$\begin{array}{lllllllll}96 & 170 & 266 & -8.5 & -3.8-1937.26 & 0.50 & 0.00 & -3.11-1939.87 & 131.24\end{array}$ $\begin{array}{llllllllll}96 & 171 & 267 & -8.7 & -3.8-1941.07 & 0.06 & 0.86 & -3.07-1943.21 & 135.96\end{array}$ $\begin{array}{lllllllll}96 & 172 & 268 & -8.8 & -3.7-1944.78 & -0.44 & 0.00 & -3.04-1948.26 & 138.99\end{array}$ $\begin{array}{lllllllll}96 & 173 & 269 & -9.0 & -3.6-1948.40 & -0.38 & 0.86 & -3.01-1950.92 & 144.40\end{array}$ $\begin{array}{lllllllll}96 & 174 & 270 & -9.2 & -3.5-1951.93 & -0.31 & 0.00 & -2.97-1955.21 & 148.18\end{array}$ $\begin{array}{llllllllll}96 & 175 & 271 & -9.3 & -3.4-1955.37 & -0.79 & 0.86 & -2.94-1958.25 & 153.22\end{array}$ $96 \quad 176.272 \quad-9.5 \quad-3.3-1958.73 \quad-1.100 .00-2.91-1962.74 \quad 156.80$

$\begin{array}{rrr}0.00 & 0.00 & 0.00 \\ 0.00 & 0.00 & 0.00 \\ 0.00 & 0.00 & 0.00 \\ 0.00 & 0.00 & 0.00 \\ 0.00 & 0.00 & 0.00 \\ 0.00 & 0.00 & 0.00 \\ 0.00 & 0.00 & 0.00 \\ 0.00 & 0.00 & 0.00 \\ 0.00 & 0.00 & 0.00 \\ 0.00 & 0.00 & 0.00 \\ 0.00 & 0.00 & 0.00 \\ 0.00 & 0.00 & 0.00 \\ 0.00 & 0.00 & 0.00 \\ 0.00 & 0.00 & 0.00 \\ 0.00 & 0.00 & 0.00 \\ 0.00 & 0.00 & 0.00 \\ 0.00 & 0.00 & 0.00 \\ 0.00 & 0.00 & 0.00 \\ 0.00 & 0.00 & 0.00 \\ 0.00 & 0.00 & 0.00 \\ 0.00 & 0.00 & 0.00 \\ 0.00 & 0.00 & 0.00 \\ 0.00 & 0.00 & 0.00 \\ 0.00 & 0.00 & 0.00 \\ 0.00 & 0.00 & 0.00 \\ 0.00 & 0.00 & 0.00 \\ 0.00 & 0.00 & 0.00 \\ 0.00 & 0.00 & 0.00 \\ 0.00 & 0.00 & 0.00 \\ 0.00 & 0.00 & 0.00 \\ 0.00 & 0.00 & 0.00 \\ 49.38 & -2.74 & -0.96 \\ 0.00 & 0.00 & 0.00 \\ 51.70 & -3.04 & 0.86\end{array}$

52.22

54.69

55.49

58.74

61.35

62.64

65.56

70.65

72.99

76.77

83.04

85.43

89.29

95.75

98.43

51.70

$53.70-3.46$

$54.80-3.07-0$

$57.18-3.35$ $\begin{array}{llll}58.45 & -3.05 & -0.29\end{array}$

$\begin{array}{llll}61.00 & -3.40 & -0.35\end{array}$

$\begin{array}{llll}62.61 & -2.99 & -0.03\end{array}$

$\begin{array}{llll}65.53 & -3.20 & -0.03\end{array}$

$\begin{array}{lll}67.39 & -2.79 & 0.21\end{array}$

$\begin{array}{lll}70.75 & -2.79 & 0.10\end{array}$

$\begin{array}{llll}72.98 & -2.23 & -0.01\end{array}$

$\begin{array}{llll}76.64 & -2.15 & -0.13\end{array}$

$\begin{array}{lll}0.00 & 0.00 & 0.00\end{array}$

$\begin{array}{lll}0.00 & 0.00 & 0.00\end{array}$

$\begin{array}{lll}0.00 & 0.00 & 0.00\end{array}$

$\begin{array}{llll}0.00 & 0.00 & 0.00\end{array}$

$\begin{array}{lll}0.00 & 0.00 & 0.00\end{array}$

$\begin{array}{lll}0.00 & 0.00 & 0.00\end{array}$

$\begin{array}{lll}0.00 & 0.00 & 0.00\end{array}$

$\begin{array}{llll}0.00 & 0.00 & 0.00\end{array}$

$\begin{array}{llll}0.00 & 0.00 & 0.00\end{array}$

$\begin{array}{lll}0.00 & 0.00 & 0.00\end{array}$

$\begin{array}{llll}0.00 & 0.00 & 0.00\end{array}$

$\begin{array}{llll}0.00 & 0.00 & 0.00\end{array}$

$\begin{array}{llll}0.00 & 0.00 & 0.00\end{array}$

$\begin{array}{lll}0.00 & 0.00 & 0.00\end{array}$

$\begin{array}{llll}0.00 & 0.00 & 0.00\end{array}$

$0.00 \quad 0.00 \quad 0.00$

$\begin{array}{llll}0.00 & 0.00 & 0.00\end{array}$

$\begin{array}{llll}0.00 & 0.00 & 0.00\end{array}$

$\begin{array}{lll}0.00 & 0.00 & 0.00\end{array}$

$\begin{array}{llll}0.00 & 0.00 & 0.00\end{array}$

$\begin{array}{lll}0.00 & 0.00 & 0.00\end{array}$ 
$\begin{array}{llllllllllll}96 & 177 & 273 & -9.7 & -3.2-1962.00 & -1.52 & 0.85 & -2.88-1965.54 & 162.07\end{array}$ $\begin{array}{llllllllll}96 & 178 & 274 & -9.8 & -3.1-1965.18 & -1.41 & 0.00 & -2.85-1969.44 & 166.24\end{array}$ $96179275-10.0 \quad-3.1-1968.28-1.47 \quad 0.85-2.81-1971.72 \quad 172.03$ $96 \quad 180 \quad 276-10.1 \quad-3.0-1971.30-1.41 \quad 0.00-2.79-1975.49 \quad 176.33$ $96 \quad 181 \quad 277-10.3 \quad-2.9-1974.23-1.73 \quad 0.85-2.76-1977.87 \quad 182.02$ $96 \quad 182 \quad 278-10.5 \quad-2.8-1977.09-1.53 \quad 0.00-2.73-1981.34 \quad 186.62$ $96.183 \quad 279-10.6 \quad-2.7-1979.86-1.71 \quad 0.85-2.70-1983.42 \quad 192.61$ $96184280-10.8 \quad-2.7-1982.55-1.23 \quad 0.00-2.67-1986.45 \quad 197.65$ $96 \quad 185 \quad 281-10.9-2.6-1985.17-0.53 \quad 0.84-2.64-1987.50204 .68$ $96 \quad 186 \quad 282-11.1-2.5-1987.70$ $96 \quad 187283-11.2-2.4-1990.17$ $96 \quad 188 \quad 284-11.4-2.3-1992.55$ $96 \quad 189285-11.5-2.3-1994.86$ $96 \quad 190286-11.7 \quad-2.2-1997.10$ $96191287-11.8-2.1-1999.26$ $96192288-12.0-2.1-2001.35$ $96 \quad 193 \quad 289-12.1 \quad-2.0-2003.37$ $0.010 .00-2.62-1990.31209 .94$ $0.44 \quad 0.84-2.59-1991.48 \quad 216.84$ $\begin{array}{llllll}0.96 & 0.00 & -2.57-1994.16 & 222.24\end{array}$ $\begin{array}{llllll}0.52 & 0.84 & -2.54-1996.04 & 228.42\end{array}$ $\begin{array}{llllll}0.43 & 0.00 & -2.51-1999.18 & 233.36\end{array}$ $\begin{array}{lllll}0.12 & 0.83 & -2.49-2000.80 & 239.81\end{array}$ $0.09 \quad 0.00-2.47-2003.73 \quad 244.95$

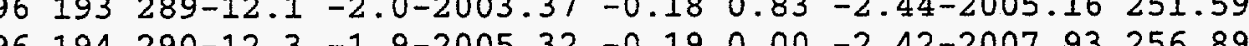
$96195291-12.4-1.8-2007.20-0.43 \quad 0.83-2.40-2009.20263 .69$ $96196292-12.6-1.8-2009.01-0.43 \quad 0.00-2.37-2011.82 \quad 269.15$ $96197293-12.7-1.7-2010.76-0.73 \quad 0.82 \quad-2.35-2013.01276 .02$ $96 \quad 198 \quad 294-12.9-1.6-2012.43-0.75 \quad 0.00-2.33-2015.51281 .59$ $96199295-13.0-1.6-2014.05-1.110 .82-2.31-2016.64288 .54$ $96200296-13.1-1.5-2015.59-1.110 .00-2.29-2018.99294 .26$ $96201297-13.3 \quad-1.4-2017.07-1.48 \quad 0.82 \quad-2.27-2020.00 \quad 301.32$ $96202 \quad 298-13.4-1.4-2018.49-1.50 \quad 0.00-2.24-2022.23 \quad 307.16$ $96203 \quad 299-13.5-1.3-2019.84 \quad-1.97 \quad 0.82 \quad-2.22-2023.22 \quad 314.24$ $96204300-13.7-1.3-2021.13-1.910 .00-2.20-2025.25320 .29$ $96205 \quad 301-13.8-1.2-2022.36-2.24 \quad 0.81-2.19-2025.97 \quad 327.63$ $96206 \quad 302-13.9 \quad-1.1-2023.53 \quad-2.18 \quad 0.00 \quad-2.17-2027.88 \quad 333.80$ $96207303-14.1-1.1-2024.64-2.58 \quad 0.81-2.15-2028.56 \quad 341.19$ $96208304-14.2 \quad-1.0-2025.69-2.66 \quad 0.00-2.13-2030.48 \quad 347.34$ $96209305-14.3-1.0-2026.68-3.08 \quad 0.81-2.11-2031.06 \quad 354.83$ $\begin{array}{lllllllll}96 & 210 & 306-14.5 & -0.9-2027.62 & -3.09 & 0.00 & -2.09-2032.80 & 361.16\end{array}$ $96211307-14.6 \quad-0.8-2028.49-3.17 \quad 0.81-2.07-2032.93 \quad 369.10$ $96212308-14.7-0.8-2029.32-2.91 \quad 0.00-2.06-2034.28 \quad 375.83$ $96213 \quad 309-14.9 \quad-0.7-2030.08 \quad-3.08 \quad 0.80-2.04-2034.39 \quad 383.78$ $\begin{array}{lllllllll}96 & 214 & 310-15.0 & -0.7-2030.79 & -2.77 & 0.00 & -2.02-2035.58 & 390.67\end{array}$ $96215 \quad 311-15.1-0.6-2031.45-3.00 \quad 0.80-2.00-2035.65 \quad 398.67$ $96216 \quad 312-15.2-0.6-2032.05-2.79 \quad 0.00-1.99-2036.83 \quad 405.56$ $\begin{array}{llllllll}96 & 217 & 313-15.3 & -0.5-2032.60 & -3.12 & 0.80 & -1.97-2036.89 & 413.57\end{array}$ $\begin{array}{lllllllll}96 & 218 & 314-15.5 & -0.5-2033.10 & -3.13 & 0.00 & -1.96-2038.18 & 420.35\end{array}$ $96219 \quad 315-15.6-0.4-2033.55-3.36 \quad 0.80-1.94-2038.05 \quad 428.56$ $96 \begin{array}{llllllll}920 & 316-15.7 & -0.4-2033.94 & -3.33 & 0.00 & -1.92-2039.20 & 435.48\end{array}$ $\begin{array}{llllllll}96 & 221 & 317-15.8 & -0.3-2034.29 & -3.55 & 0.79 & -1.91-2038.95 & 443.80\end{array}$ $96222 \quad 318-15.9-0.3-2034.59-3.24 \quad 0.00-1.89-2039.72 \quad 451.10$ $97121218 \quad 1.7-10.3-160935-0.161 .83-6.30-1613.98 \quad 68.90$ $97 \begin{array}{lllllll}122 & 219 & 1.5-10.2-1619.61 & -0.26 & 1.04 & -6.19-1625.01\end{array}$ $97123220 \quad 1.3-10.0-1629.69-0.651 .83-6.09-1634.60$ $\begin{array}{llllllllll}97 & 124 & 221 & 1.1 & -9.8-1639.61 & -0.83 & 1.04 & -5.99-1645.38\end{array}$ $\begin{array}{lllllllll}97 & 125 & 222 & 0.8 & -9.7-1649.36 & -1.27 & 1.82 & -5.89-1654.69\end{array}$ $\begin{array}{lllllllll}97 & 126 & 223 & 0.6 & -9.5-1658.94 & -1.47 & 1.04 & -5.79-1665.16\end{array}$ $\begin{array}{lllllllll}97 & 127 & 224 & 0.4 & -9.3-1668.37 & -0.38 & 1.82 & -5.70-1672.62\end{array}$ $\begin{array}{lllllllll}97 & 128 & 225 & 0.2 & -9.2-1677.63 & -0.25 & 1.04 & -5.61-1682.44\end{array}$ $97 \begin{array}{lllll}129 & 226 & -0.1 & -9.0-1686.74\end{array}$ $97 \begin{array}{lllll}130 & 227 & -0.3 & -8.9-1695.68\end{array}$ $97 \begin{array}{lllll}131 & 228 & -0.5 & -8.7 & -1704.48\end{array}$ $97 \begin{array}{lllll}132 & 229 & -0.7 & -8.6-1713.12\end{array}$ $97 \begin{array}{lllll}933 & 230 & -0.9 & -8.4-1721.61\end{array}$ $97 \begin{array}{lllll}134 & 231 & -1.1 & -8.3-1729.95\end{array}$ $97 \begin{array}{lllll}135 & 232 & -1.4 & -8.1-1738.15\end{array}$ $1.351 .82-5.52-1689.09$ $0.841 .04-5.43-1699.23$ $0.621 .81-5.35-1707.39$ $0.471 .04-5.26-1716.87$ $0.281 .81-5.18-1724.70$ $0.08 \quad 1.04-5.10-1733.93$ $1.6-8.0 .241 .81-5.03-1741.61$ $97136233-1.6-8.0-1746.20-0.391 .04-4.95-1750.50$ $\begin{array}{llllllllll}97 & 137 & 234 & -1.8 & -7.8-1754.11 & -0.75 & 1.80 & -4.88-1757.94\end{array}$ $97 \begin{array}{llllllll}138 & 235 & -2.0 & -7.7-1761.88 & -0.92 & 1.04 & -4.81-1766.56\end{array}$ $97 \begin{array}{llllllll}139 & 236 & -2.2 & -7.6-1769.51 & -1.32 & 1.80 & -4.74-1773.77\end{array}$ $\begin{array}{lllllll}97 & 140 & 237 & -2.4 & -7.4-1777.00 & -1.52 & 1.04 \\ 103 & -4.67-1782.15\end{array}$
64.42

61.71

60.47

58.07

58.68

56.94

58.36

56.29

56.20

54.80

55.03

53.87

54.26

53.45

54.08

53.53

54.39

54.09

\begin{tabular}{|c|c|c|}
\hline 00 & 00 & 00 \\
\hline 0.00 & 0.00 & 0.00 \\
\hline 00 & 00 & .00 \\
\hline .00 & .00 & .00 \\
\hline 0.00 & 0.00 & 0.00 \\
\hline 0 & 0.00 & 0 \\
\hline 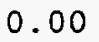 & 00 & \\
\hline .00 & .00 & 0.00 \\
\hline 0.00 & 0.00 & 0.00 \\
\hline .00 & 0.00 & 0.00 \\
\hline . & 0.00 & \\
\hline .00 & 0.00 & 0.00 \\
\hline 0.00 & 0.00 & 0.00 \\
\hline 0.00 & 0.00 & 0.00 \\
\hline 0.0 & 0.00 & 0.00 \\
\hline .00 & .00 & 0.00 \\
\hline 0.00 & 0.00 & 0.00 \\
\hline 0.00 & 0.00 & 0.00 \\
\hline 0 & 0. & 0.00 \\
\hline 0.0 & 0.00 & 0.00 \\
\hline 0.00 & 0.00 & 0.00 \\
\hline 0.00 & 0.00 & 0.00 \\
\hline 0.00 & 0. & 0.00 \\
\hline 0.0 & 0.00 & 0.00 \\
\hline 0.00 & 0.00 & 0.00 \\
\hline 0.00 & 0.00 & 0.00 \\
\hline 0.0 & 0. & 0.00 \\
\hline 0.0 & 0.00 & 0.00 \\
\hline 0.00 & 0.00 & 0.00 \\
\hline 0.00 & 0.00 & 0.00 \\
\hline 0.0 & 0.00 & 0.00 \\
\hline 0.00 & 0.00 & 0.00 \\
\hline 0.00 & 0.00 & 0.00 \\
\hline 0.0 & 0.00 & 0.00 \\
\hline 0.0 & 0. & 0.00 \\
\hline 0.00 & 0.00 & 0.00 \\
\hline 0.0 & 0.00 & 0.00 \\
\hline 0.0 & 0.00 & 0.00 \\
\hline 0.0 & 0.0 & 0.00 \\
\hline 0.00 & 0.00 & 0.00 \\
\hline 0.00 & 0.00 & 0.00 \\
\hline 0.0 & 0. & 0.00 \\
\hline 0.0 & 0.00 & 0.00 \\
\hline 0.00 & 0.00 & 0.00 \\
\hline 0.0 & 0.0 & 0.00 \\
\hline 0 . & 0.0 & 0.00 \\
\hline 0.0 & 0.00 & 0.00 \\
\hline 0.0 & 0.00 & 0.00 \\
\hline 0.0 & 0.0 & 0.00 \\
\hline 0.0 & 0.0 & 0.00 \\
\hline 0.0 & 0.00 & 0.00 \\
\hline 0.0 & 0.00 & 0.00 \\
\hline 0.0 & 0. & 0.00 \\
\hline 0.0 & 0. & 0. \\
\hline 0.0 & $0 . c$ & 0. \\
\hline 0.00 & 0.00 & 0.00 \\
\hline 0.0 & 0.0 & 0.00 \\
\hline 0.0 & 0.0 & 0.00 \\
\hline 0.00 & 0.00 & 0.00 \\
\hline 0.0 & 0.0 & 0.00 \\
\hline 0.0 & 0.0 & 0.00 \\
\hline 0.0 & 0.00 & 0.00 \\
\hline 0.00 & 0.00 & 0.00 \\
\hline & & \\
\hline 0.0 & 0.00 & 0.0 \\
\hline 0.00 & 0.00 & 0.00 \\
\hline
\end{tabular}


$97141238-2.6 \quad-7.3-1784.36-1.85 \quad 1.80-4.60-1789.01$

$97 \begin{array}{llllllll}942 & 239 & -2.8 & -7.2-1791.58 & -2.03 & 1.04 & -4.53-1797.10\end{array}$

$97 \begin{array}{lllllllll}143 & 240 & -3.0 & -7.0 & -1798.67 & -2.48 & 1.79 & -4.47-1803.83\end{array}$

$97 \begin{array}{lllllllll}944 & 241 & -3.2 & -6.9-1805.63 & -2.48 & 1.04 & -4.41-1811.47\end{array}$

$97 \begin{array}{llllllll}945 & 242 & -3.4 & -6.8-1812.46 & -2.79 & 1.79 & -4.35-1817.81\end{array}$

$97146243-3.6 \quad-6.6-1819.16-2.94 \quad 1.04-4.29-1825.34$

$97 \begin{array}{lllllllll}947 & 244 & -3.8 & -6.5-1825.74 & -3.27 & 1.79 & -4.23-1831.45\end{array}$

$97148 \quad 245-4.0 \quad-6.4-1832.19-3.21 \quad 1.04-4.17-1838.53$

$97 \begin{array}{llllllll}149 & 246 & -4.2 & -6.3-1838.52 & -3.52 & 1.78 & -4.12-1844.37\end{array}$

$97150 \quad 247 \quad-4.4 \quad-6.1-1844.72-3.46 \quad 1.04 \quad-4.06-1851.20$

$97151248-4.6-6.0-1850.81-3.71 \quad 1.78-4.01-1856.75$

$\begin{array}{lllllllll}97 & 152 & 249 & -4.8 & -5.9-1856.78 & -3.55 & 1.04 & -3.95-1863.24\end{array}$

$\begin{array}{lllllllll}97 & 153 & 250 & -5.0 & -5.8-1862.63 & -3.45 & 1.78 & -3.90-1868.21\end{array}$

$\begin{array}{lllllllll}97 & 154 & 251 & -5.2 & -5.7-1868.37 & -3.03 & 1.04 & -3.85-1874.21\end{array}$

$\begin{array}{lllllllll}97 & 155 & 252 & -5.4 & -5.6 & -1873.99 & -2.55 & 1.77 & -3.80-1878.57\end{array}$

$97 \begin{array}{llllllll}156 & 253 & -5.6 & -5.5-1879.50 & -2.17 & 1.04 & -3.76-1884.38\end{array}$

$97 \begin{array}{llllllll}957 & 254 & -5.7 & -5.3-1884.90 & -1.95 & 1.77 & -3.71-1888.79\end{array}$

$97 \begin{array}{llllllll}158 & 255 & -5.9 & -5.2-1890.19 & -1.69 & 1.04 & -3.66-1894.50\end{array}$

$97159256-6.1 \quad-5.1-1895.37-1.82 \quad 1.77-3.62-1899.04$

$97160257-6.3-5.0-1900.44-1.69 \quad 1.04-3.57-1904.66$

$97161258-6.5-4.9-1905.41-1.891 .76-3.53-1909.06$

$\begin{array}{lllllllll}97 & 162 & 259 & -6.7 & -4.8-1910.27 & -1.76 & 1.04 & -3.49-1914.47\end{array}$

55.29

55.28

56.62

57.05

58.78

59.32

61.28

62.28

64.51

65.75

68.27

69.85

72.95

75.02

78.73

80.99

84.66

87.02

90.55

93.00

96.67

$-6.8-4.7-1915.03-1.761 .76-3.44-1918.47103 .40$

$97 \begin{array}{lllllllll}164 & 261 & -7.0 & -4.6-1919.69 & -1.27 & 1.04 & -3.40-1923.32 & 106.63\end{array}$

$97 \begin{array}{lllllllll}165 & 262 & -7.2 & -4.5-1924.24 & -1.11 & 1.76 & -3.36-1926.96 & 111.06\end{array}$

$97 \begin{array}{lllllllll}966 & 263 & -7.4 & -4.4-1928.70 & -0.67 & 1.04 & -3.32-1931.65 & 114.45\end{array}$

$\begin{array}{llllllllll}97 & 167 & 264 & -7.5 & -4.3-1933.06 & -0.35 & 1.76 & -3.28-1934.93 & 119.23\end{array}$

$97 \begin{array}{lllllllll}168 & 265 & -7.7 & -4.2-1937.31 & -0.21 & 1.04 & -3.25-1939.73 & 122.51\end{array}$

$97 \begin{array}{lllllllll}969 & 266 & -7.9 & -4.1-1941.48 & -0.47 & 1.75 & -3.21-1943.40 & 126.90\end{array}$

$97 \begin{array}{llllllll}970 & 267 & -8.1 & -4.0-1945.55 & 0.22 & 1.04 & -3.17-1947.45 & 130.92\end{array}$

$\begin{array}{llllllllll}97 & 171 & 268 & -8.2 & -3.9-1949.52 & 0.00 & 1.75 & -3.14-1950.91 & 135.54\end{array}$

$97 \begin{array}{llllllll}972 & 269 & -8.4 & -3.8-1953.40 & -0.45 & 1.04 & -3.10-1955.91 & 138.61\end{array}$

$\begin{array}{llllllllll}97 & 173 & 270 & -8.6 & -3.7-1957.19 & -0.44 & 1.75 & -3.07-1958.95 & 143.64\end{array}$

$\begin{array}{lllllllllll}97 & 174 & 271 & -8.7 & -3.7-1960.89 & -0.40 & 1.04 & -3.03-1963.28 & 147.38\end{array}$

$\begin{array}{llllllllll}97 & 175 & 272 & -8.9 & -3.6-1964.50 & -0.81 & 1.75 & -3.00-1966.56 & 152.17\end{array}$

$97 \begin{array}{lllllllll}176 & 273 & -9.1 & -3.5-1968.02 & -1.03 & 1.04 & -2.97-1970.97 & 155.83\end{array}$

$97 \begin{array}{lllllllll}977 & 274 & -9.2 & -3.4-1971.45 & -1.46 & 1.74 & -2.93-1974.11 & 160.77\end{array}$

$97 \begin{array}{llllllllll}178 & 275 & -9.4 & -3.3-1974.80 & -1.31 & 1.04 & -2.90-1977.97 & 164.98\end{array}$

$\begin{array}{llllllllll}97 & 179 & 276 & -9.6 & -3.2-1978.06 & -1.29 & 1.74 & -2.87-1980.48 & 170.54\end{array}$

$97 \begin{array}{llllllllll}180 & 277 & -9.7 & -3.1-1981.24 & -1.23 & 1.04 & -2.84-1984.27 & 174.82\end{array}$

$97 \begin{array}{llllllll}181 & 278 & -9.9 & -3.0-1984.34 & -1.52 & 1.74 & -2.81-1986.93 & 180.23\end{array}$

$97182279-10.1 \quad-3.0-1987.35-1.261 .04-2.78-1990.34184 .89$

$97183280-10.2 \quad-2.9-1990.28-1.391 .74-2.75-1992.68 \quad 190.62$

$97184281-10.4 \quad-2.8-1993.13-0.90 \quad 1.04-2.72-1995.71195 .67$

$97185282-10.5 \quad-2.7-1995.90-0.381 .73-2.70-1997.24202 .21$

$97186283-10.7-2.6-1998.59 \quad 0.13 \quad 1.04-2.67-2000.08 \quad 207.44$

$97187284-10.8 \quad-2.6-2001.20 \quad 0.47 \quad 1.73-2.64-2001.65 \quad 213.95$

$97188285-11.0-2.5-2003.74$

$97189286-11.1-2.4-2006.20$

$97 \quad 190287-11.3-2.3-2008.59$

$97191288-11.4 \quad-2.3-2010.90-0.14 \quad 1.73-2.54-2011.86236 .02$

$97192289-11.6-2.2-2013.14-0.16 \quad 1.04-2.51-2014.77241 .18$

$97193290-11.7-2.1-2015.31-0.44 \quad 1.72-2.49-2016.51247 .51$

$97194291-11.9-2.1-2017.41-0.45 \quad 1.04-2.47-2019.28 \quad 252.81$

$97195292-12.0-2.0-2019.43 \quad-0.72 \quad 1.72 \quad-2.44-2020.87 \quad 259.29$

$97196 \quad 293-12.2-1.9-2021.39-0.73 \quad 1.04-2.42-2023.49264 .74$

$97197294-12.3 \quad-1.8-2023.27-1.09 \quad 1.72 \quad-2.40-2025.04 \quad 271.27$

$97198295-12.5-1.8-2025.09-1.06 \quad 1.04-2.37-2027.48 \quad 276.89$

$97199296-12.6-1.7-2026.84-1.50 \quad 1.72 \quad-2.35-2028.98 \quad 283.47$

$97200297-12.7-1.6-2028.53-1.57 \quad 1.04-2.33-2031.38 \quad 289.14$

$97201298-12.9-1.6-2030.15-1.95 \quad 1.72-2.31-2032.69295 .90$

$97202299-13.0-1.5-2031.70-1.93 \quad 1.04-2.29-2034.88301 .79$

$97203 \quad 300-13.1-1.5-2033.19-2.44 \quad 1.71-2.27-2036.19308 .55$

$97204301-13.3-1.4-2034.62-2.381 .04-2.25-2038.20 \quad 314.60$

$97205 \quad 302-13.4 \quad-1.3-2035.99-2.74 \quad 1.71-2.23-2039.24 \quad 321.64$

$97206 \quad 303-13.6-1.3-2037.29-2.44 \quad 1.04-2.21-2040.89328 .06$

104 $\begin{array}{lll}0.00 & 0.00 & 0.00 \\ 0.00 & 0.00 & 0.00 \\ 0.00 & 0.00 & 0.00 \\ 0.00 & 0.00 & 0.00 \\ 0.00 & 0.00 & 0.00\end{array}$

$58.68-3.58-0.64$

$\begin{array}{llll}60.70 & -3.85 & -0.58\end{array}$

$61.81-3.68-0.47$

$\begin{array}{llll}0.00 & 0.00 & 0.00\end{array}$

$\begin{array}{llll}65.48 & -3.73 & -0.27\end{array}$

$68.11-3.87-0.16$

$69.84-3.56-0.01$

$\begin{array}{llll}72.95 & -3.45 & 0.00\end{array}$

$75.22-2.83 \quad 0.20$

$0.00 \quad 0.00 \quad 0.00$

$0.00 \quad 0.00 \quad 0.00$

$0.00 \quad 0.00 \quad 0.00$

$0.00 \quad 0.00 \quad 0.00$

$\begin{array}{llll}0.00 & 0.00 & 0.00\end{array}$

$0.00 \quad 0.00 \quad 0.00$

$0.00 \quad 0.00 \quad 0.00$

$0.00 \quad 0.00 \quad 0.00$

$0.00 \quad 0.00 \quad 0.00$

$0.00 \quad 0.00 \quad 0.00$

$\begin{array}{llll}0.00 & 0.00 & 0.00\end{array}$

$0.00 \quad 0.00 \quad 0.00$

$\begin{array}{lll}0.00 & 0.00 & 0.00\end{array}$

$0.00 \quad 0.00 \quad 0.00$

$0.00 \quad 0.00 \quad 0.00$

$0.00 \quad 0.00 \quad 0.00$

$0.00 \quad 0.00 \quad 0.00$

$\begin{array}{lll}0.00 & 0.00 & 0.00\end{array}$

$0.00 \quad 0.00 \quad 0.00$

$\begin{array}{llll}0.00 & 0.00 & 0.00\end{array}$

$0.00 \quad 0.00 \quad 0.00$

$0.00 \quad 0.00 \quad 0.00$

$0.00 \quad 0.00 \quad 0.00$

$\begin{array}{llll}0.00 & 0.00 & 0.00\end{array}$

$\begin{array}{llll}0.00 & 0.00 & 0.00\end{array}$

$0.00 \quad 0.00 \quad 0.00$

$0.00 \quad 0.00 \quad 0.00$

$\begin{array}{llll}0.00 & 0.00 & 0.00\end{array}$

$0.00 \quad 0.00 \quad 0.00$

$0.00 \quad 0.00 \quad 0.00$

$\begin{array}{llll}0.00 & 0.00 & 0.00\end{array}$

$0.00 \quad 0.00 \quad 0.00$

$0.00 \quad 0.00 \quad 0.00$

$0.00 \quad 0.00 \quad 0.00$

$0.00 \quad 0.00 \quad 0.00$

$0.00 \quad 0.00 \quad 0.00$

$\begin{array}{llll}0.00 & 0.00 & 0.00\end{array}$

$\begin{array}{llll}0.00 & 0.00 & 0.00\end{array}$

$0.00 \quad 0.00 \quad 0.00$

$0.00 \quad 0.00 \quad 0.00$

$0.00 \quad 0.00 \quad 0.00$

$0.00 \quad 0.00 \quad 0.00$

$0.00 \quad 0.00 \quad 0.00$

$0.00 \quad 0.00 \quad 0.00$

$\begin{array}{llll}0.00 & 0.00 & 0.00\end{array}$

$\begin{array}{llll}0.00 & 0.00 & 0.00\end{array}$

$\begin{array}{llll}0.00 & 0.00 & 0.00\end{array}$

$0.00 \quad 0.00 \quad 0.00$

$\begin{array}{llll}0.00 & 0.00 & 0.00\end{array}$

$\begin{array}{llll}0.00 & 0.00 & 0.00\end{array}$

$0.00 \quad 0.00 \quad 0.00$

$0.00 \quad 0.00 \quad 0.00$ 
$97207304-13.7-1.2-2038.53-2.77 \quad 1.71-2.19-2041.78 \quad 335.24$ $97208 \quad 305-13.8-1.1-2039.71-2.88 \quad 1.04-2.17-2043.72 \quad 341.38$ $97209306-13.9-1.1-2040.83-3.31 \quad 1.71-2.15-2044.58 \quad 348.58$ $97210 \quad 307-14.1-1.0-2041.90-3.32 \quad 1.04-2.13-2046.30354 .93$ $97211308-14.2-1.0-2042.90-3.58 \quad 1.71-2.11-2046.89362 .42$ $97212309-14.3 \quad-0.9-2043.85-3.35 \quad 1.04-2.09-2048.25 \quad 369.13$ $97213 \quad 310-14.5 \quad-0.9-2044.74-3.381 .70-2.08-2048.49376 .96$ $97214311-14.6-0.8-2045.58-3.13 \quad 1.04-2.06-2049.72 \quad 383.80$ $97215 \quad 312-14.7-0.7-2046.35-3.30 \quad 1.70-2.04-2049.99 \quad 391.60$ $97216 \quad 313-14.8 \quad-0.7-2047.08-3.11 \quad 1.04-2.03-2051.17398 .49$ $97217314-15.0 \quad-0.6-2047.75-3.52 \quad 1.70-2.01-2051.58 \quad 406.15$ $97218 \quad 315-15.1 \quad-0.6-2048.37-3.52 \quad 1.04-1.99-2052.84 \quad 412.97$ $97219316-15.2-0.5-2048.94-3.70 \quad 1.70-1.98-2052.91420 .96$ $97220 \quad 317-15.3 \quad-0.5-2049.45-3.67 \quad 1.04-1.96-2054.04 \quad 427.91$ $97221318-15.4 \quad-0.4-2049.91-3.891 .70-1.94-2054.05 \quad 435.97$ $97222 \quad 319-15.6 \quad-0.4-2050.33-3.61 \quad 1.04-1.93-2054.82 \quad 443.27$ $97223.320-15.7-0.3-2050.69-3.73 \quad 1.70-1.91-2054.64 \quad 451.52$ $97224 \quad 321-15.8 \quad-0.3-2051.01-3.37 \quad 1.04-1.90-2055.23 \quad 459.00$ $\begin{array}{lllllllll}98 & 123 & 221 & 1.7-10.2-1628.21 & -0.73 & 0.97 & -6.22-1634.19 & 72.10\end{array}$ $98124 \quad 222 \quad 1.5-10.1-1638.35-0.93 \quad 0.00-6.11-1645.39$ $\begin{array}{lllllllll}98 & 125 & 223 & 1.2 & -9.9-1648.32 & -1.37 & 0.96 & -6.01-1654.75\end{array}$ $\begin{array}{lllllllll}98 & 126 & 224 & 1.0 & -9.7-1658.13 & -1.52 & 0.00 & -5.92-1665.57\end{array}$ $\begin{array}{lllllllll}98 & 127 & 225 & 0.8 & -9.6-1667.78 & -0.54 & 0.95 & -5.82-1673.18\end{array}$ $\begin{array}{lllllllll}98 & 128 & 226 & 0.6 & -9.4-1677.26 & -0.23 & 0.00 & -5.73-1683.22\end{array}$ $\begin{array}{llllllll}98 & 129 & 227 & 0.4 & -9.2-1686.59 & 1.28 & 0.95 & -5.64-1689.99\end{array}$ $\begin{array}{lllllllll}98 & 130 & 228 & 0.1 & -9.1-1695.76 & 0.86 & 0.00 & -5.55-1700.44\end{array}$ $\begin{array}{lllllllll}98 & 131 & 229 & -0.1 & -8.9-1704.77 & 0.70 & 0.95 & -5.46-1708.58\end{array}$ $\begin{array}{lllllllll}98 & 132 & 230 & -0.3 & -8.8-1713.63 & 0.61 & 0.00 & -5.37-1718.39\end{array}$ $\begin{array}{lllllllll}98 & 133 & 231 & -0.5 & -8.6-1722.33 & 0.39 & 0.94 & -5.29-1726.30\end{array}$ $\begin{array}{lllllllll}98 & 134 & 232 & -0.7 & -8.5-1730.89 & 0.24 & 0.00 & -5.21-1735.86\end{array}$ $\begin{array}{lllllllll}98 & 135 & 233 & -0.9 & -8.3-1739.30 & 0.00 & 0.94 & -5.13-1743.50\end{array}$ $\begin{array}{llllllllll}98 & 136 & 234 & -1.1 & -8.2-1747.56 & -0.18 & 0.00 & -5.06-1752.80\end{array}$ $\begin{array}{lllllllll}98 & 137 & 235 & -1.4 & -8.0-1755.68 & -0.53 & 0.93 & -4.98-1760.26\end{array}$ $\begin{array}{llllllllll}98 & 138 & 236 & -1.6 & -7.9-1763.66 & -0.70 & 0.00 & -4.91-1769.27\end{array}$ $98 \begin{array}{llllllll}139 & 237 & -1.8 & -7.8-1771.50 & -1.11 & 0.93 & -4.84-1776.52\end{array}$ $98 \begin{array}{lllllllll}940 & 238 & -2.0 & -7.6-1779.20 & -1.32 & 0.00 & -4.77-1785.28\end{array}$ $\begin{array}{llllllll}98 & 141 & 239 & -2.2 & -7.5-1786.76 & -1.65 & 0.92 & -4.70-1792.19\end{array}$ $\begin{array}{lllllllll}98 & 142 & 240 & -2.4 & -7.4-1794.19 & -1.86 & 0.00 & -4.63-1800.68\end{array}$ $\begin{array}{lllllllll}98 & 143 & 241 & -2.6 & -7.2-1801.48 & -2.34 & 0.92 & -4.56-1807.47\end{array}$

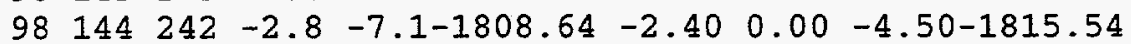
$\begin{array}{llllllllll}98 & 145 & 243 & -3.0 & -7.0 & -1815.67 & -2.92 & 0.91 & -4.44-1822.12\end{array}$ $98146 \quad 244-3.2-6.8-1822.57-2.94 \quad 0.00-4.38-1829.89$ $\begin{array}{lllllllll}98 & 147 & 245 & -3.4 & -6.7 & -1829.35 & -3.27 & 0.91 & -4.32-1836.03\end{array}$ $98148246-3.6-6.6-1836.00-3.25 \quad 0.00-4.26-1843.51$ $\begin{array}{lllllllll}98 & 149 & 247 & -3.8 & -6.5-1842.52 & -3.61 & 0.91 & -4.20-1849.43\end{array}$ $98150 \quad 248-4.0 \quad-6.3-1848.93-3.57 \quad 0.00-4.15-1856.64$ $\begin{array}{llllllllll}98 & 151 & 249 & -4.2 & -6.2 & -1855.21 & -3.87 & 0.90 & -4.09-1862.27\end{array}$ $98 \begin{array}{lllllllll}152 & 250 & -4.4 & -6.1-1861.37 & -3.87 & 0.00 & -4.04-1869.28\end{array}$ $\begin{array}{lllllllll}98 & 153 & 251 & -4.6 & -6.0 & -1867.41 & -3.65 & 0.90 & -3.98-1874.15\end{array}$ $\begin{array}{lllllllll}98 & 154 & 252 & -4.8 & -5.9-1873.34 & -3.23 & 0.00 & -3.93-1880.50\end{array}$ $\begin{array}{lllllllll}98 & 155 & 253 & -4.9 & -5.8-1879.15 & -2.77 & 0.89 & -3.88-1884.91\end{array}$ $98 \quad 156 \quad 254 \quad-5.1 \quad-5.6-1884.85-2.390 .00-3.83-1891.07$ $\begin{array}{lllllllll}98 & 157 & 255 & -5.3 & -5.5-1890.44 & -2.40 & 0.89 & -3.78-1895.73\end{array}$ $\begin{array}{lllllllll}98 & 158 & 256 & -5.5 & -5.4-1895.91 & -1.97 & 0.00 & -3.74-1901.62\end{array}$ $\begin{array}{lllllllll}98 & 159 & 257 & -5.7 & -5.3-1901.28 & -2.11 & 0.89 & -3.69-1906.19\end{array}$ $98 \quad 160 \quad 258 \quad-5.9 \quad-5.2-1906.53 \quad-2.00 \quad 0.00-3.64-1912.18$ $98161259-6.1-5.1-1911.68-2.21 \quad 0.88-3.60-1916.61$ $\begin{array}{lllllllll}98 & 162 & 260 & -6.2 & -5.0-1916.72 & -2.12 & 0.00 & -3.56-1922.40\end{array}$ 68.97 67.68 64.93 65.39 63.43 64.72 62.35 62.28 60.54 60.71 59.21 59.65 58.42 59.02 58.09 58.91 58.22 59.39 58.97 60.25 60.25 61.74 62.04 63.98 64.57 66.72 67.58 70.02 71.08 74.28 76.00 79.66 81.57 84.99 87.17 90.67 92.75 96.39 $98 \quad \begin{array}{llllllllll}163 & 261 & -6.4 & -4.9-1921.66 & -2.16 & 0.88 & -3.51-1926.46 & 102.69\end{array}$

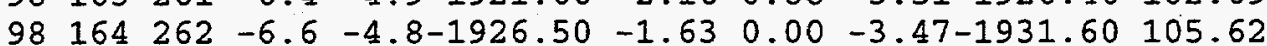
$\begin{array}{llllllllll}98 & 165 & 263 & -6.8 & -4.7-1931.23 & -1.42 & 0.88 & -3.43-1935.21 & 110.08\end{array}$ $\begin{array}{lllllllllll}98 & 166 & 264 & -7.0 & -4.6-1935.86 & -0.94 & 0.00 & -3.39-1940.19 & 113.17\end{array}$ $\begin{array}{llllllllll}98 & 167 & 265 & -7.1 & -4.5-1940.40 & -0.58 & 0.87 & -3.35-1943.46 & 117.98\end{array}$ $\begin{array}{lllllllllll}98 & 168 & 266 & -7.3 & -4.4-1944.83 & -0.49 & 0.00 & -3.31-1948.63 & 120.87\end{array}$ $\begin{array}{llllllllll}98 & 169 & 267 & -7.5 & -4.3-1949.17 & -0.66 & 0.87 & -3.27-1952.23 & 125.34\end{array}$ $98 \quad 170268-7.6-4.2-1953.41 \quad 0.04 \quad 0.00-3.24-1956.60129 .04$

0.00

0.00 0.00

0.00 0.00

0.00

0.00

0.00

0.00

0.00 0.00

0.00 0.00 0.00

0.00 0.00

0.00 0.00

0.00

0.00 0.00

0.00 0.00 0.00

0.00 0.00

0.00

0.00 0.00

0.00 0.00

0.00 0.00 0.00

0.00 0.00

0.00 0.00

0.00

0.00

0.00 0.00

0.00 0.00

0.00 0.00

0.00 0.00

0.00

0.00

0.00 0.00

0.00 0.00

0.00 0.00

0.00

0.00

0.00 0.00

0.00 0.00

0.00 0.00

0.00

0.00

0.00

0.00

0.00 0.00

0.00 0.00

0.00

0.00

0.00 0.00

0.00 0.00

$59.32-3.33$

0.00 0.00

$61.46-3.52$

$63.38-3.87$

$64.09-3.73$

$66.13-4.20$

67.24

$-3.91$

69.72

71.17

74.13

$-4.17$

-3.80
-3.20

$79.29-3.14$

81.34

$-2.62$

0.00

0.00

0.00

0.00

0.00

0.00

0.00

0.00

0.00

0.00

0.00

0.00

0.00

0.00

0.00

0.00

0.00

0.00

0.00

0.00

0.00

0.00

0.00

0.00

0.00

0.00

0.00

0.00
0.00

0.00

0.00

0.00

0.00

0.00

0.00

0.00

0.00

0.00

0.00

0.00

0.00

0.00

0.00

0.00

0.00

0.00

0.00

0.00

0.00

0.00

0.00

0.00

0.00

0.00

0.00

0.00

0.00

0.00

0.00

0.00

0.00

0.00

0.00

0.00

0.00

0.00

0.00

$-0.93$

0.00 $-0.58$ $-0.60$ $-0.48$ $-0.34$ $-0.30$ 0.09

0.03 $-0.37$ $-0.23$

0.00

0.00

0.00

0.00

0.00

0.00

0.00

0.00

0.00

0.00

0.00

0.00

0.00

0.00 
$\begin{array}{lllllllllllll}98 & 171 & 269 & -7.8 & -4.1-1957.55 & -0.19 & 0.86 & -3.20-1960.08 & 133.64\end{array}$ $\begin{array}{llllllllll}98 & 172 & 270 & -8.0 & -4.0-1961.61 & -0.45 & 0.00 & -3.16-1965.22 & 136.57\end{array}$ $\begin{array}{llllllllll}98 & 173 & 271 & -8.2 & -3.9-1965.56 & -0.62 & 0.86 & -3.13-1968.45 & 141.41\end{array}$ $\begin{array}{llllllllll}98 & 174 & 272 & -8.3 & -3.8-1969.43 & -0.58 & 0.00 & -3.09-1973.10 & 144.83\end{array}$ $\begin{array}{llllllllll}98 & 175 & 273 & -8.5 & -3.7-1973.21 & -0.96 & 0.86 & -3.06-1976.37 & 149.63\end{array}$ $\begin{array}{lllllllllll}98 & 176 & 274 & -8.7 & -3.6-1976.89 & -1.08 & 0.00 & -3.03-1981.00 & 153.07\end{array}$ $\begin{array}{llllllllll}98 & 177 & 275 & -8.8 & -3.6-1980.49 & -1.49 & 0.85 & -2.99-1984.12 & 158.03\end{array}$

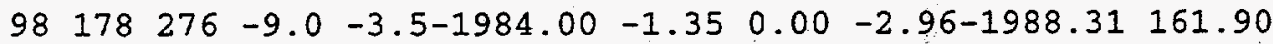
$\begin{array}{llllllllll}98 & 179 & 277 & -9.2 & -3.4-1987.43 & -1.39 & 0.85 & -2.93-1990.89 & 167.39\end{array}$ $\begin{array}{llllllllll}98 & 180 & 278 & -9.3 & -3.3-1990.77 & -1.29 & 0.00 & -2.90-1994.96 & 171.40\end{array}$ $\begin{array}{llllllllll}98 & 181 & 279 & -9.5 & -3.2-1994.02 & -1.57 & 0.85 & -2.87-1997.61 & 176.82\end{array}$ $\begin{array}{llllllllll}98 & 182 & 280 & -9.6 & -3.1-1997.19 & -1.31 & 0.00 & -2.84-2001.34 & 181.16\end{array}$ $\begin{array}{lllllllll}98 & 183 & 281 & -9.8 & -3.0-2000.28 & -1.41 & 0.85 & -2.81-2003.65 & 186.92\end{array}$ $98184282-10.0 \quad-3.0-2003.29-0.90 \quad 0.00-2.78-2006.97 \quad 191.68$ $98185283-10.1 \quad-2.9-2006.22-0.34 \quad 0.84-2.75-2008.46 \quad 198.25$ $98186284-10.3 \quad-2.8-2009.06 \quad 0.16 \quad 0.00-2.72-2011.62 \quad 203.16$ $98 \quad 187285-10.4-2.7-2011.83$ $98 \quad 188286-10.6-2.6-2014.52$ $0.410 .84-2.69-2013.27 \quad 209.59$ $0.620 .00-2.67-2016.57214 .36$ $98 \quad 189 \quad 287-10.7-2.6-2017.14$ $0.310 .84-2.64-2018.63 \quad 220.37$

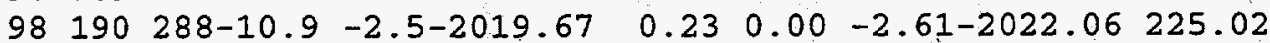
$98191289-11.0-2.4-2022.14-0.09 \quad 0.83-2.59-2023.98231 .17$ $98192 \quad 290-11.2-2.3-2024.52-0.11 \quad 0.00-2.56-2027.20236 .02$ $98 \quad 193291-11.3 \quad-2.3-2026.84-0.42 \quad 0.83-2.54-2028.97 \quad 242.32$ $98 \quad 194 \quad 292-11.5 \quad-2.2-2029.08-0.43 \quad 0.00-2.51-2032.03 \quad 247.33$ $98 \quad 195 \quad 293-11.6 \quad-2.1-2031.25 \quad-0.72 \quad 0.83-2.49-2033.64253 .80$ $98196294-11.8 \quad-2.1-2033.35-0.74 \quad 0.00-2.47-2036.56 \quad 258.94$ $98 \quad 197295-11.9-2.0-2035.38-1.03 \quad 0.82 \quad-2.44-2038.03 \quad 265.54$ $98198296-12.0 \cdot-1.9-2037.35-1.13 \quad 0.00-2.42-2040.90 \quad 270.75$ $98 \quad 199 \quad 297-12.2 \quad-1.9-2039.24 \quad-1.52 \quad 0.82 \quad-2.40-2042.33 \quad 277.38$ $98200298-12.3-1.8-2041.06-1.65 \quad 0.00-2.38-2045.09282 .70$ $98201299-12.5 \quad-1.7-2042.82 \quad-2.06 \quad 0.82 \quad-2.35-2046.42 \quad 289.44$ $98202300-12.6 \quad-1.7-2044.52 \quad-2.06 \quad 0.00-2.33-2048.91295 .02$ $98203 \quad 301-12.8-1.6-2046.15-2.47 \quad 0.82 \quad-2.31-2050.11301 .89$ $98204302-12.9-1.5-2047.71-2.38 \quad 0.00-2.29-2052.38 \quad 307.70$ $98205303-13.0-1.5-2049.21-2.80 \quad 0.81-2.27-2053.46314 .68$ $98206 \quad 304-13.2-1.4-2050.65-2.65 \quad 0.00-2.25-2055.54 \quad 320.67$ $98207305-13.3-1.3-2052.02-2.91 \quad 0.81-2.23-2056.35327 .94$ $98208 \quad 306-13.4-1.3-2053.34-2.74 \quad 0.00-2.21-2058.29334 .07$ $98209307-13.6-1.2-2054.59-3.27 \quad 0.81 \quad-2.19-2059.24341 .19$ $98210 \quad 308-13.7-1.2-2055.78-3.190 .00-2.17-2061.14 \quad 347.36$ $98211 \quad 309-13.8-1.1-2056.92 \quad-3.47 \quad 0.81-2.15-2061.73 \quad 354.84$ $98212310-13.9-1.0-2057.99-3.23 \quad 0.00-2.13-2063.36 \quad 361.29$ $98213 \quad 311-14.1-1.0-2059.01 \quad-3.30 \quad 0.80-2.12-2063.62 \quad 369.09$ $98 \quad 214 \quad 312-14.2 \quad-0.9-2059.97-3.06 \quad 0.00-2.10-2065.13 \quad 375.66$ $98 \quad 215 \quad 313-14.3 \quad-0.9-2060.88-3.290 .80-2.08-2065.45 \quad 383.41$ $98216 \quad 314-14.5 \quad-0.8-2061.73-3.09 \quad 0.00-2.06-2066.88 \quad 390.05$ $98217 \quad 315-14.6 \quad-0.8-2062.52-3.57 \quad 0.80-2.05-2067.34397 .66$ $98218 \quad 316-14.7-0.7-2063.26-3.32 \quad 0.00-2.03-2068.61 \quad 404.46$ $98 \quad 219 \quad 317-14.8 \quad-0.7-2063.95 \quad-3.79 \quad 0.80-2.01-2068.96 \quad 412.19$ $98 \quad 220 \quad 318-14.9-0.6-2064.58-3.81 \quad 0.00-2.00-2070.39418 .83$ $98221 \quad 319-15.1 \quad-0.6-2065.17-4.05 \quad 0.79-1.98-2070.40 \quad 426.89$ $98222 \quad 320-15.2 \quad-0.5-2065.70-3.71 \quad 0.00-1.96-2071.37 \quad 433.99$ $\begin{array}{lllllllll}98 & 223 & 321-15.3 & -0.5-2066.18 & -3.91 & 0.79 & -1.95-2071.25 & 442.19\end{array}$ $98 \quad 224 \quad 322-15.4 \quad-0.4-2066.61-3.55 \quad 0.00-1.93-2072.09 \quad 449.41$ $\begin{array}{lllllllll}98 & 225 & 323-15.5 & -0.4-2066.99 & -3.64 & 0.79 & -1.92-2071.76 & 457.82\end{array}$ $\begin{array}{lllllllll}98 & 226 & 324-15.6 & -0.3-2067.32 & -0.03 & 0.00 & -1.90-2069.25 & 468.39\end{array}$ $\begin{array}{lllllllll}98 & 227 & 325-15.8 & -0.3-2067.61 & -0.88 & 0.79 & -1.89-2069.59 & 476.13\end{array}$ $99 \begin{array}{lllllllll}125 & 224 & 1.7-10.1-1646.87 & -1.22 & 1.82 & -6.14-1652.42 & 77.28\end{array}$ $99 \begin{array}{llllllll}126 & 225 & 1.4 & -9.9-1656.90 & -1.34 & 1.04 & -6.04-1663.25\end{array}$ 99127226 $99 \begin{array}{lll}928 & 227\end{array}$ $1.2-9.8-1666.77-0.371 .81-5.94-1671.27$ $1.0-9.6-1676.48-0.261 .04-5.85-1681.55$ $\begin{array}{lll}99 & 129 & 228\end{array}$ $0.8-9.5-1686.02$ $99130 \quad 229$ $0.6-9.3-1695.41$ $99 \quad 131230 \quad 0.3-9.1-1704.64$ $\begin{array}{llllll}99 & 132 & 231 & 0.1 & -9.0-1713.71\end{array}$ $99 \begin{array}{lllll}933 & 232 & -0.1 & -8.8 & -1722.63\end{array}$ $1.371 .81-5.75-1688.60$ $0.551 .04-5.66-1699.48$ $0.401 .81-5.57-1708.01$ $0.341 .04-5.49-1717.82$ $0.131 .80-5.40-1726.10$

74.52 74.57 72.37 73.39

70.57

70.12 68.38 68.17

\begin{tabular}{|c|c|c|}
\hline 00 & 0.00 & 0.00 \\
\hline .00 & 0.00 & 0.00 \\
\hline .00 & 0.00 & 0.00 \\
\hline 0.00 & 0.00 & 0.00 \\
\hline 0.00 & 0.00 & 0.00 \\
\hline 0.00 & 0.00 & 0.00 \\
\hline 0.00 & 0.00 & 0.00 \\
\hline 0.00 & 0.00 & 0.00 \\
\hline 0.00 & 0.00 & 0.00 \\
\hline 0.00 & 0.00 & 0.00 \\
\hline 0.00 & 0.00 & 0.00 \\
\hline 0.00 & 0.00 & 0.00 \\
\hline 0.00 & 0.00 & 0.00 \\
\hline 0.00 & 0.00 & 0.00 \\
\hline 0.00 & 0.00 & 0.00 \\
\hline 0.00 & 0.00 & 0.00 \\
\hline 0.00 & 0.00 & 0.00 \\
\hline 0.00 & 0.00 & 0.00 \\
\hline 0.00 & 0.00 & 0.00 \\
\hline 0.00 & 0.00 & 0.00 \\
\hline 0.00 & 0.00 & 0.00 \\
\hline .00 & 0.00 & 0.00 \\
\hline 0.00 & 0.00 & 0.00 \\
\hline 0.00 & 0.00 & 0.00 \\
\hline .00 & 0.00 & 0.00 \\
\hline .00 & 0.00 & 0.00 \\
\hline 0.00 & 0.00 & 0.00 \\
\hline 0.00 & 0.00 & 0.00 \\
\hline .00 & 0.00 & 0.00 \\
\hline .00 & 0.00 & 0.00 \\
\hline 0.00 & 0.00 & 0.00 \\
\hline 0.00 & 0.00 & 0.00 \\
\hline .00 & 0.00 & 0.00 \\
\hline .00 & 0.00 & 0.00 \\
\hline 0.00 & 0.00 & 0.00 \\
\hline 0.00 & 0.00 & 0.00 \\
\hline .00 & 0.00 & 0.00 \\
\hline .00 & 0.00 & 0.00 \\
\hline 0.00 & 0.00 & 0.00 \\
\hline 0.00 & 0.00 & 0.00 \\
\hline 0.00 & 0.00 & 0.00 \\
\hline .00 & 0.00 & 0.00 \\
\hline 0.00 & 0.00 & 0.00 \\
\hline 0.00 & 0.00 & 0.00 \\
\hline .00 & 0.00 & 0.00 \\
\hline 0.00 & 0.00 & 0.00 \\
\hline 0.00 & 0.00 & 0.00 \\
\hline 0.00 & 0.00 & 0.00 \\
\hline .00 & 0.00 & 0.00 \\
\hline 0.00 & 0.00 & 0.00 \\
\hline 0.00 & 0.00 & 0.00 \\
\hline 0.00 & 0.00 & 0.00 \\
\hline 0.00 & 0.00 & 0.0 \\
\hline 0.00 & 0.00 & 0.00 \\
\hline 0.00 & 0.00 & 0.00 \\
\hline 0.00 & 0.00 & 0.00 \\
\hline 0.00 & 0.00 & 0.00 \\
\hline 0.00 & 0.00 & 0.00 \\
\hline 0.00 & 0.00 & 0.00 \\
\hline 0.00 & 0.00 & 0.00 \\
\hline .00 & 0.00 & 0.00 \\
\hline 0.00 & 0.00 & 0.00 \\
\hline 0.00 & 0.00 & 0.00 \\
\hline & 0.00 & 0.00 \\
\hline .00 & 0.00 & 0.0 \\
\hline & 0.00 & 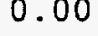 \\
\hline
\end{tabular}
106 
$99 \begin{array}{llllllll}934 & 233 & -0.3 & -8.7-1731.40 & 0.06 & 1.04 & -5.32-1735.63\end{array}$ $99 \begin{array}{llllllll}935 & 234 & -0.5 & -8.5-1740.03 & -0.22 & 1.80 & -5.24-1743.69\end{array}$ $\begin{array}{lllllllll}99 & 136 & 235 & -0.7 & -8.4-1748.50 & -0.39 & 1.04 & -5.16-1753.02\end{array}$ $\begin{array}{lllllllll}99 & 137 & 236 & -0.9 & -8.3-1756.83 & -0.72 & 1.80 & -5.09-1760.84\end{array}$ $\begin{array}{lllllllll}99 & 138 & 237 & -1.1 & -8.1-1765.02 & -0.94 & 1.04 & -5.01-1769.93\end{array}$ $99 \begin{array}{llllllll}939 & 238 & -1.4 & -8.0-1773.07 & -1.32 & 1.79 & -4.94-1777.53\end{array}$ $99 \begin{array}{llllllll}140 & 239 & -1.6 & -7.8-1780.97 & -1.60 & 1.04 & -4.87-1786.40\end{array}$ $\begin{array}{llllllllll}99 & 141 & 240 & -1.8 & -7.7-1788.74 & -1.94 & 1.79 & -4.80-1793.69\end{array}$ $\begin{array}{llllllllll}99 & 142 & 241 & -2.0 & -7.6-1796.37 & -2.22 & 1.04 & -4.73-1802.28\end{array}$ $\begin{array}{lllllllllll}99 & 143 & 242 & -2.2 & -7.4-1803.87 & -2.58 & 1.79 & -4.66-1809.32\end{array}$ $99 \begin{array}{lllllllll}944 & 243 & -2.4 & -7.3-1811.23 & -2.64 & 1.04 & -4.59-1817.43\end{array}$ $\begin{array}{lllllllll}99 & 145 & 244 & -2.6 & -7.2-1818.46 & -3.22 & 1.78 & -4.53-1824.43\end{array}$ $\begin{array}{lllllllll}99 & 146 & 245 & -2.8 & -7.0-1825.56 & -3.26 & 1.04 & -4.47-1832.25\end{array}$ $99147246-3.0 \quad-6.9-1832.54-3.63 \quad 1.78-4.41-1838.79$ $\begin{array}{lllllllll}99 & 148 & 247 & -3.2 & -6.8-1839.38 & -3.64 & 1.04 & -4.35-1846.33\end{array}$ $99 \begin{array}{llllllll}949 & 248 & -3.4 & -6.7-1846.10 & -4.01 & 1.78 & -4.29-1852.63\end{array}$ $\begin{array}{lllllllll}99 & 150 & 249 & -3.6 & -6.5-1852.70 & -4.03 & 1.04 & -4.23-1859.93\end{array}$ $\begin{array}{lllllllll}99 & 151 & 250 & -3.8 & -6.4-1859.18 & -4.52 & 1.77 & -4.17-1866.10\end{array}$ $99 \begin{array}{llllllll}952 & 251 & -3.9 & -6.3-1865.53 & -4.38 & 1.04 & -4.12-1872.99\end{array}$ $\begin{array}{llllllllll}99 & 153 & 252 & -4.1 & -6.2-1871.77 & -4.14 & 1.77 & -4.07-1878.20\end{array}$ $99 \begin{array}{llllllll}954 & 253 & -4.3 & -6.1-1877.89 & -3.73 & 1.04 & -4.01-1884.59\end{array}$ $\begin{array}{lllllllll}99 & 155 & 254 & -4.5 & -5.9-1883.89 & -3.28 & 1.77 & -3.96-1889.36\end{array}$ $99 \begin{array}{llllllll}956 & 255 & -4.7 & -5.8-1889.77 & -2.90 & 1.04 & -3.91-1895.55\end{array}$ $99157256-4.9-5.7-1895.55-2.92 \quad 1.76-3.86-1900.56$ $\begin{array}{lllllllll}99 & 158 & 257 & -5.1 & -5.6-1901.21 & -2.62 & 1.04 & -3.81-1906.60\end{array}$ $\begin{array}{lllllllll}99 & 159 & 258 & -5.3 & -5.5 & -1906.76 & -2.62 & 1.76 & -3.77-1911.38\end{array}$ $99 \begin{array}{llllllll}960 & 259 & -5.5 & -5.4-1912.20 & -2.51 & 1.04 & -3.72-1917.39\end{array}$ $\begin{array}{lllllllll}99 & 161 & 260 & -5.6 & -5.3-1917.53 & -2.75 & 1.76 & -3.67-1922.20\end{array}$ $99 \begin{array}{llllllll}162 & 261 & -5.8 & -5.2-1922.75 & -2.65 & 1.04 & -3.63-1927.99\end{array}$ $99163262-6.0-5.1-1927.87-2.651 .76-3.58-1932.35104 .06$ $\begin{array}{llllllllll}99 & 164 & 263 & -6.2 & -5.0-1932.89 & -2.17 & 1.04 & -3.54-1937.56 & 106.92\end{array}$ $99 \begin{array}{lllllllll}165 & 264 & -6.4 & -4.9-1937.80 & -1.93 & 1.75 & -3.50-1941.48 & 111.08\end{array}$ $99166265-6.5-4.8-1942.61-1.46 \quad 1.04-3.46-1946.49114 .14$ $99167266-6.7-4.7-1947.32-0.95 \quad 1.75-3.42-1949.94 \quad 118.76$ $\begin{array}{llllllllll}99 & 168 & 267 & -6.9 & -4.6-1951.93 & -0.85 & 1.04 & -3.38-1955.12 & 121.65\end{array}$ $\begin{array}{llllllllll}99 & 169 & 268 & -7.1 & -4.5-1956.44 & -0.99 & 1.75 & -3.34-1959.02 & 125.82\end{array}$ $\begin{array}{lllllllllll}99 & 170 & 269 & -7.2 & -4.4-1960.85 & -0.40 & 1.04 & -3.30-1963.51 & 129.40\end{array}$ $99 \begin{array}{llllllllll}171 & 270 & -7.4 & -4.3-1965.17 & -0.61 & 1.74 & -3.26-1967.30 & 133.69\end{array}$ $\begin{array}{lllllllllll}99 & 172 & 271 & -7.6 & -4.2-1969.39 & -0.39 & 1.04 & -3.23-1971.97 & 137.09\end{array}$ $99 \begin{array}{llllllllll}173 & 272 & -7.7 & -4.1-1973.52 & -0.79 & 1.74 & -3.19-1975.76 & 141.37\end{array}$

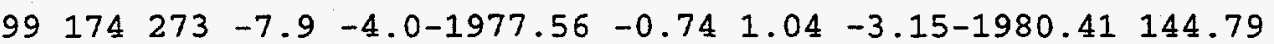
$99 \begin{array}{llllllllll}175 & 274 & -8.1 & -3.9-1981.50 & -1.13 & 1.74 & -3.12-1984.01 & 149.26\end{array}$ $\begin{array}{llllllllll}99 & 176 & 275 & -8.2 & -3.8-1985.35 & -0.89 & 1.04 & -3.09-1988.29 & 153.05\end{array}$ $99177276-8.4 \quad-3.7-1989.11-1.51 \quad 1.74-3.05-1991.94 .157 .48$ $99 \begin{array}{lllllllll}178 & 277 & -8.6 & -3.6-1992.79 & -1.36 & 1.04 & -3.02-1996.13 & 161.35\end{array}$ $99 \begin{array}{lllllllll}179 & 278 & -8.7 & -3.5-1996.38 & -1.41 & 1.73 & -2.99-1999.04 & 166.52\end{array}$ $99 \begin{array}{llllllllll}180 & 279 & -8.9 & -3.5-1999.88 & -1.24 & 1.04 & -2.95-2003.04 & 170.59\end{array}$ $\begin{array}{llllllllll}99 & 181 & 280 & -9.1 & -3.4-2003.29 & -1.49 & 1.73 & -2.92-2005.98 & 175.72\end{array}$ $99 \begin{array}{lllllllll}182 & 281 & -9.2 & -3.3-2006.63 & -1.15 & 1.04 & -2.89-2009.63 & 180.14\end{array}$ $99 \begin{array}{lllllllll}983 & 282 & -9.4 & -3.2-2009.87 & -1.30 & 1.73 & -2.86-2012.31 & 185.54\end{array}$ $99 \begin{array}{lllllllll}184 & 283 & -9.5 & -3.1-2013.04 & -0.79 & 1.04 & -2.83-2015.62 & 190.29\end{array}$ $\begin{array}{llllllllll}99 & 185 & 284 & -9.7 & -3.0-2016.12 & -0.45 & 1.73 & -2.80-2017.65 & 196.34\end{array}$ $99 \begin{array}{lllll}986 & 285 & -9.9 & -3.0-2019.12\end{array}$ $99 \quad 187286-10.0-2.9-2022.05$ $99 \quad 188 \quad 287-10.2 \quad-2.8-2024.89$ $99189288-10.3-2.7-2027.66$ $99 \quad 190 \quad 289-10.5 \quad-2.6-2030.35$ $99191290-10.6-2.6-2032.96$ $99 \quad 192 \quad 291-10.8 \quad-2.5-2035.50$ $99193 \quad 292-10.9-2.4-2037.96$ $99194293-11.1-2.3-2040.35$ $99195294-11.2-2.3-2042.67-0.98 \quad 1.72-2.54-2044.47250 .23$ $99196295-11.4 \quad-2.2-2044.92 \quad-1.00 \quad 1.04-2.51-2047.39255 .38$ $99197296-11.5 \quad-2.1-2047.09-1.39 \quad 1.71-2.49-2049.26 \quad 261.58$ $99198297-11.6 \quad-2.1-2049.20-1.44 \quad 1.04-2.47-2052.07266 .85$ $99199298-11.8-2.0-2051.23-1.901 .71-2.44-2053.86 \quad 273.12$
0.00

0.00

0.00

0.00

0.00

0.00

0.00

0.00

0.00

0.00

0.00

0.00

0.00

0.00

0.00

0.00

0.00

0.00

0.00

0.00

0.00

0.00

0.00

0.00

0.00

0.00

0.00

0.00

0.00

0.00

0.00

0.00

0.00

0.00

0.00

0.00

0.00

0.00

0.00

0.00

0.00

$70.29-4.51-0.50$

77

\section{9}

$84: 08$

0.00

0.00

0.00

$-4.48$

-0.50
-0.45

0.0

$$
0
$$

0.00
$-4.50-0$

0.00

$\begin{array}{ll}-4.35 & -0.21 \\ -3.90 & -0.17\end{array}$

$-3.77-0.49$

0.00

0.00

0.00

$-3.19$

$-0.29$

0.00

$0.00 \quad 0.00$

0.00

$0.00 \quad 0.00$

$0.00 \quad 0.00$

$0.00 \quad 0.00$

0.00

0.00

0.00

0.00

0.00

0.00

0.00

0.00

0.00

0.00

0.00

0.00

0.00

0.00

0.00

0.00

0.00

0.00

0.00

0.00

0.00

0.00

0.00

0.00

0.00

0.00

0.00

0.00

0.00

0.00

0.00

0.00

0.00

0.00

0.00

0.00

0.00

0.00

0.00

0.00

0.00

0.00

0.00

0.00

0.00

0.00

0.00

0.00

0.00

0.00

0.00

0.00

0.00

0.00

0.00

0.00

0.00

0.00

0.00

.00

0.00

0.00

0.00

$\begin{array}{llllll}0.10 & 1.04 & -2.77-2020.76 & 201.30 \\ 0.55 & 1.72 & -2.75-2022.52 & 207.61\end{array}$ $0.40 \quad 1.04-2.72-2026.17212 .03$ $\begin{array}{llllll}0.09 & 1.72 & -2.69-2028.54 & 217.74\end{array}$ $0.011 .04-2.66-2031.96222 .38$ $-0.301 .72-2.64-2034.18 \quad 228.24$ $\begin{array}{lllll}-0.35 & 1.04 & -2.61-2037.42 & 233.06\end{array}$ $\begin{array}{lllll}-0.66 & 1.72 & -2.59-2039.49 & 239.07\end{array}$

0.00

0.00

0.00

0.00

.00

0.00

0.00

0.00

0.00

0.00

0.00

0.00

0.00

0.00

0.00

0.00

0.00

0.00

0.00

0.00

0.00

0.00

0.00

0.00

0.00

0.00

0.00

0.00

0.00

0.00

0.00

0.00

0.00

0.00

0.00

0.00

0.00 
$99200299-11.9-1.9-2053.20-1.98 \quad 1.04-2.42-2056.56 \quad 278.50$ $99201300-12.1-1.9-2055.10-2.45 \quad 1.71-2.40-2058.24 \quad 284.89$ $99202301-12.2-1.8-2056.93-2.47 \quad 1.04-2.38-2060.74290 .46$ $99203 \quad 302-12.4-1.7-2058.70-2.861 .71-2.35-2062.21297 .07$ $99204 \quad 303-12.5 \quad-1.7-2060.40-2.78 \quad 1.04-2.33-2064.48 \quad 302.87$ $99205 \quad 304-12.6-1.6-2062.04-3.06 \quad 1.71-2.31-2065.70 \quad 309.71$ $99206305-12.8-1.5-2063.61-2.91 \quad 1.04-2.29-2067.77315 .71$ $99207306-12.9-1.5-2065.12-3.12 \quad 1.70-2.27-2068.81322 .75$ $99208307-13.0-1.4-2066.57-3.01 \quad 1.04-2.25-2070.79 \quad 328.84$ $99209 \quad 308-13.2-1.4-2067.95-3.46 \quad 1.70-2.23-2071.94 \quad 335.76$ $99210 \quad 309-13.3 \quad-1.3-2069.28-3.35 \quad 1.04-2.21-2073.80 \quad 341.97$ $99211310-13.4-1.2-2070.54-3.66 \quad 1.70-2.19-2074.70 \quad 349.15$ $99212311-13.6-1.2-2071.75-3.43 \quad 1.04-2.17-2076.31355 .60$ $99213 \quad 312-13.7-1.1-2072.90-3.64 \quad 1.70-2.16-2076.99 \quad 362.99$ $\begin{array}{llllllllll}99 & 214 & 313-13.8 & -1.1-2073.98 & -3.55 & 1.04 & -2.14-2078.63 & 369.42\end{array}$ $99215314-13.9-1.0-2075.02-3.641 .70-2.12-2079.08 \quad 377.05$ $99216315-14.1-0.9-2075.99-3.491 .04-2.10-2080.54383 .66$ $99217316-14.2-0.9-2076.91-3.96 \quad 1.69-2.08-2081.26 \quad 391.01$ $99218 \quad 317-14.3 \quad-0.8-2077.77-3.68 \quad 1.04-2.07-2082.48 \quad 397.86$ $99219318-14.4-0.8-2078.58-4.14 \quad 1.69-2.05-2083.08 \quad 405.34$ $99220319-14.6 \quad-0.7-2079.34 \quad-4.18 \quad 1.04 \quad-2.03-2084.51411 .97$ $99221320-14.7-0.7-2080.04 \quad-4.44 \quad 1.69-2.02-2084.81 \quad 419.75$ $99222 \quad 321-14.8-0.6-2080.69-4.13 \quad 1.04-2.00-2085.78 \quad 426.85$ $99223 \quad 322-14.9-0.6-2081.29-4.29 \quad 1.69-1.98-2085.87 \quad 434.83$ $99.224323-15.0 \quad-0.5-2081.84-3.94 \quad 1.04-1.97-2086.71 \quad 442.07$ $99225 \quad 324-15.2 \quad-0.5-2082.33-4.06 \quad 1.69-1.95-2086.66 \quad 450.18$ $99226 \quad 325-15.3 \quad-0.4-2082.78-3.691 .04-1.94-2087.37 \quad 457.54$ $99227326-15.4-0.4-2083.18-1.00 \quad 1.69-1.92-2084.41468 .57$ $99 \quad 228 \quad 327-15.5 \quad-0.3-2083.53-1.78 \quad 1.04-1.91-2086.18 \quad 474.88$ $99229328-15.6-0.3-2083.83-2.23 \quad 1.68-1.89-2086.27 \quad 482.86$ $\begin{array}{llllllll}100 & 126 & 226 & 1.9-10.2-1655.26 & -1.62 & 0.00 & -6.17-1663.05\end{array}$ $\begin{array}{llllllll}100 & 127 & 227 & 1.6-10.0-1665.35 & -0.65 & 0.95 & -6.07-1671.11\end{array}$ $\begin{array}{lllllllll}100 & 128 & 228 & 1.4 & -9.8-1675.28 & -0.30 & 0.00 & -5.97-1681.55\end{array}$ 100129229 $100 \quad 130 \quad 230$ $1.2-9.7-1685.04$ $1.0-9.5-1694.64$ $100 \quad 131231$ $\begin{array}{lll}100 & 132 & 232\end{array}$ $0.8-9.4-1704.09$ $0.5-9.2-1713.38$ $100133233 \quad 0.3-9.1-1722.52$ $100 \quad 134 \quad 234 \quad 0.1-8.9-1731.50$ $\begin{array}{lllll}100 & 135 & 235 & -0.1 & -8.8-1740.34\end{array}$ $1.130 .95-5.88-1688.84$ $0.53 \quad 0.00-5.78-1699.90$ $0.37 \quad 0.95-5.69-1708.47$ $0.330 .00-5.60-1718.65$ $0.110 .94-5.52-1726.98$ $0.04 \quad 0.00-5.43-1736.89$ $-0.120 .94-5.35-1744.87$ $\begin{array}{lllllllll}100 & 136 & 236 & -0.3 & -8.6-1749.02 & -0.28 & 0.00 & -5.27-1754.57\end{array}$ $\begin{array}{lllllllll}100 & 137 & 237 & -0.5 & -8.5-1757.56 & -0.56 & 0.93 & -5.19-1762.38\end{array}$ $\begin{array}{llllllll}100 & 138 & 238 & -0.7 & -8.3-1765.96 & -0.80 & 0.00 & -5.11-1771.87\end{array}$ $\begin{array}{lllllllll}100 & 139 & 239 & -0.9 & -8.2-1774.21 & -1.17 & 0.93 & -5.04-1779.50\end{array}$ $\begin{array}{lllllllll}100 & 140 & 240 & -1.1 & -8.0-1782.33 & -1.36 & 0.00 & -4.97-1788.65\end{array}$ $\begin{array}{lllllllll}100 & 141 & 241 & -1.3 & -7.9-1790.30 & -1.72 & 0.92 & -4.89-1795.99\end{array}$ $\begin{array}{llllllllll}100 & 142 & 242 & -1.6 & -7.8-1798.13 & -1.99 & 0.00 & -4.82-1804.95\end{array}$ $\begin{array}{lllllllll}100 & 143 & 243 & -1.8 & -7.6-1805.83 & -2.36 & 0.92 & -4.76-1812.03\end{array}$ $\begin{array}{llllllllll}100 & 144 & 244 & -2.0 & -7.5-1813.40 & -2.47 & 0.00 & -4.69-1820.56\end{array}$ $\begin{array}{lllllllll}100 & 145 & 245 & -2.2 & -7.4-1820.83 & -3.07 & 0.91 & -4.62-1827.61\end{array}$ $\begin{array}{lllllllll}100 & 146 & 246 & -2.4 & -7.2-1828.13 & -3.13 & 0.00 & -4.56-1835.82\end{array}$

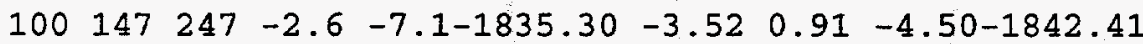
$\begin{array}{lllllllll}100 & 148 & 248 & -2.8 & -7.0-1842.35 & -3.57 & 0.00 & -4.44-1850.35\end{array}$ $\begin{array}{lllllllll}100 & 149 & 249 & -2.9 & -6.9-1849.27 & -3.97 & 0.91 & -4.38-1856.71\end{array}$ $\begin{array}{lllllllll}100 & 150 & 250 & -3.1 & -6.7-1856.06 & -4.04 & 0.00 & -4.32-1864.41\end{array}$ $\begin{array}{lllllllll}100 & 151 & 251 & -3.3 & -6.6-1862.73 & -4.57 & 0.90 & -4.26-1870.66\end{array}$ $\begin{array}{lllllllll}100 & 152 & 252 & -3.5 & -6.5-1869.28 & -4.47 & 0.00 & -4.20-1877.95\end{array}$ $\begin{array}{lllllllll}100 & 153 & 253 & -3.7 & -6.4-1875.70 & -4.24 & 0.90 & -4.15-1883.20\end{array}$ $100 \quad 154 \quad 254-3.9-6.2-1882.01-3.88 \quad 0.00-4.09-1889.99$ $100 \begin{array}{llllllll}155 & 255 & -4.1 & -6.1-1888.20 & -3.71 & 0.89 & -4.04-1895.06\end{array}$ $\begin{array}{lllllllll}100 & 156 & 256 & -4.3 & -6.0-1894.28 & -3.09 & 0.00 & -3.99-1901.36\end{array}$ $\begin{array}{lllllllll}100 & 157 & 257 & -4.5 & -5.9-1900.24 & -3.13 & 0.89 & -3.94-1906.42\end{array}$ $\begin{array}{lllllllll}100 & 158 & 258 & -4.7 & -5.8-1906.09 & -2.88 & 0.00 & -3.89-1912.86\end{array}$ $\begin{array}{lllllllll}100 & 159 & 259 & -4.8 & -5.7-1911.82 & -3.08 & 0.89 & -3.84-1917.86\end{array}$ $\begin{array}{lllllllll}100 & 160 & 260 & -5.0 & -5.6-1917.44 & -2.88 & 0.00 & -3.79-1924.12\end{array}$ $\begin{array}{llllllll}100 & 161 & 261 & -5.2 & -5.5-1922.96 & -3.12 & 0.88 & -3.75-1928.94\end{array}$
0.00

0.00

0.00

0.00

0.00

0.00

0.00

0.00

0.00

0.00

0.00

0.00

0.00

0.00

0.00

0.00

0.00

0.00

0.00

0.00

0.00

0.00

0.00

0.00

0.00

0.00

0.00

0.00

0.00

0.00

0.00

0.00

0.00

0.00

0.00

0.00

0.00

0.00

0.00

0.00

0.00

0.00

0.00

0.00

0.00

0.00

0.00

0.00

0.00

0.00

0.00

0.00

0.00

0.00

0.00

0.00

0.00

0.00

0.00

81.99

82.00

79.64

80.42

77.43

76.93

74.82

74.56

72.72

72.81

71.18

71.44

70.02

70.47

69.39

70.12

69.24

70.22

69.77

70.79

70.65

72.13

72.26

73.98

74.34

76.17

76.95

79.77

81.05

84.05

85.82

88.84

90.47

93.54

95.35

98.60
0.00

0.00

0.00

0.00

0.00

0.00

0.00

0.00

0.00

0.00

0.00

0.00

0.00

0.00

0.00

0.00

0.00

0.00

0.00

70.12

0.00

71.89

0.00

0.00

0.00

0.00

0.00

0.00

0.00

0.00

0.00

0.00

0.00

0.00

0.00

0.00

0.00

0.00

0.00

0.00

0.00

0.00

74.07

$-3.66$

0.00

$-3.94$

$76.81-4.61$

79.34

80.90

83.79

85.48

88.58

0.00

0.00

0.00

0.00
0.00

0.00

0.00

0.00

0.00

0.00

0.00

0.00

0.00

0.00

0.00

0.00

0.00

0.00

0.00

0.00

0.00

0.00

0.00

0.00

0.00

0.00

0.00

0.00

0.00

0.00

0.00

0.00

0.00

0.00

0.00

0.00

0.00

0.00

0.00

0.00

0.00

0.00

0.00

0.00

0.00

0.00

0.00

0.00

0.00

0.00

0.00

0.00

0.00

0.00 $-0.53$

$-0.37$ 108 
$\begin{array}{lllllllllll}100 & 162 & 262 & -5.4 & -5.3-1928.37 & -3.05 & 0.00 & -3.70-1935.12 & 100.49\end{array}$ $\begin{array}{llllllllll}100 & 163 & 263 & -5.6 & -5.2-1933.67 & -3.08 & 0.88 & -3.66-1939.52 & 104.16\end{array}$ $\begin{array}{lllllllllll}100 & 164 & 264 & -5.8 & -5.1-1938.86 & -2.60 & 0.00 & -3.61-1945.07 & 106.68\end{array}$ $\begin{array}{llllllllll}100 & 165 & 265 & -5.9 & -5.0-1943.95 & -2.31 & 0.88 & -3.57-1948.95 & 110.87\end{array}$ $100166266-6.1-4.9-1948.94-1.83 \quad 0.00-3.53-1954.29113 .60$ $\begin{array}{lllllllllll}100 & 167 & 267 & -6.3 & -4.8-1953.82 & -1.30 & 0.87 & -3.49-1957.74 & 118.23\end{array}$ $100168268-6.5-4.7-1958.61-1.16 \quad 0.00-3.44-1963.21 \quad 120.83$ $\begin{array}{lllllllllll}100 & 169 & 269 & -6.6 & -4.6-1963.29 & -1.30 & 0.87 & -3.41-1967.13 & 124.98\end{array}$ $\begin{array}{lllllllllll}100 & 170 & 270 & -6.8 & -4.5-1967.88 & -0.72 & 0.00 & -3.37-1971.96 & 128.22\end{array}$ $\begin{array}{llllllllll}100 & 171 & 271 & -7.0 & -4.4-1972.37 & -0.88 & 0.86 & -3.33-1975.71 & 132.54\end{array}$ $\begin{array}{llllllllll}100 & 172 & 272 & -7.2 & -4.3-1976.76 & -0.68 & 0.00 & -3.29-1980.73 & 135.60\end{array}$ $\begin{array}{llllllllll}100 & 173 & 273 & -7.3 & -4.2-1981.06 & -1.11 & 0.86 & -3.25-1984.56 & 139.84\end{array}$ $\begin{array}{lllllllllll}100 & 174 & 274 & -7.5 & -4.2 & -1985.26 & -1.05 & 0.00 & -3.22-1989.53 & 142.94\end{array}$ $\begin{array}{lllllllllll}100 & 175 & 275 & -7.7 & -4.1-1989.37 & -1.43 & 0.86 & -3.18-1993.13 & 147.41\end{array}$ $\begin{array}{llllllllllll}100 & 176 & 276 & -7.8 & -4.0-1993.39 & -1.17 & 0.00 & -3.15-1997.71 & 150.90\end{array}$ $\begin{array}{lllllllll}100 & 177 & 277 & -8.0 & -3.9-1997.32 & -1.68 & 0.85 & -3.11-2001.26 & 155.42\end{array}$ $\begin{array}{llllllllll}100 & 178 & 278 & -8.2 & -3.8-2001.16 & -1.53 & 0.00 & -3.08-2005.77 & 158.98\end{array}$ $\begin{array}{lllllllllll}100 & 179 & 279 & -8.3 & -3.7-2004.91 & -1.63 & 0.85 & -3.04-2008.74 & 164.09\end{array}$ $100 \quad 180280-8.5-3.6-2008.58-1.45 \quad 0.00-3.01-2013.04167 .86$ $\begin{array}{llllllllll}100 & 181 & 281 & -8.7 & -3.5-2012.15 & -1.69 & 0.85 & -2.98-2015.98 & 172.99\end{array}$ $\begin{array}{llllllllll}100 & 182 & 282 & -8.8 & -3.4-2015.65 & -1.29 & 0.00 & -2.95-2019.89 & 177.16\end{array}$ $\begin{array}{llllllllll}100 & 183 & 283 & -9.0 & -3.4-2019.05 & -1.43 & 0.85 & -2.92-2022.56 & 182.56\end{array}$ $\begin{array}{lllllllllll}100 & 184 & 284 & -9.1 & -3.3-2022.38 & -0.93 & 0.00 & -2.89-2026.19 & 186.99\end{array}$ $\begin{array}{lllllllll}100 & 185 & 285 & -9.3 & -3.2-2025.62 & -0.52 & 0.84 & -2.86-2028.15 & 193.10\end{array}$ $\begin{array}{lllll}100 & 186 & 286 & -9.4 & -3.1-2028.78\end{array}$ $100187287-9.6-3.0-2031.86$ $\begin{array}{lllll}100 & 188 & 288 & -9.8 & -3.0-2034.85\end{array}$ $\begin{array}{lllll}100 & 189 & 289 & -9.9 & -2.9-2037.77\end{array}$ $100190 \quad 290-10.1-2.8-2040.62$ $100191 \quad 291-10.2-2.7-2043.38$ $100192 \quad 292-10.4-2.6-2046.07$ $100193293-10.5-2.6-2048.68$ $100194294-10.7-2.5-2051.22$ $100195 \quad 295-10.8-2.4-2053.69$ $100196296-11.0-2.4-2056.08$ $0.610 .00-2.83-2031.00 \quad 198.33$ $0.28 \quad 0.84-2.80-2033.54203 .86$ $0.540 .00-2.77-2037.09208 .38$ $0.230 .84-2.74-2039.45 \quad 214.09$ $0.13 \quad 0.00-2.72-2043.20 \quad 218.41$ $\begin{array}{llll}-0.19 & 0.83 & -2.69-2045.43224 .26\end{array}$ $-0.220 .00-2.66-2048.95228 .80$ $-0.540 .83-2.64-2051.03234 .80$ $\begin{array}{llll}-0.57 & 0.00 & -2.61-2054.40 & 239.50\end{array}$ $-0.880 .83-2.59-2056.32 \quad 245.64$ $100197297-11.1 \quad-2.3-2058.40-1.33 \quad 0.82-2.54-2061.44256 .67$ $100198 \quad 298-11.2 \quad-2.2-2060.65-1.36 \quad 0.00-2.51-2064.52261 .66$ $100199299-11.4-2.1-2062.83-1.86 \quad 0.82 \quad-2.49-2066.35 \quad 267.90$ $100200 \quad 300-11.5 \quad-2.1-2064.94-2.03 \quad 0.00-2.47-2069.43 \quad 272.89$ $100201301-11.7 \quad-2.0-2066.98-2.47 \quad 0.82 \quad-2.44-2071.07 \quad 279.33$ $100202 \quad 302-11.8 \quad-1.9-2068.95-2.46 \quad 0.00-2.42-2073.83 \quad 284.64$ $100203 \quad 303-12.0 \quad-1.9-2070.86-2.94 \quad 0.82 \quad-2.40-2075.38 \quad 291.16$ $100204304-12.1-1.8-2072.70-2.85 \quad 0.00-2.38-2077.92 \quad 296.69$ $100205 \quad 305-12.2-1.7-2074.47-2.85 \quad 0.81-2.36-2078.86 \quad 303.82$ $100206 \quad 306-12.4-1.7-2076.18-2.90 \quad 0.00-2.33-2081.41309 .34$ $100207 \quad 307-12.5 \quad-1.6-2077.82 \quad-2.94 \quad 0.81-2.31-2082.27 \quad 316.56$ $100208 \quad 308-12.6-1.5-2079.41-2.79 \quad 0.00-2.29-2084.49 \quad 322.41$ $100209 \quad 309-12.8-1.5-2080.93-3.23 \quad 0.81-2.27-2085.62 \quad 329.35$ $100210 \quad 310-12.9-1.4-2082.38-3.10 \quad 0.00-2.25-2087.74 \quad 335.30$ $100211311-13.0 \quad-1.4-2083.78-3.43 \quad 0.81-2.23-2088.64 \quad 342.47$ $100212312-13.2-1.3-2085.11-3.25 \quad 0.00-2.21-2090.58 \quad 348.60$ $100213 \quad 313-13.3-1.2-2086.39-3.27 \quad 0.80-2.20-2091.05 \quad 356.20$ $100214 \quad 314-13.4 \quad-1.2-2087.61-3.42 \quad 0.00-2.18-2093.21 \quad 362.12$ $100215315-13.6-1.1-2088.77-3.55 \quad 0.80-2.16-2093.68 \quad 369.72$ $100216 \quad 316-13.7-1.1-2089.87-3.48 \quad 0.00-2.14-2095.49 \quad 375.98$ $100 \quad 217317-13.8-1.0-2090.91-3.65 \quad 0.80-2.12-2095.89 \quad 383.65$ $100218 \quad 318-13.9-1.0-2091.90-3.72 \quad 0.00-2.10-2097.73 \quad 389.89$ $\begin{array}{lllllllll}100 & 219 & 319-14.1 & -0.9-2092.84 & -4.22 & 0.80 & -2.09-2098.35 & 397.34\end{array}$ $100220320-14.2 \quad-0.8-2093.71-4.27 \quad 0.00-2.07-2100.05403 .70$ $100221 \quad 321-14.3 \quad-0.8-2094.54-4.56 \quad 0.79-2.05-2100.36 \quad 411.47$ $100 \quad 222 \quad 322-14.4 \quad-0.7-2095.31-4.23 \quad 0.00-2.04-2101.57 \quad 418.32$ $\begin{array}{lllllllll}100 & 223 & 323-14.5 & -0.7-2096.02 & -4.45 & 0.79 & -2.02-2101.70 & 426.27\end{array}$ $100224324-14.7 \quad-0.6-2096.69-4.11 \quad 0.00-2.00-2102.80 \quad 433.24$

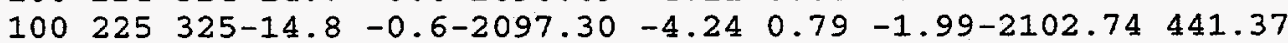
$100226 \quad 326-14.9-0.5-2097.87-3.90 \quad 0.00-1.97-2103.74 \quad 448.44$ $100227327-15.0 \quad-0.5-2098.38-1.290 .79-1.96-2100.84 \quad 459.41$
0.00

0.00

$0.00 \quad 0.00$

0.00

0.00

0.00

0.00

0.00

0.00

0.00

0.00

0.00

0.00

0.00

0.00

0.00

0.00

0.00

0.00

0.00

0.00

0.00

0.00

0.00

0.00

0.00

0.00

0.00

0.00

0.00

0.00

0.00

0.00

0.00

0.00

0.00

0.00

0.00

0.00

0.00

0.00

0.00

0.00

0.00

0.00

0.00

0.00

0.00

0.00

0.00

0.00

0.00

0.00

0.00

0.00

0.00

0.00

0.00

0.00

0.00

0.00

0.00

0.00

0.00

0.00

0.00

0.00

0.00

0.00

0.00

0.00

0.00

0.00

0.00

0.00

0.00

0.00

0.00

0.00

0.00

0.00

0.00

0.00

0.00

0.00

0.00

0.00

0.00

0.00

0.00

0.00

0.00

0.00

0.00

0.00

0.00

0.00

0.00

0.00

0.00

0.00

0.00

0.00

0.00

0.00

0.00

0.00

0.00

0.00

0.00

0.00

0.00

0.00

0.00

0.00

0.00

0.00

0.00

0.00

0.00

0.00

0.00

0.00

0.00

0.00

0.00

0.00

0.00

0.00

0.00

0.00

0.00

0.00

0.00

0.00

0.00

0.00

0.00

0.00

0.00

0.00

0.00

0.00

0.00

0.00

0.00

0.00

0.00

0.00

0.00

0.00

0.00

0.00

0.00

0.00

0.00

0.00

0.00

0.00

0.00

0.00

0.00

0.00

0.00

0.00

0.00

0.00

0.00

0.00

0.00

0.00

0.00

0.00

0.00

0.00

0.00

0.00

0.00

0.00

0.00

0.00

0.00

0.00

0.00

0.00

0.00

0.00 
$\begin{array}{llllllllll}100 & 228 & 328-15.1 & -0.4-2098.84 & -2.07 & 0.00 & -1.94-2102.86 & 465.47\end{array}$

$\begin{array}{lllllllll}100 & 229 & 329-15.2 & -0.4-2099.26 & -2.51 & 0.78 & -1.93-2102.91 & 473.49\end{array}$

$100230.330-15.4 \quad-0.3-2099.63-2.50 \quad 0.00-1.91-2104.04 \quad 480.43$

$\begin{array}{lllllllll}100 & 231 & 331-15.5 & -0.3-2099.94 & -2.64 & 0.78 & -1.90-2103.70 & 488.84\end{array}$

$101128229 \quad 1.8-10.1-1673.66-0.431 .03-6.09-1679.16 \quad 89.30$

101129230

$1.8-10.1-1673.66-0.43 \quad 1.03-6.09-1679.16$

89.30

$\begin{array}{lll}101 & 130 & 231\end{array}$

$1.6-9.9-1683.65$

$1.071 .80-6.00-1686.77$

86.72

$\begin{array}{lll}101 & 131 & 232\end{array}$

$1.4-9.7-1693.47$

$0.461 .03-5.90-1697.88$

$1.2-9.6-1703.13$

$0.251 .80-5.81-1706.89$

85.78

$101 \quad 132 \quad 233$

$1.0-9.4-1712.64$

$0.20 \quad 1.03-5.72-1717.12$

83.61

$\begin{array}{lll}101 & 133 & 234\end{array}$

$0.7-9.3-1721.99$

$-0.021 .80-5.63-1725.84$

101134235

$0.5-9.1-1731.18-0.13 \quad 1.03-5.54-1735.83$

$101135 \quad 236$

$0.3-9.0-1740.23-0.26 \quad 1.79-5.46-1744.16$

$\begin{array}{lllllllll}101 & 136 & 237 & 0.1 & -8.8-1749.13 & -0.35 & 1.03 & -5.38-1753.83\end{array}$

$\begin{array}{lllllllll}101 & 137 & 238 & -0.1 & -8.7-1757.88 & -0.60 & 1.79 & -5.30-1761.99\end{array}$

$\begin{array}{lllllllll}101 & 138 & 239 & -0.3 & -8.5-1766.48 & -0.72 & 1.03 & -5.22-1771.39\end{array}$

$\begin{array}{llllllllll}101 & 139 & 240 & -0.5 & -8.4-1774.94 & -1.09 & 1.79 & -5.14-1779.39\end{array}$

$\begin{array}{lllllllll}101 & 140 & 241 & -0.7 & -8.2-1783.26 & -1.37 & 1.03 & -5.07-1788.67\end{array}$

$\begin{array}{lllllllll}101 & 141 & 242 & -0.9 & -8.1-1791.44 & -1.73 & 1.78 & -4.99-1796.38\end{array}$

$\begin{array}{lllllllll}101 & 142 & 243 & -1.1 & -8.0-1799.48 & -1.99 & 1.03 & -4.92-1805.36\end{array}$

$\begin{array}{lllllllll}101 & 143 & 244 & -1.3 & -7.8-1807.38 & -2.36 & 1.78 & -4.85-1812.82\end{array}$

$\begin{array}{lllllllll}101 & 144 & 245 & -1.5 & -7.7-1815.15 & -2.47 & 1.03 & -4.78-1821.37\end{array}$

$\begin{array}{lllllllll}101 & 145 & 246 & -1.7 & -7.6-1822.78 & -2.81 & 1.78 & -4.72-1828.53\end{array}$

$\begin{array}{lllllllll}101 & 146 & 247 & -1.9 & -7.4-1830.28 & -3.16 & 1.03 & -4.65-1837.06\end{array}$

$\begin{array}{llllllllll}101 & 147 & 248 & -2.1 & -7.3-1837.65 & -3.59 & 1.77 & -4.59-1844.06\end{array}$

$\begin{array}{lllllllll}101 & 148 & 249 & -2.3 & -7.2-1844.90 & -3.68 & 1.03 & -4.53-1852.07\end{array}$

$\begin{array}{llllllllll}101 & 149 & 250 & -2.5 & -7.0-1852.01 & -4.10 & 1.77 & -4.46-1858.81\end{array}$

$\begin{array}{lllllllll}101 & 150 & 251 & -2.7 & -6.9-1859.00 & -4.23 & 1.03 & -4.40-1866.60\end{array}$

$\begin{array}{lllllllll}101 & 151 & 252 & -2.9 & -6.8-1865.86 & -4.76 & 1.77 & -4.35-1873.20\end{array}$

$\begin{array}{llllllllll}101 & 152 & 253 & -3.1 & -6.7-1872.60 & -4.71 & 1.03 & -4.29-1880.57\end{array}$

$\begin{array}{llllllllll}101 & 153 & 254 & -3.3 & -6.6-1879.22 & -4.53 & 1.76 & -4.23-1886.22\end{array}$

$\begin{array}{lllllllll}101 & 154 & 255 & -3.5 & -6.4-1885.72 & -4.20 & 1.03 & -4.18-1893.07\end{array}$

$\begin{array}{lllllllll}101 & 155 & 256 & -3.7 & -6.3-1892.10 & -4.13 & 1.76 & -4.12-1898.60\end{array}$

$\begin{array}{lllllllll}101 & 156 & 257 & -3.9 & -6.2-1898.36 & -3.51 & 1.03 & -4.07-1904.91\end{array}$

$\begin{array}{llllllllll}101 & 157 & 258 & -4.1 & -6.1-1904.51 & -3.64 & 1.76 & -4.02-1910.41\end{array}$

$\begin{array}{lllllllll}101 & 158 & 259 & -4.2 & -6.0-1910.55 & -3.43 & 1.03 & -3.97-1916.91\end{array}$

$\begin{array}{lllllllll}101 & 159 & 260 & -4.4 & -5.9-1916.47 & -3.65 & 1.75 & -3.92-1922.28\end{array}$

$\begin{array}{lllllllll}101 & 160 & 261 & -4.6 & -5.7-1922.27 & -3.46 & 1.03 & -3.87-1928.57\end{array}$

82.97

81.05

80.79

79.20

79.11

77.77

77.85

76.64

77.00

76.09

76.71

76.22

77.13

76.67

77.75

77.81

79.15

79.42

80.89

81.60

84.02

85.24

87.78

89.54

92.11

93.68

96.39

$\begin{array}{llllllllll}101 & 161 & 262 & -4.8 & -5.6-1927.97 & -3.72 & 1.75 & -3.82 & -1933.76 & 101.05\end{array}$

$101162263-5.0 \quad-5.5-1933.56-3.66 \quad 1.03-3.78-1939.97 \quad 102.91$

$\begin{array}{lllllllllll}101 & 163 & 264 & -5.2 & -5.4-1939.04 & -3.70 & 1.75 & -3.73-1944.72 & 106.23\end{array}$

$\begin{array}{llllllllll}101 & 164 & 265 & -5.3 & -5.3-1944.42 & -3.20 & 1.03 & -3.68-1950.27 & 108.75\end{array}$

$\begin{array}{lllllllllll}101 & 165 & 266 & -5.5 & -5.2-1949.68 & -2.89 & 1.75 & -3.64-1954.47 & 112.63\end{array}$

$\begin{array}{llllllllll}101 & 166 & 267 & -5.7 & -5.1-1954.85 & -2.42 & 1.03 & -3.60-1959.84 & 115.33\end{array}$

$\begin{array}{lllllllllll}101 & 167 & 268 & -5.9 & -5.0-1959.91 & -2.12 & 1.74 & -3.55-1963.84 & 119.40\end{array}$

$\begin{array}{llllllllll}101 & 168 & 269 & -6.1 & -4.9-1964.87 & -1.55 & 1.03 & -3.51-1968.90 & 122.41\end{array}$

$\begin{array}{llllllllll}101 & 169 & 270 & -6.2 & -4.8-1969.73 & -1.66 & 1.74 & -3.47-1973.12 & 126.26\end{array}$

$101170271-6.4-4.7-1974.49-1.40 \quad 1.03-3.43-1978.29129 .16$

$\begin{array}{lllllllllll}101 & 171 & 272 & -6.6 & -4.6-1979.15 & -1.17 & 1.74 & -3.39-1981.98 & 133.55\end{array}$

$\begin{array}{lllllllllll}101 & 172 & 273 & -6.7 & -4.5-1983.72 & -1.15 & 1.03 & -3.35-1987.19 & 136.40\end{array}$

$\begin{array}{lllllllllll}101 & 173 & 274 & -6.9 & -4.4-1988.18 & -1.38 & 1.74 & -3.32-1991.14 & 140.52\end{array}$

$\begin{array}{llllllllll}101 & 174 & 275 & -7.1 & -4.3-1992.56 & -1.32 & 1.03 & -3.28-1996.13 & 143.61\end{array}$

$101 \quad 175 \quad 276-7.3-4.2-1996.84-1.68 \quad 1.73-3.24-2000.03 \quad 147.78$

$\begin{array}{lllllllllll}101 & 176 & 277 & -7.4 & -4.1-2001.02 & -1.42 & 1.03 & -3.21-2004.62 & 151.26\end{array}$

$\begin{array}{llllllllll}101 & 177 & 278 & -7.6 & -4.0-2005.12 & -1.81 & 1.73 & -3.17-2008.37 & 155.58\end{array}$

$\begin{array}{llllllllll}101 & 178 & 279 & -7.8 & -4.0-2009.12 & -1.67 & 1.03 & -3.14-2012.90 & 159.12\end{array}$

$\begin{array}{llllllllll}101 & 179 & 280 & -7.9 & -3.9-2013.04 & -1.72 & 1.73 & -3.10-2016.14 & 163.96\end{array}$

$\begin{array}{llllllllllll}101 & 180 & 281 & -8.1 & -3.8-2016.87 & -1.53 & 1.03 & -3.07-2020.44 & 167.73\end{array}$

$\begin{array}{lllllllllll}101 & 181 & 282 & -8.2 & -3.7-2020.61 & -1.74 & 1.73 & -3.04-2023.66 & 172.58\end{array}$

$\begin{array}{llllllllll}101 & 182 & 283 & -8.4 & -3.6-2024.26 & -1.33 & 1.03 & -3.01-2027.56 & 176.75\end{array}$

$\begin{array}{llllllllll}101 & 183 & 284 & -8.6 & -3.5-2027.82 & -1.46 & 1.72 & -2.97-2030.54 & 181.84\end{array}$

$\begin{array}{llllllllll}101 & 184 & 285 & -8.7 & -3.4-2031.31 & -0.99 & 1.03 & -2.94-2034.21 & 186.24\end{array}$

$\begin{array}{llllllllll}101 & 185 & 286 & -8.9 & -3.4-2034.71 & 0.06 & 1.72 & -2.91-2035.84 & 192.68\end{array}$

$\begin{array}{llllllllll}101 & 186 & 287 & -9.0 & -3.3-2038.02 & -0.12 & 1.03 & -2.88-2039.99 & 196.60\end{array}$

$101 \quad 187288-9.2-3.2-2041.26$

$\begin{array}{lllll}101 & 188 & 289 & -9.4 & -3.1-2044.41\end{array}$

$0.101 .72-2.85-2042.29202 .38$

$0.361 .03-2.82-2045.84206 .89$

$\begin{array}{lllll}101 & 189 & 290 & -9.5 & -3.0-2047.48\end{array}$

$0.061 .72-2.80-2048.50212 .31$

0.00

0.00

0.00

0.00

0.00

0.00

0.00

0.00

0.00

0.00

0.00

0.00

0.00

0.00

0.00

0.00

0.00

0.00

0.00

0.00

0.00

0.00

0.00

0.00

0.00

0.00

0.00

0.00

0.00

0.00

0.00

0.00

0.00

0.00

0.00

0.00

0.00

0.00

0.00

0.00

0.00

0.00

0.00

0.00

0.00

0.00

0.00

0.00

0.00

0.00

0.00

0.00

0.00

0.00

0.00

0.00

0.00

0.00

.00

0.00

0.00

0.00

0.00

0.00

0.00

0.00

0.00

0.00

0.00

0.00

0.00

0.00

0.00

0.00

0.00

0.00

0.00

0.00

0.00

0.00

0.00

0.00

0.00

0.00

0.00

0.00

$84.83-4.61-0.41$

$87.55-4.36-0.23$

$88.98-4.07-0.56$

$91.68-4.07-0.43$

0.00

0.00

0.00

0.00

0.00

0.00

0.00

0.00

0.00

0.00

0.00

0.00

0.00

0.00

0.00

0.00

0.00

0.00

0.00

0.00

0.00

0.00

0.00

0.00

0.00

0.00

0.00

0.00

0.00

0.00

0.00

0.00

0.00

0.00

0.00

0.00

0.00

0.00

0.00

0.00

0.00

0.00

0.00

0.00

0.00

0.00

0.00

0.00

0.00

0.00

0.00

0.00

0.00

0.00

0.00

0.00

0.00

0.00

0.00

0.00

0.00

0.00

0.00

0.00

0.00

0.00

0.00

0.00

0.00

0.00

0.00

0.00

0.00

0.00

0.00

0.00

0.00

0.00

0.00

0.00

0.00

0.00

0.00

0.00

0.00

0.00

0.00

0.00

0.00

0.00

0.00

0.00

0.00 
$101 \quad 190291-9.7-3.0-2050.48-0.06 \quad 1.03 \quad-2.77-2052.28 \quad 216.60$ $\begin{array}{lllllllllll}101 & 191 & 292 & -9.8 & -2.9-2053.39 & -0.38 & 1.71 & -2.74-2054.80 & 222.15\end{array}$ $101192 \quad 293-10.0-2.8-2056.23-0.42 \quad 1.03-2.71-2058.34226 .69$ $101 \quad 193294-10.1 \quad-2.7-2059.00-0.73 \quad 1.71-2.69-2060.70 \quad 232.39$ $101194295-10.3-2.6-2061.69-0.76 \quad 1.03-2.66-2064.08 \quad 237.09$ $101195296-10.4-2.6-2064.30-1.08 \quad 1.71-2.63-2066.30242 .93$ $101196297-10.6-2.5-2066.84-1.10 \quad 1.03 \quad-2.61-2069.52 \quad 247.79$ $101197298-10.7-2.4-2069.30-1.52 .1 .71-2.58-2071.70253 .68$ $101198299-10.8-2.4-2071.70-1.62 \quad 1.03-2.56-2074.85258 .60$ $101199300-11.0-2.3-2074.02-2.11 \quad 1.71-2.54-2076.96 \quad 264.56$ $101200301-11.1-2.2-2076.27-2.27 \quad 1.03-2.51-2080.03 \quad 269.57$ $101201302-11.3-2.1-2078.46-2.741 .70-2.49-2081.98275 .68$ $101202303-11.4-2.1-2080.57-2.77 \quad 1.03-2.47-2084.78 \quad 280.96$ $101203 \quad 304-11.6-2.0-2082.62-3.14 \quad 1.70-2.44-2086.50 \quad 287.31$ $101204305-11.7-1.9-2084.60-3.03 \quad 1.03-2.42-2089.02 \quad 292.86$ $101205306-11.8-1.9-2086.51-3.04 \quad 1.70-2.40-2090.25299 .70$ $101206 \quad 307-12.0-1.8-2088.36-2.99 \quad 1.03-2.38-2092.69305 .33$ $101207308-12.1-1.7-2090.14-3.21 \quad 1.70-2.36-2094.00 \quad 312.09$ $101208 \quad 309-12.2-1.7-2091.85-3.03 \quad 1.03-2.34-2096.19317 .98$ $101209 \quad 310-12.4 \quad-1.6-2093.51-3.26 \quad 1.70-2.31-2097.39324 .85$ $101210311-12.5-1.6-2095.10-3.15 \quad 1.03-2.29-2099.51330 .80$ $\begin{array}{lllllllll}101 & 211 & 312-12.7 & -1.5-2096.63 & -3.50 & 1.69 & -2.27-2100.71 & 337.67\end{array}$ $101212313-12.8-1.4-2098.09-3.18 \quad 1.03 \quad-2.25-2102.50 \quad 343.95$ $101213 \quad 314-12.9-1.4-2099.50-3.57 \quad 1.69-2.24-2103.61350 .91$ $101214315-13.0-1.3-2100.85-3.581 .03-2.22-2105.61356 .98$ $101215 \quad 316-13.2-1.3-2102.14-3.83 \quad 1.69-2.20-2106.47364 .19$ $101216 \quad 317-13.3-1.2-2103.37-3.76 \quad 1.03-2.18-2108.27370 .46$ 101 $217318-13.4-1.1-2104.54-4.27 \quad 1.69-2.16-2109.28 \quad 377.53$ $101218319-13.6-1.1-2105.65-4.03 \quad 1.03-2.14-2110.79 \quad 384.09$ $101219320-13.7-1.0-2106.71-4.57 \quad 1.69-2.13-2111.72 \quad 391.23$ $101220321-13.8-1.0-2107.71-4.57 \quad 1.03-2.11-2113.36 \quad 397.67$ $\begin{array}{lllllllll}101 & 221 & 322-13.9 & -0.9-2108.66 & -4.91 & 1.68 & -2.09-2113.97 & 405.12\end{array}$ $101222 \quad 323-14.0-0.9-2109.55-4.66 \quad 1.03-2.07-2115.25 \quad 411.92$ $101223 \quad 324-14.2-0.8-2110.39-4.851 .68-2.06-2115.61419 .63$ $\begin{array}{lllllllll}101 & 224 & 325-14.3 & -0.8-2111.17 & -4.54 & 1.03 & -2.04-2116.72 & 426.59\end{array}$ $101225326-14.4 \quad-0.7-2111.90-4.64 \quad 1.68-2.02-2116.89 \quad 434.49$ $101226327-14.5-0.7-2112.58-4.29 \quad 1.03-2.01-2117.85 \quad 441.60$ $101227328-14.6-0.6-2113.21-1.541 .68-1.99-2115.07 \quad 452.46$ $101228 \quad 329-14.8-0.5-2113.79-2.31 \quad 1.03-1.98-2117.05 \quad 458.55$ $\begin{array}{lllllllll}101 & 229 & 330-14.9 & -0.5-2114.32 & -2.75 & 1.68 & -1.96-2117.36 & 466.31\end{array}$ $101230331-15.0-0.5-2114.80-2.71 \quad 1.03-1.95-2118.42 \quad 473.31$ $\begin{array}{lllllllll}101 & 231 & 332-15.1 & -0.4-2115.23 & -2.86 & 1.68 & -1.93-2118.35 & 481.46\end{array}$ $101232333-15.2-0.4-2115.61-2.82 \quad 1.03-1.92-2119.32 \quad 488.56$ $\begin{array}{lllllllll}101 & 233 & 334-15.3 & -0.3-2115.95^{\prime}-3.15 & 1.67 & -1.90-2119.33 & 496.62\end{array}$ $102130232 \quad 1.8-10.0-1691.88$ $\begin{array}{lllll}102 & 131 & 233 & 1.6 & -9.8-1701.76\end{array}$ $\begin{array}{llllll}102 & 132 & 234 & 1.4 & -9.6-1711.48\end{array}$ $1.00 \quad 0.00-6.02-1696.90 \quad 94.96$ $0.35 \quad 0.95-5.93-1706.39 \quad 93.54$ $0.30 \quad 0.00-5.84-1717.02 \quad 90.99$ $\begin{array}{llll}102 & 133 & 235\end{array}$ $1.2-9.5-1721.05 \quad 0.08 \quad 0.94-5.75-1725.77$ $102 \quad 134236$ $0.9-9.3-1730.45-0.01 \quad 0.00-5.66-1736.12$ $\begin{array}{lllllllll}102 & 135 & 237 & 0.7 & -9.2-1739.71 & -0.22 & 0.94 & -5.57-1744.57\end{array}$ $\begin{array}{lllllllll}102 & 136 & 238 & 0.5 & -9.0-1748.82 & -0.27 & 0.00 & -5.49-1754.58\end{array}$ $\begin{array}{lllllllll}102 & 137 & 239 & 0.3 & -8.9-1757.78 & -0.39 & 0.93 & -5.41-1762.65\end{array}$ $102138240 \quad 0.1-8.7-1766.59-0.57 \quad 0.00-5.33-1772.49$ $\begin{array}{lllllllll}102.139 & 241 & -0.1 & -8.6-1775.26 & -0.88 & 0.93 & -5.25-1780.46\end{array}$ $\begin{array}{lllllllllll}102 & 140 & 242 & -0.3 & -8.4-1783.79 & -1.08 & 0.00 & -5.17-1790.04\end{array}$ $\begin{array}{lllllllll}102 & 141 & 243 & -0.5 & -8.3-1792.17 & -1.42 & 0.92 & -5.10-1797.76\end{array}$ $\begin{array}{lllllllll}102 & 142 & 244 & -0.7 & -8.2-1800.41 & -1.69 & 0.00 & -5.02-1807.13\end{array}$ $\begin{array}{lllllllll}102 & 143 & 245 & -0.9 & -8.0-1808.52 & -2.06 & 0.92 & -4.95-1814.61\end{array}$ $\begin{array}{llllllllll}102 & 144 & 246 & -1.1 & -7.9-1816.49 & -2.18 & 0.00 & -4.88-1823.55\end{array}$ $\begin{array}{llllllllll}102 & 145 & 247 & -1.3 & -7.8-1824.32 & -2.55 & 0.91 & -4.81-1830.77\end{array}$ $102146 \quad 248-1.5-7.6-1832.02-2.90 \quad 0.00-4.75-1839.67$ $\begin{array}{lllllllll}102 & 147 & 249 & -1.7 & -7.5 & -1839.59 & -3.31 & 0.91 & -4.68-1846.67\end{array}$ $\begin{array}{lllllllll}102 & 148 & 250 & -1.9 & -7.4-1847.03 & -3.44 & 0.00 & -4.62-1855.09\end{array}$ $102149251-2.1-7.2-1854.34-3.87 \quad 0.91-4.55-1861.86$ $\begin{array}{lllllllll}102 & 150 & 252 & -2.3 & -7.1-1861.52 & -4.08 & 0.00 & -4.49-1870.10\end{array}$ $\begin{array}{llllllll}102 & 151 & 253 & -2.5 & -7.0-1868.58 & -4.49 & 0.90 & -4.43-1876.60\end{array}$

90.30 88.02 87.65 85.71 85.72 83.94 84.04 82.54 82.88 81.59 82.18 81.31 82.16 81.34 82.41 82.06 83.36 83.19 84.76

\begin{tabular}{|c|c|c|}
\hline & & \\
\hline 0.00 & 0.00 & \\
\hline & 0.00 & \\
\hline 0 & 00 & \\
\hline & 00 & \\
\hline .00 & & \\
\hline & & \\
\hline & & \\
\hline & & \\
\hline & & \\
\hline & & \\
\hline & & \\
\hline & & \\
\hline & & \\
\hline . & 0.00 & \\
\hline & & \\
\hline 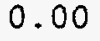 & 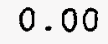 & \\
\hline & & \\
\hline 0 & & \\
\hline 0 & 0. & \\
\hline & & \\
\hline & & \\
\hline 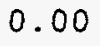 & & \\
\hline & & \\
\hline 0 & 0. & \\
\hline 0. & & \\
\hline$\theta$ & 0. & \\
\hline & & \\
\hline & & \\
\hline & 0 . & 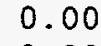 \\
\hline & & \\
\hline & & 0. \\
\hline .0 & $c$ & 0 \\
\hline & & \\
\hline & 0 & \\
\hline & & \\
\hline & & \\
\hline & & \\
\hline & & \\
\hline & & \\
\hline & & \\
\hline & & 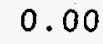 \\
\hline & & \\
\hline & 0 & \\
\hline 0 & & \\
\hline & & \\
\hline 0 & & \\
\hline & 0. & \\
\hline & 0 & \\
\hline & & \\
\hline & & \\
\hline & & \\
\hline 0. & 0 & \\
\hline & & \\
\hline 0. & & \\
\hline & 0 . & \\
\hline & & \\
\hline 0.0 & 0. & \\
\hline .0 & 0. & \\
\hline & & \\
\hline 0.0 & 0. & \\
\hline & & \\
\hline & & \\
\hline & 0. & 0. \\
\hline & & \\
\hline & & \\
\hline
\end{tabular}


$\begin{array}{llllllllll}102 & 152 & 254 & -2.7 & -6.9-1875.51 & -4.65 & 0.00 & -4.37-1884.54\end{array}$ $\begin{array}{lllllllll}102 & 153 & 255 & -2.9 & -6.7-1882.33 & -4.44 & 0.90 & -4.32-1890.18\end{array}$ $\begin{array}{lllllllll}102 & 154 & 256 & -3.1 & -6.6-1889.02 & -4.19 & 0.00 & -4.26-1897.47\end{array}$ $\begin{array}{lllllllll}102 & 155 & 257 & -3.3 & -6.5-1895.59 & -4.11 & 0.89 & -4.21-1903.01\end{array}$ $\begin{array}{lllllllll}102 & 156 & 258 & -3.5 & -6.4-1902.04 & -3.57 & 0.00 & -4.15-1909.76\end{array}$ $\begin{array}{llllllllll}102 & 157 & 259 & -3.6 & -6.3-1908.37 & -3.74 & 0.89 & -4.10-1915.32\end{array}$ $\begin{array}{lllllllll}102 & 158 & 260 & -3.8 & -6.2-1914.59 & -3.57 & 0.00 & -4.05-1922.21\end{array}$ $\begin{array}{lllllllll}102 & 159 & 261 & -4.0 & -6.0-1920.70 & -3.73 & 0.89 & -4.00-1927.54\end{array}$ $\begin{array}{llllllllll}102 & 160 & 262 & -4.2 & -5.9-1926.69 & -3.73 & 0.00 & -3.95-1934.37\end{array}$ $102161263-4.4-5.8-1932.57-4.030 .88-3.90-1939.62102 .46$ $102 \quad 162264-4.6-5.7-1938.34-4.010 .00-3.85-1946.20103 .95$ $\begin{array}{llllllllll}102 & 163 & 265 & -4.8 & -5.6-1944.00 & -4.06 & 0.88 & -3.80-1950.99 & 107.23\end{array}$ $\begin{array}{llllllllll}102 & 164 & 266 & -4.9 & -5.5-1949.56 & -3.58 & 0.00 & -3.76-1956.89 & 109.40\end{array}$ $\begin{array}{llllllllll}102 & 165 & 267 & -5.1 & -5.4-1955.01 & -3.24 & 0.88 & -3.71-1961.08 & 113.28\end{array}$ $\begin{array}{llllllllll}102 & 166 & 268 & -5.3 & -5.3-1960.35 & -2.80 & 0.00 & -3.67-1966.82 & 115.62\end{array}$ $\begin{array}{llllllllll}102 & 167 & 269 & -5.5 & -5.2-1965.59 & -2.55 & 0.87 & -3.62-1970.89 & 119.62\end{array}$ $\begin{array}{llllllllll}102 & 168 & 270 & -5.6 & -5.1-1970.72 & -1.94 & 0.00 & -3.58-1976.24 & 122.33\end{array}$ $\begin{array}{llllllllll}102 & 169 & 271 & -5.8 & -5.0-1975.76 & -2.06 & 0.87 & -3.54-1980.49 & 126.16\end{array}$ $\begin{array}{lllllllllll}102 & 170 & 272 & -6.0 & -4.9-1980.69 & -1.77 & 0.00 & -3.50-1985.96 & 128.76\end{array}$ $102171273-6.2-4.8-1985.52-1.590 .86-3.46-1989.71133 .08$ $\begin{array}{lllllllllll}102 & 172 & 274 & -6.3 & -4.7-1990.26 & -1.53 & 0.00 & -3.42-1995.21 & 135.65\end{array}$ $\begin{array}{llllllllll}102 & 173 & 275 & -6.5 & -4.6-1994.90 & -1.78 & 0.86 & -3.38-1999.20 & 139.74\end{array}$ $\begin{array}{lllllllllll}102 & 174 & 276 & -6.7 & -4.5-1999.44 & -1.72 & 0.00 & -3.34-2004.51 & 142.50\end{array}$ $\begin{array}{llllllllll}102 & 175 & 277 & -6.8 & -4.4-2003.89 & -2.08 & 0.86 & -3.31-2008.42 & 146.66\end{array}$ $\begin{array}{llllllllll}102 & 176 & 278 & -7.0 & -4.3-2008.24 & -1.81 & 0.00 & -3.27-2013.32 & 149.82\end{array}$ $\begin{array}{lllllllll}102 & 177 & 279 & -7.2 & -4.2-2012.51 & -2.05 & 0.85 & -3.23-2016.94 & 154.28\end{array}$ $102178280-7.3-4.1-2016.68-1.910 .00-3.20-2021.78 \quad 157.51$ $\begin{array}{llllllllll}102 & 179 & 281 & -7.5 & -4.0-2020.76 & -2.06 & 0.85 & -3.16-2025.13 & 162.23\end{array}$ $\begin{array}{lllllllllll}102 & 180 & 282 & -7.7 & -3.9-2024.75 & -1.86 & 0.00 & -3.13-2029.74 & 165.70\end{array}$ $\begin{array}{llllllllll}102 & 181 & 283 & -7.8 & -3.9-2028.65 & -2.01 & 0.85 & -3.10-2032.90 & 170.60\end{array}$ $\begin{array}{lllllllllll}102 & 182 & 284 & -8.0 & -3.8-2032.46 & -1.65 & 0.00 & -3.06-2037.17 & 174.40\end{array}$ $\begin{array}{llllllllll}102 & 183 & 285 & -8.2 & -3.7-2036.19 & -1.72 & 0.85 & -3.03-2040.10 & 179.55\end{array}$ $102184286-8.3-3.6-2039.83-1.17 \quad 0.00-3.00-2044.00 \quad 183.72$ $\begin{array}{llllllllll}102 & 185 & 287 & -8.5 & -3.5-2043.39 & -0.87 & 0.84 & -2.97-2046.38 & 189.41\end{array}$ $\begin{array}{lllllllllll}102 & 186 & 288 & -8.6 & -3.4-2046.86 & -0.29 & 0.00 & -2.94-2050.09 & 193.77\end{array}$ $\begin{array}{llllllllll}102 & 187 & 289 & -8.8 & -3.3-2050.25 & -0.05 & 0.84 & -2.91-2052.37 & 199.56\end{array}$ $\begin{array}{lllllllll}102 & 188 & 290 & -8.9 & -3.3-2053.56 & 2.18 & 0.00 & -2.88-2054.26 & 205.75\end{array}$ $\begin{array}{lllllllll}102 & 189 & 291 & -9.1 & -3.2-2056.79 & 0.23 & 0.84 & -2.85-2058.57 & 209.50\end{array}$ $\begin{array}{llllllllll}102 & 190 & 292 & -9.3 & -3.1-2059.94 & 0.12 & 0.00 & -2.82 & -2062.64 & 213.51\end{array}$ $\begin{array}{lllllllllll}102 & 191 & 293 & -9.4 & -3.0-2063.01 & -0.20 & 0.83 & -2.79-2065.17 & 219.05\end{array}$ $\begin{array}{lllllllllll}102 & 192 & 294 & -9.6 & -2.9-2066.00 & -0.26 & 0.00 & -2.76-2069.02 & 223.27\end{array}$ $\begin{array}{llllllllll}102 & 193 & 295 & -9.7 & -2.9-2068.91 & -0.58 & 0.83 & -2.74-2071.40 & 228.96\end{array}$ $102194296-9.9-2.8-2071.75-0.590 .00-2.71-2075.05 \quad 233.38$ $102195297-10.0 \quad-2.7-2074.51-0.91 \quad 0.83-2.68-2077.28 \quad 239.23$ $102196298-10.2-2.6-2077.20-0.95 \quad 0.00-2.66-2080.81243 .77$ $\begin{array}{lllllllll}102 & 197 & 299-10.3 & -2.6-2079.81 & -1.38 & 0.82 & -2.63-2083.00 & 249.65\end{array}$ $102198 \quad 300-10.4 \quad-2.5-2082.35-1.49 \quad 0.00-2.61-2086.45 \quad 254.27$ $102199301-10.6-2.4-2084.82-2.00 \quad 0.82-2.58-2088.58 \quad 260.21$ $102200302-10.7-2.4-2087.21-2.15 \quad 0.00-2.56-2091.92 \quad 264.94$ $102201303-10.9-2.3-2089.54-2.64 \quad 0.82 \quad-2.54-2093.90271 .04$ $102202 \quad 304-11.0-2.2-2091.80-2.64 \quad 0.00-2.51-2096.95276 .06$ $102203 \quad 305-11.2-2.1-2093.98-2.97 \quad 0.82-2.49-2098.63282 .45$ $102204 \quad 306-11.3 \quad-2.1-2096.10-2.86 \quad 0.00-2.47-2101.43 \quad 287.72$ $102205307-11.4 \quad-2.0-2098.15-2.890 .81-2.44-2102.67294 .55$ $102206308-11.6-1.9-2100.14-2.56 \quad 0.00-2.42-2105.12300 .17$ $102207309-11.7-1.9-2102.06-2.76 \quad 0.81-2.40-2106.40 \quad 306.96$ $102208 \quad 310-11.9-1.8-2103.91 .2 .56 \quad 0.00-2.38-2108.85312 .58$ $102209311-12.0-1.8-2105.70-2.770 .81-2.36-2110.02 \quad 319.49$ $102210 \quad 312-12.1-1.7-2107.42-2.64 \quad 0.00-2.34-2112.40 \quad 325.18$ $102211313-12.3-1.6-2109.09-2.95 \quad 0.81-2.32-2113.55332 .10$ $102212314-12.4-1.6-2110.69-2.91 \quad 0.00-2.30-2115.89337 .83$ $102213315-12.5-1.5-2112.22-3.310 .80-2.28-2117.01344 .78$ $102214316-12.7-1.4-2113.70-3.360 .00-2.26-2119.32 \quad 350.54$ $102215 \quad 317-12.8-1.4-2115.12-3.650 .80-2.24-2120.20 \quad 357.73$ $102216 \quad 318-12.9-1.3-2116.48-3.640 .00-2.22-2122.33 \quad 363.67$ $102217319-13.0-1.3-2117.78-4.140 .80-2.20-2123.32 \quad 370.76$
$84.72-4.82-0.17$ $\begin{array}{llll}86.85 & -4.91 & -0.47\end{array}$ $87.82-4.48-0.29$ $90.22-4.53-0.42$ $0.00 \quad 0.00 \quad 0.00$

$94.02-4.19-0.45$

$0.00 \quad 0.00 \quad 0.00$

$\begin{array}{lll}0.00 & 0.00 & 0.00\end{array}$

$\begin{array}{llll}0.00 & 0.00 & 0.00\end{array}$

$0.00 \cdot 0.00 \quad 0.00$

$0.00 \quad 0.00 \quad 0.00$

$\begin{array}{llll}0.00 & 0.00 & 0.00\end{array}$

$\begin{array}{llll}0.00 & 0.00 & 0.00\end{array}$

$0.00 \quad 0.00 \quad 0.00$

$0.00 \quad 0.00 \quad 0.00$

$\begin{array}{llll}0.00 & 0.00 & 0.00\end{array}$

$\begin{array}{llll}0.00 & 0.00 & 0.00\end{array}$

$\begin{array}{llll}0.00 & 0.00 & 0.00\end{array}$

$\begin{array}{llll}0.00 & 0.00 & 0.00\end{array}$

$0.00 \quad 0.00 \quad 0.00$

$0.00 \quad 0.00 \quad 0.00$

$\begin{array}{llll}0.00 & 0.00 & 0.00\end{array}$

$\begin{array}{llll}0.00 & 0.00 & 0.00\end{array}$

$\begin{array}{llll}0.00 & 0.00 & 0.00\end{array}$

$0.00 \quad 0.00 \quad 0.00$

$0.00 \quad 0.00 \quad 0.00$

$0.00 \quad 0.00 \quad 0.00$

$\begin{array}{llll}0.00 & 0.00 & 0.00\end{array}$

$0.00 \quad 0.00 \quad 0.00$

$0.00 \quad 0.00 \quad 0.00$

$0.00 \quad 0.00 \quad 0.00$

$\begin{array}{llll}0.00 & 0.00 & 0.00\end{array}$

$\begin{array}{llll}0.00 & 0.00 & 0.00\end{array}$

$\begin{array}{llll}0.00 & 0.00 & 0.00\end{array}$

$\begin{array}{llll}0.00 & 0.00 & 0.00\end{array}$

$\begin{array}{llll}0.00 & 0.00 & 0.00\end{array}$

$\begin{array}{llll}0.00 & 0.00 & 0.00\end{array}$

$0.00 \quad 0.00 \quad 0.00$

$\begin{array}{llll}0.00 & 0.00 & 0.00\end{array}$

$\begin{array}{llll}0.00 & 0.00 & 0.00\end{array}$

$\begin{array}{llll}0.00 & 0.00 & 0.00\end{array}$

$0.00 \quad 0.00 \quad 0.00$

$\begin{array}{llll}0.00 & 0.00 & 0.00\end{array}$

$\begin{array}{llll}0.00 & 0.00 & 0.00\end{array}$

$\begin{array}{llll}0.00 & 0.00 & 0.00\end{array}$

$0.00 \quad 0.00,0.00$

$\begin{array}{llll}0.00 & 0.00 & 0.00\end{array}$

$\begin{array}{llll}0.00 & 0.00 & 0.00\end{array}$

$\begin{array}{llll}0.00 & 0.00 & 0.00\end{array}$

$\begin{array}{llll}0.00 & 0.00 & 0.00\end{array}$

$\begin{array}{llll}0.00 & 0.00 & 0.00\end{array}$

$\begin{array}{llll}0.00 & 0.00 & 0.00\end{array}$

$\begin{array}{llll}0.00 & 0.00 & 0.00\end{array}$

$0.00 \quad 0.00 \quad 0.00$

$\begin{array}{llll}0.00 & 0.00 & 0.00\end{array}$

$\begin{array}{llll}0.00 & 0.00 & 0.00\end{array}$

$0.00 \quad 0.00 \quad 0.00$

$0.00 \quad 0.00 \quad 0.00$

$0.00 \quad 0.00 \quad 0.00$

$0.00 \quad 0.00 \quad 0.00$

$0.00 \quad 0.00 \quad 0.00$

$\begin{array}{llll}0.00 & 0.00 & 0.00\end{array}$

$\begin{array}{llll}0.00 & 0.00 & 0.00\end{array}$

$\begin{array}{llll}0.00 & 0.00 & 0.00\end{array}$

$\begin{array}{llll}0.00 & 0.00 & 0.00\end{array}$

$0.00 \quad 0.00 \quad 0.00$ 
$102218 \quad 320-13.2-1.2-2119.02-4.240 .00-2.18-2125.44376 .71$ $\begin{array}{lllllllll}102 & 219 & 321-13.3 & -1.2-2120.20 & -4.52 & 0.80 & -2.16-2126.09 & 384.13\end{array}$ $\begin{array}{lllllllll}102 & 220 & 322-13.4 & -1.1-2121.32 & -4.82 & 0.00 & -2.15-2128.29 & 390.00\end{array}$ $\begin{array}{lllllllll}102 & 221 & 323-13.5 & -1.0-2122.40 & -4.95 & 0.79 & -2.13-2128.68 & 397.68\end{array}$ $\begin{array}{llllllllll}102 & 222 & 324-13.7 & -1.0-2123.41 & -4.86 & 0.00 & -2.11-2130.38 & 404.05\end{array}$ $\begin{array}{lllllllll}102 & 223 & 325-13.8 & -0.9-2124.37 & -5.03 & 0.79 & -2.09-2130.70 & 411.80\end{array}$ $\begin{array}{lllllllll}102 & 224 & 326-13.9 & -0.9-2125.28 & -4.70 & 0.00 & -2.08-2132.05 & 418.52\end{array}$ $\begin{array}{lllllllll}102 & 225 & 327-14.0 & -0.8-2126.13 & -4.88 & 0.79 & -2.06-2132.28 & 426.37\end{array}$ $\begin{array}{lllllllll}102 & 226 & 328-14.2 & -0.8-2126.93 & -4.55 & 0.00 & -2.04-2133.52 & 433.20\end{array}$ $\begin{array}{lllllllll}102 & 227 & 329-14.3 & -0.7-2127.68 & -1.96 & 0.79 & -2.03-2130.88 & 443.91\end{array}$ $102228 \quad 330-14.4 \quad-0.7-2128.37-2.69 \quad 0.00-2.01-2133.07 \quad 449.79$ $\begin{array}{lllllllll}102 & 229 & 331-14.5 & -0.6-2129.01 & -3.09 & 0.78 & -2.00-2133.32 & 457.62\end{array}$ $102230332-14.6-0.6-2129.61-3.09 \quad 0.00-1.98-2134.68 \quad 464.33$ $\begin{array}{llllllllll}102 & 231 & 333-14.7 & -0.5-2130.16 & -3.20 & 0.78 & -1.97-2134.54 & 472.54\end{array}$ $102232334-14.9-0.5-2130.65-3.100 .00-1.95-2135.70 \quad 479.45$ $102233335-15.0-0.4-2131.10-3.460 .78-1.94-2135.71487 .51$ $102234336-15.1 \quad-0.4-2131.50-3.33 \quad 0.00-1.92-2136.75 \quad 494.54$ $102235337-15.2-0.3-2131.85-3.77 \quad 0.78-1.91-2136.75 \quad 502.61$ $102236 \quad 338-15.3-0.3-2132.16-3.620 .00-1.89-2137.67 \quad 509.76$ $\begin{array}{llllllllll}103 & 132 & 235 & 1.8 & -9.9-1709.92 & 0.14 & 1.02 & -5.96-1714.71 & 100.56\end{array}$ $\begin{array}{lllllllllll}103 & 133 & 236 & 1.6 & -9.7-1719.70 & -0.07 & 1.79 & -5.86-1723.84 & 99.51\end{array}$ $\begin{array}{lllllllll}103 & 134 & 237 & 1.3 & -9.5-1729.32 & -0.17 .1 .02 & -5.77-1734.24\end{array}$ $\begin{array}{lllllllll}103 & 135 & 238 & 1.1 & -9.4-1738.79 & -0.37 & 1.79 & -5.69-1743.06\end{array}$ 103136239 $0.9-9.2-1748.11-0.47 .1 .02-5.60-1753.15$ 103137240 $0.7-9.1-1757.28-0.671 .78-5.52-1761.68$ $\begin{array}{llllllll}103 & 138 & 241 & 0.5 & -8.9-1766.30 & -0.73 & 1.02 & -5.43-1771.44\end{array}$ $\begin{array}{lllllllll}103 & 139 & 242 & 0.3 & -8.8-1775.17 & -0.95 & 1.78 & -5.35-1779.69\end{array}$ $\begin{array}{lllllllll}103 & 140 & 243 & 0.1 & -8.7-1783.90 & -1.13 & 1.02 & -5.28-1789.28\end{array}$ $\begin{array}{lllllllll}103 & 141 & 244 & -0.1 & -8.5-1792.49 & -1.49 & 1.78 & -5.20-1797.40\end{array}$ $\begin{array}{lllllllll}103 & 142 & 245 & -0.3 & -8.4-1800.94 & -1.72 & 1.02 & -5.12-1806.76\end{array}$ $\begin{array}{lllllllll}103 & 143 & 246 & -0.5 & -8.2-1809.24 & -2.09 & 1.77 & -5.05-1814.61\end{array}$ $\begin{array}{llllllllll}103 & 144 & 247 & -0.7 & -8.1 & -1817.41 & -2.25 & 1.02 & -4.98-1823.62\end{array}$ $\begin{array}{lllllllll}103 & 145 & 248 & -0.9 & -8.0 & -1825.45 & -2.61 & 1.77 & -4.91-1831.20\end{array}$ $\begin{array}{lllllllll}103 & 146 & 249 & -1.1 & -7.8-1833.35 & -2.97 & 1.02 & -4.84-1840.14\end{array}$ $\begin{array}{llllllllll}103 & 147 & 250 & -1.3 & -7.7-1841.11 & -3.42 & 1.77 & -4.77-1847.54\end{array}$ $\begin{array}{llllllllll}103 & 148 & 251 & -1.5 & -7.6-1848.75 & -3.61 & 1.02 & -4.71-1856.05\end{array}$ $\begin{array}{llllllllll}103 & 149 & 252 & -1.7 & -7.4-1856.26 & -4.04 & 1.76 & -4.65-1863.18\end{array}$ $\begin{array}{llllllllll}103 & 150 & 253 & -1.9 & -7.3-1863.64 & -4.32 & 1.02 & -4.58-1871.51\end{array}$ $\begin{array}{lllllllll}103 & 151 & 254 & -2.1 & -7.2-1870.89 & -4.76 & 1.76 & -4.52-1878.41\end{array}$ $\begin{array}{llllllllll}103 & 152 & 255 & -2.3 & -7.1-1878.01 & -4.94 & 1.02 & -4.46-1886.39\end{array}$ $\begin{array}{lllllllll}103 & 153 & 256 & -2.5 & -6.9 & -1885.02 & -4.76 & 1.76 & -4.40-1892.42\end{array}$ $\begin{array}{llllllllll}103 & 154 & 257 & -2.7 & -6.8-1891.90 & -4.57 & 1.02 & -4.35-1899.79\end{array}$ $\begin{array}{lllllllll}103 & 155 & 258 & -2.9 & -6.7-1898.66 & -4.53 & 1.75 & -4.29-1905.72\end{array}$ $\begin{array}{lllllllll}103 & 156 & 259 & -3.1 & -6.6-1905.30 & -4.08 & 1.02 & -4.23-1912.59\end{array}$ $103157260-3.2-6.5-1911.82-4.24 \quad 1.75-4.18-1918.49$ $103158 \quad 261-3.4-6.3-1918.23-4.13 \quad 1.02-4.13-1925.46$ $\begin{array}{lllllllll}103 & 159 & 262 & -3.6 & -6.2-1924.52 & -4.31 & 1.75 & -4.08-1931.15 & 102.05\end{array}$ $\begin{array}{llllllllll}103 & 160 & 263 & -3.8 & -6.1-1930.69 & -4.36 & 1.02 & -4.02-1938.05 & 103.22\end{array}$ $\begin{array}{llllllllll}103 & 161 & 264 & -4.0 & -6.0 & -1936.76 & -4.64 & 1.75 & -3.97-1943.63 & 105.72\end{array}$ $\begin{array}{llllllllll}103 & 162 & 265 & -4.2 & -5.9-1942.71 & -4.66 & 1.02 & -3.93-1950.27 & 107.14\end{array}$ $\begin{array}{llllllllll}103 & 163 & 266 & -4.3 & -5.8-1948.56 & -4.76 & 1.74 & -3.88-1955.45 & 110.04\end{array}$ $\begin{array}{lllllllllll}103 & 164 & 267 & -4.5 & -5.7-1954.29 & -4.27 & 1.02 & -3.83-1961.36 & 112.19\end{array}$ $\begin{array}{llllllllll}103 & 165 & 268 & -4.7 & -5.6-1959.92 & -3.93 & 1.74 & -3.78-1965.89 & 115.74\end{array}$ $\begin{array}{llllllllll}103 & 166 & 269 & -4.9 & -5.5 & -1965.44 & -3.42 & 1.02 & -3.74-1971.57 & 118.13\end{array}$ $\begin{array}{llllllllll}103 & 167 & 270 & -5.1 & -5.4-1970.85 & -3.08 & 1.74 & -3.70-1975.89 & 121.88\end{array}$ $\begin{array}{llllllllll}103 & 168 & 271 & -5.2 & -5.3-1976.16 & -2.45 & 1.02 & -3.65-1981.24 & 124.60\end{array}$ $\begin{array}{lllllllll}103 & 169 & 272 & -5.4 & -5.2-1981.37 & -2.58 & 1.73 & -3.61-1985.83 & 128.09\end{array}$ $\begin{array}{llllllllll}103 & 170 & 273 & -5.6 & -5.1-1986.48 & -2.35 & 1.02 & -3.57-1991.37 & 130.61\end{array}$ $\begin{array}{llllllllll}103 & 171 & 274 & -5.8 & -5.0-1991.49 & -2.23 & 1.73 & -3.53-1995.51 & 134.55\end{array}$ $\begin{array}{llllllllll}103 & 172 & 275 & -5.9 & -4.9-1996.40 & -2.12 & 1.02 & -3.49-2000.98 & 137.15\end{array}$ $103173 \quad 276-6.1-4.8-2001.20-2.17 \quad 1.73-3.45-2005.09141 .11$ $\begin{array}{lllllllllll}103 & 174 & 277 & -6.3 & -4.7-2005.92 & -2.10 & 1.02 & -3.41-2010.40 & 143.87\end{array}$ $103175278-6.4-4.6-2010.53-2.451 .73-3.37-2014.63147 .72$ $\begin{array}{llllllllll}103 & 176 & 279 & -6.6 & -4.5-2015.06 & -2.17 & 1.02 & -3.33-2019.53 & 150.88\end{array}$ $\begin{array}{llllllllll}103 & 177 & 280 & -6.8 & -4.4-2019.49 & -2.30 & 1.72 & -3.30-2023.36 & 155.13\end{array}$ $\begin{array}{llllllllll}103 & 178 & 281 & -6.9 & -4.3-2023.82 & -2.16 & 1.02 & -3.26-2028.22 & 158.34\end{array}$

0.00

0.00

0.00

0.00

0.00

0.00

0.00

0.00

0.00

0.00

0.00

0.00

0.00

0.00

0.00

0.00

0.00

0.00

0.00

0.00

0.00

0.00

0.00

0.00

0.00

0.00

0.00

0.00

0.00

0.00

0.00

0.00

0.00

0.00

0.00

0.00

0.00

0.00

0.00

0.00

0.00

0.00

0.00

95.84

98.13

0.00

0.00

0.00

0.00

0.00

0.00

0.00

0.00

0.00

0.00

0.00

0.00

0.00

0.00

0.00

0.00

0.00

0.00

0.00

0.00

0.00
0.00 0.00

0.00 0.00 $0.00 \quad 0.00$ $0.00 \quad 0.00$ $0.00 \quad 0.00$ $0.00 \quad 0.00$ $0.00 \quad 0.00$ $0.00 \quad 0.00$ 0.00 0.00 0.00 0.00 0.00 0.00 0.00 0.00 0.00 0.00 0.00 0.00 0.00 0.00 0.00 0.00 0.00 0.00 0.00 0.00 0.00 0.00 0.00 0.00 0.00 0.00 0.00 0.00 0.00 0.00 0.00 0.00 0.00 0.00 0.00 0.00 0.00 0.00 0.00 0.00 0.00 0.00 0.00 0.00 0.00 0.00

0.00 0.00 0.00 0.00 0.00 0.00 0.00 0.00 0.00 0.00 0.00 0.00 0.00 0.00 0.00 0.00 0.00 0.00 0.00 $0.00 \quad 0.00$ $-4.64-0.56$ $-4.68-0.44$ $0.00 \quad 0.00$ $0.00 \quad 0.00$ $0.00 \quad 0.00$ $0.00 \quad 0.00$ $0.00 \quad 0.00$ $0.00 \quad 0.00$ $0.00 \quad 0.00$ $0.00 \quad 0.00$ $0.00 \quad 0.00$ $0.00 \quad 0.00$ $0.00 \quad 0.00$ $0.00 \quad 0.00$ $0.00 \quad 0.00$ $0.00 \quad 0.00$ $0.00 \quad 0.00$ $0.00 \quad 0.00$ $0.00 \quad 0.00$ $0.00 \quad 0.00$ $0.00 \quad 0.00$ $0.00 \quad 0.00$ $0.00 \quad 0.00$ 
$\begin{array}{lllllllllll}103 & 179 & 282 & -7.1 & -4.2-2028.07 & -2.28 & 1.72 & -3.22-2031.85 & 162.78\end{array}$ $\begin{array}{llllllllll}103 & 180 & 283 & -7.3 & -4.1-2032.22 & -2.08 & 1.02 & -3.19-2036.47 & 166.24\end{array}$ $\begin{array}{lllllllllll}103 & 181 & 284 & -7.4 & -4.0-2036.28 & -2.21 & 1.72 & -3.16-2039.93 & 170.84\end{array}$ $\begin{array}{lllllllllll}103 & 182 & 285 & -7.6 & -3.9-2040.26 & -1.83 & 1.02 & -3.12-2044.19 & 174.66\end{array}$ $\begin{array}{llllllllll}103 & 183 & 286 & -7.8 & -3.8-2044.15 & -1.87 & 1.72 & -3.09-2047.39 & 179.53\end{array}$ $\begin{array}{llllllllll}103 & 184 & 287 & -7.9 & -3.8-2047.95 & -1.42 & 1.02 & -3.06-2051.40 & 183.59\end{array}$ $\begin{array}{lllllllllll}103 & 185 & 288 & -8.1 & -3.7-2051.67 & -1.13 & 1.72 & -3.02-2054.10 & 188.95\end{array}$ $\begin{array}{lllllllllll}103 & 186 & 289 & -8.2 & -3.6-2055.30 & -0.58 & 1.02 & -2.99-2057.85 & 193.28\end{array}$ $\begin{array}{llllllllll}103 & 187 & 290 & -8.4 & -3.5-2058.85 & 1.29 & 1.71 & -2.96-2058.80 & 200.40\end{array}$ $\begin{array}{llllllllll}103 & 188 & 291 & -8.5 & -3.4-2062.31 & 2.05 & 1.02 & -2.93-2062.17 & 205.10\end{array}$ $\begin{array}{llllllllll}103 & 189 & 292 & -8.7 & -3.3-2065.69 & 0.11 & 1.71 & -2.90-2066.77 & 208.57\end{array}$ $\begin{array}{lllllllllll}103 & 190 & 293 & -8.9 & -3.3-2069.00 & -0.03 & 1.02 & -2.87-2070.87 & 212.54\end{array}$ $\begin{array}{lllllllllll}103 & 191 & 294 & -9.0 & -3.2-2072.22 & -0.36 & 1.71 & -2.84-2073.71 & 217.77\end{array}$ $\begin{array}{llllllllll}103 & 192 & 295 & -9.2 & -3.1-2075.36 & -0.40 & 1.02 & -2.82 & -2077.55 & 222.01\end{array}$ $\begin{array}{lllllllllll}103 & 193 & 296 & -9.3 & -3.0-2078.42 & -0.70 & 1.71 & -2.79-2080.21 & 227.42\end{array}$ $\begin{array}{llllllllll}103 & 194 & 297 & -9.5 & -2.9-2081.41 & -0.71 & 1.02 & -2.76-2083.86 & 231.84\end{array}$ $\begin{array}{llllllllll}103 & 195 & 298 & -9.6 & -2.9-2084.32 & -1.08 & 1.70 & -2.73-2086.43 & 237.34\end{array}$ $\begin{array}{llllllllll}103 & 196 & 299 & -9.8 & -2.8-2087.16 & -1.14 & 1.02 & -2.71-2089.98 & 241.86\end{array}$ $\begin{array}{llllllllll}103 & 197 & 300 & -9.9 & -2.7 & -2089.92 & -1.50 & 1.70 & -2.68-2092.40 & 247.52\end{array}$ $\begin{array}{llllllllll}103 & 198 & 301-10.1 & -2.6-2092.60 & -1.73 & 1.02 & -2.66-2095.97 & 252.02\end{array}$ $\begin{array}{lllllllll}103 & 199 & 302-10.2 & -2.6-2095.22 & -2.17 & 1.70 & -2.63-2098.32 & 257.74\end{array}$ $103200 \quad 303-10.3 \quad-2.5-2097.76-2.42 \quad 1.02 \quad-2.61-2101.76 \quad 262.37$ $103201304-10.5-2.4-2100.23-2.90 \quad 1.70-2.58-2104.01268 .19$ $\begin{array}{lllllllll}103 & 202 & 305-10.6 & -2.4-2102.63 & -2.99 & 1.02 & -2.56-2107.15 & 273.12\end{array}$ $103203 \quad 306-10.8-2.3-2104.96-3.32 \quad 1.70-2.53-2109.12 \quad 279.23$ $103204307-10.9 \cdot-2.2-2107.21-3.12 \quad 1.02-2.51-2111.82 \quad 284.59$ $103205308-11.1-2.2-2109.41-3.17 \quad 1.69-2.49-2113.37291 .12$ $103206 \quad 309-11.2-2.1-2111.53-2.98 \quad 1.02-2.47-2115.95296 .61$ $103207310-11.3-2.0-2113.59-3.131 .69-2.44-2117.47 \quad 303.16$ $103208 \quad 311-11.5 \quad-2.0-2115.58-2.99 \quad 1.02-2.42-2119.96 \quad 308.74$ $103209312-11.6-1.9-2117.50-3.13 \quad 1.69-2.40-2121.34315 .43$ $103210 \quad 313-11.7-1.8-2119.36-3.08 \quad 1.02-2.38-2123.80 \quad 321.05$ $103211314-11.9-1.8-2121.16-3.291 .69-2.36-2125.12 \quad 327.80$ $103212315-12.0-1.7-2122.89-3.17 \quad 1.02 \quad-2.34-2127.37 \quad 333.61$ $103213 \quad 316-12.1-1.6-2124.56-3.51 \quad 1.69-2.32-2128.70 \quad 340.36$ $103214317-12.3-1.6-2126.17-3.591 .02-2.30-2131.03 \quad 346.10$ $103215318-12.4-1.5-2127.72-3.891 .68-2.28-2132.20 \quad 353.00$ $\begin{array}{lllllllll}103 & 216 & 319-12.5 & -1.5-2129.20 & -3.94 & 1.02 & -2.26-2134.38 & 358.89\end{array}$ $103217320-12.7-1.4-2130.63-4.45 \quad 1.68-2.24-2135.64365 .71$ $\begin{array}{lllllllll}103 & 218 & 321-12.8 & -1.3-2132.00 & -4.58 & 1.02 & -2.22-2137.78 & 371.64\end{array}$ $\begin{array}{llllllllll}103 & 219 & 322-12.9 & -1.3-2133.31 & -5.12 & 1.68 & -2.20-2138.95 & 378.54\end{array}$ $103220323-13.0-1.2-2134.56-5.15 \quad 1.02-2.18-2140.87 \quad 384.69$ $\begin{array}{lllllllll}103 & 221 & 324-13.2 & -1.2-2135.76 & -5.51 & 1.68 & -2.17-2141.75 & 391.88\end{array}$ $\begin{array}{lllllllll}103 & 222 & 325-13.3 & -1.1-2136.90 & -5.23 & 1.02 & -2.15-2143.25 & 398.45\end{array}$ $\begin{array}{lllllllll}103 & 223 & 326-13.4 & -1.1-2137.98 & -5.46 & 1.68 & -2.13-2143.89 & 405.88\end{array}$ $\begin{array}{llllllll}103 & 224 & 327-13.5 & -1.0-2139.01 & -5.13 & 1.02 & -2.11-2145.23 & 412.62\end{array}$ $\begin{array}{lllllllll}103 & 225 & 328-13.7 & -0.9-2139.98 & -5.31 & 1.67 & -2.10-2145.71 & 420.20\end{array}$ $\begin{array}{lllllllll}103 & 226 & 329-13.8 & -0.9-2140.90 & -4.99 & 1.02 & -2.08-2146.95 & 427.04\end{array}$ $\begin{array}{lllllllll}103 & 227 & 330-13.9 & -0.8-2141.77 & -2.30 & 1.67 & -2.06-2144.46 & 437.60\end{array}$ $103228 \quad 331-14.0 \quad-0.8-2142.58-3.00 \quad 1.02-2.05-2146.60 \quad 443.53$ $103229 \quad 332-14.1-0.7-2143.34-3.41 \quad 1.67-2.03-2147.11451 .09$ $\begin{array}{lllllllll}103 & 230 & 333-14.3 & -0.7-2144.05 & -3.39 & 1.02 & -2.02-2148.43 & 457.84\end{array}$ $\begin{array}{llllllllll}103 & 231 & 334-14.4 & -0.6-2144.71 & -3.53 & 1.67 & -2.00-2148.57 & 465.77\end{array}$ $\begin{array}{lllllllll}103 & 232 & 335-14.5 & -0.6-2145.32 & -3.40 & 1.02 & -1.98-2149.68 & 472.73\end{array}$ $\begin{array}{lllllllll}103 & 233 & 336-14.6 & -0.5-2145.88 & -3.70 & 1.67 & -1.97-2149.88 & 480.60\end{array}$ $\begin{array}{lllllllll}103 & 234 & 337-14.7 & -0.5-2146.40 & -3.69 & 1.02 & -1.95-2151.02 & 487.54\end{array}$ $\begin{array}{lllllllll}103 & 235 & 338-14.8 & -0.4-2146.86 & -4.09 & 1.67 & -1.94-2151.22 & 495.41\end{array}$ $\begin{array}{lllllllll}103 & 236 & 339-14.9 & -0.4-2147.28 & -3.94 & 1.02 & -1.92-2152.12 & 502.59\end{array}$ $\begin{array}{llllllllll}104 & 134 & 238 & 1.7 & -9.8-1727.78 & -0.02 & 0.00 & -5.89-1733.69 & 105.00\end{array}$ $\begin{array}{llllllllll}104 & 135 & 239 & 1.5 & -9.6-1737.46 & -0.31 & 0.94 & -5.80-1742.63 & 104.12\end{array}$ $\begin{array}{llllllllll}104 & 136 & 240 & 1.3 & -9.4-1746.99 & -0.28 & 0.00 & -5.71-1752.98 & 101.85\end{array}$ $\begin{array}{lllllllll}104 & 137 & 241 & 1.1 & -9.3-1756.36 & -0.47 & 0.93 & -5.63-1761.53 & 101.37\end{array}$ $104 \quad 138 \quad 242$ 104139243 $104140 \quad 244$ 104141245
$0.9-9.2-1765.59-0.44 \quad 0.00-5.54-1771.57$ $0.7-9.0-1774.67-0.640 .93-5.46-1779.85$ $0.5-8.9-1783.61-0.82 \quad 0.00-5.38-1789.81$ $0.3-8.7-1792.40-1.15 \quad 0.92-5.30-1797.93$ 114
0.00

0.00

0.00

0.00

0.00

0.00

0.00

0.00

0.00

0.00

0.00

0.00

0.00

0.00

0.00

0.00

0.00

0.00

0.00

0.00

0.00

0.00

0.00

0.00

0.00

0.00

0.00

0.00

0.00

0.00

0.00

0.00

0.00

0.00

0.00

0.00

0.00

0.00

0.00

0.00

0.00

0.00

0.00

0.00

0.00

0.00

0.00

0.00

0.00

0.00

0.00

0.00

0.00

0.00

0.00

0.00

0.00

0.00

0.00

0.00

0.00

0.00

0.00

0.00

0.00

0.00

0.00

0.00

0.00

0.00

0.00

0.00

0.00

0.00

0.00

0.00

0.00

0.00

0.00

0.00

0.00

0.00

0.00

0.00

0.00

0.00

0.00

0.00

0.00

0.00

0.00

0.00

0.00

0.00

0.00

0.00

0.00

0.00

0.00

0.00

0.00

0.00

0.00

0.00

0.00

0.00

0.00

0.00

0.00

0.00

0.00

0.00

0.00

0.00

0.00

0.00

0.00

0.00

0.00

0.00

0.00

0.00

0.00

0.00

0.00

0.00

0.00

0.00

0.00

0.00

0.00

0.00

0.00

0.00

0.00

0.00

0.00

0.00

0.00

0.00

0.00

0.00

0.00

0.00

0.00

0.00

0.00

0.00

0.00

0.00

0.00

0.00

0.00

0.00

0.00

0.00

0.00

0.00

0.00

0.00

0.00

0.00

0.00

0.00

0.00

0.00

0.00

0.00

99.39

99.19

97.30

97.25

0.00

0.00

0.00

0.00

$0.00 \quad 0.00 \quad 0.00$

0.00

0.00

0.00 
$104142246 \quad 0.1-8.6-1801.05-1.40 \quad 0.00-5.23-1807.68$ $\begin{array}{llllllllll}104 & 143 & 247 & -0.1 & -8.4-1809.56 & -1.74 & 0.92 & -5.15-1815.54\end{array}$ $\begin{array}{lllllllll}104 & 144 & 248 & -0.3 & -8.3-1817.93 & -1.92 & 0.00 & -5.08-1824.93\end{array}$ $\begin{array}{lllllllll}104 & 145 & 249 & -0.5 & -8.2-1826.17 & -2.30 & 0.91 & -5.01-1832.56\end{array}$ $\begin{array}{lllllllll}104 & 146 & 250 & -0.7 & -8.0-1834.27 & -2.67 & 0.00 & -4.94-1841.87\end{array}$ $\begin{array}{llllllll}104 & 147 & 251 & -0.9 & -7.9-1842.23 & -3.12 & 0.91 & -4.87-1849.31\end{array}$ $\begin{array}{lllllllll}104 & 148 & 252 & -1.1 & -7.8-1850.06 & -3.34 & 0.00 & -4.80-1858.21\end{array}$ $\begin{array}{lllllllll}104 & 149 & 253 & -1.3 & -7.6-1857.77 & -3.83 & 0.91 & -4.74-1865.43\end{array}$ $\begin{array}{lllllllll}104 & 150 & 254 & -1.5 & -7.5-1865.34 & -4.12 & 0.00 & -4.67-1874.13\end{array}$ $\begin{array}{lllllllll}104 & 151 & 255 & -1.7 & -7.4-1872.78 & -4.70 & 0.90 & -4.61-1881.19\end{array}$ $\begin{array}{lllllllll}104 & 152 & 256 & -1.9 & -7.3-1880.10 & -4.76 & 0.00 & -4.55-1889.41\end{array}$ $\begin{array}{lllllllll}104 & 153 & 257 & -2.1 & -7.1-1887.30 & -4.68 & 0.90 & -4.49-1895.57\end{array}$ $\begin{array}{llllllll}104 & 154 & 258 & -2.3 & -7.0-1894.37 & -4.49 & 0.00 & -4.43-1903.29\end{array}$ $\begin{array}{llllllll}104 & 155 & 259 & -2.5 & -6.9-1901.32 & -4.49 & 0.89 & -4.37-1909.29\end{array}$ $104156260-2.6-6.8-1908.15-4.120 .00-4.32-1916.59$ $\begin{array}{lllllllll}104 & 157 & 261 & -2.8 & -6.6-1914.86 & -4.34 & 0.89 & -4.26-1922.57 & 101.76\end{array}$ $\begin{array}{lllllllll}104 & 158 & 262 & -3.0 & -6.5-1921.45 & -4.26 & 0.00 & -4.21-1929.92 & 102.48\end{array}$ $\begin{array}{llllllllll}104 & 159 & 263 & -3.2 & -6.4-1927.93 & -4.60 & 0.89 & -4.15-1935.80 & 104.67\end{array}$ $\begin{array}{llllllllll}104 & 160 & 264 & -3.4 & -6.3-1934.29 & -4.57 & 0.00 & -4.10-1942.96 & 105.58\end{array}$ $\begin{array}{llllllllll}104 & 161 & 265 & -3.6 & -6.2-1940.54 & -4.92 & 0.88 & -4.05-1948.63 & 107.99\end{array}$ $\begin{array}{lllllllllll}104 & 162 & 266 & -3.8 & -6.1-1946.67 & -5.04 & 0.00 & -4.00-1955.71 & 108.97\end{array}$ $\begin{array}{llllllllll}104 & 163 & 267 & -3.9 & -6.0-1952.70 & -5.08 & 0.88 & -3.95-1960.85 & 111.90\end{array}$ $\begin{array}{lllllllllll}104 & 164 & 268 & -4.1 & -5.9-1958.61 & -4.61 & 0.00 & -3.91-1967.13 & 113.70\end{array}$ $\begin{array}{llllllllll}104 & 165 & 269 & -4.3 & -5.7-1964.42 & -4.28 & 0.88 & -3.86-1971.68 & 117.22\end{array}$ $\begin{array}{llllllllll}104 & 166 & 270 & -4.5 & -5.6-1970.12 & -3.75 & 0.00 & -3.81-1977.68 & 119.29\end{array}$ $\begin{array}{llllllllll}104 & 167 & 271 & -4.7 & -5.5-1975.71 & -3.39 & 0.87 & -3.77-1981.99 & 123.05\end{array}$ $\begin{array}{llllllllll}104 & 168 & 272 & -4.8 & -5.4-1981.20 & -2.75 & 0.00 & -3.72-1987.67 & 125.44\end{array}$ $\begin{array}{llllllllll}104 & 169 & 273 & -5.0 & -5.3-1986.58 & -2.90 & 0.87 & -3.68-1992.29 & 128.89\end{array}$ $\begin{array}{llllllllll}104 & 170 & 274 & -5.2 & -5.2-1991.86 & -2.63 & 0.00 & -3.64-1998.13 & 131.13\end{array}$ $\begin{array}{llllllllll}104 & 171 & 275 & -5.3 & -5.1-1997.04 & -2.41 & 0.86 & -3.59-2002.18 & 135.14\end{array}$ $\begin{array}{llllllllll}104 & 172 & 276 & -5.5 & -5.0-2002.12 & -2.37 & 0.00 & -3.55-2008.04 & 137.35\end{array}$ $\begin{array}{llllllllll}104 & 173 & 277 & -5.7 & -4.9-2007.10 & -2.63 & 0.86 & -3.51-2012.38 & 141.08\end{array}$ $\begin{array}{lllllllll}104 & 174 & 278 & -5.9 & -4.8-2011.99 & -2.59 & 0.00 & -3.47-2018.05 & 143.49\end{array}$ $\begin{array}{llllllllll}104 & 175 & 279 & -6.0 & -4.7-2016.77 & -2.91 & 0.86 & -3.43-2022.26 & 147.35\end{array}$ $\begin{array}{llllllllll}104 & 176 & 280 & -6.2 & -4.6-2021.46 & -2.64 & 0.00 & -3.40-2027.50 & 150.18\end{array}$ $\begin{array}{llllllllll}104 & 177 & 281 & -6.4 & -4.5-2026.06 & -2.65 & 0.85 & -3.36-2031.21 & 154.54\end{array}$ $\begin{array}{lllllllll}104 & 178 & 282 & -6.5 & -4.5-2030.56 & -2.52 & 0.00 & -3.32-2036.40 & 157.42\end{array}$ $\begin{array}{llllllllll}104 & 179 & 283 & -6.7 & -4.4-2034.97 & -2.70 & 0.85 & -3.29-2040.10 & 161.79\end{array}$ $104 \quad 180284-6.9-4.3-2039.29-2.40 \quad 0.00-3.25-2044.94 \quad 165.03$ $\begin{array}{lllllllllll}104 & 181 & 285 & -7.0 & -4.2-2043.52 & -2.59 & 0.85 & -3.22-2048.47 & 169.57\end{array}$ $\begin{array}{llllllllll}104 & 182 & 286 & -7.2 & -4.1-2047.65 & -2.25 & 0.00 & -3.18-2053.08 & 173.03\end{array}$ $\begin{array}{llllllllll}104 & 183 & 287 & -7.4 & -4.0-2051.70 & -2.28 & 0.85 & -3.15-2056.28 & 177.90\end{array}$ $\begin{array}{lllllllll}104 & 184 & 288 & -7.5 & -3.9-2055.66 & -1.75 & 0.00 & -3.11-2060.53 & 181.73\end{array}$ $\begin{array}{llllllllll}104 & 185 & 289 & -7.7 & -3.8-2059.54 & -1.38 & 0.84 & -3.08-2063.16 & 187.17\end{array}$ $\begin{array}{lllllllll}104 & 186 & 290 & -7.8 & -3.7-2063.33 & -0.19 & 0.00 & -3.05-2066.57 & 191.83\end{array}$ $\begin{array}{lllllllll}104 & 187 & 291 & -8.0 & -3.7-2067.03 & 0.89 & 0.84 & -3.02-2068.32 & 198.15\end{array}$ $104 \quad 188 \quad 292-8.1-3.6-2070.66$ $104189293-8.3-3.5-2074.19$ $0.090 .00-2.99-2073.55200 .99$ $0.320 .84-2.96-2075.99206 .62$

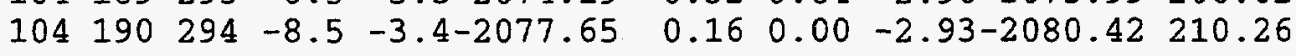
$\begin{array}{lllllllll}104 & 191 & 295 & -8.6 & -3.3-2081.03 & -0.18 & 0.83 & -2.90-2083.27 & 215.48\end{array}$ $\begin{array}{llllllllll}104 & 192 & 296 & -8.8 & -3.3-2084.32 & -0.22 & 0.00 & -2.87-2087.41 & 219.42\end{array}$ $\begin{array}{llllllllll}104 & 193 & 297 & -8.9 & -3.2-2087.54 & -0.52 & 0.83 & -2.84-2090.07 & 224.83\end{array}$ $\begin{array}{lllllllll}104 & 194 & 298 & -9.1 & -3.1-2090.68 & -0.59 & 0.00 & -2.81-2094.08 & 228.89\end{array}$ $\begin{array}{llllllllll}104 & 195 & 299 & -9.2 & -3.0-2093.74 & -0.92 & 0.83 & -2.79-2096.61 & 234.43\end{array}$ $\begin{array}{llllllllll}104 & 196 & 300 & -9.4 & -2.9-2096.72 & -0.98 & 0.00 & -2.76-2100.46 & 238.65\end{array}$ $\begin{array}{lllllllll}104 & 197 & 301 & -9.5 & -2.9-2099.63 & -1.46 & 0.82 & -2.73-2103.00 & 244.19\end{array}$ $\begin{array}{lllllllll}104 & 198 & 302 & -9.7 & -2.8-2102.46 & -1.59 & 0.00 & -2.71-2106.76 & 248.50\end{array}$ $\begin{array}{llllllllll}104 & 199 & 303 & -9.8 & -2.7-2105.22 & -2.08 & 0.82 & -2.68-2109.16 & 254.17\end{array}$ $\begin{array}{llllllllll}104 & 200 & 304 & -9.9 & -2.6-2107.91 & -2.35 & 0.00 & -2.65-2112.91 & 258.48\end{array}$ $104201305-10.1-2.6-2110.52-2.790 .82-2.63-2115.12 \quad 264.35$ $104202306-10.2-2.5-2113.06-2.88 \quad 0.00-2.61-2118.55 \quad 268.99$ $104203 \quad 307-10.4 \quad-2.4-2115.53-3.17 \quad 0.82-2.58-2120.47 \quad 275.14$ $104204308-10.5-2.4-2117.94-2.97 \quad 0.00-2.56-2123.46 \quad 280.22$ $104205 \quad 309-10.7-2.3-2120.27-3.15 \quad 0.81-2.53-2125.14 \quad 286.62$ $104206 \quad 310-10.8 \quad-2.2-2122.53-2.95 \quad 0.00-2.51-2127.99291 .84$ $104207311-10.9-2.2-2124.72 \quad-3.10 \quad 0.81-2.49-2129.50 \quad 298.40$

0.00

0.00

0.00

0.00

0.00

0.00

0.00

0.00 .

0.00

0.00

0.00

0.00

0.00

0.00

0.00

0.00

0.00

0.00

0.00

94.24

0.00

0.00

0.00

0.00

0.00

98.28

$-5.10$

0.00

0.00

0.00

0.00

0.00

0.00

0.00

0.00

0.00

0.00

0.00

0.00

0.00

0.00

0.00

0.00

0.00

0.00

0.00

0.00

0.00

0.00

0.00

0.00

0.00

0.00

0.00

0.00

0.00

0.00

0.00

0.00

0.00

0.00

0.00

0.00

0.00

0.00

0.00

0.00

0.00

0.00

0.00

0.00

0.00

0.00

0.00

0.00

0.00

0.00

0.00

0.00

0.00

0.00

0.00

0.00

0.00

0.00

0.00

0.00

0.00

0.00

0.00

0.00

0.00

0.00

0.00

0.00

0.00

0.00

0.00

0.00

0.00

0.00

0.00

0.00

0.00

0.00

0.00

0.00

0.00

0.00

0.00

0.00

0.00

0.00

0.00

0.00

0.00

0.00

0.00

0.00

0.00

0.00

0.00

0.00

0.00

0.00

0.00

0.00
0.00

0.00

0.00

0.00

0.00

0.00

0.00

0.00

0.00

0.00

$-0.31$

0.00

0.00

$-0.61$

0.00

0.00

0.00

0.00

0.00

0.00

0.00

0.00

0.00

0.00

0.00

0.00

0.00

0.00

0.00

0.00

0.00

0.00

0.00

0.00

0.00

0.00

0.00

0.00

0.00

0.00

0.00

0.00

0.00

0.00

0.00

0.00

0.00

0.00

0.00

0.00

0.00

0.00

0.00

0.00

0.00

0.00

0.00

0.00

0.00

0.00

0.00

0.00

0.00

0.00

0.00

0.00 
$104208 \quad 312-11.1 \quad-2.1-2126.85 \quad-2.93 \quad 0.00 \quad-2.47-2132.25 \quad 303.72$ $\begin{array}{llllllll}104 & 209 & 313-11.2 & -2.0-2128.91 & -3.15 & 0.81 & -2.44-2133.70 & 310.34\end{array}$ $\begin{array}{llllllll}104 & 210 & 314-11.4 & -2.0-2130.91 & -3.05 & 0.00 & -2.42-2136.38 & 315.73\end{array}$ $\begin{array}{llllllll}104 & 211 & 315-11.5 & -1.9-2132.84 & -3.33 & 0.81 & -2.40-2137.77 & 322.42\end{array}$ $104212 \quad 316-11.6 \quad-1.8-2134.71-3.22 \quad 0.00 \quad-2.38-2140.31 \quad 327.95$ $104 \quad 213 \quad 317-11.8 \quad-1.8-2136.51 \quad-3.64 \quad 0.80 \quad-2.36-2141.71 \quad 334.62$ $\begin{array}{llllllll}104 & 214 & 318-11.9 & -1.7-2138.25 & -3.49 & 0.00 & -2.34-2144.08 & 340.32\end{array}$ $104215 \quad 319-12.0 \quad-1.6-2139.93 \quad-3.96 \quad 0.80 \quad-2.32-2145.41 \quad 347.06$ $\begin{array}{llllllll}104 & 216 & 320-12.2 & -1.6-2141.55 & -3.89 & 0.00 & -2.30-2147.74 & 352.80\end{array}$ $\begin{array}{llllllll}104 & 217 & 321-12.3 & -1.5-2143.11 & -4.43 & 0.80 & -2.28-2149.02 & 359.60\end{array}$ $\begin{array}{lllllllll}104 & 218 & 322-12.4 & -1.5-2144.60 & -4.54 & 0.00 & -2.26-2151.40 & 365.28\end{array}$ $\begin{array}{llllllll}104 & 219 & 323-12.5 & -1.4-2146.04 & -5.10 & 0.80 & -2.24-2152.59 & 372.17\end{array}$ $\begin{array}{lllllllll}104 & 220 & 324-12.7 & -1.3-2147.42 & -5.15 & 0.00 & -2.22-2154.79 & 378.03\end{array}$ $\begin{array}{llllllll}104 & 221 & 325-12.8 & -1.3-2148.74 & -5.52 & 0.79 & -2.20-2155.67 & 385.23\end{array}$ $\begin{array}{llllllll}104 & 222 & 326-12.9 & -1.2-2150.00 & -5.28 & 0.00 & -2.19-2157.47 & 391.50\end{array}$ $\begin{array}{llllllll}104 & 223 & 327-13.0 & -1.2-2151.21 & -5.48 & 0.79 & -2.17-2158.07 & 398.97\end{array}$ $\begin{array}{llllllll}104 & 224 & 328-13.2 & -1.1-2152.36 & -5.16 & 0.00 & -2.15-2159.67 & 405.44\end{array}$ $\begin{array}{llllllll}104 & 225 & 329-13.3 & -1.1-2153.46 & -5.35 & 0.79 & -2.13-2160.15 & 413.03\end{array}$ $\begin{array}{llllllll}104 & 226 & 330-13.4 & -1.0-2154.50 & -5.12 & 0.00 & -2.12-2161.73 & 419.52\end{array}$ $\begin{array}{llllllll}104 & 227 & 331-13.5 & -1.0-2155.49 & -2.76 & 0.79 & -2.10-2159.56 & 429.77\end{array}$

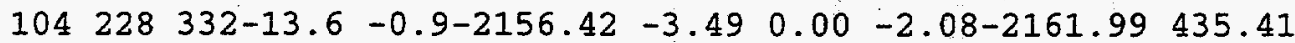
$\begin{array}{llllllll}104 & 229 & 333-13.8 & -0.8-2157.30 & -3.87 & 0.78 & -2.07-2162.45 & 443.02\end{array}$ $\begin{array}{llllllll}104 & 230 & 334-13.9 & -0.8-2158.13 & -3.83 & 0.00 & -2.05-2164.01 & 449.53\end{array}$ $\begin{array}{llllllll}104 & 231 & 335-14.0 & -0.7-2158.90 & -3.95 & 0.78 & -2.03-2164.10 & 457.51\end{array}$ $\begin{array}{llllllll}104 & 232 & 336-14.1 & -0.7-2159.63 & -3.79 & 0.00 & -2.02-2165.44 & 464.25\end{array}$ $\begin{array}{llllllll}104 & 233 & 337-14.2 & -0.6-2160.30 & -4.12 & 0.78 & -2.00-2165.65 & 472.11\end{array}$ $\begin{array}{llllllll}104 & 234 & 338-14.3 & -0.6-2160.93 & -3.98 & 0.00 & -1.99-2166.90 & 478.93\end{array}$ $\begin{array}{llllllll}104 & 235 & 339-14.5 & -0.5-2161.51 & -4.36 & 0.78 & -1.97-2167.06 & 486.84\end{array}$ $\begin{array}{llllllllll}105 & 136 & 241 & 1.7 & -9.7 & -1745.46 & -0.41 & 1.02 & -5.83-1750.68 & 111.41\end{array}$ $\begin{array}{lllllllll}105 & 137 & 242 & 1.5 & -9.5-1755.05 & -0.64 & 1.78 & -5.74-1759.65 & 110.51\end{array}$ 105138243 105139244 105140245 105141246 $105142 \quad 247$ 105143248 105144249 105145250 105146251 $105147 \quad 252$ $105148253-0$ $\begin{array}{llllllllll}105 & 149 & 254 & -0.9 & -7.8-1858.87 & -4.01 & 1.76 & -4.83 & -1865.95 & 101.07\end{array}$ $\begin{array}{llllllllll}105 & 150 & 255 & -1.1 & -7.7-1866.64 & -4.41 & 1.02 & -4.77-1874.80 & 100.30\end{array}$ $\begin{array}{llllllllll}105 & 151 & 256 & -1.3 & -7.6 & -1874.28 & -4.99 & 1.76 & -4.70-1882.21 & 100.95\end{array}$ $\begin{array}{llllllllll}105 & 152 & 257 & -1.5 & -7.4-1881.79 & -5.10 & 1.02 & -4.64-1890.51 & 100.73\end{array}$ $\begin{array}{llllllllll}105 & 153 & 258 & -1.7 & -7.3-1889.18 & -5.07 & 1.75 & -4.58-1897.07 & 102.24\end{array}$ $\begin{array}{llllllllll}105 & 154 & 259 & -1.9 & -7.2 & -1896.44 & -4.91 & 1.02 & -4.52-1904.85 & 102.53\end{array}$ $\begin{array}{llllllllll}105 & 155 & 260 & -2.1 & -7.1-1903.58 & -4.98 & 1.75 & -4.46-1911.27 & 104.18\end{array}$

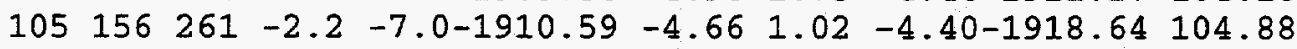
$\begin{array}{llllllllll}105 & 157 & 262 & -2.4 & -6.8 & -1917.49 & -4.89 & 1.75 & -4.34-1924.98 & 106.61\end{array}$

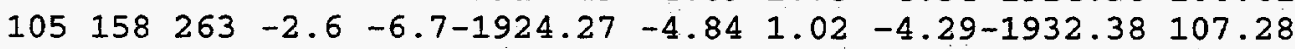
$\begin{array}{llllllllll}105 & 159 & 264 & -2.8 & -6.6 & -1930.93 & -5.21 & 1.74 & -4.24-1938.63 & 109.10\end{array}$ $\begin{array}{lllllllll}105 & 160 & 265 & -3.0 & -6.5-1937.48 & -5.32 & 1.02 & -4.18-1945.96 & 109.84\end{array}$ $\begin{array}{lllllllll}105 & 161 & 266 & -3.2 & -6.4-1943.91 & -5.57 & 1.74 & -4.13-1951.87 & 112.01\end{array}$ $\begin{array}{lllllllll}105 & 162 & 267 & -3.3 & -6.3-1950.23 & -5.75 & 1.02 & -4.08-1959.04 & 112.91\end{array}$ $\begin{array}{llllllllll}105 & 163 & 268 & -3.5 & -6.1-1956.43 & -5.79 & 1.74 & -4.03-1964.51 & 115.51\end{array}$ $\begin{array}{lllllllll}105 & 164 & 269 & -3.7 & -6.0-1962.53 & -5.28 & 1.02 & -3.98-1970.77 & 117.32\end{array}$ $\begin{array}{lllllllll}105 & 165 & 270 & -3.9 & -5.9-1968.51 & -4.97 & 1.73 & -3.93-1975.68 & 120.48\end{array}$ $\begin{array}{lllllllll}105 & 166 & 271 & -4.1 & -5.8-1974.39 & -4.40 & 1.02 & -3.89-1981.66 & 122.58\end{array}$ $\begin{array}{lllllllll}105 & 167 & 272 & -4.2 & -5.7-1980.16 & -4.06 & 1.73 & -3.84-1986.33 & 125.98\end{array}$ $\begin{array}{lllllllll}105 & 168 & 273 & -4.4 & -5.6-1985.82 & -3.29 & 1.02 & -3.79-1991.89 & 128.49\end{array}$

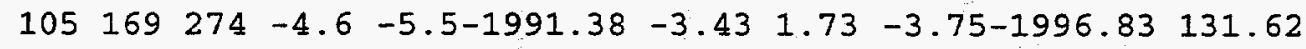
$\begin{array}{lllllllll}105 & 170 & 275 & -4.8 & -5.4-1996.84 & -3.19 & 1.02 & -3.71-2002.72 & 133.80\end{array}$ $\begin{array}{lllllllll}105 & 171 & 276 & -4.9 & -5.3-2002.19 & -3.11 & 1.73 & -3.66-2007.24 & 137.36\end{array}$ $\begin{array}{lllllllll}105 & 172 & 277 & -5.1 & -5.2-2007.44 & -2.89 & 1.02 & -3.62-2012.94 & 139.73\end{array}$ $\begin{array}{lllllllll}105 & 173 & 278 & -5.3 & -5.1-2012.60 & -3.14 & 1.72 & -3.58-2017.59 & 143.14\end{array}$
0.00

0.00

0.00

0.00

0.00

0.00

0.00

0.00

0.00

0.00

0.00

0.00

0.00

0.00

0.00

0.00

0.00

0.00

0.00

0.00

0.00

0.00

0.00

0.00

0.00

0.00

0.00

0.00

0.00

0.00

0.00

0.00

0.00

0.00

0.00

0.00

0.00

0.00

0.00

0.00

0.00

0.00

0.00

0.00

0.00

0.00

0.00

0.00

0.00

0.00

0.00

0.00

0.00

0.00

0.00

0.00

0.00

0.00

0.00

0.00

0.00

0.00

0.00

0.00

0.00

0.00
0.00

0.00

0.00

0.00

0.00

0.00

0.00

0.00

0.00

0.00

0.00

0.00

0.00

0.00

0.00

0.00

0.00

0.00

0.00

0.00

0.00

0.00

0.00

0.00

0.00

0.00

0.00

0.00

0.00

0.00

0.00

0.00

0.00

0.00

0.00

0.00

0.00

0.00

0.00

0.00

0.00

0.00

0.00

0.00

0.00

0.00

0.00

0.00

0.00

0.00

0.00

0.00

0.00

0.00

0.00

0.00

0.00

0.00

0.00

0.00

0.00

0.00

0.00

0.00

0.00

.0 .00
0.00

0.00

0.00

0.00

0.00

0.00

0.00

0.00

0.00

0.00

0.00

0.00

0.00

0.00

0.00

0.00

0.00

0.00

0.00

0.00

0.00

0.00

0.00

0.00

0.00

0.00

0.00

0.00

0.00

0.00

0.00

0.00

0.00

0.00

0.00

0.00

0.00

0.00

0.00

0.00

0.00

0.00

0.00

0.00

0.00

0.00

0.00

0.00

0.00

0.00

0.00

0.00

0.00

0.00

0.00

0.00

0.00

0.00

0.00

0.00

0.00

0.00

0.00

0.00

0.00

0.00 
$\begin{array}{llllllllllll}105 & 174 & 279 & -5.5 & -5.0-2017.65 & -3.11 & 1.02 & -3.54-2023.28 & 145.52\end{array}$ $\begin{array}{llllllllll}105 & 175 & 280 & -5.6 & -4.9-2022.61 & -3.42 & 1.72 & -3.50-2027.80 & 149.07\end{array}$ $\begin{array}{llllllllll}105 & 176 & 281 & -5.8 & -4.8-2027.46 & -3.15 & 1.02 & -3.46-2033.06 & 151.89\end{array}$ $\begin{array}{llllllllll}105 & 177 & 282 & -6.0 & -4.7-2032.23 & -3.00 & 1.72 & -3.42-2036.93 & 156.09\end{array}$ $\begin{array}{llllllllll}105 & 178 & 283 & -6.1 & -4.6-2036.90 & -2.85 & 1.02 & -3.38-2042.11 & 158.98\end{array}$ $\begin{array}{lllllllllll}105 & 179 & 284 & -6.3 & -4.5-2041.47 & -3.07 & 1.72 & -3.35-2046.17 & 162.99\end{array}$ $\begin{array}{llllllllll}105 & 180 & 285 & -6.5 & -4.4-2045.95 & -2.89 & 1.02 & -3.31-2051.14 & 166.10\end{array}$ $\begin{array}{lllllllllll}105 & 181 & 286 & -6.6 & -4.3-2050.34 & -2.86 & 1.71 & -3.28-2054.77 & 170.54\end{array}$ $\begin{array}{llllllllll}105 & 182 & 287 & -6.8 & -4.3-2054.65 & -2.51 & 1.02 & -3.24-2059.38 & 174.00\end{array}$ $\begin{array}{llllllllll}105 & 183 & 288 & -6.9 & -4.2-2058.86 & -2.56 & 1.71 & -3.21-2062.91 & 178.54\end{array}$ $\begin{array}{lllllllllll}105 & 184 & 289 & -7.1 & -4.1-2062.98 & -2.03 & 1.02 & -3.17-2067.16 & 182.36\end{array}$ $\begin{array}{llllllllll}105 & 185 & 290 & -7.3 & -4.0-2067.01 & -0.96 & 1.71 & -3.14-2069.40 & 188.19\end{array}$ $\begin{array}{llllllllll}105 & 186 & 291 & -7.4 & -3.9-2070.96 & -0.42 & 1.02 & -3.11-2073.47 & 192.19\end{array}$ $105187292-7.6-3.8-2074.82-0.881 .71-3.07-2077.07 \quad 196.66$ $\begin{array}{lllllllllll}105 & 188 & 293 & -7.7 & -3.7-2078.60 & -0.27 & 1.02 & -3.04-2080.90 & 200.91\end{array}$ $105189294-7.9-3.6-2082.30 \quad 0.19 \quad 1.70-3.01-2083.42 \quad 206.46$ $\begin{array}{llllllllll}105 & 190 & 295 & -8.1 & -3.6-2085.91 & 0.02 & 1.02 & -2.98-2087.85 & 210.10\end{array}$

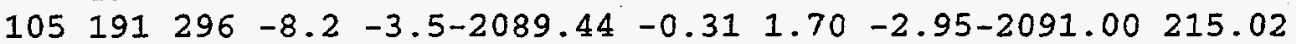
$\begin{array}{llllllllll}105 & 192 & 297 & -8.4 & -3.4-2092.89 & -0.37 & 1.02 & -2.92-2095.16 & 218.93\end{array}$ $\begin{array}{llllllllll}105 & 193 & 298 & -8.5 & -3.3-2096.26 & -0.70 & 1.70 & -2.89-2098.15 & 224.02\end{array}$ $\begin{array}{llllllllll}105 & 194 & 299 & -8.7 & -3.2-2099.54 & -0.75 & 1.02 & -2.86-2102.14 & 228.09\end{array}$ $\begin{array}{llllllllll}105 & 195 & 300 & -8.8 & -3.2 & -2102.76 & -1.09 & 1.70 & -2.84-2104.98 & 233.32\end{array}$ $\begin{array}{lllllllllll}105 & 196 & 301 & -9.0 & -3.1-2105.89 & -1.20 & 1.02 & -2.81-2108.88 & 237.50\end{array}$ $\begin{array}{llllllllll}105 & 197 & 302 & -9.1 & -3.0-2108.95 & -1.66 & 1.70 & -2.78-2111.69 & 242.76\end{array}$ $105198303-9.3-2.9-2111.93-1.841 .02-2.76-2115.50247 .02$ $\begin{array}{llllllllll}105 & 199 & 304 & -9.4 & -2.9-2114.83 & -2.30 & 1.69 & -2.73-2118.17 & 252.43\end{array}$ $\begin{array}{lllllllllll}105 & 200 & 305 & -9.6 & -2.8 & -2117.66 & -2.56 & 1.02 & -2.70-2121.91 & 256.76\end{array}$ $\begin{array}{llllllllll}105 & 201 & 306 & -9.7 & -2.7-2120.42 & -3.04 & 1.69 & -2.68-2124.45 & 262.29\end{array}$ $105202307-9.8-2.6-2123.11-3.06 \quad 1.02-2.65-2127.80267 .00$ $105203 \quad 308-10.0-2.6-2125.72-3.32 \quad 1.69-2.63-2129.98 \quad 272.90$ $105204309-10.1-2.5-2128.27-3.241 .02-2.60-2133.09277 .86$ $105205 \quad 310-10.3-2.4-2130.74-3.43 \quad 1.69-2.58-2135.06 \quad 283.96$ $105206 \quad 311-10.4-2.4-2133.14-3.201 .02-2.56-2137.88 \quad 289.21$ $105207312-10.6-2.3-2135.47-3.38 \quad 1.69-2.53-2139.70 \quad 295.46$ $105208 \quad 313-10.7-2.2-2137.74 \quad-3.26 \quad 1.02 \quad-2.51-2142.49 \quad 300.74$ $105209314-10.8-2.2-2139.94-3.53 \quad 1.68-2.49-2144.27 \quad 307.03$ $105210 \quad 315-11.0 \quad-2.1-2142.07-3.48 \quad 1.02-2.47-2147.00 \quad 312.38$ $105211316-11.1-2.0-2144.14-3.851 .68-2.44-2148.75318 .70$ $105212317-11.2-2.0-2146.14-3.72 \quad 1.02-2.42-2151.27 \quad 324.25$ $105213 \quad 318-11.4-1.9-2148.08-4.11 \quad 1.68-2.40-2152.91330 .68$ $\begin{array}{lllllllll}105 & 214 & 319-11.5 & -1.8-2149.95 & -4.16 & 1.02 & -2.38-2155.48 & 336.19\end{array}$ $105215320-11.6-1.8-2151.76-4.56 \quad 1.68-2.36-2157.01342 .73$ $105216 \quad 321-11.8-1.7-2153.51-4.64 \quad 1.02-2.34-2159.47 \quad 348.33$ $\begin{array}{lllllllll}105 & 217 & 322-11.9 & -1.7-2155.20 & -5.05 & 1.68 & -2.32-2160.89 & 354.99\end{array}$ $\begin{array}{lllllllll}105 & 218 & 323-12.0 & -1.6-2156.82 & -4.92 & 1.02 & -2.30-2163.03 & 360.92\end{array}$ $\begin{array}{lllllllll}105 & 219 & 324-12.2 & -1.5-2158.39 & -5.47 & 1.67 & -2.28-2164.47 & 367.55\end{array}$ $\begin{array}{llllllll}105 & 220 & 325-12.3 & -1.5-2159.90 & -5.54 & 1.02 & -2.26-2166.68 & 373.41\end{array}$ $\begin{array}{lllllllll}105 & 221 & 326-12.4 & -1.4-2161.35 & -5.92 & 1.67 & -2.24-2167.84 & 380.33\end{array}$ $\begin{array}{llllllllll}105 & 222 & 327-12.5 & -1.4-2162.74 & -5.70 & 1.02 & -2.23-2169.64 & 386.59\end{array}$ $\begin{array}{llllllllll}105 & 223 & 328-12.7 & -1.3-2164.07 & -5.84 & 1.67 & -2.21-2170.44 & 393.86\end{array}$ $\begin{array}{lllllllll}105 & 224 & 329-12.8 & -1.2-2165.34 & -5.64 & 1.02 & -2.19-2172.15 & 400.23\end{array}$ $\begin{array}{lllllllll}105 & 225 & 330-12.9 & -1.2-2166.56 & -5.85 & 1.67 & -2.17-2172.91 & 407.54\end{array}$ $105226331-13.0-1.1-2167.72-5.56 \quad 1.02-2.15-2174.42414 .10$ $\begin{array}{lllllllll}105 & 227 & 332-13.2 & -1.1-2168.83 & -3.26 & 1.67 & -2.14-2172.56 & 424.03\end{array}$ $\begin{array}{lllllllll}105 & 228 & 333-13.3 & -1.0-2169.89 & -3.92 & 1.02 & -2.12-2174.91 & 429.76\end{array}$ $\begin{array}{lllllllll}105 & 229 & 334-13.4 & -1.0-2170.88 & -4.30 & 1.66 & -2.10-2175.62 & 437.11\end{array}$ $\begin{array}{lllllllll}105 & 230 & 335-13.5 & -0.9-2171.83 & -4.23 & 1.02 & -2.09-2177.13 & 443.68\end{array}$ $\begin{array}{lllllllll}105 & 231 & 336-13.6 & -0.9-2172.73 & -4.35 & 1.66 & -2.07-2177.48 & 451.40\end{array}$ $\begin{array}{lllllllll}105 & 232 & 337-13.8 & -0.8-2173.57 & -4.16 & 1.02 & -2.05-2178.76 & 458.19\end{array}$ $\begin{array}{lllllllll}105 & 233 & 338-13.9 & -0.8-2174.36 & -4.51 & 1.66 & -2.04-2179.25 & 465.78\end{array}$ $105 \quad 234 \quad 339-14.0-0.7-2175.10-4.36 \quad 1.02-2.02-2180.46 \quad 472.63$ $\begin{array}{llllllllll}106 & 138 & 244 & 1.7 & -9.6-1762.98 & -0.57 & 0.00 & -5.76-1769.31 & 116.19\end{array}$ $\begin{array}{lllllllll}106 & 139 & 245 & 1.5 & -9.4-1772.47 & -0.53 & 0.93 & -5.68-1777.75 & 115.82\end{array}$ $\begin{array}{llllllllll}106 & 140 & 246 & 1.3 & -9.3-1781.82 & -0.67 & 0.00 & -5.60-1788.08 & 113.56\end{array}$ $\begin{array}{llllllllll}106 & 141 & 247 & 1.1 & -9.1-1791.02 & -1.01 & 0.92 & -5.51-1796.62 & 113.10\end{array}$ 106142248

$0.9-9.0-1800.07-1.22 \quad 0.00-5.44-1806.73 \quad 111.06$

0.00

0.00

0.00

0.00

0.00

0.00

0.00

0.00

0.00

0.00

0.00

0.00

0.00

0.00

0.00

0.00

0.00

0.00

0.00

0.00

0.00

0.00

0.00

0.00

0.00

0.00

0.00

0.00

0.00

0.00

0.00

0.00

0.00

0.00

0.00

0.00

0.00

0.00

0.00

0.00

0.00

0.00

0.00

0.00

0.00

0.00

0.00

0.00

0.00

0.00

0.00

0.00

0.00

0.00

0.00

0.00

0.00

0.00

0.00

0.00

0.00

0.00

0.00

0.00

0.00

0.00

0.00

0.00

0.00

0.00

0.00

0.00

0.00

0.00

0.00

0.00

0.00

0.00

0.00

0.00

0.00

0.00

0.00

0.00

0.00

0.00

0.00

0.00

0.00

0.00

0.00

0.00

0.00

0.00

0.00

0.00

0.00

0.00

0.00

0.00

0.00

0.00

0.00

0.00

0.00

0.00

0.00

0.00

0.00

0.00

0.00

0.00

0.00

0.00

0.00

0.00

0.00

0.00

0.00

0.00

0.00

0.00

0.00

0.00

0.00

0.00

0.00

0.00

0.00

0.00
0.00

0.00

0.00

0.00

0.00

0.00

0.00

0.00

0.00

0.00

0.00

0.00

0.00

0.00

0.00

0.00

0.00

0.00

0.00

0.00

0.00

0.00

0.00

0.00

0.00

0.00

0.00

0.00

0.00

0.00

0.00

0.00

0.00

0.00

0.00

0.00

0.00

0.00

0.00

0.00

0.00

0.00

0.00

0.00

0.00

0.00

0.00

0.00

0.00

0.00

0.00

0.00

0.00

0.00

0.00

0.00

0.00

0.00

0.00

0.00

0.00

0.00

0.00

0.00

0.00

0.00 
106143249

106144250

106145251

$106146 \quad 252$

106147253

$\begin{array}{lll}106 & 148 & 254\end{array}$

$\begin{array}{llllll}-0.5 & -8.0-1859.57 & -3.90 & 0.91 & -4.93-1867.49 & 106.80\end{array}$

$\begin{array}{llllllllll}106 & 150 & 256 & -0.7 & -7.9-1867.53 & -4.35 & 0.00 & -4.86-1876.74 & 105.62\end{array}$

$\begin{array}{llllllllll}106 & 151 & 257 & -0.9 & -7.8-1875.37 & -4.83 & 0.90 & -4.79-1884.09 & 106.34\end{array}$

$\begin{array}{llllllllll}106 & 152 & 258 & -1.1 & -7.6 & -1883.07 & -4.95 & 0.00 & -4.73-1892.75 & 105.75\end{array}$

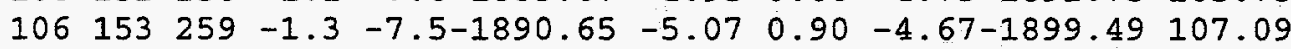

$\begin{array}{lllllllll}106 & 154 & 260 & -1.5 & -7.4-1898.10 & -4.85 & 0.00 & -4.61-1907.56 & 107.09\end{array}$

$\begin{array}{llllllllll}106 & 155 & 261 & -1.6 & -7.3-1905.43 & -4.93 & 0.89 & -4.55-1914.01 & 108.71\end{array}$

$\begin{array}{lllllllll}106 & 156 & 262 & -1.8 & -7.1-1912.64 & -4.63 & 0.00 & -4.49-1921.75 & 109.04\end{array}$

$\begin{array}{llllllllll}106 & 157 & 263 & -2.0 & -7.0 & -1919.72 & -4.90 & 0.89 & -4.43-1928.16 & 110.70\end{array}$

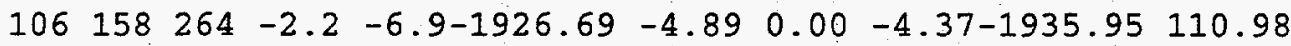

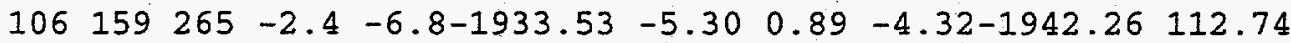

$\begin{array}{llllllllll}106 & 160 & 266 & -2.6 & -6.7-1940.26 & -5.44 & 0.00 & -4.26-1949.97 & 113.11\end{array}$

$\begin{array}{lllllllll}106 & 161 & 267 & -2.8 & -6.6-1946.88 & -5.81 & 0.88 & -4.21-1956.02 & 115.13\end{array}$

$\begin{array}{lllllllll}106 & 162 & 268 & -2.9 & -6.4-1953.38 & -5.91 & 0.00 & -4.16-1963.45 & 115.77\end{array}$

$\begin{array}{lllllllll}106 & 163 & 269 & -3.1 & -6.3-1959.76 & -5.97 & 0.88 & -4.11-1968.96 & 118.33\end{array}$

$\begin{array}{llllllllll}106 & 164 & 270 & -3.3 & -6.2-1966.04 & -5.52 & 0.00 & -4.06-1975.62 & 119.74\end{array}$

$\begin{array}{lllllllll}106 & 165 & 271 & -3.5 & -6.1-1972.21 & -5.18 & 0.88 & -4.01-1980.52 & 122.91\end{array}$

$\begin{array}{lllllllll}106 & 166 & 272 & -3.7 & -6.0-1978.26 & -4.59 & 0.00 & -3.96-1986.81 & 124.69\end{array}$

$\begin{array}{lllllllll}106 & 167 & 273 & -3.8 & -5.9-1984.21 & -4.23 & 0.87 & -3.91-1991.48 & 128.10\end{array}$

$\begin{array}{lllllllll}106 & 168 & 274 & -4.0 & -5.8-1990.05 & -3.52 & 0.00 & -3.87-1997.44 & 130.21\end{array}$

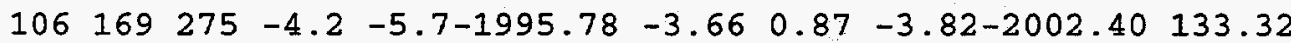

$\begin{array}{lllllllll}106 & 170 & 276 & -4.4 & -5.6-2001.41 & -3.35 & 0.00 & -3.78-2008.54 & 135.25\end{array}$

$\begin{array}{lllllllll}106 & 171 & 277 & -4.5 & -5.5-2006.94 & -3.11 & 0.86 & -3.73-2012.92 & 138.94\end{array}$

$\begin{array}{lllllllll}106 & 172 & 278 & -4.7 & -5.4-2012.37 & -3.13 & 0.00 & -3.69-2019.18 & 140.75\end{array}$

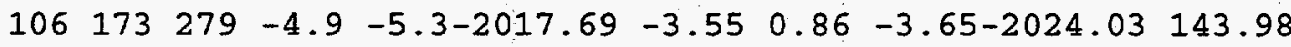

$\begin{array}{llllllllll}106 & 174 & 280 & -5.1 & -5.2 & -2022.91 & -3.56 & 0.00 & -3.61-2030.08 & 146.00\end{array}$

$\begin{array}{lllllllll}106 & 175 & 281 & -5.2 & -5.1-2028.04 & -3.92 & 0.86 & -3.57-2034.67 & 149.48\end{array}$

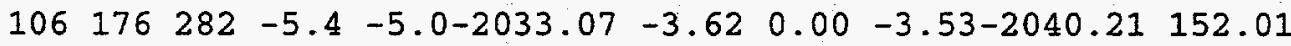

$\begin{array}{lllllllll}106 & 177 & 283 & -5.6 & -4.9-2038.00 & -3.46 & 0.85 & -3.49-2044.09 & 156.20\end{array}$

$\begin{array}{lllllllll}106 & 178 & 284 & -5.7 & -4.8-2042.83 & -3.41 & 0.00 & -3.45-2049.69 & 158.67\end{array}$

$\begin{array}{lllllllll}106 & 179 & 285 & -5.9 & -4.7-2047.57 & -3.63 & 0.85 & -3.41-2053.76 & 162.67\end{array}$

$\begin{array}{lllllllll}106 & 180 & 286 & -6.1 & -4.6-2052.22 & -3.31 & 0.00 & -3.37-2058.90 & 165.60\end{array}$

$\begin{array}{llllllllll}106 & 181 & 287 & -6.2 & -4.5 & -2056.77 & -3.47 & 0.85 & -3.34-2062.73 & 169.84\end{array}$

$\begin{array}{llllllllll}106 & 182 & 288 & -6.4 & -4.4-2061.24 & -3.10 & 0.00 & -3.30-2067.64 & 173.01\end{array}$

$\begin{array}{lllllllll}106 & 183 & 289 & -6.6 & -4.3-2065.61 & -3.15 & 0.85 & -3.27-2071.18 & 177.54\end{array}$

$\begin{array}{lllllllll}106 & 184 & 290 & -6.7 & -4.2-2069.89 & -2.54 & 0.00 & -3.23-2075.67 & 181.12\end{array}$

$\begin{array}{lllllllll}106 & 185 & 291 & -6.9 & -4.1-2074.09 & -1.52 & 0.84 & -3.20-2077.96 & 186.90\end{array}$

$\begin{array}{lllllllll}106 & 186 & 292 & -7.0 & -4.1-2078.20 & -1.42 & 0.00 & -3.16-2082.78 & 190.15\end{array}$

$\begin{array}{lllllllll}106 & 187 & 293 & -7.2 & -4.0-2082.22 & -1.10 & 0.84 & -3.13-2085.61 & 195.39\end{array}$

$\begin{array}{lllllllll}106 & 188 & 294 & -7.3 & -3.9-2086.15 & -0.43 & 0.00 & -3.10-2089.68 & 199.39\end{array}$

$\begin{array}{lllllllll}106 & 189 & 295 & -7.5 & -3.8-2090.00 & -0.27 & 0.84 & -3.07-2092.50 & 204.64\end{array}$

$\begin{array}{lllllllll}106 & 190 & 296 & -7.7 & -3.7-2093.77 & 0.25 & 0.00 & -3.04-2096.56 & 208.66\end{array}$

$\begin{array}{lllllllll}106 & 191 & 297 & -7.8 & -3.6-2097.45 & -0.02 & 0.83 & -3.01-2099.65 & 213.64\end{array}$

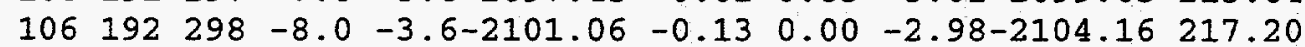

$\begin{array}{lllllllll}106 & 193 & 299 & -8.1 & -3.5-2104.58 & -0.46 & 0.83 & -2.95-2107.15 & 222.28\end{array}$

$\begin{array}{lllllllll}106 & 194 & 300 & -8.3 & -3.4-2108.02 & -0.51 & 0.00 & -2.92-2111.44 & 226.06\end{array}$

$\begin{array}{lllllllll}106 & 195 & 301 & -8.4 & -3.3-2111.38 & -0.89 & 0.83 & -2.89-2114.33 & 231.24\end{array}$

$\begin{array}{lllllllll}106 & 196 & 302 & -8.6 & -3.2-2114.66 & -1.02 & 0.00 & -2.86-2118.54 & 235.10\end{array}$

$\begin{array}{lllllllll}106 & 197 & 303 & -8.7 & -3.2-2117.87 & -1.46 & 0.82 & -2.83-2121.34 & 240.38\end{array}$

$\begin{array}{lllllllll}106 & 198 & 304 & -8.9 & -3.1-2121.00 & -1.66 & 0.00 & -2.81-2125.46 & 244.33\end{array}$

$\begin{array}{lllllllll}106 & 199 & 305 & -9.0 & -3.0-2124.05 & -2.17 & 0.82 & -2.78-2128.18 & 249.68\end{array}$

$\begin{array}{lllllllll}106 & 200 & 306 & -9.2 & -2.9-2127.03 & -2.43 & 0.00 & -2.75-2132.21 & 253.72\end{array}$

$\begin{array}{lllllllll}106 & 201 & 307 & -9.3 & -2.9-2129.93 & -2.86 & 0.82 & -2.73-2134.70 & 259.30\end{array}$

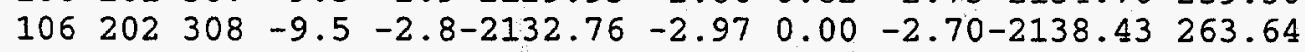

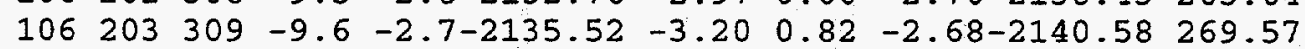
$\begin{array}{lllllllll}106 & 204 & 310 & -9.7 & -2.6-2138.20 & -3.15 & 0.00 & -2.65-2144.00 & 274.21\end{array}$

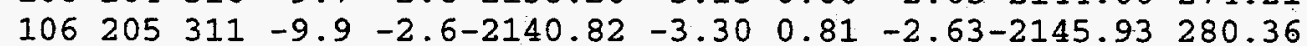

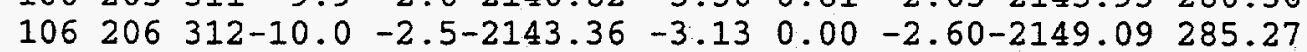
$\begin{array}{llllllll}106 & 207 & 313-10.2 & -2.4-2145.84 & -3.34 & 0.81 & -2.58-2150.94 & 291.49\end{array}$ $\begin{array}{llllllll}106 & 208 & 314-10.3 & -2.4-2148.24 & -3.24 & 0.00 & -2.56-2154.04 & 296.47\end{array}$
0.00

0.00

$0.00 \quad 0.00$

0.00

$0.00 \quad 0.00$

$0.00 \quad 0.00$

$0.00 \quad 0.00$

$0.00 \quad 0.00$

$0.00 \quad 0.00$

$0.00 \quad 0.00$

$0.00 \quad 0.00$

0.00

0.00

0.00

0.00

106.59

0.00

$-5.35$

0.00

0.00

110.10

0.00
-5.50

0.00

0.00

0.00

0.00

0.00

0.00

0.00

0.00

0.00

0.00

0.00

0.00

0.00

0.00

0.00

0.00

0.00

0.00

0.00

0.00

0.00

0.00

0.00

0.00

0.00

0.00

0.00

0.00

0.00

0.00

0.00

0.00

0.00

0.00

0.00

0.00

0.00

0.00

0.00

0.00

0.00

0.00

0.00

0.00

0.00

0.00

0.00

0.00

0.00

0.00

0.00

0.00

0.00

0.00

0.00

0.00

0.00
0.00

0.00

0.00

0.00

0.00

0.00

0.00

0.00

0.00

0.00

0.00

0.00

0.00

0.00

0.00

0.00

0.00

0.00

0.00

0.00

0.00

0.00

0.00

0.00

0.00

0.00

0.00

0.00

0.00

0.00

0.00

0.00

0.00

0.00

0.00

0.00

0.00

0.00

0.00

0.00

0.00

0.00

0.00

0.00
0.00
0.00

0.00

0.00

0.00

0.00

0.00

0.00

0.00

0.00

$-0.50$

0.00

0.00

$-0.60$

0.00

0.00

0.00

0.00

0.00

0.00

0.00

0.00

0.00

0.00

0.00

0.00

0.00

0.00

0.00

0.00

0.00

0.00

0.00

0.00

0.00

0.00

0.00

0.00

0.00

0.00
0.00
0.00

0.00

0.00

0.00

0.00

0.00

0.00

0.00

0.00

0.00

0.00

0.00

0.00

0.00

0.00

0.00

0.00

0.00

0.00

0.00

0.00

0.00

0.00

0.00

0.00
0.00 
$\begin{array}{lllllllll}106 & 209 & 315-10.4 & -2.3-2150.58 & -3.57 & 0.81 & -2.53-2155.87 & 302.70\end{array}$ $\begin{array}{lllllllll}106 & 210 & 316-10.6 & -2.2-2152.85 & -3.56 & 0.00 & -2.51-2158.92 & 307.73\end{array}$ $\begin{array}{llllllll}106 & 211 & 317-10.7 & -2.2-2155.05 & -3.93 & 0.81 & -2.49-2160.66 & 314.05\end{array}$ $\begin{array}{llllllll}106 & 212 & 318-10.9 & -2.1-2157.19 & -3.98 & 0.00 & -2.47-2163.64 & 319.15\end{array}$ $\begin{array}{llllllll}106 & 213 & 319-11.0 & -2.0-2159.26 & -4.31 & 0.80 & -2.44-2165.21 & 325.65\end{array}$

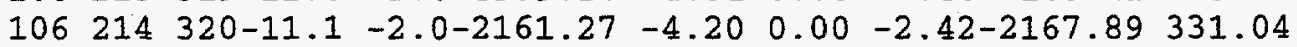

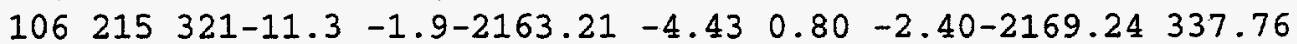
$\begin{array}{lllllllll}106 & 216 & 322 & -11.4 & -1.8-2165.09 & -4.40 & 0.00 & -2.38-2171.87 & 343.20\end{array}$ $\begin{array}{llllllll}106 & 217 & 323-11.5 & -1.8-2166.91 & -4.55 & 0.80 & -2.36-2173.02 & 350.12\end{array}$ $\begin{array}{llllllll}106 & 218 & 324-11.6 & -1.7-2168.67 & -4.89 & 0.00 & -2.34-2175.90 & 355.32\end{array}$ $\begin{array}{llllllll}106 & 219 & 325-11.8 & -1.7-2170.36 & -5.51 & 0.80 & -2.32-2177.40 & 361.89\end{array}$ $\begin{array}{llllllll}106 & 220 & 326-11.9 & -1.6-2172.00 & -5.45 & 0.00 & -2.30-2179.75 & 367.61\end{array}$ $\begin{array}{llllllll}106 & 221 & 327-12.0 & -1.5-2173.57 & -5.82 & 0.79 & -2.28-2180.88 & 374.55\end{array}$ $\begin{array}{llllllll}106 & 222 & 328-12.2 & -1.5-2175.09 & -5.47 & 0.00 & -2.26-2182.82 & 380.68\end{array}$ $\begin{array}{llllllll}106 & 223 & 329-12.3 & -1.4-2176.55 & -5.81 & 0.79 & -2.25-2183.81 & 387.76\end{array}$ $\begin{array}{lllllllll}106 & 224 & 330-12.4 & -1.4-2177.95 & -5.55 & 0.00 & -2.23-2185.72 & 393.92\end{array}$ $\begin{array}{llllllll}106 & 225 & 331-12.5 & -1.3-2179.29 & -5.76 & 0.79 & -2.21-2186.47 & 401.25\end{array}$

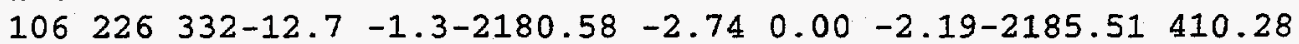
$\begin{array}{llllllll}106 & 227 & 333-12.8 & -1.2-2181.81 & -3.78 & 0.79 & -2.17-2186.97 & 416.89\end{array}$ $\begin{array}{llllllll}106 & 228 & 334-12.9 & -1.1-2182.98 & -4.53 & 0.00 & -2.16-2189.67 & 422.26\end{array}$ $\begin{array}{llllllll}106 & 2.29 & 335-13.0 & -1.1-2184.10 & -4.84 & 0.78 & -2.14-2190.30 & 429.71\end{array}$ $\begin{array}{llllllll}106 & 230 & 336-13.1 & -1.0-2185.17 & -4.77 & 0.00 & -2.12-2192.06 & 436.01\end{array}$ $\begin{array}{llllllll}106 & 231 & 337-13.3 & -1.0-2186.18 & -4.96 & 0.78 & -2.11-2192.47 & 443.68\end{array}$ $\begin{array}{llllllll}106 & 232 & 338-13.4 & -0.9-2187.14 & -4.61 & 0.00 & -2.09-2193.84 & 450.38\end{array}$

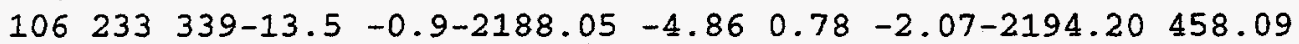

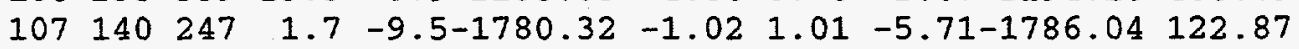

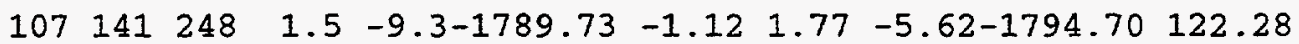

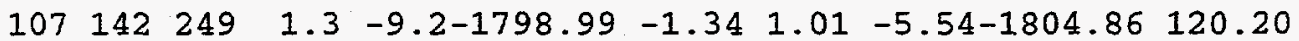
$\begin{array}{lllllllll}107 & 143 & 250 & 1.1 & -9.0-1808.10 & -1.68 & 1.76 & -5.46-1813.48 & 119.65\end{array}$ $\begin{array}{llllllllll}107 & 144 & 251 & 0.9 & -8.9-1817.07 & -2.05 & 1.01 & -5.38-1823.50 & 117.70\end{array}$

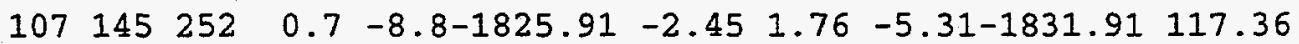

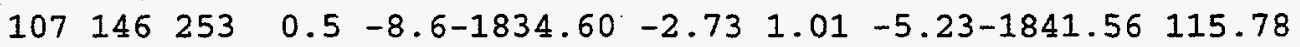

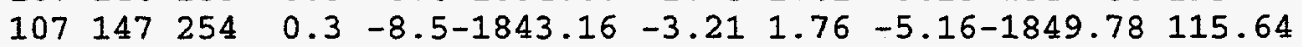
$\begin{array}{lllllllll}107 & 148 & 255 & 0.1 & -8.4-1851.58 & -3.59 & 1.01 & -5.09-1859.25 & 114.23\end{array}$

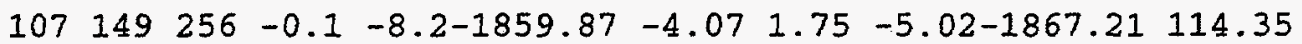
$\begin{array}{lllllllll}107 & 150 & 257 & -0.3 & -8.1-1868.03 & -4.51 & 1.01 & -4.95-1876.48 & 113.15\end{array}$

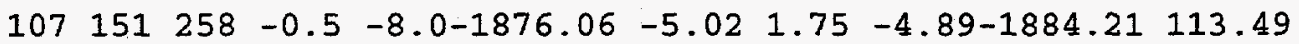
$\begin{array}{lllllllll}107 & 152 & 259 & -0.7 & -7.8-1883.95 & -5.22 & 1.01 & -4.82-1892.98 & 112.79\end{array}$ $\begin{array}{llllllllll}107 & 153 & 260 & -0.9 & -7.7-1891.72 & -5.28 & 1.75 & -4.76-1900.01 & 113.83\end{array}$ $\begin{array}{llllllllll}107 & 154 & 261 & -1.1 & -7.6-1899.36 & -5.19 & 1.01 & -4.69-1908.24 & 113.68\end{array}$ $\begin{array}{llllllllll}107 & 155 & 262 & -1.2 & -7.5 & -1906.88 & -5.31 & 1.74 & -4.63-1915.08 & 114.90\end{array}$ $\begin{array}{lllllllll}107 & 156 & 263 & -1.4 & -7.3-1914.28 & -5.17 & 1.01 & -4.57-1923.01 & 115.05\end{array}$ $\begin{array}{lllllllll}107 & 157 & 264 & -1.6 & -7.2-1921.55 & -5.37 & 1.74 & -4.51-1929.69 & 116.43\end{array}$ $\begin{array}{lllllllll}107 & 158 & 265 & -1.8 & -7.1-1928.70 & -5.40 & 1.01 & -4.46-1937.55 & 116.65\end{array}$ $\begin{array}{llllllllll}107 & 159 & 266 & -2.0 & -7.0-1935.73 & -5.84 & 1.74 & -4.40-1944.24 & 118.03\end{array}$ $\begin{array}{llllllllll}107 & 160 & 267 & -2.2 & -6.9 & -1942.65 & -6.00 & 1.01 & -4.34-1951.98 & 118.36\end{array}$ $\begin{array}{llllllllll}107 & 161 & 268 & -2.4 & -6.7-1949.45 & -6.41 & 1.73 & -4.29-1958.41 & 120.00\end{array}$ $\begin{array}{lllllllll}107 & 162 & 269 & -2.5 & -6.6-1956.13 & -6.55 & 1.01 & -4.24-1965.90 & 120.58\end{array}$ $\begin{array}{llllllllll}107 & 163 & 270 & -2.7 & -6.5-1962.70 & -6.61 & 1.73 & -4.18-1971.76 & 122.79\end{array}$

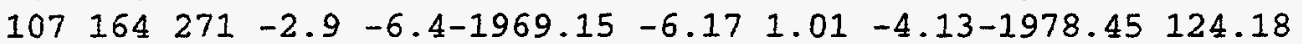
$\begin{array}{llllllllll}107 & 165 & 272 & -3.1 & -6.3-1975.50 & -5.84 & 1.73 & -4.08 & -1983.69 & 127.01\end{array}$ $\begin{array}{llllllllll}107 & 166 & 273 & -3.3 & -6.2-1981.73 & -5.26 & 1.01 & -4.03-1990.01 & 128.76\end{array}$ $\begin{array}{llllllllll}107 & 167 & 274 & -3.4 & -6.1-1987.86 & -4.93 & 1.73 & -3.99-1995.05 & 131.79\end{array}$ $\begin{array}{llllllllll}107 & 168 & 275 & -3.6 & -6.0 & -1993.87 & -4.11 & 1.01 & -3.94-2000.91 & 134.00\end{array}$ $\begin{array}{lllllllll}107 & 169 & 276 & -3.8 & -5.9-1999.78 & -4.23 & 1.72 & -3.89-2006.18 & 136.80\end{array}$ $\begin{array}{llllllllll}107 & 170 & 277 & -4.0 & -5.7-2005.59 & -4.01 & 1.01 & -3.85-2012.43 & 138.62\end{array}$ $\begin{array}{lllllllll}107 & 171 & 278 & -4.1 & -5.6-2011.29 & -3.69 & 1.72 & -3.80-2017.06 & 142.07\end{array}$ $\begin{array}{lllllllll}107 & 172 & 279 & -4.3 & -5.5-2016.89 & -3.72 & 1.01 & -3.76-2023.35 & 143.84\end{array}$ $\begin{array}{llllllllll}107 & 173 & 280 & -4.5 & -5.4-2022.38 & -4.13 & 1.72 & -3.72-2028.51 & 146.76\end{array}$ $\begin{array}{llllllllll}107 & 174 & 281 & -4.7 & -5.3-2027.78 & -4.11 & 1.01 & -3.67-2034.55 & 148.79\end{array}$ $\begin{array}{lllllllll}107 & 175 & 282 & -4.8 & -5.2-2033.07 & -4.49 & 1.72 & -3.63-2039.48 & 151.93\end{array}$ $\begin{array}{lllllllll}107 & 176 & 283 & -5.0 & -5.1-2038.27 & -4.17 & 1.01 & -3.59-2045.02 & 154.47\end{array}$ $107 \begin{array}{llllllll}177 & 284 & -5.2 & -5.0-2043.37 & -4.21 & 1.71 & -3.55-2049.41 & 158.14\end{array}$ $\begin{array}{lllllllll}107 & 178 & 285 & -5.3 & -4.9-2048.37 & -3.88 & 1.01 & -3.51-2054.75 & 160.88\end{array}$ $\begin{array}{llllllllll}107 & 179 & 286 & -5.5 & -4.9-2053.27 & -4.14 & 1.71 & -3.47-2059.18 & 164.52\end{array}$

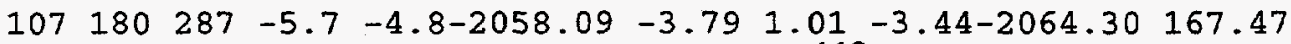

0.00 0.00 0.00 0.00 0.00

0.00 0.00

0.00

0.00

0.00

0.00

0.00

0.00

0.00

0.00

0.00

0.00

0.00

0.00

0.00

0.00

0.00

0.00

0.00

0.00

0.00

0.00

0.00

0.00

0.00

0.00

0.00

0.00

0.00

0.00

0.00

0.00

0.00

0.00

0.00

0.00

0.00

0.00

0.00

0.00

0.00

0.00

0.00

0.00

0.00

0.00

0.00

0.00

0.00

0.00

0.00

0.00

0.00

0.00

0.00

0.00

0.00

0.00

0.00

0.00

0.00

0.00

0.00

0.00

0.00

0.00

0.00

0.00

0.00

0.00

0.00

0.00

0.00

0.00

0.00

0.00

0.00

0.00

0.00

0.00

0.00

0.00

0.00

0.00

0.00

0.00

0.00

0.00

0.00

0.00

0.00

0.00

0.00

0.00

0.00

0.00

0.00

0.00

0.00

0.00

0.00

0.00

0.00

0.00

0.00

0.00

0.00

0.00

0.00

0.00

0.00

0.00

0.00

0.00

0.00

0.00

0.00

0.00

0.00

0.00

0.00

0.00

0.00

0.00

0.00

0.00

0.00

0.00

0.00

0.00

0.00

0.00

0.00

0.00

0.00

0.00

0.00

0.00

0.00

0.00

0.00

0.00

0.00

0.00

0.00

0.00

0.00

0.00

0.00

0.00

0.00

0.00

0.00

0.00

0.00

0.00

0.00

0.00

0.00

0.00

0.00

0.00

0.00

0.00

0.00

0.00

0.00

0.00

0.00

0.00

0.00

0.00

0.00

0.00

0.00

0.00

0.00

0.00

0.00

0.00

0.00

0.00

0.00

0.00

0.00

0.00

0.00

0.00

0.00

0.00

0.00

0.00

0.00 
$\begin{array}{lllllllllll}107 & 181 & 288 & -5.8 & -4.7-2062.81 & -3.96 & 1.71 & -3.40-2068.46 & 171.38\end{array}$ $\begin{array}{llllllllll}107 & 182 & 289 & -6.0 & -4.6-2067.43 & -3.61 & 1.01 & -3.36-2073.39 & 174.52\end{array}$ $\begin{array}{llllllllll}107 & 183 & 290 & -6.2 & -4.5-2071.97 & -3.63 & 1.71 & -3.33-2077.22 & 178.77\end{array}$ $\begin{array}{lllllllllll}107 & 184 & 291 & -6.3 & -4.4-2076.41 & -3.02 & 1.01 & -3.29-2081.71 & 182.34\end{array}$ $\begin{array}{llllllllll}107 & 185 & 292 & -6.5 & -4.3-2080.77 & -2.46 & 1.70 & -3.26-2084.78 & 187.35\end{array}$ $107186293-6.6-4.2-2085.03-1.851 .01-3.22-2089.09191 .10$ $107187294-6.8-4.1-2089.21-1.50 \quad 1.70-3.19-2092.20 \quad 196.07$ $\begin{array}{lllllllllll}107 & 188 & 295 & -7.0 & -4.0-2093.31 & -0.82 & 1.01 & -3.16-2096.27 & 200.07\end{array}$ $\begin{array}{lllllllllll}107 & 189 & 296 & -7.1 & -4.0-2097.31 & -0.63 & 1.70 & -3.12-2099.37 & 205.04\end{array}$ $\begin{array}{llllllllll}107 & 190 & 297 & -7.3 & -3.9-2101.24 & 0.21 & 1.01 & -3.09-2103.11 & 209.38\end{array}$ $\begin{array}{llllllllll}107 & 191 & 298 & -7.4 & -3.8-2105.07 & -0.13 & 1.70 & -3.06-2106.57 & 213.99\end{array}$ $\begin{array}{llllllllll}107 & 192 & 299 & -7.6 & -3.7-2108.83 & -0.21 & 1.01 & -3.03-2111.06 & 217.57\end{array}$ $\begin{array}{llllllllll}107 & 193 & 300 & -7.7 & -3.6-2112.50 & -0.55 & 1.69 & -3.00-2114.36 & 222.34\end{array}$ $\begin{array}{llllllllll}107 & 194 & 301 & -7.9 & -3.5-2116.10 & -0.58 & 1.01 & -2.97-2118.64 & 226.13\end{array}$ $\begin{array}{llllllllll}107 & 195 & 302 & -8.0 & -3.5-2119.61 & -0.98 & 1.69 & -2.94-2121.84 & 231.00\end{array}$ $\begin{array}{llllllllll}107 & 196 & 303 & -8.2 & -3.4-2123.04 & -1.09 & 1.01 & -2.91-2126.04 & 234.88\end{array}$ $\begin{array}{llllllllll}107 & 197 & 304 & -8.3 & -3.3-2126.40 & -1.55 & 1.69 & -2.88-2129.14 & 239.84\end{array}$ $\begin{array}{llllllllll}107 & 198 & 305 & -8.5 & -3.2-2129.68 & -1.77 & 1.01 & -2.86-2133.29 & 243.76\end{array}$ $\begin{array}{lllllllll}107 & 199 & 306 & -8.6 & -3.2-2132.88 & -2.28 & 1.69 & -2.83-2136.30 & 248.83\end{array}$ $107200307-8.8 \quad-3.1-2136.00-2.51 \quad 1.01-2.80-2140.30 \quad 252.90$ $\begin{array}{lllllllll}107 & 201 & 308 & -8.9 & -3.0-2139.05 & -2.99 & 1.69 & -2.78-2143.13 & 258.14\end{array}$ $\begin{array}{llllllllll}107 & 202 & 309 & -9.1 & -2.9-2142.02 & -3.08 & 1.01 & -2.75-2146.84 & 262.50\end{array}$ $\begin{array}{llllllllll}107 & 203 & 310 & -9.2 & -2.9-2144.93 & -3.30 & 1.68 & -2.72-2149.27 & 268.15\end{array}$ $\begin{array}{llllllllll}107 & 204 & 311 & -9.4 & -2.8-2147.75 & -3.24 & 1.01 & -2.70-2152.68 & 272.80\end{array}$ $\begin{array}{llllllllll}107 & 205 & 312 & -9.5 & -2.7-2150.51 & -3.43 & 1.68 & -2.67-2154.93 & 278.62\end{array}$ $\begin{array}{llllllllll}107 & 206 & 313 & -9.6 & -2.6-2153.20 & -3.27 & 1.01 & -2.65-2158.10 & 283.52\end{array}$ $\begin{array}{lllllllll}107 & 207 & 314 & -9.8 & -2.6-2155.81 & -3.51 & 1.68 & -2.62-2160.27 & 289.43\end{array}$ $107208 \quad 315-9.9 .-2.5-2158.36-3.43 \quad 1.01-2.60-2163.38 \quad 294.39$ $107209316-10.1-2.4-2160.83-3.79 \quad 1.68-2.58-21.65 .52 \quad 300.32$ $107210 \quad 317-10.2 \quad-2.4-2163.24-3.80 \quad 1.01-2.55-2168.58 \quad 305.33$ $107211318-10.3-2.3-2165.58-4.251 .68-2.53-2170.69311 .30$ $107212 \quad 319-10.5-2.2-2167.85-4.26 \quad 1.01-2.51-2173.61316 .44$ $107213 \quad 320-10.6-2.2-2170.06-4.61 \quad 1.67-2.49-2175.48 \quad 322.64$ $107214321-10.7-2.1-2172.20-4.451 .01-2.47-2178.11328 .09$ $107215322-10.9-2.0-2174.28-4.62 \quad 1.67-2.44-2179.67 \quad 334.60$ $\begin{array}{lllllllll}107 & 216 & 323-11.0 & -2.0-2176.29 & -4.57 & 1.01 & -2.42-2182.28 & 340.06\end{array}$ $107217324-11.1-1.9-2178.24-4.72 \quad 1.67-2.40-2183.70 \quad 346.72$ $\begin{array}{lllllllll}107 & 218 & 325-11.3 & -1.9-2180.13 & -4.77 & 1.01 & -2.38-2186.27 & 352.21\end{array}$ $107219326-11.4-1.8-2181.96-5.601 .67-2.36-2188.25358 .30$ $107220 \quad 327-11.5-1.7-2183.72-5.56 \quad 1.01-2.34-2190.61364 .01$ $\begin{array}{llllllllll}107 & 221 & 328-11.7 & -1.7-2185.42 & -5.97 & 1.67 & -2.32-2192.05 & 370.65\end{array}$ $107222329-11.8-1.6-2187.07-5.70 \quad 1.01-2.30-2194.06 \quad 376.71$ $107223 \quad 330-11.9-1.6-2188.65-6.01 \quad 1.66-2.28-2195.28 \quad 383.56$ $107224331-12.0-1.5-2190.18-5.83 \quad 1.01-2.27-2197.26 \quad 389.65$ $107225332-12.2-1.4-2191.65-6.09 \quad 1.66-2.25-2198.32 \quad 396.66$ $107226333-12.3-1.4-2193.06-3.361 .01-2.23-2197.63 \quad 405.42$ $107227334-12.4-1.3-2194.41-4.41 \quad 1.66-2.21-2199.37 \quad 411.76$ $107228 \quad 335-12.5-1.3-2195.71-5.17 \quad 1.01-2.19-2202.06 \quad 417.14$ $\begin{array}{llllllll}107229 & 336-12.7 & -1.2-2196.95 & -5.47 & 1.66 & -2.18-2202.93 & 424.33\end{array}$ $107230337-12.8-1.2-2198.14-5.37 \quad 1.01-2.16-2204.65 \quad 430.69$ $\begin{array}{lllllllll}107 & 231 & 338-12.9 & -1.1-2199.27 & -5.55 & 1.66 & -2.14-2205.30 & 438.11\end{array}$ $107232339-13.0-1.0-2200.35-5.111 .01-2.13-2206.57 \quad 444.91$ $\begin{array}{llllllllll}108 & 142 & 250 & 1.7 & -9.4-1797.50 & -1.01 & 0.00 & -5.65-1804.16 & 128.16\end{array}$ $\begin{array}{llllllllll}108 & 143 & 251 & 1.5 & -9.2-1806.82 & -1.33 & 0.92 & -5.57-1812.80 & 127.59\end{array}$ $\begin{array}{llllllllll}108 & 144 & 252 & 1.3 & -9.1-1815.99 & -1.58 & 0.00 & -5.49-1823.06 & 125.40\end{array}$ $\begin{array}{lllllllllll}108 & 145 & 253 & 1.1 & -9.0-1825.02 & -1.97 & 0.91 & -5.41-1831.49 & 125.04\end{array}$ $\begin{array}{llllllllll}108 & 146 & 254 & 0.9 & -8.8-1833.92 & -2.37 & 0.00 & -5.33-1841.62 & 122.98\end{array}$ 108147255 $\begin{array}{lll}108 & 148 & 256\end{array}$ $108 \quad 149257$ $\begin{array}{lll}108 & 150 & 258\end{array}$ $\begin{array}{lllllllll}108 & 151 & 259 & -0.1 & -8.2-1876.35 & -4.62 & 0.90 & -4.98-1885.04 & 119.92\end{array}$ $\begin{array}{lllllll}0.7 & -8.7-1842.67 & -2.85 & 0.91 & -5.26-1849.87 & 122.80\end{array}$ $\begin{array}{llllllll}0.5 & -8.5-1851.29 & -3.20 & 0.00 & -5.19-1859.68 & 121.07\end{array}$ $0.3-8.4-1859.78-3.67 \quad 0.91-5.12-1867.66 \quad 121.16$ $0.1-8.3-1868.13-4.120 .00-5.05-1877.30119 .60$ $\begin{array}{llllllllll}108 & 152 & 260 & -0.3 & -8.0-1884.44 & -4.80 & 0.00 & -4.91-1894.15 & 118.89\end{array}$ $\begin{array}{llllllllll}108 & 153 & 261 & -0.5 & -7.9-1892.40 & -4.96 & 0.90 & -4.85-1901.31 & 119.80\end{array}$ $\begin{array}{llllllllll}108 & 154 & 262 & -0.7 & -7.8-1900.23 & -4.91 & 0.00 & -4.78-1909.92 & 119.26\end{array}$ $\begin{array}{llllllllll}108 & 155 & 263 & -0.9 & -7.6-1907.94 & -5.07 & 0.89 & -4.72-1916.83 & 120.42\end{array}$
0.00 0.00

0.00

0.00

0.00

0.00

0.00

0.00

0.00

0.00

0.00

0.00

0.00

0.00

0.00

0.00

0.00

0.00

0.00

0.00

0.00

0.00

0.00

0.00

0.00

0.00

0.00

0.00

0.00

0.00

0.00

0.00

0.00

0.00

0.00

0.00

0.00

0.00

0.00

0.00

0.00

0.00

0.00

0.00

0.00

0.00

0.00

0.00

0.00

0.00

0.00

0.00

0.00

0.00

0.00

0.00

0.00

0.00

0.00

0.00

0.00

0.00

0.00

0.00

0.00

0.00

0.00

0.00

0.00

0.00

0.00

0.00

0.00

0.00

0.00

0.00

0.00

0.00

0.00

0.00

0.00

0.00

0.00

0.00

0.00

0.00

0.00

0.00

0.00

0.00

0.00

0.00

0.00

0.00

0.00

0.00

0.00

0.00

0.00

0.00

0.00

0.00

0.00

0.00

0.00

0.00

0.00

0.00

0.00

0.00

0.00

0.00

0.00

0.00

0.00

0.00

0.00

0.00

0.00

$0.00 \quad 0.00$

0.00

0.00

0.00

0.00

0.00

0.00

0.00
0.00

0.00

0.00

0.00

0.00

0.00

0.00

0.00

0.00

0.00

0.00

0.00

0.00

0.00

0.00

0.00

0.00

0.00

0.00

0.00

0.00

0.00

0.00

0.00

0.00

0.00

0.00

0.00

0.00

0.00

0.00

0.00

0.00

0.00

0.00

0.00

0.00

0.00

0.00

0.00

0.00

0.00

0.00

0.00

0.00

0.00

0.00

0.00

0.00

0.00

0.00

0.00

0.00

0.00

0.00

0.00

0.00

0.00

0.00

0.00

0.00

0.00

0.00

0.00

0.00

0.00 120

0.00 
$\begin{array}{llllllllllll}108 & 156 & 264 & -1.0 & -7.5-1915.52 & -4.86 & 0.00 & -4.66-1925.04 & 120.28\end{array}$ $\begin{array}{lllllllllll}108 & 157 & 265 & -1.2 & -7.4-1922.98 & -5.21 & 0.89 & -4.60-1931.90 & 121.49\end{array}$ $\begin{array}{llllllllll}108 & 158 & 266 & -1.4 & -7.3-1930.32 & -5.27 & 0.00 & -4.54-1940.13 & 121.34\end{array}$ $\begin{array}{lllllllllll}108 & 159 & 267 & -1.6 & -7.2-1937.54 & -5.75 & 0.89 & -4.48-1946.88 & 122.65\end{array}$ $\begin{array}{lllllllllll}108 & 160 & 268 & -1.8 & -7.0-1944.63 & -5.95 & 0.00 & -4.43-1955.01 & 122.60\end{array}$ $\begin{array}{llllllllll}108 & 161 & 269 & -2.0 & -6.9-1951.61 & -6.38 & 0.88 & -4.37-1961.48 & 124.19\end{array}$ $\begin{array}{lllllllllll}108 & 162 & 270 & -2.1 & -6.8-1958.48 & -6.54 & 0.00 & -4.32-1969.34 & 124.41\end{array}$ $\begin{array}{llllllllll}108 & 163 & 271 & -2.3 & -6.7-1965.23 & -6.64 & 0.88 & -4.26-1975.26 & 126.57\end{array}$ $\begin{array}{lllllllllll}108 & 164 & 272 & -2.5 & -6.6-1971.87 & -6.23 & 0.00 & -4.21-1982.31 & 127.58\end{array}$ $\begin{array}{lllllllllll}108 & 165 & 273 & -2.7 & -6.5-1978.39 & -5.93 & 0.88 & -4.16-1987.61 & 130.36\end{array}$ $\begin{array}{llllllllll}108 & 166 & 274 & -2.9 & -6.4-1984.80 & -5.31 & 0.00 & -4.11-1994.22 & 131.81\end{array}$ $\begin{array}{lllllllllll}108 & 167 & 275 & -3.0 & -6.2-1991.10 & -4.90 & 0.87 & -4.06-1999.19 & 134.91\end{array}$ $\begin{array}{lllllllllll}108 & 168 & 276 & -3.2 & -6.1-1997.30 & -4.24 & 0.00 & -4.01-2005.55 & 136.63\end{array}$ $\begin{array}{lllllllllll}108 & 169 & 277 & -3.4 & -6.0-2003.38 & -4.32 & 0.87 & -3.97-2010.80 & 139.45\end{array}$ $\begin{array}{llllllllll}108 & 170 & 278 & -3.6 & -5.9-2009.36 & -3.68 & 0.00 & -3.92-2016.96 & 141.36\end{array}$ $\begin{array}{llllllllll}108 & 171 & 279 & -3.7 & -5.8-2015.24 & -4.11 & 0.86 & -3.87-2022.36 & 144.04\end{array}$ $\begin{array}{llllllllll}108 & 172 & 280 & -3.9 & -5.7-2021.01 & -4.20 & 0.00 & -3.83-2029.04 & 145.43\end{array}$ $\begin{array}{lllllllllll}108 & 173 & 281 & -4.1 & -5.6-2026.68 & -4.64 & 0.86 & -3.79-2034.24 & 148.30\end{array}$ $\begin{array}{lllllllllll}108 & 174 & 282 & -4.3 & -5.5-2032.24 & -4.59 & 0.00 & -3.74-2040.57 & 150.03\end{array}$ $\begin{array}{lllllllllll}108 & 175 & 283 & -4.4 & -5.4-2037.71 & -4.97 & 0.86 & -3.70-2045.52 & 153.16\end{array}$ $108176284-4.6-5.3-2043.07-4.72 \quad 0.00-3.66-2051.45 \quad 155.30$ $\begin{array}{llllllllll}108 & 177 & 285 & -4.8 & -5.2-2048.34 & -4.79 & 0.85 & -3.62-2055.89 & 158.93\end{array}$ $\begin{array}{llllllllll}108 & 178 & 286 & -4.9 & -5.1-2053.51 & -4.44 & 0.00 & -3.58-2061.52 & 161.37\end{array}$ $\begin{array}{lllllllllll}108 & 179 & 287 & -5.1 & -5.0-2058.58 & -4.77 & 0.85 & -3.54-2066.04 & 164.93\end{array}$ $\begin{array}{llllllllll}108 & 180 & 288 & -5.3 & -4.9-2063.56 & -4.47 & 0.00 & -3.50-2071.53 & 167.51\end{array}$ $\begin{array}{lllllllllll}108 & 181 & 289 & -5.4 & -4.8-2068.44 & -4.66 & 0.85 & -3.46-2075.71 & 171.39\end{array}$ $\begin{array}{lllllllllll}108 & 182 & 290 & -5.6 & -4.7-2073.23 & -4.21 & 0.00 & -3.42-2080.86 & 174.31\end{array}$ $\begin{array}{llllllllll}108 & 183 & 291 & -5.8 & -4.6-2077.93 & -4.22 & 0.85 & -3.39-2084.69 & 178.56\end{array}$ $\begin{array}{llllllllll}108 & 184 & 292 & -5.9 & -4.6-2082.53 & -3.59 & 0.00 & -3.35-2089.48 & 181.84\end{array}$ $\begin{array}{llllllllll}108 & 185 & 293 & -6.1 & -4.5-2087.05 & -2.81 & 0.84 & -3.32-2092.33 & 187.06\end{array}$ $\begin{array}{lllllllll}108 & 186 & 294 & -6.2 & -4.4-2091.48 & -2.13 & 0.00 & -3.28-2096.89 & 190.58\end{array}$ $\begin{array}{llllllllll}108 & 187 & 295 & -6.4 & -4.3-2095.81 & -1.67 & 0.84 & -3.25-2099.89 & 195.64\end{array}$ $108 \quad 188296-6.6-4.2-2100.07-0.94 \quad 0.00-3.21-2104.22 \quad 199.39$ $\begin{array}{llllllllll}108 & 189 & 297 & -6.7 & -4.1-2104.23 & -0.67 & 0.84 & -3.18-2107.24 & 204.43\end{array}$ $\begin{array}{llllllllll}108 & 190 & 298 & -6.9 & -4.0-2108.31 & 1.49 & 0.00 & -3.15-2109.97 & 209.78\end{array}$ $\begin{array}{llllllllll}108 & 191 & 299 & -7.0 & -3.9-2112.30 & 0.11 & 0.83 & -3.12 & -2114.47 & 213.35\end{array}$ $\begin{array}{llllllllll}108 & 192 & 300 & -7.2 & -3.9-2116.21 & 0.08 & 0.00 & -3.09-2119.22 & 216.68\end{array}$ $\begin{array}{llllllllll}108 & 193 & 301 & -7.3 & -3.8-2120.04 & -0.23 & 0.83 & -3.05-2122.49 & 221.47\end{array}$ $\begin{array}{lllllllllll}108 & 194 & 302 & -7.5 & -3.7-2123.79 & -0.33 & 0.00 & -3.02-2127.14 & 224.90\end{array}$ $\begin{array}{lllllllllll}108 & 195 & 303 & -7.6 & -3.6-2127.45 & -0.71 & 0.83 & -2.99-2130.33 & 229.78\end{array}$ $\begin{array}{llllllllll}108 & 196 & 304 & -7.8 & -3.5-2131.03 & -0.82 & 0.00 & -2.96-2134.82 & 233.36\end{array}$ $\begin{array}{llllllllll}108 & 197 & 305 & -7.9 & -3.5-2134.54 & -1.28 & 0.82 & -2.94-2137.93 & 238.32\end{array}$ $\begin{array}{llllllllll}108 & 198 & 306 & -8.1 & -3.4-2137.97 & -1.48 & 0.00 & -2.91-2142.35 & 241.97\end{array}$ $\begin{array}{llllllllll}108 & 199 & 307 & -8.2 & -3.3-2141.31 & -1.98 & 0.82 & -2.88-2145.35 & 247.04\end{array}$ $\begin{array}{llllllllll}108 & 200 & 308 & -8.4 & -3.2-2144.58 & -2.18 & 0.00 & -2.85-2149.62 & 250.85\end{array}$

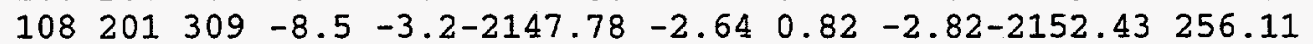
$\begin{array}{lllllllllll}108 & 202 & 310 & -8.7 & -3.1-2150.90 & -2.72 & 0.00 & -2.80-2156.42 & 260.19\end{array}$ $\begin{array}{llllllllll}108 & 203 & 311 & -8.8 & -3.0-2153.95 & -2.94 & 0.82 & -2.77-2158.84 & 265.84\end{array}$ $\begin{array}{llllllllll}108 & 204 & 312 & -9.0 & -2.9-2156.92 & -2.88 & 0.00 & -2.75-2162.54 & 270.20\end{array}$ $\begin{array}{llllllllll}108 & 205 & 313 & -9.1 & -2.9-2159.82 & -3.07 & 0.81 & -2.72-2164.80 & 276.03\end{array}$ $\begin{array}{llllllllll}108 & 206 & 314 & -9.3 & -2.8-2162.64 & -2.90 & 0.00 & -2.70-2168.24 & 280.65\end{array}$ $\begin{array}{llllllllll}108 & 207 & 315 & -9.4 & -2.7-2165.40 & -3.16 & 0.81 & -2.67-2170.42 & 286.54\end{array}$ $\begin{array}{llllllllll}108 & 208 & 316 & -9.5 & -2.6-2168.09 & -3.08 & 0.00 & -2.65-2173.81 & 291.22\end{array}$ $\begin{array}{llllllllll}108 & 209 & 317 & -9.7 & -2.6-2170.70 & -3.46 & 0.81 & -2.62-2175.98 & 297.13\end{array}$ $\begin{array}{lllllllll}108 & 210 & 318 & -9.8 & -2.5-2173.25 & -3.49 & 0.00 & -2.60-2179.34 & 301.84\end{array}$ $\begin{array}{llllllll}108 & 211 & 319-10.0 & -2.4-2175.72 & -3.93 & 0.81 & -2.58-2181.43 & 307.82\end{array}$ $\begin{array}{lllllllll}108 & 212 & 320-10.1 & -2.4-2178.13 & -3.94 & 0.00 & -2.55-2184.63 & 312.69\end{array}$ $\begin{array}{llllllll}108 & 213 & 321-10.2 & -2.3-2180.48 & -4.30 & 0.80 & -2.53-2186.51 & 318.89\end{array}$ $\begin{array}{llllllll}108 & 214 & 322-10.4 & -2.2-2182.76 & -4.12 & 0.00 & -2.51-2189.39 & 324.08\end{array}$ $\begin{array}{lllllllll}108 & 215 & 323-10.5 & -2.2-2184.97 & -4.28 & 0.80 & -2.49-2190.93 & 330.60\end{array}$ $\begin{array}{lllllllll}108 & 216 & 324-10.6 & -2.1-2187.11 & -3.99 & 0.00 & -2.47-2193.57 & 336.04\end{array}$ $\begin{array}{lllllllll}108 & 217 & 325-10.8 & -2.0-2189.20 & -4.36 & 0.80 & -2.44-2195.20 & 342.48\end{array}$ $\begin{array}{llllllll}108 & 218 & 326-10.9 & -2.0-2191.21 & -4.33 & 0.00 & -2.42-2197.97 & 347.78\end{array}$ $\begin{array}{lllllllll}108 & 219 & 327-11.0 & -1.9-2193.17 & -5.21 & 0.80 & -2.40-2199.99 & 353.83\end{array}$ $\begin{array}{lllllllll}108 & 220 & 328-11.2 & -1.9-2195.07 & -2.14 & 0.00 & -2.38-2199.59 & 362.30\end{array}$ $\begin{array}{llllllll}108 & 221 & 329-11.3 & -1.8-2196.90 & -2.40 & 0.79 & -2.36-2200.87 & 369.10\end{array}$

0.00

0.00

0.00

0.00

0.00

0.00

0.00

0.00

0.00

0.00

0.00

0.00

0.00

0.00

0.00

0.00

0.00

0.00

0.00

0.00

0.00

0.00

0.00

0.00

0.00

0.00

0.00

0.00

0.00

0.00

0.00

0.00

0.00

0.00

0.00

0.00

0.00

0.00

0.00

0.00

0.00

0.00

0.00

0.00

0.00

0.00

0.00

0.00

0.00

0.00

0.00

0.00

0.00

0.00

0.00

0.00

0.00

0.00

0.00

0.00

0.00

0.00

0.00

0.00

0.00

0.00

0.00

0.00

0.00

0.00

0.00

0.00

0.00

0.00

0.00

0.00

0.00

0.00

0.00

0.00

0.00

0.00

0.00

0.00

0.00

0.00

0.00

0.00

0.00

0.00

0.00

0.00

0.00

0.00

0.00

0.00

0.00

0.00

0.00

0.00

0.00

0.00

0.00

0.00

0.00

0.00

0.00

0.00

0.00

0.00

0.00

0.00

0.00

0.00

0.00

0.00

0.00

0.00

0.00

0.00

0.00

0.00

0.00

0.00

0.00

0.00

0.00

0.00

0.00
0.00

0.00

0.00

0.00

0.00

0.00

0.00

0.00

0.00

0.00

0.00

0.00

0.00

0.00

0.00

0.00

0.00

0.00

0.00

0.00

0.00

0.00

0.00

0.00

0.00

0.00

0.00

0.00

0.00

0.00

0.00

0.00

0.00

0.00

0.00

0.00

0.00

0.00

0.00

0.00

0.00

0.00

0.00

0.00

0.00

0.00

0.00

0.00

0.00 .

0.00

0.00

0.00

0.00

0.00

0.00

0.00

0.00

0.00

0.00

0.00

0.00 .

0.00

0.00

0.00

0.00

0.00 
$108222 \quad 330-11.4 \quad-1.7-2198.67-2.590 .00-2.34-2203.60 \quad 374.43$ $\begin{array}{lllllllll}108 & 223 & 331-11.5 & -1.7-2200.38 & -3.13 & 0.79 & -2.32-2205.04 & 381.06\end{array}$ $\begin{array}{lllllllll}108 & 224 & 332-11.7 & -1.6-2202.03 & -5.46 & 0.00 & -2.31-2209.80 & 384.38\end{array}$ $\begin{array}{lllllllll}108 & 225 & 333-11.8 & -1.6-2203.63 & -3.23 & 0.79 & -2.29-2208.35 & 393.90\end{array}$ $\begin{array}{lllllllll}108 & 226 & 334-11.9 & -1.5-2205.16 & -3.96 & 0.00 & -2.27-2211.39 & 398.93\end{array}$ $108227335-12.0-1.4-2206.64-5.01 \quad 0.79-2.25-2213.11405 .28$ $\begin{array}{llllllllll}108 & 228 & 336-12.2 & -1.4-2208.06 & -5.79 & 0.00 & -2.23-2216.08 & 410.38\end{array}$ $\begin{array}{lllllllll}108 & 229 & 337-12.3 & -1.3-2209.43 & -6.08 & 0.78 & -2.21-2216.93 & 417.60\end{array}$ $\begin{array}{llllllllll}108 & 230 & 338-12.4 & -1.3-2210.73 & -5.95 & 0.00 & -2.20-2218.88 & 423.73\end{array}$ $108231339-12.5-1.2-2211.99-6.110 .78-2.18-2219.49431 .19$ $\begin{array}{llllllllll}109 & 144 & 253 & 1.7 & -9.3-1814.52 & -1.56 & 1.00 & -5.59-1820.67 & 135.06\end{array}$ $\begin{array}{lllllllllll}109 & 145 & 254 & 1.5 & -9.2-1823.75 & -1.96 & 1.75 & -5.51-1829.47 & 134.33\end{array}$ $\begin{array}{llllllllll}109 & 146 & 255 & 1.3 & -9.0-1832.84 & -2.24 & 1.00 & -5.44-1839.51 & 132.36\end{array}$ $\begin{array}{llllllllll}109 & 147 & 256 & 1.1 & -8.9-1841.79 & -2.68 & 1.75 & -5.36-1848.08 & 131.86\end{array}$ $\begin{array}{lllllllllll}109 & 148 & 257 & 0.9 & -8.7-1850.61 & -3.17 & 1.00 & -5.29-1858.06 & 129.96\end{array}$ $\begin{array}{lllllllll}109 & 149 & 258 & 0.7 & -8.6-1859.29 & -3.63 & 1.75 & -5.21-1866.38 & 129.70\end{array}$ $109150259 \quad 0.5-8.5-1867.83-4.09 \quad 1.00-5.14-1876.06 \quad 128.10$ $\begin{array}{llllllllll}109 & 151 & 260 & 0.3 & -8.3-1876.24 & -4.58 & 1.74 & -5.07-1884.15 & 128.08\end{array}$ $\begin{array}{lllllllllll}109 & 152 & 261 & 0.1 & -8.2-1884.52 & -4.78 & 1.00 & -5.01-1893.31 & 127.00\end{array}$ $\begin{array}{llllllllll}109 & 153 & 262 & -0.1 & -8.1-1892.68 & -4.77 & 1.74 & -4.94-1900.64 & 127.73\end{array}$ $\begin{array}{llllllllll}109 & 154 & 263 & -0.3 & -8.0-1900.70 & -4.80 & 1.00 & -4.87-1909.37 & 127.08\end{array}$ $\begin{array}{llllllllllll}109 & 155 & 264 & -0.5 & -7.8-1908.60 & -5.04 & 1.74 & -4.81-1916.71 & 127.81\end{array}$ $109156265-0.6-7.7-1916.37-5.04 \quad 1.00-4.75-1925.15 \quad 127.44$ $109157266-0.8-7.6-1924.01-5.391 .74-4.69-1932.35128 .30$ $109158 \quad 267-1.0 \quad-7.5-1931.54-5.41 \quad 1.00-4.63-1940.57 \quad 128.16$ $\begin{array}{lllllllllll}109 & 159 & 268 & -1.2 & -7.3-1938.94 & -5.93 & 1.73 & -4.57-1947.71 & 129.09\end{array}$ $\begin{array}{lllllllllll}109 & 160 & 269 & -1.4 & -7.2-1946.22 & -6.25 & 1.00 & -4.51-1955.98 & 128.89\end{array}$ $\begin{array}{lllllllllll}109 & 161 & 270 & -1.6 & -7.1-1953.39 & -6.65 & 1.73 & -4.45-1962.76 & 130.18\end{array}$ $109 \quad 162 \quad 271-1.8-7.0-1960.44-6.92 \quad 1.00-4.40-1970.75 \quad 130.27$ $\begin{array}{lllllllllll}109 & 163 & 272 & -1.9 & -6.9-1967.37 & -7.06 & 1.73 & -4.34-1977.04 & 132.04\end{array}$ $109164273-2.1-6.8-1974.18-6.67 \quad 1.00-4.29-1984.14 \quad 133.02$ $\begin{array}{lllllllllll}109 & 165 & 274 & -2.3 & -6.6-1980.89 & -6.36 & 1.72 & -4.24-1989.76 & 135.47\end{array}$ $\begin{array}{llllllllll}109 & 166 & 275 & -2.5 & -6.5-1987.48 & -5.78 & 1.00 & -4.19-1996.44 & 136.86\end{array}$ $\begin{array}{llllllllll}109 & 167 & 276 & -2.7 & -6.4-1993.96 & -5.46 & 1.72 & -4.14-2001.83 & 139.54\end{array}$ $109168277-2.8-6.3-2000.33-4.81 \quad 1.00-4.09-2008.22 \quad 141.22$ $\begin{array}{llllllllll}109 & 169 & 278 & -3.0 & -6.2 & -2006.59 & -4.82 & 1.72 & -4.04-2013.73 & 143.79\end{array}$ $109170 \quad 279-3.2-6.1-2012.74-4.31 \quad 1.00-3.99-2020.04 \quad 145.55$ $\begin{array}{llllllllll}109 & 171 & 280 & -3.4 & -6.0-2018.79 & -4.77 & 1.72 & -3.95-2025.79 & 147.87\end{array}$ $\begin{array}{lllllllllll}109 & 172 & 281 & -3.5 & -5.9-2024.74 & -4.78 & 1.00 & -3.90-2032.41 & 149.32\end{array}$ $\begin{array}{llllllllll}109 & 173 & 282 & -3.7 & -5.8-2030.57 & -5.18 & 1.71 & -3.86-2037.90 & 151.90\end{array}$ $\begin{array}{lllllllllll}109 & 174 & 283 & -3.9 & -5.7-2036.31 & -5.20 & 1.00 & -3.81-2044.32 & 153.55\end{array}$ $\begin{array}{llllllllll}109 & 175 & 284 & -4.0 & -5.6-2041.95 & -5.55 & 1.71 & -3.77-2049.55 & 156.39\end{array}$ $\begin{array}{llllllllll}109 & 176 & 285 & -4.2 & -5.5-2047.48 & -5.23 & 1.00 & -3.73-2055.43 & 158.58\end{array}$ $\begin{array}{llllllllll}109 & 177 & 286 & -4.4 & -5.4-2052.91 & -5.27 & 1.71 & -3.68-2060.16 & 161.93\end{array}$ $\begin{array}{lllllllllll}109 & 178 & 287 & -4.5 & -5.3-2058.25 & -5.07 & 1.00 & -3.64-2065.96 & 164.20\end{array}$ $\begin{array}{llllllllll}109 & 179 & 288 & -4.7 & -5.2-2063.49 & -5.46 & 1.71 & -3.60-2070.85 & 167.38\end{array}$ $\begin{array}{llllllllll}109 & 180 & 289 & -4.9 & -5.1-2068.63 & -5.12 & 1.00 & -3.56-2076.31 & 169.99\end{array}$ $\begin{array}{lllllllllll}109 & 181 & 290 & -5.0 & -5.0-2073.68 & -5.32 & 1.70 & -3.52-2080.82 & 173.55\end{array}$ $\begin{array}{llllllllll}109 & 182 & 291 & -5.2 & -4.9-2078.64 & -4.86 & 1.00 & -3.49-2085.98 & 176.47\end{array}$ $\begin{array}{llllllllll}109 & 183 & 292 & -5.4 & -4.8-2083.50 & -4.86 & 1.70 & -3.45-2090.10 & 180.41\end{array}$ $\begin{array}{llllllllll}109 & 184 & 293 & -5.5 & -4.7-2088.26 & -4.23 & 1.00 & -3.41-2094.90 & 183.69\end{array}$ $109185294-5.7-4.6-2092.94-3.391 .70-3.38-2098.01 \quad 188.55$ $\begin{array}{lllllllllll}109 & 186 & 295 & -5.9 & -4.5-2097.53 & -2.70 & 1.00 & -3.34-2102.56 & 192.17\end{array}$ $109187296-6.0-4.4-2102.02-2.151 .70-3.31-2105.79 \quad 197.02$ $\begin{array}{llllllllll}109 & 188 & 297 & -6.2 & -4.4-2106.43 & -1.39 & 1.00 & -3.27-2110.09 & 200.78\end{array}$ $\begin{array}{lllllllll}109 & 189 & 298 & -6.3 & -4.3-2110.75 & 0.19 & 1.69 & -3.24-2112.11 & 206.84\end{array}$ $109190 \quad 299-6.5-4.2-2114.99-0.41 \quad 1.00-3.21-2117.60 \quad 209.42$ $109191300-6.6-4.1-2119.14-0.21 \quad 1.69-3.17-2120.83 \quad 214.26$ $109192301-6.8-4.0-2123.20-0.02 \quad 1.00-3.14-2125.36217 .80$ $\begin{array}{llllllllll}109 & 193 & 302 & -6.9 & -3.9 & -2127.19 & -0.32 & 1.69 & -3.11-2128.93 & 222.30\end{array}$ $\begin{array}{lllllllllll}109 & 194 & 303 & -7.1 & -3.9-2131.08 & -0.39 & 1.00 & -3.08-2133.55 & 225.76\end{array}$ $\begin{array}{llllllllll}109 & 195 & 304 & -7.3 & -3.8-2134.90 & -0.76 & 1.69 & -3.05-2137.02 & 230.35\end{array}$ $\begin{array}{llllllllllll}109 & 196 & 305 & -7.4 & -3.7-2138.63 & -0.86 & 1.00 & -3.02-2141.51 & 233.94\end{array}$ $\begin{array}{llllllllll}109 & 197 & 306 & -7.6 & -3.6-2142.29 & -1.27 & 1.68 & -2.99-2144.86 & 238.65\end{array}$ $\begin{array}{lllllllllll}109 & 198 & 307 & -7.7 & -3.5-2145.87 & -1.48 & 1.00 & -2.96-2149.30 & 242.29\end{array}$ $109 \quad 199308-7.9-3.5-2149.36-1.96 \quad 1.68-2.93-2152.57 \quad 247.09$

\begin{tabular}{|c|c|c|}
\hline 00 & 00 & \\
\hline .00 & 0.00 & \\
\hline & 0.00 & \\
\hline & .00 & \\
\hline & .00 & \\
\hline & 00 & \\
\hline & 0.00 & \\
\hline & 0.00 & \\
\hline & 0.00 & \\
\hline & 0.00 & \\
\hline & 0.00 & \\
\hline & 0.00 & \\
\hline & 0.00 & \\
\hline & 0.00 & \\
\hline & 0.00 & \\
\hline & 0.00 & \\
\hline & 0.00 & \\
\hline & 0.00 & \\
\hline$v$ & 0.00 & \\
\hline & 0.00 & \\
\hline & 0.00 & \\
\hline & 0.00 & \\
\hline & 0.00 & \\
\hline .0 & 0.00 & \\
\hline 0 & 0.00 & \\
\hline .0 & 0.00 & \\
\hline & 0.00 & \\
\hline & 0.00 & \\
\hline & 0.00 & \\
\hline & 0.00 & \\
\hline ( & 0.00 & \\
\hline & 0.00 & \\
\hline & 0.00 & 0 \\
\hline 0 & 0.00 & \\
\hline 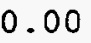 & 0.00 & \\
\hline 0 & 0.00 & \\
\hline & 0.00 & \\
\hline$\theta$ & 0.00 & 0 \\
\hline & 0.00 & \\
\hline 0 & 0.00 & \\
\hline & 0.00 & \\
\hline 0 & 0.00 & \\
\hline & 0.00 & 0. \\
\hline & 0.00 & 0 \\
\hline .0 & 0.00 & \\
\hline 0 & 0.00 & \\
\hline & 0.00 & \\
\hline & 0.00 & \\
\hline & 0.00 & \\
\hline .0 & 0.00 & \\
\hline & 0.00 & \\
\hline & 0.00 & \\
\hline & 0.00 & \\
\hline & 0.00 & \\
\hline & 0.00 & \\
\hline & 0.00 & \\
\hline & 0.00 & \\
\hline & 0.00 & \\
\hline & 0.00 & \\
\hline & 0.00 & \\
\hline & & \\
\hline & 0.00 & \\
\hline & 0.00 & \\
\hline & 0.00 & \\
\hline & 0.00 & \\
\hline & 0.00 & \\
\hline
\end{tabular}


$109200 \quad 309-8.0 \quad-3.4-2152.78-2.11 \quad 1.00-2.90-2156.79250 .94$ $\begin{array}{lllllllll}109 & 201 & 310 & -8.1 & -3.3-2156.12 & -2.65 & 1.68 & -2.88-2159.97 & 255.83\end{array}$ $\begin{array}{llllllllll}109 & 202 & 311 & -8.3 & -3.2-2159.39 & -2.61 & 1.00 & -2.85-2163.84 & 260.03\end{array}$ $\begin{array}{llllllllll}109 & 203 & 312 & -8.4 & -3.1-2162.58 & -2.82 & 1.68 & -2.82 & -2166.54 & 265.40\end{array}$ $\begin{array}{llllllllll}109 & 204 & 313 & -8.6 & -3.1-2165.70 & -2.75 & 1.00 & -2.79-2170.24 & 269.78\end{array}$ $\begin{array}{llllllllll}109 & 205 & 314 & -8.7 & -3.0 & -2168.74 & -2.96 & 1.68 & -2.77-2172.79 & 275.29\end{array}$ $\begin{array}{llllllllll}109 & 206 & 315 & -8.9 & -2.9-2171.71 & -2.83 & 1.00 & -2.74-2176.28 & 279.88\end{array}$ $109207316-9.0 \quad-2.9-2174.61-3.10 \quad 1.67-2.72-2178.75 .285 .48$ $\begin{array}{llllllllll}109 & 208 & 317 & -9.2 & -2.8-2177.43 & -3.04 & 1.00 & -2.69-2182.16 & 290.14\end{array}$ $109209.318-9.3 \quad-2.7-2180.19-3.45 \quad 1.67-2.67-2184.63 \quad 295.74$ $\begin{array}{lllllllll}109 & 210 & 319 & -9.4 & -2.6-2182.87 & -3.48 & 1.00 & -2.65-2187.99 & 300.45\end{array}$ $\begin{array}{lllllllll}109 & 211 & 320 & -9.6 & -2.6-2185.49 & -3.92 & 1.67 & -2.62-2190.36 & 306.16\end{array}$ $\begin{array}{llllllllll}109 & 212 & 321 & -9.7 & -2.5 & -2188.04 & -3.93 & 1.00 & -2.60-2193.56 & 311.03\end{array}$ $\begin{array}{llllllllll}109 & 213 & 322 & -9.8 & -2.4-2190.52 & -4.27 & 1.67 & -2.58-2195.69 & 316.97\end{array}$ $109214323-10.0 \quad-2.4-2192.93-4.07 \quad 1.00-2.55-2198.55 \quad 322.18$ $\begin{array}{lllllllll}109 & 215 & 324-10.1 & -2.3-2195.27 & -4.24 & 1.67 & -2.53-2200.38 & 328.42\end{array}$ 109. $216 \quad 325-10.2-2.2-2197.56-4.14 \quad 1.00-2.51-2203.20 \quad 333.67$ $109217326-10.4-2.2-2199.77-4.51 \quad 1.66-2.49-2205.10339 .84$ $109218 \quad 327-10.5 \quad-2.1-2201.92-4.46 \quad 1.00-2.47-2207.84 \quad 345.17$ $109219 \quad 328-10.6 \quad-2.1-2204.01-2.43 \quad 1.66-2.45-2207.22 \quad 353.86$ $109220 \quad 329-10.8-2.0-2206.03-2.35 \quad 1.00-2.42-2209.80 \quad 359.35$ $109221330-10.9-1.9-2208.00-2.591 .66-2.40-2211.33 \quad 365.90$ $109222331-11.0-1.9-2209.90-2.78 \quad 1.00-2.38-2214.06 \quad 371.24$ $109223 \quad 332-11.2-1.8-2211.74-3.28 \quad 1.66-2.36-2215.72 \quad 377.65$ $109224333-11.3-1.7-2213.52-3.47 \quad 1.00-2.34-2218.33 \quad 383.12$ $109225334-11.4-1.7-2215.24-3.95 \quad 1.66-2.33-2219.86 \quad 389.66$ $109226 \quad 335-11.5 \quad-1.6-2216.90-4.73 \quad 1.00-2.31-2222.93 \quad 394.66$ $\begin{array}{lllllllll}109 & 227 & 336-11.7 & -1.6-2218.50 & -5.79 & 1.66 & -2.29-2224.92 & 400.74\end{array}$ $109228 \quad 337-11.8-1.5-2220.05-6.58 \quad 1.00-2.27-2227.89 \quad 405.84$ $109229338-11.9-1.5-2221.53-6.85 \quad 1.65-2.25-2228.98 \quad 412.82$ $109230 \quad 339-12.0-1.4-2222.96-6.70 \quad 1.00-2.23-2230.89418 .98$ $\begin{array}{llllllllll}110 & 146 & 256 & 1.7 & -9.2-1831.37 & -1.84 & 0.00 & -5.54-1838.75 & 140.39\end{array}$ $\begin{array}{lllllllll}110 & 147 & 257 & 1.5 & -9.1-1840.52 & -2.27 & 0.91 & -5.46-1847.34 & 139.87\end{array}$ $\begin{array}{llllllllll}110 & 148 & 258 & 1.3 & -8.9-1849.53 & -2.64 & 0.00 & -5.39-1857.56 & 137.72\end{array}$ $\begin{array}{llllllllll}110 & 149 & 259 & 1.1 & -8.8-1858.41 & -3.15 & 0.91 & -5.31-1865.96 & 137.39\end{array}$ $\begin{array}{llllllllll}110 & 150 & 260 & 0.9 & -8.7-1867.14 & -3.48 & 0.00 & -5.24-1875.86 & 135.56\end{array}$ $\begin{array}{llllllllll}110 & 151 & 261 & 0.7 & -8.5-1875.75 & -3.94 & 0.90 & -5.17-1883.96 & 135.54\end{array}$ $\begin{array}{llllllllll}110 & 152 & 262 & 0.5 & -8.4-1884.22 & -4.12 & 0.00 & -5.10-1893.44 & 134.13\end{array}$ $\begin{array}{llllllllll}110 & 153 & 263 & 0.3 & -8.3-1892.56 & -4.29 & 0.90 & -5.03-1900.99 & 134.65\end{array}$ $\begin{array}{llllllllll}110 & 154 & 264 & 0.1 & -8.1-1900.78 & -4.35 & 0.00 & -4.97-1910.09 & 133.62\end{array}$ $\begin{array}{llllllllll}110 & 155 & 265 & -0.1 & -8.0-1908.86 & -4.59 & 0.89 & -4.90-1917.46 & 134.32\end{array}$ $\begin{array}{llllllllll}110 & 156 & 266 & -0.3 & -7.9-1916.82 & -4.53 & 0.00 & -4.84-1926.19 & 133.67\end{array}$ $\begin{array}{llllllllll}110 & 157 & 267 & -0.4 & -7.8-1924.65 & -4.93 & 0.89 & -4.77-1933.47 & 134.45\end{array}$ $\begin{array}{llllllllll}110 & 158 & 268 & -0.6 & -7.6-1932.36 & -5.07 & 0.00 & -4.71-1942.15 & 133.85\end{array}$ $\begin{array}{llllllllll}110 & 159 & 269 & -0.8 & -7.5-1939.95 & -5.60 & 0.89 & -4.65-1949.32 & 134.75\end{array}$ $\begin{array}{llllllllll}110 & 160 & 270 & -1.0 & -7.4-1947.42 & -5.85 & 0.00 & -4.59-1957.86 & 134.27\end{array}$ $\begin{array}{llllllllll}110 & 161 & 271 & -1.2 & -7.3-1954.77 & -6.34 & 0.88 & -4.54-1964.76 & 135.45\end{array}$ $\begin{array}{llllllllll}110 & 162 & 272 & -1.4 & -7.2-1962.00 & -6.55 & 0.00 & -4.48-1973.03 & 135.25\end{array}$ $\begin{array}{llllllllll}110 & 163 & 273 & -1.5 & -7.1-1969.11 & -6.79 & 0.88 & -4.42 & -1979.45 & 136.91\end{array}$ $\begin{array}{llllllllll}110 & 164 & 274 & -1.7 & -6.9-1976.11 & -6.40 & 0.00 & -4.37-1986.88 & 137.55\end{array}$ $\begin{array}{lllllllll}110 & 165 & 275 & -1.9 & -6.8-1982.99 & -6.15 & 0.88 & -4.32-1992.58 & 139.91\end{array}$ $\begin{array}{lllllllllll}110 & 166 & 276 & -2.1 & -6.7-1989.76 & -5.61 & 0.00 & -4.26-1999.63 & 140.93\end{array}$ $\begin{array}{llllllllll}110 & 167 & 277 & -2.3 & -6.6-1996.42 & -5.21 & 0.87 & -4.21-2004.97 & 143.67\end{array}$ $\begin{array}{lllllllll}110 & 168 & 278 & -2.4 & -6.5-2002.96 & -4.74 & 0.00 & -4.16-2011.87 & 144.84\end{array}$ $\begin{array}{llllllllll}110 & 169 & 279 & -2.6 & -6.4-2009.40 & -4.91 & 0.87 & -4.11-2017.56 & 147.22\end{array}$ $\begin{array}{lllllllll}110 & 170 & 280 & -2.8 & -6.3-2015.73 & -4.76 & 0.00 & -4.07-2024.56 & 148.30\end{array}$ $\begin{array}{lllllllll}110 & 171 & 281 & -3.0 & -6.2-2021.95 & -5.20 & 0.86 & -4.02-2030.31 & 150.62\end{array}$ $\begin{array}{llllllllll}110 & 172 & 282 & -3.1 & -6.1-2028.07 & -5.22 & 0.00 & -3.97-2037.26 & 151.73\end{array}$ $\begin{array}{llllllllll}110 & 173 & 283 & -3.3 & -6.0-2034.08 & -5.65 & 0.86 & -3.93-2042.80 & 154.27\end{array}$ $\begin{array}{llllllllll}110 & 174 & 284 & -3.5 & -5.9-2039.99 & -5.65 & 0.00 & -3.88-2049.52 & 155.62\end{array}$ $\begin{array}{llllllllll}110 & 175 & 285 & -3.6 & -5.7-2045.79 & -6.01 & 0.86 & -3.84 & -2054.78 & 158.43\end{array}$ $\begin{array}{lllllllll}110 & 176 & 286 & -3.8 & -5.6-2051.50 & -5.73 & 0.00 & -3.79-2061.02 & 160.26\end{array}$ $\begin{array}{lllllllllll}110 & 177 & 287 & -4.0 & -5.5-2057.10 & -5.74 & 0.85 & -3.75-2065.74 & 163.62\end{array}$ $\begin{array}{llllllllll}110 & 178 & 288 & -4.2 & -5.5-2062.60 & -5.83 & 0.00 & -3.71-2072.14 & 165.28\end{array}$ $\begin{array}{llllllllll}110 & 179 & 289 & -4.3 & -5.4-2068.01 & -6.16 & 0.85 & -3.67-2076.99 & 168.51\end{array}$ $\begin{array}{llllllllll}110 & 180 & 290 & -4.5 & -5.3-2073.32 & -5.83 & 0.00 & -3.63-2082.78 & 170.79\end{array}$

0.00

0.00 0.00

0.00 0.00

0.00

0.00

0.00

0.00

0.00

0.00

0.00

0.00

0.00

0.00

0.00

0.00

0.00

0.00

0.00

0.00

0.00

0.00

0.00

0.00

0.00

0.00

0.00

0.00

0.00

0.00

0.00

0.00

0.00

0.00

0.00

0.00

0.00

0.00

0.00

0.00

0.00

0.00

0.00

0.00

0.00

0.00

0.00

0.00

0.00

0.00

0.00

0.00

0.00

0.00

0.00

0.00

0.00

0.00

0.00

0.00

0.00

0.00

0.00

0.00

0.00

0.00

0.00
0.00

0.00

0.00

0.00

0.00

0.00

0.00

0.00

0.00

0.00

0.00

0.00

0.00

0.00

0.00

0.00

0.00

0.00

0.00

0.00

0.00

0.00

0.00

0.00

0.00

0.00

0.00

0.00

0.00

0.00

0.00

0.00

0.00

0.00

0.00

0.00

0.00

0.00

0.00

0.00

0.00

0.00

0.00

0.00

0.00

0.00

0.00

0.00

0.00

0.00

0.00

0.00

0.00

0.00

0.00

0.00

0.00

0.00

0.00

0.00

0.00

0.00

0.00

0.00
0.00

0.00

0.00

0.00

0.00

0.00

0.00

0.00

0.00

0.00

0.00

0.00

0.00

0.00

0.00

0.00

0.00

0.00

0.00

0.00

0.00

0.00

0.00

0.00

0.00

0.00

0.00

0.00

0.00

0.00

0.00

0.00

0.00

0.00

0.00

0.00

0.00

0.00

0.00

0.00

0.00

0.00

0.00

0.00

0.00

0.00

0.00

0.00

0.00

0.00

0.00

0.00

0.00

0.00

0.00

0.00

0.00

0.00

0.00

0.00

0.00

0.00

0.00

0.00

0.00

0.00 
$\begin{array}{llllllllll}110 & 181 & 291 & -4.7 & -5.2-2078.53 & -6.01 & 0.85 & -3.59-2087.28 & 174.36\end{array}$ $\begin{array}{llllllllll}110 & 182 & 292 & -4.8 & -5.1-2083.65 & -5.54 & 0.00 & -3.55-2092.74 & 176.97\end{array}$ $\begin{array}{llllllllll}110 & 183 & 293 & -5.0 & -5.0-2088.67 & -5.52 & 0.85 & -3.51-2096.86 & 180.92\end{array}$ $\begin{array}{lllllllll}110 & 184 & 294 & -5.1 & -4.9-2093.60 & -4.88 & 0.00 & -3.47-2101.95 & 183.90\end{array}$ $\begin{array}{lllllllll}110 & 185 & 295 & -5.3 & -4.8-2098.44 & -3.89 & 0.84 & -3.44-2104.93 & 189.00\end{array}$ $\begin{array}{llllllllll}110 & 186 & 296 & -5.5 & -4.7-2103.19 & -3.29 & 0.00 & -3.40-2109.88 & 192.12\end{array}$ $\begin{array}{llllllllll}110 & 187 & 297 & -5.6 & -4.6-2107.84 & -2.44 & 0.84 & -3.37-2112.81 & 197.26\end{array}$ $\begin{array}{lllllllll}110 & 188 & 298 & -5.8 & -4.5-2112.41 & -1.44 & 0.00 & -3.33-2117.18 & 200.96\end{array}$ $\begin{array}{lllllllll}110 & 189 & 299 & -5.9 & -4.4-2116.89 & -1.14 & 0.84 & -3.30-2120.49 & 205.72\end{array}$ $\begin{array}{lllllllll}110 & 190 & 300 & -6.1 & -4.3-2121.28 & 0.23 & 0.00 & -3.26-2124.31 & 209.97\end{array}$ $\begin{array}{llllllllll}110 & 191 & 301 & -6.3 & -4.3-2125.59 & -0.13 & 0.83 & -3.23-2128.11 & 214.24\end{array}$ $\begin{array}{lllllllll}110 & 192 & 302 & -6.4 & -4.2-2129.81 & 0.41 & 0.00 & -3.20-2132.59 & 217.83\end{array}$ $\begin{array}{llllllllll}110 & 193 & 303 & -6.6 & -4.1-2133.94 & -0.11 & 0.83 & -3.16-2136.38 & 222.11\end{array}$ $\begin{array}{llllllllll}110 & 194 & 304 & -6.7 & -4.0-2137.99 & -0.11 & 0.00 & -3.13-2141.24 & 225.33\end{array}$ $\begin{array}{llllllllll}110 & 195 & 305 & -6.9 & -3.9-2141.96 & -0.46 & 0.83 & -3.10-2144.70 & 229.94\end{array}$ $\begin{array}{llllllllll}110 & 196 & 306 & -7.0 & -3.8-2145.85 & -0.54 & 0.00 & -3.07-2149.46 & 233.25\end{array}$ $\begin{array}{llllllllll}110 & 197 & 307 & -7.2 & -3.8-2149.65 & -0.97 & 0.82 & -3.04-2152.84 & 237.94\end{array}$ $\begin{array}{llllllllll}110 & 198 & 308 & -7.3 & -3.7-2153.38 & -1.13 & 0.00 & -3.01-2157.52 & 241.33\end{array}$ $\begin{array}{llllllllll}110 & 199 & 309 & -7.5 & -3.6-2157.02 & -1.64 & 0.82 & -2.98-2160.82 & 246.10\end{array}$ $\begin{array}{llllllllll}110 & 200 & 310 & -7.6 & -3.5-2160.59 & -1.83 & 0.00 & -2.95-2165.38 & 249.62\end{array}$ $\begin{array}{llllllllll}110 & 201 & 311 & -7.8 & -3.4-2164.08 & -2.27 & 0.82 & -2.93-2168.46 & 254.61\end{array}$ $\begin{array}{llllllllll}110 & 202 & 312 & -7.9 & -3.4-2167.49 & -2.23 & 0.00 & -2.90-2172.62 & 258.52\end{array}$ $\begin{array}{lllllllllll}110 & 203 & 313 & -8.1 & -3.3-2170.83 & -2.45 & 0.82 & -2.87-2175.33 & 263.88\end{array}$ $\begin{array}{lllllllll}110 & 204 & 314 & -8.2 & -3.2-2174.09 & -2.36 & 0.00 & -2.84-2179.29 & 267.99\end{array}$

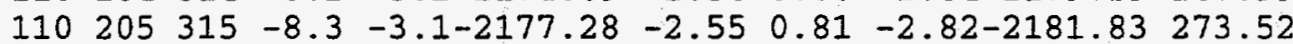
$\begin{array}{llllllllll}110 & 206 & 316 & -8.5 & -3.1-2180.39 & -2.40 & 0.00 & -2.79-2185.58 & 277.84\end{array}$ $\begin{array}{llllllllll}110 & 207 & 317 & -8.6 & -3.0-2183.43 & -2.65 & 0.81 & -2.77-2188.03 & 283.46\end{array}$

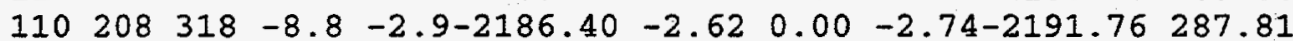
$\begin{array}{llllllllll}110 & 209 & 319 & -8.9 & -2.9-2189.29 & -2.99 & 0.81 & -2.72-2194.19 & 293.45\end{array}$ $\begin{array}{llllllllll}110 & 210 & 320 & -9.1 & -2.8-2192.12 & -3.01 & 0.00 & -2.69-2197.82 & 297.89\end{array}$ $\begin{array}{lllllllllll}110 & 211 & 321 & -9.2 & -2.7 & -2194.87 & -3.44 & 0.81 & -2.67-2200.17 & 303.61\end{array}$ $\begin{array}{lllllllll}110 & 212 & 322 & -9.3 & -2.6-2197.56 & -3.42 & 0.00 & -2.64-2203.62 & 308.23\end{array}$ $\begin{array}{lllllllllll}110 & 213 & 323 & -9.5 & -2.6-2200.17 & -3.75 & 0.80 & -2.62-2205.74 & 314.18\end{array}$ $\begin{array}{llllllllll}110 & 214 & 324 & -9.6 & -2.5-2202.72 & -3.56 & 0.00 & -2.60-2208.88 & 319.12\end{array}$ $\begin{array}{llllllllll}110 & 215 & 325 & -9.7 & -2.4-2205.20 & -3.72 & 0.80 & -2.57-2210.70 & 325.37\end{array}$

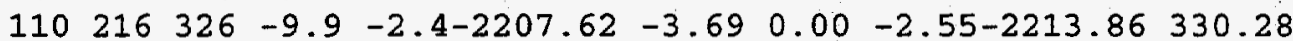
$\begin{array}{lllllllll}110 & 217 & 327-10.0 & -2.3-2209.97 & -3.96 & 0.80 & -2.53-2215.66 & 336.55\end{array}$ $\begin{array}{lllllllll}110 & 218 & 328-10.1 & -2.2-2212.25 & -2.22 & 0.00 & -2.51-2216.98 & 343.30\end{array}$ $\begin{array}{lllllllll}110 & 219 & 329-10.3 & -2.2-2214.47 & -2.42 & 0.80 & -2.49-2218.58 & 349.77\end{array}$ $\begin{array}{llllllll}110 & 220 & 330-10.4 & -2.1-2216.63 & -2.43 & 0.00 & -2.47-2221.52 & 354.90\end{array}$ $\begin{array}{lllllllll}110 & 221 & 331-10.5 & -2.1-2218.72 & -2.98 & 0.79 & -2.45-2223.35 & 361.14\end{array}$ $\begin{array}{lllllllll}110 & 222 & 332-10.7 & -2.0-2220.75 & -3.17 & 0.00 & -2.42-2226.35 & 366.22\end{array}$ $\begin{array}{lllllllll}110 & 223 & 333-10.8 & -1.9-2222.72 & -3.65 & 0.79 & -2.40-2227.98 & 372.65\end{array}$ $\begin{array}{lllllllll}110 & 224 & 334-10.9 & -1.9-2224.63 & -3.68 & 0.00 & -2.38-2230.69 & 378.02\end{array}$ $110 \quad 225 \quad 335-11.1-1.8-2226.48-4.50 \quad 0.79-2.37-2232.55 \quad 384.23$ $\begin{array}{lllllllll}110 & 226 & 336-11.2 & -1.8-2228.26 & -5.37 & 0.00 & -2.35-2235.98 & 388.87\end{array}$ $\begin{array}{llllllllll}110 & 227 & 337-11.3 & -1.7-2229.99 & -6.44 & 0.79 & -2.33-2237.97 & 394.95\end{array}$ $110228 \quad 338-11.4-1.6-2231.66-7.24 \quad 0.00-2.31-2241.21399 .79$ $110229339-11.6-1.6-2233.27-7.50 \quad 0.78-2.29-2242.28 \quad 406.79$ $\begin{array}{llllllllll}111 & 148 & 259 & 1.7 & -9.1-1848.07 & -2.52 & 1.00 & -5.49-1855.08 & 147.47\end{array}$ $\begin{array}{lllllllllll}111 & 149 & 260 & 1.5 & -9.0-1857.14 & -2.95 & 1.74 & -5.41-1863.76 & 146.86\end{array}$ $111150 \quad 261$ $\begin{array}{lll}111 & 151 & 262\end{array}$ $\begin{array}{llll}111 & 152 & 263\end{array}$ $\begin{array}{llll}111 & 153 & 264\end{array}$ 111154265 $\begin{array}{llll}111 & 155 & 266\end{array}$ $\begin{array}{llll}111 & 156 & 267\end{array}$ $1.3-8.9-1866.07-3.321 .00-5.34-1873.73 .144 .96$ $\begin{array}{llllllll}1.1 & -8.7-1874.87 & -3.78 & 1.74 & -5.27-1882.17 & 144.59\end{array}$ $0.9-8.6-1883.53-3.93 \quad 1.00-5.20-1891.66 \quad 143.18$ $0.7-8.5-1892.06-4.091 .74-5.13-1899.54143 .36$ $0.5-8.3-1900.46-4.151 .00-5.06-1908.67 \quad 142.30$ $0.3-8.2-1908.74-4.411 .73-4.99-1916.41142 .64$ $0.1-8.1-1916.88-4.44 \quad 1.00-4.93-1925.25 \quad 141.87$ $\begin{array}{llllllllll}111 & 157 & 268 & -0.1 & -8.0-1924.91 & -4.82 & 1.73 & -4.86-1932.86 & 142.33\end{array}$ $\begin{array}{llllllllll}111 & 158 & 269 & -0.2 & -7.8-1932.80 & -4.96 & 1.00 & -4.80-1941.56 & 141.70\end{array}$ $\begin{array}{llllllllll}111 & 159 & 270 & -0.4 & -7.7 & -1940.58 & -5.48 & 1.73 & -4.74-1949.07 & 142.26\end{array}$ $\begin{array}{llllllllll}111 & 160 & 271 & -0.6 & -7.6-1948.23 & -5.71 & 1.00 & -4.68-1957.62 & 141.79\end{array}$ $\begin{array}{llllllllll}111 & 161 & 272 & -0.8 & -7.5-1955.76 & -6.20 & 1.72 & -4.62-1964.85 & 142.62\end{array}$ $\begin{array}{lllllllllll}111 & 162 & 273 & -1.0 & -7.3-1963.17 & -6.41 & 1.00 & -4.56-1973.14 & 142.40\end{array}$ $\begin{array}{llllllllll}111 & 163 & 274 & -1.2 & -7.2-1970.46 & -6.70 & 1.72 & -4.51-1979.95 & 143.67\end{array}$ $\begin{array}{lllllllllll}111 & 164 & 275 & -1.3 & -7.1-1977.64 & -6.38 & 1.00 & -4.45-1987.47 & 144.22\end{array}$ 124
0.00

0.00

0.00

0.00

0.00

0.00

0.00

0.00

0.00

0.00

0.00

0.00

0.00

0.00

0.00

0.00

0.00

0.00

0.00

0.00

0.00

0.00

0.00

0.00

0.00

0.00

0.00

0.00

0.00

0.00

0.00

0.00

0.00

.00

0.00

0.00

0.00

0.00

0.00

0.00

0.00

0.00

0.00

0.00

0.00

0.00

0.00

0.00

0.00

0.00

0.00

0.00

0.00

.

0.00

0.00

0.00

0.00

0.00

0.00

0.00

0.00

0.00

0.00

0.00

0.00

0.00

0.00

0.00

0.00

0.00

0.00

0.00

0.00

0.00

0.00

0.00

0.00

0.00

0.00

0.00

0.00

0.00

0.00

0.00

0.00

0.00

0.00

0.00

0.00

0.00

0.00

0.00

0.00

0.00

0.00

0.00

0.00

0.00

$0.00 \quad 0.00$

$0.00 \quad 0.00$

$0.00 \quad 0.00$

0.00

0.00

$0.00 \quad 0.00$

$0.00 \quad 0.00$

0.00

0.00

0.00

0.00

$0.00 \quad 0.00$

$0.00 \quad 0.00$

0.00

0.00

0.00

0.00

0.00

0.00

$0.00 \quad 0.00$

0.00

0.00

0.00

0.00

$0.00 \quad 0.00$

$0.00 \quad 0.00$

0.00

0.00

0.00

0.00

$0.00 \quad 0.00$

$0.00 \quad 0.00$

$0.00 \quad 0.00$

0.00

0.00

$0.00 \quad 0.00$

$0.00 \quad 0.00$

0.00

0.00

0.00

0.00

0.00

0.00

$0.00 \quad 0.00$

0.00

0.00

0.00

0.00

0.00

0.00

$0.00 \quad 0.00$

$0.00 \quad 0.00$

0.00

0.00

$0.00 \quad 0.00$

$0.00 \quad 0.00$

$0.00 \quad 0.00$

0.00

0.00

$0.00 \quad 0.00$

$0.00 \quad 0.00$

$0.00 \quad 0.00$ 
$\begin{array}{llllllllllll}111 & 165 & 276 & -1.5 & -7.0-1984.70 & -6.19 & 1.72 & -4.40 & -1993.57 & 146.19\end{array}$ $\begin{array}{lllllllllll}111 & 166 & 277 & -1.7 & -6.9-1991.65 & -5.69 & 1.00 & -4.34-2000.68 & 147.15\end{array}$ $\begin{array}{lllllllllll}111 & 167 & 278 & -1.9 & -6.8-1998.49 & -5.36 & 1.72 & -4.29-2006.42 & 149.48\end{array}$ $\begin{array}{lllllllllll}111 & 168 & 279 & -2.0 & -6.7-2005.21 & -5.12 & 1.00 & -4.24-2013.57 & 150.40\end{array}$ $\begin{array}{llllllllll}111 & 169 & 280 & -2.2 & -6.6-2011.82 & -5.45 & 1.71 & -4.19-2019.75 & 152.30\end{array}$ $\begin{array}{lllllllllll}111 & 170 & 281 & -2.4 & -6.4-2018.33 & -5.43 & 1.00 & -4.14-2026.90 & 153.22\end{array}$ $\begin{array}{llllllllll}111 & 171 & 282 & -2.6 & -6.3-2024.72 & -5.88 & 1.71 & -4.09-2032.98 & 155.21\end{array}$ $\begin{array}{llllllllll}111 & 172 & 283 & -2.7 & -6.2-2031.01 & -5.94 & 1.00 & -4.04-2040.00 & 156.26\end{array}$ $\begin{array}{lllllllllll}111 & 173 & 284 & -2.9 & -6.1-2037.20 & -6.34 & 1.71 & -4.00-2045.83 & 158.51\end{array}$ $\begin{array}{llllllllll}111 & 174 & 285 & -3.1 & -6.0-2043.28 & -6.35 & 1.00 & -3.95-2052.58 & 159.83\end{array}$ $\begin{array}{llllllllll}111 & 175 & 286 & -3.3 & -5.9-2049.25 & -6.71 & 1.70 & -3.91-2058.16 & 162.31\end{array}$ $\begin{array}{llllllllll}111 & 176 & 287 & -3.4 & -5.8-2055.12 & -6.49 & 1.00 & -3.86-2064.48 & 164.07\end{array}$ $\begin{array}{llllllllll}111 & 177 & 288 & -3.6 & -5.7-2060.89 & -6.56 & 1.70 & -3.82-2069.57 & 167.05\end{array}$ $\begin{array}{llllllllll}111 & 178 & 289 & -3.8 & -5.6-2066.57 & -6.71 & 1.00 & -3.78-2076.05 & 168.64\end{array}$ $\begin{array}{llllllllll}111 & 179 & 290 & -3.9 & -5.5-2072.14 & -7.01 & 1.70 & -3.74-2081.18 & 171.58\end{array}$ $\begin{array}{llllllllll}111 & 180 & 291 & -4.1 & -5.4-2077.61 & -6.66 & 1.00 & -3.69-2086.97 & 173.86\end{array}$ $\begin{array}{llllllllll}111 & 181 & 292 & -4.3 & -5.3-2082.99 & -6.82 & 1.70 & -3.65-2091.77 & 177.14\end{array}$ $\begin{array}{lllllllllll}111 & 182 & 293 & -4.4 & -5.2-2088.27 & -6.35 & 1.00 & -3.61-2097.24 & 179.74\end{array}$ $\begin{array}{llllllllll}111 & 183 & 294 & -4.6 & -5.1-2093.46 & -6.29 & 1.69 & -3.58-2101.63 & 183.42\end{array}$ $\begin{array}{llllllllll}111 & 184 & 295 & -4.8 & -5.0-2098.55 & -5.65 & 1.00 & -3.54-2106.74 & 186.38\end{array}$ $\begin{array}{llllllllll}111 & 185 & 296 & -4.9 & -4.9-2103.55 & -4.70 & 1.69 & -3.50-2110.06 & 191.13\end{array}$ $\begin{array}{lllllllllll}111 & 186 & 297 & -5.1 & -4.9-2108.46 & -4.07 & 1.00 & -3.46-2114.99 & 194.27\end{array}$ $\begin{array}{llllllllll}111 & 187 & 298 & -5.2 & -4.8-2113.27 & -2.94 & 1.69 & -3.43-2117.95 & 199.38\end{array}$ $\begin{array}{lllllllllll}111 & 188 & 299 & -5.4 & -4.7-2118.00 & -2.30 & 1.00 & -3.39-2122.69 & 202.71\end{array}$ $\begin{array}{llllllllll}111 & 189 & 300 & -5.6 & -4.6-2122.64 & -1.22 & 1.69 & -3.36-2125.52 & 207.95\end{array}$ $\begin{array}{llllllllll}111 & 190 & 301 & -5.7 & -4.5-2127.18 & -0.94 & 1.00 & -3.32-2130.45 & 211.10\end{array}$ $\begin{array}{llllllllll}111 & 191 & 302 & -5.9 & -4.4-2131.65 & -0.53 & 1.69 & -3.29-2133.78 & 215.84\end{array}$ $\begin{array}{llllllllll}111 & 192 & 303 & -6.0 & -4.3-2136.02 & 0.05 & 1.00 & -3.25-2138.23 & 219.46\end{array}$ $\begin{array}{llllllllll}111 & 193 & 304 & -6.2 & -4.2-2140.31 & 0.32 & 1.68 & -3.22-2141.53 & 224.23\end{array}$ $\begin{array}{llllllllll}111 & 194 & 305 & -6.3 & -4.2-2144.52 & -0.21 & 1.00 & -3.19-2146.92 & 226.92\end{array}$ $\begin{array}{llllllllll}111 & 195 & 306 & -6.5 & -4.1-2148.64 & -0.52 & 1.68 & -3.16-2150.63 & 231.27\end{array}$ $\begin{array}{llllllllll}111 & 196 & 307 & -6.6 & -4.0-2152.68 & -0.60 & 1.00 & -3.13-2155.40 & 234.57\end{array}$ $\begin{array}{llllllllll}111 & 197 & 308 & -6.8 & -3.9-2156.63 & -1.03 & 1.68 & -3.10-2159.08 & 238.97\end{array}$ $\begin{array}{llllllllll}111 & 198 & 309 & -6.9 & -3.8-2160.51 & -1.18 & 1.00 & -3.07-2163.75 & 242.36\end{array}$ $\begin{array}{lllllllllll}111 & 199 & 310 & -7.1 & -3.8-2164.30 & -1.63 & 1.68 & -3.04-2167.29 & 246.90\end{array}$ $\begin{array}{lllllllllll}111 & 200 & 311 & -7.2 & -3.7-2168.02 & -1.81 & 1.00 & -3.01-2171.84 & 250.43\end{array}$ $\begin{array}{llllllllll}111 & 201 & 312 & -7.4 & -3.6-2171.65 & -2.21 & 1.67 & -2.98-2175.17 & 255.17\end{array}$ $\begin{array}{llllllllll}111 & 202 & 313 & -7.5 & -3.5-2175.21 & -2.14 & 1.00 & -2.95-2179.30 & 259.10\end{array}$ $\begin{array}{lllllllllll}111 & 203 & 314 & -7.7 & -3.4-2178.70 & -2.35 & 1.67 & -2.92-2182.29 & 264.18\end{array}$ $\begin{array}{llllllllll}111 & 204 & 315 & -7.8 & -3.4-2182.10 & -2.26 & 1.00 & -2.89-2186.26 & 268.29\end{array}$ $\begin{array}{llllllllll}111 & 205 & 316 & -8.0 & -3.3-2185.43 & -2.45 & 1.67 & -2.87 & -2189.08 & 273.54\end{array}$ $\begin{array}{llllllllll}111 & 206 & 317 & -8.1 & -3.2-2188.69 & -2.26 & 1.00 & -2.84-2192.79 & 277.90\end{array}$ $\begin{array}{llllllllll}111 & 207 & 318 & -8.2 & -3.1-2191.87 & -2.50 & 1.67 & -2.81-2195.52 & 283.25\end{array}$ $\begin{array}{lllllllllll}111 & 208 & 319 & -8.4 & -3.1-2194.98 & -2.41 & 1.00 & -2.79-2199.18 & 287.65\end{array}$ $\begin{array}{llllllllll}111 & 209 & 320 & -8.5 & -3.0-2198.02 & -2.72 & 1.67 & -2.76-2201.83 & 293.07\end{array}$ $\begin{array}{llllllllll}111 & 210 & 321 & -8.7 & -2.9-2200.98 & -2.74 & 1.00 & -2.74-2205.46 & 297.52\end{array}$ $\begin{array}{llllllllll}111 & 211 & 322 & -8.8 & -2.9-2203.88 & -3.12 & 1.66 & -2.71-2208.04 & 303.00\end{array}$ $\begin{array}{llllllllll}111 & 212 & 323 & -8.9 & -2.8-2206.70 & -3.13 & 1.00 & -2.69-2211.52 & 307.60\end{array}$ $\begin{array}{llllllllll}111 & 213 & 324 & -9.1 & -2.7-2209.45 & -3.40 & 1.66 & -2.67-2213.86 & 313.33\end{array}$ $\begin{array}{llllllllll}111 & 214 & 325 & -9.2 & -2.6-2212.14 & -3.05 & 1.00 & -2.64-2216.83 & 318.43\end{array}$ $\begin{array}{llllllllll}111 & 215 & 326 & -9.4 & -2.6-2214.76 & -3.35 & 1.66 & -2.62-2219.06 & 324.27\end{array}$ $\begin{array}{lllllllllll}111 & 216 & 327 & -9.5 & -2.5 & -2217.31 & -3.36 & 1.00 & -2.60 & -2222.26 & 329.14\end{array}$ $\begin{array}{lllllllllll}111 & 217 & 328 & -9.6 & -2.4-2219.79 & -3.35 & 1.66 & -2.57-2224.06 & 335.42\end{array}$

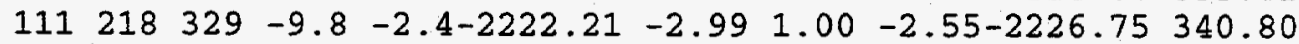
$\begin{array}{lllllllllll}111 & 219 & 330 & -9.9 & -2.3-2224.56 & -3.17 & 1.66 & -2.53 & -2228.60 & 347.02\end{array}$ $\begin{array}{llllllllll}111 & 220 & 331-10.0 & -2.3-2226.85 & -3.19 & 1.00 & -2.51-2231.55 & 352.14\end{array}$ $\begin{array}{lllllllll}111 & 221 & 332-10.2 & -2.2-2229.07 & -3.57 & 1.66 & -2.49-2233.47 & 358.29\end{array}$ $\begin{array}{lllllllll}111 & 222 & 333-10.3 & -2.1-2231.23 & -3.77 & 1.00 & -2.47-2236.47 & 363.36\end{array}$ $\begin{array}{lllllllll}111 & 223 & 334-10.4 & -2.1-2233.33 & -4.13 & 1.65 & -2.45-2238.25 & 369.65\end{array}$ $\begin{array}{llllllllll}111 & 224 & 335-10.6 & -2.0-2235.37 & -4.47 & 1.00 & -2.43-2241.26 & 374.71\end{array}$ $\begin{array}{lllllllll}111 & 225 & 336-10.7 & -1.9-2237.34 & -5.21 & 1.65 & -2.41-2243.31 & 380.74\end{array}$ $\begin{array}{lllllllll}111 & 226 & 337-10.8 & -1.9-2239.26 & -6.26 & 1.00 & -2.39-2246.90 & 385.21\end{array}$ $\begin{array}{llllllllll}111 & 227 & 338-10.9 & -1.8-2241.11 & -7.34 & 1.65 & -2.37-2249.17 & 391.02\end{array}$ $\begin{array}{lllllllll}111 & 228 & 339-11.1 & -1.8-2242.91 & -8.16 & 1.00 & -2.35-2252.41 & 395.85\end{array}$ $\begin{array}{lllllllllll}112 & 150 & 262 & 1.7 & -9.1-1864.60 & -2.80 & 0.00 & -5.44-1872.84 & 153.11\end{array}$ $\begin{array}{llllllllll}112 & 151 & 263 & 1.5 & -8.9-1873.60 & -3.21 & 0.90 & -5.36-1881.27 & 152.76\end{array}$

0.00 0.00 0.00 0.00 0.00

0.00

0.00

0.00

0.00

0.00

0.00

0.00

0.00

0.00

0.00

0.00

0.00

0.00

0.00

0.00

0.00

0.00

0.00

0.00

0.00

0.00

0.00

0.00

0.00

0.00

0.00

0.00

0.00

0.00

0.00

0.00

0.00

0.00

0.00

0.00

0.00

0.00

0.00

0.00

0.00

0.00

0.00

0.00

0.00

0.00

0.00

0.00

0.00

0.00

0.00

0.00

0.00

0.00

0.00

0.00

0.00

0.00

0.00

0.00

0.00

0.00

0.00

0.00
0.00

0.00

0.00

0.00

0.00

0.00

0.00

0.00

0.00

0.00

0.00

0.00

0.00

0.00

0.00

0.00

0.00

0.00

0.00

0.00

0.00

0.00

0.00

0.00

0.00

0.00

0.00

0.00

0.00

0.00

0.00

0.00

0.00

0.00

0.00

0.00

0.00

0.00

0.00

0.00

0.00

0.00

0.00

0.00

0.00

0.00

0.00

0.00

0.00

0.00

0.00

0.00

0.00

0.00

0.00

0.00

0.00

0.00

0.00

0.00

0.00

0.00

0.00

0.00
0.00

0.00

0.00

0.00

0.00

0.00

0.00

0.00

0.00

0.00

0.00

0.00

0.00

0.00

0.00

0.00

0.00

0.00

0.00

0.00

0.00

0.00

0.00

0.00

0.00

0.00

0.00

0.00

0.00

0.00

0.00

0.00

0.00

0.00

0.00

0.00

0.00

0.00

0.00

0.00

0.00

0.00

0.00

0.00

0.00

0.00

0.00

0.00

0.00

0.00

0.00

0.00

0.00

0.00

0.00

0.00

0.00

0.00

0.00

0.00

0.00

0.00

0.00

0.00

0.00

0.00 
$\begin{array}{lll}112 & 152 & 264\end{array}$ $\begin{array}{lll}112 & 153 & 265\end{array}$

$\begin{array}{lll}112 & 154 & 266\end{array}$

$\begin{array}{lll}112 & 155 & 267\end{array}$

$\begin{array}{lll}112 & 156 & 268\end{array}$

$\begin{array}{lll}112 & 157 & 269\end{array}$

$\begin{array}{lll}112 & 158 & 270\end{array}$

$\begin{array}{lll}112 & 159 & 271\end{array}$

$\begin{array}{lll}112 & 160 & 272\end{array}$

112161273

$\begin{array}{lll}112 & 162 & 274\end{array}$

$\begin{array}{lll}112 & 163 & 275\end{array}$

112164276

$\begin{array}{lll}112 & 165 & 277\end{array}$

$\begin{array}{lll}112 & 166 & 27\end{array}$

$\begin{array}{lllllllll}112 & 167 & 279 & -1.5 & -7.0-2000.17 & -5.14 & 0.87 & -4.37-2008.80 & 154.36\end{array}$

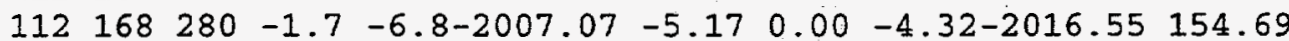

$\begin{array}{lllllllll}112 & 169 & 281 & -1.8 & -6.7-2013.86 & -5.62 & 0.87 & -4.27-2022.87 & 156.44\end{array}$

$\begin{array}{lllllllll}112 & 170 & 282 & -2.0 & -6.6-2020.54 & -5.75 & 0.00 & -4.22-2030.50 & 156.88\end{array}$

$\begin{array}{lllllllll}112 & 171 & 283 & -2.2 & -6.5-2027.10 & -6.19 & 0.86 & -4.17-2036.60 & 158.86\end{array}$

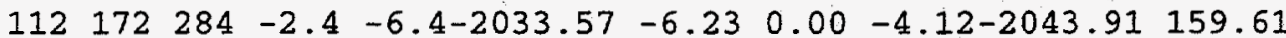

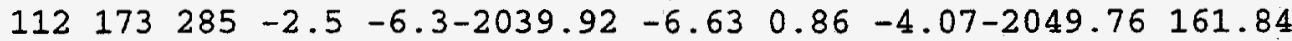

$\begin{array}{llllllllll}112 & 174 & 286 & -2.7 & -6.2-2046.17 & -6.64 & 0.00 & -4.02-2056.84 & 162.83\end{array}$

$\begin{array}{lllllllll}112 & 175 & 287 & -2.9 & -6.1-2052.32 & -6.99 & 0.86 & -3.98-2062.43 & 165.31\end{array}$

$\begin{array}{llllllllll}112 & 176 & 288 & -3.0 & -6.0 & -2058.36 & -6.93 & 0.00 & -3.93-2069.22 & 166.59\end{array}$

$\begin{array}{lllllllll}112 & 177 & 289 & -3.2 & -5.9-2064.30 & -7.29 & 0.85 & -3.89-2074.62 & 169.26\end{array}$

$\begin{array}{lllllllll}112 & 178 & 290 & -3.4 & -5.8-2070.14 & -7.44 & 0.00 & -3.84-2081.43 & 170.53\end{array}$

$\begin{array}{lllllllll}112 & 179 & 291 & -3.5 & -5.7-2075.88 & -7.71 & 0.85 & -3.80-2086.54 & 173.49\end{array}$

$\begin{array}{lllllllll}112 & 180 & 292 & -3.7 & -5.6-2081.52 & -7.39 & 0.00 & -3.76-2092.67 & 175.43\end{array}$

$\begin{array}{lllllllll}112 & 181 & 293 & -3.9 & -5.5-2087.06 & -7.58 & 0.85 & -3.72-2097.51 & 178.66\end{array}$

$\begin{array}{llllllllll}112 & 182 & 294 & -4.0 & -5.4-20.92 .51 & -7.10 & 0.00 & -3.68-2103.29 & 180.95\end{array}$

$\begin{array}{lllllllll}112 & 183 & 295 & -4.2 & -5.3-2097.86 & -7.02 & 0.85 & -3.64-2107.67 & 184.64\end{array}$

$\begin{array}{lllllllll}112 & 184 & 296 & -4.4 & -5.2-2103.11 & -6.37 & 0.00 & -3.60-2113.08 & 187.30\end{array}$

$\begin{array}{lllllllll}112 & 185 & 297 & -4.5 & -5.1-2108.27 & -5.48 & 0.84 & -3.56-2116.47 & 191.98\end{array}$

$\begin{array}{llllllllll}112 & 186 & 298 & -4.7 & -5.0-2113.34 & -4.78 & 0.00 & -3.52-2121.65 & 194.88\end{array}$

$\begin{array}{lllllllll}112 & 187 & 299 & -4.8 & -4.9-2118.32 & -3.69 & 0.84 & -3.49-2124.65 & 199.94\end{array}$

$\begin{array}{lllllllll}112 & 188 & 300 & -5.0 & -4.8-2123.20 & -2.93 & 0.00 & -3.45-2129.58 & 203.09\end{array}$

$\begin{array}{lllllllll}112 & 189 & 301 & -5.2 & -4.7-2128.00 & -2.02 & 0.84 & -3.41-2132.60 & 208.14\end{array}$

$\begin{array}{lllllllll}112 & 190 & 302 & -5.3 & -4.7-2132.70 & -1.25 & 0.00 & -3.38-2137.33 & 211.48\end{array}$

$\begin{array}{lllllllll}112 & 191 & 303 & -5.5 & -4.6-2137.32 & -0.64 & 0.83 & -3.35-2140.47 & 216.41\end{array}$

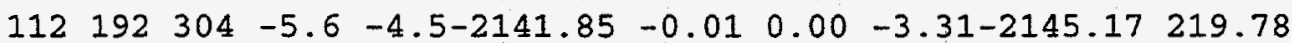

$\begin{array}{lllllllll}112 & 193 & 305 & -5.8 & -4.4-2146.30 & 0.41 & 0.83 & -3.28-2148.33 & 224.69\end{array}$

$\begin{array}{lllllllll}112 & 194 & 306 & -5.9 & -4.3-2150.65 & 1.47 & 0.00 & -3.24-2152.43 & 228.67\end{array}$

$\begin{array}{lllllllll}112 & 195 & 307 & -6.1 & -4.2-2154.93 & -0.26 & 0.83 & -3.21-2157.57 & 231.59\end{array}$

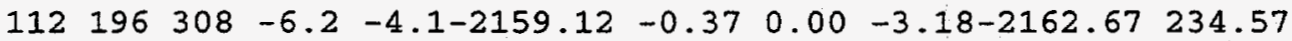

$\begin{array}{llllllllll}112 & 197 & 309 & -6.4 & -4.1-2163.23 & -0.76 & 0.82 & -3.15-2166.31 & 239.00\end{array}$

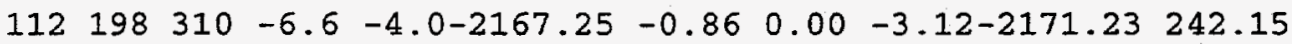

$\begin{array}{lllllllll}112 & 199 & 311 & -6.7 & -3.9-2171.20 & -1.35 & 0.82 & -3.09-2174.81 & 246.64\end{array}$

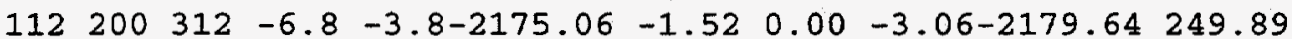

$\begin{array}{lllllllll}112 & 201 & 313 & -7.0 & -3.7-2178.85 & -1.94 & 0.82 & -3.03-2183.00 & 254.60\end{array}$

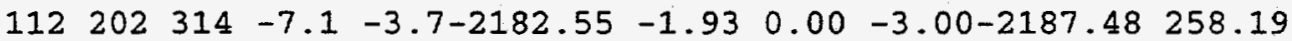

$\begin{array}{lllllllll}112 & 203 & 315 & -7.3 & -3.6-2186.18 & -2.10 & 0.82 & -2.97-2190.44 & 263.30\end{array}$

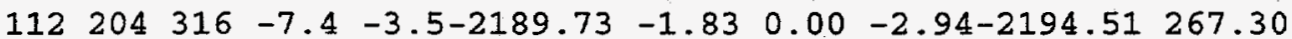

$\begin{array}{lllllllll}112 & 205 & 317 & -7.6 & -3.4-2193.21 & -2.00 & 0.81 & -2.92-2197.31 & 272.57\end{array}$

$\begin{array}{lllllllll}112 & 206 & 318 & -7.7 & -3.4-2196.61 & -1.82 & 0.00 & -2.89-2201.32 & 276.64\end{array}$

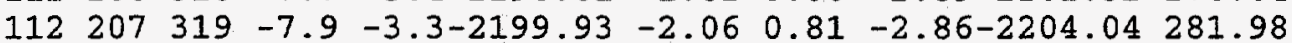

$\begin{array}{lllllllll}112 & 208 & 320 & -8.0 & -3.2-2203.18 & -1.96 & 0.00 & -2.84-2207.98 & 286.12\end{array}$

$\begin{array}{lllllllll}112 & 209 & 321 & -8.2 & -3.1-2206.36 & -2.30 & 0.81 & -2.81-2210.66 & 291.50\end{array}$

$\begin{array}{lllllllll}112 & 210 & 322 & -8.3 & -3.1-2209.47 & -2.28 & 0.00 & -2.79-2214.53 & 295.71\end{array}$

$\begin{array}{lllllllll}112 & 211 & 323 & -8.4 & -3.0-2212.50 & -2.73 & 0.81 & -2.76-2217.18 & 301.13\end{array}$

$\begin{array}{lllllllll}112 & 212 & 324 & -8.6 & -2.9-2215.46 & -2.11 & 0.00 & -2.74-2220.31 & 306.07\end{array}$

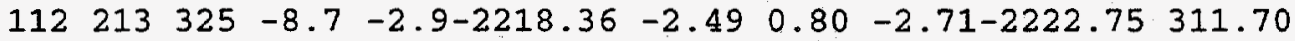

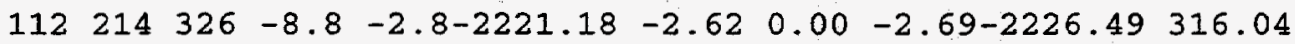

$\begin{array}{lllllllll}112 & 215 & 327 & -9.0 & -2.7-2223.93 & -2.97 & 0.80 & -2.66-2228.77 & 321.83\end{array}$

$\begin{array}{lllllllll}112 & 216 & 328 & -9.1 & -2.6-2226.62 & -2.95 & 0.00 & -2.64-2232.21 & 326.46\end{array}$

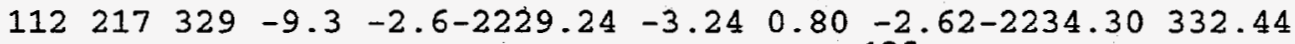

0.00

0.00

0.00

0.00

0.00

0.00

0.00

0.00

0.00

0.00

0.00

0.00

0.00

0.00

0.00

0.00

0.00

0.00

0.00

0.00

0.00

0.00

0.00

0.00

0.00

0.00

0.00

0.00

0.00

0.00

0.00

0.00

0.00

0.00

0.00

0.00

0.00

0.00

0.00

0.00

0.00

0.00

0.00

0.00

0.00

0.00

0.00

0.00

0.00

0.00

0.00

0.00

0.00

0.00

0.00

0.00

0.00

0.00

0.00

0.00

0.00

0.00

0.00

0.00

0.00

0.00
0.00

0.00

0.00

0.00

0.00

0.00

0.00

0.00

0.00

0.00

0.00

0.00

0.00

0.00

0.00

0.00

0.00

0.00

0.00

0.00

0.00

0.00

0.00

0.00

0.00

0.00

0.00

0.00

0.00

0.00

0.00

0.00

0.00

0.00

0.00

0.00

0.00

0.00

0.00

0.00

0.00

0.00

0.00

0.00

0.00

0.00

0.00

0.00

0.00

0.00

0.00

0.00

0.00

0.00

0.00

0.00

0.00

0.00

0.00

0.00

0.00

0.00

0.00

0.00

0.00

0.00
0.00

0.00

0.00

0.00

0.00

0.00

0.00

0.00

0.00

0.00

0.00

0.00

0.00

0.00

0.00

0.00

0.00

0.00

0.00

0.00

0.00

0.00

0.00

0.00

0.00

0.00

0.00

0.00

0.00

0.00

0.00

0.00

0.00

0.00

0.00

0.00

0.00

0.00

0.00

0.00

0.00

0.00

0.00

0.00

0.00

0.00

0.00

0.00

0.00

0.00

0.00

0.00

0.00

0.00

0.00

0.00

0.00

0.00

0.00

0.00

0.00

0.00

0.00

0.00

0.00

0.00 
$\begin{array}{lllllllllll}112 & 218 & 330 & -9.4 & -2.5-2231.79 & -3.13 & 0.00 & -2.59-2237.51 & 337.30\end{array}$ $\begin{array}{lllllllll}112 & 219 & 331 & -9.5 & -2.4-2234.28 & -3.23 & 0.80 & -2.57-2239.28 & 343.60\end{array}$ $\begin{array}{llllllllll}112 & 220 & 332 & -9.7 & -2.4-2236.69 & -3.33 & 0.00 & -2.55-2242.58 & 348.38\end{array}$ $\begin{array}{llllllllll}112 & 221 & 333 & -9.8 & -2.3-2239.05 & -3.72 & 0.79 & -2.53-2244.51 & 354.52\end{array}$ $\begin{array}{llllllllll}112 & 222 & 334 & -9.9 & -2.3-2241.34 & -3.94 & 0.00 & -2.51-2247.79 & 359.31\end{array}$ $\begin{array}{lllllllll}112 & 223 & 335-10.1 & -2.2-2243.57 & -4.38 & 0.79 & -2.49-2249.65 & 365.52\end{array}$ $\begin{array}{lllllllll}112 & 224 & 336-10.2 & -2.1-2245.74 & -4.96 & 0.00 & -2.47-2253.16 & 370.08\end{array}$ $\begin{array}{llllllll}112 & 225 & 337-10.3 & -2.1-2247.84 & -5.80 & 0.79 & -2.45-2255.30 & 376.02\end{array}$ $\begin{array}{llllllll}112 & 226 & 338-10.4 & -2.0-2249.88 & -6.92 & 0.00 & -2.43-2259.23 & 380.16\end{array}$ $\begin{array}{lllllllll}112 & 227 & 339-10.6 & -1.9-2251.86 & -8.00 & 0.79 & -2.41-2261.48 & 385.97\end{array}$ $\begin{array}{llllllllll}113 & 153 & 266 & 1.5 & -8.8-1889.90 & -3.10 & 1.73 & -5.32-1896.59 & 160.84\end{array}$ $\begin{array}{llllllllll}11.3 & 154 & 267 & 1.3 & -8.7-1898.68 & -3.21 & 0.99 & -5.25-1906.15 & 159.36\end{array}$ $\begin{array}{llllllllll}113 & 155 & 268 & 1.1 & -8.6-1907.33 & -3.52 & 1.73 & -5.18-1914.30 & 159.27\end{array}$ $\begin{array}{llllllllll}113 & 156 & 269 & 0.9 & -8.5-1915.86 & -3.60 & 0.99 & -5.11-1923.57 & 158.07\end{array}$ $\begin{array}{lll}113 & 157 & 270\end{array}$ $0.7-8.3-1924.25-3.98 \quad 1.72-5.04-1931.55 \quad 158.17$ $\begin{array}{lll}113 & 158 & 271\end{array}$ $0.5-8.2-1932.52-4.17 \quad 0.99-4.98-1940.67 \quad 157.12$ $\begin{array}{lll}113 & 159 & 272\end{array}$ $\begin{array}{lll}113 & 160 & 273\end{array}$ $0.3-8.1-1940.66-4.70 \quad 1.72-4.91-1948.55 \quad 157.31$ $0.2-8.0-1948.68-4.96 \quad 0.99-4.85-1957.50 \quad 156.43$ $\begin{array}{llllllllll}113 & 161 & 274 & 0.0 & -7.8-1956.57 & -5.48 & 1.72 & -4.79-1965.13 & 156.88\end{array}$ $\begin{array}{llllllllll}113 & 162 & 275 & -0.2 & -7.7-1964.35 & -5.66 & 0.99 & -4.73-1973.75 & 156.33\end{array}$ $\begin{array}{llllllllll}113 & 163 & 276 & -0.4 & -7.6-1972.01 & -6.03 & 1.72 & -4.67-1980.99 & 157.15\end{array}$ $\begin{array}{lllllllll}113 & 164 & 277 & -0.6 & -7.5-1979.55 & -5.86 & 0.99 & -4.61-1989.03 & 157.19\end{array}$ $\begin{array}{lllllllllll}113 & 165 & 278 & -0.7 & -7.4-1986.97 & -5.62 & 1.71 & -4.56-1995.43 & 158.86\end{array}$ $\begin{array}{llllllllll}113 & 166 & 279 & -0.9 & -7.2-1994.27 & -5.11 & 0.99 & -4.50-2002.89 & 159.47\end{array}$ $\begin{array}{llllllllll}113 & 167 & 280 & -1.1 & -7.1-2001.46 & -5.95 & 1.71 & -4.45-2010.15 & 160.28\end{array}$ $\begin{array}{llllllllll}113 & 168 & 281 & -1.3 & -7.0-2008.54 & -6.09 & 0.99 & -4.40-2018.03 & 160.47\end{array}$ $\begin{array}{llllllllll}113 & 169 & 282 & -1.5 & -6.9-2015.50 & -6.54 & 1.71 & -4.34-2024.68 & 161.90\end{array}$ $\begin{array}{llllllllll}113 & 170 & 283 & -1.6 & -6.8-2022.36 & -6.61 & 0.99 & -4.29-2032.26 & 162.38\end{array}$ $\begin{array}{lllllllllll}113 & 171 & 284 & -1.8 & -6.7-2029.10 & -7.00 & 1.70 & -4.24-2038.64 & 164.08\end{array}$ $\begin{array}{lllllllllll}113 & 172 & 285 & -2.0 & -6.6-2035.74 & -7.04 & 0.99 & -4.19-2045.97 & 164.82\end{array}$ $\begin{array}{llllllllll}113 & 173 & 286 & -2.1 & -6.5-2042.26 & -7.43 & 1.70 & -4.14-2052.13 & 166.73\end{array}$ $\begin{array}{lllllllll}113 & 174 & 287 & -2.3 & -6.4-2048.68 & -7.43 & 0.99 & -4.10-2059.22 & 167.72\end{array}$ $\begin{array}{llllllllll}113 & 175 & 288 & -2.5 & -6.3-2055.00 & -7.76 & 1.70 & -4.05-2065.11 & 169.90\end{array}$ $\begin{array}{lllllllll}113 & 176 & 289 & -2.7 & -6.2-2061.21 & -7.94 & 0.99 & -4.00-2072.16 & 170.91\end{array}$ $\begin{array}{llllllllll}113 & 177 & 290 & -2.8 & -6.1-2067.32 & -8.19 & 1.70 & -3.96-2077.77 & 173.38\end{array}$ $\begin{array}{llllllllll}113 & 178 & 291 & -3.0 & -6.0-2073.33 & -8.37 & 0.99 & -3.91-2084.62 & 174.60\end{array}$ $\begin{array}{llllllllll}113 & 179 & 292 & -3.2 & -5.9-2079.23 & -8.61 & 1.69 & -3.87-2090.02 & 177.27\end{array}$ $\begin{array}{llllllllll}113 & 180 & 293 & -3.3 & -5.8-2085.04 & -8.22 & 0.99 & -3.83-2096.10 & 179.27\end{array}$ $\begin{array}{llllllllll}113 & 181 & 294 & -3.5 & -5.7 & -2090.75 & -8.35 & 1.69 & -3.79-2101.19 & 182.24\end{array}$ $\begin{array}{llllllllll}113 & 182 & 295 & -3.7 & -5.6-2096.36 & -7.82 & 0.99 & -3.74-2106.93 & 184.57\end{array}$ $\begin{array}{llllllllll}113 & 183 & 296 & -3.8 & -5.5-2101.87 & -7.72 & 1.69 & -3.70-2111.60 & 187.97\end{array}$ $\begin{array}{llllllllll}113 & 184 & 297 & -4.0 & -5.4-2107.29 & -7.06 & 0.99 & -3.66-2117.02 & 190.63\end{array}$ $\begin{array}{llllllllll}113 & 185 & 298 & -4.1 & -5.3-2112.61 & -5.99 & 1.69 & -3.62-2120.54 & 195.18\end{array}$ $\begin{array}{llllllllll}113 & 186 & 299 & -4.3 & -5.2 & -2117.84 & -5.47 & 0.99 & -3.59-2125.90 & 197.89\end{array}$ $\begin{array}{llllllllll}113 & 187 & 300 & -4.5 & -5.1-2122.98 & -4.34 & 1.68 & -3.55-2129.18 & 202.68\end{array}$ $\begin{array}{llllllllll}113 & 188 & 301 & -4.6 & -5.0 & -2128.02 & -3.62 & 0.99 & -3.51-2134.16 & 205.77\end{array}$ $\begin{array}{llllllllll}113 & 189 & 302 & -4.8 & -4.9-2132.97 & -2.71 & 1.68 & -3.48-2137.48 & 210.53\end{array}$ $\begin{array}{lllllllll}113 & 190 & 303 & -4.9 & -4.8-2137.84 & -1.93 & 0.99 & -3.44-2142.21 & 213.86\end{array}$ $\begin{array}{lllllllll}113 & 191 & 304 & -5.1 & -4.7-2142.61 & -1.26 & 1.68 & -3.40-2145.60 & 218.55\end{array}$ $\begin{array}{llllllllll}113 & 192 & 305 & -5.3 & -4.6-2147.30 & -0.60 & 0.99 & -3.37-2150.28 & 221.94\end{array}$ $\begin{array}{llllllllll}113 & 193 & 306 & -5.4 & -4.6-2151.90 & 0.30 & 1.68 & -3.34-2153.25 & 227.04\end{array}$ $\begin{array}{lllllllll}113 & 194 & 307 & -5.6 & -4.5-2156.41 & 0.43 & 0.99 & -3.30-2158.29 & 230.07\end{array}$ $\begin{array}{llllllllll}113 & 195 & 308 & -5.7 & -4.4-2160.84 & -0.58 & 1.68 & -3.27-2163.01 & 233.42\end{array}$ $\begin{array}{llllllllll}113 & 196 & 309 & -5.9 & -4.3-2165.18 & -0.41 & 0.99 & -3.24-2167.83 & 236.67\end{array}$ $\begin{array}{lllllllllll}113 & 197 & 310 & -6.0 & -4.2 & -2169.44 & -0.78 & 1.67 & -3.20 & -2171.75 & 240.83\end{array}$ $\begin{array}{llllllllll}113 & 198 & 311 & -6.2 & -4.1-2173.62 & -0.90 & 0.99 & -3.17-2176.70 & 243.95\end{array}$ $\begin{array}{lllllllllll}113 & 199 & 312 & -6.3 & -4.0-2177.71 & -1.36 & 1.67 & -3.14-2180.54 & 248.18\end{array}$ $\begin{array}{llllllllll}113 & 200 & 313 & -6.5 & -4.0-2181.72 & -1.50 & 0.99 & -3.11-2185.34 & 251.45\end{array}$ $\begin{array}{llllllllll}113 & 201 & 314 & -6.6 & -3.9-2185.66 & -1.88 & 1.67 & -3.08-2188.95 & 255.91\end{array}$ $\begin{array}{lllllllll}113 & 202 & 315 & -6.8 & -3.8-2189.51 & -1.96 & 0.99 & -3.05-2193.53 & 259.40\end{array}$ $\begin{array}{llllllllll}113 & 203 & 316 & -6.9 & -3.7-2193.29 & -2.03 & 1.67 & -3.02 & -2196.67 & 264.33\end{array}$ $\begin{array}{lllllllllll}113 & 204 & 317 & -7.1 & -3.7-2196.98 & -1.86 & 0.99 & -2.99-2200.84 & 268.23\end{array}$ $\begin{array}{lllllllll}113 & 205 & 318 & -7.2 & -3.6-2200.60 & -1.86 & 1.67 & -2.97-2203.76 & 273.38\end{array}$ $\begin{array}{lllllllll}113 & 206 & 319 & -7.3 & -3.5-2204.15 & -1.65 & 0.99 & -2.94-2207.74 & 277.48\end{array}$ $\begin{array}{llllllllll}113 & 207 & 320 & -7.5 & -3.4-2207.62 & -1.88 & 1.66 & -2.91 & -2210.74 & 282.55\end{array}$ $\begin{array}{lllllllll}113 & 208 & 321 & -7.6 & -3.4-2211.01 & -1.76 & 0.99 & -2.89-2214.66 & 286.70\end{array}$

0.00

0.00

0.00

0.00

0.00

0.00

0.00

0.00

0.00

0.00

0.00

0.00

0.00

0.00

0.00

0.00

0.00

0.00

0.00

0.00

0.00

0.00

0.00

0.00

0.00

0.00

0.00

0.00

0.00

0.00

0.00

0.00

0.00

0.00

0.00

0.00

0.00

0.00

0.00

0.00

0.00

0.00

0.00

0.00

0.00

0.00

0.00

0.00

0.00

0.00

0.00

0.00

0.00

0.00

0.00

0.00

0.00

0.00

0.00

0.00

0.00

0.00

0.00

0.00

0.00

0.00
0.00

0.00

0.00

0.00

0.00

0.00

0.00

0.00

0.00

0.00

0.00

0.00

0.00

0.00

0.00

0.00

0.00

0.00

0.00

0.00

0.00

0.00

0.00

0.00

0.00

0.00

0.00

0.00

0.00

0.00

0.00

0.00

0.00

0.00

0.00

0.00

0.00

0.00

0.00

0.00

0.00

0.00

0.00

0.00

0.00

0.00

0.00

0.00

0.00

0.00

0.00

0.00

0.00

0.00

0.00

0.00

0.00

0.00

0.00

0.00

0.00

0.00

0.00

0.00

0.00

0.00
0.00

0.00

0.00

0.00

0.00

0.00

0.00

0.00

0.00

0.00

0.00

0.00

0.00

0.00

0.00

0.00

0.00

0.00

0.00

0.00

0.00

0.00

0.00

0.00

0.00

0.00

0.00

0.00

0.00

0.00

0.00

0.00

0.00

0.00

0.00

0.00

0.00

0.00

0.00

0.00

0.00

0.00

0.00

0.00

0.00

0.00

0.00

0.00

0.00

0.00

0.00

0.00

0.00

0.00

0.00

0.00

0.00

0.00

0.00

0.00

0.00

0.00

0.00

0.00

0.00

0.00 
$\begin{array}{lllllllll}113 & 209 & 322 & -7.8 & -3.3-2214.33 & -2.07 & 1.66 & -2.86-2217.60 & 291.84\end{array}$ $\begin{array}{lllllllll}113 & 210 & 323 & -7.9 & -3.2-2217.57 & -2.02 & 0.99 & -2.83-2221.43 & 296.07\end{array}$ $\begin{array}{lllllllll}113 & 211 & 324 & -8.1 & -3.1-2220.75 & -2.50 & 1.66 & -2.81-2224.40 & 301.18\end{array}$ $\begin{array}{lllllllll}113 & 212 & 325 & -8.2 & -3.1-2223.85 & -1.91 & 0.99 & -2.78-2227.55 & 306.10\end{array}$ $\begin{array}{lllllllll}113 & 213 & 326 & -8.3 & -3.0-2226.88 & -2.27 & 1.66 & -2.76-2230.25 & 311.47\end{array}$ $\begin{array}{lllllllll}113 & 214 & 327 & -8.5 & -2.9-2229.84 & -2.51 & 0.99 & -2.73-2234.09 & 315.70\end{array}$ $\begin{array}{lllllllll}113 & 215 & 328 & -8.6 & -2.9-2232.73 & -2.96 & 1.66 & -2.71-2236.75 & 321.11\end{array}$ $\begin{array}{lllllllll}113 & 216 & 329 & -8.7 & -2.8-2235.56 & -3.03 & 0.99 & -2.69-2240.28 & 325.65\end{array}$ $\begin{array}{lllllllll}113 & 217 & 330 & -8.9 & -2.7-2238.31 & -3.22 & 1.65 & -2.66-2242.54 & 331.47\end{array}$ $\begin{array}{lllllllll}113 & 218 & 331 & -9.0 & -2.6-2241.00 & -3.24 & 0.99 & -2.64-2245.88 & 336.19\end{array}$

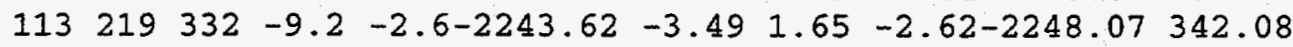

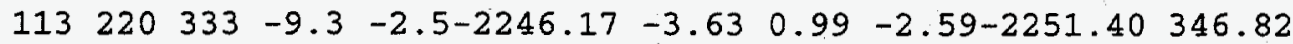
$\begin{array}{lllllllll}113 & 221 & 334 & -9.4 & -2.5-2248.66 & -4.16 & 1.65 & -2.57-2253.74 & 352.55\end{array}$ $\begin{array}{lllllllll}113 & 222 & 335 & -9.6 & -2.4-2251.08 & -4.45 & 0.99 & -2.55-2257.09 & 357.27\end{array}$ $\begin{array}{lllllllll}113 & 223 & 336 & -9.7 & -2.3-2253.44 & -5.11 & 1.65 & -2.53-2259.43 & 363.00\end{array}$

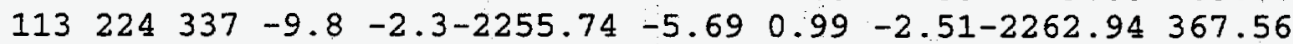

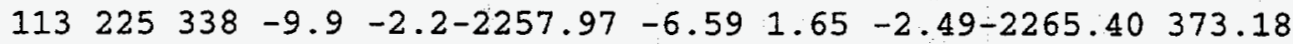
$\begin{array}{llllllll}113 & 226 & 339-10.1 & -2.1-2260.14 & -7.68 & 0.99 & -2.47-2269.29 & 377.36\end{array}$ $\begin{array}{llllllllll}114 & 155 & 269 & 1.5 & -8.8-1906.06 & -3.02 & 0.89 & -5.27-1913.46 & 167.39\end{array}$ $\begin{array}{llllllllll}114 & 156 & 270 & 1.3 & -8.6 & -1914.77 & -3.14 & 0.00 & -5.20-1923.11 & 165.80\end{array}$

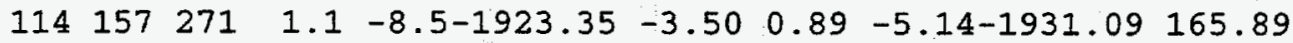
$114158 \quad 272$ 114159273 114160274 114161275 114162276 114163277 114164278 114165279 $\begin{array}{lllllll}0.9 & -8.4-1931.80 & -3.68 & 0.00 & -5.07-1940.55 & 164.51\end{array}$ $\begin{array}{lllllll}0.7 & -8.3-1940.13 & -4.16 & 0.89 & -5.00-1948.40 & 164.72\end{array}$ $\begin{array}{llllll}0.5 & -8.1-1948.33 & -4.41 & 0.00 & -4.94-1957.68 & 163.52\end{array}$ $0.4-8.0-1956.41-4.84 \quad 0.88-4.88-1965.24 \quad 164.03$ $\begin{array}{lllllll}0.2 & -7.9-1964.37 & -5.10 & 0.00 & -4.82-1974.28 & 163.06\end{array}$ $\begin{array}{lllllll}0.0 & -7.8-1972.20 & -5.47 & 0.88 & -4.76-1981.55 & 163.86\end{array}$ $\begin{array}{llllllll}79 & -0.4 & -7.5-1987.52 & -6.01 & 0.88 & -4.64-1997.30 & 164.26\end{array}$ $\begin{array}{llllllllll}114 & 166 & 280 & -0.5 & -7.4-1995.00 & -6.07 & 0.00 & -4.58-2005.66 & 163.97\end{array}$ $\begin{array}{llllllllll}114 & 167 & 281 & -0.7 & -7.3-2002.37 & -6.47 & 0.87 & -4.53-2012.50 & 165.20\end{array}$ $\begin{array}{lllllllll}114 & 168 & 282 & -0.9 & -7.2-2009.63 & -6.57 & 0.00 & -4.47-2020.67 & 165.10\end{array}$

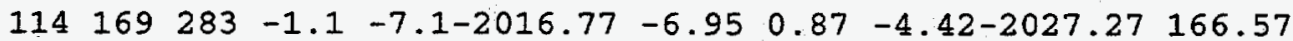
$\begin{array}{llllllllll}114 & 170 & 284 & -1.2 & -7.0-2023.79 & -7.00 & 0.00 & -4.37-2035.16 & 166.75\end{array}$ $\begin{array}{llllllllll}114 & 171 & 285 & -1.4 & -6.9-2030.71 & -7.41 & 0.86 & -4.32 & -2041.57 & 168.41\end{array}$

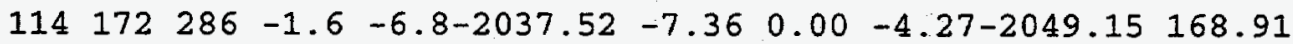
$\begin{array}{llllllllll}114 & 173 & 287 & -1.8 & -6.6-2044.22 & -7.74 & 0.86 & -4.22 & -2055.32 & 170.81\end{array}$ $\begin{array}{lllllllll}114 & 174 & 288 & -1.9 & -6.5-2050.81 & -7.80 & 0.00 & -4.17-2062.78 & 171.42\end{array}$

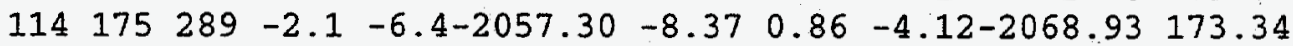
$\begin{array}{lllllllll}114 & 176 & 290 & -2.3 & -6.3-2063.68 & -8.61 & 0.00 & -4.07-2076.36 & 173.98\end{array}$

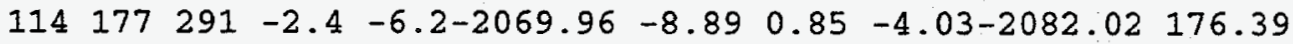
$\begin{array}{lllllllll}114 & 178 & 292 & -2.6 & -6.1-2076.13 & -8.89 & 0.00 & -3.98-2089.01 & 177.48\end{array}$ $\begin{array}{lllllllll}114 & 179 & 293 & -2.8 & -6.0-2082.21 & -9.12 & 0.85 & -3.94-2094.41 & 180.14\end{array}$ $\begin{array}{lllllllll}114 & 180 & 294 & -2.9 & -5.9-2088.18 & -8.76 & 0.00 & -3.90-2100.83 & 181.79\end{array}$ $\begin{array}{lllllllll}114 & 181 & 295 & -3.1 & -5.8-2094.05 & -8.88 & 0.85 & -3.85-2105.94 & 184.76\end{array}$ $\begin{array}{lllllllll}114 & 182 & 296 & -3.3 & -5.7-2099.83 & -8.39 & 0.00 & -3.81-2112.03 & 186.74\end{array}$ $\begin{array}{lllllllll}114 & 183 & 297 & -3.4 & -5.6-2105.50 & -8.26 & 0.85 & -3.77-2116.69 & 190.15\end{array}$ $\begin{array}{lllllllll}114 & 184 & 298 & -3.6 & -5.5-2111.08 & -7.59 & 0.00 & -3.73-2122.40 & 192.51\end{array}$ $\begin{array}{lllllllll}114 & 185 & 299 & -3.8 & -5.4-2116.57 & -6.51 & 0.84 & -3.69-2125.92 & 197.06\end{array}$ $\begin{array}{lllllllll}114 & 186 & 300 & -3.9 & -5.3-2121.96 & -5.99 & 0.00 & -3.65-2131.60 & 199.46\end{array}$ $\begin{array}{llllllllll}114 & 187 & 301 & -4.1 & -5.2 & -2127.25 & -4.84 & 0.84 & -3.61-2134.86 & 204.26\end{array}$

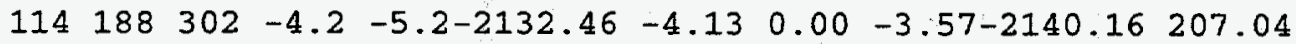
$\begin{array}{lllllllll}114 & 189 & 303 & -4.4 & -5.1-2137.57 & -3.10 & 0.84 & -3.54-2143.37 & 211.90\end{array}$

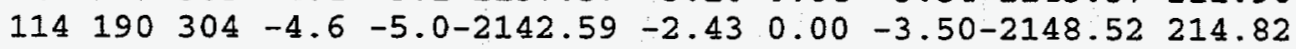
$\begin{array}{llllllllll}114 & 191 & 305 & -4.7 & -4.9-2147.52 & -1.52 & 0.83 & -3.46-2151.67 & 219.74\end{array}$ $\begin{array}{lllllllll}114 & 192 & 306 & -4.9 & -4.8-2152.36 & -0.95 & 0.00 & -3.43-2156.74 & 222.74\end{array}$ $\begin{array}{lllllllll}114 & 193 & 307 & -5.0 & -4.7-2157.12 & -0.15 & 0.83 & -3.39-2159.83 & 227.73\end{array}$ $\begin{array}{lllllllll}114 & 194 & 308 & -5.2 & -4.6-2161.78 & 0.31 & 0.00 & -3.36-2164.83 & 230.79\end{array}$ $\begin{array}{lllllllll}114 & 195 & 309 & -5.3 & -4.5-2166.37 & 0.99 & 0.83 & -3.33-2167.87 & 235.82\end{array}$

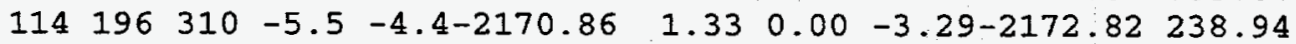

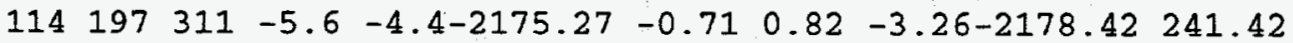
$\begin{array}{lllllllll}114 & 198 & 312 & -5.8 & -4.3-2179.60 & -0.77 & 0.00 & -3.23-2183.60 & 244.31\end{array}$ $\begin{array}{llllllllll}114 & 199 & 3.13 & -5.9 & -4.2-2183.84 & -1.16 & 0.82 & -3.20-2187.38 & 248.61\end{array}$ $\begin{array}{lllllllll}114 & 200 & 314 & -6.1 & -4.1-2188.01 & -1.32 & 0.00 & -3.17-2192.49 & 251.56\end{array}$ $\begin{array}{lllllllll}114 & 201 & 315 & -6.2 & -4.0-2192.09 & -1.69 & 0.82 & -3.13-2196.09 & 256.03\end{array}$ $\begin{array}{llllllllll}114 & 202 & 316 & -6.4 & -4.0-2196.09 & -1.72 & 0.00 & -3.10-2200.92 & 259.28\end{array}$
0.00

0.00

0.00

0.00

0.00

0.00

0.00

0.00

0.00

0.00

0.00

0.00

0.00

0.00

0.00

0.00

0.00

0.00

0.00

0.00

0.00

0.00

0.00

0.00

0.00

0.00

0.00

0.00

0.00

0.00

0.00

0.00

0.00

0.00

0.00

0.00

0.00

0.00

0.00

0.00

0.00

0.00

0.00

0.00

0.00

0.00

0.00

0.00

0.00

0.00

0.00

0.00

0.00

0.00

0.00

0.00

0.00

0.00

0.00

0.00

0.00

0.00

0.00

0.00

0.00

0.00
0.00

0.00

0.00

0.00

0.00

0.00

0.00

0.00

0.00

0.00

0.00

0.00

0.00

0.00

0.00

0.00

0.00

0.00

0.00

0.00

0.00

0.00

0.00

0.00

0.00

0.00

0.00

0.00

0.00

0.00

0.00

0.00

0.00

0.00

0.00

0.00

0.00

0.00

0.00

0.00

0.00

0.00

0.00

0.00

0.00

0.00

0.00

0.00

0.00

0.00

0.00

0.00

0.00

0.00

0.00

0.00

0.00

0.00

0.00

0.00

0.00

0.00

0.00

0.00

0.00

0.00
0.00

0.00

0.00

0.00

0.00

0.00

0.00

0.00

0.00

0.00 0.00 0.00

0.00

0.00 0.00 0.00 0.00 0.00 0.00 0.00 0.00

0.00 0.00 0.00 0.00 0.00 0.00 0.00 0.00 0.00 0.00 0.00 0.00 0.00 0.00 0.00 0.00 0.00 0.00 0.00 0.00 0.00 0.00 0.00 0.00 0.00 0.00 0.00 0.00 0.00 0.00 0.00 0.00 0.00 0.00 0.00 0.00 0.00 0.00 0.00 0.00 0.00 0.00 0.00 0.00 128 
$\begin{array}{lllllllllll}114 & 203 & 317 & -6.5 & -3.9-2200.01 & -1.87 & 0.82 & -3.08-2204.14 & 264.13\end{array}$ $\begin{array}{lllllllll}114 & 204 & 318 & -6.7 & -3.8-2203.85 & -1.58 & 0.00 & -3.05-2208.48 & 267.86\end{array}$ $\begin{array}{llllllllll}114 & 205 & 319 & -6.8 & -3.7-2207.62 & -1.68 & 0.81 & -3.02-2211.51 & 272.91\end{array}$ $\begin{array}{llllllllll}114 & 206 & 320 & -7.0 & -3.6-2211.31 & -1.29 & 0.00 & -2.99-2215.59 & 276.89\end{array}$ $\begin{array}{llllllllll}114 & 207 & 321 & -7.1 & -3.6-2214.92 & -1.49 & 0.81 & -2.96-2218.56 & 281.99\end{array}$ $\begin{array}{llllllllll}114 & 208 & 322 & -7.3 & -3.5-2218.46 & -1.34 & 0.00 & -2.93-2222.73 & 285.89\end{array}$ $\begin{array}{llllllllll}114 & 209 & 323 & -7.4 & -3.4-2221.92 & -1.62 & 0.81 & -2.91-2225.64 & 291.06\end{array}$ $\begin{array}{lllllllllll}114 & 210 & 324 & -7.5 & -3.3-2225.31 & -1.57 & 0.00 & -2.88-2229.76 & 295.01\end{array}$ $\begin{array}{llllllllll}114 & 211 & 325 & -7.7 & -3.3-2228.62 & -2.07 & 0.81 & -2.85-2232.74 & 300.10\end{array}$ $\begin{array}{llllllllll}114 & 212 & 326 & -7.8 & -3.2-2231.86 & -1.64 & 0.00 & -2.83-2236.33 & 304.58\end{array}$ $\begin{array}{llllllllll}114 & 213 & 327 & -8.0 & -3.1-2235.03 & -2.01 & 0.80 & -2.80-2239.04 & 309.94\end{array}$ $\begin{array}{llllllllll}114 & 214 & 328 & -8.1 & -3.1-2238.13 & -2.26 & 0.00 & -2.78-2243.17 & 313.88\end{array}$ $\begin{array}{llllllllll}114 & 215 & 329 & -8.2 & -3.0-2241.16 & -2.69 & 0.80 & -2.75-2245.80 & 319.32\end{array}$ $\begin{array}{lllllllllll}114 & 216 & 330 & -8.4 & -2.9-2244.12 & -2.64 & 0.00 & -2.73-2249.49 & 323.71\end{array}$ $\begin{array}{llllllllll}114 & 217 & 331 & -8.5 & -2.9-2247.01 & -2.91 & 0.80 & -2.71-2251.83 & 329.44\end{array}$ $\begin{array}{llllllllll}114 & 218 & 332 & -8.6 & -2.8-2249.83 & -2.98 & 0.00 & -2.68-2255.49 & 333.85\end{array}$ $\begin{array}{lllllllll}114 & 219 & 333 & -8.8 & -2.7-2252.59 & -3.26 & 0.80 & -2.66-2257.71 & 339.70\end{array}$ $\begin{array}{llllllllll}114 & 220 & 334 & -8.9 & -2.6-2255.27 & -3.53 & 0.00 & -2.64-2261.44 & 344.04\end{array}$ $\begin{array}{llllllllll}114 & 221 & 335 & -9.0 & -2.6-2257.89 & -4.03 & 0.79 & -2.61-2263.74 & 349.81\end{array}$ $\begin{array}{llllllllll}114 & 222 & 336 & -9.2 & -2.5-2260.45 & -4.54 & 0.00 & -2.59-2267.58 & 354.05\end{array}$ $\begin{array}{llllllllll}114 & 223 & 337 & -9.3 & -2.5-2262.94 & -5.34 & 0.79 & -2.57-2270.06 & 359.64\end{array}$ $\begin{array}{llllllllll}114 & 224 & 338 & -9.4 & -2.4-2265.36 & -6.18 & 0.00 & -2.55-2274.09 & 363.68\end{array}$ $\begin{array}{llllllllll}114 & 225 & 339 & -9.6 & -2.3-2267.73 & -7.12 & 0.79 & -2.53-2276.59 & 369.26\end{array}$ $\begin{array}{lllllllll}115 & 157 & 272 & 1.5 & -8.7-1922.06 & -3.33 & 1.72 & -5.23-1928.90 & 175.35\end{array}$ $\begin{array}{llllllllll}115 & 158 & 273 & 1.3 & -8.6-1930.70 & -3.52 & 0.99 & -5.16-1938.40 & 173.92\end{array}$ $\begin{array}{lllllllllll}115 & 159 & 274 & 1.1 & -8.4-1939.21 & -3.96 & 1.72 & -5.09-1946.55 & 173.84\end{array}$ $\begin{array}{llllllllll}115 & 160 & 275 & 0.9 & -8.3-1947.60 & -4.21 & 0.99 & -5.03-1955.85 & 172.61\end{array}$ $\begin{array}{llllllllll}115 & 161 & 276 & 0.7 & -8.2-1955.86 & -4.70 & 1.71 & -4.97-1963.81 & 172.72\end{array}$ $\begin{array}{llllllllll}115 & 162 & 277 & 0.6 & -8.1-1964.00 & -4.96 & 0.99 & -4.90-1972.88 & 171.73\end{array}$ $\begin{array}{llllllllll}115 & 163 & 278 & 0.4 & -8.0-1972.02 & -5.33 & 1.71 & -4.84-1980.48 & 172.20\end{array}$ $\begin{array}{llllllllll}115 & 164 & 279 & 0.2 & -7.8-1979.92 & -5.79 & 0.99 & -4.78-1989.50 & 171.25\end{array}$ $\begin{array}{llllllllll}115 & 165 & 280 & 0.0 & -7.7-1987.69 & -6.30 & 1.71 & -4.72-1997.01 & 171.81\end{array}$ $\begin{array}{llllllllll}115 & 166 & 281 & -0.2 & -7.6-1995.36 & -6.45 & 0.99 & -4.67-2005.48 & 171.41\end{array}$ $\begin{array}{lllllllllll}115 & 167 & 282 & -0.3 & -7.5-2002.90 & -6.86 & 1.71 & -4.61-2012.67 & 172.30\end{array}$ $\begin{array}{llllllllll}115 & 168 & 283 & -0.5 & -7.4-2010.33 & -6.95 & 0.99 & -4.55-2020.85 & 172.19\end{array}$ $\begin{array}{llllllllll}115 & 169 & 284 & -0.7 & -7.3-2017.65 & -7.40 & 1.70 & -4.50-2027.85 & 173.26\end{array}$ $\begin{array}{llllllllll}115 & 170 & 285 & -0.9 & -7.1-2024.85 & -7.43 & 0.99 & -4.45-2035.74 & 173.44\end{array}$ $\begin{array}{lllllllllll}115 & 171 & 286 & -1.0 & -7.0-2031.94 & -7.84 & 1.70 & -4.39-2042.48 & 174.77\end{array}$ $\begin{array}{llllllllll}115 & 172 & 287 & -1.2 & -6.9-2038.92 & -7.76 & 0.99 & -4.34-2050.04 & 175.28\end{array}$ $\begin{array}{llllllllll}115 & 173 & 288 & -1.4 & -6.8-2045.80 & -8.16 & 1.70 & -4.29-2056.55 & 176.84\end{array}$ $\begin{array}{llllllllll}115 & 174 & 289 & -1.6 & -6.7-2052.56 & -8.17 & 0.99 & -4.24-2063.98 & 177.48\end{array}$ $\begin{array}{llllllllll}115 & 175 & 290 & -1.7 & -6.6-2059.22 & -8.52 & 1.69 & -4.19-2070.24 & 179.30\end{array}$ $\begin{array}{llllllllll}115 & 176 & 291 & -1.9 & -6.5-2065.77 & -8.66 & 0.99 & -4.15-2077.59 & 180.02\end{array}$ $\begin{array}{llllllllll}115 & 177 & 292 & -2.1 & -6.4-2072.21 & -8.98 & 1.69 & -4.10-2083.60 & 182.07\end{array}$ $\begin{array}{llllllllll}115 & 178 & 293 & -2.2 & -6.3-2078.56 & -9.10 & 0.99 & -4.05-2090.72 & 183.02\end{array}$ $\begin{array}{llllllllll}115 & 179 & 294 & -2.4 & -6.2-2084.80 & -9.44 & 1.69 & -4.01-2096.56 & 185.26\end{array}$ $\begin{array}{llllllllll}115 & 180 & 295 & -2.6 & -6.1-2090.94 & -9.09 & 0.99 & -3.96-2103.00 & 186.89\end{array}$ $\begin{array}{llllllllll}115 & 181 & 296 & -2.7 & -6.0 & -2096.97 & -9.16 & 1.69 & -3.92-2108.37 & 189.60\end{array}$ $\begin{array}{lllllllll}115 & 182 & 297 & -2.9 & -5.9-2102.91 & -8.59 & 0.99 & -3.88-2114.39 & 191.64\end{array}$ $\begin{array}{llllllllll}115 & 183 & 298 & -3.1 & -5.8-2108.75 & -8.46 & 1.68 & -3.84-2119.36 & 194.74\end{array}$ $\begin{array}{llllllllll}115 & 184 & 299 & -3.2 & -5.7-2114.50 & -7.78 & 0.99 & -3.79-2125.08 & 197.09\end{array}$ $\begin{array}{llllllllll}115 & 185 & 300 & -3.4 & -5.6-2120.14 & -6.70 & 1.68 & -3.75-2128.91 & 201.33\end{array}$ $\begin{array}{llllllllll}115 & 186 & 301 & -3.5 & -5.5-2125.69 & -6.19 & 0.99 & -3.71-2134.61 & 203.71\end{array}$ $\begin{array}{lllllllllll}115 & 187 & 302 & -3.7 & -5.4-2131.15 & -5.06 & 1.68 & -3.67 & -2138.20 & 208.19\end{array}$ $\begin{array}{llllllllll}115 & 188 & 303 & -3.9 & -5.3-2136.51 & -4.34 & 0.99 & -3.64-2143.50 & 210.96\end{array}$ $\begin{array}{llllllllll}115 & 189 & 304 & -4.0 & -5.2-2141.78 & -3.33 & 1.68 & -3.60-2147.03 & 215.50\end{array}$ $\begin{array}{lllllllll}115 & 190 & 305 & -4.2 & -5.1-2146.96 & -2.66 & 0.99 & -3.56-2152.20 & 218.41\end{array}$ $\begin{array}{llllllllll}115 & 191 & 306 & -4.3 & -5.0-2152.05 & -1.76 & 1.68 & -3.52-2155.66 & 223.02\end{array}$ $\begin{array}{llllllllll}115 & 192 & 307 & -4.5 & -4.9-2157.05 & -1.19 & 0.99 & -3.49-2160.74 & 226.01\end{array}$ $\begin{array}{lllllllll}115 & 193 & 308 & -4.6 & -4.9-2161.96 & -0.50 & 1.67 & -3.45-2164.24 & 230.58\end{array}$ $\begin{array}{llllllllll}115 & 194 & 309 & -4.8 & -4.8-2166.78 & 0.01 & 0.99 & -3.42-2169.20 & 233.69\end{array}$ $\begin{array}{lllllllllll}115 & 195 & 310 & -5.0 & -4.7-2171.52 & -1.02 & 1.67 & -3.38-2174.25 & 236.71\end{array}$ $\begin{array}{llllllllll}115 & 196 & 311 & -5.1 & -4.6-2176.16 & -0.70 & 0.99 & -3.35-2179.22 & 239.81\end{array}$ $\begin{array}{lllllllll}115 & 197 & 312 & -5.3 & -4.5-2180.73 & -0.95 & 1.67 & -3.32-2183.32 & 243.78\end{array}$ $\begin{array}{llllllllll}115 & 198 & 313 & -5.4 & -4.4-2185.21 & -0.98 & 0.99 & -3.28-2188.48 & 246.69\end{array}$ $\begin{array}{llllllllll}115 & 199 & 314 & -5.6 & -4.3-2189.60 & -1.31 & 1.67 & -3.25-2192.50 & 250.75\end{array}$
0.00

0.00

0.00

0.00

0.00

0.00

0.00

0.00

0.00

0.00

0.00

0.00

0.00

0.00

0.00

0.00

0.00

0.00

0.00

0.00

0.00

0.00

0.00

0.00

0.00

0.00

0.00

0.00

0.00

0.00

0.00

0.00

0.00

0.00

0.00

0.00

0.00

0.00

0.00

0.00

0.00

0.00

0.00

0.00

0.00

0.00

0.00

0.00

0.00

0.00

0.00

0.00

0.00

0.00

0.00

0.00

0.00

0.00

0.00

0.00

0.00

0.00

0.00

0.00

0.00

0.00

0.00

0.00

0.00

$0: 00$

0.00

0.00

0.00

0.00

0.00

0.00

0.00

0.00

0.00

0.00

0.00

0.00

0.00

0.00

0.00

0.00

0.00

0.00

0.00

0.00

0.00

0.00

0.00

0.00

0.00

0.00

0.00

0.00

0.00

0.00

0.00

0.00

0.00

0.00

0.00

0.00

0.00

0.00

0.00

0.00

0.00

0.00

0.00

0.00

0.00

0.00

0.00

0.00

0.00

0.00

0.00

0.00

0.00

0.00

0.00

0.00

0.00

0.00

0.00

$0.00 \quad 0.00$

0.00
0.00

0.00

0.00

0.00

0.00

0.00

0.00

0.00

0.00

0.00

0.00

0.00

0.00

0.00

0.00

0.00

0.00

0.00

0.00

0.00

0.00

0.00

0.00

0.00

0.00

0.00

0.00

0.00

0.00

0.00

0.00

0.00

0.00

0.00

0.00

0.00

0.00

0.00

0.00

0.00

0.00

0.00

0.00

0.00

0.00

0.00

0.00

0.00

0.00

0.00

0.00

0.00

0.00

0.00

0.00

0.00

0.00

0.00

0.00

0.00

0.00

0.00

0.00

0.00

0.00

0.00 
$\begin{array}{lllllllllll}115 & 200 & 315 & -5.7 & -4.3-2193.91 & -1.40 & 0.99 & -3.22-2197.55 & 253.77\end{array}$ $\begin{array}{llllllllll}115 & 201 & 316 & -5.9 & -4.2-2198.14 & -1.78 & 1.66 & -3.19-2201.45 & 257.94\end{array}$ $\begin{array}{llllllllll}115 & 202 & 317 & -6.0 & -4.1-2202.29 & -1.80 & 0.99 & -3.16-2206.26 & 261.20\end{array}$ $\begin{array}{llllllllll}115 & 203 & 318 & -6.2 & -4.0-2206.36 & -1.92 & 1.66 & -3.13-2209.75 & 265.79\end{array}$ $\begin{array}{lllllllll}115 & 204 & 319 & -6.3 & -3.9-2210.35 & -1.66 & 0.99 & -3.10-2214.12 & 269.48\end{array}$ $\begin{array}{lllllllllll}115 & 205 & 320 & -6.5 & -3.9-2214.26 & -1.71 & 1.66 & -3.07-2217.38 & 274.29\end{array}$ $\begin{array}{lllllllll}115 & 206 & 321 & -6.6 & -3.8-2218.10 & -1.32 & 0.99 & -3.04-2221.47 & 278.28\end{array}$ $\begin{array}{llllllllll}115 & 207 & 322 & -6.7 & -3.7-2221.85 & -1.47 & 1.66 & -3.01-2224.68 & 283.14\end{array}$ $\begin{array}{llllllllll}115 & 208 & 323 & -6.9 & -3.6-2225.53 & -1.13 & 0.99 & -2.98-2228.66 & 287.23\end{array}$ $\begin{array}{llllllllll}115 & 209 & 324 & -7.0 & -3.6-2229.14 & -1.52 & 1.66 & -2.96-2231.96 & 292.00\end{array}$ $\begin{array}{llllllllll}115 & 210 & 325 & -7.2 & -3.5-2232.67 & -1.43 & 0.99 & -2.93-2236.04 & 295.99\end{array}$ $\begin{array}{llllllllll}115 & 211 & 326 & -7.3 & -3.4-2236.12 & -1.73 & 1.65 & -2.90-2239.10 & 301.00\end{array}$ $\begin{array}{llllllllll}115 & 212 & 327 & -7.5 & -3.3-2239.50 & -1.81 & 0.99 & -2.88-2243.20 & 304.97\end{array}$ $\begin{array}{llllllllll}115 & 213 & 328 & -7.6 & -3.3-2242.81 & -2.06 & 1.65 & -2.85-2246.07 & 310.18\end{array}$ $\begin{array}{llllllllll}115 & 214 & 329 & -7.7 & -3.2-2246.05 & -2.23 & 0.99 & -2.83-2250.12 & 314.20\end{array}$ $\begin{array}{lllllllllll}115 & 215 & 330 & -7.9 & -3.1-2249.22 & -2.54 & 1.65 & -2.80-2252.91 & 319.48\end{array}$ $\begin{array}{llllllllll}115 & 216 & 331 & -8.0 & -3.1-2252.31 & -2.73 & 0.99 & -2.78-2256.83 & 323.63\end{array}$ $\begin{array}{llllllllll}115 & 217 & 332 & -8.1 & -3.0-2255.34 & -3.04 & 1.65 & -2.75-2259.48 & 329.05\end{array}$ $\begin{array}{llllllllll}115 & 218 & 333 & -8.3 & -2.9-2258.30 & -3.11 & 0.99 & -2.73-2263.15 & 333.46\end{array}$ $\begin{array}{llllllllll}115 & 219 & 334 & -8.4 & -2.9-2261.19 & -3.54 & 1.65 & -2.70-2265.78 & 338.89\end{array}$ $\begin{array}{llllllllll}115 & 220 & 335 & -8.5 & -2.8-2264.01 & -3.82 & 0.99 & -2.68-2269.52 & 343.23\end{array}$ $\begin{array}{llllllllll}115 & 221 & 336 & -8.7 & -2.7-2266.76 & -4.34 & 1.64 & -2.66-2272.12 & 348.70\end{array}$ $\begin{array}{llllllllll}115 & 222 & 337 & -8.8 & -2.7-2269.45 & -4.87 & 0.99 & -2.64-2275.97 & 352.92\end{array}$ $\begin{array}{llllllllll}115 & 223 & 338 & -8.9 & -2.6-2272.07 & -5.76 & 1.64 & -2.61-2278.80 & 358.16\end{array}$ $\begin{array}{lllllllll}115 & 224 & 339 & -9.1 & -2.5-2274.63 & -6.39 & 0.99 & -2.59-2282.62 & 362.41\end{array}$ $\begin{array}{llllllllll}116 & 159 & 275 & 1.5 & -8.6-1937.92 & -3.42 & 0.89 & -5.19-1945.64 & 182.01\end{array}$ $\begin{array}{llllllllll}116 & 160 & 276 & 1.3 & -8.5-1946.49 & -3.66 & 0.00 & -5.12-1955.27 & 180.45\end{array}$ $\begin{array}{llllllllll}116 & 161 & 277 & 1.1 & -8.4-1954.94 & -4.15 & 0.88 & -5.05-1963.26 & 180.54\end{array}$ $\begin{array}{llllllllll}116 & 162 & 278 & 0.9 & -8.3-1963.26 & -4.41 & 0.00 & -4.99-1972.66 & 179.21\end{array}$ $\begin{array}{llllllllll}116 & 163 & 279 & 0.8 & -8.1-1971.46 & -4.77 & 0.88 & -4.93-1980.28 & 179.66\end{array}$ $\begin{array}{llllllllll}116 & 164 & 280 & 0.6 & -8.0-1979.53 & -5.48 & 0.00 & -4.87-1989.88 & 178.13\end{array}$ $\begin{array}{lllllllllll}116 & 165 & 281 & 0.4 & -7.9-1987.49 & -5.99 & 0.88 & -4.81-1997.41 & 178.67\end{array}$ $\begin{array}{lllllllllll}116 & 166 & 282 & 0.2 & -7.8-1995.33 & -6.18 & 0.00 & -4.75-2006.26 & 177.90\end{array}$ $\begin{array}{llllllllll}116 & 167 & 283 & 0.0 & -7.7-2003.05 & -6.64 & 0.87 & -4.69-2013.51 & 178.71\end{array}$ $\begin{array}{lllllllllll}116 & 168 & 284 & -0.1 & -7.5-2010.66 & -6.78 & 0.00 & -4.63-2022.07 & 178.22\end{array}$ $\begin{array}{lllllllllll}116 & 169 & 285 & -0.3 & -7.4-2018.15 & -7.21 & 0.87 & -4.58-2029.07 & 179.30\end{array}$ $\begin{array}{lllllllllll}116 & 170 & 286 & -0.5 & -7.3-2025.53 & -7.22 & 0.00 & -4.52-2037.27 & 179.17\end{array}$ $\begin{array}{llllllllll}116 & 171 & 287 & -0.7 & -7.2-2032.79 & -7.60 & 0.86 & -4.47-2044.00 & 180.51\end{array}$ $\begin{array}{lllllllllll}116 & 172 & 288 & -0.8 & -7.1-2039.95 & -7.50 & 0.00 & -4.42-2051.87 & 180.72\end{array}$ $\begin{array}{llllllllllll}116 & 173 & 289 & -1.0 & -7.0-2046.99 & -7.83 & 0.86 & -4.37-2058.33 & 182.33\end{array}$ $\begin{array}{lllllllllll}116 & 174 & 290 & -1.2 & -6.9-2053.93 & -7.81 & 0.00 & -4.32-2066.05 & 182.67\end{array}$ $\begin{array}{lllllllllll}116 & 175 & 291 & -1.3 & -6.8-2060.75 & -8.07 & 0.86 & -4.27-2072.23 & 184.56\end{array}$ $\begin{array}{lllllllllll}116 & 176 & 292 & -1.5 & -6.7-2067.48 & -8.30 & 0.00 & -4.22-2079.99 & 184.87\end{array}$ $\begin{array}{llllllllll}116 & 177 & 293 & -1.7 & -6.6-2074.09 & -8.62 & 0.85 & -4.17-2086.03 & 186.91\end{array}$ $\begin{array}{llllllllll}116 & 178 & 294 & -1.9 & -6.5-2080.60 & -8.70 & 0.00 & -4.12-2093.43 & 187.59\end{array}$ $\begin{array}{llllllllll}116 & 179 & 295 & -2.0 & -6.4-2087.01 & -8.98 & 0.85 & -4.08-2099.22 & 189.87\end{array}$ $\begin{array}{lllllllllll}116 & 180 & 296 & -2.2 & -6.2-2093.31 & -8.58 & 0.00 & -4.03-2105.93 & 191.23\end{array}$

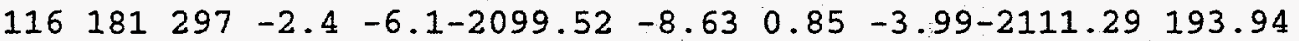
$\begin{array}{llllllllll}116 & 182 & 298 & -2.5 & -6.0-2105.62 & -8.08 & 0.00 & -3.94-2117.64 & 195.65\end{array}$ $\begin{array}{llllllllll}116 & 183 & 299 & -2.7 & -6.0-2111.62 & -7.94 & 0.85 & -3.90-2122.62 & 198.75\end{array}$ $\begin{array}{llllllllll}116 & 184 & 300 & -2.8 & -5.9-2117.53 & -7.25 & 0.00 & -3.86-2128.64 & 200.80\end{array}$ $\begin{array}{lllllllllll}116 & 185 & 301 & -3.0 & -5.8-2123.34 & -6.39 & 0.84 & -3.82 & -2132.70 & 204.81\end{array}$ $\begin{array}{llllllllll}116 & 186 & 302 & -3.2 & -5.7 & -2129.05 & -5.66 & 0.00 & -3.78-2138.49 & 207.10\end{array}$ $\begin{array}{lllllllllll}116 & 187 & 303 & -3.3 & -5.6-2134.67 & -4.54 & 0.84 & -3.74-2142.11 & 211.55\end{array}$ $\begin{array}{llllllllll}116 & 188 & 304 & -3.5 & -5.5 & -2140.19 & -3.82 & 0.00 & -3.70-2147.71 & 214.02\end{array}$ $\begin{array}{llllllllll}116 & 189 & 305 & -3.6 & -5.4-2145.62 & -2.83 & 0.84 & -3.66-2151.27 & 218.53\end{array}$ $\begin{array}{llllllllll}116 & 190 & 306 & -3.8 & -5.3-2150.96 & -2.15 & 0.00 & -3.62-2156.73 & 221.14\end{array}$ $\begin{array}{lllllllllll}116 & 191 & 307 & -4.0 & -5.2-2156.20 & -1.32 & 0.83 & -3.58-2160.27 & 225.67\end{array}$ $\begin{array}{llllllllll}116 & 192 & 308 & -4.1 & -5.1-2161.36 & -0.62 & 0.00 & -3.55-2165.52 & 228.49\end{array}$ $\begin{array}{llllllllll}116 & 193 & 309 & -4.3 & -5.0-2166.42 & -0.11 & 0.83 & -3.51-2169.21 & 232.87\end{array}$ $\begin{array}{lllllllllll}116 & 194 & 310 & -4.4 & -4.9-2171.40 & 0.53 & 0.00 & -3.48-2174.34 & 235.81\end{array}$ $\begin{array}{llllllllll}116 & 195 & 311 & -4.6 & -4.8-2176.29 & -0.67 & 0.83 & -3.44-2179.57 & 238.66\end{array}$ $\begin{array}{llllllllll}116 & 196 & 312 & -4.7 & -4.8-2181.09 & -0.55 & 0.00 & -3.41-2185.05 & 241.25\end{array}$ $\begin{array}{llllllllll}116 & 197 & 313 & -4.9 & -4.7-2185.80 & -0.58 & 0.82 & -3.37-2188.93 & 245.44\end{array}$ $\begin{array}{lllllllllll}116 & 198 & 314 & -5.0 & -4.6-2190.43 & -0.94 & 0.00 & -3.34-2194.71 & 247.73\end{array}$ $116199315-5.2-4.5-2194.98-1.30 \quad 0.82-3.31-2198.76 \quad 251.75$ 130

\begin{tabular}{|c|c|c|}
\hline 00 & .00 & 00 \\
\hline 0.00 & 0.00 & 0.00 \\
\hline 0.00 & 0.00 & 0.00 \\
\hline 0.00 & 0.00 & 0.00 \\
\hline 0.00 & 0.00 & 00 \\
\hline 0.00 & 0.00 & 0.00 \\
\hline 0.00 & 0.00 & 0.00 \\
\hline 0.00 & 0.00 & 0.00 \\
\hline 0.00 & 0.00 & 0.00 \\
\hline 0.00 & 0.00 & 0.00 \\
\hline 0.00 & 0.00 & 0.00 \\
\hline 0.00 & 0.00 & 0.00 \\
\hline 0.00 & 0.00 & 0.00 \\
\hline 0.00 & 0.00 & 0.00 \\
\hline 0.00 & 0.00 & 0.00 \\
\hline 0.00 & 0.00 & 0.00 \\
\hline 0.00 & 0.00 & 0.00 \\
\hline 0.00 & 0.00 & 0.00 \\
\hline 0.00 & 0.00 & 0.00 \\
\hline 0.00 & 0.00 & 0.00 \\
\hline 0.00 & 0.00 & 0.00 \\
\hline 0.00 & 0.00 & 0.00 \\
\hline 0.00 & 0.00 & 0.00 \\
\hline 0.00 & 0.00 & 0.00 \\
\hline 0.00 & 0.00 & 0.00 \\
\hline 0.00 & 0.00 & 0.00 \\
\hline 0.00 & 0.00 & 0.00 \\
\hline 0.00 & 0.00 & 0.00 \\
\hline 0.00 & 0.00 & 0.00 \\
\hline 0.00 & 0.00 & 0.00 \\
\hline 0.0 & 0.00 & 0.00 \\
\hline 0.00 & 0.00 & 0.00 \\
\hline 0.00 & 0.00 & 0.00 \\
\hline 0.00 & 0.00 & 0.00 \\
\hline 0.0 & 0.00 & 0.00 \\
\hline 0.00 & 0.00 & 0.00 \\
\hline 0.00 & 0.00 & 0.00 \\
\hline 0.00 & 0.00 & 0.00 \\
\hline 0.0 & 0.00 & 0.00 \\
\hline 0.00 & 0.00 & 0.00 \\
\hline 0.00 & 0.00 & 0.00 \\
\hline 0.0 & 0.00 & 0.00 \\
\hline 0.0 & 0.00 & 0.00 \\
\hline 0.00 & 0.00 & 0.00 \\
\hline 0.00 & 0.00 & 0.00 \\
\hline 0.0 & 0.0 & 0.00 \\
\hline 0.00 & 0.00 & 0.00 \\
\hline 0.00 & 0.00 & 0.00 \\
\hline 0.00 & 0.00 & 0.00 \\
\hline 0.0 & 0.00 & 0.00 \\
\hline 0.00 & 0.00 & 0.00 \\
\hline 0.00 & 0.00 & 0.00 \\
\hline 0.0 & 0.00 & 0.00 \\
\hline 0.00 & 0.00 & 0.00 \\
\hline 0.00 & 0.00 & 0.00 \\
\hline 0.00 & 0.00 & 0.00 \\
\hline 0.06 & 0.0 & 0.00 \\
\hline 0.00 & 0.00 & 0.00 \\
\hline 0.00 & 0.00 & 0.00 \\
\hline 0.00 & 0.00 & 0.00 \\
\hline 0.00 & 0.00 & 0.00 \\
\hline 0.00 & 0.00 & 0.00 \\
\hline 0.00 & 0.00 & 0.00 \\
\hline 0.00 & 0.00 & 0.00 \\
\hline 0.00 & 0.00 & 0.00 \\
\hline 0 & ?0 & \\
\hline
\end{tabular}


$\begin{array}{llllllllllll}116 & 200 & 316 & -5.3 & -4.4-2199.44 & -1.34 & 0.00 & -3.27-2204.06 & 254.53\end{array}$ $\begin{array}{llllllllll}116 & 201 & 317 & -5.5 & -4.3-2203.82 & -1.69 & 0.82 & -3.24-2207.94 & 258.72\end{array}$ $\begin{array}{llllllllll}116 & 202 & 318 & -5.6 & -4.3-2208.12 & -1.71 & 0.00 & -3.21-2213.04 & 261.68\end{array}$ $\begin{array}{llllllllll}116 & 203 & 319 & -5.8 & -4.2-2212.34 & -1.83 & 0.82 & -3.18-2216.53 & 266.27\end{array}$ $\begin{array}{llllllllll}116 & 204 & 320 & -5.9 & -4.1-2216.47 & -1.56 & 0.00 & -3.15-2221.19 & 269.68\end{array}$ $\begin{array}{llllllllll}116 & 205 & 321 & -6.1 & -4.0-2220.53 & -1.53 & 0.81 & -3.12 & -2224.37 & 274.57\end{array}$ $\begin{array}{llllllllll}116 & 206 & 322 & -6.2 & -3.9-2224.51 & -1.21 & 0.00 & -3.09-2228.81 & 278.20\end{array}$ $\begin{array}{llllllllll}116 & 207 & 323 & -6.4 & -3.9-2228.41 & -1.25 & 0.81 & -3.06-2231.91 & 283.17\end{array}$ $\begin{array}{llllllllll}116 & 208 & 324 & -6.5 & -3.8-2232.23 & -1.04 & 0.00 & -3.03-2236.31 & 286.85\end{array}$ $\begin{array}{llllllllll}116 & 209 & 325 & -6.7 & -3.7-2235.98 & -1.39 & 0.81 & -3.01-2239.57 & 291.66\end{array}$ $\begin{array}{lllllllll}116 & 210 & 326 & -6.8 & -3.6-2239.65 & -1.27 & 0.00 & -2.98-2243.90 & 295.40\end{array}$ $\begin{array}{llllllllll}116 & 211 & 327 & -6.9 & -3.6-2243.25 & -1.60 & 0.81 & -2.95-2246.99 & 300.37\end{array}$ $\begin{array}{llllllllll}116 & 212 & 328 & -7.1 & -3.5-2246.77 & -1.40 & 0.00 & -2.93-2251.10 & 304.34\end{array}$ $\begin{array}{lllllllll}116 & 213 & 329 & -7.2 & -3.4-2250.22 & -1.76 & 0.80 & -2.90-2254.08 & 309.44\end{array}$ $\begin{array}{llllllllllll}116 & 214 & 330 & -7.4 & -3.3-2253.60 & -1.87 & 0.00 & -2.87-2258.34 & 313.24\end{array}$ $\begin{array}{llllllllll}116 & 215 & 331 & -7.5 & -3.3-2256.90 & -2.24 & 0.80 & -2.85-2261.19 & 318.47\end{array}$ $\begin{array}{llllllllll}116 & 216 & 332 & -7.6 & -3.2-2260.14 & -2.25 & 0.00 & -2.82 & -2265.21 & 322.52\end{array}$ $\begin{array}{llllllllll}116 & 217 & 333 & -7.8 & -3.1-2263.30 & -2.53 & 0.80 & -2.80 & -2267.83 & 327.97\end{array}$ $\begin{array}{llllllllll}116 & 218 & 334 & -7.9 & -3.1-2266.39 & -2.80 & 0.00 & -2.77 & -2271.97 & 331.90\end{array}$ $\begin{array}{llllllllll}116 & 219 & 335 & -8.0 & -3.0-2269.42 & -3.27 & 0.80 & -2.75-2274.64 & 337.30\end{array}$ $\begin{array}{llllllllll}116 & 220 & 336 & -8.2 & -2.9-2272.37 & -3.55 & 0.00 & -2.73-2278.65 & 341.36\end{array}$ $\begin{array}{llllllllll}116 & 221 & 337 & -8.3 & -2.9-2275.26 & -4.10 & 0.79 & -2.70-2281.27 & 346.82\end{array}$ $\begin{array}{llllllllll}116 & 222 & 338 & -8.4 & -2.8-2278.08 & -4.51 & 0.00 & -2.68-2285.27 & 350.89\end{array}$ $\begin{array}{llllllllll}116 & 223 & 339 & -8.6 & -2.7 & -2280.83 & -5.55 & 0.79 & -2.66-2288.25 & 355.98\end{array}$ $\begin{array}{llllllllll}117 & 161 & 278 & 1.5 & -8.6-1953.63 & -4.01 & 1.71 & -5.14-1961.08 & 189.98\end{array}$ $\begin{array}{llllllllll}117 & 162 & 279 & 1.3 & -8.4-1962.14 & -4.61 & 0.98 & -5.08-1970.84 & 188.29\end{array}$ $\begin{array}{llllllllll}117 & 163 & 280 & 1.1 & -8.3-1970.52 & -4.55 & 1.71 & -5.02-1978.38 & 188.83\end{array}$ $\begin{array}{llllllllll}117 & 164 & 281 & 1.0 & -8.2-1978.77 & -5.52 & 0.98 & -4.95-1988.27 & 187.01\end{array}$ $\begin{array}{llllllllll}117 & 165 & 282 & 0.8 & -8.1-1986.91 & -6.09 & 1.70 & -4.89-1996.19 & 187.16\end{array}$ $\begin{array}{llllllllll}117 & 166 & 283 & 0.6 & -8.0-1994.93 & -6.25 & 0.98 & -4.83-2005.03 & 186.39\end{array}$ $\begin{array}{llllllllll}117 & 167 & 284 & 0.4 & -7.8-2002.83 & -6.72 & 1.70 & -4.77-2012.62 & 186.87\end{array}$ $\begin{array}{llllllllll}117 & 168 & 285 & 0.2 & -7.7-2010.61 & -6.87 & 0.98 & -4.72-2021.21 & 186.35\end{array}$ $\begin{array}{llllllllll}117 & 169 & 286 & 0.1 & -7.6-2018.28 & -7.27 & 1.70 & -4.66-2028.51 & 187.12\end{array}$ $\begin{array}{llllllllll}117 & 170 & 287 & -0.1 & -7.5-2025.83 & -7.33 & 0.98 & -4.60-2036.78 & 186.92\end{array}$ $\begin{array}{llllllllll}117 & 171 & 288 & -0.3 & -7.4-2033.27 & -7.72 & 1.69 & -4.55-2043.84 & 187.93\end{array}$ $\begin{array}{llllllllll}117 & 172 & 289 & -0.5 & -7.3-2040.60 & -7.68 & 0.98 & -4.50-2051.79 & 188.06\end{array}$ $\begin{array}{llllllllll}117 & 173 & 290 & -0.6 & -7.2 & -2047.81 & -8.03 & 1.69 & -4.44-2058.59 & 189.33\end{array}$ $\begin{array}{llllllllll}117 & 174 & 291 & -0.8 & -7.0-2054.92 & -8.02 & 0.98 & -4.39-2066.35 & 189.64\end{array}$ $\begin{array}{lllllllllll}117 & 175 & 292 & -1.0 & -6.9-2061.92 & -8.30 & 1.69 & -4.34-2072.87 & 191.19\end{array}$ $\begin{array}{llllllllll}117 & 176 & 293 & -1.1 & -6.8-2068.81 & -8.21 & 0.98 & -4.29-2080.33 & 191.81\end{array}$ $\begin{array}{llllllllll}117 & 177 & 294 & -1.3 & -6.7-2075.59 & -8.44 & 1.69 & -4.24-2086.59 & 193.62\end{array}$ $\begin{array}{llllllllll}117 & 178 & 295 & -1.5 & -6.6-2082.27 & -8.55 & 0.98 & -4.20-2094.03 & 194.24\end{array}$ $\begin{array}{llllllllll}117 & 179 & 296 & -1.6 & -6.5-2088.84 & -8.96 & 1.68 & -4.15-2100.27 & 196.08\end{array}$ $\begin{array}{llllllllll}117 & 180 & 297 & -1.8 & -6.4-2095.31 & -8.57 & 0.98 & -4.10-2107.01 & 197.41\end{array}$ $\begin{array}{llllllllll}117 & 181 & 298 & -2.0 & -6.3-2101.68 & -8.59 & 1.68 & -4.06-2112.65 & 199.84\end{array}$ $\begin{array}{llllllllll}117 & 182 & 299 & -2.1 & -6.2-2107.95 & -8.02 & 0.98 & -4.01-2119.00 & 201.56\end{array}$ $\begin{array}{llllllllll}117 & 183 & 300 & -2.3 & -6.1-2114.12 & -7.85 & 1.68 & -3.97-2124.26 & 204.37\end{array}$ $\begin{array}{llllllllll}117 & 184 & 301 & -2.5 & -6.0-2120.19 & -7.16 & 0.98 & -3.93-2130.29 & 206.41\end{array}$ $\begin{array}{lllllllll}117 & 185 & 302 & -2.6 & -5.9-2126.16 & -6.29 & 1.68 & -3.88-2134.66 & 210.12\end{array}$ $\begin{array}{llllllllll}117 & 186 & 303 & -2.8 & -5.8-2132.03 & -5.57 & 0.98 & -3.84 & -2140.46 & 212.38\end{array}$ $\begin{array}{lllllllllll}117 & 187 & 304 & -2.9 & -5.7-2137.81 & -4.47 & 1.67 & -3.80-2144.41 & 216.51\end{array}$ $\begin{array}{llllllllll}117 & 188 & 305 & -3.1 & -5.6-2143.49 & -3.74 & 0.98 & -3.76-2150.01 & 218.98\end{array}$ $\begin{array}{llllllllll}117 & 189 & 306 & -3.3 & -5.5-2149.08 & -2.72 & 1.67 & -3.72-2153.85 & 223.21\end{array}$ $\begin{array}{llllllllll}117 & 190 & 307 & -3.4 & -5.4-2154.58 & -2.07 & 0.98 & -3.68-2159.35 & 225.78\end{array}$ $\begin{array}{llllllllll}117 & 191 & 308 & -3.6 & -5.4-2159.98 & -1.32 & 1.67 & -3.65-2163.27 & 229.93\end{array}$ $\begin{array}{lllllllll}117 & 192 & 309 & -3.7 & -5.3-2165.29 & -2.24 & 0.98 & -3.61-2170.16 & 231.12\end{array}$ $\begin{array}{lllllllllll}117 & 193 & 310 & -3.9 & -5.2-2170.51 & 0.11 & 1.67 & -3.57-2172.30 & 237.04\end{array}$ $\begin{array}{lllllllll}117 & 194 & 311 & -4.1 & -5.1-2175.64 & 0.53 & 0.98 & -3.54-2177.66 & 239.75\end{array}$ $\begin{array}{llllllllll}117 & 195 & 312 & -4.2 & -5.0-2180.68 & -0.83 & 1.67 & -3.50-2183.35 & 242.14\end{array}$ $\begin{array}{llllllllll}117 & 196 & 313 & -4.4 & -4.9-2185.64 & -0.43 & 0.98 & -3.46-2188.55 & 245.01\end{array}$ $\begin{array}{llllllllll}117 & 197 & 314 & -4.5 & -4.8-2190.51 & -0.19 & 1.66 & -3.43-2192.46 & 249.17\end{array}$ $\begin{array}{llllllllll}117 & 198 & 315 & -4.7 & -4.7-2195.29 & -1.26 & 0.98 & -3.40-2198.96 & 250.74\end{array}$ $\begin{array}{llllllllll}117 & 199 & 316 & -4.8 & -4.6-2199.99 & -1.53 & 1.66 & -3.36-2203.22 & 254.56\end{array}$ $\begin{array}{llllllllll}117 & 200 & 317 & -5.0 & -4.6-2204.60 & -1.58 & 0.98 & -3.33-2208.53 & 257.32\end{array}$ $\begin{array}{llllllllll}117 & 201 & 318 & -5.1 & -4.5-2209.13 & -1.87 & 1.66 & -3.30-2212.64 & 261.28\end{array}$

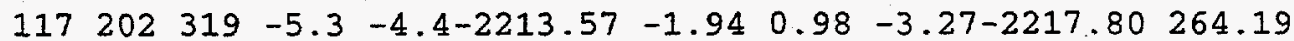

0.00

0.00

0.00

0.00

0.00

0.00

0.00

0.00

0.00

0.00

0.00

0.00

0.00

0.00

0.00

0.00

0.00

0.00

0.00

0.00

0.00

0.00

0.00

0.00

0.00

0.00

0.00

0.00

0.00

0.00

0.00

0.00

0.00

0.00

0.00

0.00

0.00

0.00

0.00

0.00

0.00

0.00

0.00

0.00

0.00

0.00

0.00

0.00

0.00

0.00

0.00

0.00

0.00

0.00

0.00

0.00

0.00

0.00

0.00

0.00

.00

0.00

0.00

0.00

0.00

0.00

0.00

0.00

0.00

0.00

0.00

0.00

0.00

0.00

0.00

0.00

0.00

0.00

0.00

0.00

0.00

0.00

0.00

0.00

0.00

0.00

0.00

0.00

0.00

0.00

0.00

0.00

0.00

0.00

0.00

0.00

0.00

0.00

0.00

0.00

0.00

0.00

0.00

0.00

0.00

0.00

0.00

0.00

0.00

0.00

0.00

0.00

0.00

0.00

0.00

0.00

0.00

0.00

0.00

0.00

0.00

0.00

0.00

0.00

0.00

0.00

0.00

0.00

0.00

0.00

0.00

0.00

0.00

0.00

0.00

0.00

0.00

0.00

0.00

0.00

0.00

0.00

0.00

0.00

0.00

0.00

0.00

0.00

0.00

0.00

0.00

0.00

0.00

0.00

0.00

0.00

0.00

0.00

0.00

0.00

0.00

0.00

0.00

0.00

0.00

0.00

0.00

0.00

0.00

0.00

0.00

0.00

0.00

0.00

0.00

0.00

0.00

0.00 
$117203 \quad 320-5.4-4.3-2217.94-1.991 .66-3.23-2221.51268 .55$ $\begin{array}{lllllllll}117 & 204 & 321 & -5.6 & -4.2-2222.22 & -1.70 & 0.98 & -3.20-2226.14 & 271.99\end{array}$ $\begin{array}{llllllllll}117 & 205 & 322 & -5.7 & -4.2 & -2226.43 & -1.66 & 1.65 & -3.17-2229.60 & 276.60\end{array}$ $\begin{array}{llllllllll}117 & 206 & 323 & -5.9 & -4.1-2230.55 & -1.31 & 0.98 & -3.14-2234.02 & 280.25\end{array}$ $\begin{array}{llllllllll}117 & 207 & 324 & -6.0 & -4.0-2234.60 & -1.34 & 1.65 & -3.11-2237.40 & 284.95\end{array}$ $\begin{array}{llllllllll}117 & 208 & 325 & -6.1 & -3.9-2238.56 & -1.09 & 0.98 & -3.09-2241.76 & 288.66\end{array}$ $117209326-6.3-3.8-2242.45-1.39 .1 .65-3.06-2245.25 \quad 293.24$ $\begin{array}{llllllllll}117 & 210 & 327 & -6.4 & -3.8-2246.27 & -1.21 & 0.98 & -3.03-2249.52 & 297.04\end{array}$ $\begin{array}{llllllllll}117 & 211 & 328 & -6.6 & -3.7-2250.01 & -1.51 & 1.65 & -3.00-2252.87 & 301.76\end{array}$ $\begin{array}{llllllllll}117 & 212 & 329 & -6.7 & -3.6-2253.67 & -1.43 & 0.98 & -2.97-2257.09 & 305.61\end{array}$ $\begin{array}{llllllllll}117 & 213 & 330 & -6.9 & -3.5-2257.26 & -1.74 & 1.65 & -2.95-2260.30 & 310.48\end{array}$ $\begin{array}{lllllllllll}117 & 214 & 331 & -7.0 & -3.5-2260.78 & -1.81 & 0.98 & -2.92-2264.53 & 314.32\end{array}$ $\begin{array}{llllllllll}117 & 215 & 332 & -7.1 & -3.4-2264.22 & -2.20 & 1.64 & -2.89-2267.67 & 319.25\end{array}$ $\begin{array}{llllllllll}117 & 216 & 333 & -7.3 & -3.3-2267.59 & -2.26 & 0.98 & -2.87-2271.74 & 323.25\end{array}$ $\begin{array}{lllllllllll}117 & 217 & 334 & -7.4 & -3.3-2270.89 & -2.71 & 1.64 & -2.84-2274.80 & 328.26\end{array}$ $\begin{array}{llllllllll}117 & 218 & 335 & -7.5 & -3.2-2274.12 & -2.98 & 0.98 & -2.82 & -2278.94 & 332.20\end{array}$ $\begin{array}{llllllllll}117 & 219 & 336 & -7.7 & -3.1-2277.28 & -3.46 & 1.64 & -2.79-2281.89 & 337.31\end{array}$ $\begin{array}{llllllllll}117 & 220 & 337 & -7.8 & -3.1-2280.37 & -3.73 & 0.98 & -2.77-2285.89 & 341.39\end{array}$ $\begin{array}{llllllllll}117 & 221 & 338 & -7.9 & -3.0-2283.39 & -4.32 & 1.64 & -2.75-2288.82 & 346.53\end{array}$ $\begin{array}{llllllllll}117 & 222 & 339 & -8.1 & -2.9-2286.35 & -4.72 & 0.98 & -2.72-2292.81 & 350.61\end{array}$ $\begin{array}{llllllllll}118 & 163 & 281 & 1.5 & -8.5-1969.20 & -4.04 & 0.88 & -5.10-1977.47 & 197.00\end{array}$ $\begin{array}{llllllllll}118 & 164 & 282 & 1.3 & -8.4-1977.64 & -5.07 & 0.00 & -5.04-1987.75 & 194.79\end{array}$ $\begin{array}{llllllllll}118 & 165 & 283 & 1.1 & -8.3-1985.96 & -5.52 & 0.88 & -4.98-1995.58 & 195.03\end{array}$ $\begin{array}{llllllllll}118 & 166 & 284 & 1.0 & -8.1-1994.15 & -5.72 & 0.00 & -4.92-2004.79 & 193.89\end{array}$ $\begin{array}{llllllllll}118 & 167 & 285 & 0.8 & -8.0-2002.23 & -6.15 & 0.87 & -4.86-2012.36 & 194.39\end{array}$ $\begin{array}{llllllllll}118 & 168 & 286 & 0.6 & -7.9-2010.19 & -6.25 & 0.00 & -4.80-2021.24 & 193.59\end{array}$ $\begin{array}{llllllllll}118 & 169 & 287 & 0.4 & -7.8-2018.03 & -6.75 & 0.87 & -4.74-2028.65 & 194.24\end{array}$ $\begin{array}{llllllllll}118 & 170 & 288 & 0.3 & -7.7-2025.75 & -6.78 & 0.00 & -4.68-2037.22 & 193.75\end{array}$ $\begin{array}{lllllllllll}118 & 171 & 289 & 0.1 & -7.6-2033.37 & -7.20 & 0.86 & -4.63-2044.33 & 194.71\end{array}$ $\begin{array}{lllllllllll}118 & 172 & 290 & -0.1 & -7.4-2040.87 & -7.04 & 0.00 & -4.57-2052.48 & 194.63\end{array}$ $\begin{array}{lllllllllll}118 & 173 & 291 & -0.3 & -7.3-2048.26 & -7.55 & 0.86 & -4.52-2059.47 & 195.72\end{array}$ $\begin{array}{llllllllll}118 & 174 & 292 & -0.4 & -7.2-2055.53 & -7.52 & 0.00 & -4.47-2067.52 & 195.73\end{array}$ $\begin{array}{llllllllll}118 & 175 & 293 & -0.6 & -7.1-2062.70 & -7.82 & 0.86 & -4.42-2074.08 & 197.24\end{array}$ $\begin{array}{llllllllll}118 & 176 & 294 & -0.8 & -7.0-2069.76 & -7.67 & 0.00 & -4.37-2081.80 & 197.60\end{array}$ $\begin{array}{llllllllll}118 & 177 & 295 & -0.9 & -6.9-2076.71 & -7.89 & 0.85 & -4.32-2088.07 & 199.40\end{array}$ $\begin{array}{llllllllll}118 & 178 & 296 & -1.1 & -6.8-2083.56 & -7.90 & 0.00 & -4.27-2095.73 & 199.81\end{array}$ $\begin{array}{llllllllll}118 & 179 & 297 & -1.3 & -6.7-2090.30 & -8.27 & 0.85 & -4.22-2101.94 & 201.67\end{array}$ $\begin{array}{llllllllll}118 & 180 & 298 & -1.4 & -6.6-2096.94 & -7.83 & 0.00 & -4.17-2108.94 & 202.74\end{array}$ $\begin{array}{llllllllll}118 & 181 & 299 & -1.6 & -6.5-2103.47 & -7.84 & 0.85 & -4.13-2114.59 & 205.16\end{array}$ $\begin{array}{llllllllll}118 & 182 & 300 & -1.8 & -6.4-2109.91 & -7.24 & 0.00 & -4.08-2121.23 & 206.59\end{array}$ $\begin{array}{llllllllll}118 & 183 & 301 & -1.9 & -6.3-2116.24 & -7.05 & 0.85 & -4.04-2126.48 & 209.41\end{array}$ $\begin{array}{llllllllll}118 & 184 & 302 & -2.1 & -6.2-2122.47 & -6.36 & 0.00 & -3.99-2132.82 & 211.14\end{array}$ $\begin{array}{llllllllll}118 & 185 & 303 & -2.3 & -6.1-2128.60 & -5.29 & 0.84 & -3.95-2137.00 & 215.04\end{array}$ $\begin{array}{llllllllll}118 & 186 & 304 & -2.4 & -6.0-2134.64 & -4.78 & 0.00 & -3.91-2143.33 & 216.78\end{array}$ $\begin{array}{llllllllll}118 & 187 & 305 & -2.6 & -5.9-2140.58 & -3.67 & 0.84 & -3.87-2147.27 & 220.91\end{array}$ $\begin{array}{lllllllll}118 & 188 & 306 & -2.7 & -5.8-2146.42 & -2.95 & 0.00 & -3.83-2153.19 & 223.06\end{array}$ $\begin{array}{llllllllll}118 & 189 & 307 & -2.9 & -5.7-2152.17 & -2.05 & 0.84 & -3.79-2157.17 & 227.16\end{array}$ $\begin{array}{lllllllll}118 & 190 & 308 & -3.1 & -5.6-2157.82 & -1.31 & 0.00 & -3.75-2162.88 & 229.52\end{array}$ $\begin{array}{lllllllll}118 & 191 & 309 & -3.2 & -5.5-2163.38 & -0.66 & 0.83 & -3.71-2166.91 & 233.55\end{array}$ $\begin{array}{llllllllll}118 & 192 & 310 & -3.4 & -5.4-2168.85 & 0.16 & 0.00 & -3.67-2172.36 & 236.18\end{array}$ $\begin{array}{llllllllll}118 & 193 & 311 & -3.5 & -5.3-2174.22 & 0.38 & 0.83 & -3.63-2176.65 & 239.96\end{array}$ $\begin{array}{lllllllll}118 & 194 & 312 & -3.7 & -5.2-2179.51 & 0.76 & 0.00 & -3.59-2182.35 & 242.34\end{array}$ $\begin{array}{llllllllll}118 & 195 & 313 & -3.8 & -5.1-2184.71 & -5.04 & 0.83 & -3.56-2192.48 & 240.27\end{array}$ $\begin{array}{llllllllll}118 & 196 & 314 & -4.0 & -5.1-2189.82 & -4.91 & 0.00 & -3.52-2198.25 & 242.58\end{array}$ $\begin{array}{lllllllll}118 & 197 & 315 & -4.1 & -5.0-2194.84 & -5.13 & 0.82 & -3.49-2202.63 & 246.26\end{array}$ $\begin{array}{llllllllll}118 & 198 & 316 & -4.3 & -4.9-2199.77 & -1.41 & 0.00 & -3.45-2204.63 & 252.33\end{array}$ $\begin{array}{llllllllll}118 & 199 & 317 & -4.4 & -4.8-2204.62 & -1.55 & 0.82 & -3.42-2208.77 & 256.27\end{array}$ $\begin{array}{llllllllll}118 & 200 & 318 & -4.6 & -4.7-2209.38 & -1.59 & 0.00 & -3.39-2214.36 & 258.75\end{array}$ $\begin{array}{llllllllll}118 & 201 & 319 & -4.7 & -4.6-2214.06 & -1.88 & 0.82 & -3.35-2218.47 & 262.71\end{array}$ $\begin{array}{llllllllll}118 & 202 & 320 & -4.9 & -4.6-2218.66 & -1.82 & 0.00 & -3.32-2223.80 & 265.46\end{array}$ $\begin{array}{llllllllll}118 & 203 & 321 & -5.0 & -4.5-2223.17 & -1.99 & 0.82 & -3.29-2227.63 & 269.69\end{array}$ $\begin{array}{llllllllll}118 & 204 & 322 & -5.2 & -4.4-2227.60 & -1.69 & 0.00 & -3.26-2232.55 & 272.85\end{array}$ $\begin{array}{llllllllll}118 & 205 & 323 & -5.3 & -4.3-2231.95 & -1.64 & 0.81 & -3.23-2236.00 & 277.46\end{array}$ $\begin{array}{lllllllll}118 & 206 & 324 & -5.5 & -4.2-2236.22 & -1.28 & 0.00 & -3.20-2240.70 & 280.84\end{array}$ $\begin{array}{llllllllll}118 & 207 & 325 & -5.6 & -4.1-2240.41 & -1.31 & 0.81 & -3.17-2244.08 & 285.53\end{array}$ $\begin{array}{llllllllll}118 & 208 & 326 & -5.8 & -4.1-2244.52 & -1.04 & 0.00 & -3.14-2248.70 & 288.98\end{array}$
0.00

0.00

0.00

0.00

0.00

0.00

0.00

0.00

0.00

0.00

0.00

0.00

0.00

0.00

0.00

0.00

0.00

0.00

0.00

0.00

0.00

0.00

0.00

0.00

0.00

0.00

0.00

0.00

0.00

0.00

0.00

0.00

0.00

0.00

0.00

0.00

0.00

0.00

0.00

0.00

0.00

0.00

0.00

0.00

0.00

0.00

0.00

0.00

0.00

0.00

0.00

0.00

0.00

0.00

0.00

0.00

0.00

0.00

0.00

0.00

0.00

0.00

0.00

0.00

0.00

0.00

0.00

0.00

0.00

0.00

0.00

0.00

0.00

0.00

0.00

0.00

0.00

0.00

0.00

0.00

0.00

0.00

0.00

0.00

0.00

0.00

0.00

0.00

0.00

0.00

0.00

0.00

0.00

0.00

0.00

0.00

0.00

0.00

0.00

0.00

0.00

0.00

0.00

0.00

0.00

0.00

0.00

0.00

0.00

0.00

0.00

0.00

0.00

0.00

0.00

0.00

0.00

0.00

0.00

0.00

0.00

0.00

0.00

0.00

0.00

0.00

0.00

0.00

0.00

0.00

0.00

0.00

0.00

0.00

0.00

0.00

0.00

0.00

0.00

$0.00 \quad 0.00$

$0.00 \quad 0.00$

0.00

0.00

0.00

0.00

$0.00 \quad 0.00$

$0.00 \quad 0.00$

$0.00 \quad 0.00$

$0.00 \quad 0.00$

$0.00 \quad 0.00$

$0.00 \quad 0.00$

$0.00 \quad 0.00$

$0.00 \quad 0.00$

$0.00 \quad 0.00$

$0.00 \quad 0.00$

$0.00 \quad 0.00$

$0.00 \quad 0.00$

$0.00 \quad 0.00$

$0.00 \quad 0.00$

$0.00 \quad 0.00$

0.00

0.00
0.00

$0.00 \quad 0.00$ 
$\begin{array}{llllllllllll}118 & 209 & 327 & -5.9 & -4.0-2248.56 & -1.20 & 0.81 & -3.11-2252.06 & 293.70\end{array}$ $\begin{array}{llllllllll}118 & 210 & 328 & -6.1 & -3.9-2252.51 & -0.99 & 0.00 & -3.08-2256.58 & 297.24\end{array}$ $\begin{array}{llllllllll}118 & 211 & 329 & -6.2 & -3.8-2256.39 & -2.63 & 0.81 & -3.05 & -2261.27 & 300.63\end{array}$ $\begin{array}{llllllllll}118 & 212 & 330 & -6.3 & -3.8 & -2260.20 & -2.64 & 0.00 & -3.02-2265.86 & 304.11\end{array}$ $\begin{array}{llllllllll}118 & 213 & 331 & -6.5 & -3.7 & -2263.93 & -2.86 & 0.80 & -3.00-2268.98 & 309.06\end{array}$ $\begin{array}{lllllllll}118 & 214 & 332 & -6.6 & -3.6-2267.58 & -1.44 & 0.00 & -2.97-2271.99 & 314.12\end{array}$

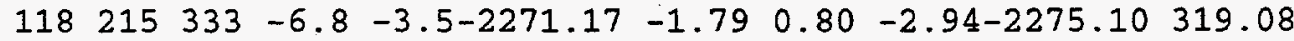
$\begin{array}{lllllllllll}118 & 216 & 334 & -6.9 & -3.5 & -2274.68 & -1.79 & 0.00 & -2.92 & -2279.38 & 322.87\end{array}$ $\begin{array}{lllllllllll}118 & 217 & 335 & -7.0 & -3.4-2278.11 & -2.28 & 0.80 & -2.89 & -2282.49 & 327.84\end{array}$ $\begin{array}{llllllllll}118 & 218 & 336 & -7.2 & -3.3-2281.48 & -2.56 & 0.00 & -2.87-2286.91 & 331.49\end{array}$ $\begin{array}{lllllllllll}118 & 219 & 337 & -7.3 & -3.3-2284.77 & -3.04 & 0.80 & -2.84-2289.86 & 336.61\end{array}$ $\begin{array}{llllllllll}118 & 220 & 338 & -7.4 & -3.2-2288.00 & -3.35 & 0.00 & -2.82-2294.17 & 340.37\end{array}$ $\begin{array}{lllllllllll}118 & 221 & 339 & -7.6 & -3.1-2291.16 & -3.95 & 0.79 & -2.79-2297.11 & 345.50\end{array}$ $\begin{array}{llllllllll}119 & 165 & 284 & 1.5 & -8.4-1984.63 & -5.36 & 1.70 & -5.06-1993.36 & 204.52\end{array}$ $\begin{array}{llllllllll}119 & 166 & 285 & 1.3 & -8.3-1993.00 & -5.45 & 0.98 & -5.00-2002.48 & 203.46\end{array}$ $\begin{array}{llllllllll}119 & 167 & 286 & 1.2 & -8.2-2001.26 & -5.96 & 1.70 & -4.94-2010.46 & 203.55\end{array}$ $\begin{array}{llllllllll}119 & 168 & 287 & 1.0 & -8.1-2009.39 & -6.29 & 0.98 & -4.88-2019.59 & 202.50\end{array}$ $\begin{array}{llllllllll}119 & 169 & 288 & 0.8 & -8.0-2017.41 & -6.71 & 1.69 & -4.82 & -2027.25 & 202.91\end{array}$ $\begin{array}{llllllllll}119 & 170 & 289 & 0.6 & -7.8-2025.31 & -6.58 & 0.98 & -4.77-2035.68 & 202.55\end{array}$ $\begin{array}{llllllllll}119 & 171 & 290 & 0.5 & -7.7-2033.10 & -7.05 & 1.69 & -4.71-2043.16 & 203.14\end{array}$ $\begin{array}{llllllllll}119 & 172 & 291 & 0.3 & -7.6-2040.77 & -7.15 & 0.98 & -4.65-2051.60 & 202.78\end{array}$ $\begin{array}{llllllllll}119 & 173 & 292 & 0.1 & -7.5-2048.33 & -7.45 & 1.69 & -4.60-2058.69 & 203.75\end{array}$ $\begin{array}{llllllllll}119 & 174 & 293 & -0.1 & -7.4-2055.78 & -7.58 & 0.98 & -4.55-2066.93 & 203.59\end{array}$ $\begin{array}{lllllllllll}119 & 175 & 294 & -0.2 & -7.3-2063.12 & -7.89 & 1.68 & -4.49-2073.81 & 204.77\end{array}$ $\begin{array}{llllllllll}119 & 176 & 295 & -0.4 & -7.2 & -2070.34 & -7.67 & 0.98 & -4.44-2081.48 & 205.18\end{array}$ $\begin{array}{llllllllll}119 & 177 & 296 & -0.6 & -7.1-2077.47 & -7.63 & 1.68 & -4.39-2087.81 & 206.92\end{array}$ $\begin{array}{llllllllll}119 & 178 & 297 & -0.7 & -7.0-2084.48 & -7.75 & 0.98 & -4.34-2095.60 & 207.20\end{array}$ $\begin{array}{llllllllll}119 & 179 & 298 & -0.9 & -6.9-2091.39 & -8.11 & 1.68 & -4.29-2102.11 & 208.76\end{array}$ $\begin{array}{lllllllllll}119 & 180 & 299 & -1.1 & -6.7-2098.19 & -7.79 & 0.98 & -4.24-2109.25 & 209.69\end{array}$ $\begin{array}{llllllllll}119 & 181 & 300 & -1.2 & -6.6-2104.89 & -7.73 & 1.68 & -4.20 & -2115.14 & 211.87\end{array}$ $\begin{array}{lllllllllll}119 & 182 & 301 & -1.4 & -6.5-2111.49 & -7.09 & 0.98 & -4.15-2121.76 & 213.33\end{array}$ $\begin{array}{lllllllllll}119 & 183 & 302 & -1.6 & -6.4-2117.99 & -6.88 & 1.67 & -4.11-2127.30 & 215.86\end{array}$ $\begin{array}{llllllllll}119 & 184 & 303 & -1.7 & -6.3-2124.38 & -6.18 & 0.98 & -4.06-2133.65 & 217.58\end{array}$

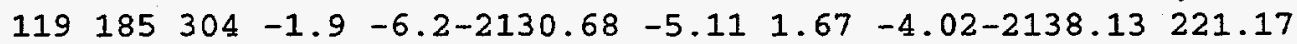
$\begin{array}{llllllllll}119 & 186 & 305 & -2.0 & -6.1-2136.87 & -4.60 & 0.98 & -3.97-2144.47 & 222.90 .\end{array}$ $\begin{array}{lllllllllll}119 & 187 & 306 & -2.2 & -6.0-2142.97 & -3.47 & 1.67 & -3.93-2148.70 & 226.74\end{array}$ $\begin{array}{llllllllll}119 & 188 & 307 & -2.4 & -5.9-2148.97 & -2.78 & 0.98 & -3.89-2154.67 & 228.85\end{array}$ $\begin{array}{llllllllll}119 & 189 & 308 & -2.5 & -5.9-2154.88 & -1.93 & 1.67 & -3.85-2158.99 & 232.59\end{array}$ $\begin{array}{llllllllll}119 & 190 & 309 & -2.7 & -5.8-2160.69 & -1.18 & 0.98 & -3.81-2164.70 & 234.95\end{array}$ $\begin{array}{llllllllll}119 & 191 & 310 & -2.8 & -5.7-2166.41 & -0.59 & 1.67 & -3.77-2169.10 & 238.63\end{array}$ $\begin{array}{llllllllll}119 & 192 & 311 & -3.0 & -5.6-2172.03 & -0.08 & 0.98 & -3.73-2174.87 & 240.93\end{array}$ $\begin{array}{llllllllll}119 & 193 & 312 & -3.2 & -5.5-2177.57 & 0.43 & 1.66 & -3.69-2179.17 & 244.70\end{array}$ $\begin{array}{llllllllll}119 & 194 & 313 & -3.3 & -5.4-2183.01 & 0.77 & 0.98 & -3.66-2184.92 & 247.03\end{array}$ $\begin{array}{llllllllll}119 & 195 & 314 & -3.5 & -5.3-2188.36 & -5.33 & 1.66 & -3.62-2195.65 & 244.37\end{array}$

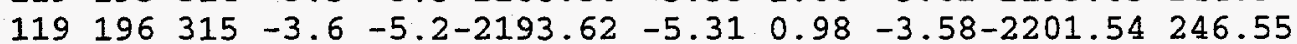
$\begin{array}{llllllllll}119 & 197 & 316 & -3.8 & -5.1-2198.80 & -5.53 & 1.66 & -3.55-2206.21 & 249.94\end{array}$ $\begin{array}{llllllllll}119 & 198 & 317 & -3.9 & -5.0-2203.88 & -1.99 & 0.98 & -3.51-2208.41 & 255.82\end{array}$ $\begin{array}{llllllllll}119 & 199 & 318 & -4.1 & -5.0-2208.88 & -1.93 & 1.66 & -3.48-2212.63 & 259.67\end{array}$ $\begin{array}{llllllllll}119 & 200 & 319 & -4.2 & -4.9-2213.80 & -1.96 & 0.98 & -3.44-2218.22 & 262.15\end{array}$ $\begin{array}{lllllllll}119 & 201 & 320 & -4.4 & -4.8-2218.62 & -2.15 & 1.65 & -3.41-2222.53 & 265.92\end{array}$ $\begin{array}{lllllllllll}119 & 202 & 321 & -4.5 & -4.7-2223.37 & -2.04 & 0.98 & -3.38-2227.81 & 268.71\end{array}$ $\begin{array}{llllllllll}119 & 203 & 322 & -4.7 & -4.6-2228.03 & -2.18 & 1.65 & -3.34-2231.90 & 272.69\end{array}$ $\begin{array}{llllllllll}119 & 204 & 323 & -4.8 & -4.5-2232.61 & -1.86 & 0.98 & -3.31-2236.80 & 275.85\end{array}$ $\begin{array}{llllllllll}119 & 205 & 324 & -5.0 & -4.5-2237.11 & -1.76 & 1.65 & -3.28-2240.50 & 280.23\end{array}$ $\begin{array}{llllllllll}119 & 206 & 325 & -5.1 & -4.4-2241.52 & -1.39 & 0.98 & -3.25-2245.18 & 283.62\end{array}$ $\begin{array}{llllllllll}119 & 207 & 326 & -5.3 & -4.3-2245.86 & -1.43 & 1.65 & -3.22-2248.86 & 288.02\end{array}$ $\begin{array}{llllllllll}119 & 208 & 327 & -5.4 & -4.2-2250.11 & -1.13 & 0.98 & -3.19-2253.46 & 291.49\end{array}$ $\begin{array}{llllllllll}119 & 209 & 328 & -5.5 & -4.1-2254.29 & -1.29 & 1.65 & -3.16-2257.09 & 295.92\end{array}$ $\begin{array}{lllllllllll}119 & 210 & 329 & -5.7 & -4.1-2258.39 & -1.10 & 0.98 & -3.13-2261.64 & 299.44\end{array}$ $\begin{array}{llllllllll}119 & 211 & 330 & -5.8 & -4.0-2262.41 & -3.10 & 1.64 & -3.10-2266.97 & 302.19\end{array}$ $\begin{array}{llllllllll}119 & 212 & 331 & -6.0 & -3.9-2266.36 & -3.00 & 0.98 & -3.07-2271.46 & 305.77\end{array}$ $\begin{array}{lllllllll}119 & 213 & 332 & -6.1 & -3.8-2270.23 & -3.21 & 1.64 & -3.04-2274.84 & 310.46\end{array}$ $\begin{array}{lllllllll}119 & 214 & 333 & -6.3 & -3.8-2274.03 & -1.15 & 0.98 & -3.02-2277.22 & 316.16\end{array}$ $\begin{array}{llllllllll}119 & 215 & 334 & -6.4 & -3.7-2277.75 & -1.57 & 1.64 & -2.99-2280.67 & 320.78\end{array}$ $\begin{array}{llllllllll}119 & 216 & 335 & -6.5 & -3.6-2281.40 & -1.66 & 0.98 & -2.96-2285.04 & 324.47\end{array}$ $\begin{array}{llllllllll}119 & 217 & 336 & -6.7 & -3.5 & -2284.97 & -2.18 & 1.64 & -2.94-2288.45 & 329.14\end{array}$ 133
0.00

0.00

0.00

0.00

0.00

0.00

0.00

0.00

0.00

0.00

0.00

0.00

0.00

0.00

0.00

0.00

0.00

0.00

0.00

0.00

0.00

0.00

0.00

0.00

0.00

0.00

0.00

0.00

0.00

0.00

0.00

0.00

0.00

0.00

0.00

0.00

$\begin{array}{llll}0.00 & 0.00 & 0.00\end{array}$

$\begin{array}{lll}0.00 & 0.00 & 0.00\end{array}$

$\begin{array}{llll}0.00 & 0.00 & 0.00\end{array}$

$\begin{array}{llll}0.00 & 0.00 & 0.00\end{array}$

$\begin{array}{llll}0.00 & 0.00 & 0.00\end{array}$

$\begin{array}{llll}0.00 & 0.00 & 0.00\end{array}$

$\begin{array}{lll}0.00 & 0.00 & 0.00\end{array}$

$\begin{array}{llll}0.00 & 0.00 & 0.00\end{array}$

$\begin{array}{llll}0.00 & 0.00 & 0.00\end{array}$

$\begin{array}{lll}0.00 & 0.00 & 0.00\end{array}$

$\begin{array}{llll}0.00 & 0.00 & 0.00\end{array}$

$0.00 \quad 0.00 \quad 0.00$

$\begin{array}{llll}0.00 & 0.00 & 0.00\end{array}$

$\begin{array}{llll}0.00 & 0.00 & 0.00\end{array}$

$\begin{array}{llll}0.00 & 0.00 & 0.00\end{array}$

$\begin{array}{lll}0.00 & 0.00 & 0.00\end{array}$

$\begin{array}{llll}0.00 & 0.00 & 0.00\end{array}$

$\begin{array}{llll}0.00 & 0.00 & 0.00\end{array}$

$\begin{array}{llll}0.00 & 0.00 & 0.00\end{array}$

$\begin{array}{llll}0.00 & 0.00 & 0.00\end{array}$

$\begin{array}{llll}0.00 & 0.00 & 0.00\end{array}$

$\begin{array}{llll}0.00 & 0.00 & 0.00\end{array}$

$\begin{array}{llll}0.00 & 0.00 & 0.00\end{array}$

$\begin{array}{llll}0.00 & 0.00 & 0.00\end{array}$

$\begin{array}{llll}0.00 & 0.00 & 0.00\end{array}$

$\begin{array}{llll}0.00 & 0.00 & 0.00\end{array}$

$\begin{array}{llll}0.00 & 0.00 & 0.00\end{array}$

$\begin{array}{llll}0.00 & 0.00 & 0.00\end{array}$

$\begin{array}{llll}0.00 & 0.00 & 0.00\end{array}$

$\begin{array}{lll}0.00 & 0.00 & 0.00\end{array}$

$\begin{array}{llll}0.00 & 0.00 & 0.00\end{array}$

$\begin{array}{llll}0.00 & 0.00 & 0.00\end{array}$

$\begin{array}{lll}0.00 & 0.00 & 0.00\end{array}$

$\begin{array}{llll}0.00 & 0.00 & 0.00\end{array}$

$\begin{array}{llll}0.00 & 0.00 & 0.00\end{array}$

$\begin{array}{llll}0.00 & 0.00 & 0.00\end{array}$

$\begin{array}{llll}0.00 & 0.00 & 0.00\end{array}$

$\begin{array}{lll}0.00 & 0.00 & 0.00\end{array}$

$\begin{array}{llll}0.00 & 0.00 & 0.00\end{array}$

$\begin{array}{llll}0.00 & 0.00 & 0.00\end{array}$

$\begin{array}{llll}0.00 & 0.00 & 0.00\end{array}$

$\begin{array}{lll}0.00 & 0.00 & 0.00\end{array}$

$\begin{array}{llll}0.00 & 0.00 & 0.00\end{array}$

$\begin{array}{llll}0.00 & 0.00 & 0.00\end{array}$

$\begin{array}{llll}0.00 & 0.00 & 0.00\end{array}$

$\begin{array}{lll}0.00 & 0.00 & 0.00\end{array}$

$\begin{array}{llll}0.00 & 0.00 & 0.00\end{array}$

$\begin{array}{lll}0.00 & 0.00 & 0.00\end{array}$

$\begin{array}{lll}0.00 & 0.00 & 0.00\end{array}$

$\begin{array}{llll}0.00 & 0.00 & 0.00\end{array}$

$\begin{array}{llll}0.00 & 0.00 & 0.00\end{array}$

$\begin{array}{lll}0.00 & 0.00 & 0.00\end{array}$

$\begin{array}{lll}0.00 & 0.00 & 0.00\end{array}$ 


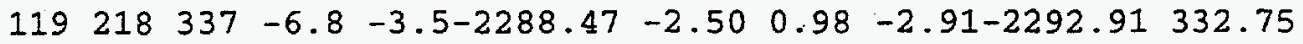
$\begin{array}{lllllllll}119 & 219 & 338 & -6.9 & -3.4-2291.91 & -3.03 & 1.64 & -2.89-2296.19 & 337.54\end{array}$ $\begin{array}{lllllllll}119 & 220 & 339 & -7.1 & -3.3-2295.27 & -3.34 & 0.98 & -2.86-2300.49 & 341.31\end{array}$ $\begin{array}{llllllllll}120 & 167 & 287 & 1.5 & -8.4-1999.92 & -5.53 & 0.87 & -5.03-2009.60 & 211.68\end{array}$ $\begin{array}{lllllllll}120 & 168 & 288 & 1.4 & -8.2-2008.23 & -5.68 & 0.0 .0 & -4.97-2018.87 & 210.48\end{array}$ 120169289 120170290 120171291 120172292 120173293 120174294 120175295 120176296 $120177 \quad 297$ $120178 \quad 298$

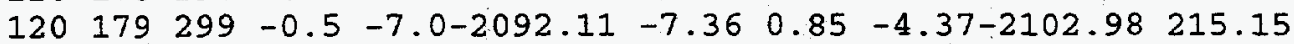
$\begin{array}{llllllllll}120 & 180 & 300 & -0.7 & -6.9-2099.08 & -6.95 & 0.00 & -4.32-2110.34 & 215.86\end{array}$

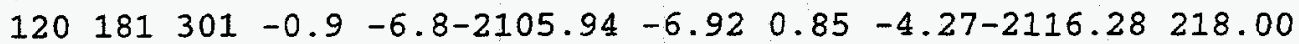
$\begin{array}{lllllllll}120 & 182 & 302 & -1.0 & -6.7-2112.70 & -6.31 & 0.00 & -4.22-2123.24 & 219.11\end{array}$

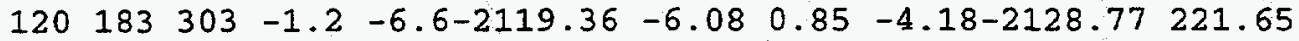
$\begin{array}{lllllllll}120 & 184 & 304 & -1.4 & -6.5-2125.92 & -5.37 & 0.00 & -4.13-2135.42 & 223.07\end{array}$ $\begin{array}{lllllllll}120 & 185 & 305 & -1.5 & -6.4-2132.38 & -4.39 & 0.84 & -4.09-2140.01 & 226.55\end{array}$

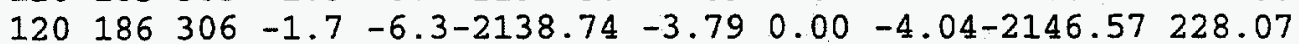
$\begin{array}{llllllllll}120 & 187 & 307 & -1.8 & -6.2 & -2145.00 & -2.71 & 0.84 & -4.00-2150.87 & 231.84\end{array}$ $\begin{array}{lllllllll}120 & 188 & 308 & -2.0 & -6.1-2151.16 & -1.93 & 0.00 & -3.96-2157.04 & 233.73\end{array}$ $\begin{array}{lllllllll}120 & 189 & 309 & -2.2 & -6.0-2157.22 & -1.25 & 0.84 & -3.91-2161.55 & 237.30\end{array}$ $\begin{array}{lllllllll}120 & 190 & 310 & -2.3 & -5.9-2163.19 & -0.51 & 0.00 & -3.87-2167.58 & 239.34\end{array}$ $\begin{array}{lllllllll}120 & 191 & 311 & -2.5 & -5.8-2169.07 & 0.00 & 0.83 & -3.83-2172.07 & 242.92\end{array}$ $\begin{array}{lllllllll}120 & 192 & 312 & -2.6 & -5.7-2174.85 & 0.43 & 0.00 & -3.79-2178.22 & 244.85\end{array}$ $\begin{array}{lllllllll}120 & 193 & 313 & -2.8 & -5.6-2180.54 & 0.80 & 0.83 & -3.75-2182.66 & 248.47\end{array}$ $\begin{array}{lllllllll}120 & 194 & 314 & -2.9 & -5.5-2186.14 & 1.09 & 0.00 & -3.72-2188.76 & 250.44\end{array}$

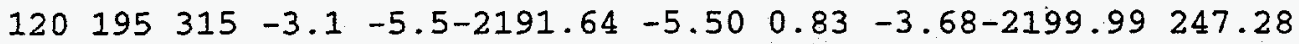
$\begin{array}{lllllllll}120 & 196 & 316 & -3.2 & -5.4-2197.06 & -5.49 & 0.00 & -3.64-2206.19 & 249.16\end{array}$ $\begin{array}{lllllllll}120 & 197 & 317 & -3.4 & -5.3-2202.39 & -5.71 & 0.82 & -3.61-2210.88 & 252.54\end{array}$

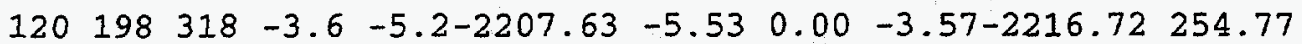
$\begin{array}{lllllllll}120 & 199 & 319 & -3.7 & -5.1-2212.78 & -1.98 & 0.82 & -3.53-2217.47 & 262.10\end{array}$

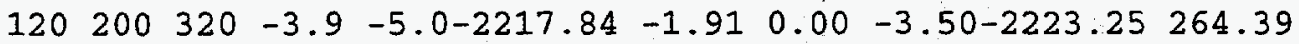
$\begin{array}{lllllllll}120 & 201 & 321 & -4.0 & -4.9-2222.82 & -2.19 & 0.82 & -3.47-2227.65 & 268.05\end{array}$

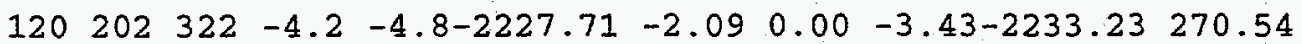

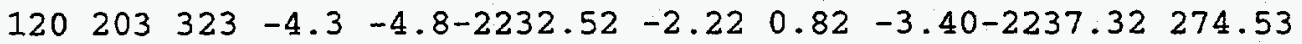
$\begin{array}{lllllllll}120 & 204 & 324 & -4.5 & -4.7-2237.25 & -1.85 & 0.00 & -3.37-2242.46 & 277.46\end{array}$ $\begin{array}{lllllllll}120 & 205 & 325 & -4.6 & -4.6-2241.89 & -1.78 & 0.81 & -3.33-2246.19 & 281.80\end{array}$ $\begin{array}{lllllllll}120 & 206 & 326 & -4.7 & -4.5-2246.45 & -1.43 & 0.00 & -3.30-2251.19 & 284.88\end{array}$ $\begin{array}{llllllllll}120 & 207 & 327 & -4.9 & -4.4-2250.94 & -1.46 & 0.81 & -3.27-2254.85 & 289.28\end{array}$ $\begin{array}{lllllllll}120 & 208 & 328 & -5.0 & -4.4-2255.34 & -1.17 & 0.00 & -3.24-2259.75 & 292.46\end{array}$ $\begin{array}{lllllllll}120 & 209 & 329 & -5.2 & -4.3-2259.66 & -1.30 & 0.81 & -3.21-2263.36 & 296.92\end{array}$

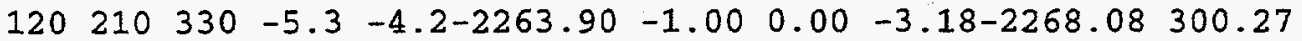
$\begin{array}{lllllllll}120 & 211 & 331 & -5.5 & -4.1-2268.07 & -1.23 & 0.81 & -3.15-2271.64 & 304.78\end{array}$

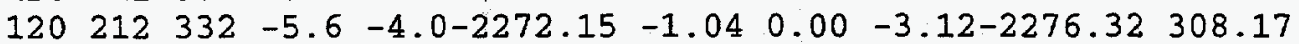
$\begin{array}{lllllllll}120 & 213 & 333 & -5.8 & -4.0-2276.17 & -3.22 & 0.80 & -3.09-2281.68 & 310.89\end{array}$ $\begin{array}{lllllllll}120 & 214 & 334 & -5.9 & -3.9-2280.10 & -0.83 & 0.00 & -3.07-2284.00 & 316.64\end{array}$

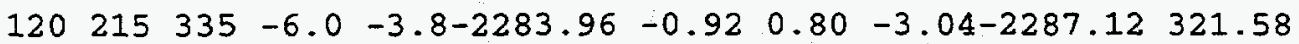
$\begin{array}{lllllllll}120 & 216 & 336 & -6.2 & -3.7-2287.75 & -1.16 & 0.00 & -3.01-2291.92 & 324.86\end{array}$ $\begin{array}{lllllllll}120 & 217 & 337 & -6.3 & -3.7-2291.46 & -1.56 & 0.80 & -2.99-2295.21 & 329.64\end{array}$ $\begin{array}{lllllllll}120 & 218 & 338 & -6.4 & -3.6-2295.11 & -1.87 & 0.00 & -2.96-2299.93 & 332.99\end{array}$ $\begin{array}{lllllllll}120 & 219 & 339 & -6.6 & -3.5-2298.67 & -2.41 & 0.80 & -2.93-2303.22 & 337.77\end{array}$ $\begin{array}{lllllllll}121 & 169 & 290 & 1.5 & -8.3-2015.06 & -5.87 & 1.69 & -4.99-2024.23 & 220.45\end{array}$ $\begin{array}{lllllllll}121 & 170 & 291 & 1.4 & -8.2-2023.31 & -5.98 & 0.97 & -4.93-2033.25 & 219.50\end{array}$ $\begin{array}{lll}121 & 171 & 292\end{array}$ 121172293 121173294 $121 \quad 174295$ 121175296 121176297 121177298 121178299

$1.2-8.1-2031.44 \quad-6.30 \quad 1.69-4.87-2040.93 \quad 219.89$ $\begin{array}{lllllll}1.0 & -8.0-2039.46 & -6.24 & 0.97 & -4.81-2049.55 & 219.35\end{array}$ $0.9-7.8-2047.37-6.57 \quad 1.68-4.76-2057.01219 .96$ $\begin{array}{llllll}0.7 & -7.7-2055.16 & -6.50 & 0.97 & -4.70-2065.39 & 219.65\end{array}$ $\begin{array}{llllll}0.5 & -7.6-2062.83 & -6.80 & 1.68 & -4.65-2072.60 & 220.51\end{array}$ $\begin{array}{lllllll}0.3 & -7.5-2070.40 & -6.54 & 0.97 & -4.59-2080.57 & 220.62\end{array}$ $\begin{array}{lllllll}0.2 & -7.4-2077.86 & -6.75 & 1.68 & -4.54-2087.48 & 221.78\end{array}$ $\begin{array}{lllllll}0.0 & -7.3-2085.21 & -6.73 & 0.97 & -4.49-2095.46 & 221.86\end{array}$
0.00

0.00

0.00

0.00

0.00

0.00

0.00

0.00

0.00

0.00

0.00

0.00

0.00

0.00

0.00

0.00

0.00

0.00

0.00

0.00

0.00

0.00

0.00

0.00

0.00

0.00

0.00

0.00

0.00

0.00

0.00

0.00

0.00

0.00

0.00

0.00

0.00

0.00

0.00

0.00

0.00

0.00

0.00

0.00

0.00

0.00

0.00

0.00

0.00

0.00

0.00

0.00

0.00

0.00

0.00

0.00

0.00

0.00

0.00

0.00

0.00 0.00 0.00

0.00 0.00 0.00 0.00 0.00 0.00 0.00 0.00 0.00 0.00 0.00 0.00 0.00 0.00 0.00 0.00 0.00 0.00 0.00 0.00 0.00 0.00 0.00 0.00 0.00 0.00 0.00 0.00 0.00 0.00 0.00 0.00 0.00 0.00 0.00 0.00 0.00 0.00 0.00 0.00 0.00 0.00 0.00 0.00 0.00 0.00 0.00 0.00 0.00 0.00 0.00 0.00 0.00 0.00 0.00 0.00 0.00 0.00 0.00

0.00 0.00

0.00 0.00

0.00 0.00 0.00

$0.00 \quad 0.00$ $0.00 \quad 0.00$

0.00 0.00 0.00 0.00 0.00 0.00 0.00 0.00 0.00 0.00 0.00 0.00 0.00 0.00 0.00 0.00 0.00 0.00 0.00 0.00 0.00 0.00 0.00 0.00 0.00 0.00 0.00 0.00 0.00 0.00 0.00 0.00 0.00 0.00 0.00 0.00 0.00 0.00 0.00 0.00 0.00 0.00 0.00 0.00 0.00 0.00 0.00 0.00 0.00 0.00 0.00 0.00 0.00 0.00 0.00 0.00 0.00 0.00 0.00 0.00 0.00 0.00 
$\begin{array}{lllllllll}121 & 179 & 300 & -0.2 & -7.2-2092.45 & -7.11 & 1.67 & -4.44-2102.33 & 223.07\end{array}$ $\begin{array}{lllllllll}121 & 180 & 301 & -0.3 & -7.1-2099.59 & -6.75 & 0.97 & -4.39-2109.76 & 223.71\end{array}$

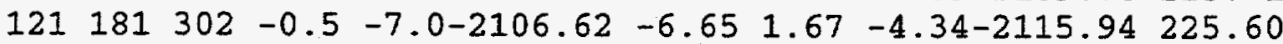
$\begin{array}{llllllllll}121 & 182 & 303 & -0.7 & -6.9-2113.55 & -6.02 & 0.97 & -4.29-2122.89 & 226.72\end{array}$

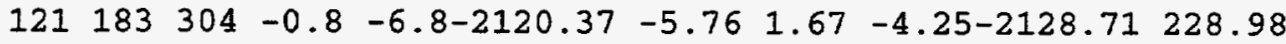
$\begin{array}{lllllllll}121 & 184 & 305 & -1.0 & -6.7-2127.09 & -5.05 & 0.97 & -4.20-2135.37 & 230.38\end{array}$ $\begin{array}{llllllllll}121 & 185 & 306 & -1.1 & -6.6-2133.71 & -3.80 & 1.67 & -4.15-2140.00 & 233.83\end{array}$ $\begin{array}{llllllllll}121 & 186 & 307 & -1.3 & -6.5-2140.23 & -3.39 & 0.97 & -4.11-2146.76 & 235.14\end{array}$ $\begin{array}{llllllll}121 & 187.308 & -1.5 & -6.4-2146.65 & -2.42 & 1.67 & -4.07-2151.47 & 238.50\end{array}$ $\begin{array}{lllllllll}121 & 188 & 309 & -1.6 & -6.3-2152.97 & -1.69 & 0.97 & -4.02-2157.72 & 240.32\end{array}$ $\begin{array}{lllllllll}121 & 189 & 310 & -1.8 & -6.2-2159.20 & -1.17 & 1.66 & -3.98-2162.69 & 243.43\end{array}$ $\begin{array}{llllllllll}121 & 190 & 311 & -1.9 & -6.1-2165.33 & -0.48 & 0.97 & -3.94-2168.78 & 245.41\end{array}$ $\begin{array}{llllllllll}121 & 191 & 312 & -2.1 & -6.0 & -2171.36 & -0.09 & 1.66 & -3.90-2173.69 & 248.57\end{array}$ $\begin{array}{lllllllll}121 & 192 & 313 & -2.3 & -5.9-2177.30 & 0.30 & 0.97 & -3.86-2179.89 & 250.44\end{array}$ $\begin{array}{lllllllll}121 & 193 & 314 & -2.4 & -5.8-2183.15 & 0.60 & 1.66 & -3.82-2184.70 & 253.69\end{array}$ $\begin{array}{lllllllll}121 & 194 & 315 & -2.6 & -5.7-2188.90 & 0.86 & 0.97 & -3.78-2190.85 & 255.62\end{array}$

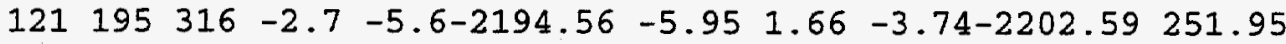

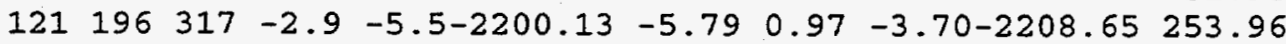

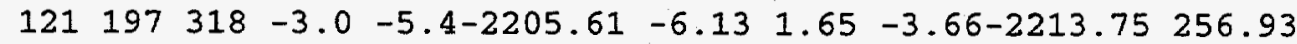
$\begin{array}{lllllllll}121 & 198 & 319 & -3.2 & -5.3-2211.00 & -5.94 & 0.97 & -3.63-2219.60 & 259.16\end{array}$ $\begin{array}{lllllllll}121 & 199 & 320 & -3.3 & -5.3-2216.30 & -2.43 & 1.65 & -3.59-2220.67 & 266.15\end{array}$ $\begin{array}{lllllllll}121 & 200 & 321 & -3.5 & -5.2-2221.52 & -2.30 & 0.97 & -3.56-2226.40 & 268.49\end{array}$ $\begin{array}{lllllllll}121 & 201 & 322 & -3.6 & -5.1-2226.65 & -2.49 & 1.65 & -3.52-2231.01 & 271.96\end{array}$ $\begin{array}{lllllllll}121 & 202 & 323 & -3.8 & -5.0-2231.69 & -2.46 & 0.97 & -3.49-2236.67 & 274.37\end{array}$ $\begin{array}{lllllllll}121 & 203 & 324 & -3.9 & -4.9-2236.65 & -2.47 & 1.65 & -3.45-2240.92 & 278.19\end{array}$ $\begin{array}{lllllllll}121 & 204 & 325 & -4.1 & -4.8-2241.52 & -2.14 & 0.97 & -3.42-2246.11 & 281.07\end{array}$ $\begin{array}{lllllllll}121 & 205 & 326 & -4.2 & -4.7-2246.31 & -1.98 & 1.65 & -3.39-2250.04 & 285.22\end{array}$ $\begin{array}{lllllllll}121 & 206 & 327 & -4.4 & -4.7-2251.02 & -1.67 & 0.97 & -3.36-2255.08 & 288.25\end{array}$

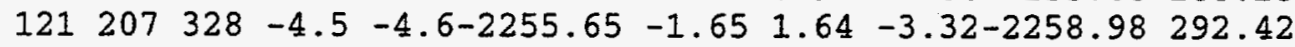
$\begin{array}{lllllllll}121 & 208 & 329 & -4.7 & -4.5-2260.19 & -1.13 & 0.97 & -3.29-2263.65 & 295.82\end{array}$ $\begin{array}{lllllllll}121 & 209 & 330 & -4.8 & -4.4-2264.66 & -1.47 & 1.64 & -3.26-2267.75 & 299.79\end{array}$

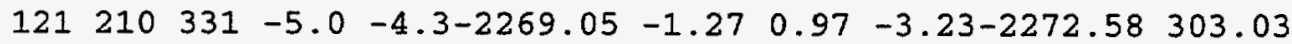
$\begin{array}{lllllllll}121 & 211 & 332 & -5.1 & -4.3-2273.35 & -1.40 & 1.64 & -3.20-2276.32 & 307.36\end{array}$

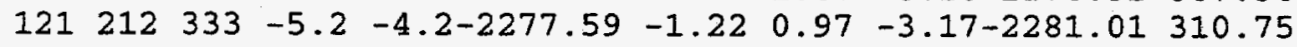
$\begin{array}{lllllllll}121 & 213 & 334 & -5.4 & -4.1-2281.74 & -3.33 & 1.64 & -3.14-2286.58 & 313.25\end{array}$

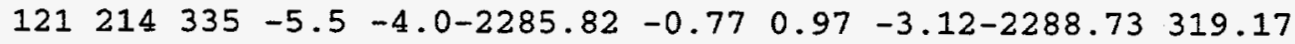
$\begin{array}{llllllllll}121 & 215 & 336 & -5.7 & -4.0 & -2289.82 & -1.04 & 1.64 & -3.09-2292.31 & 323.66\end{array}$ $\begin{array}{lllllllll}121 & 216 & 337 & -5.8 & -3.9-2293.74 & -0.87 & 0.97 & -3.06-2296.70 & 327.34\end{array}$

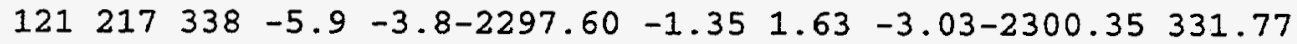
$\begin{array}{lllllllll}121 & 218 & 339 & -6.1 & -3.7-2301.37 & -1.49 & 0.97 & -3.01-2304.90 & 335.28\end{array}$ $\begin{array}{lllllllll}122 & 172 & 294 & 1.4 & -8.1-2038.26 & -5.67 & 0.00 & -4.90-2048.83 & 227.33\end{array}$ $\begin{array}{lllllllll}122 & 173 & 295 & 1.2 & -8.0-2046.34 & -5.92 & 0.86 & -4.84-2056.23 & 228.00\end{array}$ $\begin{array}{llllllllll}122 & 174 & 296 & 1.0 & -7.9-2054.30 & -5.76 & 0.00 & -4.78-2064.84 & 227.46\end{array}$ $\begin{array}{llllllllll}122 & 175 & 297 & 0.9 & -7.8-2062.15 & -5.84 & 0.86 & -4.73-2071.85 & 228.52\end{array}$ $\begin{array}{llllllllll}122 & 176 & 298 & 0.7 & -7.7-2069.88 & -5.73 & 0.00 & -4.67-2080.29 & 228.16\end{array}$ $\begin{array}{lllllllll}122 & 177 & 299 & 0.5 & -7.6-2077.51 & -5.85 & 0.85 & -4.62-2087.12 & 229.39\end{array}$ $\begin{array}{llllllllll}122 & 178 & 300 & 0.4 & -7.5 & -2085.03 & -6.27 & 0.00 & -4.57-2095.86 & 228.72\end{array}$ $\begin{array}{llllllllll}122 & 179 & 301 & 0.2 & -7.4-2092.44 & -6.56 & 0.85 & -4.51-2102.66 & 230.00\end{array}$ $\begin{array}{llllllllll}122 & 180 & 302 & 0.0 & -7.2-2099.74 & -6.21 & 0.00 & -4.46-2110.41 & 230.32\end{array}$

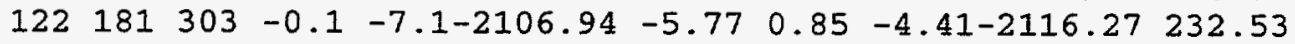

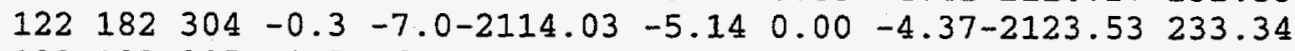
$\begin{array}{llllllllll}122 & 183 & 305 & -0.5 & -6.9-2121.01 & -4.88 & 0.85 & -4.32-2129.37 & 235.58\end{array}$

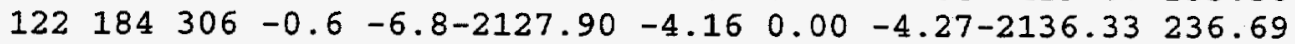

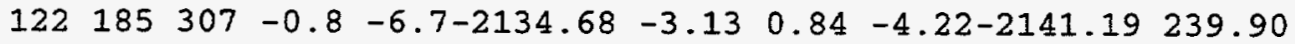
$\begin{array}{lllllllll}122 & 186 & 308 & -0.9 & -6.6-2141.36 & -2.44 & 0.00 & -4.18-2147.98 & 241.18\end{array}$ $\begin{array}{llllllllll}122 & 187 & 309 & -1.1 & -6.5-2147.94 & -1.73 & 0.84 & -4.13-2152.97 & 244.26\end{array}$

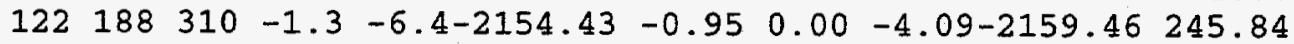
$\begin{array}{lllllllll}122 & 189 & 311 & -1.4 & -6.3-2160.81 & -0.59 & 0.84 & -4.05-2164.61 & 248.76\end{array}$ $122 \quad 190 \quad 312-1.6 \quad-6.2-2167.10$ $\begin{array}{lllll}122 & 191 & 313 & -1.7 & -6.1-2173.29\end{array}$ $\begin{array}{lllll}122 & 192 & 314 & -1.9 & -6.0-2179.38\end{array}$ $\begin{array}{lllll}122 & 193 & 315 & -2.1 & -6.0-2185.39\end{array}$ $122 \quad 194316-2.2-5.9-2191.29$ $\begin{array}{lllll}122 & 195 & 317 & -2.4 & -5.8-2197.11\end{array}$ $\begin{array}{lllll}122 & 196 & 318 & -2.5 & -5.7-2202.83\end{array}$ $\begin{array}{lllll}122 & 197 & 319 & -2.7 & -5.6-2208.46\end{array}$
$0.00 \quad 0.00-4.00-2171.10 \quad 250.34$ $\begin{array}{llll}0.33 & 0.83 & -3.96-2176.08 & 253.43\end{array}$ $\begin{array}{lllll}0.66 & 0.00 & -3.92-2182.64 & 254.94\end{array}$ $\begin{array}{lllll}0.88 & 0.83 & -3.88-2187.56 & 258.10\end{array}$ $\begin{array}{llll}1.10 & 0.00 & -3.84-2194.03 & 259.70\end{array}$ $\begin{array}{llll}-6.16 & 0.83 & -3.80-2206.24 & 255.56\end{array}$ $\begin{array}{lllll}-6.13 & 0.00 & -3.76-2212.72 & 257.15\end{array}$ $\begin{array}{lllll}-6.33 & 0.82 & -3.73-2217.70 & 260.25\end{array}$ 135
0.00

0.00

0.00

0.00

0.00

0.00

0.00

0.00

0.00

0.00

0.00

0.00

0.00

0.00

0.00

0.00

0.00

0.00

0.00

0.00

0.00

0.00

0.00

0.00

0.00

0.00

0.00

0.00

0.00

0.00

0.00

0.00

0.00

0.00

0.00

0.00

0.00

0.00

0.00

0.00

0.00

0.00

0.00

0.00

0.00

0.00

0.00

0.00

0.00

0.00

0.00

0.00

0.00

0.00

0.00

0.00

0.00

0.00

0.00

0.00

0.00

0.00

0.00

0.00

0.00

0.00

0.00

0.00

0.00

0.00

0.00

0.00

0.00

0.00

0.00

0.00

0.00

0.00

0.00

$0.00 \quad 0.00$

0.00

0.00

0.00

0.00

0.00

0.00

0.00

0.00

0.00

0.00

0.00

0.00

0.00

0.00

0.00

0.00

0.00

0.00

0.00

0.00

0.00

0.00

0.00

0.00

0.00

0.00

0.00

0.00

0.00

0.00

0.00

0.00

0.00

0.00

0.00

0.00

0.00

0.00

0.00

0.00

0.00

0.00

0.00

0.00

0.00

0.00

0.00

$0.00 \quad 0.00$

$0.00 \quad 0.00$

0.00

0.00

0.00

0.00

$0.00 \quad 0.00$

0.00

0.00

0.00

0.00

$0.00 \quad 0.00$

$0.00 \quad 0.00$

$0.00 \quad 0.00$

0.00

0.00

$0.00 \quad 0.00$

$0.00 \quad 0.00$

$0.00 \quad 0.00$

0.00

0.00

$0.00 \quad 0.00$

$0.00 \quad 0.00$

0.00

0.00

0.00

0.00

$0.00 \quad 0.00$

$0.00 \quad 0.00$

$0.00 \quad 0.00$

$0.00 \quad 0.00$

$0.00 \quad 0.00$

$0.00 \quad 0.00$

0.00

0.00

$0.00 \quad 0.00$

$0.00 \quad 0.00$

0.00

0.00 
$\begin{array}{llllllllll}122 & 198 & 320 & -2.8 & -5.5-2214.01 & -6.14 & 0.00 & -3.69-2223.84 & 262.18\end{array}$ $\begin{array}{llllllllll}122 & 199 & 321 & -3.0 & -5.4-2219.46 & -6.34 & 0.82 & -3.65-2228.63 & 265.46\end{array}$ $\begin{array}{llllllllll}122 & 200 & 322 & -3.1 & -5.3-2224.83 & -2.38 & 0.00 & -3.62-2230.83 & 271.33\end{array}$ $\begin{array}{llllllllll}122 & 201 & 323 & -3.3 & -5.2-2230.11 & -2.57 & 0.82 & -3.58-2235.44 & 274.79\end{array}$ $\begin{array}{llllllllll}122 & 202 & 324 & -3.4 & -5.1-2235.30 & -2.56 & 0.00 & -3.55-2241.41 & 276.90\end{array}$ $\begin{array}{llllllllll}122 & 203 & 325 & -3.6 & -5.1-2240.41 & -2.57 & 0.82 & -3.51-2245.67 & 280.70\end{array}$ $\begin{array}{lllllllll}122 & 204 & 326 & -3.7 & -5.0-2245.43 & -2.24 & 0.00 & -3.48-2251.15 & 283.30\end{array}$ $\begin{array}{llllllllll}122 & 205 & 327 & -3.9 & -4.9-2250.37 & -2.10 & 0.81 & -3.44-2255.10 & 287.42\end{array}$ $\begin{array}{llllllllll}122 & 206 & 328 & -4.0 & -4.8-2255.22 & -1.75 & 0.00 & -3.41-2260.39 & 290.20\end{array}$ $\begin{array}{llllllllll}122 & 207 & 329 & -4.2 & -4.7-2260.00 & -1.75 & 0.81 & -3.38-2264.31 & 294.34\end{array}$ $\begin{array}{llllllllll}122 & 208 & 330 & -4.3 & -4.6-2264.69 & -1.45 & 0.00 & -3.35-2269.49 & 297.25\end{array}$ $\begin{array}{llllllllll}122 & 209 & 331 & -4.5 & -4.6-2269.30 & -1.54 & 0.81 & -3.32-2273.35 & 301.46\end{array}$ $\begin{array}{llllllllll}122 & 210 & 332 & -4.6 & -4.5-2273.83 & -1.34 & 0.00 & -3.28-2278.46 & 304.42\end{array}$ $\begin{array}{lllllllllll}122 & 211 & 333 & -4.7 & -4.4-2278.28 & -1.48 & 0.81 & -3.25-2282.21 & 308.74\end{array}$ $\begin{array}{llllllllll}122 & 212 & 334 & -4.9 & -4.3-2282.65 & -1.27 & 0.00 & -3.22 & -2287.15 & 311.87\end{array}$ $\begin{array}{lllllllll}122 & 213 & 335 & -5.0 & -4.3-2286.95 & -1.25 & 0.80 & -3.20-2290.59 & 316.50\end{array}$ $\begin{array}{llllllllll}122 & 214 & 336 & -5.2 & -4.2-2291.17 & -0.57 & 0.00 & -3.17-2294.90 & 320.26\end{array}$ $\begin{array}{llllllllll}122 & 215 & 337 & -5.3 & -4.1-2295.31 & -0.81 & 0.80 & -3.14-2298.45 & 324.78\end{array}$ $\begin{array}{llllllllll}122 & 216 & 338 & -5.4 & -4.0-2299.37 & -0.81 & 0.00 & -3.11-2303.29 & 328.01\end{array}$ $\begin{array}{llllllllll}122 & 217 & 339 & -5.6 & -3.9-2303.36 & -0.96 & 0.80 & -3.08-2306.61 & 332.77\end{array}$ $\begin{array}{lllllllll}123 & 174 & 297 & 1.4 & -8.1-2053.07 & -5.59 & 0.97 & -4.86-2062.56 & 237.00\end{array}$ $\begin{array}{llllllllll}123 & 175 & 298 & 1.2 & -8.0-2061.09 & -5.51 & 1.68 & -4.81-2069.73 & 237.90\end{array}$ $\begin{array}{lllllllllll}123 & 176 & 299 & 1.1 & -7.8-2069.00 & -5.46 & 0.97 & -4.75-2078.24 & 237.46\end{array}$ 123177300 $123178 \quad 301$ $\begin{array}{lll}123 & 179 & 302\end{array}$ $123180 \quad 303$ $\begin{array}{lll}123 & 181 & 304\end{array}$ $\begin{array}{llll}123 & 182 & 305\end{array}$ $0.9-7.7-2076.79-5.63 \quad 1.67-4.70-2085.45 \quad 238.33$ $\begin{array}{llllllll}0.7 & -7.6-2084.48 & -6.15 & 0.97 & -4.64-2094.31 & 237.54\end{array}$ $0.6-7.5-2092.06-6.56 \quad 1.67-4.59-2101.53 \quad 238.39$ $0.4-7.4-2099.52-6.690 .97-4.54-2109.79 \quad 238.21$ $0.2-7.3-2106.89-5.29 \quad 1.67-4.49-2114.99 \quad 241.07$ $0.1-7.2-2114.14-4.66 \quad 0.97-4.44-2122.27 \quad 241.86$ $\begin{array}{llllllllll}123 & 183 & 306 & -0.1 & -7.1-2121.29 & -4.39 & 1.67 & -4.39-2128.41 & 243.80\end{array}$ $\begin{array}{llllllllll}123 & 184 & 307 & -0.3 & -7.0-2128.34 & -3.62 & 0.97 & -4.34-2135.34 & 244.94\end{array}$ $\begin{array}{llllllllll}123 & 185 & 308 & -0.4 & -6.9-2135.28 & -2.63 & 1.66 & -4.29-2140.54 & 247.81\end{array}$ $\begin{array}{llllllllll}123 & 186 & 309 & -0.6 & -6.8-2142.13 & -1.98 & 0.97 & -4.25-2147.39 & 249.03\end{array}$ $\begin{array}{llllllllll}123 & 187 & 310 & -0.7 & -6.7-2148.87 & -1.44 & 1.66 & -4.20 & -2152.85 & 251.64\end{array}$ $\begin{array}{lllllllllll}123 & 188 & 311 & -0.9 & -6.6-2155.51 & -0.80 & 0.97 & -4.16-2159.50 & 253.06\end{array}$ $\begin{array}{llllllllll}123 & 189 & 312 & -1.1 & -6.5-2162.05 & -0.54 & 1.66 & -4.11-2165.05 & 255.59\end{array}$ $\begin{array}{llllllllll}123 & 190 & 313 & -1.2 & -6.4-2168.50 & -0.01 & 0.97 & -4.07-2171.61 & 257.09\end{array}$ $\begin{array}{lllllllll}123 & 191 & 314 & -1.4 & -6.3-2174.85 & 0.27 & 1.66 & -4.03-2176.95 & 259.83\end{array}$ $123192315-1.5-6.2-2181.10$ $123193 \quad 316-1.7-6.1-2187.26$ $\begin{array}{lllll}123 & 194 & 317 & -1.8 & -6.0-2193.32\end{array}$ $0.56 \quad 0.97-3.99-2183.56 \quad 261.29$ $0.751 .65-3.94-2188.80264 .12$ $0.960 .97-3.90-2195.30265 .69$ $\begin{array}{llllllllll}123 & 195 & 318 & -2.0 & -5.9-2199.29 & -6.64 & 1.65 & -3.86-2208.15 & 260.92\end{array}$ $\begin{array}{llllllllll}123 & 196 & 319 & -2.2 & -5.8-2205.17 & -6.48 & 0.97 & -3.82 & -2214.51 & 262.62\end{array}$ $\begin{array}{llllllllll}123 & 197 & 320 & -2.3 & -5.7-2210.96 & -6.80 & 1.65 & -3.79-2219.90 & 265.31\end{array}$ $\begin{array}{lllllllll}123 & 198 & 321 & -2.5 & -5.6-2216.65 & -6.62 & 0.97 & -3.75-2226.06 & 267.22\end{array}$ $\begin{array}{lllllllll}123 & 199 & 322 & -2.6 & -5.6-2222.26 & -6.80 & 1.65 & -3.71-2231.12 & 270.23\end{array}$ $\begin{array}{llllllllll}123 & 200 & 323 & -2.8 & -5.5-2227.78 & -2.85 & 0.97 & -3.67-2233.34 & 276.08\end{array}$ $\begin{array}{llllllllll}123 & 201 & 324 & -2.9 & -5.4-2233.21 & -2.91 & 1.64 & -3.64-2238.11 & 279.38\end{array}$ $\begin{array}{llllllllll}123 & 202 & 325 & -3.1 & -5.3-2238.55 & -2.91 & 0.97 & -3.60-2244.10 & 281.47\end{array}$ $\begin{array}{llllllllll}123 & 203 & 326 & -3.2 & -5.2 & -2243.81 & -2.93 & 1.64 & -3.57-2248.66 & 284.97\end{array}$ $\begin{array}{llllllllll}123 & 204 & 327 & -3.4 & -5.1-2248.98 & -2.58 & 0.97 & -3.53-2254.13 & 287.58\end{array}$ $\begin{array}{lllllllll}123 & 205 & 328 & -3.5 & -5.0-2254.06 & -2.44 & 1.64 & -3.50-2258.36 & 291.42\end{array}$ $\begin{array}{llllllllll}123 & 206 & 329 & -3.7 & -5.0-2259.06 & -2.10 & 0.97 & -3.47-2263.67 & 294.18\end{array}$ $\begin{array}{llllllllll}123 & 207 & 330 & -3.8 & -4.9-2263.98 & -2.06 & 1.64 & -3.43-2267.84 & 298.08\end{array}$ $\begin{array}{llllllllll}123 & 208 & 331 & -3.9 & -4.8-2268.82 & -1.75 & 0.97 & -3.40-2273.01 & 300.99\end{array}$ $\begin{array}{llllllllll}123 & 209 & 332 & -4.1 & -4.7-2273.58 & -1.78 & 1.64 & -3.37-2277.09 & 304.98\end{array}$ $\begin{array}{llllllllll}123 & 210 & 333 & -4.2 & -4.6-2278.25 & -1.57 & 0.97 & -3.34-2282.19 & 307.94\end{array}$ $\begin{array}{llllllllll}123 & 211 & 334 & -4.4 & -4.6-2282.85 & -1.69 & 1.63 & -3.31-2286.21 & 312.00\end{array}$ $\begin{array}{llllllllll}123 & 212 & 335 & -4.5 & -4.5-2287.36 & -1.51 & 0.97 & -3.28-2291.18 & 315.10\end{array}$ $\begin{array}{llllllllll}123 & 213 & 336 & -4.7 & -4.4-2291.80 & -1.47 & 1.63 & -3.25-2294.88 & 319.47\end{array}$ $\begin{array}{lllllllllll}123 & 214 & 337 & -4.8 & -4.3-2296.16 & -0.74 & 0.97 & -3.22 & -2299.15 & 323.27\end{array}$ $\begin{array}{llllllllll}123 & 215 & 338 & -4.9 & -4.2-2300.44 & -0.87 & 1.63 & -3.19-2302.87 & 327.63\end{array}$ $\begin{array}{llllllllll}123 & 216 & 339 & -5.1 & -4.2-2304.64 & -0.88 & 0.97 & -3.16-2307.72 & 330.85\end{array}$ $\begin{array}{llllllllll}124 & 176 & 300 & 1.4 & -8.0-2067.75 & -4.62 & 0.00 & -4.83-2077.20 & 245.76\end{array}$ $124 \quad 177 \quad 301$ $124178 \quad 302$
$1.3-7.9-2075.72-4.94 \quad 0.85-4.77-2084.58 \quad 246.46$ $1.1-7.8-2083.57-6.10 \quad 0.00-4.72-2094.39 \quad 244.72$
0.00

0.00

0.00

0.00

0.00

0.00

0.00

0.00

0.00

0.00

0.00

0.00

0.00

0.00

0.00

0.00

0.00

0.00

0.00

0.00

0.00

0.00

0.00

0.00

0.00

0.00

0.00

0.00

0.00

0.00

0.00

0.00

0.00

0.00

0.00

0.00

0.00

0.00

0.00

0.00

0.00

0.00

0.00

0.00

0.00

0.00

0.00

0.00

0.00

0.00

0.00

0.00

0.00

0.00

0.00

0.00

0.00

0.00

0.00

0.00

0.00

0.00

0.00

0.00

0.00

0.00

0.00
0.00

0.00

0.00

0.00

0.00

0.00

0.00

0.00

0.00

0.00

0.00

0.00

0.00

0.00

0.00

0.00

0.00

0.00

0.00

0.00

0.00

0.00

0.00

0.00

0.00

0.00

0.00

0.00

0.00

0.00

0.00

0.00

0.00

0.00

0.00

0.00

0.00

0.00

0.00

0.00

0.00

0.00

0.00

0.00

0.00

0.00

0.00

0.00

0.00

0.00

0.00

0.00

0.00

0.00

0.00

0.00

0.00

0.00

0.00

0.00

0.00

0.00

0.00

0.00

0.00
0.00

0.00

0.00

0.00

0.00

0.00

0.00

0.00

0.00

0.00

0.00

0.00

0.00

0.00

0.00

0.00

0.00

0.00

0.00

0.00

0.00

0.00

0.00

0.00

0.00

0.00

0.00

0.00

0.00

0.00

0.00

0.00

0.00

0.00

0.00

0.00

0.00

0.00

0.00

0.00

0.00

0.00

0.00

0.00

0.00

0.00

0.00

0.00

0.00

0.00

0.00

0.00

0.00

0.00

0.00

0.00

0.00

0.00

0.00

0.00

0.00

0.00

0.00

0.00

0.00

0.00 
124179303 $124180 \quad 304$ 124181305 124182306 $124 \quad 183 \quad 307$ $124 \quad 184308$ 124185309 124186310 124187.311 $124 \quad 188 \quad 312$ $124 \quad 189313$ $124190 \quad 314$ 124191315 $\begin{array}{llll}192 & 316 & -1.2 & -6.4-2182.46\end{array}$ $\begin{array}{lllll}124 & 193 & 317 & -1.3 & -6.3-2188.77\end{array}$ $\begin{array}{lllll}124 & 194 & 318 & -1.5 & -6.2-2194.99\end{array}$ $124195319-1.6 \quad-6.1-2201.12$ $\begin{array}{lllll}124 & 196 & 320 & -1.8 & -6.0-2207.15\end{array}$ $\begin{array}{lllll}124 & 197 & 321 & -1.9 & -5.9-2213.09\end{array}$ $\begin{array}{lllll}124 & 198 & 322 & -2.1 & -5.8-2218.94\end{array}$ $\begin{array}{llllll}124 & 199 & 323 & -2.3 & -5.7 & -2224.70\end{array}$ $\begin{array}{lllll}124 & 200 & 324 & -2.4 & -5.6-2230.37\end{array}$ $\begin{array}{lllllllll}201 & 325 & -2.6 & -5.5 & -2235.95 & -2.99 & 0.82 & -3.70-2241.81 & 282.94\end{array}$

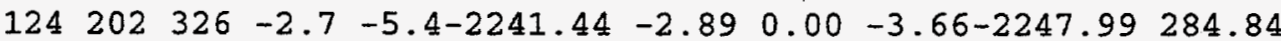
$\begin{array}{lllllllll}124 & 203 & 327 & -2.9 & -5.4-2246.84 & -3.00 & 0.82 & -3.63-2252.65 & 288.25\end{array}$

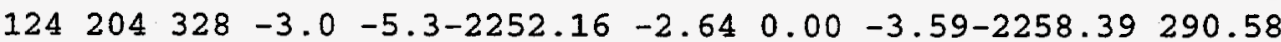

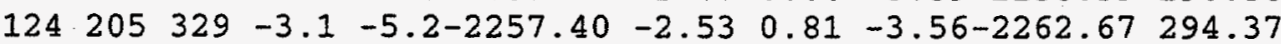
$\begin{array}{lllllllll}124 & 206 & 330 & -3.3 & -5.1-2262.54 & -2.18 & 0.00 & -3.52-2268.25 & 296.86\end{array}$

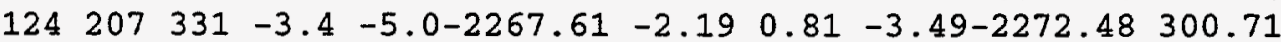
$\begin{array}{lllllllll}124 & 208 & 332 & -3.6 & -4.9-2272.59 & -1.86 & 0.00 & -3.46-2277.91 & 303.35\end{array}$ $\begin{array}{lllllllll}124 & 209 & 333 & -3.7 & -4.9-2277.49 & -1.91 & 0.81 & -3.42-2282.02 & 307.31\end{array}$

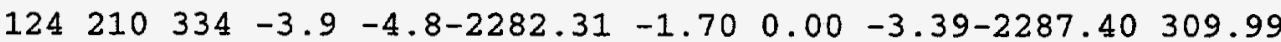
$\begin{array}{lllllllll}124 & 211 & 335 & -4.0 & -4.7-2287.05 & -1.82 & 0.81 & -3.36-2291.42 & 314.05\end{array}$

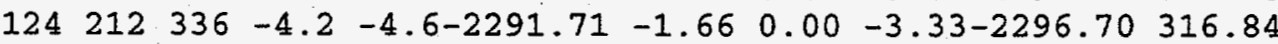

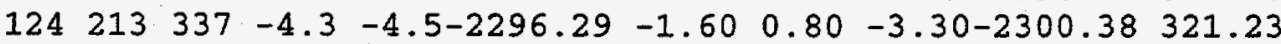

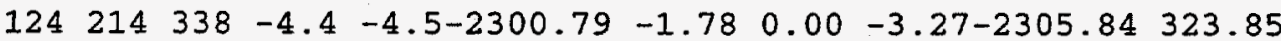
$\begin{array}{lllllllll}124 & 215 & 339 & -4.6 & -4.4-2305.21 & -0.77 & 0.80 & -3.24-2308.42 & 329.34\end{array}$ $\begin{array}{lllllllll}125 & 178 & 303 & 1.5 & -8.0-2082.30 & -6.61 & 0.96 & -4.80-2092.75 & 253.62\end{array}$ $\begin{array}{llllllllll}125 & 179 & 304 & 1.3 & -7.9-2090.21 & -6.97 & 1.67 & -4.74-2100.26 & 254.19\end{array}$ $\begin{array}{llllllllll}125 & 180 & 305 & 1.1 & -7.7-2098.01 & -7.17 & 0.96 & -4.69-2108.91 & 253.61\end{array}$ 125181306 $\begin{array}{llll}125 & 182 & 307\end{array}$ 125183308 125184309 $125185 \quad 310$ 125186311 $\begin{array}{llll}125 & 187 & 312\end{array}$ $125188 \quad 313$ $\begin{array}{llllll}1.0 & -7.6-2105.70 & -3.64 & 1.66 & -4.64-2112.32 & 258.27 \\ 0.8 & -7.5-2113.29 & -2.96 & 0.96 & -4.58-2119.87 & 258.79\end{array}$ $0.6-7.4-2120.77-2.591 .66-4.53-2126.23 \quad 260.50$ $0.5-7.3-2128.14-1.740 .96-4.48-2133.40 \quad 261.40$ $0.3-7.2-2135.41-1.151 .66-4.44-2139.33 \quad 263.54$ $\begin{array}{lllllll}0.1 & -7.1-2142.57 & -0.36 & 0.96 & -4.39 & -2146.36 & 264.58\end{array}$ $0.0-7.0-2149.64-0.37 \quad 1.66-4.34-2152.69266 .32$ $-0.2-6.9-2156.60$ $\begin{array}{lllll}125 & 189 & 314 & -0.3 & -6.8-2163.46\end{array}$ $\begin{array}{lllll}125 & 190 & 315 & -0.5 & -6.7-2170.22\end{array}$ $125191316-0.7-6.6-2176.89$ $\begin{array}{lllll}125 & 192 & 317 & -0.8 & -6.5-2183.45\end{array}$ $125193 \quad 318-1.0-6.4-2189.93$ $\begin{array}{llllllllll}125 & 194 & 319 & -1.1 & -6.3-2196.30 & -7.19 & 0.96 & -4.03-2206.56 & 268.95\end{array}$ $\begin{array}{lllllllll}125 & 195 & 320 & -1.3 & -6.2-2202.58 & -7.39 & 1.65 & -3.99-2212.31 & 271.27\end{array}$ $\begin{array}{lllllllll}125 & 196 & 321 & -1.4 & -6.1-2208.77 & -7.26 & 0.96 & -3.95-2219.02 & 272.64\end{array}$ $\begin{array}{lllllllll}125 & 197 & 322 & -1.6 & -6.0-2214.86 & -7.60 & 1.64 & -3.91-2224.73 & 275.00\end{array}$

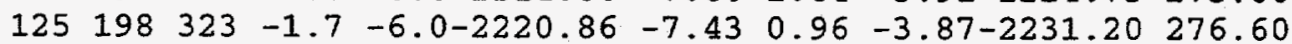

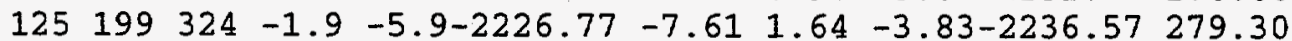
$\begin{array}{lllllllll}125 & 200 & 325 & -2.0 & -5.8-2232.59 & -7.40 & 0.96 & -3.79-2242.83 & 281.12\end{array}$ $\begin{array}{lllllllll}125 & 201 & 326 & -2.2 & -5.7-2238.32 & -3.35 & 1.64 & -3.76-2243.79 & 288.22\end{array}$

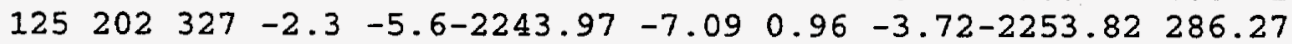
$\begin{array}{lllllllll}125 & 203 & 328 & -2.5 & -5.5-2249.52 & -3.38 & 1.64 & -3.68-2254.95 & 293.21\end{array}$ $\begin{array}{lllllllll}125 & 204 & 329 & -2.6 & -5.4-2254.99 & -3.02 & 0.96 & -3.65-2260.70 & 295.53\end{array}$ $\begin{array}{lllllllll}125 & 205 & 330 & -2.8 & -5.3-2260.37 & -2.89 & 1.64 & -3.61-2265.24 & 299.06\end{array}$ $\begin{array}{lllllllll}125 & 206 & 331 & -2.9 & -5.2-2265.67 & -2.62 & 0.96 & -3.58-2270.90 & 301.47\end{array}$
0.00 0.00

0.00 0.00

0.00 0.00

0.00 0.00

0.00 0.00

0.00

0.00 0.00

0.00 0.00

0.00

0.00

0.00

0.00 0.00 0.00

0.00 0.00

0.00

0.00

0.00

0.00

0.00

0.00

0.00

0.00

0.00

0.00

0.00

0.00

0.00

0.00

0.00

0.00

0.00

0.00

0.00

0.00

0.00

0.00

0.00

0.00

0.00

0.00

0.00

0.00

0.00

0.00

0.00

0.00

0.00

0.00

0.00

0.00

0.00

0.00

0.00

0.00

0.00

0.00

0.00

0.00

0.00

0.00

0.00

0.00

0.00

0.00

0.00

0.00

0.00

0.00

0.00 0.00 0.00 0.00 0.00 0.00 0.00 0.00 0.00 0.00 0.00 0.00 0.00 0.00 0.00 0.00 0.00 0.00
0.00 0.00 0.00 0.00 0.00 0.00 0.00 0.00 0.00 0.00 0.00 0.00 0.00 $0.00 \quad 0.00$ $\begin{array}{lll}0.00 & 0.00\end{array}$ $0.00 \quad 0.00$ $0.00 \quad 0.00$ $0.00 \quad 0.00$ $0.00 \quad 0.00$ $0.00 \quad 0.00$ $0.00 \quad 0.00$ $0.00 \quad 0.00$ $0.00 \quad 0.00$ $0.00 \quad 0.00$ $0.00 \quad 0.00$ $0.00 \quad 0.00$ $0.00 \quad 0.00$ $0.00 \quad 0.00$ $0.00 \quad 0.00$ $0.00 \quad 0.00$ $0.00 \quad 0.00$ $0.00 \quad 0.00$ $0.00 \quad 0.00$ $0.00 \quad 0.00$ $0.00 \quad 0.00$ $0.00 \quad 0.00$ 0.00 $0.00 \quad 0.00$ 0.00 0.00 0.00 0.00 0.00 0.00 0.00 $0.00 \quad 0.00$ 0.00 0.00 $0.00 \quad 0.00$ $0.00 \quad 0.00$ 0.00 0.00 $0.00 \quad 0.00$ $0.00 \quad 0.00$ $0.00 \quad 0.00$ $0.00 \quad 0.00$ $0.00 \quad 0.00$ $0.00 \quad 0.00$ $0.00 \quad 0.00$ $0.00 \quad 0.00$ $0.00 \quad 0.00$ 0.00 0.00 0.00 
$\begin{array}{lllllllllll}125 & 207 & 332 & -3.1 & -5.2 & -2270.88 & -2.55 & 1.63 & -3.54-2275.34 & 305.11\end{array}$ $\begin{array}{llllllllll}125 & 208 & 333 & -3.2 & -5.1-2276.01 & -2.32 & 0.96 & -3.51-2280.88 & 307.64\end{array}$

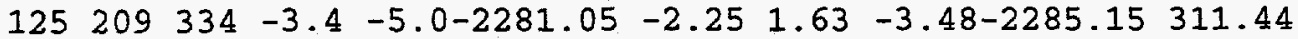
$\begin{array}{llllllllll}125 & 210 & 335 & -3.5 & -4.9-2286.01 & -2.02 & 0.96 & -3.44-2290.52 & 314.14\end{array}$ $\begin{array}{llllllllll}125 & 211 & 336 & -3.7 & -4.8-2290.90 & -2.11 & 1.63 & -3.41-2294.79 & 317.94\end{array}$ $\begin{array}{llllllllll}125 & 212 & 337 & -3.8 & -4.8-2295.70 & -1.95 & 0.96 & -3.38-2300.07 & 320.73\end{array}$ $\begin{array}{llllllllll}125 & 213 & 338 & -3.9 & -4.7-2300.42 & -1.91 & 1.63 & -3.35-2304.05 & 324.82\end{array}$ $\begin{array}{llllllllll}125 & 214 & 339 & -4.1 & -4.6-2305.06 & -1.90 & 0.96 & -3.32 & -2309.32 & 327.62\end{array}$ $\begin{array}{lllllllllll}126 & 180 & 306 & 1.5 & -7.9-2096.72 & -7.21 & 0.00 & -4.77-2108.69 & 261.08\end{array}$ $\begin{array}{llllllllll}126 & 181 & 307 & 1.3 & -7.8-2104.57 & -7.58 & 0.85 & -4.71-2116.02 & 261.83\end{array}$ $\begin{array}{llllllllll}126 & 182 & 308 & 1.1 & -7.7-2112.32 & -1.91 & 0.00 & -4.66-2118.89 & 267.03\end{array}$ $\begin{array}{llllllllll}126 & 183 & 309 & 1.0 & -7.6-2119.96 & -1.57 & 0.85 & -4.61-2125.30 & 268.69\end{array}$ $\begin{array}{llll}126 & 184 & 310\end{array}$

126185311 $126186 \quad 312$ $\begin{array}{lll}126 & 187 & 313\end{array}$ $126 \quad 188 \quad 314$ 126189315 $126190 \quad 316$ $\begin{array}{lllll}126 & 191 & 317 & -0.3 & -6.8-2177.37\end{array}$ $126192318-0.5 \quad-6.7-2184.09$ $126193 \quad 319$ $126194320-0.8-6.5-2197.25-5.600 .00-4.10-2206.95275 .83$ $\begin{array}{llllllllll}126 & 195 & 321 & -0.9 & -6.4-2203.69 & -7.66 & 0.83 & -4.05-2214.57 & 276.27\end{array}$ $\begin{array}{llllllllll}126 & 196 & 322 & -1.1 & -6.3-2210.03 & -7.67 & 0.00 & -4.01-2221.71 & 277.21\end{array}$ $\begin{array}{llllllllll}126 & 197 & 323 & -1.2 & -6.2-2216.27 & -7.91 & 0.82 & -3.97-2227.33 & 279.66\end{array}$ $\begin{array}{llllllllll}126 & 198 & 324 & -1.4 & -6.1-2222.43 & -7.76 & 0.00 & -3.93-2234.12 & 280.94\end{array}$ $\begin{array}{llllllllll}126 & 199 & 325 & -1.5 & -6.0-2228.49 & -7.96 & 0.82 & -3.89-2239.52 & 283.61\end{array}$ $\begin{array}{llllllllll}126 & 200 & 326 & -1.7 & -5.9-2234.46 & -7.75 & 0.00 & -3.85-2246.07 & 285.14\end{array}$ $\begin{array}{lllllllll}126 & 201 & 327 & -1.8 & -5.8-2240.34 & -3.44 & 0.82 & -3.82-2246.78 & 292.49\end{array}$ $\begin{array}{llllllllll}126 & 202 & 328 & -2.0 & -5.7 & -2246.14 & -3.35 & 0.00 & -3.78-2253.27 & 294.08\end{array}$ $\begin{array}{llllllllll}126 & 203 & 329 & -2.1 & -5.7-2251.84 & -3.44 & 0.82 & -3.74-2258.21 & 297.21\end{array}$ $\begin{array}{lllllllll}126 & 204 & 330 & -2.3 & -5.6-2257.46 & -3.11 & 0.00 & -3.71-2264.27 & 299.22\end{array}$ $\begin{array}{llllllllll}126 & 205 & 331 & -2.4 & -5.5-2262.99 & -2.99 & 0.81 & -3.67-2268.83 & 302.73\end{array}$ $\begin{array}{llllllllll}126 & 206 & 332 & -2.6 & -5.4-2268.43 & -2.66 & 0.00 & -3.63-2274.72 & 304.91\end{array}$ $\begin{array}{llllllllll}126 & 207 & 333 & -2.7 & -5.3-2273.79 & -2.66 & 0.81 & -3.60-2279.24 & 308.47\end{array}$ $\begin{array}{lllllllll}126 & 208 & 334 & -2.9 & -5.2-2279.06 & -2.29 & 0.00 & -3.57-2284.92 & 310.86\end{array}$ $\begin{array}{llllllllll}126 & 209 & 335 & -3.0 & -5.1-2284.25 & -2.40 & 0.81 & -3.53-2289.38 & 314.47\end{array}$ $\begin{array}{lllllllll}126 & 210 & 336 & -3.2 & -5.1-2289.36 & -2.17 & 0.00 & -3.50-2295.03 & 316.89\end{array}$ $\begin{array}{llllllllll}126 & 211 & 337 & -3.3 & -5.0 & -2294.38 & -2.28 & 0.81 & -3.47-2299.33 & 320.66\end{array}$ $\begin{array}{llllllllll}126 & 212 & 338 & -3.5 & -4.9-2299.33 & -2.11 & 0.00 & -3.43-2304.87 & 323.19\end{array}$ $\begin{array}{llllllllll}126 & 213 & 339 & -3.6 & -4.8-2304.19 & -2.03 & 0.80 & -3.40-2308.82 & 327.31\end{array}$ $\begin{array}{llllllllll}127 & 183 & 310 & 1.3 & -7.7-2118.81 & -0.75 & 1.66 & -4.68-2122.58 & 278.67\end{array}$ $\begin{array}{llllllllll}127 & 184 & 311 & 1.2 & -7.6-2126.51 & -0.26 & 0.95 & -4.63-2130.44 & 278.88\end{array}$ $\begin{array}{llllllllll}127 & 185 & 312 & 1.0 & -7.5-2134.10 & 0.06 & 1.65 & -4.58-2136.97 & 280.43\end{array}$ $\begin{array}{llllllllll}127 & 186 & 313 & 0.9 & -7.4-2141.59 & 0.38 & 0.95 & -4.53-2144.78 & 280.68\end{array}$ $\begin{array}{llllllllll}127 & 187 & 314 & 0.7 & -7.3-2148.97 & 0.65 & 1.65 & -4.48-2151.15 & 282.38\end{array}$ $\begin{array}{llllllllll}127 & 188 & 315 & 0.5 & -7.2-2156.25 & 0.99 & 0.95 & -4.43-2158.74 & 282.87\end{array}$ $\begin{array}{llllllllll}127 & 189 & 316 & 0.4 & -7.1-2163.44 & 1.16 & 1.65 & -4.39-2165.01 & 284.67\end{array}$ $\begin{array}{llllllllll}127 & 190 & 317 & 0.2 & -7.0-2170.51 & 1.54 & 0.95 & -4.34-2172.36 & 285.39\end{array}$ $\begin{array}{llllllllll}127 & 191 & 318 & 0.1 & -6.9-2177.49 & -7.44 & 1.65 & -4.29-2187.58 & 278.24\end{array}$ $\begin{array}{llllllllll}127 & 192 & 319 & -0.1 & -6.8-2184.38 & -7.01 & 0.95 & -4.25-2194.68 & 279.21\end{array}$ $\begin{array}{llllllllll}127 & 193 & 320 & -0.3 & -6.7-2191.16 & -6.71 & 1.64 & -4.21-2200.43 & 281.54\end{array}$ $\begin{array}{lllllllll}127 & 194 & 321 & -0.4 & -6.6-2197.85 & -6.16 & 0.95 & -4.16-2207.21 & 282.82\end{array}$ $\begin{array}{lllllllll}127 & 195 & 322 & -0.6 & -6.5-2204.44 & -8.18 & 1.64 & -4.12-2215.09 & 283.01\end{array}$ $\begin{array}{lllllllll}127 & 196 & 323 & -0.7 & -6.4-2210.93 & -8.21 & 0.95 & -4.08-2222.26 & 283.92\end{array}$ $\begin{array}{llllllllll}127 & 197 & 324 & -0.9 & -6.3-2217.33 & -8.44 & 1.64 & -4.04-2228.17 & 286.08\end{array}$ $\begin{array}{llllllllll}127 & 198 & 3.25 & -1.0 & -6.3-2223.64 & -8.29 & 0.95 & -4.00-2234.97 & 287.35\end{array}$ $\begin{array}{llllllllll}127 & 199 & 326 & -1.2 & -6.2-2229.85 & -8.46 & 1.64 & -3.95-2240.63 & 289.76\end{array}$ $\begin{array}{llllllllll}127 & 200 & 327 & -1.3 & -6.1-2235.98 & -8.27 & 0.95 & -3.92-2247.21 & 291.26\end{array}$ $127201328-1.5 \quad-6.0-2242.01-8.31 \quad 1.64-3.88-2252.56 \quad 293.98$ $\begin{array}{llllllllll}127 & 202 & 329 & -1.6 & -5.9-2247.95 & -7.94 & 0.95 & -3.84-2258.78 & 295.83\end{array}$ $\begin{array}{llllllllll}127 & 203 & 330 & -1.8 & -5.8-2253.80 & -3.81 & 1.63 & -3.80-2259.78 & 302.90\end{array}$ $\begin{array}{llllllllll}127 & 204 & 331 & -1.9 & -5.7 & -2259.57 & -3.48 & 0.95 & -3.76-2265.86 & 304.89\end{array}$ $\begin{array}{lllllllll}127 & 205 & 332 & -2.1 & -5.6-2265.25 & -3.37 & 1.63 & -3.73-2270.72 & 308.11\end{array}$ $127206 \quad 333-2.2 \quad-5.5-2270.84-3.04 \quad 0.95 \quad-3.69-2276.62 \quad 310.28$ 138

\begin{tabular}{|c|c|c|}
\hline $0 C$ & 0.00 & 0.00 \\
\hline 0.00 & 0.00 & 0.00 \\
\hline 0.00 & 0.00 & 0.00 \\
\hline 0.00 & 0.00 & 0.00 \\
\hline 0.00 & 0.00 & 0.00 \\
\hline 0.00 & 0.00 & 0.00 \\
\hline 0.00 & 0.00 & 0.00 \\
\hline 0.00 & 0.00 & 0.00 \\
\hline 0.00 & 0.00 & 0.00 \\
\hline 0.00 & 0.00. & 0.00 \\
\hline 0.00 & 0.00 & 0.00 \\
\hline 0.00 & 0.00 & 0.00 \\
\hline 0.00 & 0.00 & 0.00 \\
\hline 0.00 & 0.00 & 0.00 \\
\hline 0.00 & 0.00 & 0.00 \\
\hline 0.00 & 0.00 & 0.00 \\
\hline 0.00 & 0.00 & 0.00 \\
\hline 0.00 & 0.00 & 0.00 \\
\hline 0.00 & 0.00 & 0.00 \\
\hline 0.00 & 0.00 & 0.00 \\
\hline 0.00 & 0.00 & 0.00 \\
\hline 0.00 & 0.00 & 0.00 \\
\hline 0.00 & 0.00 & 0.00 \\
\hline 0.00 & 0.00 & 0.00 \\
\hline 0.00 & 0.00 & 0.00 \\
\hline 0.00 & 0.00 & 0.00 \\
\hline 0.00 & 0.00 & 0.00 \\
\hline 0.00 & 0.00 & 0.00 \\
\hline 0.00 & 0.00 & 0.00 \\
\hline 0.00 & 0.00 & 0.00 \\
\hline 0.00 & 0.00 & 0.00 \\
\hline 0.00 & 0.00 & 0.00 \\
\hline 0.00 & 0.00 & 0.00 \\
\hline 0.00 & 0.00 & 0.00 \\
\hline 0.00 & 0.00 & 0.00 \\
\hline 0.00 & 0.00 & 0.00 \\
\hline 0.00 & 0.00 & 0.00 \\
\hline 0.00 & 0.00 & 0.00 \\
\hline 0.00 & 0.00 & 0.00 \\
\hline 0.00 & 0.00 & 0.00 \\
\hline 0.00 & 0.00 & 0.00 \\
\hline 0.00 & 0.00 & 0.00 \\
\hline 0.00 & 0.00 & 0.00 \\
\hline 0.00 & 0.00 & 0.00 \\
\hline 0.00 & 0.00 & 0.00 \\
\hline 0.00 & 0.00 & 0.00 \\
\hline 0.00 & 0.00 & 0.00 \\
\hline 0.00 & 0.00 & 0.00 \\
\hline 0.00 & 0.00 & 0.00 \\
\hline 0.00 & 0.00 & 0.00 \\
\hline 0.00 & 0.00 & 0.00 \\
\hline 0.00 & 0.00 & 0.00 \\
\hline 0.00 & 0.00 & 0.00 \\
\hline 0.00 & 0.00 & 0.00 \\
\hline 0.00 & 0.00 & 0.00 \\
\hline 0.00 & 0.00 & 0.00 \\
\hline .00 & 0.00 & 0.00 \\
\hline 0.00 & 0.00 & 0.00 \\
\hline 0.00 & 0.00 & 0.00 \\
\hline 0.00 & 0.00 & 0.00 \\
\hline 0.00 & 0.00 & 0.00 \\
\hline 0.00 & 0.00 & 0.00 \\
\hline 0.00 & 0.00 & 0.00 \\
\hline 0.00 & 0.00 & 0.00 \\
\hline 0.00 & 0.00 & 0.00 \\
\hline & 0 & \\
\hline
\end{tabular}

0.00 .00 .00 .00 .00 .00 .00 .00
.00 .00 .00 .00
.00 .00 .00 .00
.00 .00 .00 .00
.00 .00 .00 .00 .00 .00
.00 .00 .00 .00
.00 .00 .00 .00
.00 .00 .00 .00
.00 .00 .00 .00
.00 .00 .00
.00 .00 .00 .00
.00 .00 .00
.00 .00 .00 .00
.00 .00 .00 .00
.00 .00 .00
.00 
$\begin{array}{llllllllll}127 & 207 & 334 & -2.4 & -5.5-2276.34 & -3.01 & 1.63 & -3.66-2281.38 & 313.58\end{array}$ $\begin{array}{lllllllll}127 & 208 & 335 & -2.5 & -5.4-2281.76 & -2.78 & 0.95 & -3.62-2287.21 & 315.83\end{array}$ $\begin{array}{lllllllll}127 & 209 & 336 & -2.7 & -5.3-2287.10 & -2.83 & 1.63 & -3.59-2291.89 & 319.22\end{array}$

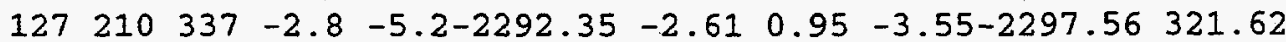
$\begin{array}{lllllllll}127 & 211 & 338 & -3.0 & -5.1-2297.52 & -2.65 & 1.63 & -3.52-2302.07 & 325.18\end{array}$ $\begin{array}{lllllllll}127 & 212 & 339 & -3.1 & -5.0-2302.61 & -2.48 & 0.95 & -3.49-2307.62 & 327.70\end{array}$ $\begin{array}{lllllllll}128 & 185 & 313 & 1.4 & -7.7-2132.91 & 0.50 & 0.84 & -4.65-2136.23 & 288.43\end{array}$ $128 \quad 186 \quad 314$ 128187315 $128 \quad 188 \quad 316$ $\begin{array}{lll}128 & 189 & 317\end{array}$ 128190318 128191319 128192320 $\begin{array}{lll}128 & 193 & 321\end{array}$ $128 \quad 194322$ $128 \quad 195 \quad 323$ 128196324 128197325 128198326 128199327 $128 \quad 200328$ $\begin{array}{lllllllll}128 & 201 & 329 & -1.1 & -6.1-2243.32 & -8.71 & 0.82 & -3.94-2255.15 & 298.65\end{array}$ $\begin{array}{llllllllll}128 & 202 & 330 & -1.3 & -6.0-2249.41 & -8.34 & 0.00 & -3.90-2261.65 & 300.22\end{array}$ $\begin{array}{lllllllll}128 & 203 & 331 & -1.4 & -6.0-2255.42 & -3.79 & 0.82 & -3.86-2262.25 & 307.69\end{array}$ $\begin{array}{lllllllll}128 & 204 & 332 & -1.6 & -5.9-2261.33 & -3.49 & 0.00 & -3.82-2268.64 & 309.37\end{array}$ $\begin{array}{lllllllll}128 & 205 & 333 & -1.7 & -5.8-2267.15 & -3.45 & 0.81 & -3.79-2273.58 & 312.51\end{array}$ $\begin{array}{lllllllll}128 & 206 & 334 & -1.9 & -5.7-2272.89 & -3.10 & 0.00 & -3.75-2279.74 & 314.41\end{array}$ $\begin{array}{llllllllll}128 & 207 & 335 & -2.0 & -5.6-2278.54 & -3.14 & 0.81 & -3.71-2284.59 & 317.64\end{array}$ $\begin{array}{lllllllll}128 & 208 & 336 & -2.2 & -5.5-2284.11 & -2.83 & 0.00 & -3.68-2290.62 & 319.68\end{array}$

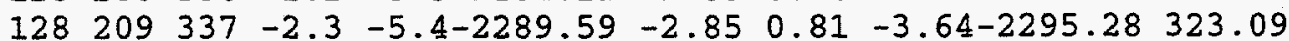
$\begin{array}{lllllllll}128 & 210 & 338 & -2.5 & -5.4-2294.99 & -2.61 & 0.00 & -3.61-2301.21 & 325.23\end{array}$ $\begin{array}{lllllllll}128 & 211 & 339 & -2.6 & -5.3-2300.30 & -2.85 & 0.81 & -3.58-2305.92 & 328.59\end{array}$ $\begin{array}{lllllllll}129 & 187 & 316 & 1.4 & -7.6-2146.89 & 1.43 & 1.65 & -4.63-2148.44 & 299.61\end{array}$ $129188 \quad 317$

129189318 $\begin{array}{lll}129 & 190 & 319\end{array}$ 129191320 $\begin{array}{lll}129 & 192 & 321\end{array}$ $129 \quad 193 \quad 322$ $129194 \quad 323$ $129 \quad 195 \quad 324$ $129 \quad 196 \quad 325$ $129 \quad 197 \quad 326$ $129 \quad 198 \quad 327$ 129199328 $129200 \quad 329$ 129201330 $129202 \quad 331$ $\begin{array}{lll}129 & 203 \quad 332\end{array}$ 129204333 $129205334-1.2-6.0-2262.73-3.880 .95-3.88-2269.55315 .72$ $\begin{array}{lllllllll}129 & 206 & 335 & -1.5 & -5.8-2274.60 & -3.48 & 0.95 & -3.81-2280.93 & 320.48\end{array}$ $\begin{array}{llllllllll}129 & 207 & 336 & -1.7 & -5.8-2280.39 & -3.44 & 1.62 & -3.77-2285.98 & 323.50\end{array}$ $\begin{array}{llllllllll}129 & 208 & 337 & -1.8 & -5.7-2286.11 & -3.05 & 0.95 & -3.74-2291.94 & 325.61\end{array}$ $\begin{array}{lllllllll}129 & 209 & 338 & -2.0 & -5.6-2291.73 & -2.91 & 1.62 & -3.70-2296.72 & 328.91\end{array}$ $\begin{array}{lllllllll}129 & 210 & 339 & -2.1 & -5.5-2297.27 & -3.21 & 0.95 & -3.67-2303.20 & 330.50\end{array}$ 130189319 130190320 $\begin{array}{lll}130 & 191 & 321\end{array}$ $130 \quad 192 \quad 322$ 130193323 $130 \quad 194 \quad 324$ $130 \quad 195 \quad 325$ 130196326 $\begin{array}{lll}130 & 197 \quad 327\end{array}$
$1.2-7.5-2154.49$

$1.1-7.4-2161.99$ $0.9-7.3-2169.39$ $0.8-7.2-2176.68$ $0.6-7.1-2183.88$ $0.4-7.0-2190.98$ $0.3-6.9-2197.97$ $0.1-6.8-2204.87$ $0.0-6.8-2211.68$ $-0.2-6.7-2218.39$ $\begin{array}{llll}1.74 & 0.95 & -4.58-2156.38 & 299.75\end{array}$ $\begin{array}{llll}-8.34 & 1.64 & -4.53-2173.22 & 290.98\end{array}$ $\begin{array}{lllll}-8.06 & 0.95 & -4.48-2180.98 & 291.29\end{array}$ $\begin{array}{llll}-7.87 & 1.64 & -4.43-2187.34 & 293.00\end{array}$ $\begin{array}{lllll}-7.38 & 0.95 & -4.39-2194.69 & 293.72\end{array}$ $\begin{array}{llll}-7.19 & 1.64 & -4.34-2200.86 & 295.62\end{array}$ $\begin{array}{lllll}-6.65 & 0.95 & -4.29-2207.97 & 296.59\end{array}$ $\begin{array}{llll}-6.28 & 1.64 & -4.25-2213.77 & 298.86\end{array}$ $-9.361 .64-4.16-2230.27 \quad 298.50$ $\begin{array}{llllll}-0.3 & -6.6-2225.00 & -9.23 & 0.95 & -4.12-2237.40 & 299.44\end{array}$ $\begin{array}{llllll}0.5 & -6.5-2231.52 & -9.45 & 1.63 & -4.08-2243.41 & 301.50\end{array}$ $\begin{array}{llllll}-0.8 & -6.3-2244.28 & -9.29 & 1.63 & -4.00-2255.94 & 305.12\end{array}$ $\begin{array}{llllll}-0.9 & -6.2-2250.52 & -8.93 & 0.95 & -3.96-2262.46 & 306.67\end{array}$

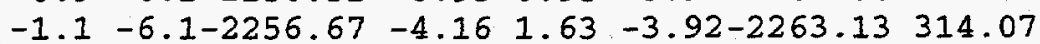
$1.2-6.0-2262.73-3.88 \quad 0.95-3.88-2269.55 \quad 315.72$ $\begin{array}{rrrrrr}-2.1 & -5.5-2297.27 & -3.21 & 0.95 & -3.67-2303.20 & 330.50 \\ 1.4 & -7.6-2160.75 & 2.10 & 0.84 & -4.60-2162.41 & 309.05\end{array}$ $\begin{array}{llllll}1.3 & -7.5-2168.30 & 2.42 & 0.00 & -4.55-2170.43 & 309.10\end{array}$ $\begin{array}{llllll}1.1 & -7.4-2175.75 & -7.71 & 0.83 & -4.50-2187.13 & 300.47\end{array}$ $\begin{array}{llllll}1.0 & -7.3-2183.11 & -7.32 & 0.00 & -4.45-2194.88 & 300.79\end{array}$ $\begin{array}{lllllll}0.8 & -7.2-2190.36 & -7.07 & 0.83 & -4.41-2201.01 & 302.74\end{array}$ $\begin{array}{llllll}0.6 & -7.1-2197.51 & -6.61 & 0.00 & -4.36-2208.48 & 303.33\end{array}$ $\begin{array}{llllll}0.5 & -7.0-2204.57 & -9.49 & 0.83 & -4.32-2217.54 & 302.34\end{array}$ $\begin{array}{lllllll}0.3 & -6.9-2211.52 & -9.41 & 0.00 & -4.27-2225.21 & 302.75\end{array}$ $\begin{array}{lllllll}0.2 & -6.8-2218.39 & -9.68 & 0.82 & -4.23-2231.47 & 304.56\end{array}$
0.00

0.00

0.00

0.00

0.00

0.00

0.00

0.00

0.00

0.00

0.00

0.00

0.00

0.00

0.00

0.00

0.00

0.00

0.00

0.00

0.00

0.00

0.00

0.00

0.00

0.00

0.00

0.00

0.00

0.00

0.00

0.00

0.00

0.00

0.00

0.00

0.00

0.00

0.00

0.00

0.00

0.00

0.00

0.00

0.00

0.00

0.00

0.00

0.00

0.00

0.00

0.00

0.00

0.00

0.00

0.00

0.00

0.00

0.00

0.00

0.00

0.00

0.00

0.00

0.00

0.00
0.00

0.00

0.00

0.00

0.00

0.00

0.00

0.00

0.00

0.00

0.00

0.00

0.00

0.00

0.00

0.00

0.00

0.00

0.00

0.00

0.00

0.00

0.00

0.00

0.00

0.00

0.00

0.00

0.00

0.00

0.00

0.00

0.00

0.00

0.00

0.00

0.00

0.00

0.00

0.00

0.00

0.00

0.00

0.00

0.00

0.00

0.00

0.00

0.00

0.00

0.00

0.00

0.00

0.00

0.00

0.00

0.00

0.00

0.00

0.00

0.00

0.00

0.00

0.00

0.00

0.00

0.00

0.00

0.00

0.00

0.00

0.00

0.00

0.00

0.00

0.00

0.00

0.00

0.00

0.00

0.00

0.00

0.00

0.00

0.00

0.00

0.00

0.00

0.00

0.00

0.00

0.00

0.00

0.00

0.00

0.00

0.00

0.00

0.00

0.00

0.00

0.00

0.00

0.00

0.00

0.00

0.00

0.00

0.00

0.00

0.00

0.00

0.00

0.00

0.00

0.00

0.00

0.00

0.00

0.00

0.00

0.00

0.00 
$\begin{array}{lll}130 & 198 & 328\end{array}$ $\begin{array}{lll}130 & 199 & 329\end{array}$ $130200 \quad 330$ 130201331 $\begin{array}{lll}130 & 202 & 332\end{array}$ $130203 \quad 333$ 130204334 130205335 130206336 130207337 130208338 130209339 $\begin{array}{lll}131 & 192 & 323\end{array}$ $\begin{array}{lll}131 & 193 & 324\end{array}$ $\begin{array}{lll}131 & 194 & 325\end{array}$ $\begin{array}{lll}131 & 195 & 326\end{array}$ $\begin{array}{lll}131 & 196 & 327\end{array}$ $\begin{array}{lll}131 & 197 & 328\end{array}$ $\begin{array}{lll}131 & 198 & 329\end{array}$ $\begin{array}{lll}131 & 199 & 330\end{array}$ $\begin{array}{lll}131 & 200 & 3.31\end{array}$ $\begin{array}{lll}131 & 201 & 332\end{array}$ 131202333 $\begin{array}{lll}131 & 203 \quad 334\end{array}$ 131204335 $\begin{array}{llll}131 & 205 & 336\end{array}$ 131206337 131207338 $\begin{array}{lll}131 & 208 & 339\end{array}$ $\begin{array}{lll}132 & 194 & 326\end{array}$ $\begin{array}{lll}132 & 195 & 327\end{array}$ $\begin{array}{lll}132 & 196 & 328\end{array}$ $\begin{array}{lll}132 & 197 & 329\end{array}$ $\begin{array}{lll}132 & 198 & 330\end{array}$ $\begin{array}{lll}132 & 199 & 331\end{array}$ $\begin{array}{lll}132 & 200 & 332\end{array}$ $\begin{array}{lll}132 & 201 & 333\end{array}$ $\begin{array}{lll}132 & 202 & 334\end{array}$ $\begin{array}{lll}132 & 203 \quad 335\end{array}$ $132 \quad 204336$ $\begin{array}{lll}132 & 205 & 337\end{array}$ 132206338 $132 \quad 207339$ $\begin{array}{lll}133 & 196 & 329\end{array}$ $\begin{array}{lll}133 & 197 & 330\end{array}$ $\begin{array}{lll}133 & 198 & 331\end{array}$ $\begin{array}{lll}133 & 199 & 332\end{array}$ $\begin{array}{lll}133 & 200 & 333\end{array}$ $\begin{array}{lll}133 & 201 & 334\end{array}$ $\begin{array}{lll}133 & 202 & 335\end{array}$ $\begin{array}{lll}133 & 203 \quad 336\end{array}$ $\begin{array}{lll}133 & 204 & 337\end{array}$ $\begin{array}{llll}133 & 205 & 338\end{array}$ $\begin{array}{lll}133 & 206 & 339\end{array}$ $\begin{array}{lll}134 & 198 & 332\end{array}$ $\begin{array}{lll}134 & 199 & 333\end{array}$ $134 \quad 200 \quad 334$ 134201335 $134 \quad 202 \quad 336$ $\begin{array}{lll}134 & 203 & 337\end{array}$ 134204338

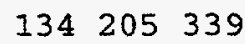
$\begin{array}{lll}135 & 201 & 336\end{array}$ $\begin{array}{lll}135 & 202 & 337\end{array}$ $\begin{array}{lll}135 & 203 & 338\end{array}$ $135 \quad 204339$
$0.0-6.7-2225.15-9.55 \quad 0.00-4.19-2238.89 \quad 305.21$ $\begin{array}{lllllll}-0.1 & -6.6-2231.82 & -9.76 & 0.82 & -4.14-2244.90 & 307.27\end{array}$ $\begin{array}{lllllll}-0.3 & -6.5-2238.40 & -9.57 & 0.00 & -4.10-2252.07 & 308.17\end{array}$ $\begin{array}{lllllll}-0.4 & -6.4-2244.89 & -4.22 & 0.82 & -4.06-2252.35 & 315.97\end{array}$ $\begin{array}{lllllll}-0.6 & -6.3-2251.28 & -9.24 & 0.00 & -4.02-2264.54 & 311.85\end{array}$ $\begin{array}{lllllll}-0.7 & -6.3-2257.58 & -4.26 & 0.82 & -3.98-2265.01 & 319.45\end{array}$ $\begin{array}{lllllll}-0.9 & -6.2-2263.79 & -3.96 & 0.00 & -3.94-2271.70 & 320.83\end{array}$ $\begin{array}{lllllll}-1.0 & -6.1-2269.91 & -3.90 & 0.81 & -3.91-2276.91 & 323.70\end{array}$ $\begin{array}{lllllll}-1.2 & -6.0-2275.95 & -3.56 & 0.00 & -3.87-2283.38 & 325.30\end{array}$ $\begin{array}{lllllll}-1.3 & -5.9-2281.89 & -3.69 & 0.81 & -3.83-2288.60 & 328.14\end{array}$ $\begin{array}{llllllll}-1.5 & -5.8-2287.75 & -3.40 & 0.00 & -3.79-2294.95 & 329.87\end{array}$ $\begin{array}{lllllll}-1.6 & -5.7-2293.53 & -3.19 & 0.81 & -3.7 .6-2299.66 & 333.22\end{array}$ $1.3-7.5-2181.98-7.70 \quad 0.95-4.52-2193.26 \quad 309.67$ $1.1-7.4-2189.39-7.39 \quad 1.64-4.48-2199.62 \quad 311.38$ $1.0-7.3-2196.70-6.89 \quad 0.95-4.43-2207.07 \quad 312.00$ $0.8-7.2-2203.91-10.07 \quad 1.63-4.38-2216.73 \quad 310.42$ $0.7-7.1-2211.02-9.99 \quad 0.95-4.34-2224.41310 .81$ $0.5-7.0-2218.04-10.27 \quad 1.63-4.30-2230.97 \quad 312.32$ $0.4-6.9-2224.96-10.15 \quad 0.95-4.25-2238.41312 .95$ $0.2-6.8-2231.78-10.361 .63-4.21-2244.72314 .71$ $0.1-6.7-2238.51-10.18 \quad 0.95-4.17-2251.91315 .59$ $-0.1-6.6-2245.14-10.21 \quad 1.63-4.12-2257.85 \quad 317.72$ $\begin{array}{llllll}-0.2 & -6.5-2251.69 & -9.87 & 0.95 & -4.08-2264.70 & 318.95\end{array}$ $-0.4-6.4-2258.14-4.64 \quad 1.62-4.04-2265.20 \quad 326.52$ $\begin{array}{lllllll}-0.5 & -6.3-2264.50 & -4.26 & 0.95 & -4.00-2271.82 & 327.97\end{array}$ $\begin{array}{llllll}-0.7 & -6.2-2270.77 & -4.29 & 1.62 & -3.97-2277.40 & 330.46\end{array}$ $-0.8-6.1-2276.95-4.38 \quad 0.95-3.93-2284.31331 .62$ $-1.0-6.0-2283.04-4.301 .62-3.89-2289.61334 .39$ $\begin{array}{lllllll}-1.1 & -6.0-2289.05 & -3.95 & 0.95 & -3.85-2295.91 & 336.17\end{array}$ $1.3-7.4-2195.54-6.79 \quad 0.00-4.50-2206.83 \quad 319.50$ $1.2-7.3-2202.91-10.390 .83-4.45-2216.92 \quad 317.48$ $1.0-7.2-2210.17-10.30 \quad 0.00-4.41-2224.88 \quad 317.60$ $0.9-7.1-2217.34-10.58 \quad 0.82 \quad-4.36-2231.46 \quad 319.09$ $0.7-7.0-2224.41-10.46 \quad 0.00-4.32-2239.19 \quad 319.43$ $0.6-6.9-2231.39-10.68 \quad 0.82-4.27-2245.52 \quad 321.17$ $0.4-6.8-2238.27-10.50 \quad 0.00-4.23-2253.00 \quad 321.76$ $\begin{array}{lllllll}0.3 & -6.7-2245.06 & -4.70 & 0.82 & -4.19-2253.13 & 329.71\end{array}$ $0.1-6.6-2251.75-4.60 \quad 0.00-4.15-2260.50 \quad 330.41$ $0.0-6.6-2258.35-4.80 \quad 0.82-4.11-2266.44 \quad 332.54$ $\begin{array}{lllllll}-0.2 & -6.5-2264.86 & -4.38 & 0.00 & -4.07-2273.31 & 333.74\end{array}$ $\begin{array}{lllllll}-0.3 & -6.4-2271.28 & -4.36 & 0.81 & -4.03-2278.85 & 336.27\end{array}$ $\begin{array}{llllll}-0.5 & -6.3-2277.61 & -4.03 & 0.00 & -3.99-2285.63 & 337.56\end{array}$ $\begin{array}{lllllll}-0.6 & -6.2-2283.85 & -4.55 & 0.81 & -3.95-2291.54 & 339.73\end{array}$ $1.4-7.4-2208.98-10.92 \quad 0.94-4.47-2223.43 \quad 326.30$ $1.2-7.3-2216.30-11.191 .63-4.43-2230.29327 .51$ $1.1-7.2-2223.53-11.08 \quad 0.94-4.38-2238.05 \quad 327.83$ $0.9-7.1-2230.65-11.30 \quad 1.62-4.34-2244.67 \quad 329.28$ $\begin{array}{lllllll}0.8 & -7.0-2237.69 & -5.68 & 0.94 & -4.30-2246.72 & 335.30\end{array}$ $0.6-6.9-2244.62-5.08 \quad 1.62-4.25-2252.33 \quad 337.76$ $\begin{array}{lllllll}0.5 & -6.8-2251.47 & -4.87 & 0.94 & -4.21-2259.61 & 338.56\end{array}$ $0.3-6.7-2258.22-5.20 \quad 1.62-4.17-2265.97 \quad 340.27$ $0.2-6.6-2264.88-4.92 \quad 0.94-4.13-2272.98 \quad 341.32$ $0.0-6.5-2271.44-4.78 \quad 1.62-4.09-2278.69 \quad 343.68$ $\begin{array}{llllll}-0.1 & -6.4-2277.92 & -5.26 & 0.94 & -4.05-2286.29 & 344.16\end{array}$ $1.4-7.3-2222.29-6.14 \quad 0.00-4.45-2232.89 \quad 340.25$ $1.3-7.2-2229.57-6.06 \quad 0.82 \quad-4.41-2239.22 \quad 341.99$ $1.1-7.1-2236.76-5.81 \quad 0.00-4.36-2246.93 \quad 342.35$ $1.0-7.0-2243.85-5.17 \quad 0.82 \quad-4.32-2252.52 \quad 344.83$ $0.8-6.9-2250.84-5.15 \quad 0.00-4.27-2260.27 \quad 345.16$ $0.7-6.8-2257.74-5.34 \quad 0.82-4.23-2266.50 \quad 347.00$ $0.5-6.8-2264.55-5.10 \quad 0.00-4.19-2273.84 \quad 347.73$ $\begin{array}{lllllll}0.4 & -6.7-2271.26 & -5.12 & 0.81 & -4.15-2279.72 & 349.92\end{array}$ $1.3-7.2-2242.73-5.61 \quad 1.62-4.38-2251.10 \quad 353.50$ $\begin{array}{lllllll}1.1 & -7.1-2249.87 & -5.59 & 0.94 & -4.34 & -2258.87 & 353.81\end{array}$ $1.0-7.0-2256.92-5.84 \quad 1.62-4.30-2265.44 \quad 355.31$ $\begin{array}{lllllll}0.8 & -6.9-2263.88 & -5.59 & 0.94 & -4.25-2272.79 & 356.03\end{array}$ 140
0.00

0.00

0.00

0.00

0.00

0.00

0.00

0.00

0.00

0.00

0.00

0.00

0.00

0.00

0.00

0.00

0.00

0.00

0.00

0.00

0.00

0.00

0.00

0.00

0.00

0.00

0.00

0.00

0.00

0.00

0.00

0.00

0.00

0.00

0.00

0.00

0.00

0.00

0.00

0.00

0.00

0.00

0.00

0.00

0.00

0.00

0.00

0.00

0.00

0.00

0.00

0.00

0.00

0.00

0.00

0.00

0.00

0.00

0.00

0.00

0.00

0.00

0.00

0.00

0.00

0.00
0.00

$0.00 \quad 0.00$

$0.00 \quad 0.00$

$0.00 \quad 0.00$

$0.00 \quad 0.00$

$0.00 \quad 0.00$

$0.00 \quad 0.00$

$0.00 \quad 0.00$

$0.00 \quad 0.00$

$0.00 \quad 0.00$

$0.00 \quad 0.00$

$0.00 \quad 0.00$

$0.00 \quad 0.00$

$0.00 \quad 0.00$

$0.00 \quad 0.00$

$0.00 \quad 0.00$

$0.00 \quad 0.00$

$0.00 \quad 0.00$

$0.00 \quad 0.00$

$0.00 \quad 0.00$

$0.00 \quad 0.00$

$0.00 \quad 0.00$

$0.00 \quad 0.00$

$0.00 \quad 0.00$

$0.00 \quad 0.00$

$0.00 \quad 0.00$

$0.00 \quad 0.00$

$0.00 \quad 0.00$

$0.00 \quad 0.00$

$0.00 \quad 0.00$

$0.00 \quad 0.00$

$0.00 \quad 0.00$

$0.00 \quad 0.00$

$0.00 \quad 0.00$

$0.00 \quad 0.00$

$0.00 \quad 0.00$

$0.00 \quad 0.00$

$0.00 \quad 0.00$

$0.00 \quad 0.00$

$0.00 \quad 0.00$

$0.00 \quad 0.00$

$0.00 \quad 0.00$

$0.00 \quad 0.00$

$0.00 \quad 0.00$

$0.00 \quad 0.00$

$0.00 \quad 0.00$

$0.00 \quad 0.00$

$0.00 \quad 0.00$

$0.00 \quad 0.00$

$0.00 \quad 0.00$

$0.00 \quad 0.00$

$0.00 \quad 0.00$

$0.00 \quad 0.00$

$0.00 \quad 0.00$

$0.00 \quad 0.00$

$0.00 \quad 0.00$

$0.00 \quad 0.00$

$0.00 \quad 0.00$

$0.00 \quad 0.00$

$0.00 \quad 0.00$

$0.00 \quad 0.00$

$0.00 \quad 0.00$

$0.00 \quad 0.00$

$0.00 \quad 0.00$

$0.00 \quad 0.00$

$0.00 \quad 0.00$ 